\title{
S
}

598.29

PN1112BD P. SKAAR'S

Montana

Bira Distribution

Seventh Edition

2012

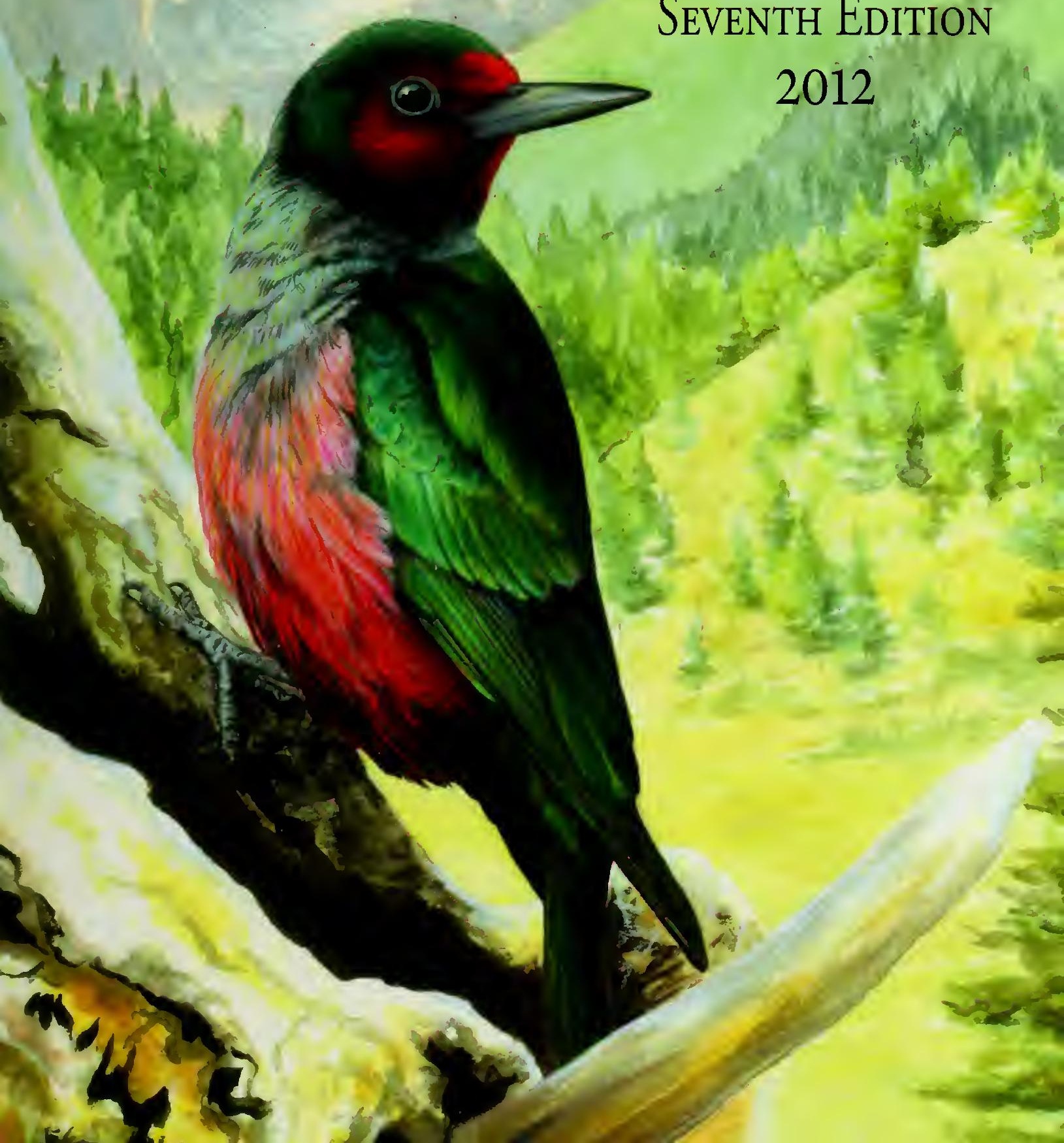




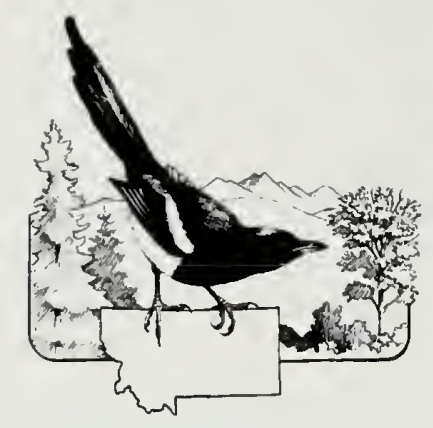

Montana Audubon
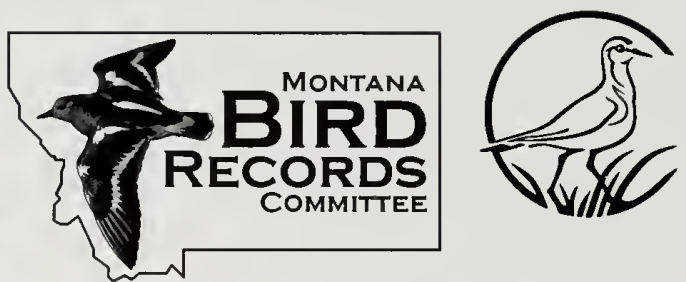

30864100622698

Natural Heritage Program

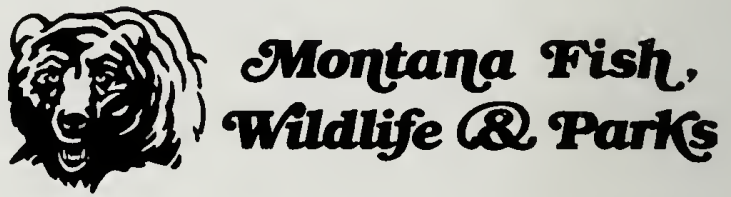

\section{Thank You}

The following businesses, organizations, and agencies contributed significantly to this book. We are pleased to display their logos in appreciation of their support.
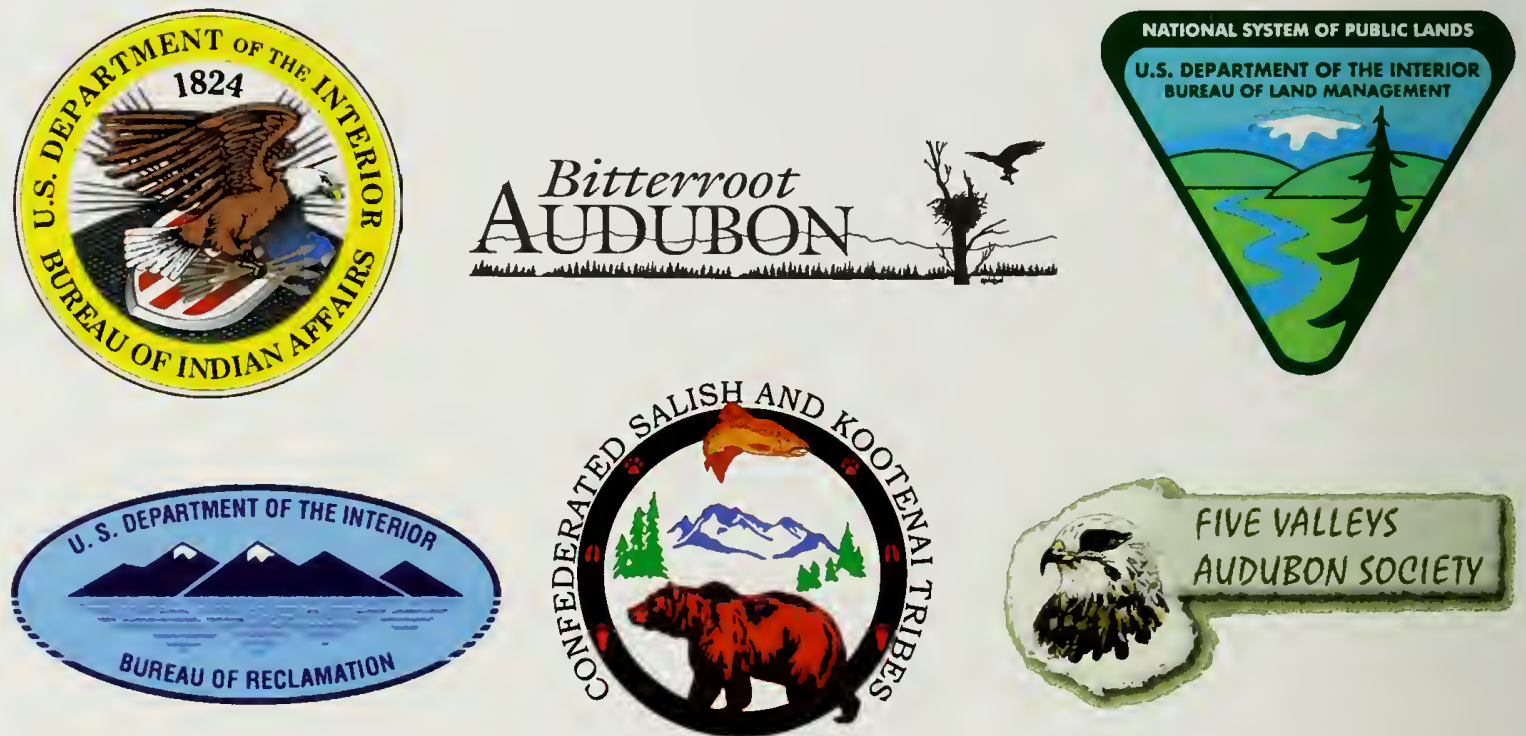

DIVISION OF FISH, WILDLIFE, RECREATION \& CONSERVATION
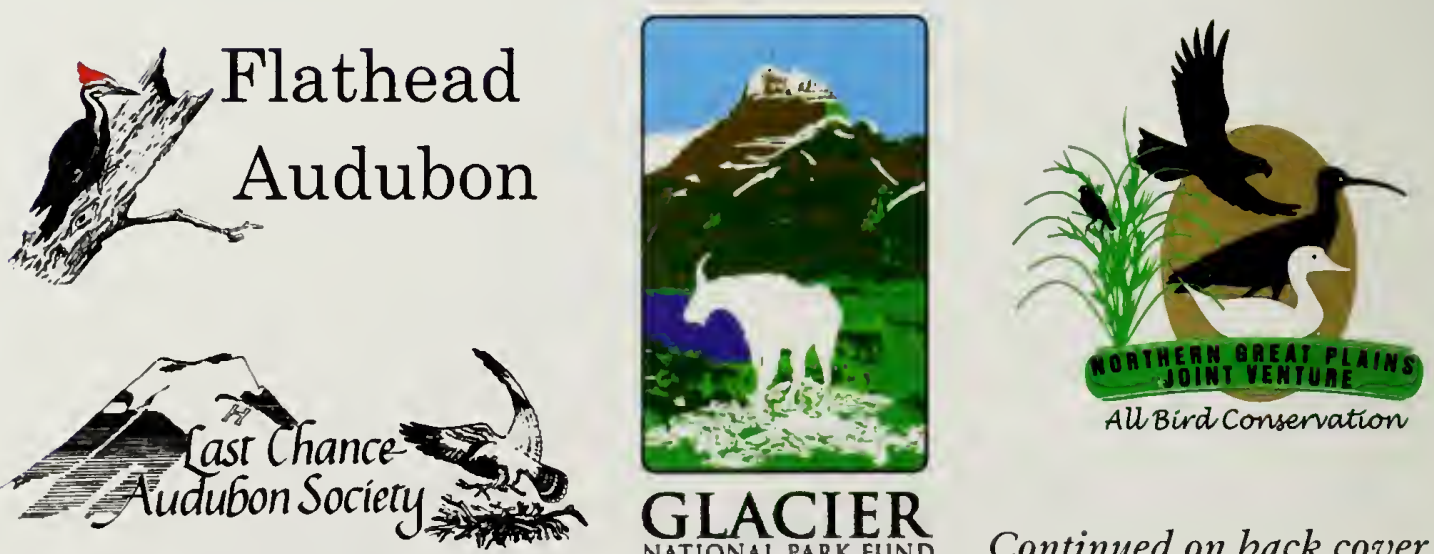


\section{CHECKLIST OF MONTANA BIRDS}
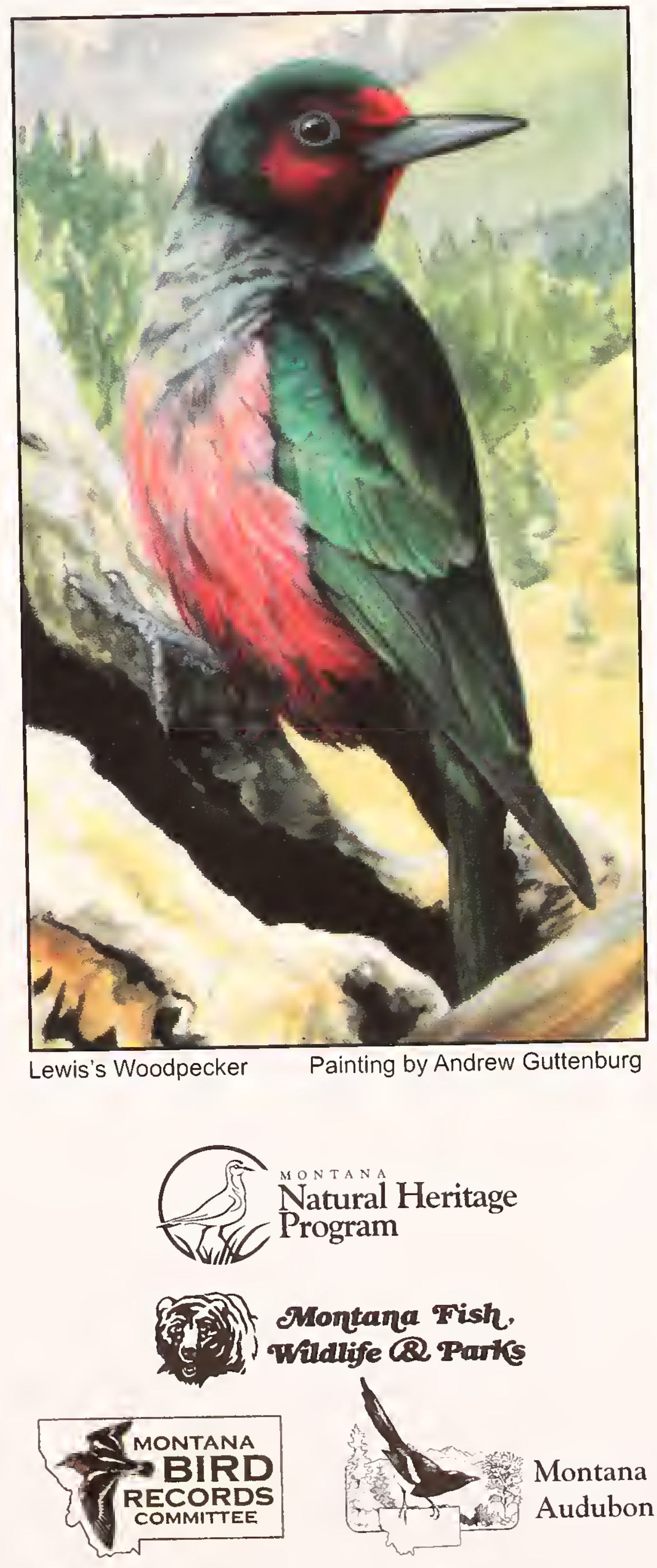


\section{Bird Species Observed in}

\section{Montana through April 2012}

Notes: $\begin{aligned} \mathrm{B} & =\text { Verified breeding records for MT } \\ \mathrm{b} & =\text { Indirect evidence of breeding for MT }\end{aligned}$ $\begin{aligned} b & =\text { Indirect evidence of breeding } \mathrm{MT} \text {, transient } \\ & =\text { No evidence of breeding for } \mathrm{M} \text {, }\end{aligned}$ $W=$ Verified overwinter records for $M T$ $(12 / 15-2 / 15)$

$w=$ Present during winter season

Species with fewer than 20 accepted records are listed in italic 1 - Greater White-fronted Goose t w

2 - Snow Goose

4 - Cackling Goose

5 - Canada Goose

- Brant

8 - Trumpeter Swan

9 - Tundra Swan

11 - Gadwall

12 _Eurasian Wigeon

- American Wigeon

_ Mallard

16 - Blue-winged Teal

- Cinnamon Teal

19 - Northern Pintail

20 - Garganey

21 - Green-winged Teal

22 - Canvasback

24 - Ring-necked Duck

25 - Tufted Duck

26 - Greater Scaup

27 - Lesser Scaup

28 - Harlequin Duck

30 - Surf Scoter

31 - Black Scoter

32 二Long-tailed Duck

34 - Common Goldeneye

35 - Barrow's Goldeneye

36 - Hooded Merganser

38 - Red-breasted Merganser

39 - Ruddy Duck

40 - Chukar

42 - Ring-necked Pheasan

43 _ Ruffed Grouse

44 - Greater Sage-Grouse

45 - Spruce Grouse

47 - White-tailed Ptarmiga

48 - Dusky Grouse

49 - Sharp-tailed Grouse

50 - Greater Prairie-Chicken

52 - Red-throated

53 _- Pacific Loon

w
54 _ Common Loon 119 - Willet 56 - Pied-billed Greb

77 - Horned Grebe

59 - Eared Grebe

60 - Western Grebe

21 - Upland Sandpiper

122 - Whimbrel

124 — Long-billed Curlew

125 - Marbled Godwit

- Manx Shearw

126 - Ruddy Turnstone

128 - Red Knot

64 - Double-crested Cormorant B w

65 - American White Pelican

67 - American Bitter

68 - Least Biltern

129 - Sanderling

130 - Semipalmated Sandpipe

131 _ Western Sandpipe

133 — White-rumped Sandpiper

134 - Baird's Sandpiper

70 - Snowy Egret

71 - Little Blue Heron

72 - Cattle Egret

74 - Black-crowned Night-Heron

75 Yellow-crowned Night-Heron

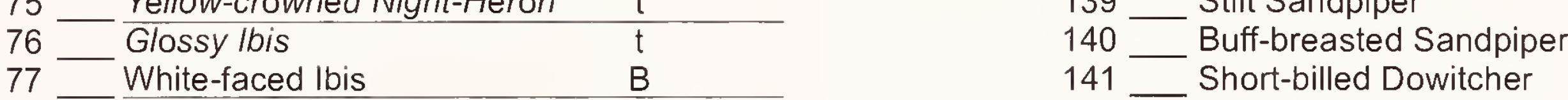

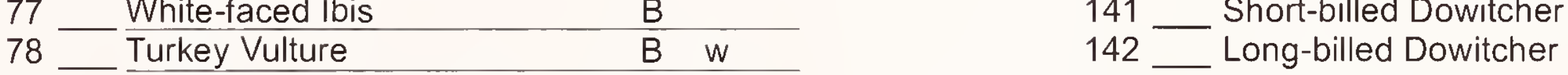

79 - Osprey

80 - White-tailed Kite

81 - Mississippi Kite

83 - Northern Harrier

84 - Sharp-shinned Hawk

85 - Cooper's Hawk

- Bed-shoushawk W

88 - Broad-winged Hawk

89 - Swainson's Hawk

90 — Red-tailed Hawk

91 - Ferruginous Hawk

93 - Golden Eagle

95 - American Kestrel

96 - Merlin

97 - Gyrfalcon

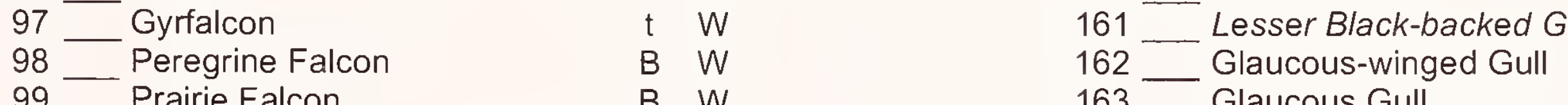

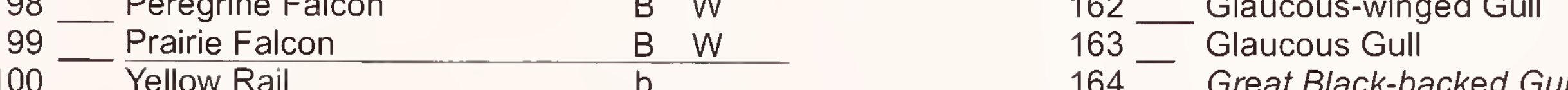

101 - Virginia Rail

102 - Sora

103 - Common Gallinule B $\quad$ B w

104 - American Coot

106 - Whooping Crane

107 - Black-bellied Plover

108 - American Golden-Plove

109 - Snowy Plover

110 - Semipalmated Plove

111 _ Piping Plover

113 - Mountain Plover

114 - Black-necked Stilt

115 - American Avocet

117 - Solitary Sandpiper

144 - American Woodcock

145 — Wilson's Phalarope

${ }_{147}^{146}$ — Red-necked Phalarope

148 - Black-legged Kittiwake

150 - Bonaparte's Gull

151 — Little Gull

153 _ Laughing Gull

155 - Franklin's

156 - Ring-billed Gull

157 _ California Gul

158 _ Herring Gull

160 - Iceland Gull

165 - $\frac{\text { Great Black-bach }}{165}$

166 _ Caspian Tern

167 - Black Tern

168 - Common Te

169 - Arctic Tern

171 - $\frac{\text { Forster's Tern }}{\text { Pomarine Jaeger }}$

172 - Parasitic Jaeger

173 - Long-tailed Jaeger

175 - Ancient Murrelet

177 - Rock Pigeon

178 - Eurasian Collared-Dove

179 - White-winged Dove

181 - Inca Dove 


\begin{tabular}{|c|c|c|c|}
\hline 246 & Scissor-tailed Flycatcher & $\mathrm{t}$ & \\
\hline 247 & Loggerhead Shrike & B & W \\
\hline 248 & Northern Shrike & $t$ & W \\
\hline 249 & White-eyed Vireo & $\mathrm{b}$ & \\
\hline 250 & Yellow-throated Vireo & $\mathrm{b}$ & \\
\hline 251 & Plumbeous Vireo & B & \\
\hline 252 & Cassin's Vireo & B & \\
\hline 253 & Blue-headed Vireo & $\mathrm{t}$ & \\
\hline 254 & Warbling Vireo & B & \\
\hline 255 & Philadelphia Vireo & $\mathrm{t}$ & \\
\hline 256 & Red-eyed Vireo & B & \\
\hline 257 & Gray Jay & B & W \\
\hline 258 & Pinyon Jay & B & W \\
\hline 259 & Steller's Jay & B & W \\
\hline 260 & Blue Jay & B & W \\
\hline 261 & Western Scrub-Jay & $t$ & W \\
\hline 262 & Clark's Nutcracker & B & W \\
\hline 263 & Black-billed Magpie & B & W \\
\hline 264 & American Crow & B & W \\
\hline 265 & Common Raven & B & W \\
\hline 266 & Horned Lark & B & W \\
\hline 267 & Purple Martin & $B$ & \\
\hline 268 & Tree Swallow & B & \\
\hline 269 & Violet-green Swallow & B & \\
\hline 270 & Northern Rough-winged Swallow & B & \\
\hline 271 & Bank Swallow & B & \\
\hline 272 & Cliff Swallow & B & \\
\hline 273 & Barn Swallow & B & \\
\hline 274 & Black-capped Chickadee & $B$ & W \\
\hline $275^{-}$ & Mountain Chickadee & $B$ & W \\
\hline 276 & Chestnut-sided Chickadee & B & W \\
\hline 277 & Boreal Chickadee & B & W \\
\hline 278 & Red-breasted Nuthatch & $B$ & W \\
\hline 279 & White-breasted Nuthatch & B & W \\
\hline 280 & Pygmy Nuthatch & $B$ & W \\
\hline 281 & Brown Creeper & $B$ & W \\
\hline 282 & Rock Wren & B & $w$ \\
\hline 283 & Canyon Wren & B & W \\
\hline 284 & Carolina Wren & $\mathrm{t}$ & \\
\hline 285 & Bewick's Wren & $t$ & W \\
\hline 286 & House Wren & B & w \\
\hline 287 & Pacific Wren & B & W \\
\hline 288 & Winter Wren & $t$ & \\
\hline 289 & Sedge Wren & B & \\
\hline 290 & Marsh Wren & B & W \\
\hline 291 & Blue-gray Gnatcatcher & B & \\
\hline 292 & American Dipper & B & W \\
\hline 293 & Golden-crowned Kinglet & B & W \\
\hline 294 & Ruby-crowned Kinglet & B & W \\
\hline 295 & Eastern Bluebird & B & W \\
\hline 296 & Western Bluebird & B & w \\
\hline 297 & Mountain Bluebird & B & w \\
\hline 298 & Townsend's Solitaire & $B$ & W \\
\hline 299 & Veery & B & \\
\hline 300 & Gray-cheeked Thrush & $t$ & \\
\hline 301 & Swainson's Thrush & B & \\
\hline 302 & Hermit Thrush & B & w \\
\hline 303 & Wood Thrush & $t$ & \\
\hline 304 & American Robin & B & W \\
\hline 305 & Varied Thrush & B & W \\
\hline 306 & Gray Catbird & $B$ & \\
\hline 307 & Northern Mockingbird & B & W \\
\hline 308 & Sage Thrasher & B & \\
\hline 309 & Brown Thrasher & $B$ & w \\
\hline
\end{tabular}

\begin{tabular}{|c|c|c|c|}
\hline 310 & Curve-billed Thrasher & $\mathrm{t}$ & W \\
\hline 311 & European Starling & $B$ & W \\
\hline 312 & Siberian Accentor & $\mathrm{t}$ & W \\
\hline 313 & American Pipit & $B$ & W \\
\hline 314 & Sprague's Pipit & B & \\
\hline 315 & Bohemian Waxwing & $\mathrm{b}$ & W \\
\hline 316 & Cedar Waxwing & $B$ & W \\
\hline 317 & Lapland Longspur & $\mathrm{t}$ & W \\
\hline 318 & Chestnut-collared Longspur & B & \\
\hline 319 & Smith's Longspur & $\mathrm{t}$ & \\
\hline 320 & McCown's Longspur & B & \\
\hline 321 & Snow Bunting & $\mathrm{t}$ & W \\
\hline 322 & Ovenbird & $B$ & \\
\hline 323 & Northern Waterthrush & $B$ & \\
\hline 324 & Golden-winged Warbler & $\mathrm{t}$ & \\
\hline 325 & Blue-winged Warbler & $\mathrm{t}$ & \\
\hline 326 & Black-and-White Warbler & B & \\
\hline 327 & Prothonotary Warbler & $\mathrm{t}$ & \\
\hline 328 & Tennessee Warbler & B & \\
\hline 329 & Orange-crowned Warbler & B & \\
\hline 330 & Nashville Warbler & B & \\
\hline 331 & Connecticut Warbler & $\mathrm{t}$ & \\
\hline 332 & MacGillivray's Warbler & B & \\
\hline 333 & Mourning Warbler & $\mathrm{t}$ & \\
\hline 334 & Kentucky Warbler & $\mathrm{t}$ & \\
\hline 335 & Common Yellowthroat & B & w \\
\hline 336 & Hooded Warbler & $\mathrm{t}$ & \\
\hline 337 & American Redstart & B & \\
\hline 338 & Cape May Warbler & $\mathrm{t}$ & \\
\hline 339 & Northern Parula & $\mathrm{t}$ & \\
\hline 340 & Magnolia Warbler & $\mathrm{t}$ & \\
\hline 341 & Bay-breasted Warbler & $\mathrm{t}$ & \\
\hline 342 & Blackburnian Warbler & $\mathrm{t}$ & \\
\hline 343 & Yellow Warbler & B & \\
\hline 344 & Chestnut-sided Warbler & $\mathrm{t}$ & \\
\hline 345 & Blackpoll Warbler & $\mathrm{t}$ & \\
\hline 346 & Black-throated Blue Warbler & $\mathrm{t}$ & w \\
\hline 347 & Palm Warbler & $\mathrm{t}$ & w \\
\hline 348 & Pine Warbler & $\mathrm{t}$ & \\
\hline 349 & Yellow-rumped Warbler & B & W \\
\hline 350 & Yellow-throated Warbler & $\mathrm{t}$ & W \\
\hline 351 & Prairie Warbler & $t$ & \\
\hline 352 & Black-throated Gray Warbler & B & w \\
\hline 353 & Townsend's Warbler & B & \\
\hline 354 & Black-throated Green Warbler & $\mathrm{t}$ & \\
\hline 355 & Canada Warbler & $\mathrm{t}$ & \\
\hline 356 & Wilson's Warbler & B & \\
\hline 357 & Painted Redstart & $\mathrm{t}$ & \\
\hline 358 & Yellow-breasted Chat & B & \\
\hline 359 & Green-tailed Towhee & $B$ & $\mathrm{~W}$ \\
\hline 360 & Spotted Towhee & B & W \\
\hline 361 & Eastern Towhee & $\mathrm{t}$ & \\
\hline 362 & American Tree Sparrow & $\mathrm{t}$ & W \\
\hline 363 & Chipping Sparrow & B & W \\
\hline 364 & Clay-colored Sparrow & B & \\
\hline 365 & Brewer's Sparrow & B & \\
\hline 366 & Field Sparrow & $B$ & \\
\hline 367 & Vesper Sparrow & $B$ & w \\
\hline 368 & Lark Sparrow & B & \\
\hline 369 & Black-throated Sparrow & $\mathrm{t}$ & \\
\hline 370 & Sage Sparrow & B & \\
\hline 371 & Lark Bunting & $B$ & \\
\hline 372 & Savannah Sparrow & B & w \\
\hline 373 & Grasshopper Sparrow & B & \\
\hline
\end{tabular}

\begin{tabular}{|c|c|c|c|}
\hline 374 & Baird's Sparrow & B & \\
\hline 375 & Le Conte's Sparrow & $\mathrm{B}$ & w \\
\hline 376 & Nelson's Sparrow & $\mathrm{B}$ & \\
\hline 377 & Fox Sparrow & B & w \\
\hline 378 & Song Sparrow & B & W \\
\hline 379 & Lincoln's Sparrow & B & w \\
\hline 380 & Swamp Sparrow & $\mathrm{t}$ & W \\
\hline 381 & White-throated Sparrow & $\mathrm{t}$ & W \\
\hline 382 & Harris's Sparrow & $\mathrm{t}$ & W \\
\hline 383 & White-crowned Sparrow & B & W \\
\hline 384 & Golden-crowned Sparrow & $\mathrm{b}$ & w \\
\hline 385 & Dark-eyed Junco & B & W \\
\hline 386 & Summer Tanager & $\mathrm{t}$ & \\
\hline 387 & Scarlet Tanager & $\mathrm{t}$ & \\
\hline 388 & Western Tanager & B & \\
\hline 389 & Northern Cardinal & $\mathrm{t}$ & W \\
\hline 390 & Pyrrhuloxia & $\mathrm{t}$ & \\
\hline 391 & Rose-breasted Grosbeak & B & w \\
\hline 392 & Black-headed Grosbeak & B & W \\
\hline 393 & Blue Grosbeak & B & \\
\hline 394 & Lazuli Bunting & B & \\
\hline 395 & Indigo Bunting & $\mathrm{b}$ & \\
\hline 396 & Painted Bunting & $\mathrm{t}$ & w \\
\hline 397 & Dickcissel & B & \\
\hline 398 & Bobolink & $B$ & \\
\hline 399 & Red-winged Blackbird & B & W \\
\hline 400 & Eastern Meadowlark & $\mathrm{t}$ & \\
\hline 401 & Western Meadowlark & B & W \\
\hline 402 & Yellow-headed Blackbird & B & W \\
\hline 403 & Rusty Blackbird & $\mathrm{t}$ & W \\
\hline 404 & Brewer's Blackbird & B & W \\
\hline 405 & Common Grackle & B & W \\
\hline 406 & Great-tailed Grackle & $\mathrm{b}$ & \\
\hline 407 & Brown-headed Cowbird & B & w \\
\hline 408 & Orchard Oriole & B & \\
\hline 409 & Hooded Oriole & $\mathrm{t}$ & \\
\hline 410 & Bullock's Oriole & B & w \\
\hline 411 & Baltimore Oriole & B & \\
\hline 412 & Brambling & $\mathrm{t}$ & W \\
\hline 413 & Gray-crowned Rosy-Finch & B & W \\
\hline 414 & Black Rosy-Finch & B & W \\
\hline 415 & Pine Grosbeak & B & W \\
\hline 416 & Purple Finch & $t$ & W \\
\hline 417 & Cassin's Finch & B & W \\
\hline 418 & House Finch & B & W \\
\hline 419 & Red Crossbill & B & W \\
\hline 420 & White-winged Crossbill & $\mathrm{b}$ & W \\
\hline 421 & Common Redpoll & $\mathrm{t}$ & W \\
\hline 422 & Hoary Redpoll & $\mathrm{t}$ & W \\
\hline 423 & Pine Siskin & B & W \\
\hline 424 & Lesser Goldfinch & B & w \\
\hline 425 & American Goldfinch & B & W \\
\hline 42 & Evening Grosbeak & B & W \\
\hline+27 & & $\mathrm{~B}$ & W \\
\hline
\end{tabular}

This checklist is based on the Montana Bird Records Committee official list, available online at: $h$ ttp://mtaudubon.org/birds/ mbrc.html and the Montana Bird Distribution Database, also available online at: http://mtnhp.org/tracker. This list can lo be nongameCheckoff/birds.html and http://www.mtnhp. org/animal/. 


\section{MT Bird Distribution}

This edition of P.D. Skaar's Montana

1 Bird Distribution presents the most current information available on the distribution of birds in Montana, representing hundreds of thousands of observations reported by thousands of individuals across the state.

\section{This edition:}

- Presents breeding and wintering maps for 427 bird species.

- Introduces maps for 18 new species.

- Provides a state check-list of all species included in this edition in taxonomic order.

- Displays current, previous edition, and historical information on each breeding season map.

- Presents a list of rare birds for Montana (those species reported fewer than 20 times).

- Includes new travel maps to aid field observations.

To submit observations, investigate the records used to generate the maps in this book, and contribute to a better understanding of the birds of Montana, log online:

http://mtnhp.org/observations.asp.

\section{This book was produced by:}

Montana Audubon

Montana Bird Records Committee

Montana Department of Fish, Wildlife \& Parks

Montana Natural Heritage Program 


\title{
P. D. Skaar's \\ Montana Bird Distribution \\ Seventh Edition
}

\author{
Updated and revised by the \\ Montana Bird Distribution Committee:
}

Montana Audubon

Amy Cilimburg

Janet Ellis
Montana Bird Records Committee
Coburn Currier
Dan Sullivan

Montana Department of Fish, Wildlife \& Parks

Scott Story

Catherine Wightman

\author{
Montana Natural Heritage Program \\ Paul Hendricks \\ Susan Lenard \\ Bryce Maxell
}



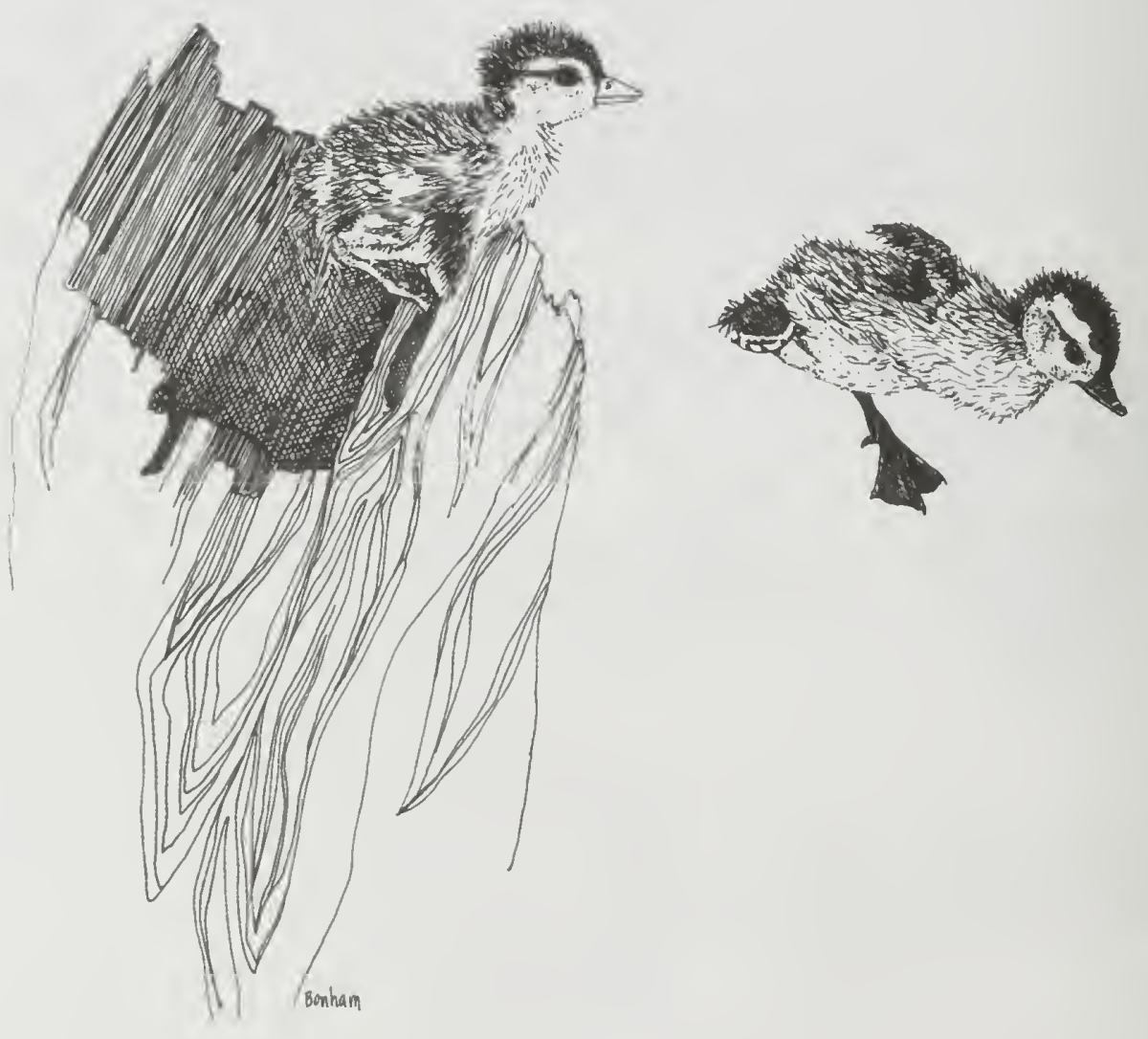

The price of this book is $\$ 15$ plus $\$ 5$ shipping and handling. Copies may be purchased through Montana Audubon, P.O. Box 595, Helena, MT 59624, 406-443-3949.

(C) 2012 Montana Audubon

This publication should be cited as:

Montana Bird Distribution Committee. 2012. P.D. Skaar's Montana Bird Distribution, $7^{\text {th }}$ Edition. Montana Audubon, Helena, Montana 208 pp + foldout map. 


\section{Table of Contents}

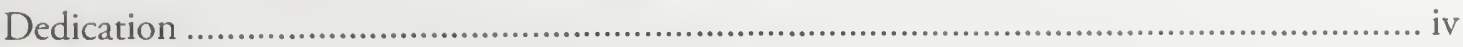

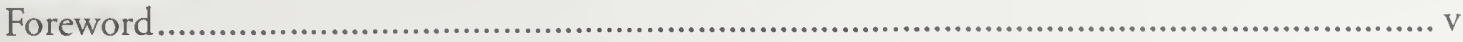

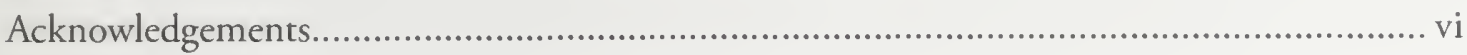

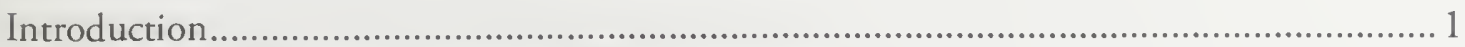

History of P.D. Skaar's Montana Bird Distribution ................................................ 1

Montana Bird Distribution Partnership .......................................................2

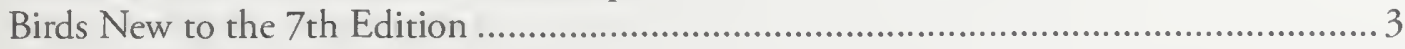

Taxonomic Changes .......................................................................... 3

The Official Bird List for the State of Montana...................................................... 4

Latilongs, Quarter Latilongs, and Quarter-quarter Latilongs ................................ 5

Montana Bird Distribution Database ................................................................6

Montana Bird Distribution on the Internet .......................................................6

Interpreting the Maps................................................................................... 7

Status Bars, Number of Records, and Species Status ........................................... 9

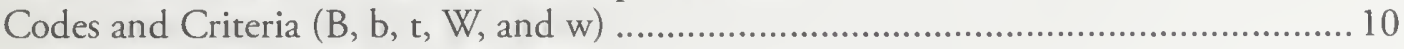

Legend

Species Distribution Maps.

Appendix A. Submission of New Records........................................................ 173

Appendix B. Bird Observation Report Form ....................................................... 176

Appendix C. Rare Birds ................................................................................ 177

Appendix D. Montana Rare Bird Report Form .......................................................... 179

Appendix E. Threatened (T), Endangered (E), Candidate Species (C), other Species of Concern (SOC), and Species of Interest (SI) ...................................... 180

Appendix F. Subspecies.................................................................................... 182

Appendix G. Introduced Species................................................................. 183

Appendix H. Hypothetical Records ..................................................................... 184

Appendix I. Extinct Species........................................................................ 185

Appendix J. A Summary of Bird Record Information by Latilong ............................ 185

Appendix K. Why Bird Distribution Information is Important ................................. 186

Appendix L. Contributors to this Edition ........................................................... 190

Appendix M. Checklist of Birds of Montana ........................................................ 192

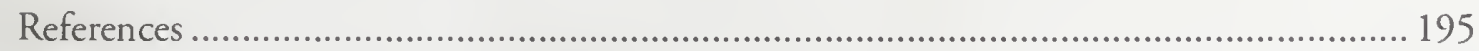

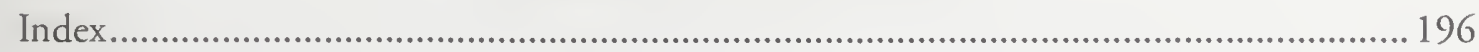

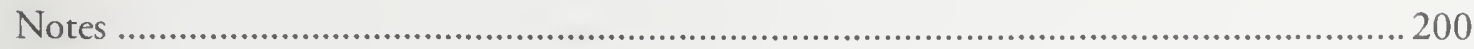

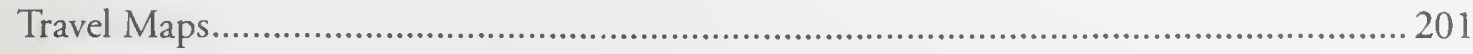




\section{Dedication}

\section{Don Stoecker}

Don Stoecker was born in 1933 and grew up during the Depression. His parents moved often in search of work and most of Don's childhood was spent on small farms in California and Louisiana. During those years, Don developed an appreciation for nature and hard work. After serving in the Navy from 1953 to 1961, he moved to Montana and worked as a farm laborer in the Big Hole and Georgetown Lake areas until he retired in 1995.

During his time as a ranch-hand Don developed his interest in birds. In order to follow his passion, upon retirement he took to a life on the road, living out of his self-contained "home on wheels" van, a mobile library of bird books and references. His license plate "Birder" attested to the focus of his pursuits. Don was the first person to develop a bird list for the "Settling Ponds" as they were then known, now the Warm Springs Wildlife Management Area near Anaconda. He also developed the first published bird checklist for the Deer Lodge National Forest, taught adult education classes in Deer Lodge and Anaconda on birding, and together with a few other interested folks in the Butte and Dillon areas, helped to establish the Pintler Audubon Society. In addition to his bird work in southwestern Montana, Don also felt it was important to gather bird observations throughout the state. As a result, he contributed thousands of records from across Montana for earlier editions of $P . D$. Skaar's Montana Bird Distribution.

When summer birds migrated south for the winter, so did Don. In his retirement, he would leave Montana in his van by late September and spend winters as a volunteer in National Wildlife Refuges in Texas, Arizona, and California. Don relished the opportunity to do helpful manual labor and guide folks on bird walks. In 2009, Don found he was not well enough to travel; he is now in a retirement center in Deer Lodge.

Don was not a formally trained ornithologist, but an avid and enthusiastic self-taught birder. His interests propelled him across thousands of miles and prompted him to record hundreds of pages of handwritten observation notes. Don combined his love for birds and people to become a significant citizen scientist who gathered thousands of records in out-of-the-way places. In 2005, he was recognized for his efforts with a Liferime Achievement award from Montana Audubon. Don is a great example of a common man who made a positive, lasting contribution toward understanding birds and their distributions in Montana.

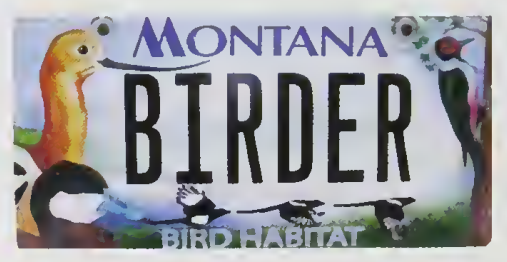




\section{Foreword}

The periodic publication of Montana Bird Distribution has provided birders and conservationists direct access to the state of our knowledge of bird distribution for more than three decades. This $7^{\text {th }}$ edition, representing the cumulative efforts of thousands of amateur birders and professional ornithologists across the state, gives us insight not only to the changing distribution of birds, but the extraordinary growth in our ability to capture knowledge. In his forward to the Sixth Edition, Jeff Marks referred to the "mind-boggling" increase of 39,000 bird records that were entered between the fifth and sixth editions. This edition includes more than 800,000 new records, and soon the Montana Bird Distribution (MBD) database will break the one million record barrier! This is a testament not only to the diligence of the Natural Heritage Program staff and MBD partners in incorporating additional agency and volunteer datasets (e.g., the Christmas Bird Count, Breeding Bird Survey, and colonial waterbird surveys), but also to the nature of birding in the information age.

Online data entry, social networks, handheld data devices with GPS and bird apps, and digital cameras have greatly increased the quantity and quality of distribution data submitted by amateur birders. We now have a data entry web portal, the Montana's Outdoor Birding Group (MOB-Montana) listserv with more than 500 members, more than 250 eBird users registered in the state, and a continually growing base of informed and engaged amateur naturalists. More than 2,700 people contributed new records to this edition; there are 85 individuals with more than 1,000 records, and four with more than 10,000 . This $7^{\text {th }}$ edition is nothing short of an amazing team effort.

This book includes 15 new species to the Official State Bird List since the $6^{\text {th }}$ edition, including unexpected vagrants from afar (e.g., Manx Shearwater, Siberian Accentor, Ross's Gull), as well as species from the Montana Bird Records Committee's hypothetical list added because of new records (e.g., Glossy Ibis, Iceland Gull). Eight new species have been confirmed breeding in the state (e.g. Gray Flycatcher, Purple Martin, Ovenbird), and 11 species have been newly confirmed as winter visitors (e.g., Eastern Bluebird, Bewick's Wren). The Eurasian Collared-Dove, reported just five times as of the $6^{\text {th }}$ edition, is now a confirmed breeder and a year-round resident statewide. The Montana Bird Records Committee has dropped documentation requirements for this and at least seven other species formerly considered rare (e.g., Thayer's Gull, Lesser Goldfinch).

But let us not take it for granted that we know all we need to know. Confirmed breeding (B) records are still scarce or lacking for many species, even for some with a long history of suspected or probable breeding (b) records in the state (e.g., Alder Flycatcher, Yellow-billed Cuckoo, White-winged Crossbill). There are still gaps in our knowledge in those places visited infrequently, particularly during winter. Browsing the maps will help you see where your own time in the field can help fill these gaps. And as we move forward, one thing is certain: bird populations and communities will respond as our human population continues to grow, and as climate and land use changes alter the Montana landscape. You and I have the opportunity to track these changes to better inform conservation and management and to continually refine our knowledge of (and connection to) Montana's avian riches.

It has taken more than the expected five years to produce this edition of Montana Bird Distribution. I think you will agree with me that it has been well worth the wait. I hope that having this edition in our glove box or daypack, next to our computer, and in our classroom will continue to inspire us to explore, look closely, and share our experiences.

\section{Dan Casey}

Northern Rockies Bird Conservation Region Coordinator, American Bird Conservancy

Chair, Montana Bird Records Committee 


\section{Acknowledgements}

The Montana Bird Distribution Committee is responsible for the production of this edition of Montana Bird Distribution. Members include Amy Cilimburg, Coburn Currier, Janet Ellis, Paul Hendricks, Susan Lenard, Bryce Maxell, Scott Story, Dan Sullivan, and Catherine Wightman.

The Montana Bird Records Committee reviewed rare bird sightings and provided advice and comment on a number of issues for this edition. The Records Committee is currently comprised of Dan Casey, Chuck Carlson, John Carlson, Coburn Currier, Nate Kohler, Rose Leach, Harriet Marble, Jeff Marks, Bob Martinka, Ted Nordhagen, Fritz Prellwitz, Mike Schwitters, and Dan Sullivan. We also thank past members Bob Eng and Terry McEneaney for their contributions since the last edition of this book. Thanks to the committee for its continuing efforts and dedication to a better understanding of Montana birds.

We are grateful to the thousands of contributors who submitted hundreds of thousands of recent records to make this edition possible (see Appendix L). Jock Young, Dick Hutto, and Kristina Smucker contributed data from the University of Montana Landbird Monitoring Program. Brian Sullivan of the Cornell Lab of Ornithology arranged the contribution of data from eBird. Denver Holt contributed data from the Owl Research Institute. Thousands of records have also been added from the U.S. Geological Survey Breeding Bird Surveys; Montana Fish, Wildlife \& Parks Bald Eagle, Waterbird, Common Loon, and Lek Surveys; National Audubon Society's Christmas Bird Counts; U.S. Fish \& Wildlife Service Shorebird Surveys; Confederated Salish and Kootenai Tribes Marsh Bird Surveys; Rocky Mountain Bird Observatory surveys; and numerous other federal, state, tribal, and non-governmental efforts on behalf of birds and their distributions in Montana.

Thank you to Montana Natural Heritage Program employees Scott Blum and Martin Miller for entering records and answering data requests; Karen Coleman and Dave Ratz for maintaining the database structure and various internet interfaces; and past employees Dan Bachen, Alexis McEwan, and Kathy Martin for their work on records entry. Past and current Montana Fish, Wildlife \& Parks staff Steve Carson, Emily Dixon, Andrew Jakes, Adam Messer, and Adam Petersen all contributed to entering records into the MBD database. A special thank you is extended to Dr. Kim Scott, Archivist at the Merrill G. Burlingame Special Collections at Montana State University Library, for allowing us to photograph P.D. Skaar's original records. Thanks also to Cedron Jones and Dan Sullivan for entering data. Gary Swant drafted the dedication and provided the photograph of Don's license plate; Jack Kirkley edited the dedication and provided additional background information. Jeff Marks edited this edition.

Financial support for this edition came with the cooperation of the following individuals and organizations: Bill Kitterman Audubon Endowment Fund and the Audubon Wildlife Fund of Montana (Montana Audubon); Montana Fish, Wildlife \& Parks (Wildlife Tax Checkoff); Bitterroot Audubon Society; Bureau of Indian Affairs (Jarvis Gust); Bureau of Land Management (John Carlson); Bureau of Reclamation (Jeff Baumberger/Justin Kucera); Confederated Salish and Kootenai Tribes' Division of Fish, Wildlife, Recreation \& Conservation (Dale Becker); Five Valleys Audubon Society; Flathead Audubon Society; Glacier National Park Fund (Merry Lynn Southers/Jane Ratzlaff); Intermountain West Joint Venture (Dave Smith); Last Chance Audubon Society; Mission Mountain Audubon Society; Montana Audubon; Montana Natural Heritage Program; MPG Ranch; National Park Service (Lisa Bate/Mark Biel); The Nature Conservancy (Kat Imhoff); Northern Great Plains Joint Venture (Ken Sambor); Northwestern Energy (Sam Milodragovich); Pintler Audubon Society; Plum Creek Foundation; Prairie Pothole Joint Venture (Casey Stemler); Sacajawea Audubon Society in memory of Margaret Hileman; U.S. Fish \& Wildlife Service (Shawn Sartorius); U.S. Forest 
Service - Region 1 (Beth Hahn); Upper Missouri Breaks Audubon Society; Westech Environmental Services, Inc. (Pat Farmer); Western Area Power Administration (Lou Hanebury); and Yellowstone Valley Audubon Society. The Bill Kitterman Audubon Endowment Fund, located at the Montana Community Foundation, is a permanent endowment dedicated to supporting Audubon's Montana Bird Distribution program. For more information about making contributions to this fund, contact Montana Audubon, P.O. Box 595, Helena, MT 59624, 406-443-3949.

The work of 12 artists appears in this publication. Their generosity, time, and talent have significantly improved the book's appearance. Andrew Guttenburg: Lewis's Woodpecker, cover; Yellow Rail, p. 49; White-throated Swift, p. 86; Northern Shrike, p. 102; Wilson's Warbler, p. 143; Red Crossbill, p. 168, Blue-gray Gnatcatcher, p. 181. Sharon Browder: Yellow-headed Blackbird, p. 161. John Carlson: Rough-legged Hawk, p. 200. Marsha Davis: Downy Woodpecker, p. 198; Evening Grosbeak, p. 170. Sid Frissell: Great Blue Heron, p. 37; Northern Flicker, p. 94. Ron Jenkins: Cooper's Hawk, p. 43; Wilson's Snipe, p. 64; Great Horned Owl, pp. 80 and 172; Black-chinned Hummingbird, p. vii; Bullock's Oriole, p. 164. James Jokerst: Downy Woodpecker, p. 92; American Dipper, p. 119. Phyllis Kober: Green-winged Teal, p. 20; Dusky Grouse, p. 30; Whimbrel, p. 57; Bonaparte's Gull, p. 67; Black-billed Cuckoo, p. 78; Gray Jay, p. 106; Chestnut-collared Longspur, p. 128; Field Sparrow, p. 147, Northern Hawk Owl, p. 194. Jerry Kustich: Red-necked Grebe, p. 4; Killdeer, p. 53; American Tree Sparrow, p. 145. Susan Lenard: House Wren, p. 117; Black-and-white Warbler, p. 131. Jan Metzmaker: Canada Goose, p. 15; Wood Duck, p. ii; Common Loon, p. 32; Osprey, p. 41; Calliope Hummingbird, pp. 88 and 190; Black-capped Chickadee, p. 112; Yellow Warbler, p. 137. We also used the Common Tern illustration (p. 73) from an anonymous artist. We appreciate the use of their illustration although they are not specifically identified. We also thank DD Dowden for her book cover design.

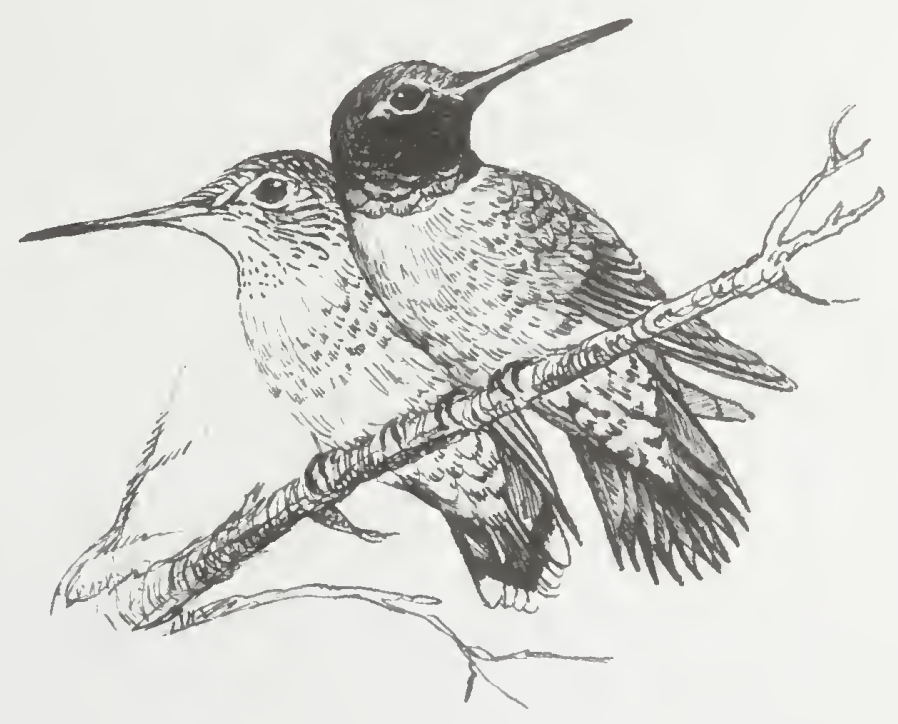



This publication is a summary of observation records of all birds currently found on Montana's Official State Bird List, with maps indicating breeding, migration, and wintering distribution areas for each species. The species are listed in taxonomic order. The maps portray the data contained in the database and do not presume to be a complete representation of the distribution of all species across the state. Instead, they provide a picture of where birds have been seen and reported. Two maps appear for each bird species, one representing observations made during the breeding season, the other representing observations recorded during the wintering season.

This book, as well as Montana's official state bird list, currently has:

- 427 species recorded for the state;

- 260 confirmed breeders; and

- 233 species reported during winter.

Of these, 104 bird species are considered rare in the state, which generally means that they have been recorded fewer than 20 times in Montana (Appendix C). An additional 11 species are of hypothetical occurrence (Appendix $\mathrm{H}$ ) and may make their way into future editions of this book. One species is known extinct in Montana (Appendix I).

For the first time, this book includes "status bars" for each bird species, a graph showing the relative number of bird observations recorded for each species for each month of the year.

Montana's size and diverse topography, ecology, and climate all contribute to the great variety and abundance of birds across the state. Distribution and abundance of the avifauna, however, is affected by increases in Montana's human population and changes in the Montana landscape. Some generalist species, those that tend to thrive in human-altered landscapes, are exhibiting population increases and associated range expansions. Specialized species such as Mountain Plover and Yellow-billed Cuckoo that require specific habitats have been negatively affected by human development. Alteration of their natural habitat reduces the ability of these birds to survive and reproduce, ultimately making them increasingly rare. Gathering more complete information about the distribution of birds across Montana can help us gain a better understanding of human impacts on the landscape and can contribute to more successful conservation efforts.

Everyone's submissions of bird observations can help bird conservation in Montana. By submitting bird observations to the MBD database, each of us is ensuring that the best source of reliable, objective information about the status and distribution of bird species found in Montana stays current over time. For more information on submitting new records to the database, see Appendix A; for information on why bird distribution information is important, see Appendix K.

\section{History of P.D. Skaar's Montana Bird Distribution}

This book bears the name of Dr. Palmer David Skaar (1923-1983), who arrived in Montana in 1957 to join the faculty of Montana State College (now Montana State University). Although his professional field was microbial genetics, Dr. Skaar did more to further the study of Montana birds than any other individual since Aretas A. Saunders published A Distributional List of the Birds of Montana in 1921. Dr. Skaar's contagious enthusiasm and his involvement in the study of Montana bird distribution earned him great respect throughout the state. 
The maps in this book use a unit of area called a "latilong," which represents the rectangular area of land formed by the intersection of latitude and longitude mapping lines (see Latilongs, Quarter Latilongs, and Quarter-quarter Latilongs, page 5). Having pioneered the latilong concept to depict animal distribution, Dr. Skaar's definitive Birds of the Bozeman Latilong, published in 1969, is a model regional bird account. The influence of his first edition of Montana Bird Distribution, published in 1975, was tremendous. It motivated volunteers and professionals across the state, and resulted in careful ornithological scrutiny of vast areas of Montana that had never before been studied. Since the publication of the first edition of Montana Bird Distribution, several classes of animals in other states have been mapped using the latilong system. In addition, the annual Breeding Bird Surveys coordinated by the U.S. Geological Survey and Canadian Wildlife Service use this system.

Dr. Skaar published the 2nd edition of Montana Bird Distribution in 1980. The 3rd edition was published in 1985 by his son, Don Skaar, Dennis Flath, and Larry Thompson (Skaar et al. 1985). The 4th edition appeared in March 1992 (Bergeron et al. 1992), with two innovations. First, it introduced quarter latilongs to better define species' distributions. Second, and more significantly, the maps in the 4 th edition were generated from a database of distribution records. The 5 th (Montana Bird Distribution Committee 1996) and 6th editions (Lenard et al. 2003) also used this database to generate distribution maps (see Montana Bird Distribution Database, page 6). The 5th edition included subspecies maps for a few selected species (e.g., Dark-eyed Junco), as well as information on the number of sightings reported for each species since the previous edition. The 6 th edition (Lenard et al. 2003) introduced color-coded species maps, showing bird observations for three timeframes.

Each update of P.D. Skaar's Montana Bird Distribution is cumulative, adding new bird records to those documented historically. Recently, 12 boxes of Dr. Skaar's personal records, accumulated observations, correspondence, and notes were deposited in the Special Collections department of the Montana State University Library and made available to the Montana Bird Distribution Committee. This ornithological archive provided valuable details concerning specific location, date, and observer information of historical observations. It also added many previously unknown records for this edition. Thus, this 7 th edition is, in the truest sense, one of Dr. Skaar's ongoing legacies. Future editions will continue as a direct result of his inspirational and visionary efforts.

\section{Montana Bird Distribution Partnership}

The Montana Bird Distribution Partnership, comprised of Montana Audubon, the Montana Bird Records Committee, the Montana Department of Fish, Wildlife \& Parks, and the Montana Natural Heritage Program, oversees the Montana Bird Distribution database and its associated products. These organizations have been collaborating informally since 1991, and under a Memorandum of Understanding (MOU) since 2007. The main goal of the MBD partnership is to track the distribution of all bird species in the state over time in order to promote the stewardship of bird species and their habitats. The partnership does this by: (1) facilitating the collection of high quality observation records by both professional and non-professional birders; (2) maintaining records in a high quality, accurate, and comprehensive database; and (3) providing meaningful access to bird distribution information. Products of the partnership include Montana Bird Distribution, created by the MBD Committee, as well as web-based information sources such as the Montana Field Guide, Natural Heritage Tracker, Natural Heritage MapViewer, Montana Audubon web pages, and Montana Bird Record Committee web pages. 


\section{Birds New to the 7 th Edition}

In the nine years since the 6th edition of Montana Bird Distribution, 18 new species have been added to Montana's official checklist. These include 15 species never before documented in the state and three species added because of a taxonomic change. The new species are: Cackling Goose (a split from Canada Goose), Tufted Duck, Manx Shearwater, Glossy Ibis, White-tailed Kite, Crested Caracara, Ross's Gull, Iceland Gull, Lesser Black-backed Gull, Long-billed Murrelet (a split from Ancient Murrelet), Inca Dove, Carolina Wren, Pacific Wren (a split from Winter Wren), Curve-billed Thrasher, Siberian Accentor, Blue-winged Warbler, Eastern Towhee, and Eastern Meadowlark.

Confirmed breeding (represented by a "B" on the maps) has been recorded for the first time in eight species: Eurasian Collared-Dove, Gray Flycatcher, Plumbeous Vireo, Purple Martin, Ovenbird, Rosebreasted Grosbeak, Blue Grosbeak, and Lesser Goldfinch. The breeding Rose-breasted Grosbeak was a male bird attending a nest and paired with a female Black-headed Grosbeak. Also, 11 species have been reported during "winter" (represented by a "w" or "W" on the maps) for the first time: Ross's Goose, Eurasian Wigeon, Surf Scoter, Greater Yellowlegs, Eurasian Collared-Dove, Western Scrub-Jay, Bewick's Wren, Eastern Bluebird, Common Yellowthroat, Northern Cardinal, and Lincoln's Sparrow.

\section{Taxonomic Changes}

All common and scientific names, as well as taxonomic sequences, follow the seventh edition of the Check-list of North American Birds (AOU 1998) and all supplements to the check-list published through 2011 (AOU 2011).

Prior to the publication of the 6th edition of Montana Bird Distribution, the AOU began producing annual supplements to the Check-list of North American Birds. The frequency with which taxonomic changes were occurring demanded a quicker response from the Committee on Classification and Nomenclature. This frequency is evident in the significant and numerous taxonomic changes appearing in this edition of $M B D$. Some of the most significant taxonomic changes affecting this book include:

- The Anseriformes (Ducks, geese, and swans) and the Galliformes (Grouse and pheasants) were moved in front of the Gaviiformes (Loons).

- The common name of the Rock Dove became Rock Pigeon.

- The Three-toed Woodpecker became the American Three-toed Woodpecker, and its Latin name changed from Picoides tridactylus to Picoides doralis.

- The Cackling Goose (Branta hutchinsii) was elevated to species status after splitting from the Canada Goose.

- The Blue Grouse was split into two species, the coastal Sooty Grouse and the interior Dusky Grouse (the Latin name of Dusky Grouse remains Dendragapus obscurus).

- The common name of Nelson's Sharp-tailed Sparrow changed to Nelson's Sparrow.

- The Winter Wren was split into three species, two of which, the Pacific Wren (Troglodytes pacificus) and Winter Wren (Troglodytes hiemalis), occur in Montana.

- The Common Moorhen was split into two species, Common Moorhen (Old World) and Common Gallinule (New World). The scientific name of Common Gallinule changed from Gallinula chloropus to Gallinula galeata.

- Many changes occurred within the Parulidae (wood-warblers), including generic reassignments and taxonomic sequence changes.

For a complete list of all taxonomic changes between the 6th and 7th Edition of $M B D$, visit http:// www.mtaudubon.org/birds/distribution.heml. 


\section{The Official Bird List for the State of Montana}

Montana's official state bird list is maintained by the Montana Bird Records Committee (MBRC). Because birders play a critical role in furthering our understanding of bird distributions in the state, it is important to have rigorous review of bird records to maintain a meaningful list of Montana birds. Consequently, the MBRC reviews documentation submitted for species new to the state or of sufficient rarity (locally, regionally, or statewide). Observations for these species require a Rare Bird Report Form (found in Appendix D). This review occurs in order to provide an objective opinion on the validity of reports of unusual species, or species presence during unusual times of the year, by a panel of professional and non-professional expert birders. Rare bird reports are evaluated by the MBRC before the observations can be entered into the MBD database. For a list of rare birds reviewed by the MBRC, including regional exceptions for reporting requirements, see Appendix $\mathrm{C}$. Based on records evaluated through January 2012, the state list stands at 427 species (Appendix M).

In addition to reviewing rare bird records, the MBRC reviews information on introduced species, with specific requirements that must be met before a species is considered established and placed on the official state bird list. For this reason, neither Northern Bobwhite nor California Quail is currently on Montanas official state bird list (see Appendix G). The MBRC also maintains a list of Hypothetical Records (also known as the Supplemental Bird List) (see Appendix H). This list contains: (1) species reported by a single observer only (with no photo or specimen); (2) species for which only one independent write-up was received even though multiple observers were present; and (3) species of uncertain origin that may have escaped captivity. These species are not yet on the official state list.

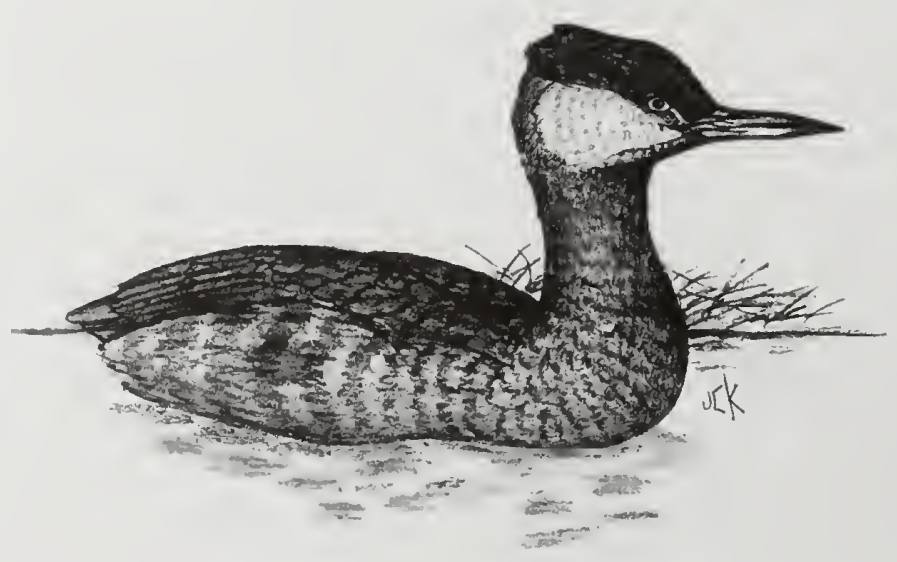




\section{Latilongs, Quarter Latilongs, and Quarter-Quarter Latilongs}

The maps in this book are the outline of Montana, with a grid pattern overlaying the state in units called latilongs and quarter latilongs. The back cover of this book illustrates this grid pattern and how the latilong system is positioned across the state. Montana is divided into 49 latilongs, 185 quarter latilongs, and more than 700 quarter-quarter latilongs (a unit for which data is collected, but because of their small size, are not shown in this book). The Travel Maps, found on pages 200 to 207 depict latilongs, quarter latilongs, and quarter-quarter latilongs.

Latilongs (LL) are assigned a number, 1 through 49 , starting at the northwest corner of the state. The average dimension of each latilong is 47 miles $(76 \mathrm{~km})$ wide and 69 miles $(111 \mathrm{~km})$ long, representing an average area of approximately 3,200 square miles $(8,436 \mathrm{sq} . \mathrm{km})$. Because of the earth's curvature, latilongs along the Canadian border are 5.4 percent smaller than those along the Wyoming border. Latilongs 48 and 49 cover only small portions of southwestern Montana.

Quarter Latilongs (QLL) are created by evenly dividing each latilong unit into four quarters. Each quarter latilong is identified by its associated latilong number, with the addition of a letter (A, B, C, or D), ordered left to right and from top to bottom (see Figure 1). Thus, latilong 19 is broken into 4 QLLs: 19A, 19B, 19C, and 19D. QLLs cover an area roughly 24 miles (38 km) by 35 miles (56 $\mathrm{km})$.

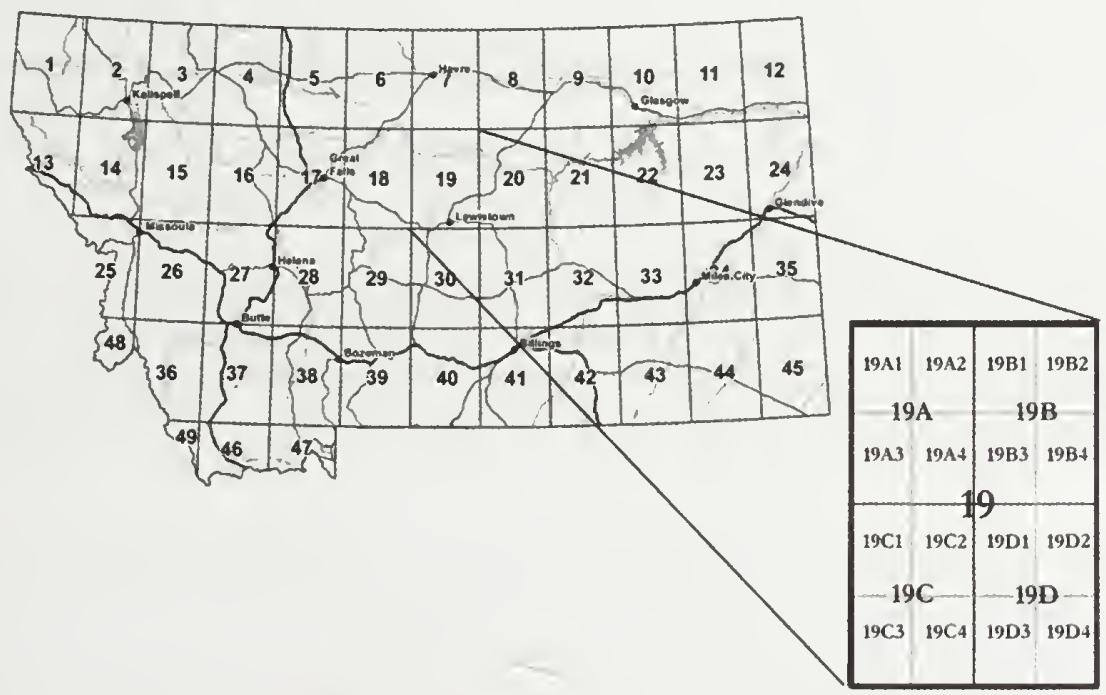

Figure 1. Quarter latilongs are created by dividing each latilong unit into four quarters; quarter-quarter latilongs by dividing each quarter latilong into four quarters.

Quarter-Quarter Latilongs (QQLL) are a further subdivision of quarter latilongs. Although QQLLs are too detailed for presentation in this publication, records in the MBD database include a QQLL designation if the original report has detailed location information allowing this to be determined. QQLLs are created by evenly dividing each quarter latilong into four quarters. Each QQLL is identified by its associated LL number and QLL letter in the same manner as for QLLs, with the addition of a number $(1,2,3$, or 4$)$, ordered left to right and from top to bottom. Thus, latilong 19 is divided into 16 QQLLs: 19A1, 19A2, 19A3, 19A4, 19B1, etc. QQLLs cover an area roughly 12 miles $(19 \mathrm{~km})$ by 17 miles $(28 \mathrm{~km})$. Montana Audubon is sponsoring an "adopt a QQLL" program to gain a more complete picture of the bird species found in specific areas. For more information on this program, visit http://www.mtaudubon.org/birds/distributionQQLL.html. 


\section{Montana Bird Distribution Database}

The MBD database contains observation records for the birds of Montana submitted by organizations, agencies, and volunteer contributors from across the state. Starting in 1991, observations were entered into an electronic database housed at the Montana Natural Heritage Program in the Montana State Library in Helena. Since then the database has become quite complex in order to deal with a wide variety of survey information and to power a number of web applications (see Montana Bird Distribution on the Internet below).

The number of observations in the database has grown dramatically since its inception, resulting from increased electronic storage and processing capabilities. This has made it possible to handle large data sets of standardized surveys from the Breeding Bird Survey, Christmas Bird Count, and Avian Science Center (Missoula), among others. As of 13 March 2012, there were 871,511 records in the database. These are from 78,300 unique locations across Montana, with anywhere from 1 to 3,358 records associated with individual locations. For a summary of the (1) total number of bird species that have been reported in each latilong, and (2) the total number of bird records in the MBD database for each latilong, see Appendix J.

\section{Montana Bird Distribution on the Internet}

A number of excellent internet resources are available to individuals interested in learning more about bird distribution and/or birding in Montana. Links to these resources can be found at: $\underline{\text { http:// }}$ mtaudubon.org/birds/resources.html.

The following websites are based on the MBD database and are updated daily:

Montana Field Guide: Perhaps the easiest way to access a variety of information on individual species known to occur in Montana is through the Montana Field Guide http://fieldguide.mt.gov/. Individual bird species accounts on this site provide photos, bird calls, range maps, natural history information, habitat use and associations, food habits, and more. The accounts also include daily updated maps and charts compatible with the maps found in this book.

Natural Heritage MapViewer and Tracker websites: Individuals interested in more specific information about the distribution of birds in the state should use the Natural Heritage MapViewer http://mtnhp.org/mapviewer// and/or Natural Heritage Tracker http://mtnhp.org/tracker/ websites. These interactive websites allow users to submit their observations electronically, as well as perform individual tailored searches of the records. These searches could, for example, answer such questions as: which species occur in the Miles City area; what is the current distribution of the Indigo Bunting in Montana; how many times, and where, has the American Bittern been reported since 2003; and what species are reported as breeding during the month of July in the Polson area? Individuals may also query the database by species, breeding or wintering status, location (LL, QLL, or QQLL), month, year, and more. Tutorials on the use of either of these websites can be found at http://mtnhp.org/ webinars/ or under the Help menus. 


\section{Interpreting the Maps}

The maps on the following pages indicate the distribution of birds based upon observations made during two distinct seasons:

- BREEDING SEASON (16 February to 14 December, which includes spring and fall migrations for many species); and

- WINTERING SEASON (15 December to 15 February).

The breeding-season maps are the larger maps located to the right on each page; the winteringseason maps are found on the left.

The letter displayed in each of the QLL blocks describes the status of the bird species observed in that particular QLL. The status shows whether the species recorded for that QLL exhibited behavior indicating "breeding," "indirect evidence of breeding," "no evidence of breeding (transient)," "wintering," or was "observed at least once during the wintering season." The status of bird observations is indicated by a lettered symbol:

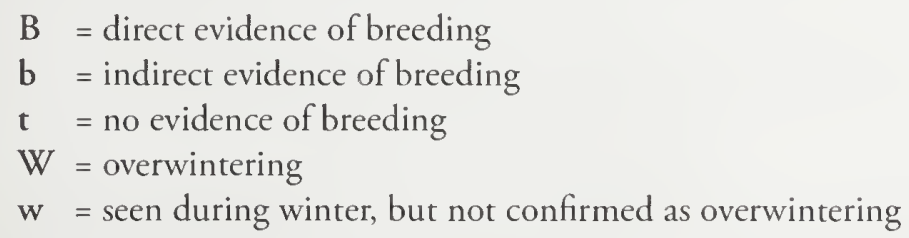

More complete definitions for $\mathrm{B}, \mathrm{b}, \mathrm{t}, \mathrm{W}$, and $\mathrm{w}$ can be found under Codes and Criteria $(\mathrm{B}, \mathrm{b}, \mathrm{t}, \mathrm{W}$, and w) on page 10. As of 13 March 2012, the database contained the following number of records: B $=29,338 ; \mathbf{b}=545,974 ; \mathrm{W}=3,697 ; \mathbf{w}=53,911$; and $\mathbf{t}=238,591$ (total records 871,511).

\section{Breeding-Season Maps}

The breeding maps in this book depict data in three different time frames:

- "New" observations (2003-2011) represented by a symbol (B, b, or t) in the center of each block.*

- "Previous" observations (1991-2002) represented by the background color in each block ( $\mathrm{B}=$ dark gray, $\mathrm{b}=$ light green, $\mathrm{t}=$ yellow).*

- "Historical" observations (data prior to 1991) represented by solid gray in LL blocks and as hatching in QLL blocks. The solid gray or hatch marks do not denote status of the observations: direct evidence of breeding, indirect evidence of breeding, or transient ( $B, b$, or t). Instead, they simply indicate an observation of this species prior to 1991 , but not since. This information is provided to help understand where birds were seen historically, but have not been reported for the past twenty years.*

The symbol within each quarter latilong rectangle displays the highest documented status for that species for that quarter latilong ("B" higher than " $b$ " higher than " $t$ "). Different from the previous editions, observations with transient (" $\mathrm{t}$ ") status are represented by the color yellow, rather than gray. 


\section{Wintering-Season Maps}

The wintering maps are smaller in size. In order for them to be readable, the data are presented in latilong blocks. As with the breeding maps, the symbol within each latilong rectangle displays the highest documented status for that species for that latilong ("W" is higher than "w"). The wintering maps in this book depict data in two different time frames:

- "New" observations (2003-2011) are represented by a symbol (W or w) in the center of each block.*

- "Previous edition" observations (historical to 2002) are represented by the background color in each block ( $\mathrm{W}=$ darker blue, $\mathrm{w}=$ lighter blue).*

* For an explanation of map colors and symbols see Figure 2 below and pages 10 and 11.

Note: Readers with an interest in specific information on observations, new and historical, should visit the MBD website and investigate the database by performing their own customized searches. For historical records, readers may also refer to previous editions of P.D. Skaar's Montana Bird Distribution.

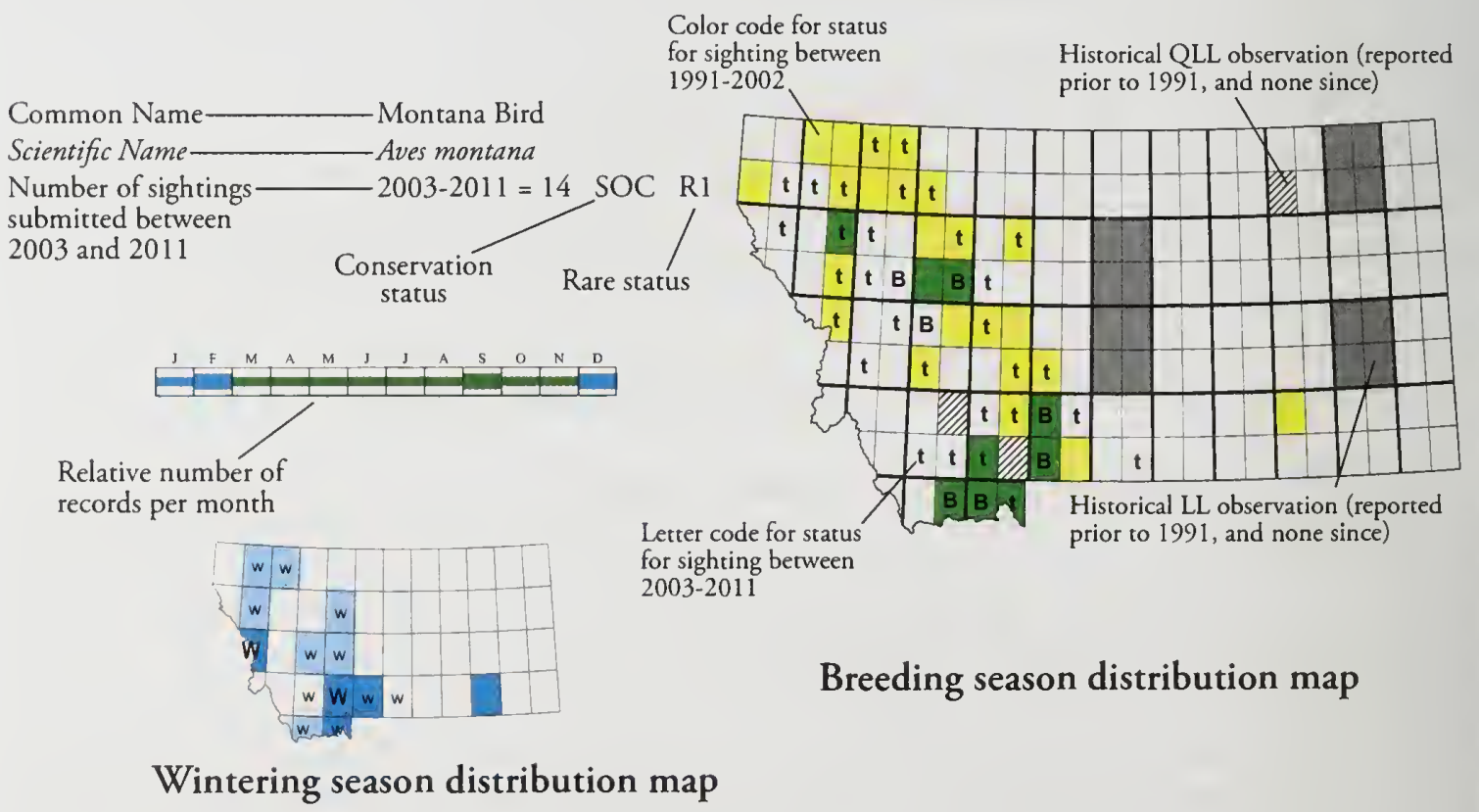

Figure 2. Example of Distribution Maps and Status Bar. 


\section{Status Bars, Number of Records, and Species Status}

For the first time in $M B D$, status bars appear for each species. These box-plot graphs show the relative number of records for each month (January through December). Records are identified for two seasons (breeding and wintering). Four separate categories for number of observations per month are defined: 1-4 records per month (thinnest line), 5-20 records, 21-75 records, to more than 75 records (thickest line). For a few rare birds, such as Western Scrub-Jay and Carolina Wren, where the bird was observed for more than one month, all months of occurrence are shown even though the total record count for 2003-2011 equals one. Observations from December through February are shown in blue; observations from March through November are shown in green, with colors generally corresponding to the winter and breeding season maps.

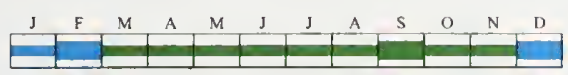

The number of records and status of each species are provided on the lines beneath the species name. Symbols and their definitions are explained below.

2003-2011 = Total number of observations reported for this species in the last nine years, 2003 $-2011$

TE C Threatened (T), Endangered (E), or Candidate (C) species under the federal Endangered Species Act (Appendix E)

SOC

Species of Concern. These species have been identified by the Montana Natural Heritage Program and Montana Department of Fish, Wildlife \& Park as Montana Species of Concern because they are at risk owing to declining population trends, threats to their habitats, restricted distribution, and/or other factors. (For more information, see Appendix E)

SI

Species of Interest. Because of specific circumstances, supplemental information is requested for these species. (For a complete list of SI species, see Appendix E)

R1, R2, R3 Rare species status. Rare species are those based on fewer than 20 documented observations for the state (R1; most species), regularly observed in a very localized part of the state but fewer than 20 records outside that area (R2; e.g. Blue-gray Gnatcatcher), and Yellow-billed Cuckoo (R3) with more than 30 records, but few in recent decades (For more complete definitions, see Appendix C) 
Codes and Criteria (B, b, t, W, and w)

The lettered symbols are defined as follows:

BREEDING SEASON (16 February - 14 December)

For observations of birds during the breeding season, the letters "B," "b," or " $t$ " are used to indicate the bird's breeding status.

\section{“B” DIRECT EVIDENCE OF BREEDING}

" $\mathrm{B}$ " is used to indicate direct evidence of breeding: evidence that eggs or young have been produced.

Breeding is not assumed simply by the presence of adults, singing and territorial behavior, copulation, or nest-building. " $\mathrm{B}$ " is used only if one or more of the following criteria are met:

1. Occupied nest - adult attending a nest with eggs or nestlings (incubating or brooding), or adults entering or leaving nest site in circumstances indicating an occupied nest (includes high nests or cavities, the contents of which cannot be seen).

2. Recently fledged young (of altricial species) incapable of sustained flight or downy young (of precocial species) restricted to the area by dependence on adults or limited mobility.

3. Adults attending young - adult feeding recently fledged young or carrying food for young, or adult carrying fecal sac.

4. Nest with egg(s) that can be clearly identified. The presence of cowbird eggs or young is confirmation of breeding for both cowbird and host species.

5. Used nest with eggshell, or just eggshell found (identification must be convincing for such records to be accepted, and cannot be based on the nest alone).

NOTE: Records of direct evidence of breeding ("B") are only accepted when at least one of the five criteria listed above is observed and information for a " $\mathrm{B}$ " designation is included in the Comments section of the Report Form. If no supporting information is given, the record will be designated a "b" (see following).

\section{“b” INDIRECT or CIRCUMSTANTIAL EVIDENCE OF BREEDING}

" $b$ " is used to indicate indirect evidence of breeding. This status applies if one or more of the following criteria are met:

1. Singing males or territorial birds observed in suitable nesting habitat during the breeding season.

2. Courtship behavior or copulation.

3. Adults visiting a probable nest site.

4. Agitation behavior, distraction display, feigning injury, or anxiety call from an adult.

5. Nest building.

6. Physiological evidence of breeding (incubation patch or egg in oviduct) based on bird in hand.

7. Independent young of the year unaccompanied by adult (for non-migratory species or prior to normal migration). 
" $\mathrm{t}$ " is used to indicate presence of any species (resident, migrant, or transient) that exhibits no breeding behavior at the time observed.

Behavioral activities that do not fit "B" or "b" should be considered "t." See "B" and "b" above to determine if a behavior constitutes direct or indirect evidence of breeding.

\section{WINTERING SEASON (15 December - 15 February)}

For observations of birds during the wintering season, the letters "W" or " $w$ " are used to indicate wintering status.

\section{"W" OVERWINTERING}

"W" is used to indicate regular observations of the species during the winter period.

Regular is defined as at least 3 days between 15 December and 14 January and 3 days between 15 January and 15 February. "W" can be documented two ways: (1) an individual observer submits data with dates for when the bird was observed during the winter months, following the above criteria, or (2) multiple "w" records in the MBD database for the same $\mathrm{LL}$ and from the same winter season are used to generate a "W", following the above date criteria. (Individuals submitting data need to include the dates for which the bird was observed during the winter months, 15 December - 15 February, in the Comments section of the Report Form.

\section{"w" OBSERVED DURING WINTER SEASON, but NOT confirmed OVERWINTERING}

"w" is used to indicate that the species was observed at least once between 15 December and 15 February, but not regularly during the winter season. 


\section{Legend}

\section{BREEDING SEASON (16 February through 14 December)}

Direct evidence of successful breeding / evidence of young:

B Observation records between 2003-2011 are represented with the capital letter B.

D Observation records between 1991-2002 are represented as the dark green background in each QLL block.

Indirect or circumstantial evidence of breeding:

b Observation records between 2003-2011 are represented with the lower letter b.

$\square$ Observation records between 1991-2002 are represented as the light green background in each QLL block.

No evidence of breeding, no breeding behavior

t Observation records between 2003-2011 are represented with the lower letter $\mathbf{t}$.

$\square$ Observation records between 1991-2002 are represented as the yellow background in each QLL block.

\section{Historical Information:}

Observation records prior to 1991 and not since are represented as the gray background in each LL block, unless mapping can be assigned accurately to the QLL block (see below). No specific breeding status is inferred.

Observation records prior to 1991 and not since, and that can be accurately identified to the QLL block, are represented with gray hatching. No specific breeding status is inferred.

\section{WINTERING SEASON (15 December through 15 February)}

Overwintering; regular sightings:

W Observation records between 2003-2011 are represented with the capital letter W.

$\square$ Observation records prior to 2003 are represented as dark blue in each LL block.

Not confirmed as overwintering but observed during wintering season:

w Observation records between 2003-2011 are represented with the lower letter $\mathbf{w}$. Observation records prior to 2003 are represented as light blue in each LL block.

2003-2011 = total number of new sightings submitted for this edition

T E C Threatened (T), endangered (E), or candidate species (C)

SOC Species of Concern

SI Species of Interest

R1 Rare throughout Montana

R2 Regionally rare within Montana

R3 Rare status retained beyond 20 observations due to infrequency of encounters, previously more common 
ANSERIFORMES Anatidae

Greater White-fronted Goose

Anser albifrons

2003-2011 = 30
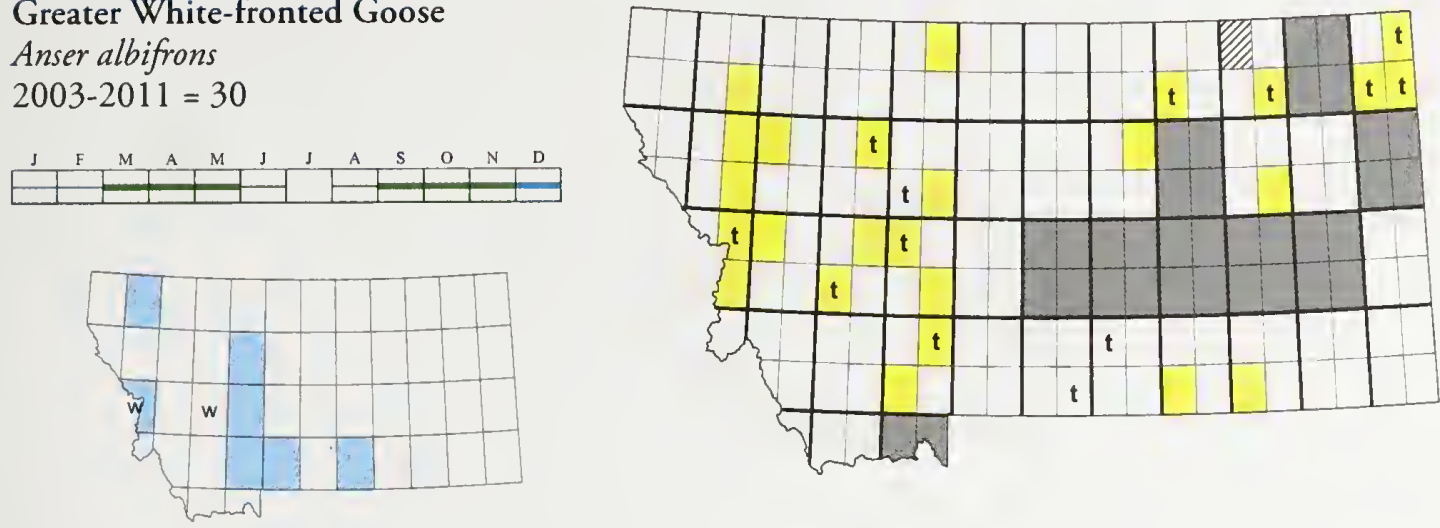

\section{Snow Goose}

Chen caerulescens

2003-2011 = 116
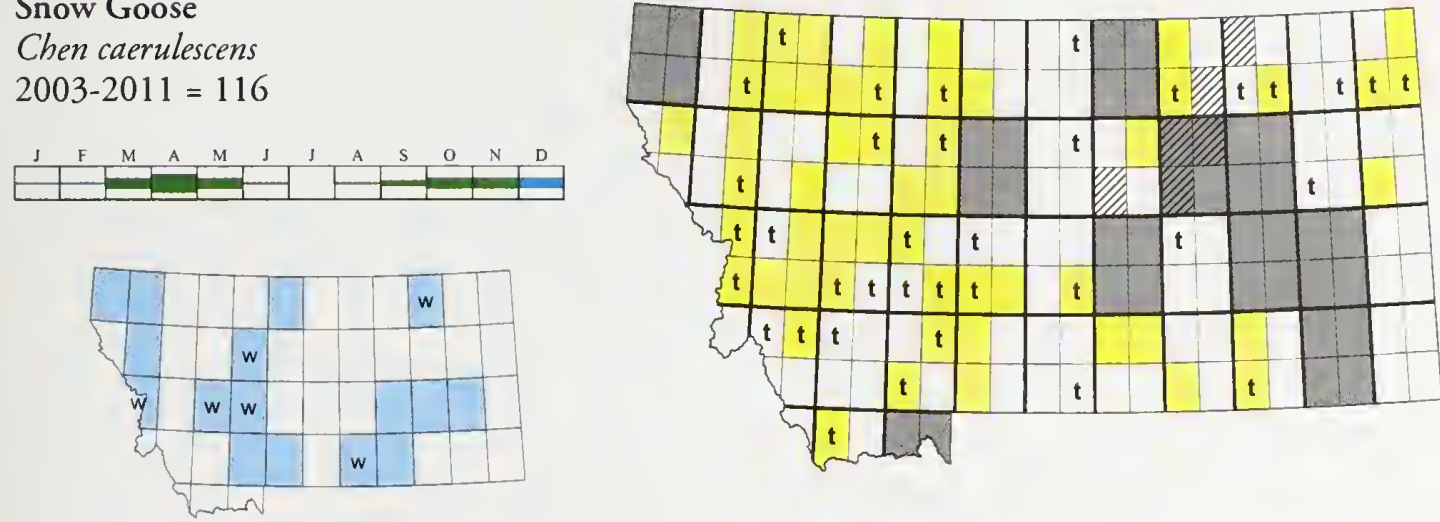

\section{Ross's Goose}

Chen rossii

2003-2011 = 42
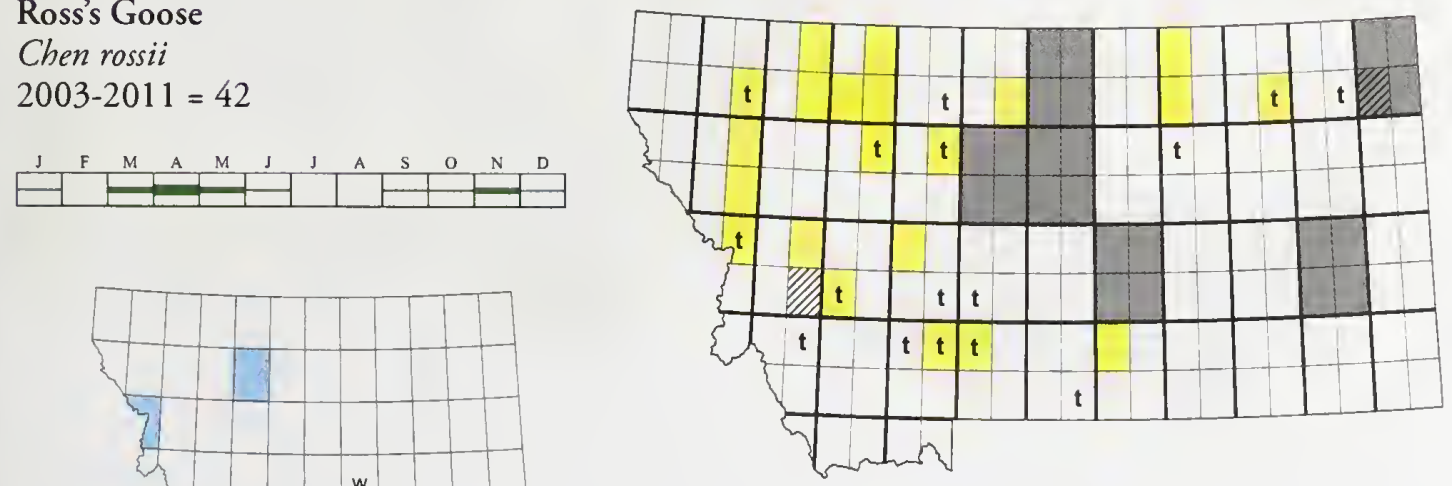
Brant

Branta bernicla

2003-2011 = 2 R1
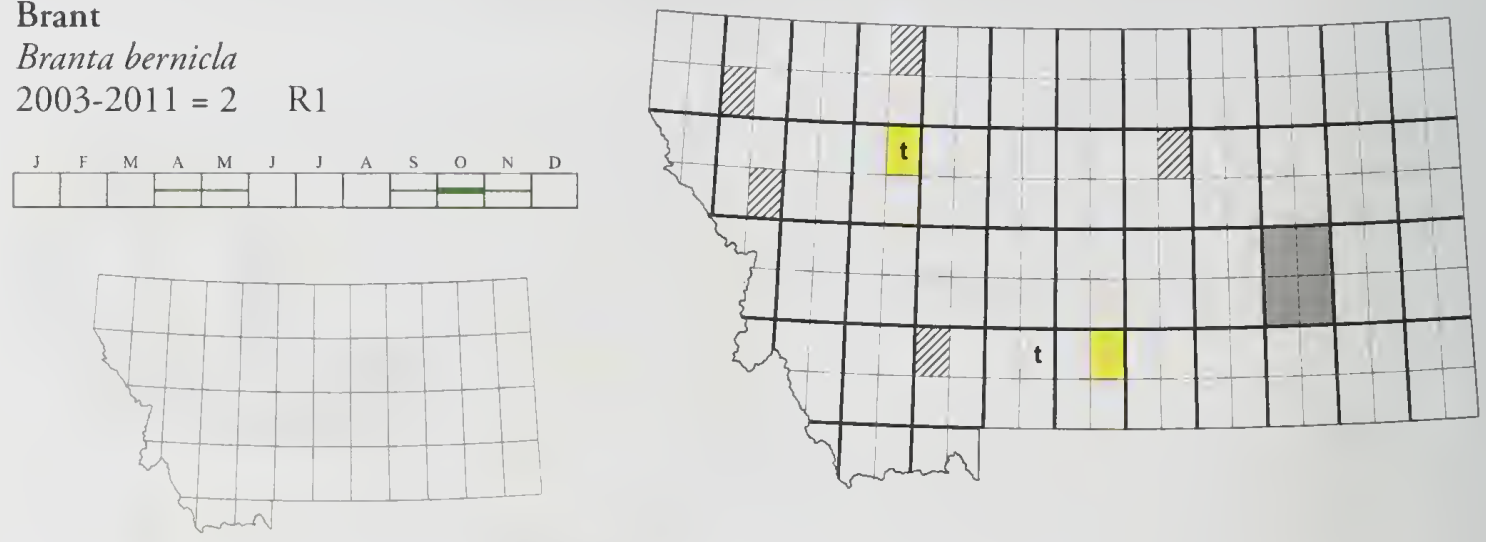

Cackling Goose

Branta hutchinsii

$2003-2011=18 \quad$ R2

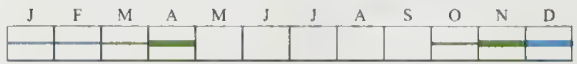
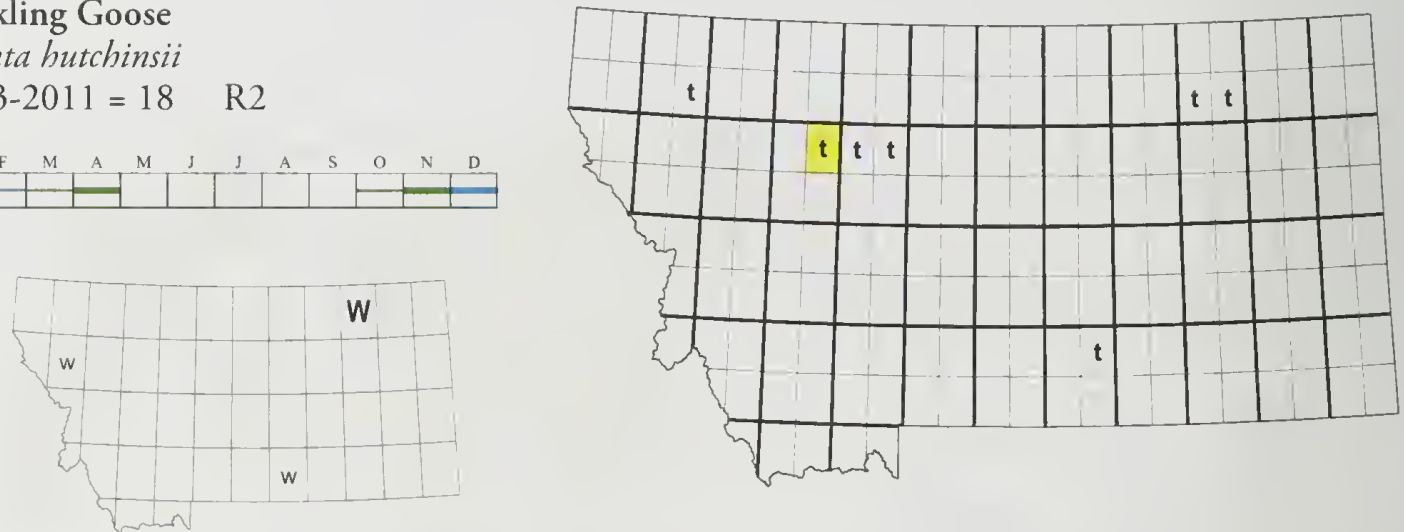

Canada Goose

Branta canadensis

2003-2011 = 3220
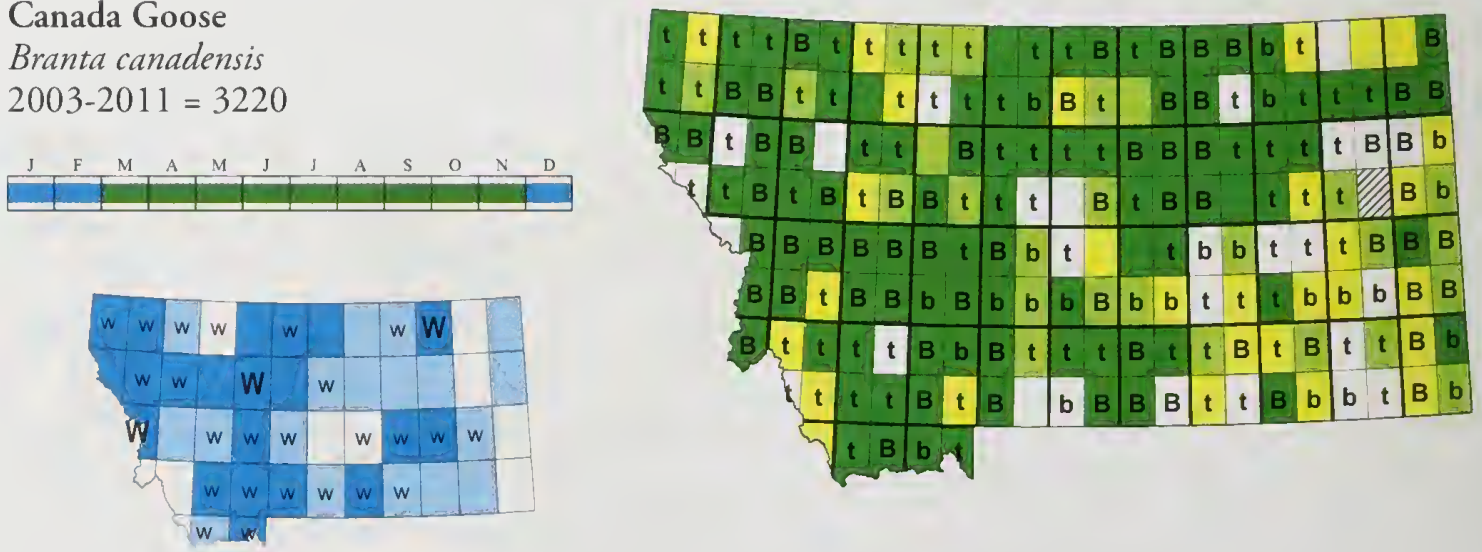


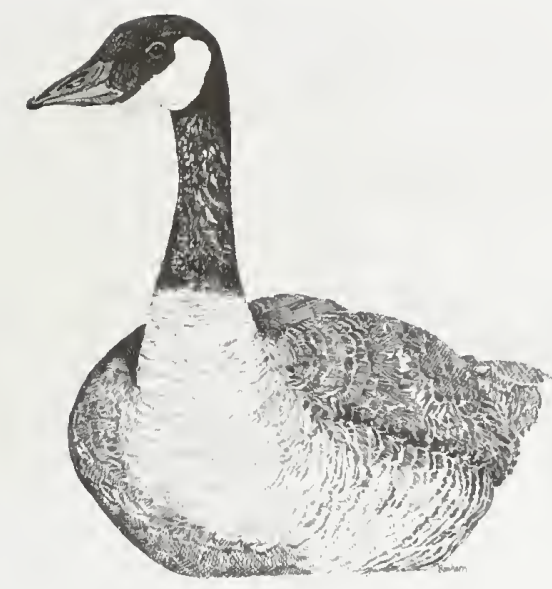

\section{Mute Swan}

Cygnus olor

2003-2011 = 0
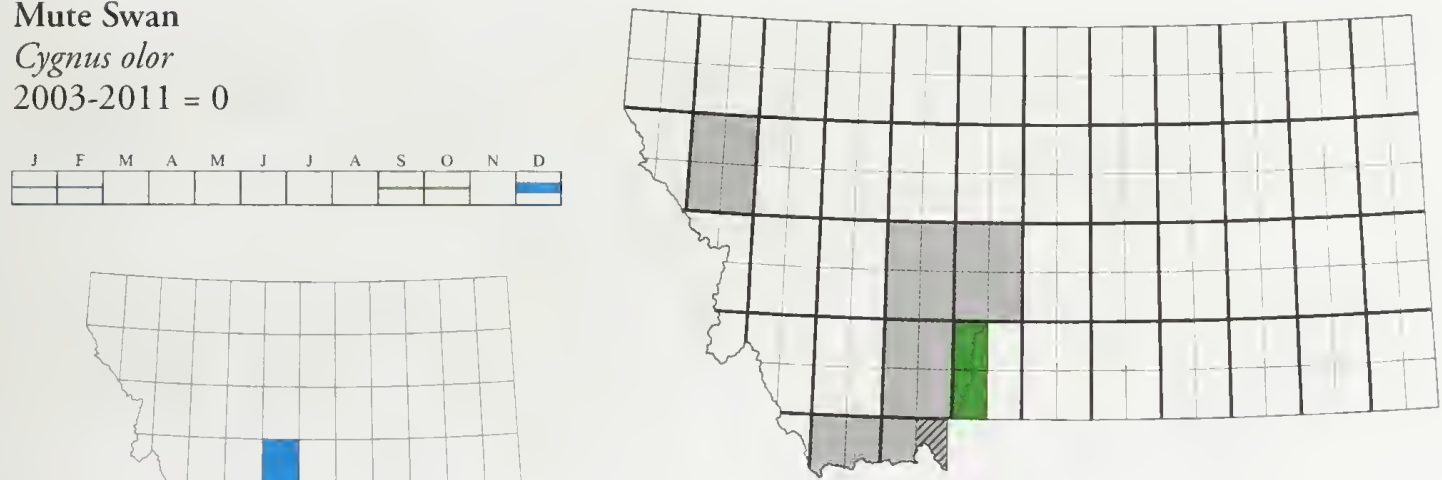

Trumpeter Swan

Cygnus buccinator

$2003-2011=420 \quad$ SOC
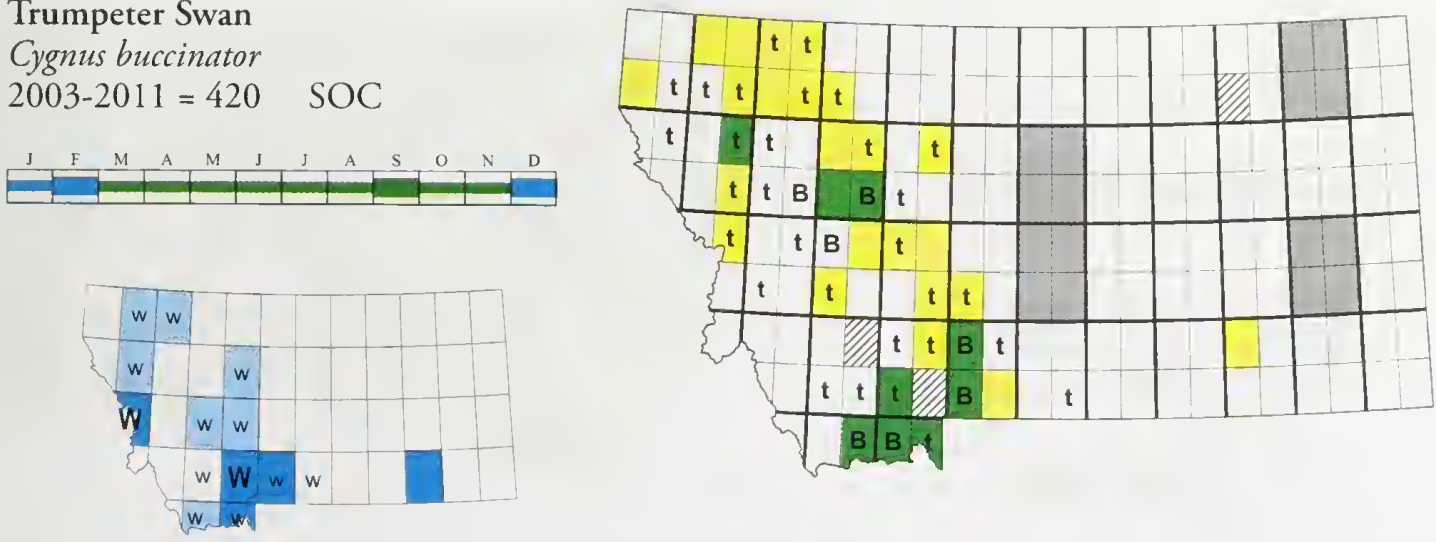
Tundra Swan

Cygnus columbianus

2003-2011 = 229
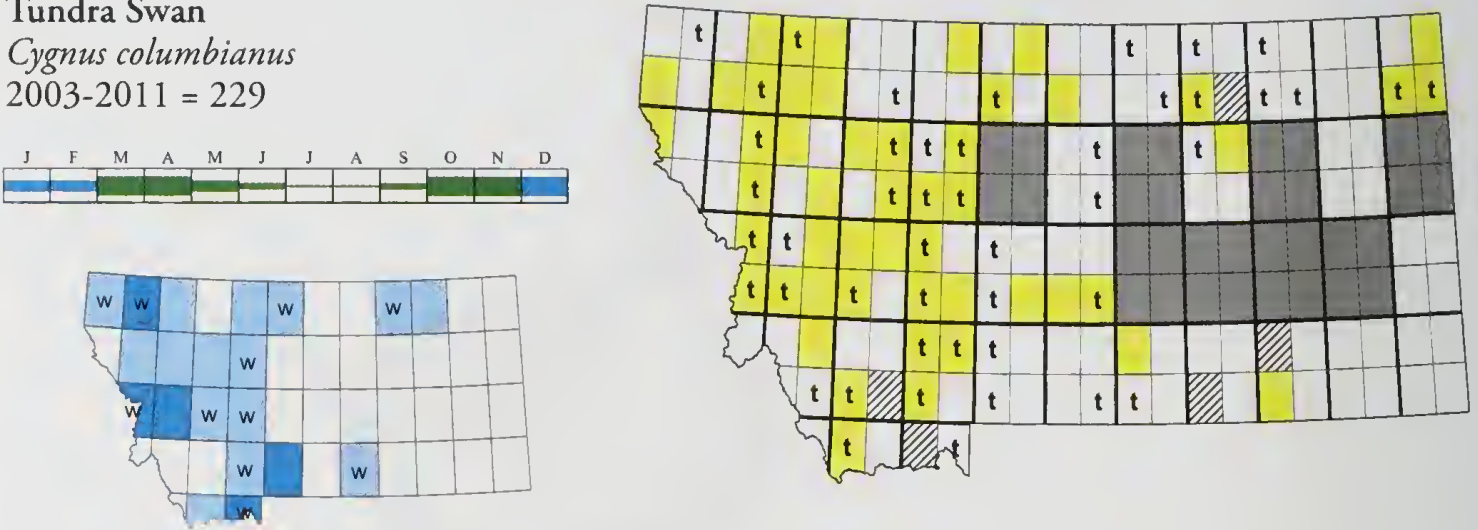

Wood Duck

Aix sponsa

$2003-2011=373$
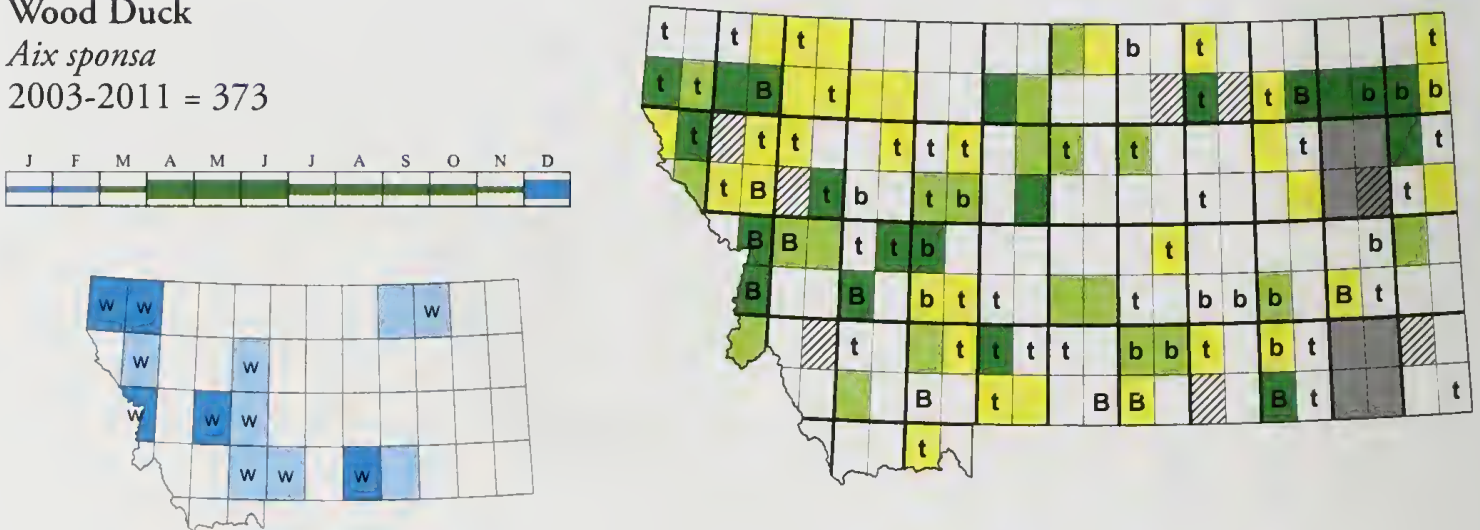

Gadwall

Anas strepera

2003-2011 = 1419
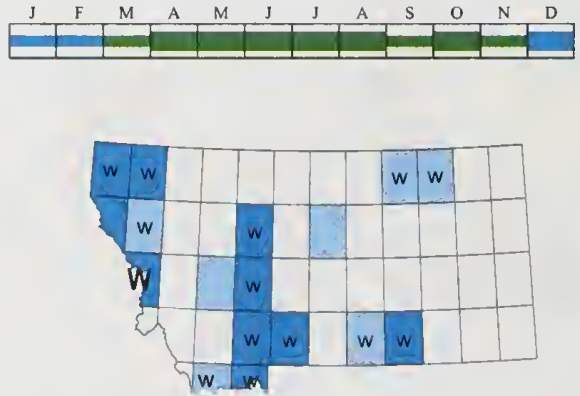

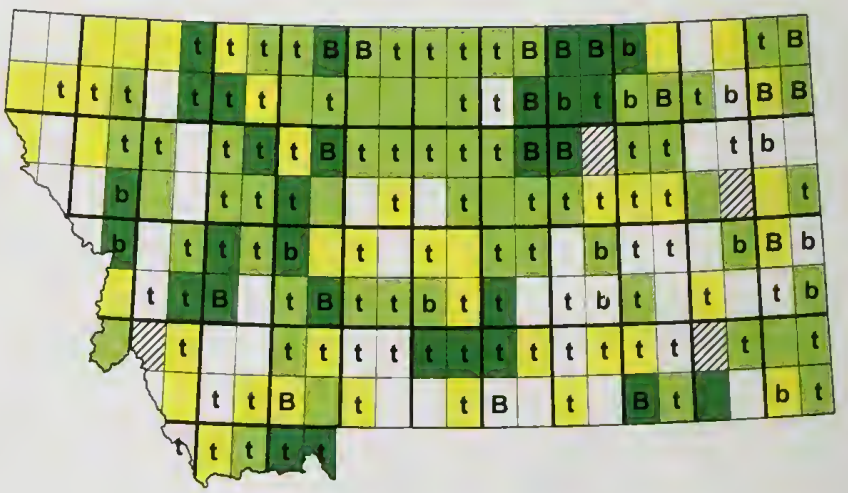


Eurasian Wigeon

Anas penelope

2003-2011 = 46
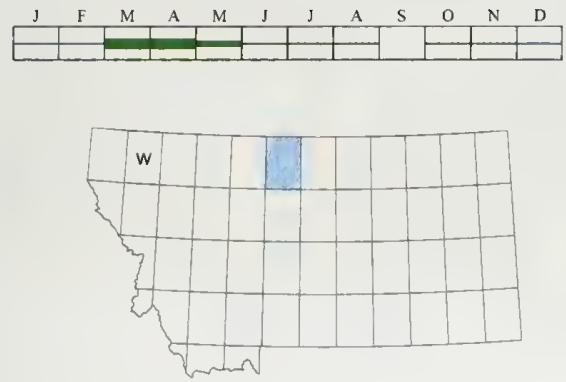

\section{American Wigeon}

Anas americana

2003-2011 = 1302
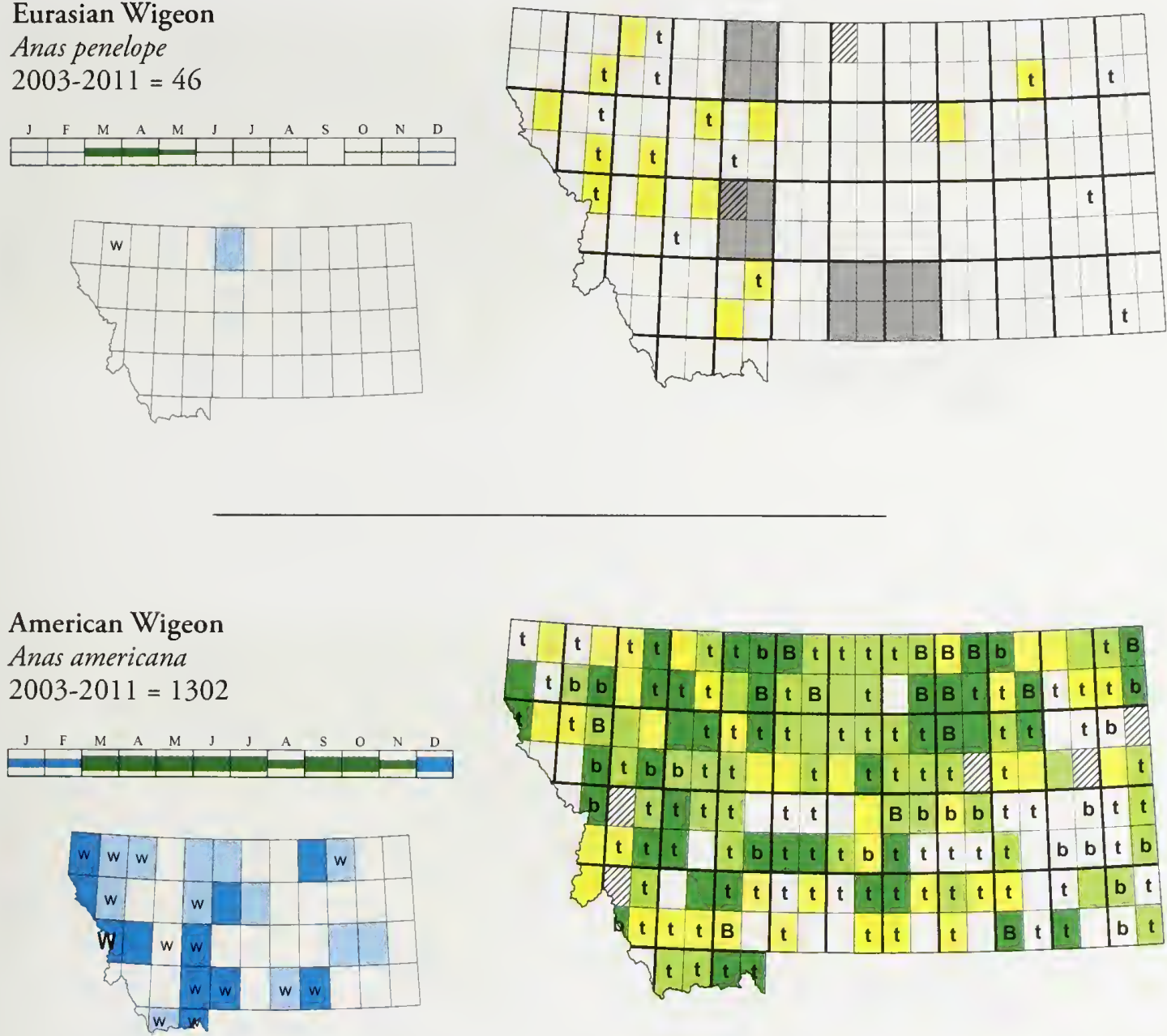

American Black Duck

Anas rubripes

2003-2011 = 3
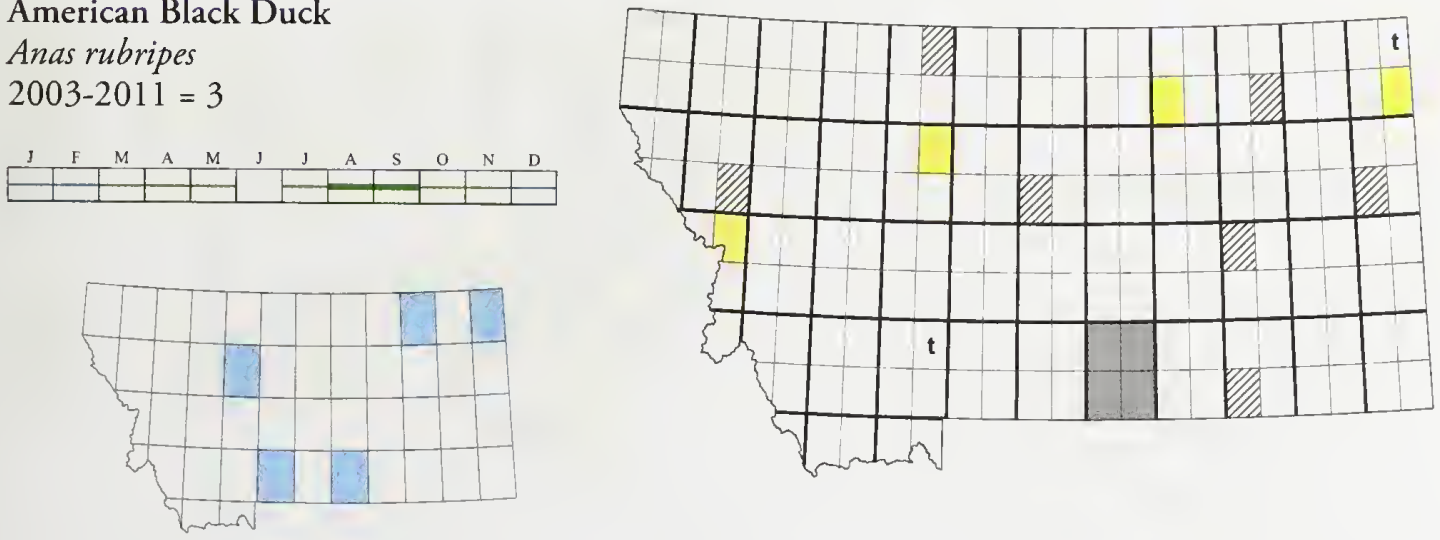
Mallard

Anas platyrhynchos

2003-2011 = 3975

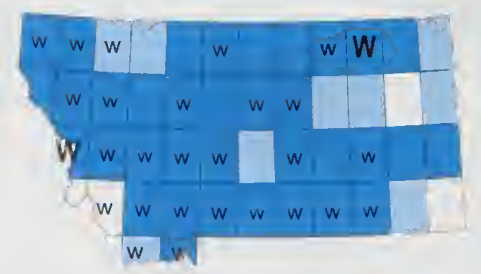

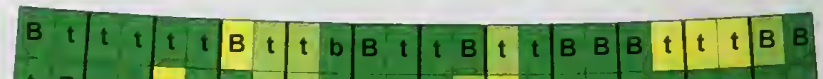

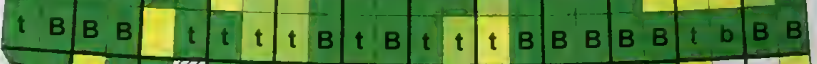

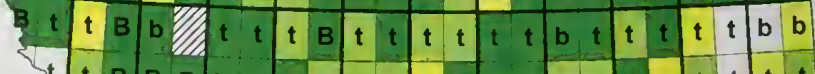

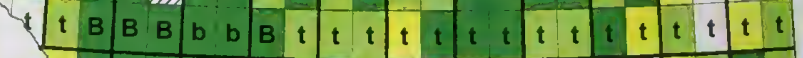

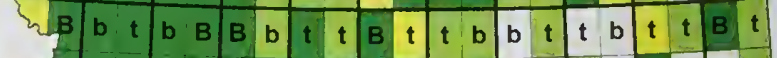

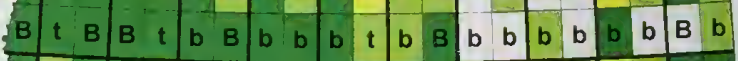

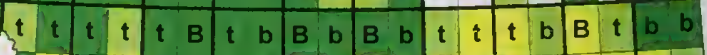

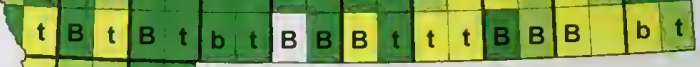
$t t a b y$
Blue-winged Teal

Anas discors

2003-2011 = 992
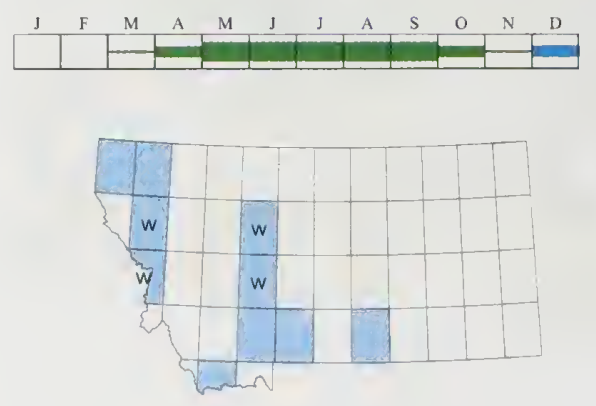

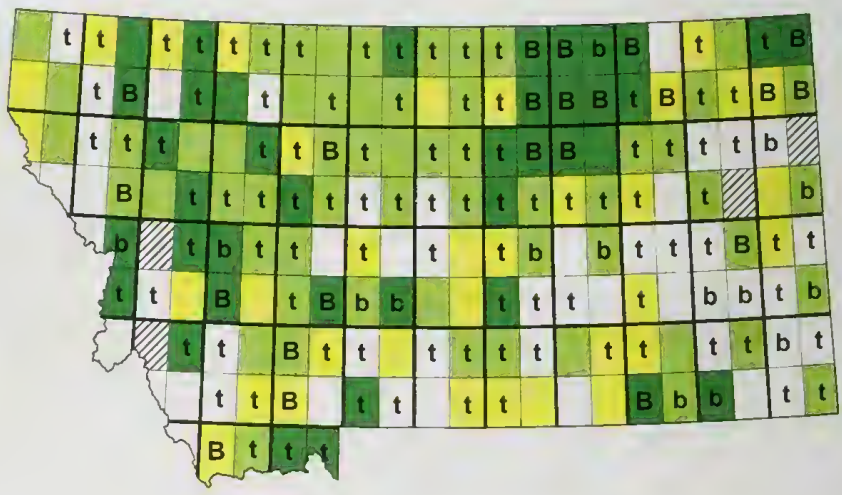

Cinnamon Teal

Anas cyanoptera

2003-2011 = 619

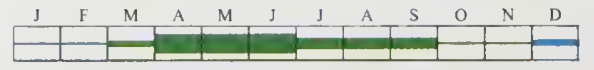

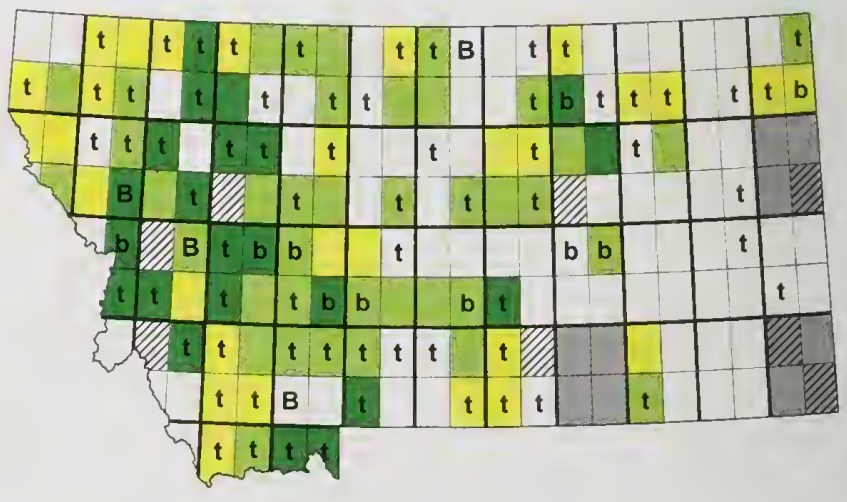


Northern Shoveler

Anas clypeata

$2003-2011=1251$
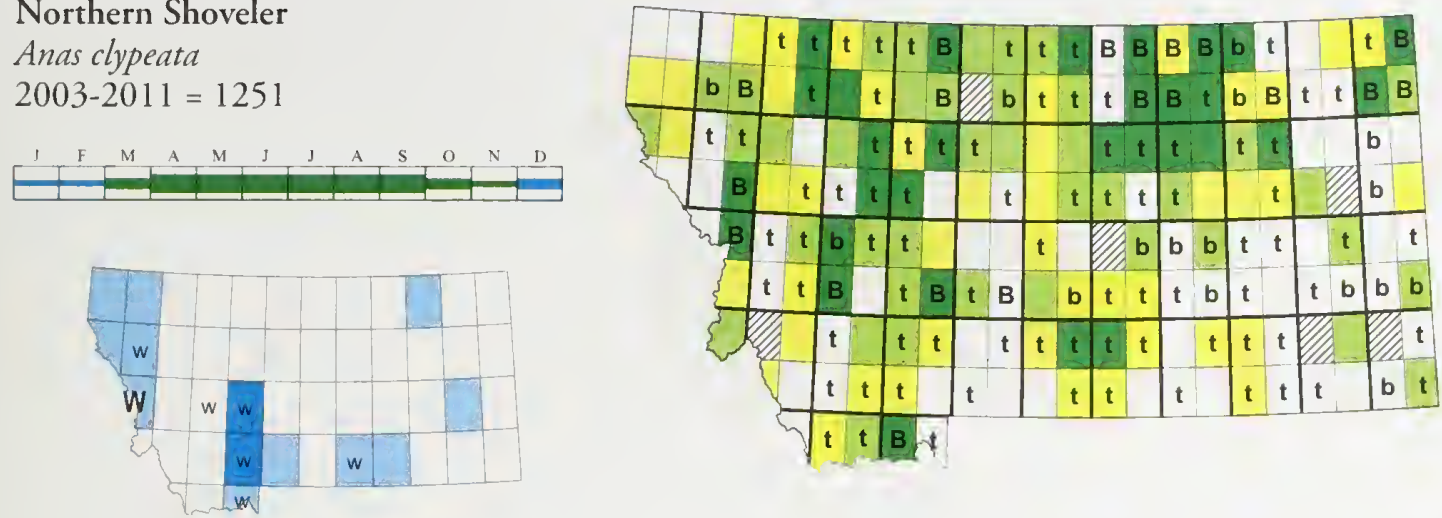

Northern Pintail

Anas acuta

$2003-2011=852$
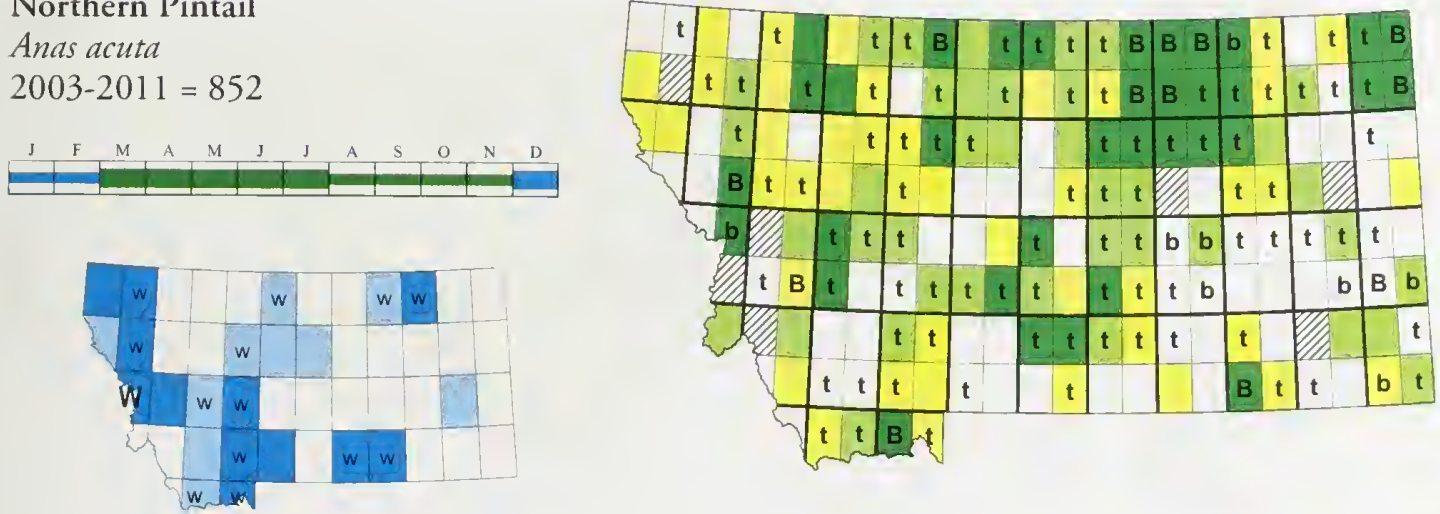

Garganey

Anas querquedula

2003-2011 = 0 R1

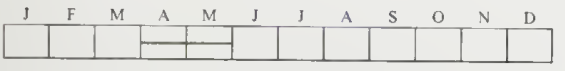

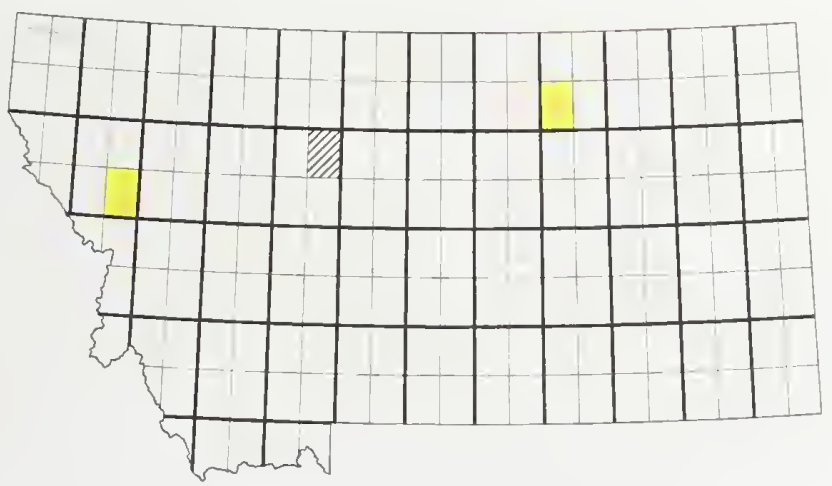




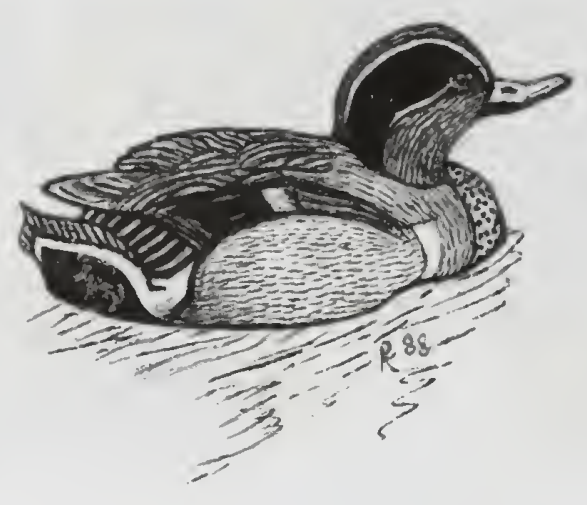

Green-winged Teal

Anas crecca

2003-2011 $=667$
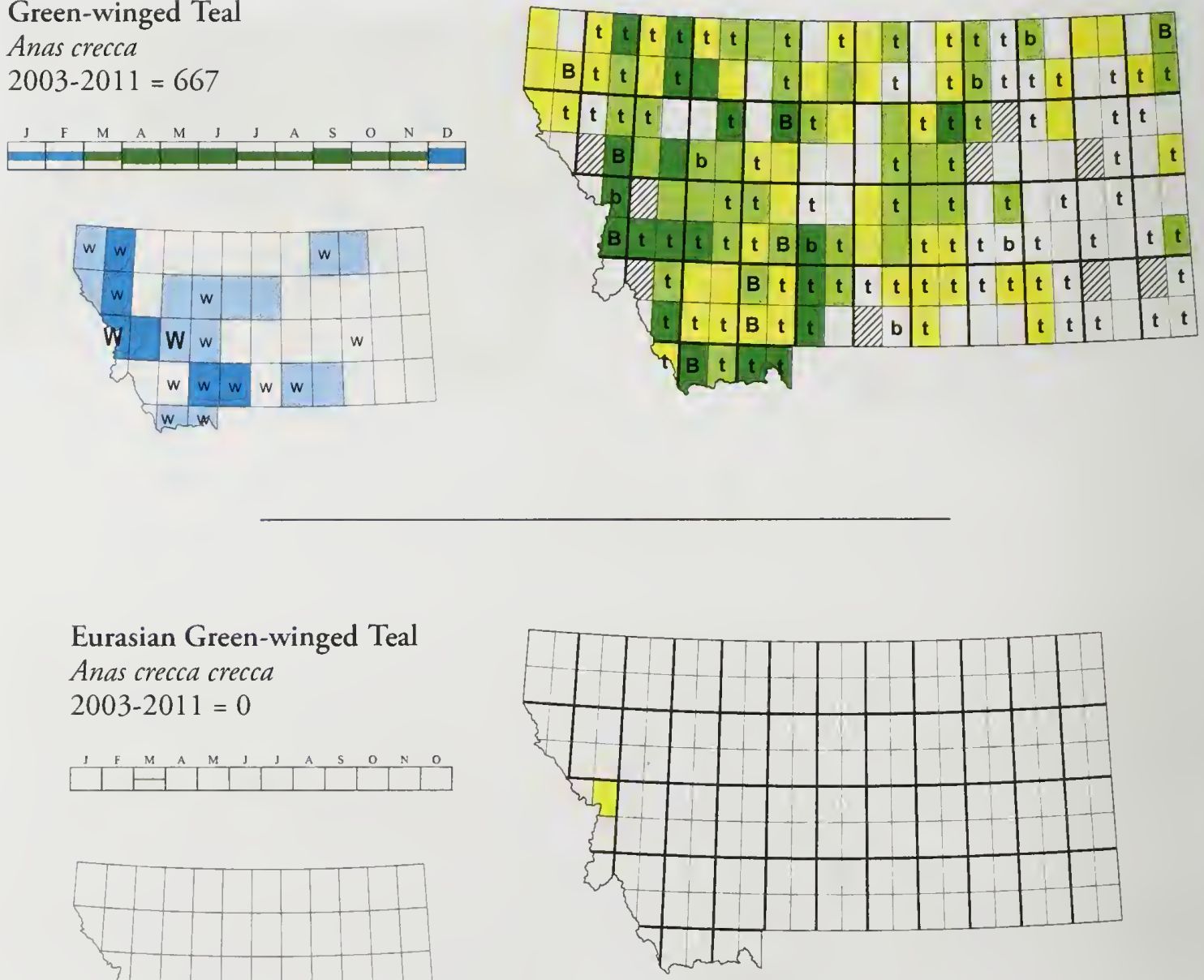

Eurasian Green-winged Teal Anas crecca crecca

2003-2011 = 0
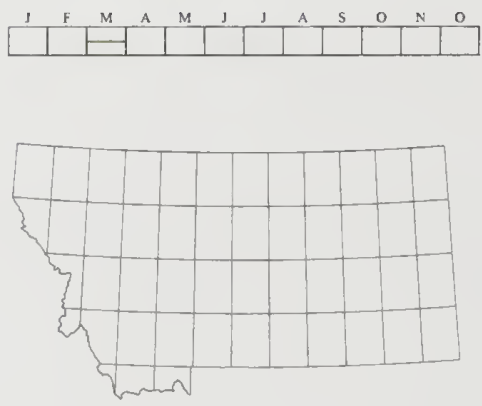
Canvasback

Aythya valisineria

$2003-2011=720$
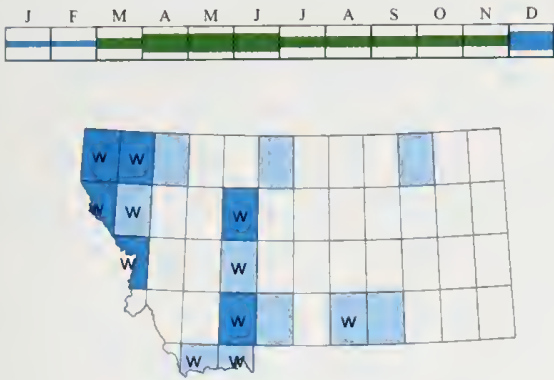

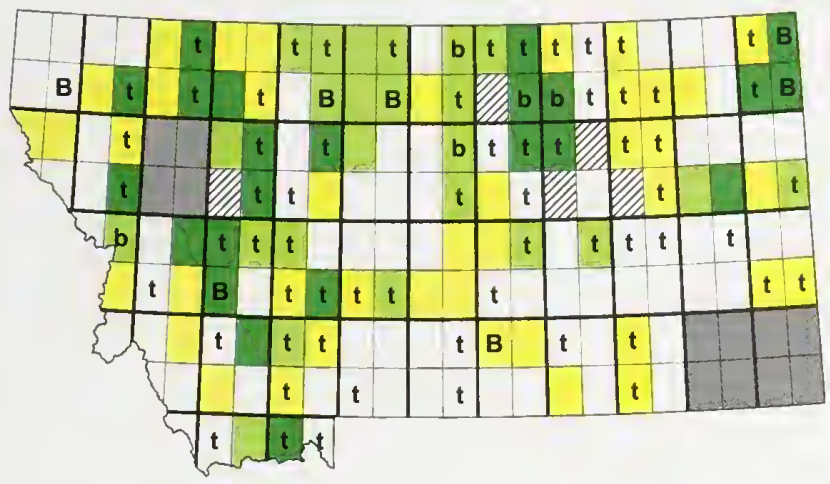

Redhead

Aythya americana

2003-2011 = 909
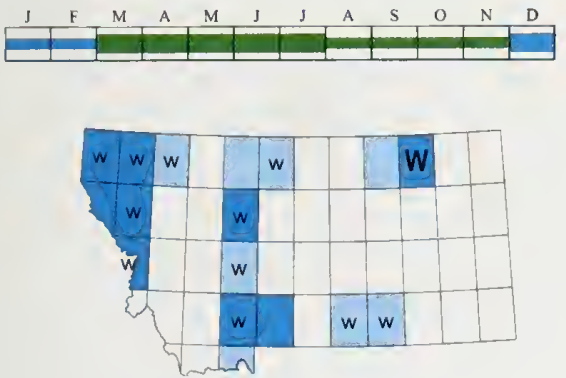

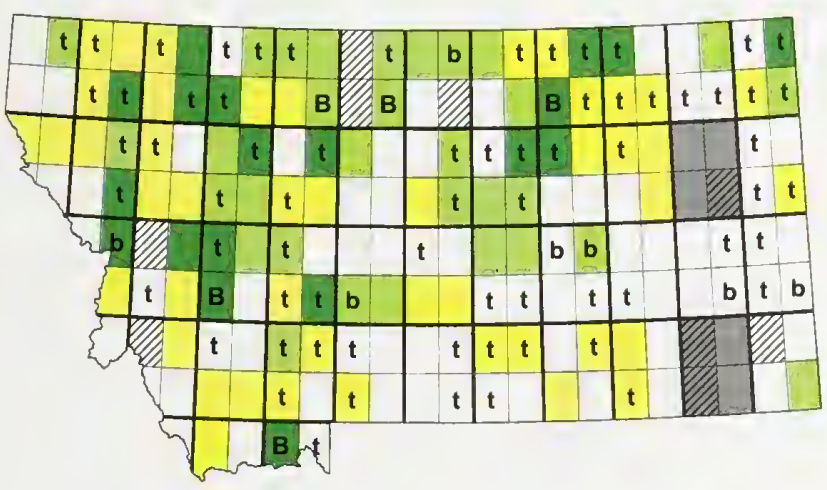

Ring-necked Duck

Aythya collaris

2003-2011 $=624$
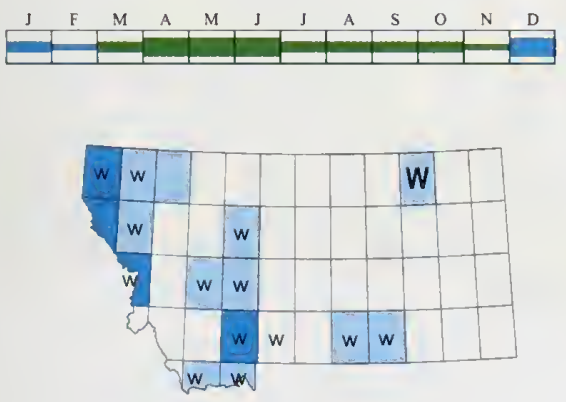

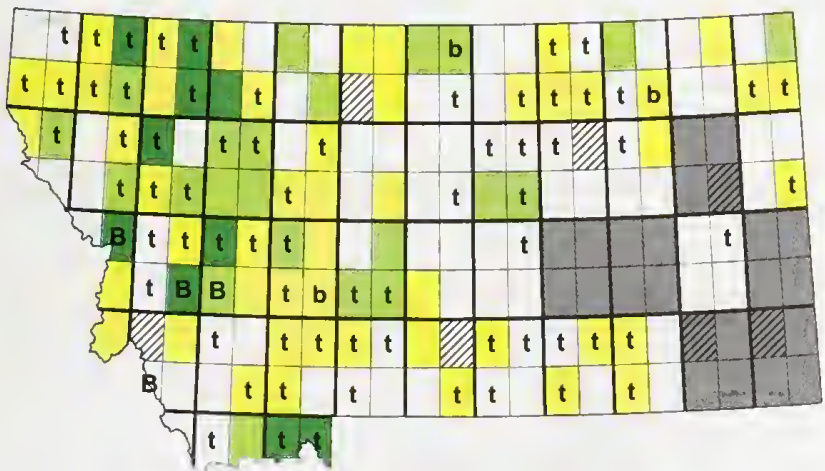


Tufted Duck

Aythya fuligula

2003-2011 = 2 R1

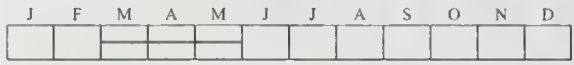

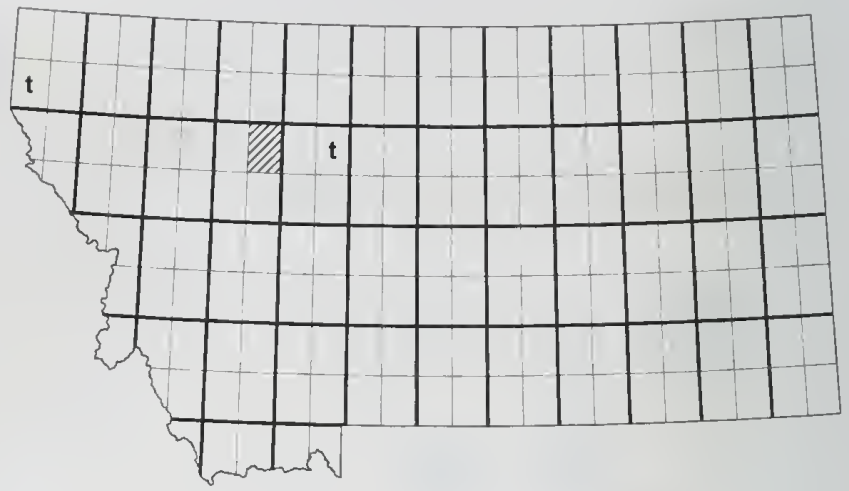

\section{Greater Scaup}

Aythya marila

2003-2011 = 197
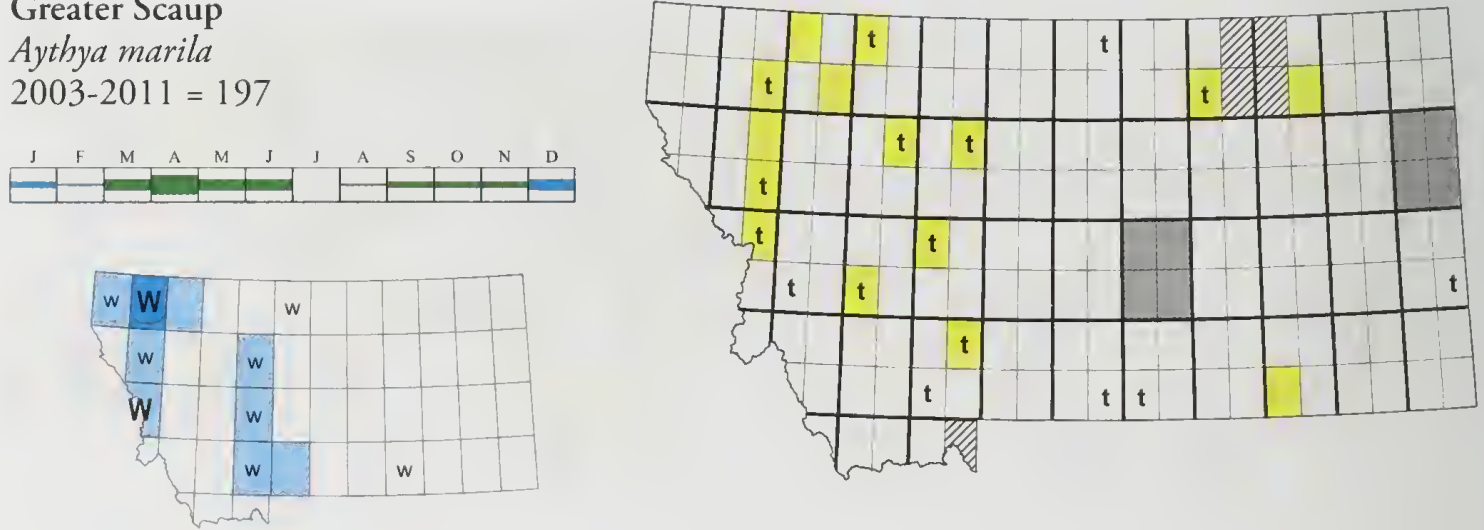

\section{Lesser Scaup}

Aythya affinis

$2003-2011=1076$
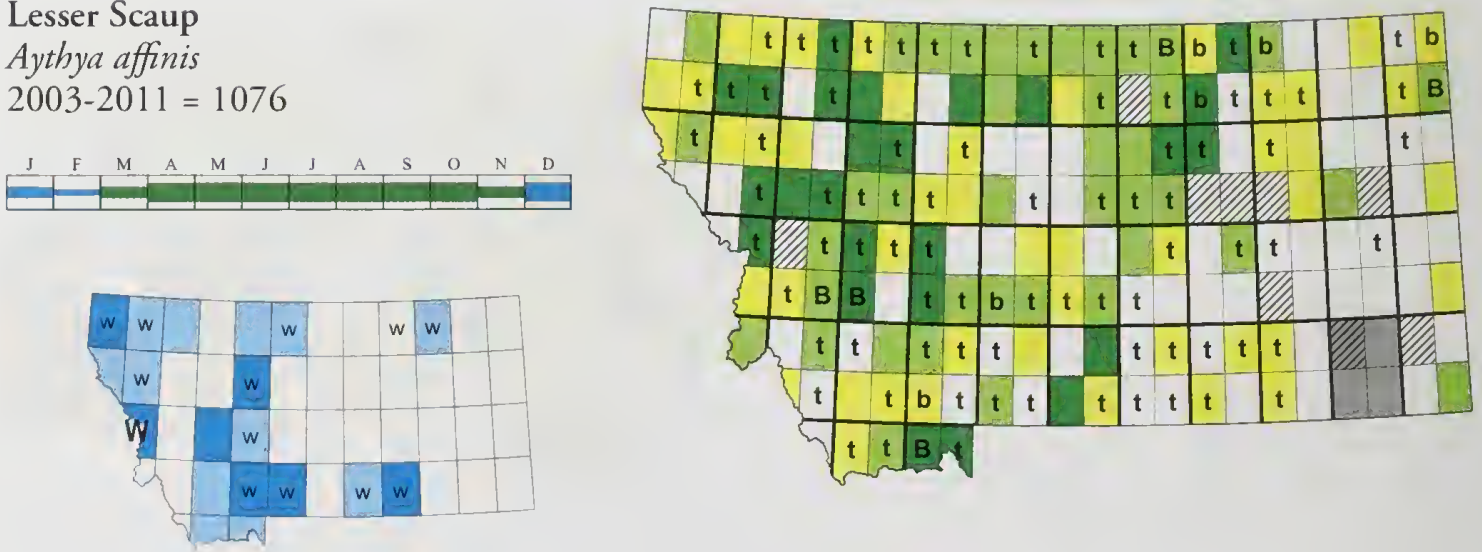
Harlequin Duck

Histrionicus histrionicus

2003-2011 = 145 SOC
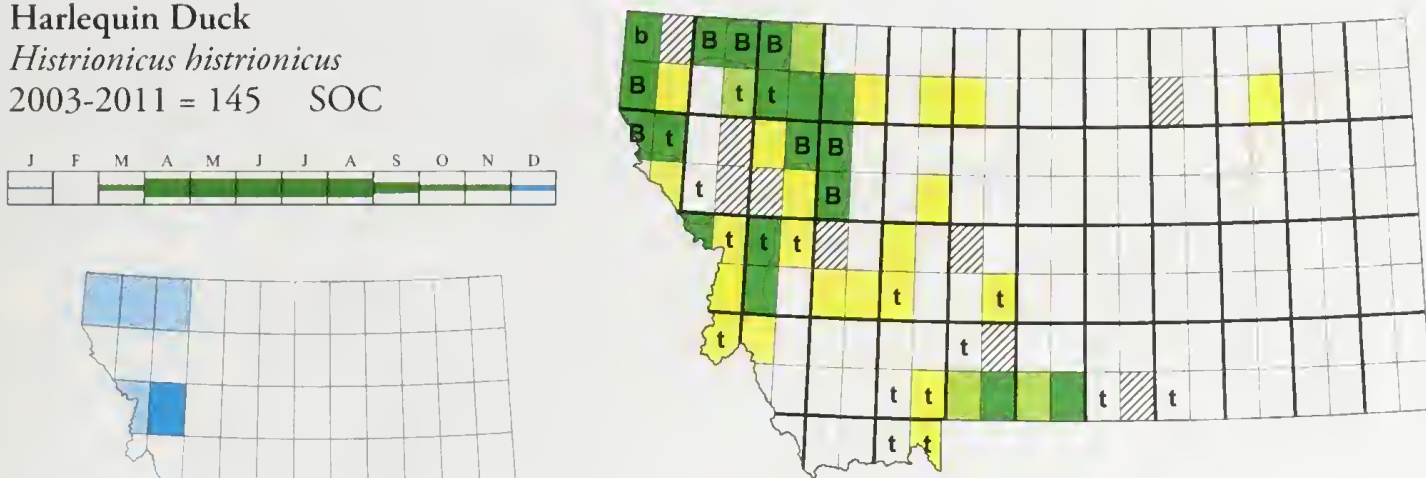

\section{Surf Scoter}

Melanitta perspicillata

2003-2011 = 30
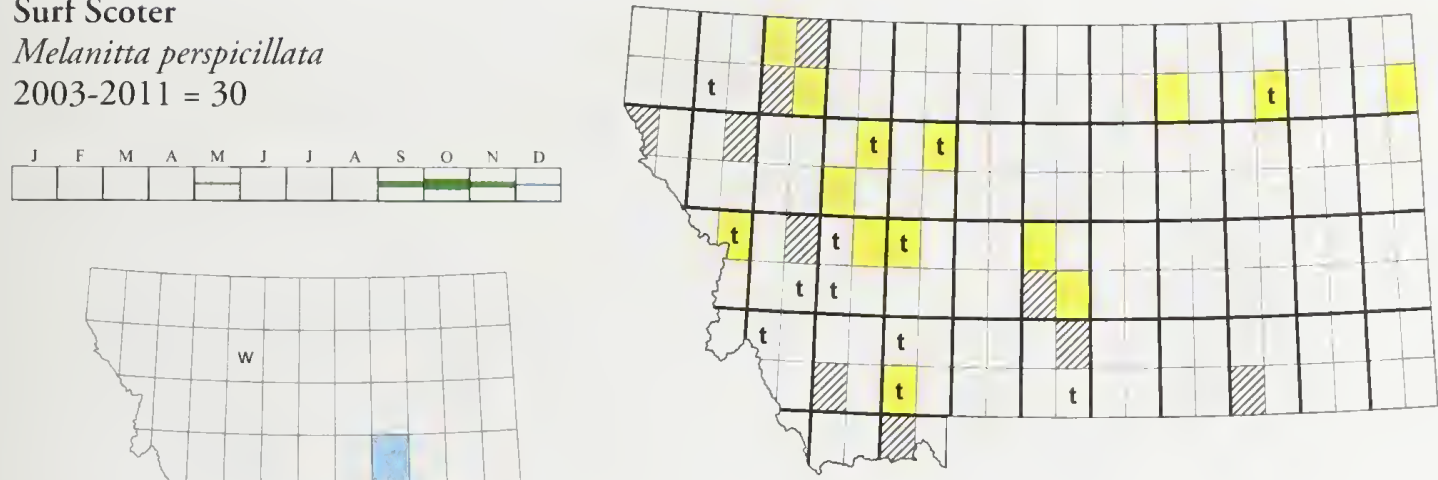

White-winged Scoter

Melanitta fusca

2003-2011 = 19
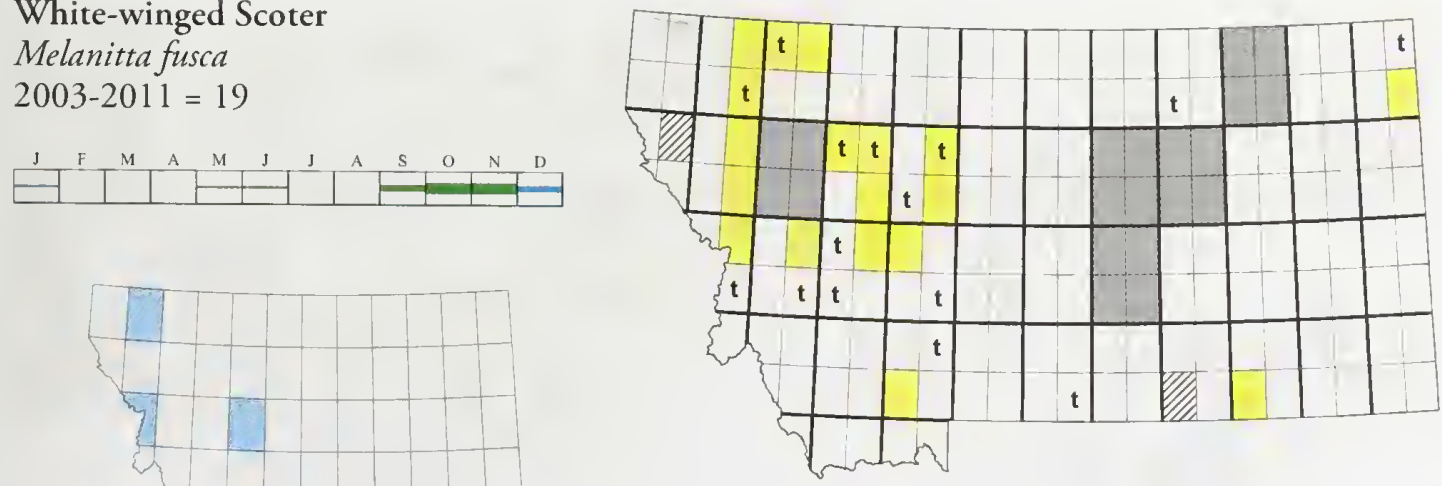
Black Scoter

Melanitta americana

2003-2011 = 9 R1

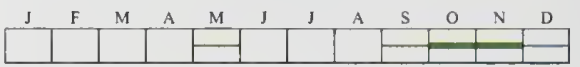
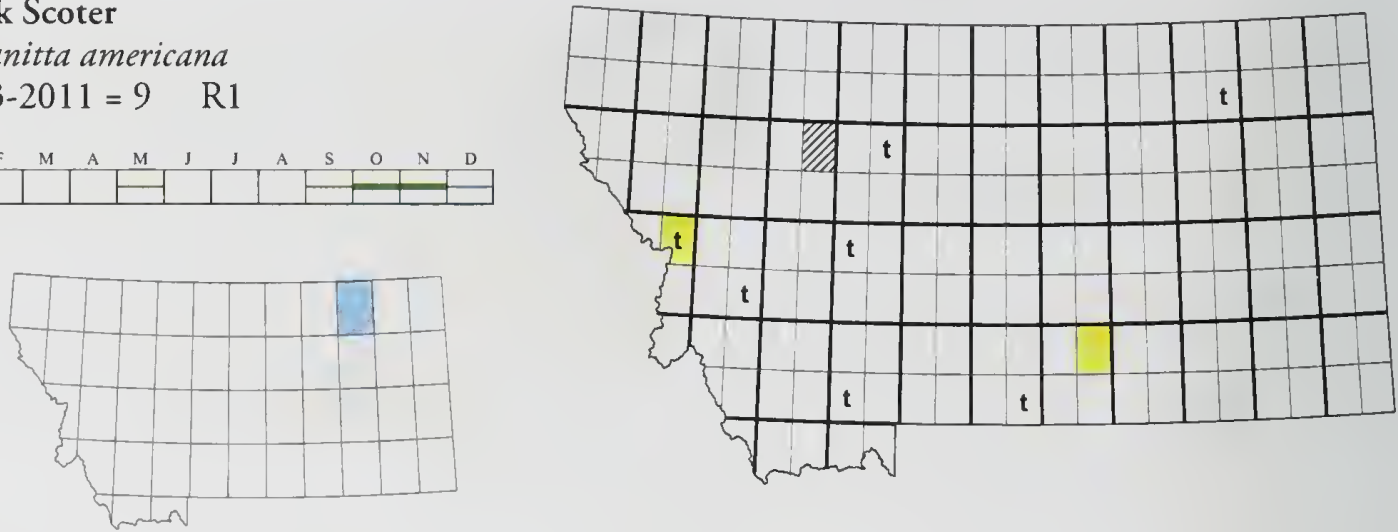

Long-tailed Duck

Clangula hyemalis

2003-2011 = 42
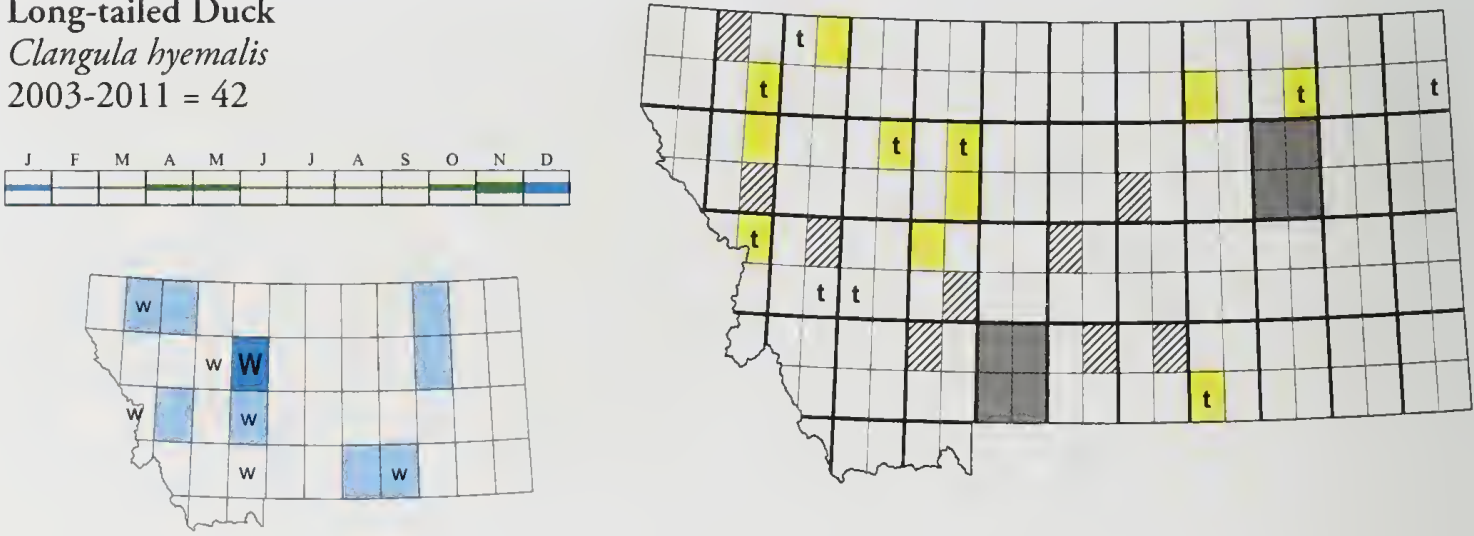

Bufflehead

Bucephala albeola

2003-2011 = 779
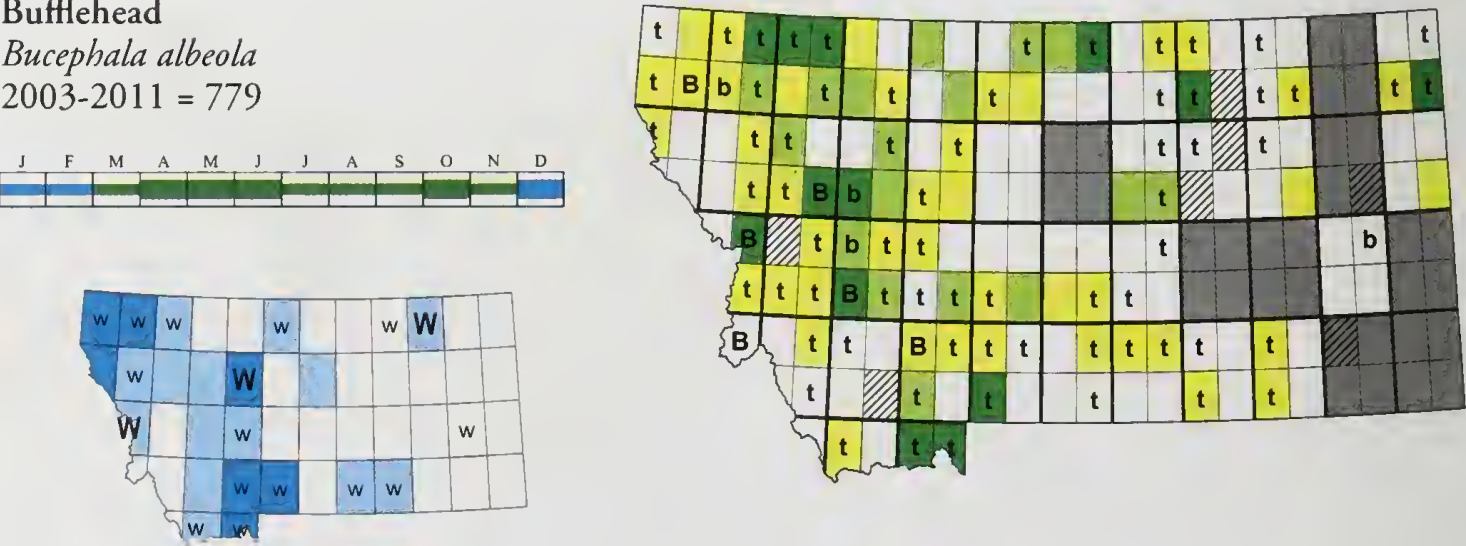
Common Goldeneye

Bucephala clangula

2003-2011 = 936
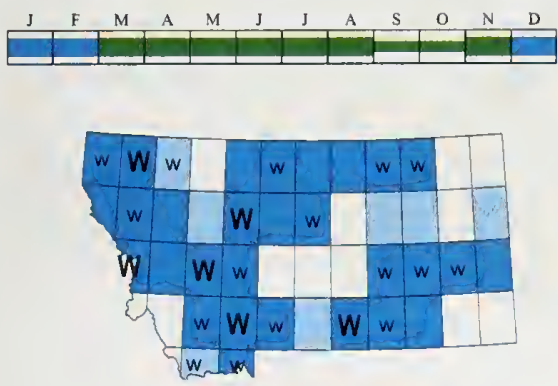

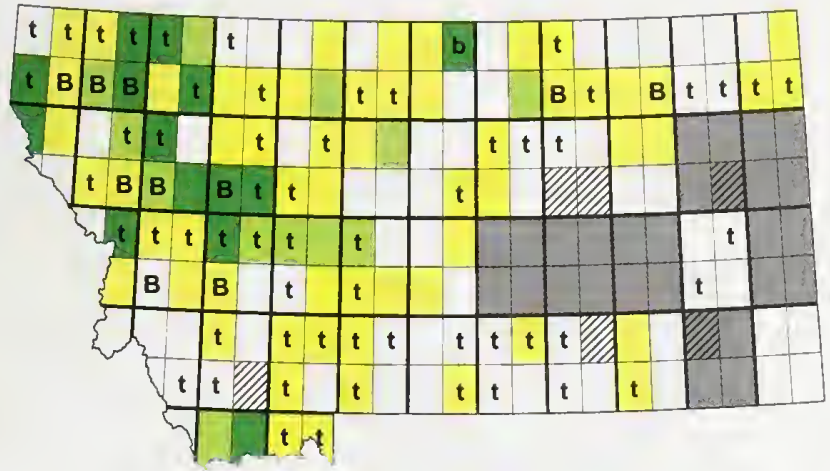

Barrow's Goldeneye

Bucephala islandica

2003-2011 = 369 SI
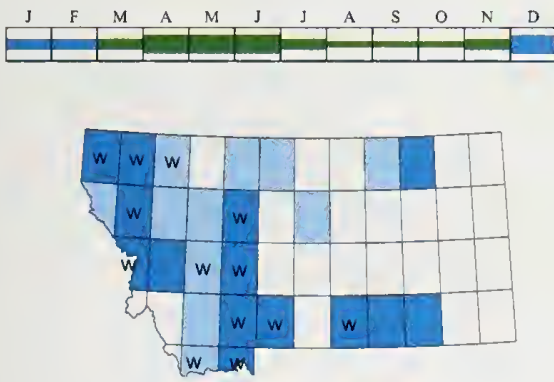

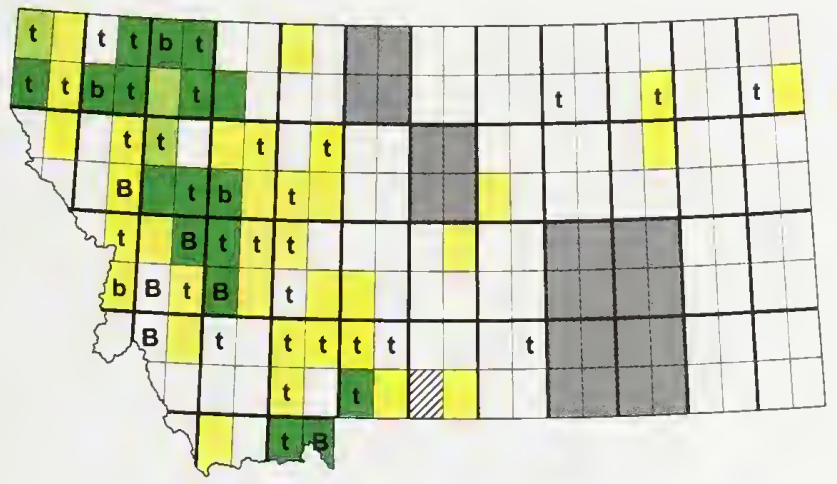

Hooded Merganser

Lophodytes cucullatus

$2003-2011=323$ SI
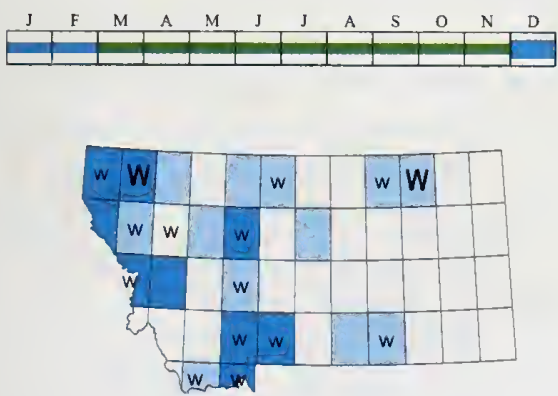

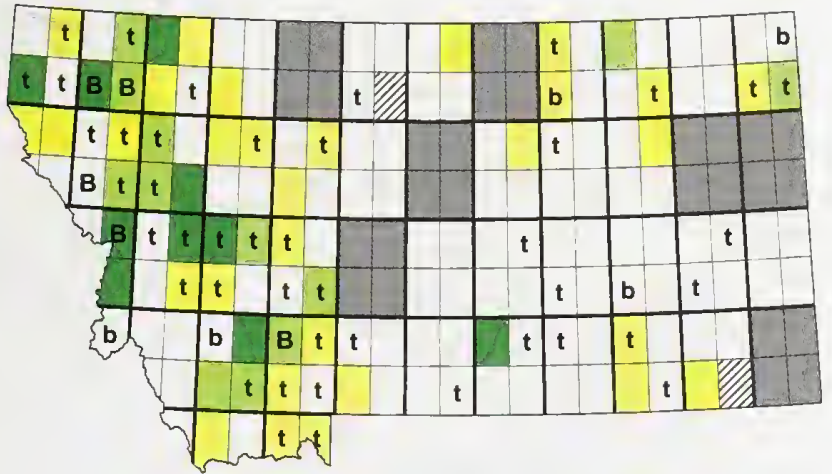


Common Merganser

Mergus merganser

$2003-2011=950$

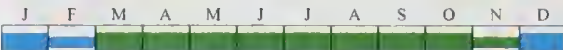

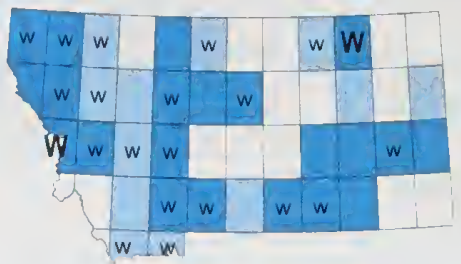

Red-breasted Merganser

Mergus serrator

2003-2011 = 98
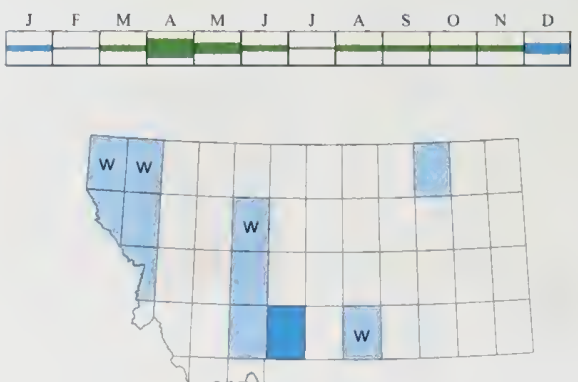

Ruddy Duck

Oxyura jamaicensis

2003-2011 = 786
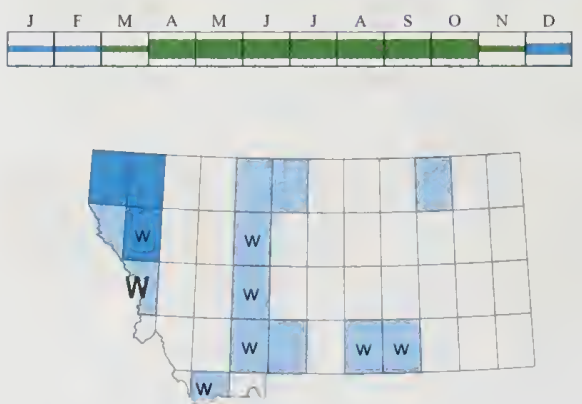
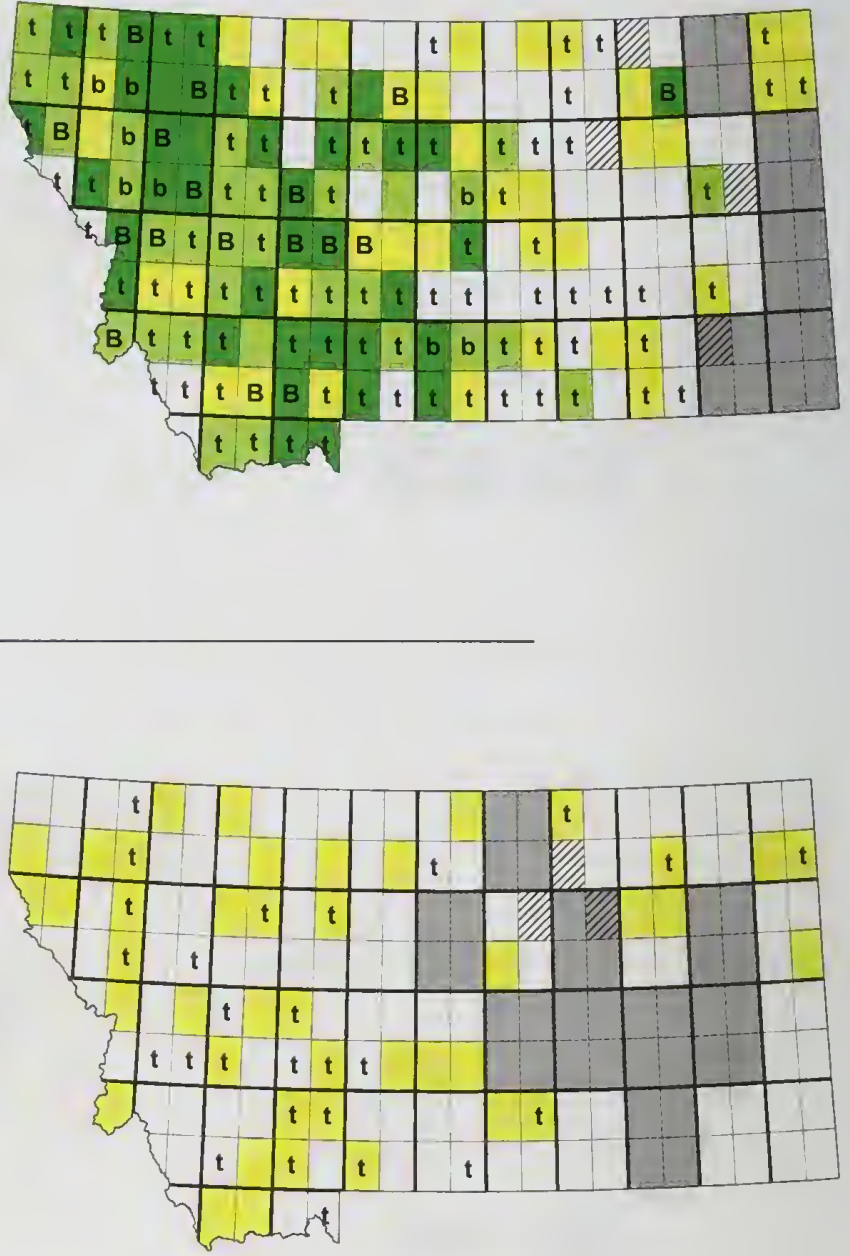

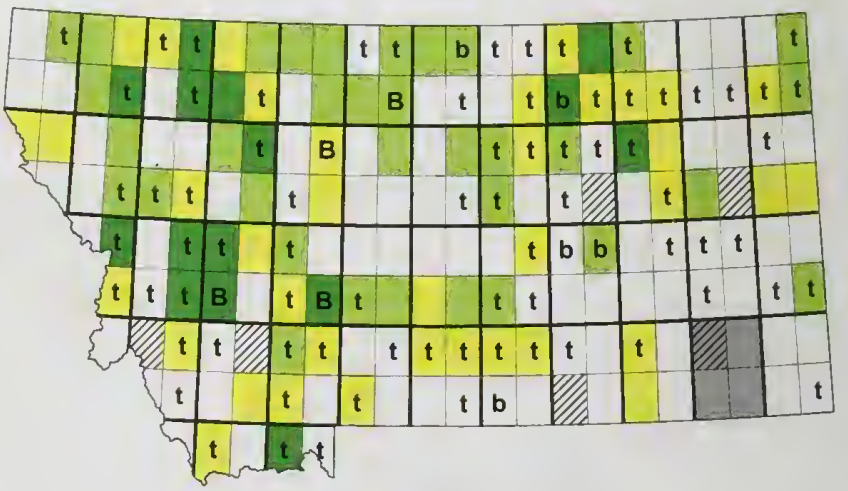




\section{Chukar}

Alectoris chukar

2003-2011 = 21
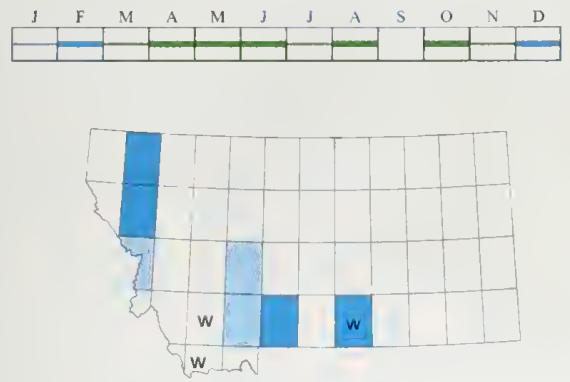

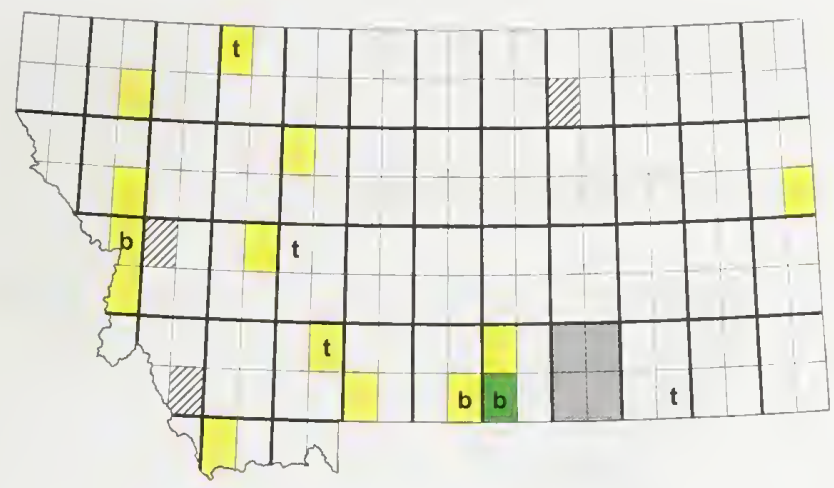

Gray Partridge

Perdix perdix

2003-2011 = 513
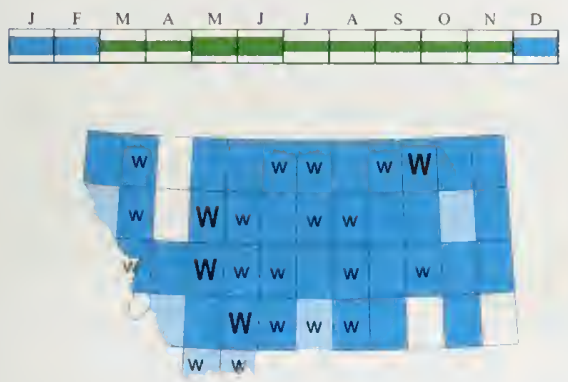

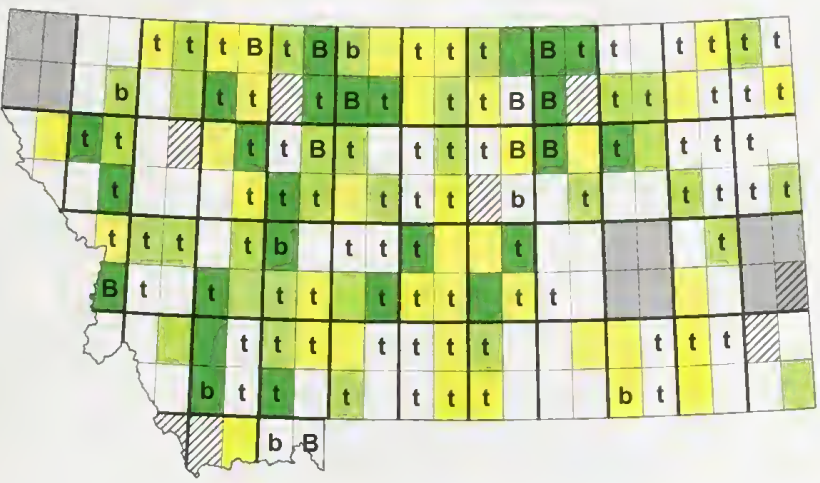

Ring-necked Pheasant

Phasianus colchicus

2003-2011 = 2911
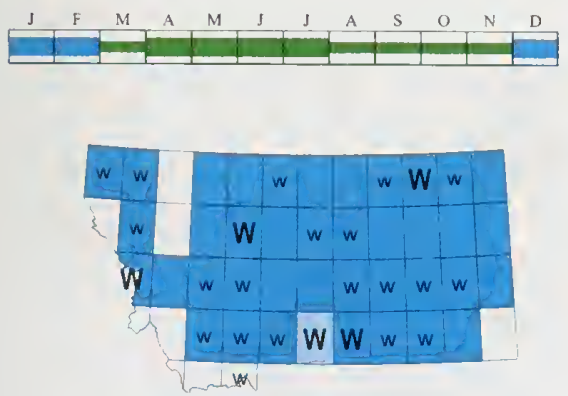

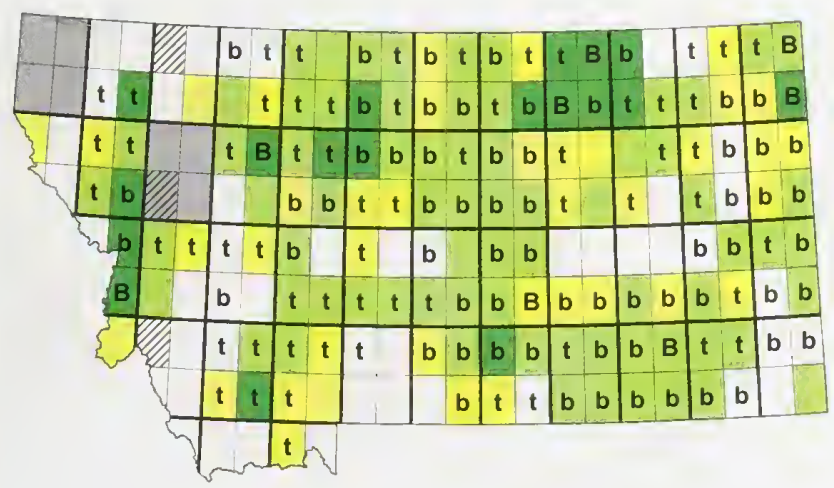


Ruffed Grouse

Bonasa umbellus

2003-2011 = 737
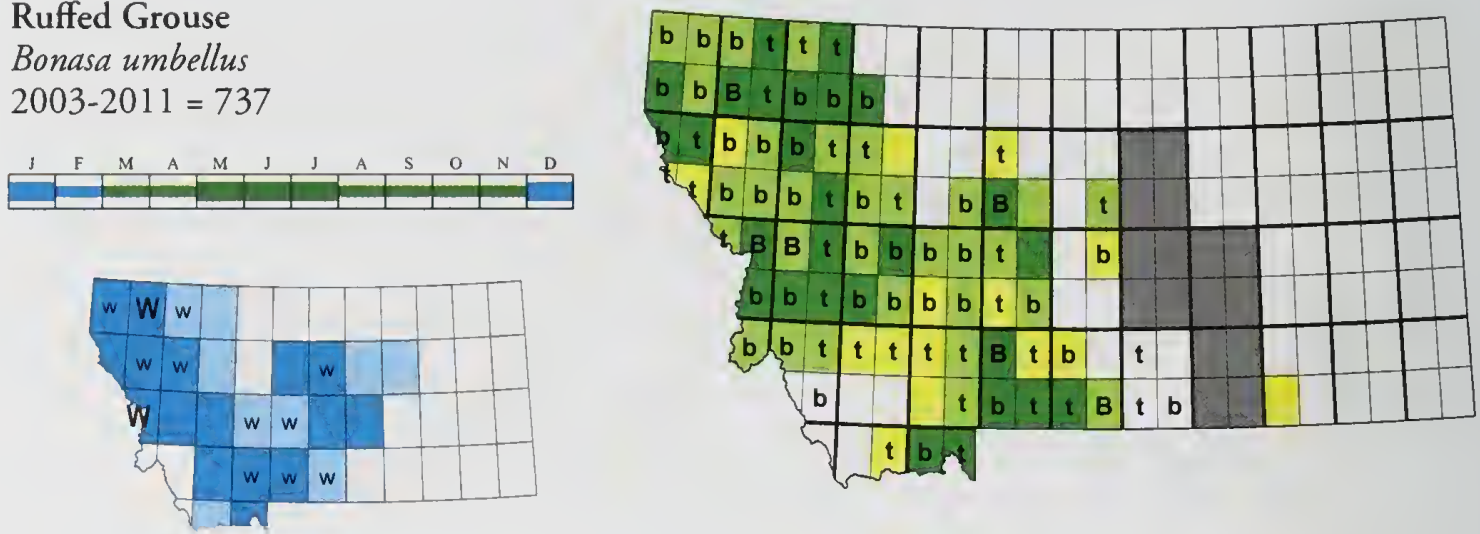

\section{Greater Sage-Grouse}

Centrocercus urophasianus

$2003-2011=4178 \quad \mathrm{C}$
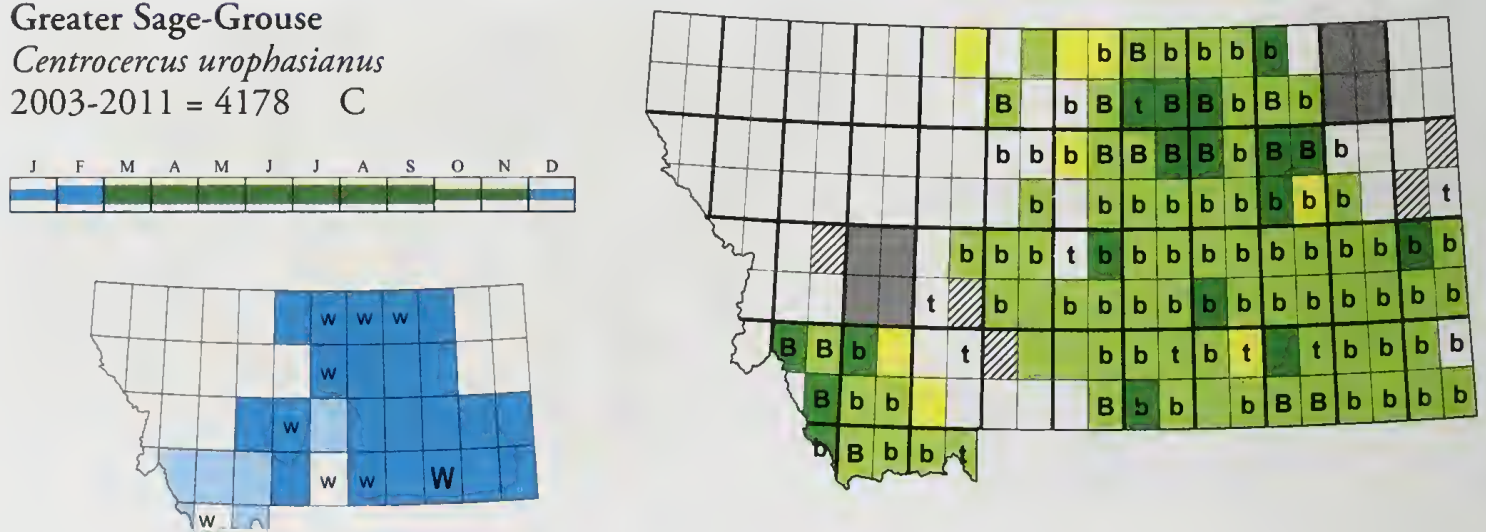

Spruce Grouse

Falcipennis canadensis

2003-2011 = 84
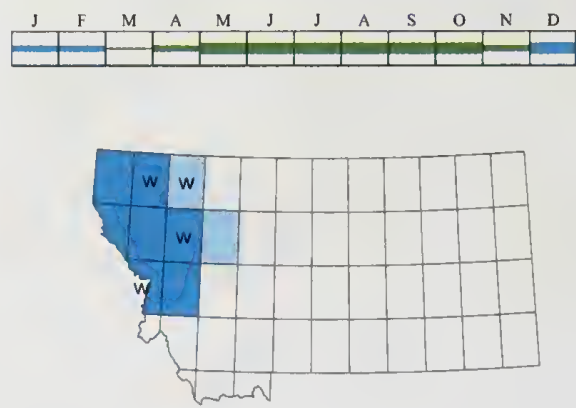

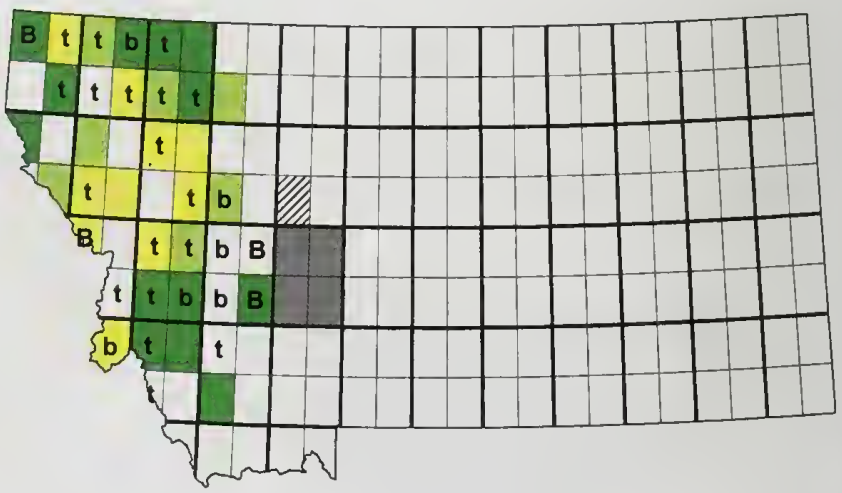


Willow Ptarmigan

Lagopus lagopus

2003-2011 = 0 R1

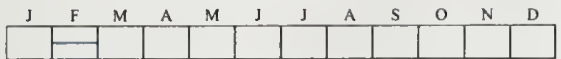
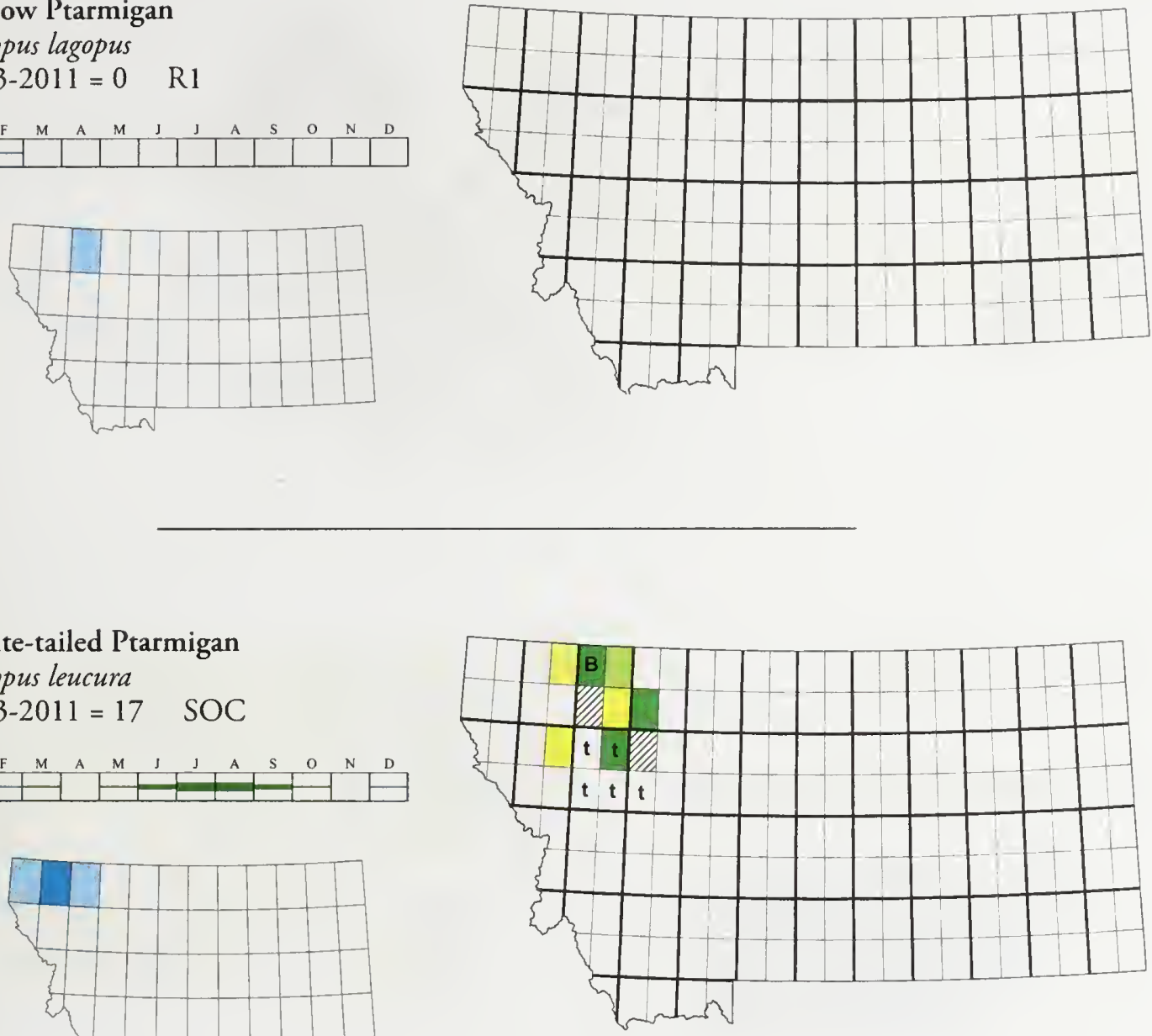

White-tailed Ptarmigan

Lagopus leucura

$2003-2011=17 \quad$ SOC

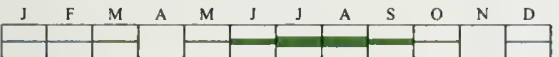

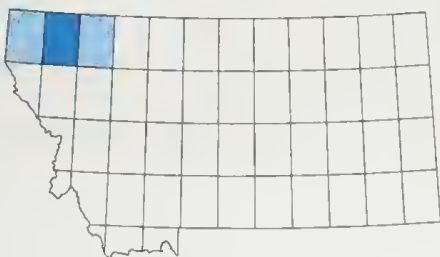

\section{Dusky Grouse}

Dendragapus obscurus

2003-2011 = 226
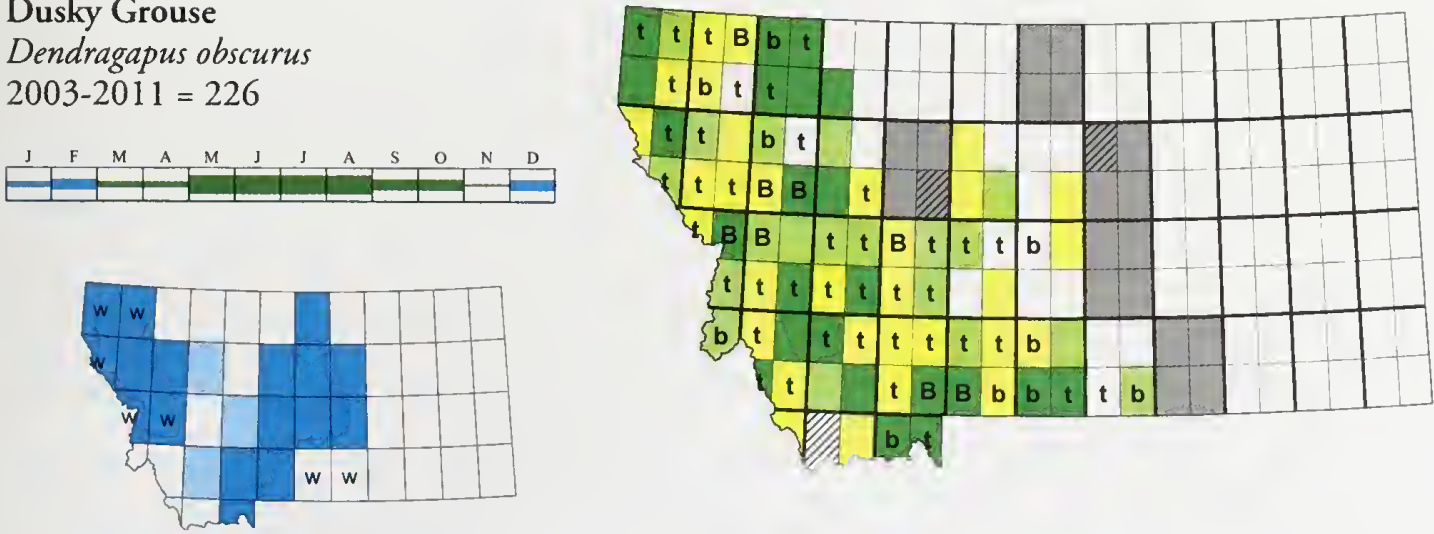


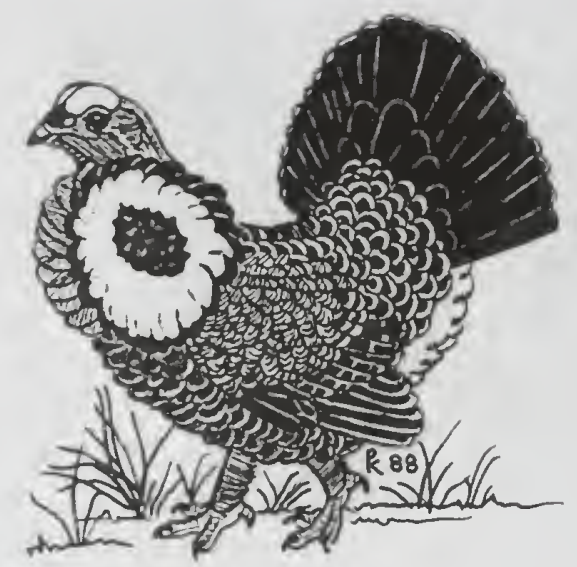

Sharp-tailed Grouse

Tympanuchus phasianellus

2003-2011 = 603
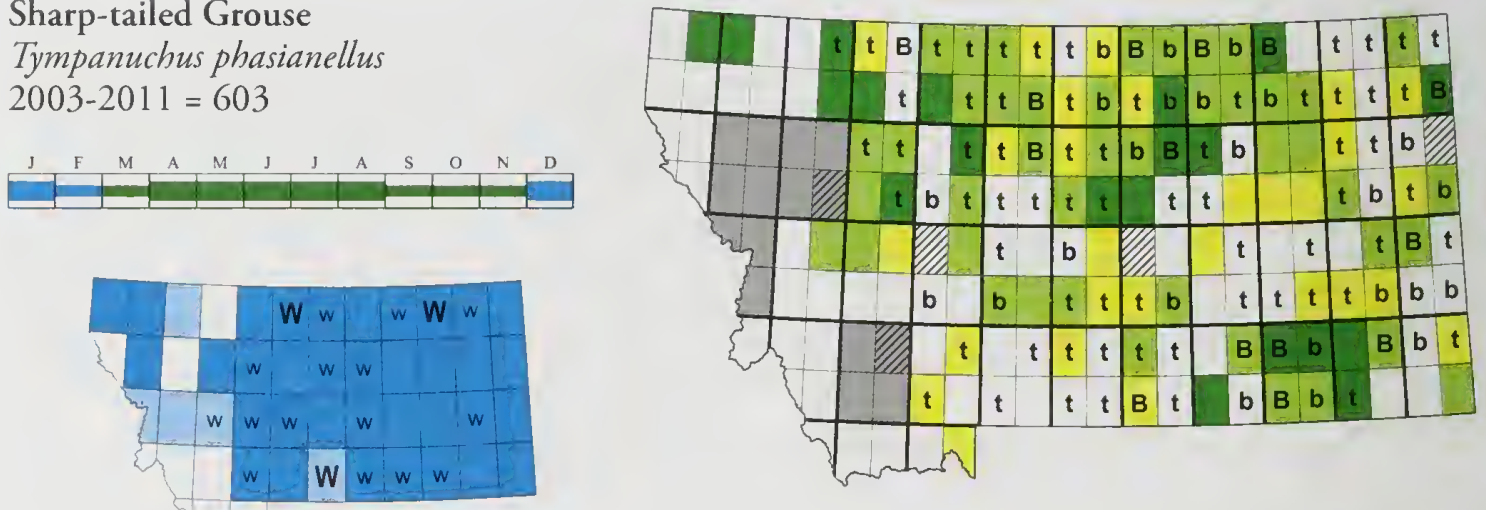

Greater Prairie-Chicken

Tympanuchus cupido

2003-2011 = 0 R1
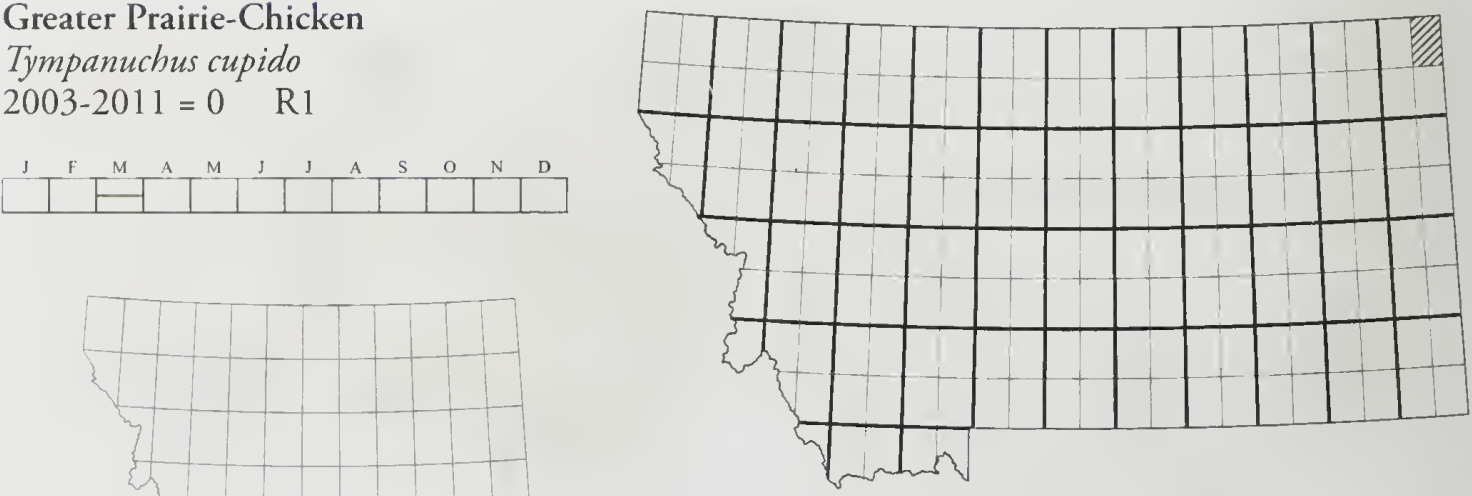
Wild Turkey

Meleagris gallopavo

2003-2011 = 614

J F M A M I J A S O N D

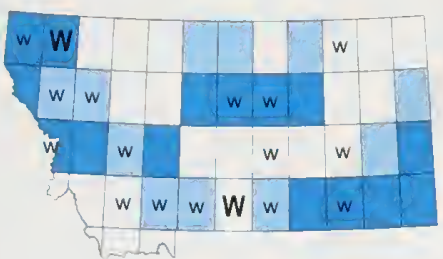

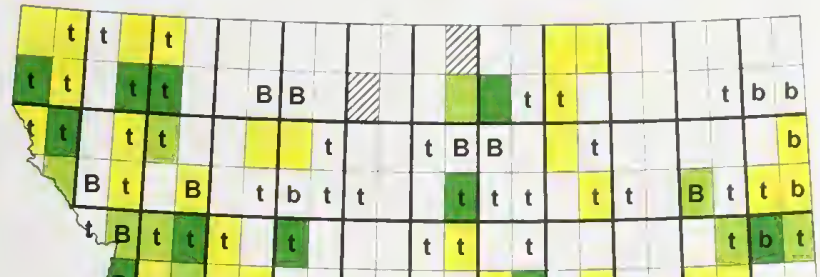

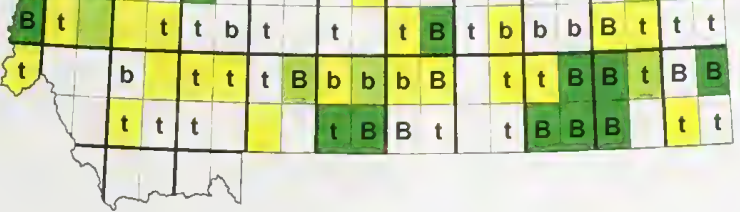

GAVIIFORMES Gaviidae

Red-throated Loon

Gavia stellata

2003-2011 = 5 R1
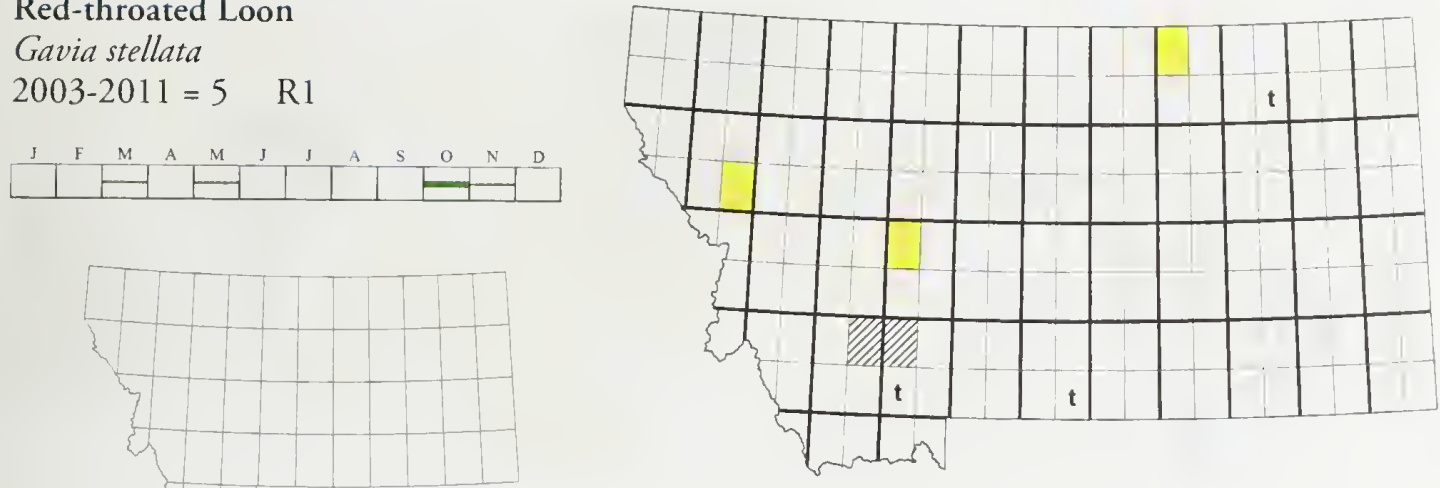

\section{Pacific Loon}

Gavia pacifica

2003-2011 = 18
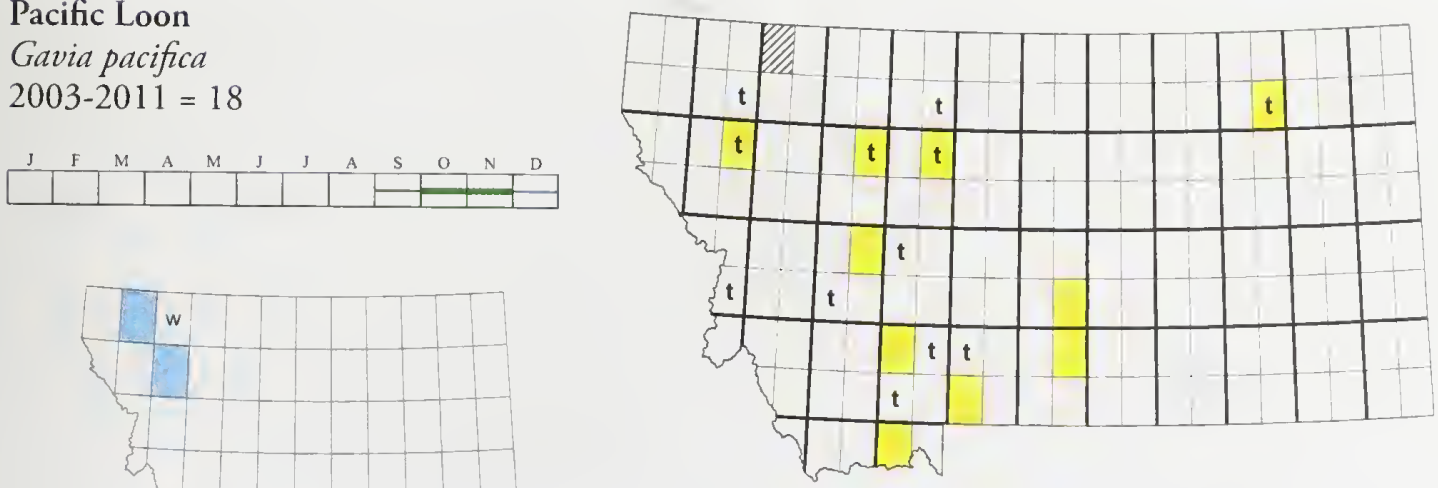


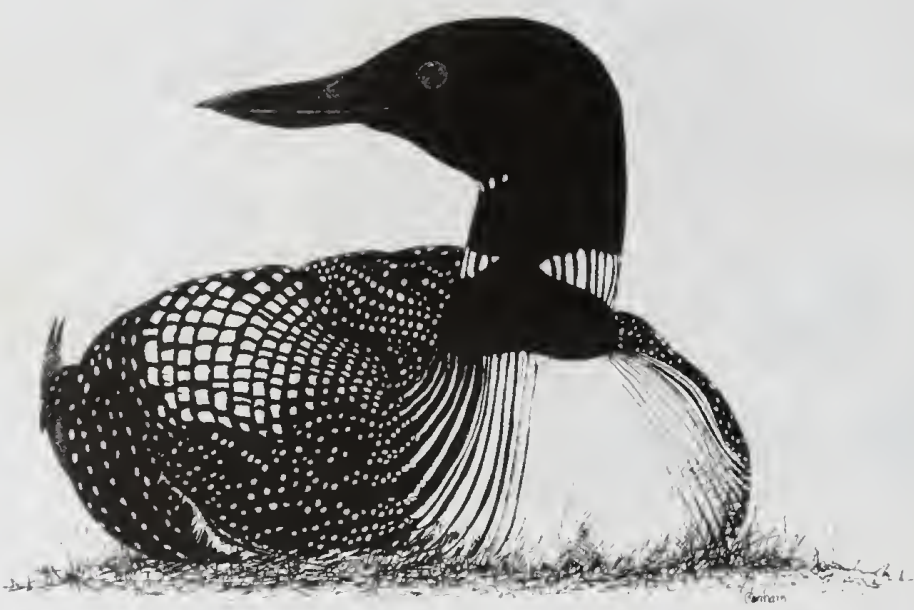

Common Loon

Gavia immer

2003-2011 = 1020 SOC
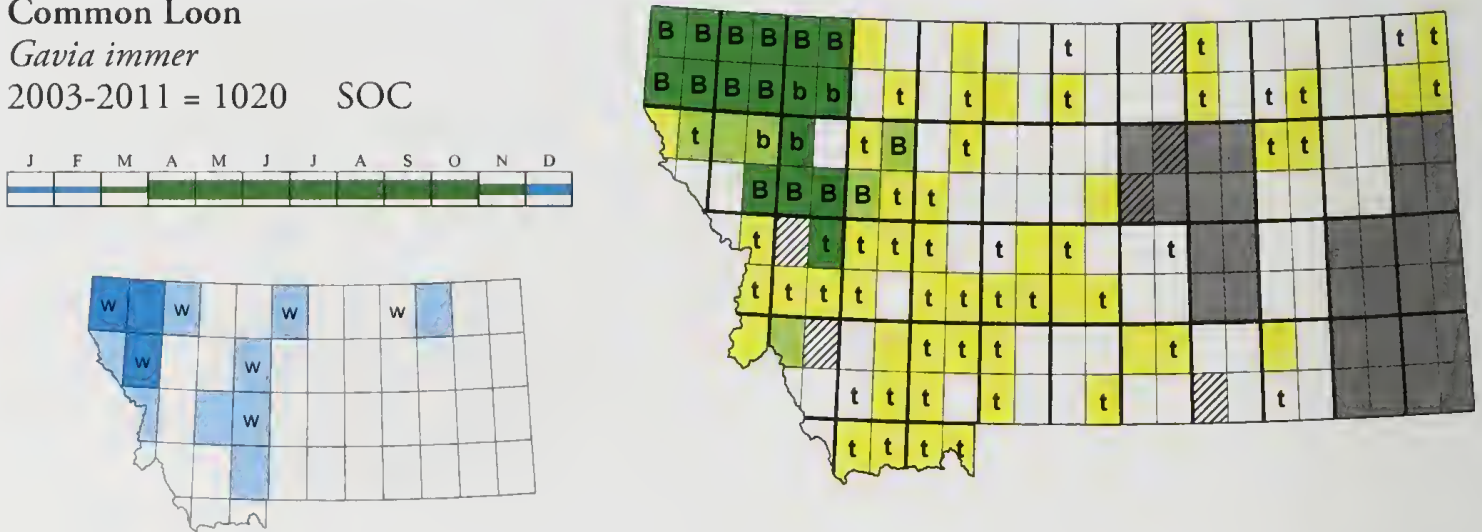

Yellow-billed Loon

Gavia adamsii

2003-2011 = $3 \quad$ R1
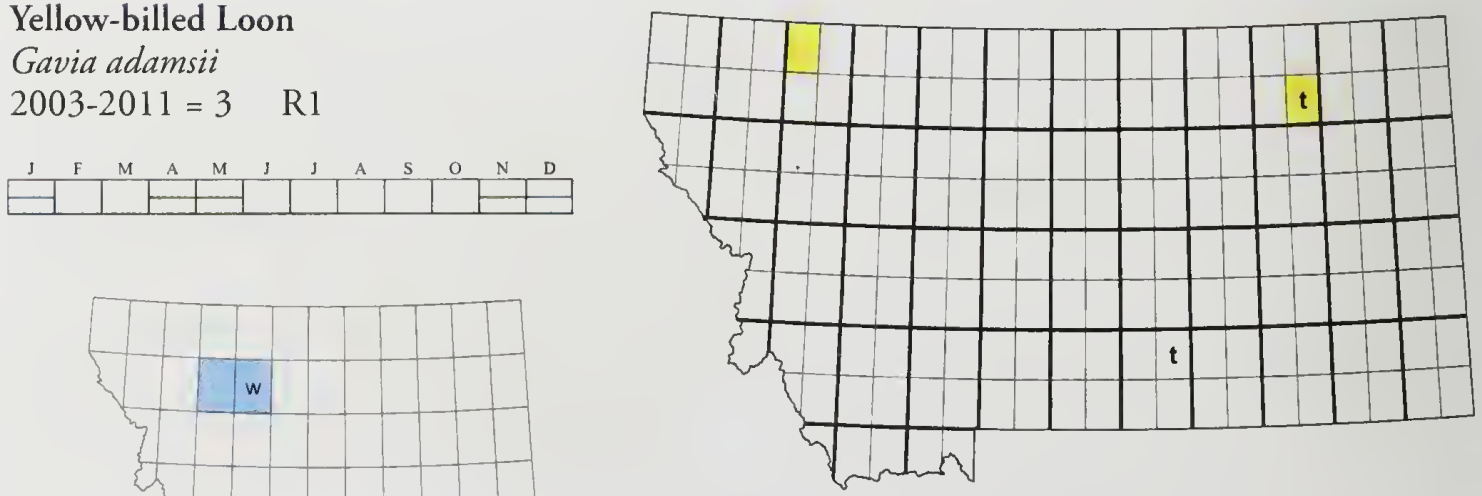
PODICIPEDIFORMES Podicipedidae

\section{Pied-billed Grebe}

Podilymbus podiceps

2003-2011 = 386
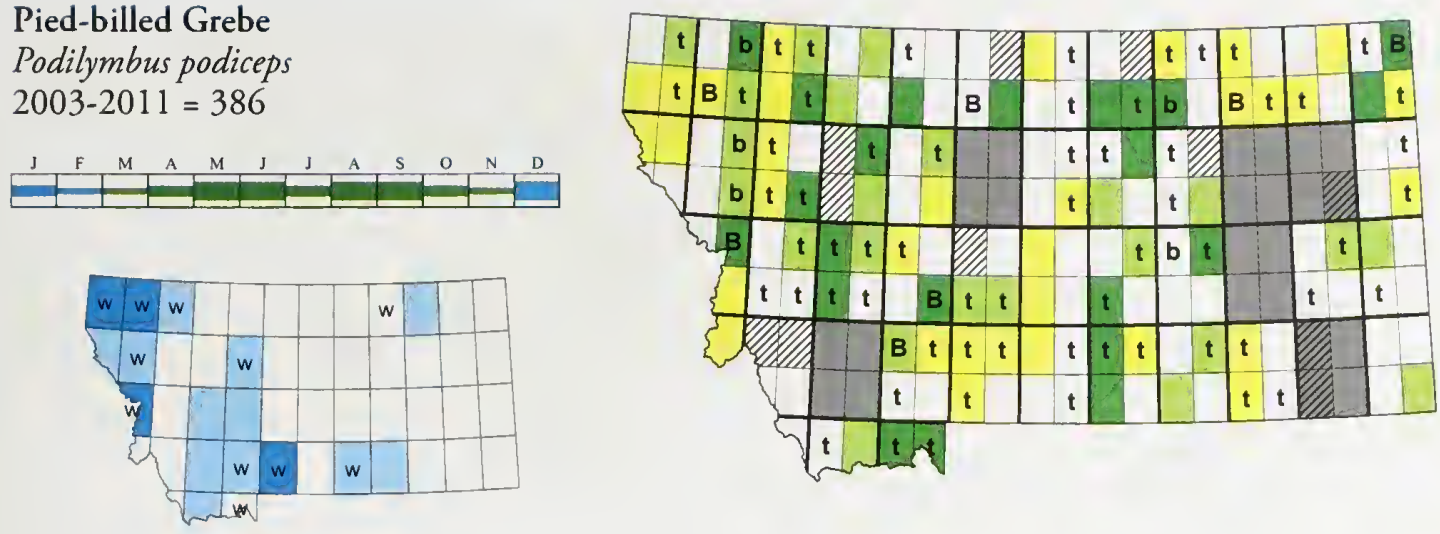

Horned Grebe

Podiceps auritus

2003-2011 = 163 SOC
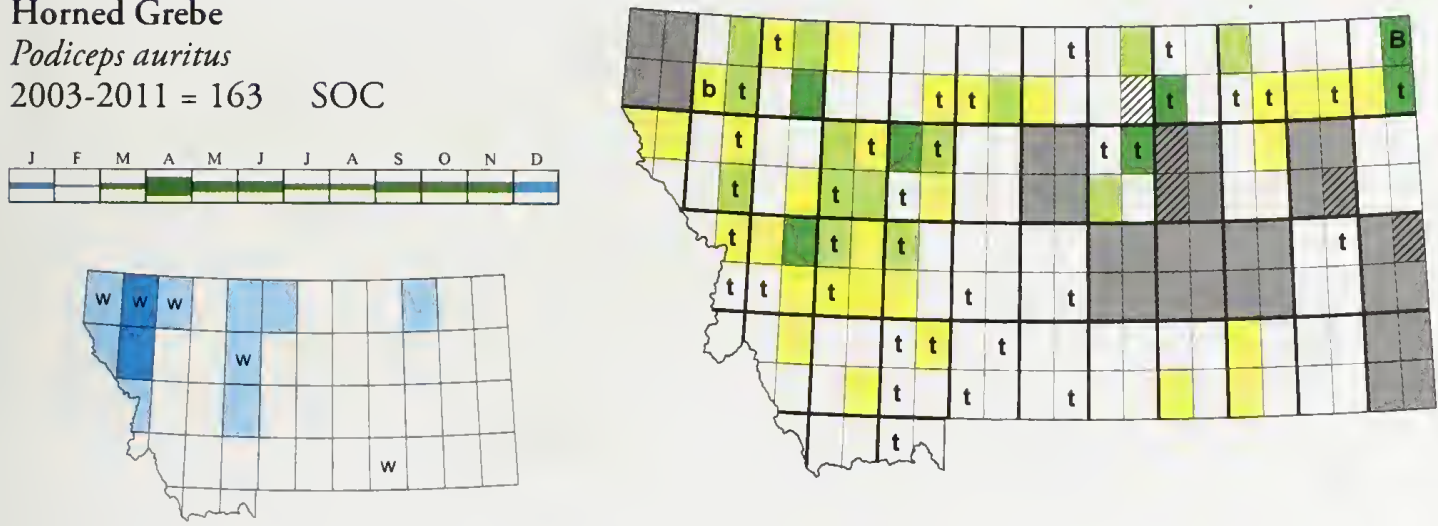

Red-necked Grebe

Podiceps grisegena

2003-2011 $=523$
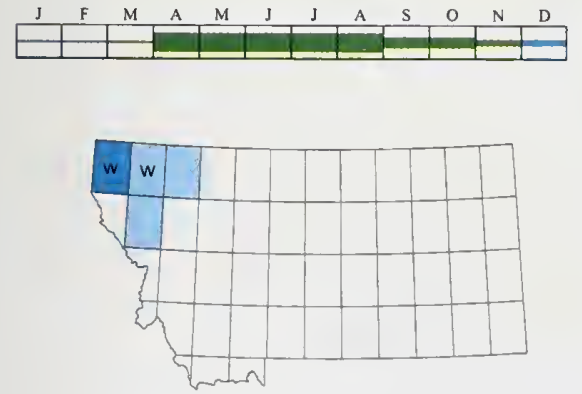
Eared Grebe

Podiceps nigricollis

2003-2011 = 622 SI

\begin{tabular}{|l|l|llllllllll}
$\mathrm{J}$ & $\mathrm{F}$ & $\mathrm{M}$ & $\mathrm{A}$ & $\mathrm{M}$ & $\mathrm{J}$ & $\mathrm{J}$ & $\mathrm{A}$ & $\mathrm{S}$ & $\mathrm{O}$ & $\mathrm{N}$ & $\mathrm{D}$ \\
\hline & & & & & & & & & & & \\
\hline
\end{tabular}

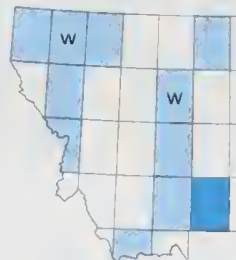

Western Grebe

Aechmophorus occidentalis

2003-2011 = 571
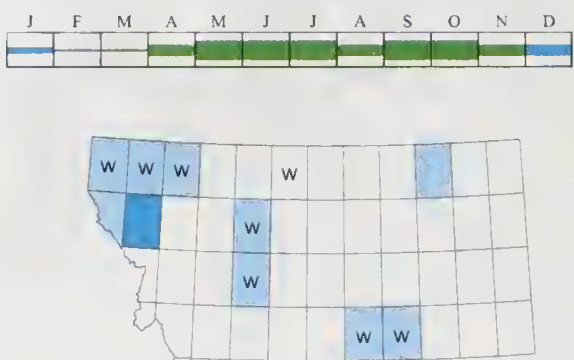

Clark's Grebe

Aechmophorus clarkii

$2003-2011=89$ SOC

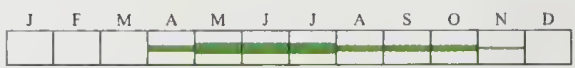

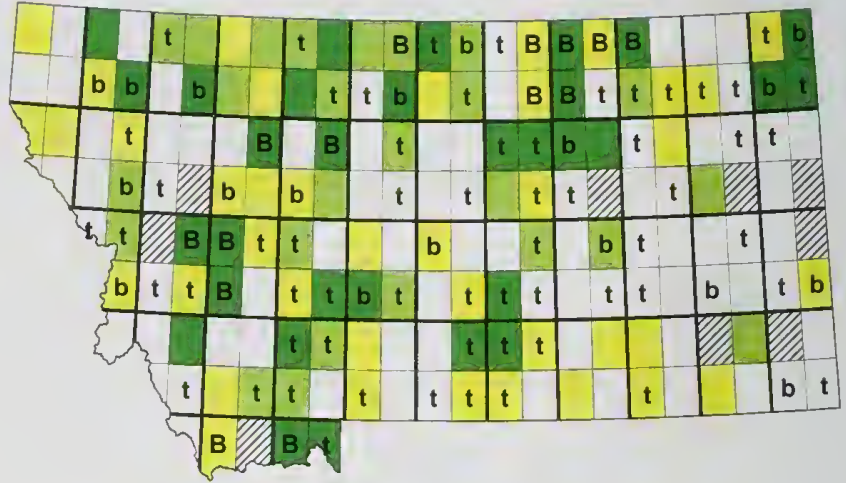

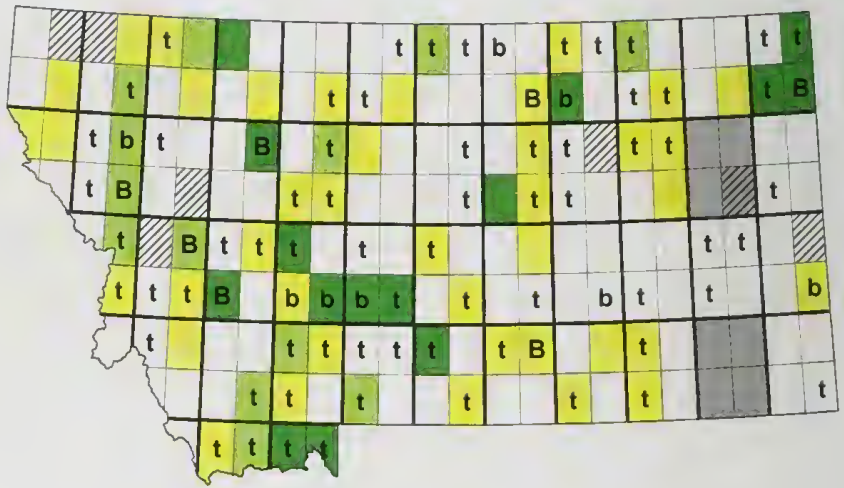


PROCELLARIIFORMES Procellariidae

Manx Shearwater

Puffinus puffinus

2003-2011 = 1 R1
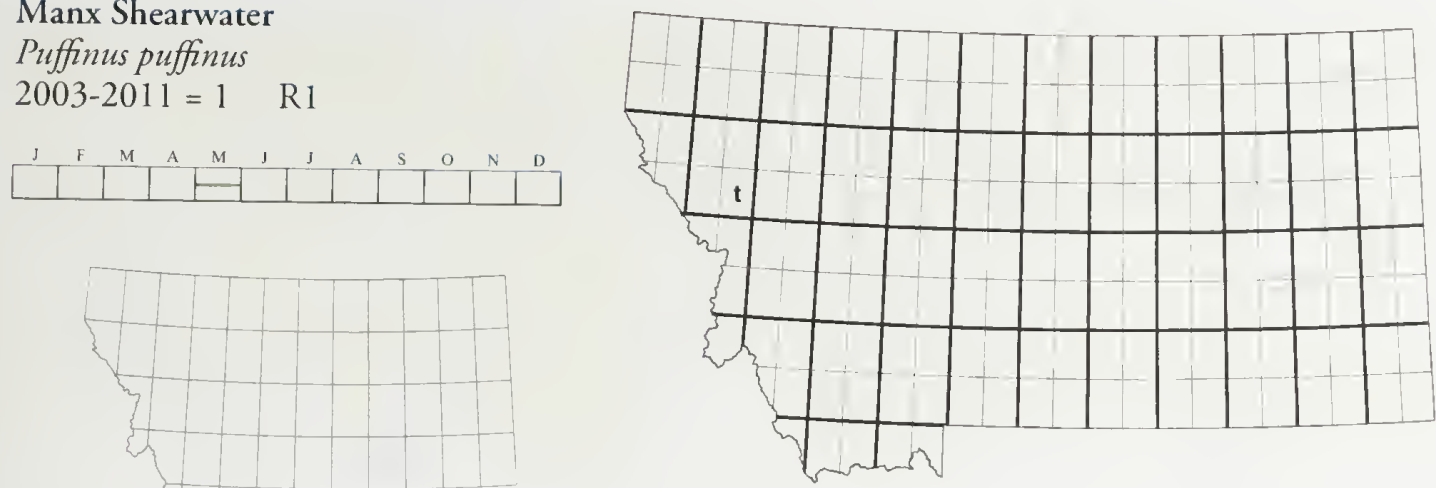

CICONIIFORMES Ciconiidae

\section{Wood Stork}

Mycteria americana

2003-2011 = $0 \quad \mathrm{R} 1$
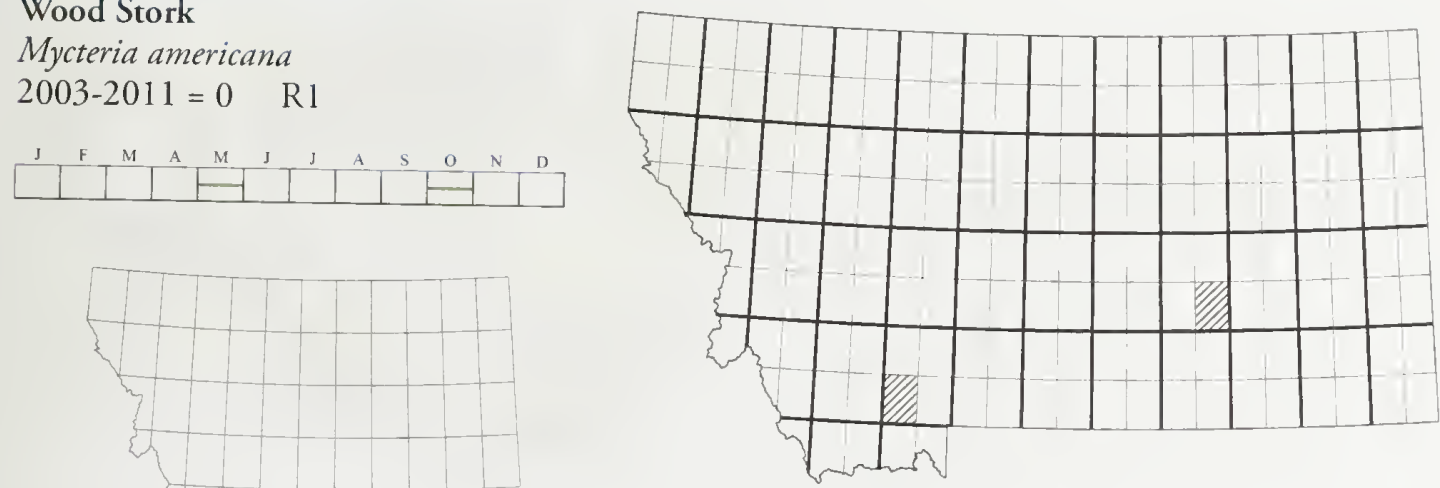

SULIFORMES Phalacrocoracidae

\section{Double-crested Cormorant}

Phalacrocorax auritus

2003-2011 = 966
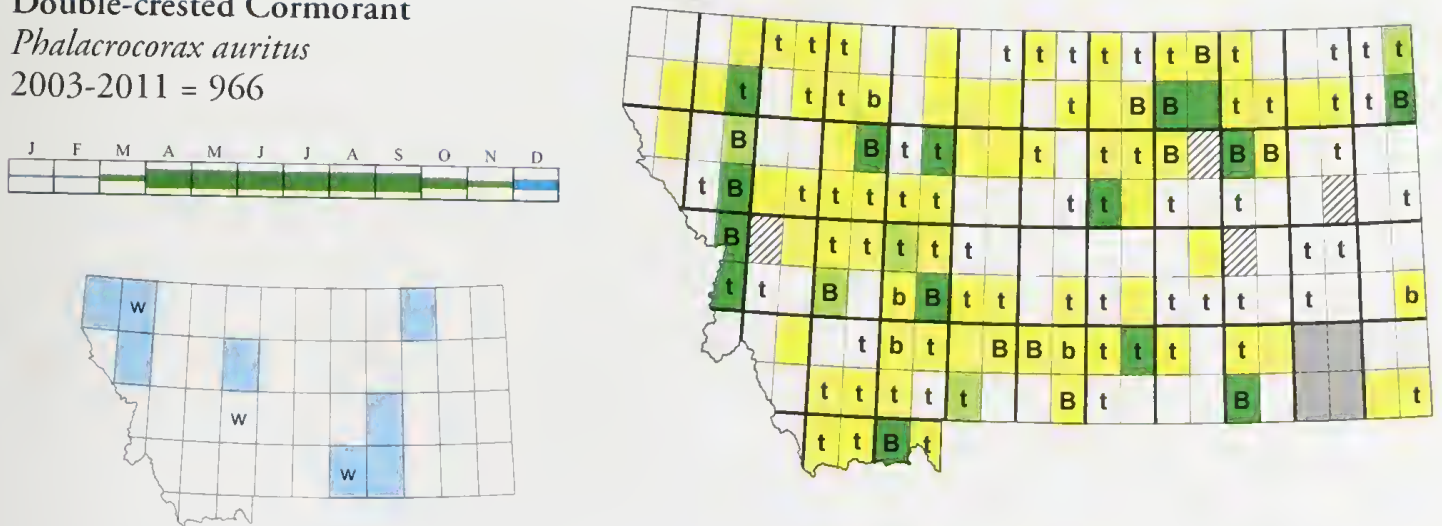
American White Pelican

Pelecanus erythrorhynchos

2003-2011 = $1146 \quad$ SOC
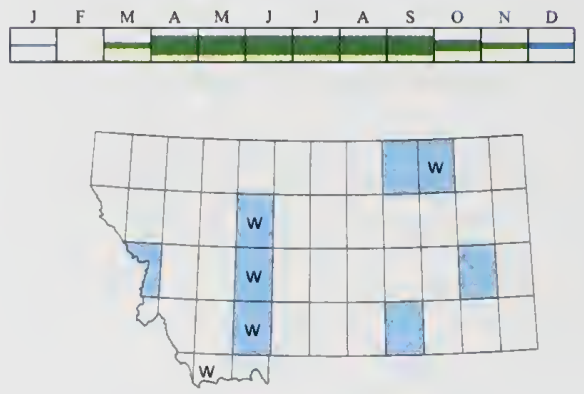

Ardeidae

\section{American Bittern}

Botaurus lentiginosus

2003-2011 = 75 SOC
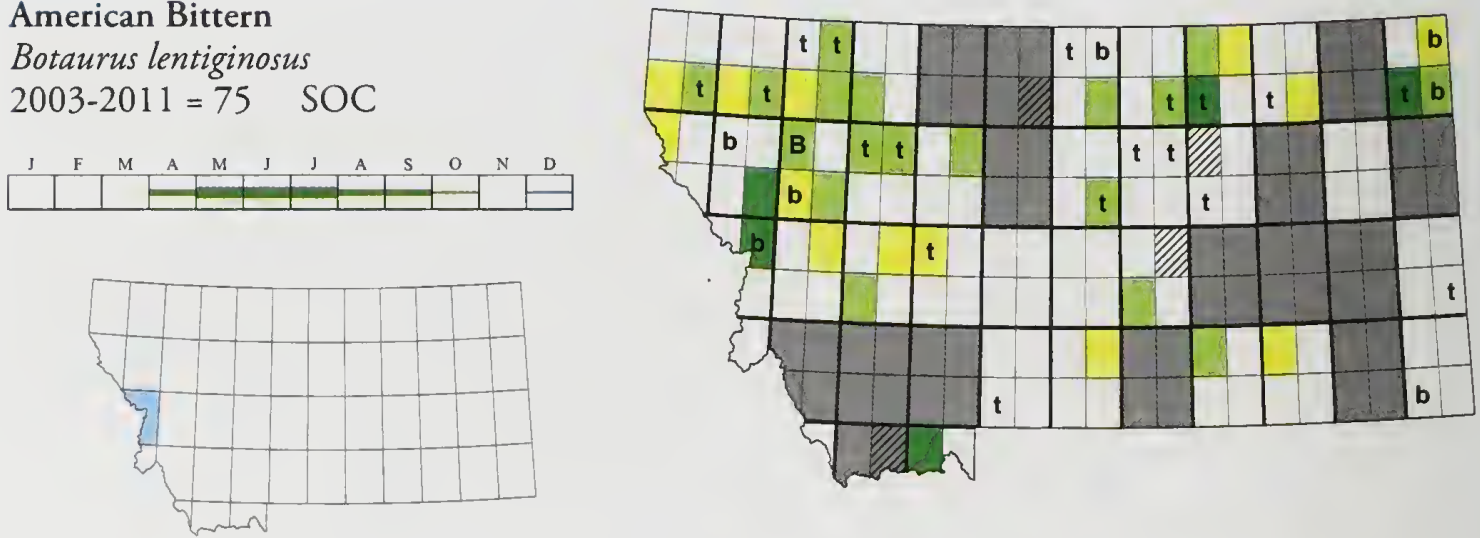

\section{Least Bittern}

Ixobrychus exilis

2003-2011 = 0 R1
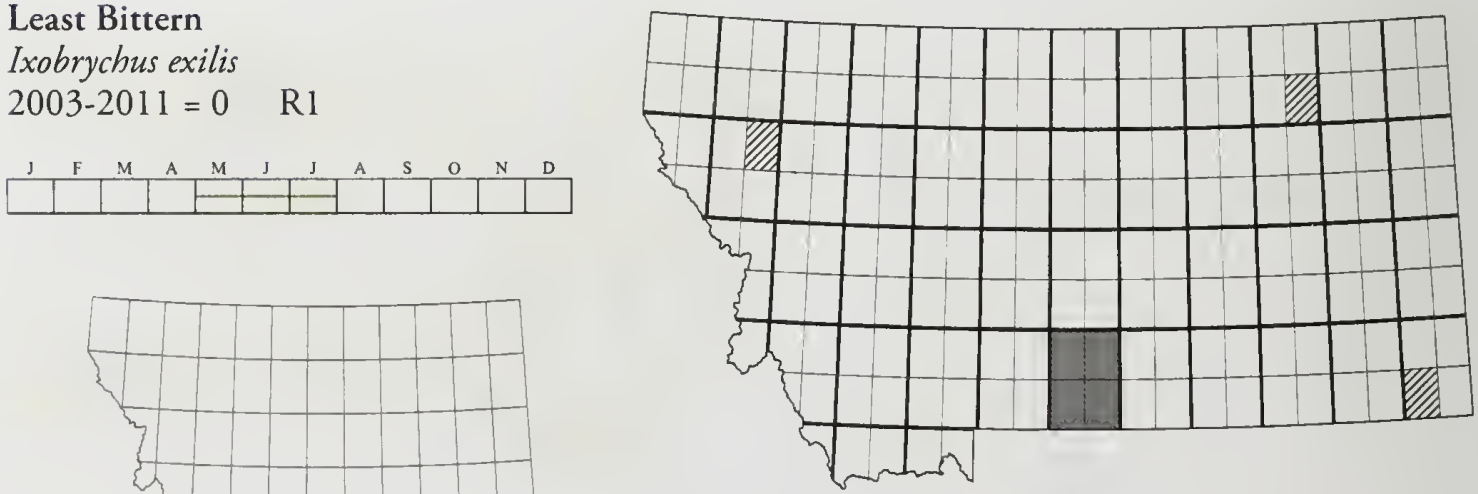
Great Blue Heron

Ardea herodias

$2003-2011=1670 \quad$ SOC
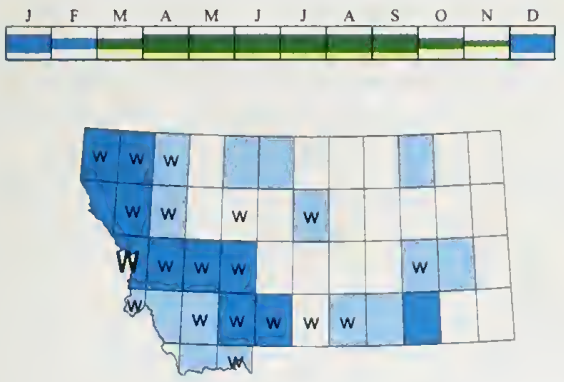

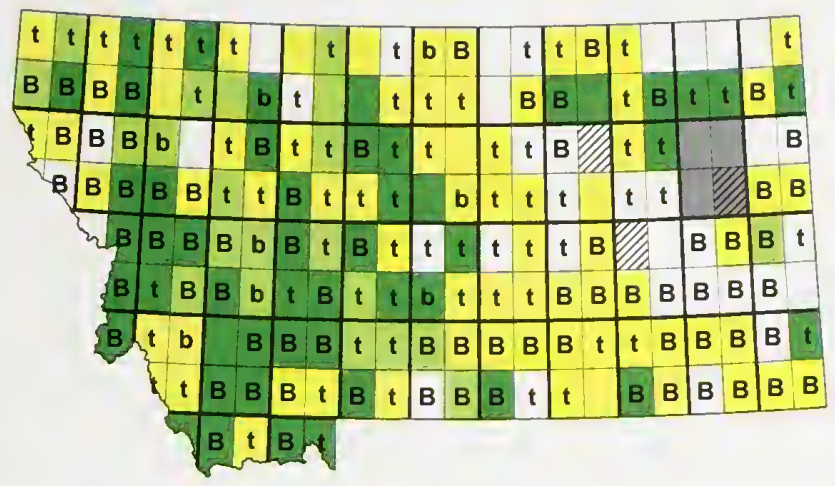

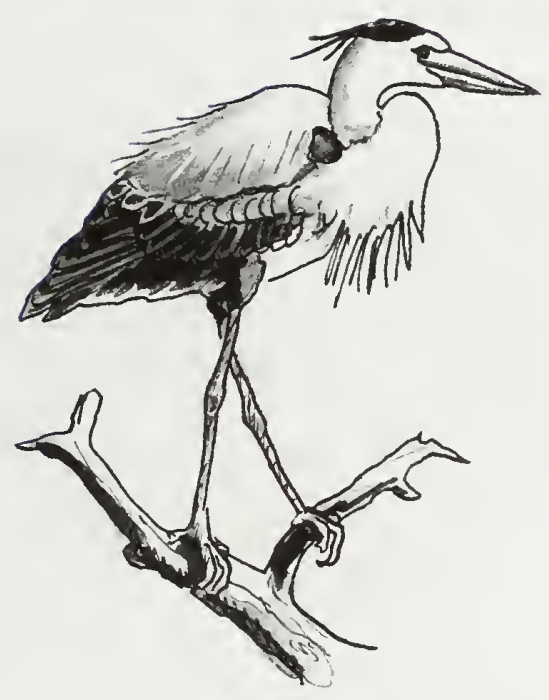

\section{Great Egret}

Ardea alba

2003-2011 = 41
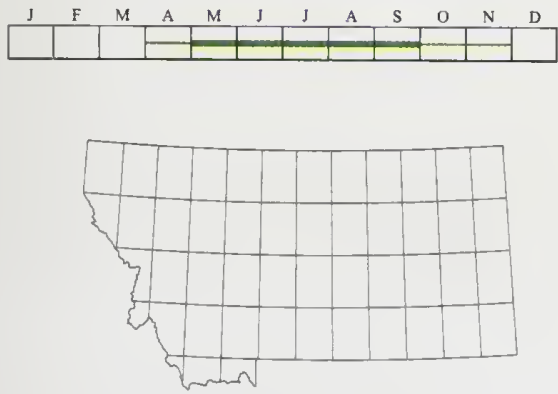

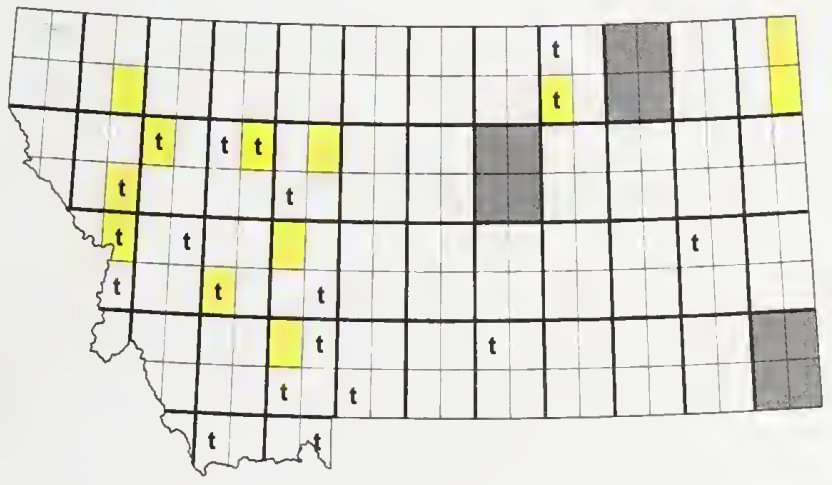


Snowy Egret

Egretta thula

$2003-2011=8$
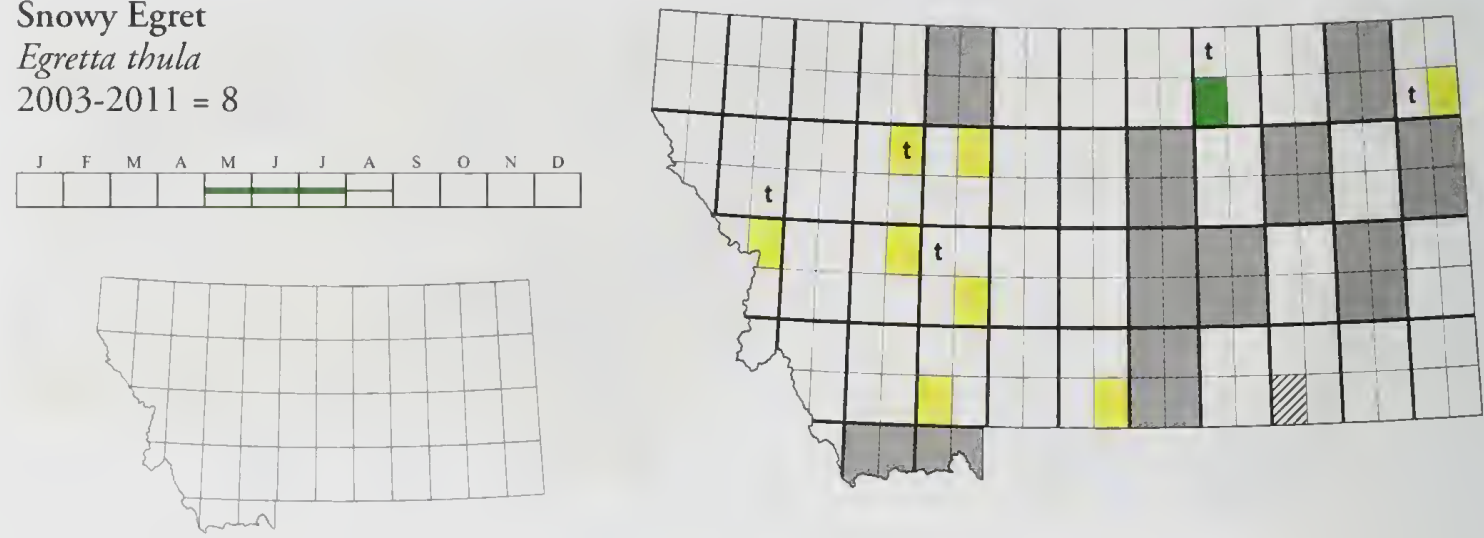

Little Blue Heron

Egretta caerulea

$2003-2011=4 \quad \mathrm{R} 1$

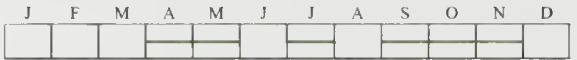

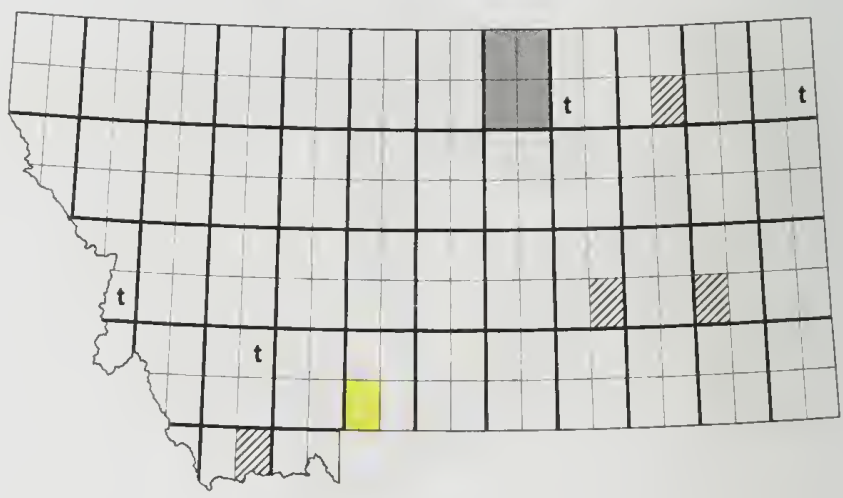

Cattle Egret

Bubulcus ibis

2003-2011 = 10

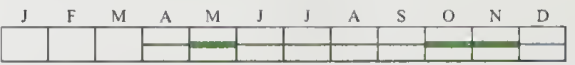

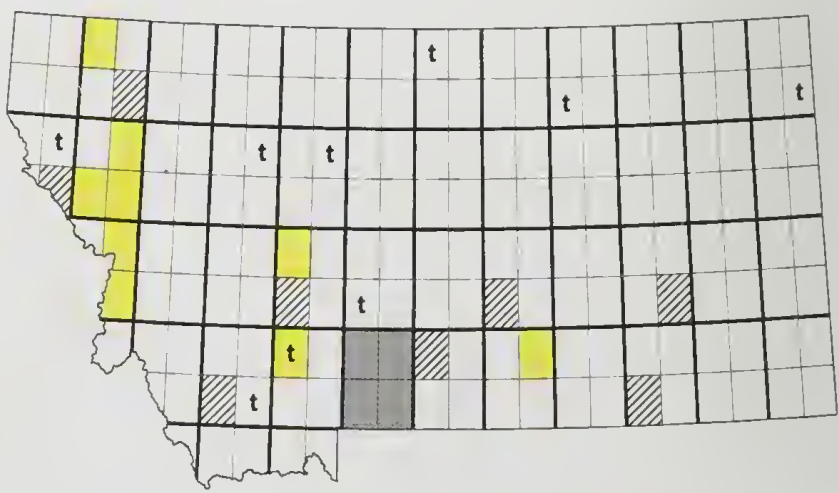




\section{Green Heron}

Butorides virescens

2003-2011 = $4 \quad$ R 1
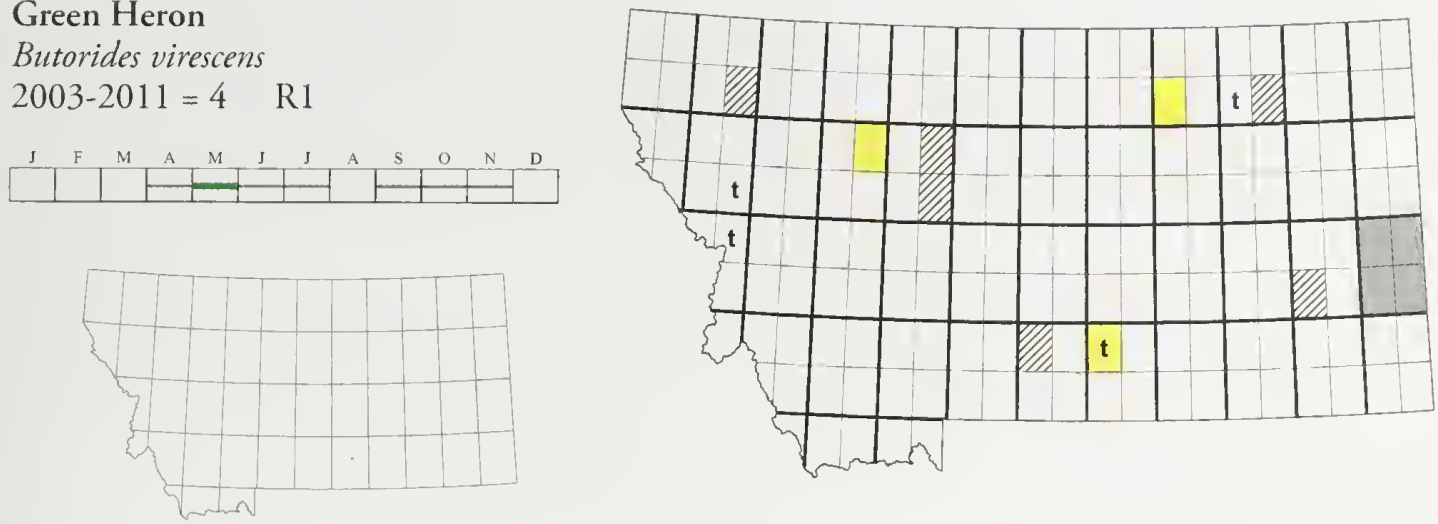

Black-crowned Night-Heron

Nycticorax nycticorax

$2003-2011=133 \quad$ SOC
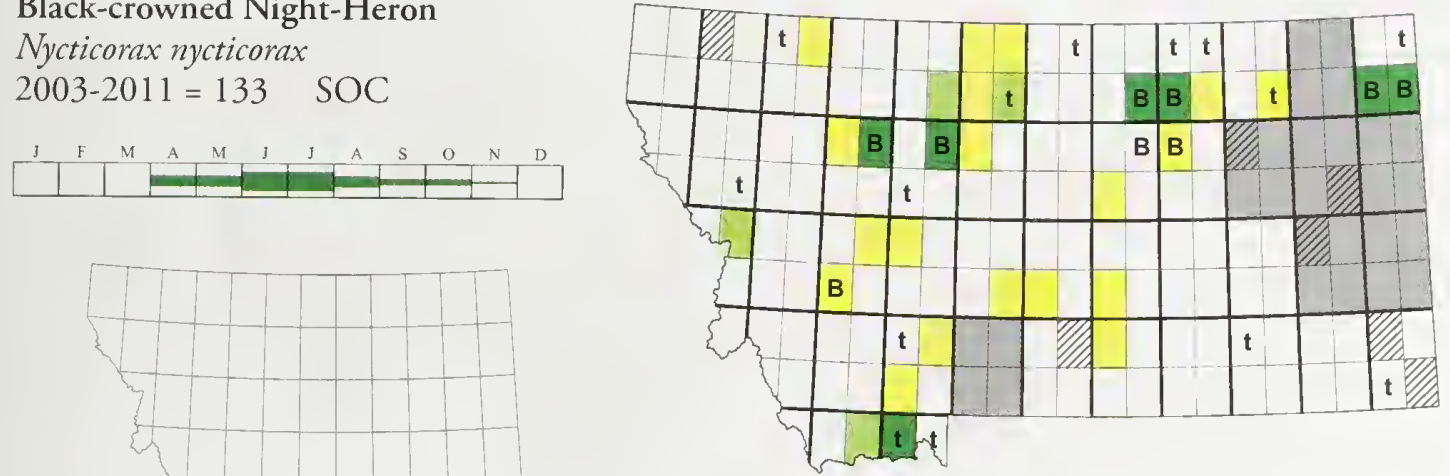

Yellow-crowned Night-Heron

Nyctanassa violacea

2003-2011 = 0 R1

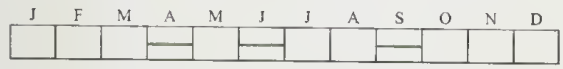

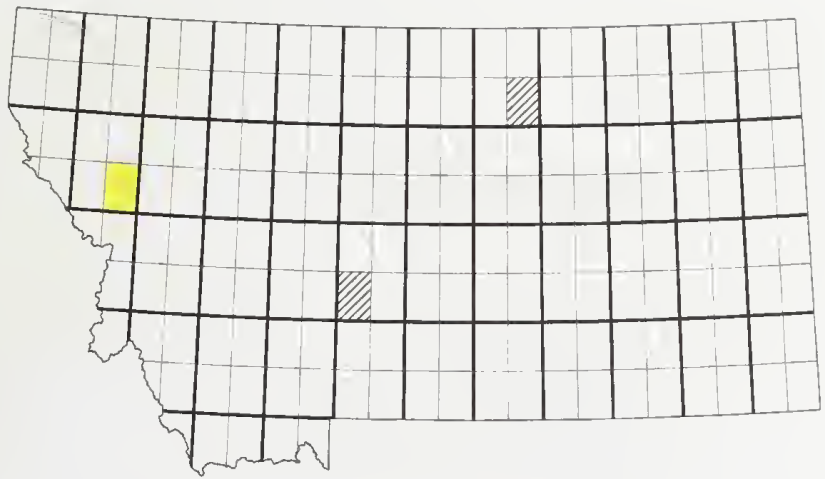


Threskiornithidae

Glossy Ibis

Plegadis falcinellus

2003-2011 = 4 R1

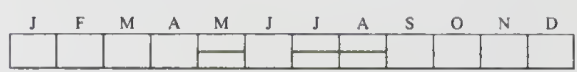
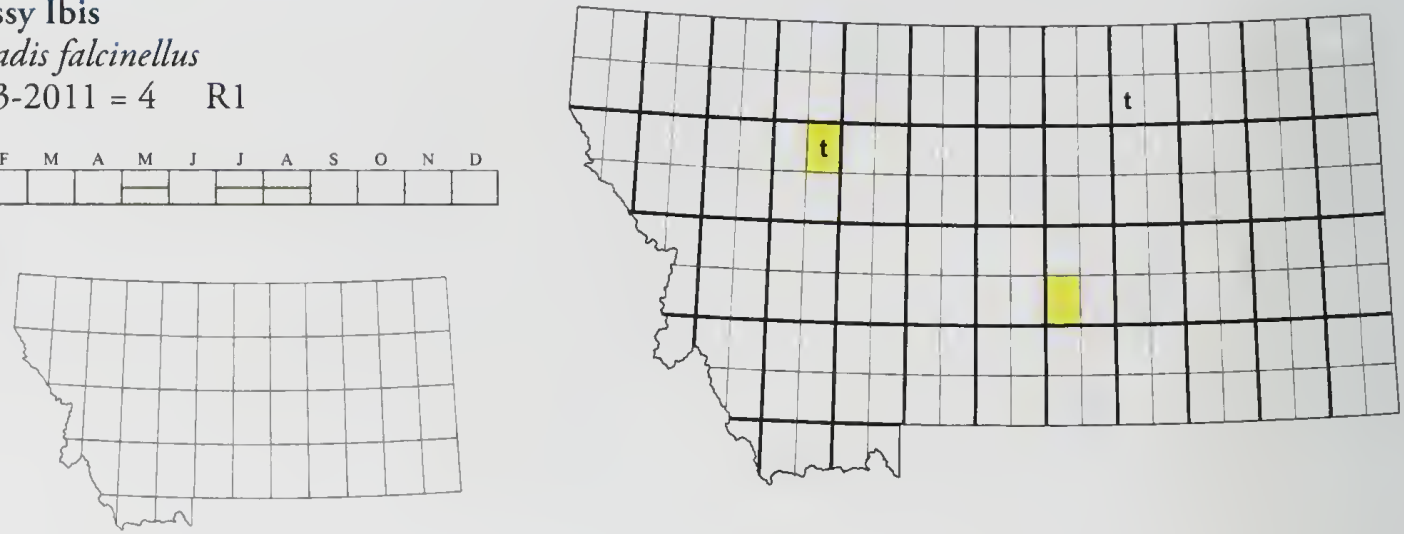

White-faced Ibis

Plegadis chibi

$2003-2011=113 \quad$ SOC
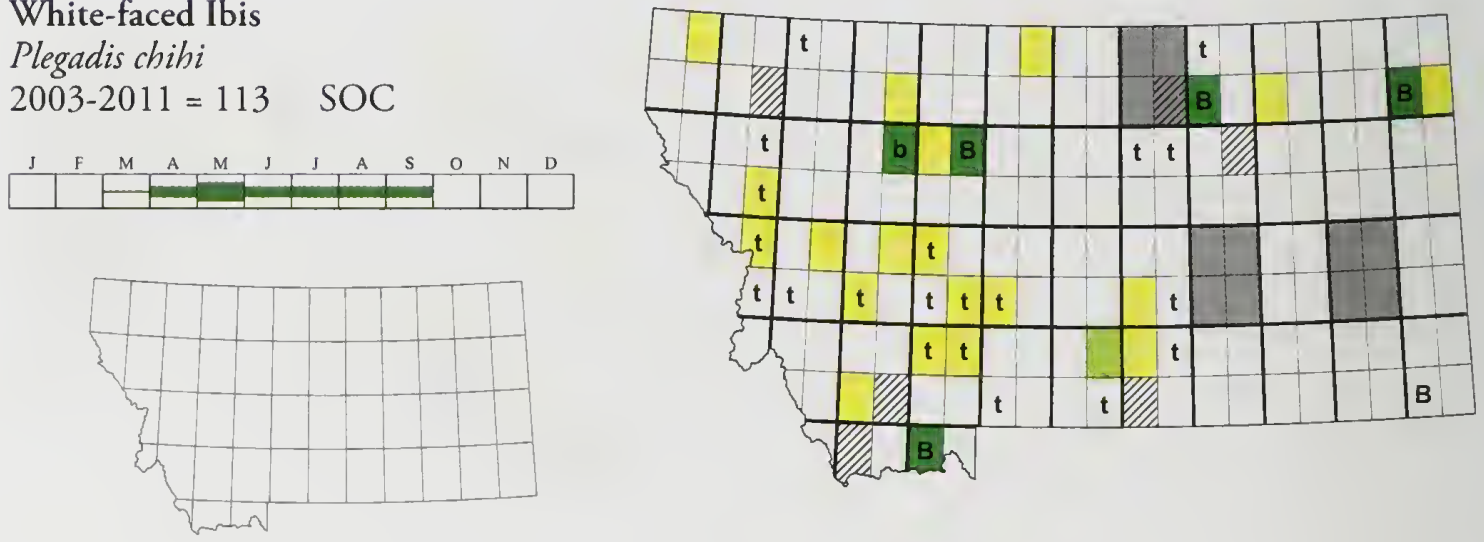

ACCIPITRIFORMES Cathartidae

\section{Turkey Vulture}

\section{Cathartes aura}

$2003-2011=897$
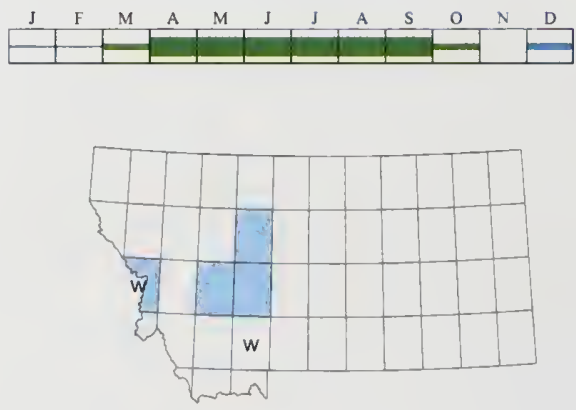

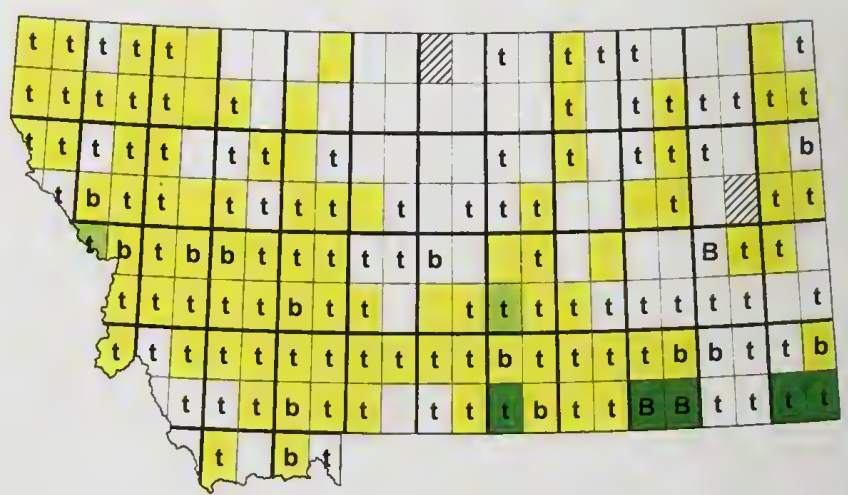


Pandionidae

Osprey

Pandion haliaetus

2003-2011 = 1408
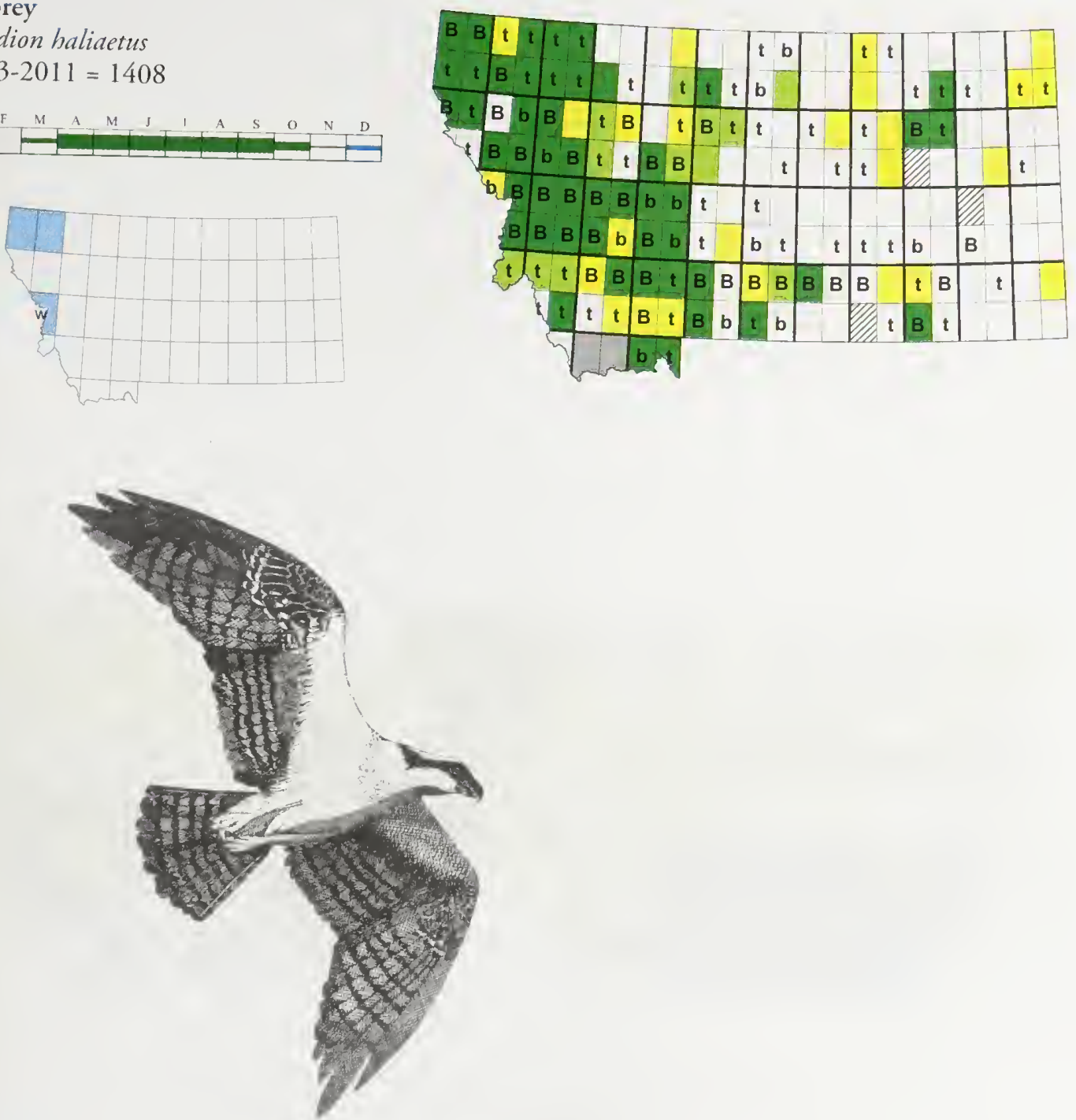

Accipitridae

White-tailed Kite

Elanus Leucurus

2003-2011=3 R1
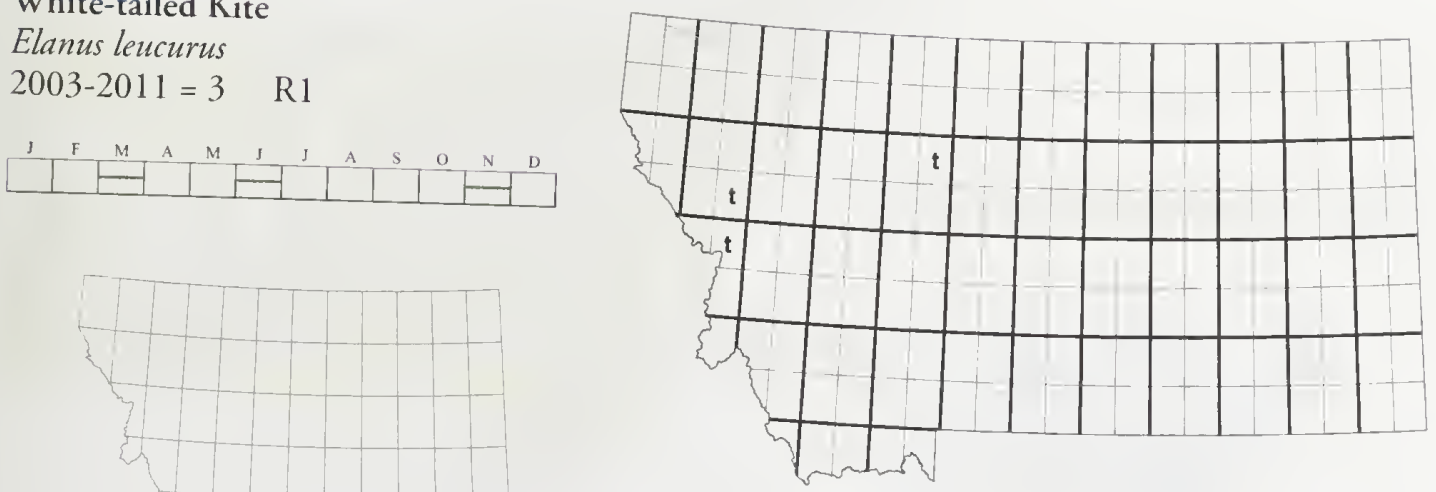
Mississippi Kite

Ictinia mississippiensis

2003-2011 = 1 R1
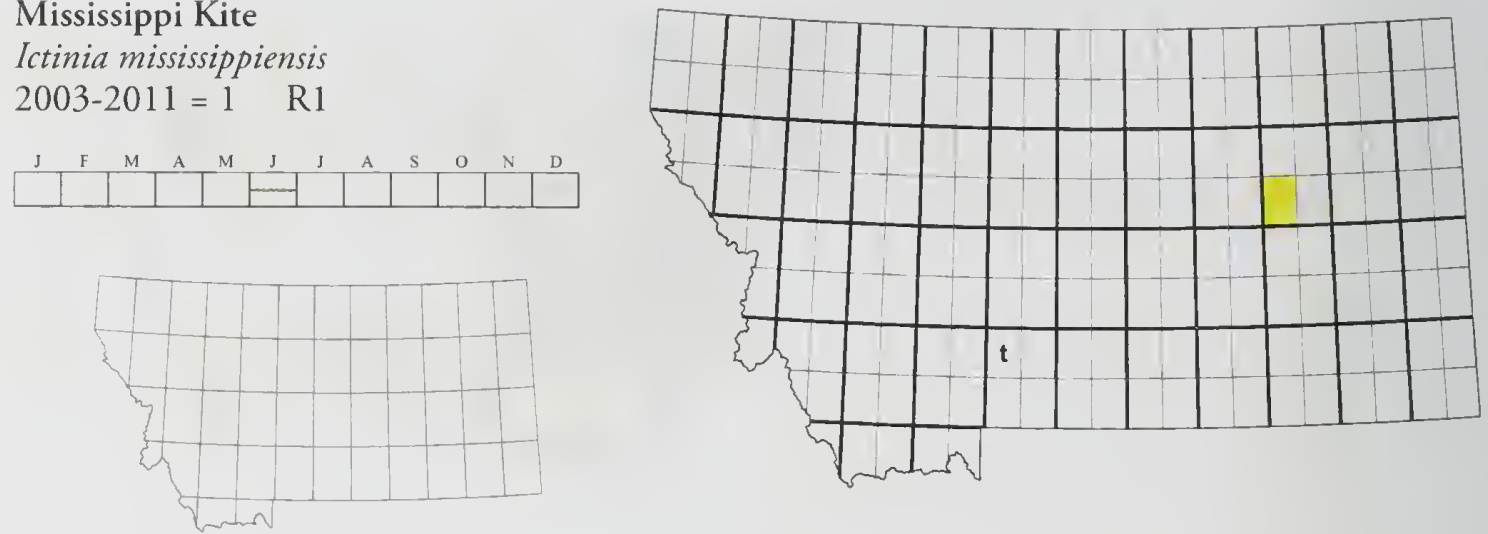

Bald Eagle

Haliaeetus leucocephalus

2003-2011 = 4165 SOC
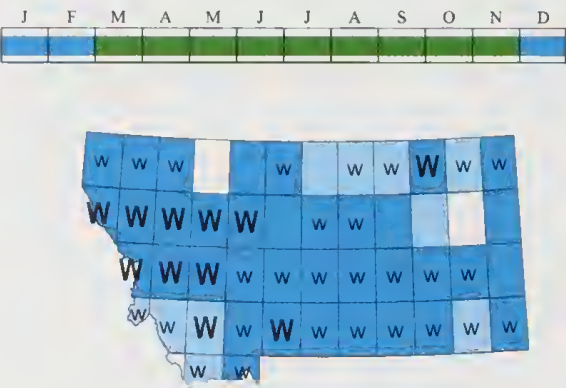

Northern Harrier

Circus cyaneus

2003-2011 = 2158
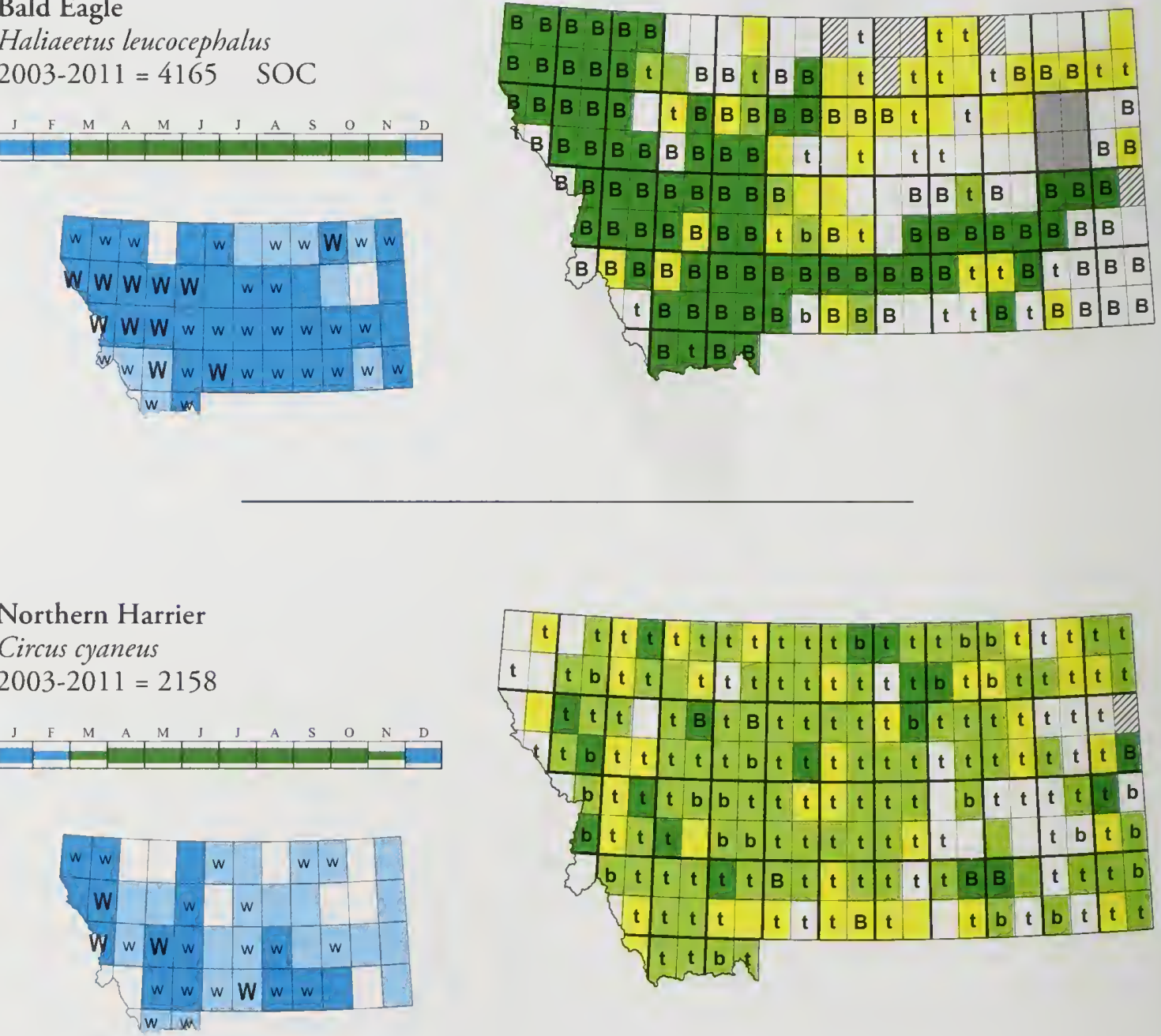
Sharp-shinned Hawk

Accipiter striatus

2003-2011 = 414
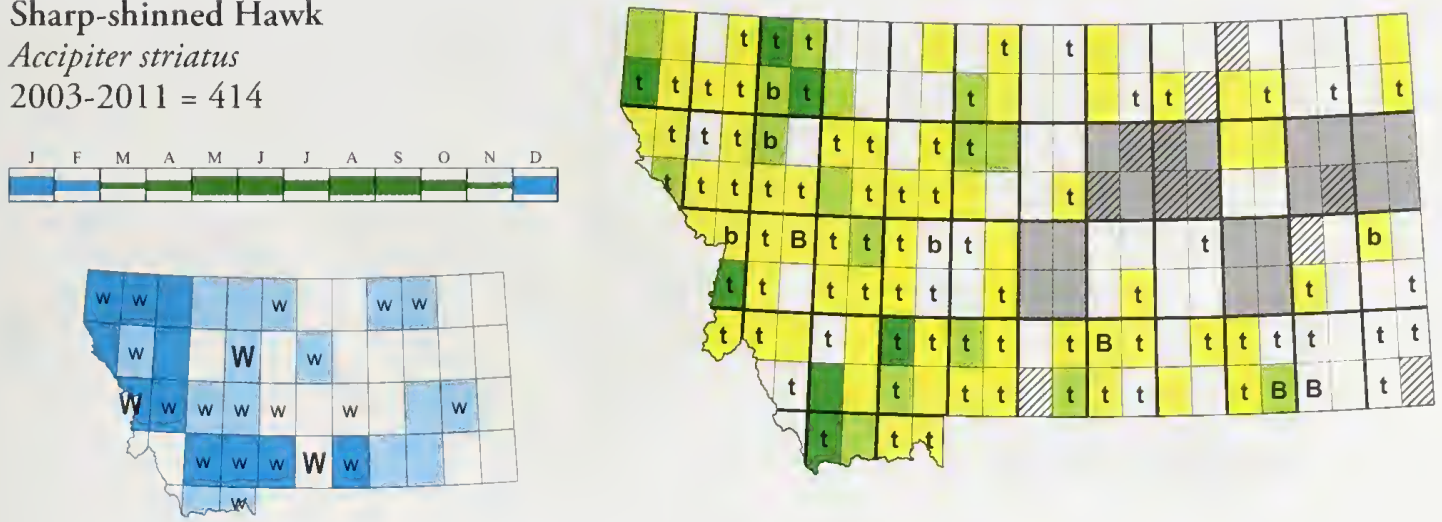

Cooper's Hawk

Accipiter cooperii

2003-2011 = 347
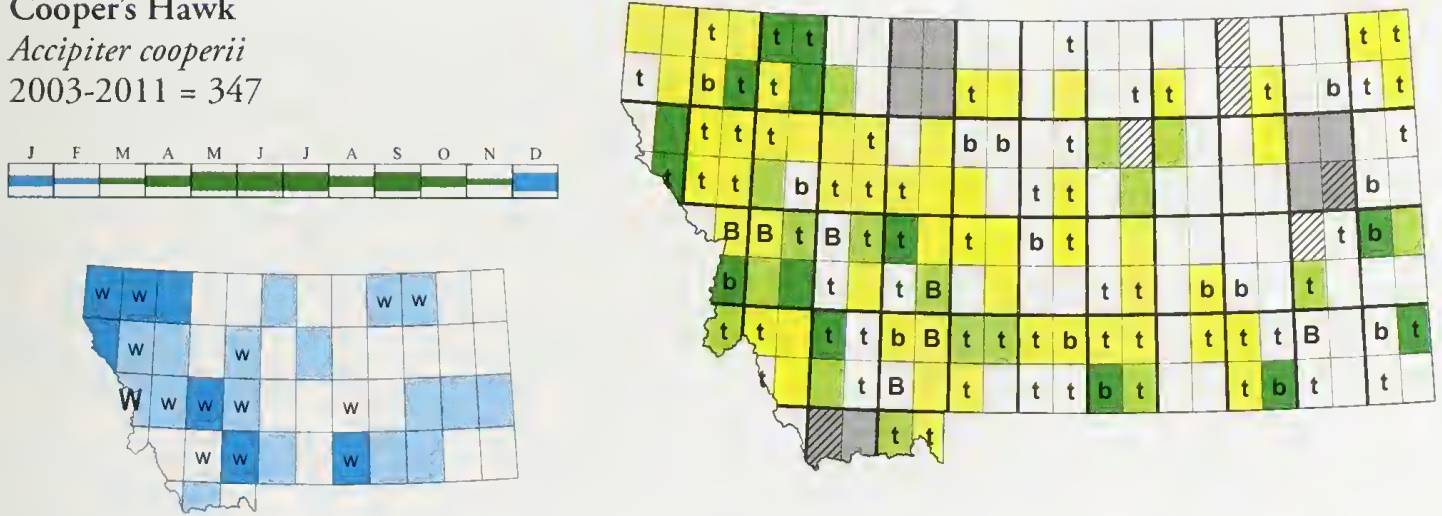

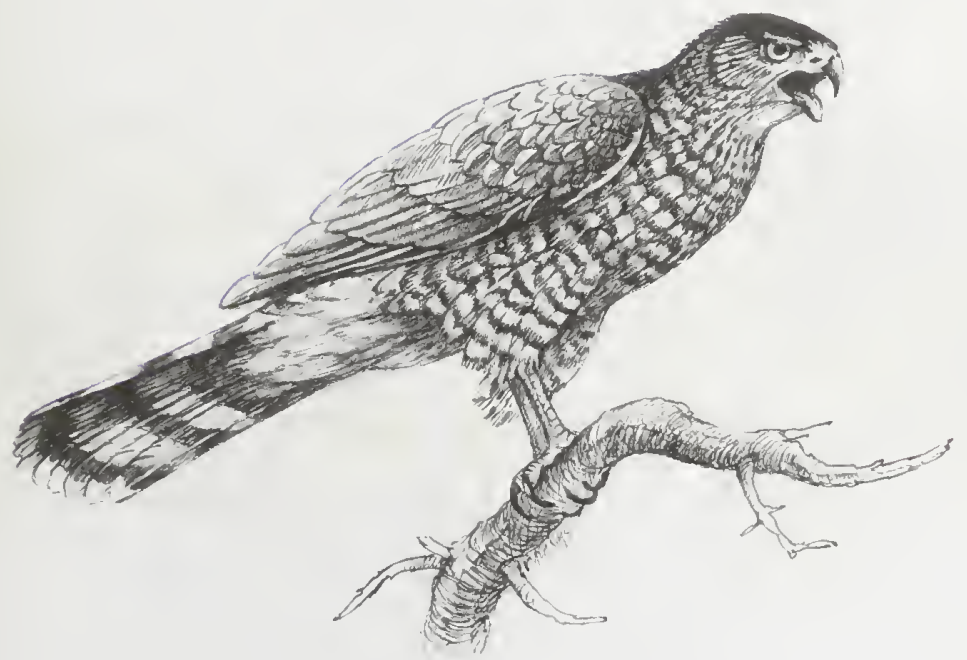


Northern Goshawk

Accipiter gentilis

2003-2011 = 540 SOC

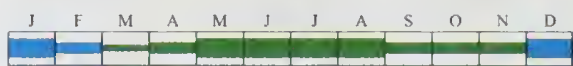
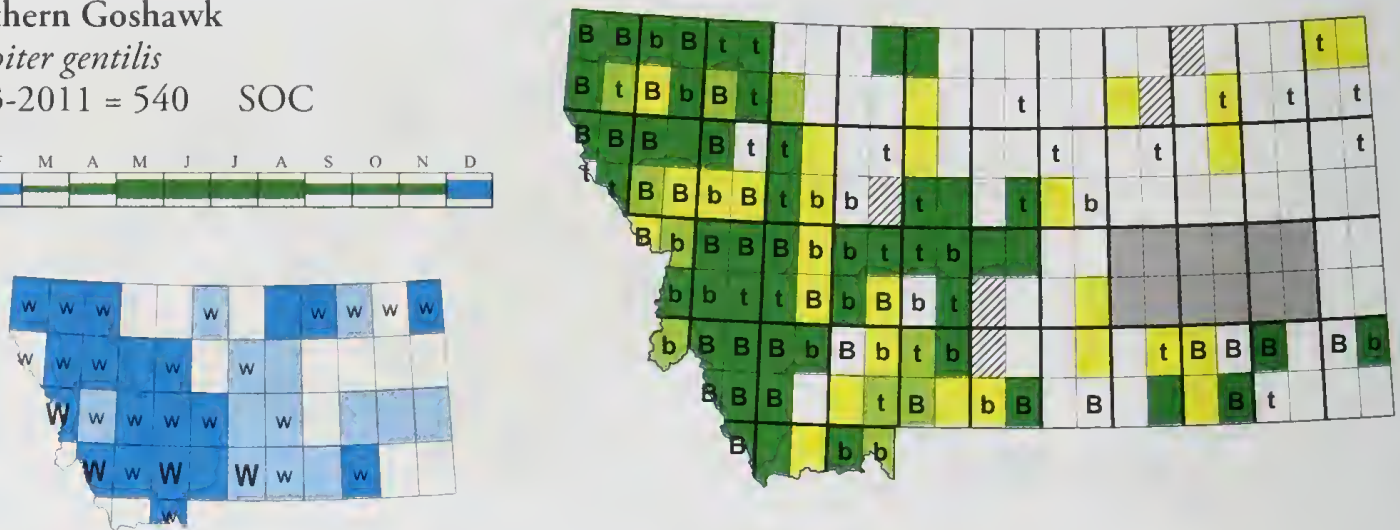

Red-shouldered Hawk

Buteo lineatus

2003-2011 = 1 R1

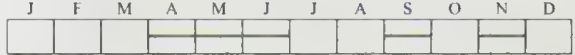

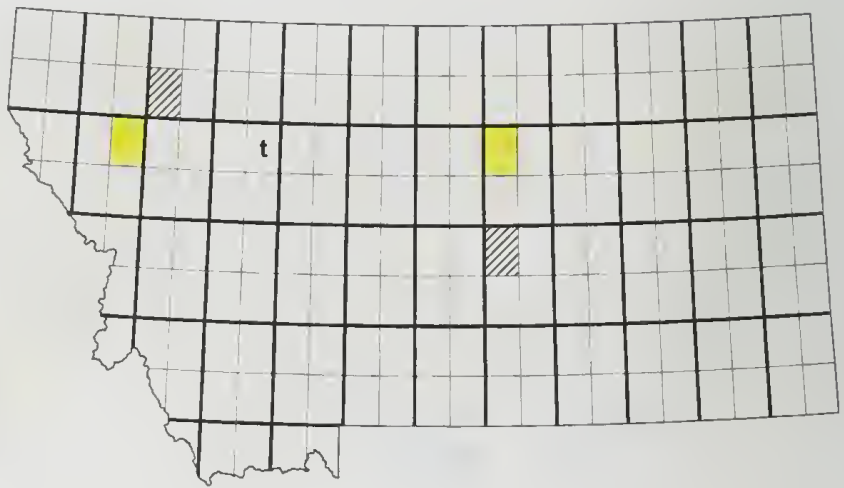

Broad-winged Hawk

Buteo platypterus

2003-2011 = 25
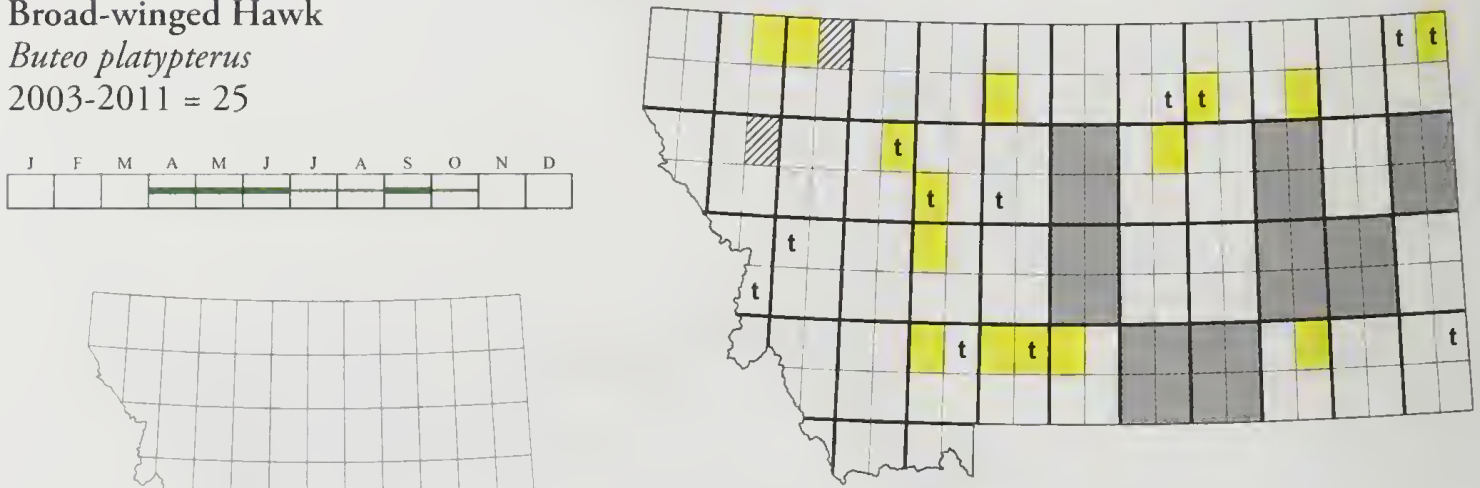
Swainson's Hawk

Buteo swainsoni

$2003-2011=762$ SI
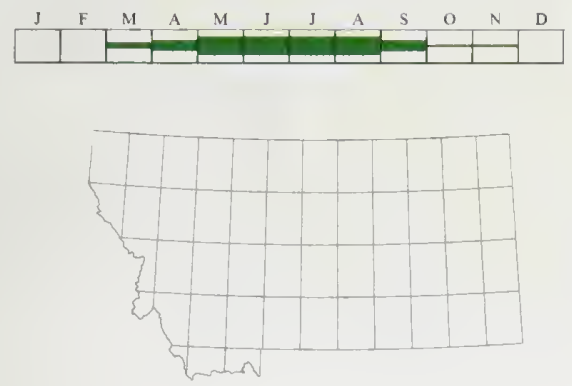

Red-tailed Hawk

Buteo jamaicensis

2003-2011 = 2947
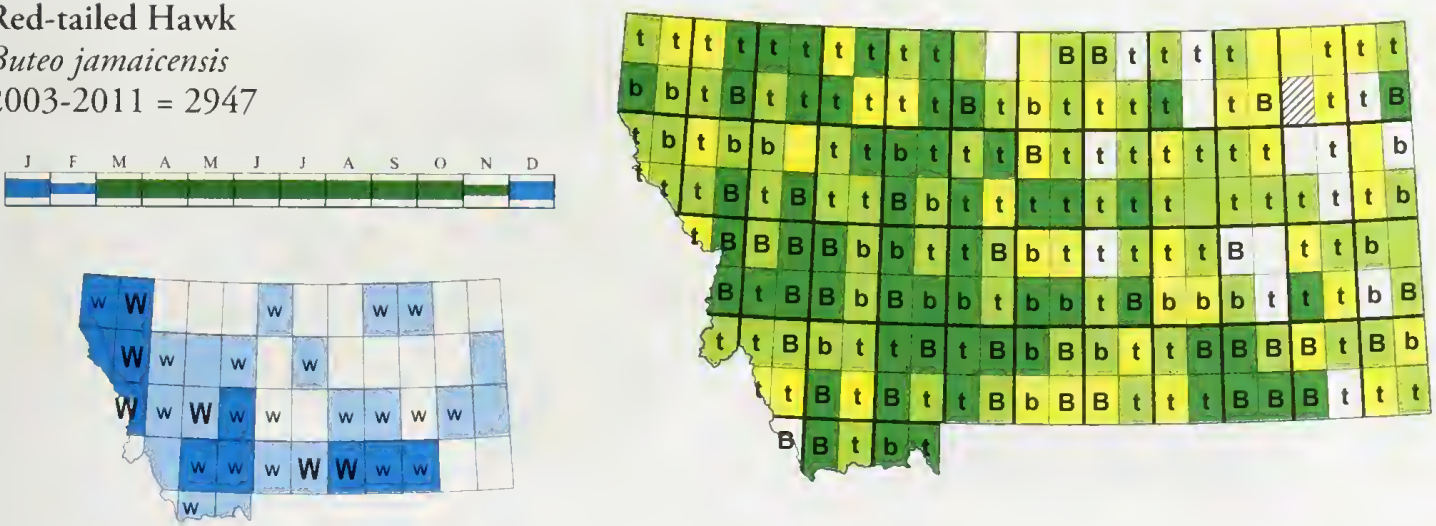

Ferruginous Hawk

Buteo regalis

$2003-2011=548 \quad$ SOC
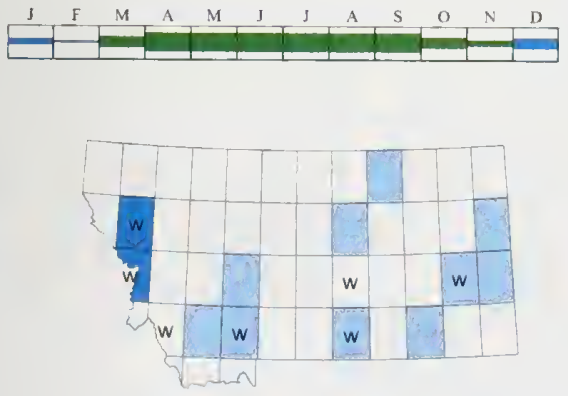

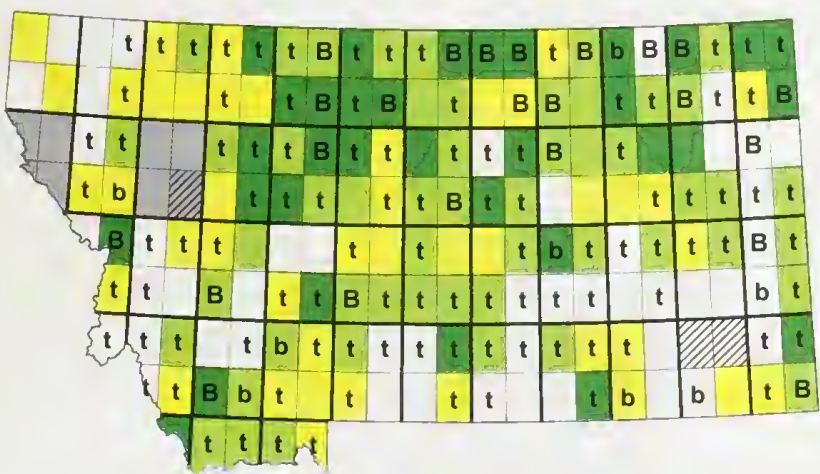


Rough-legged Hawk

Buteo lagopus

2003-2011 = 786
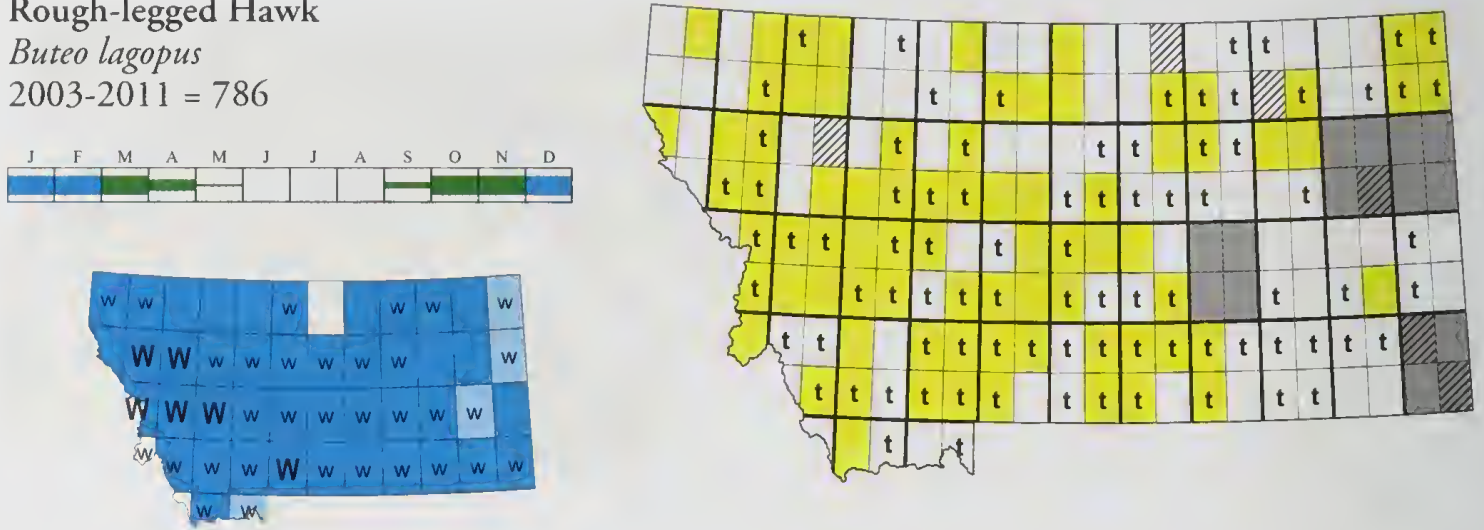

Golden Eagle

Aquila chrysaetos

$2003-2011=1088 \quad$ SOC
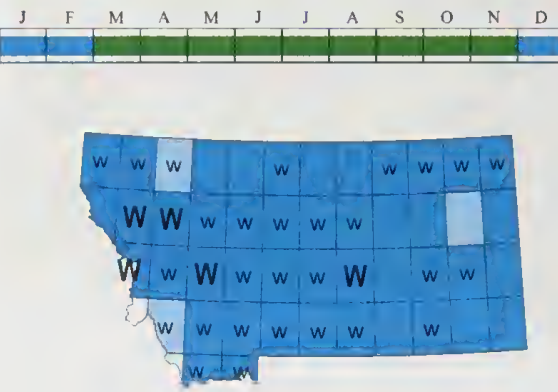

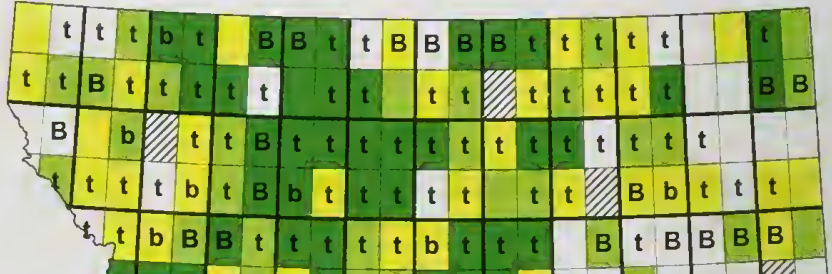
$t B B t_{t} B_{b} t t_{t} t_{t} B t_{t}$

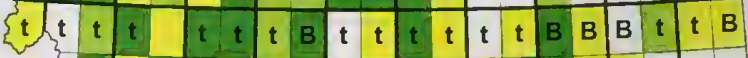
\begin{tabular}{|l|lllllllllllllllll}
\hline$y$ & $B$ & $t$ & $t$ & $t$ & $B$ & $t$ & $B$ & $B$ & $t$ & $t$ & $t$ & $B$ & $B$ & $B$ & $B$ \\
\hline
\end{tabular} th t b

FALCONIFORMES Falconidae

\section{Crested Caracara}

Caracara cheriway

$2003-2011=1 \quad R 1$

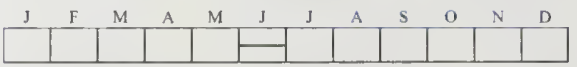
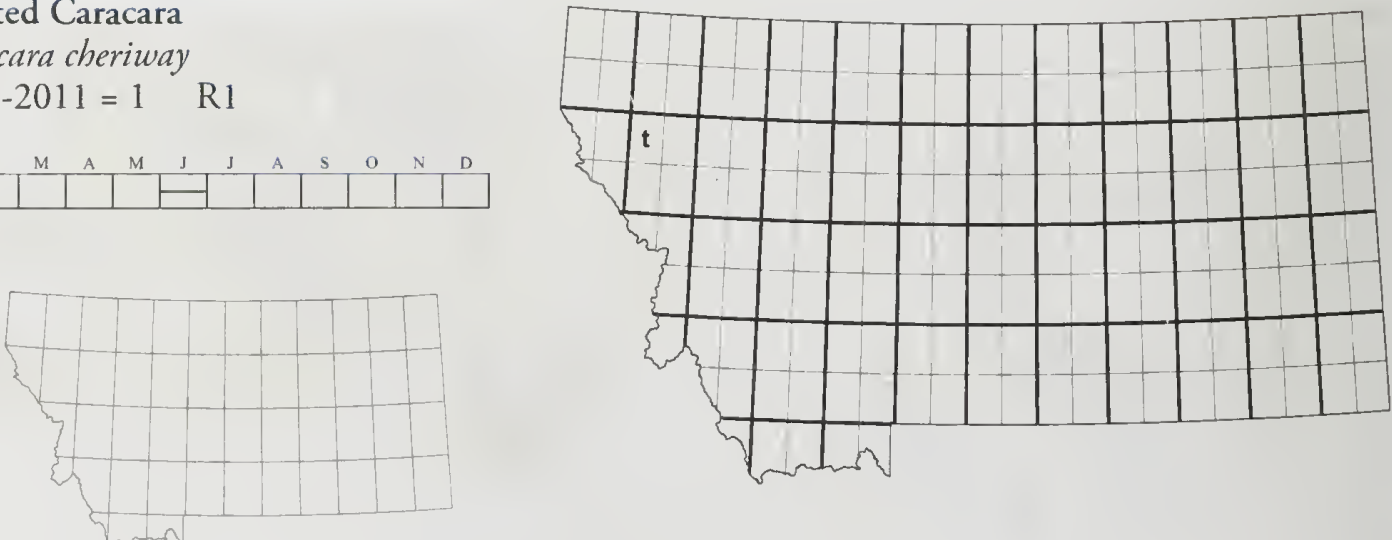
American Kestrel

Falco sparverius

$2003-2011=2038$
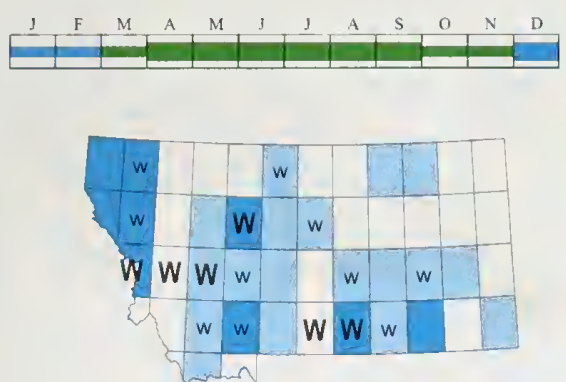

\section{Merlin}

\section{Falco columbarius}

2003-2011 = 354
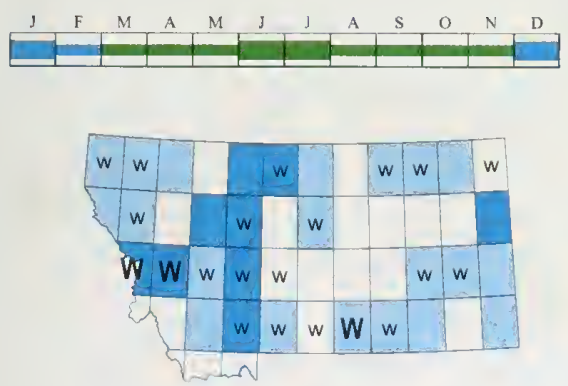
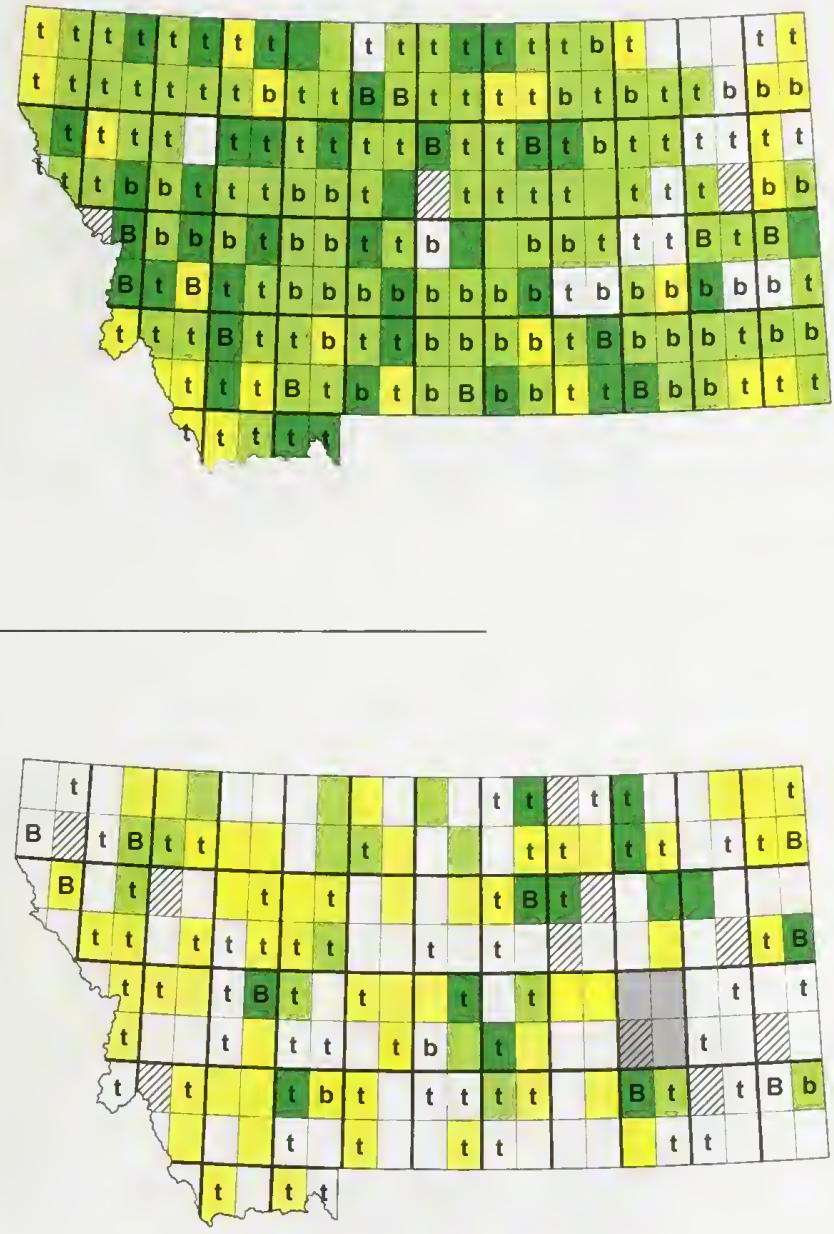

Gyrfalcon

Falco rusticolus

2003-2011 = 66
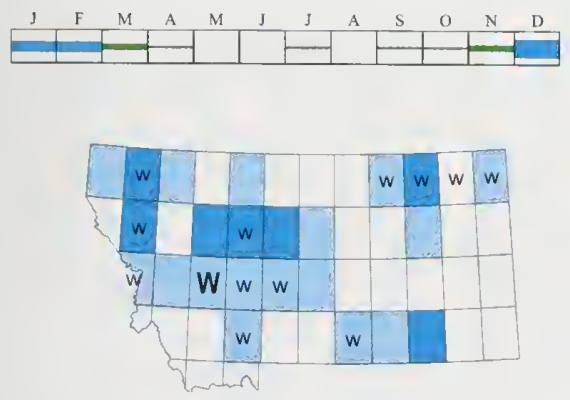

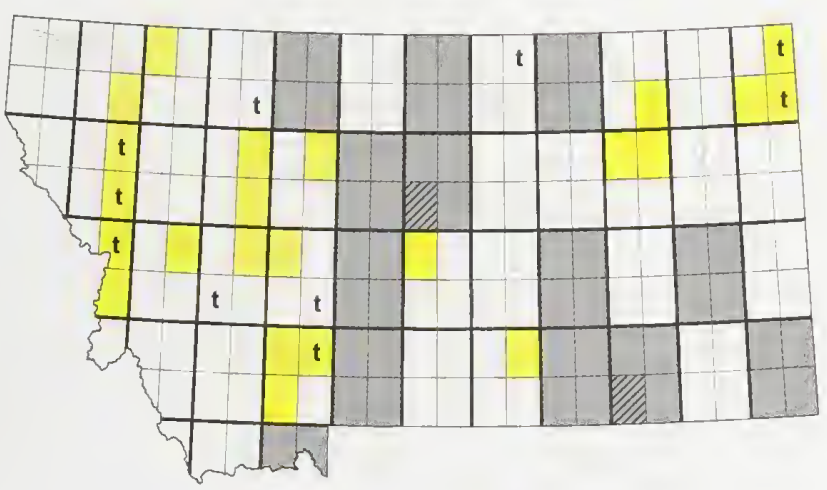


Peregrine Falcon

Falco peregrinus

$2003-2011=395 \quad$ SOC
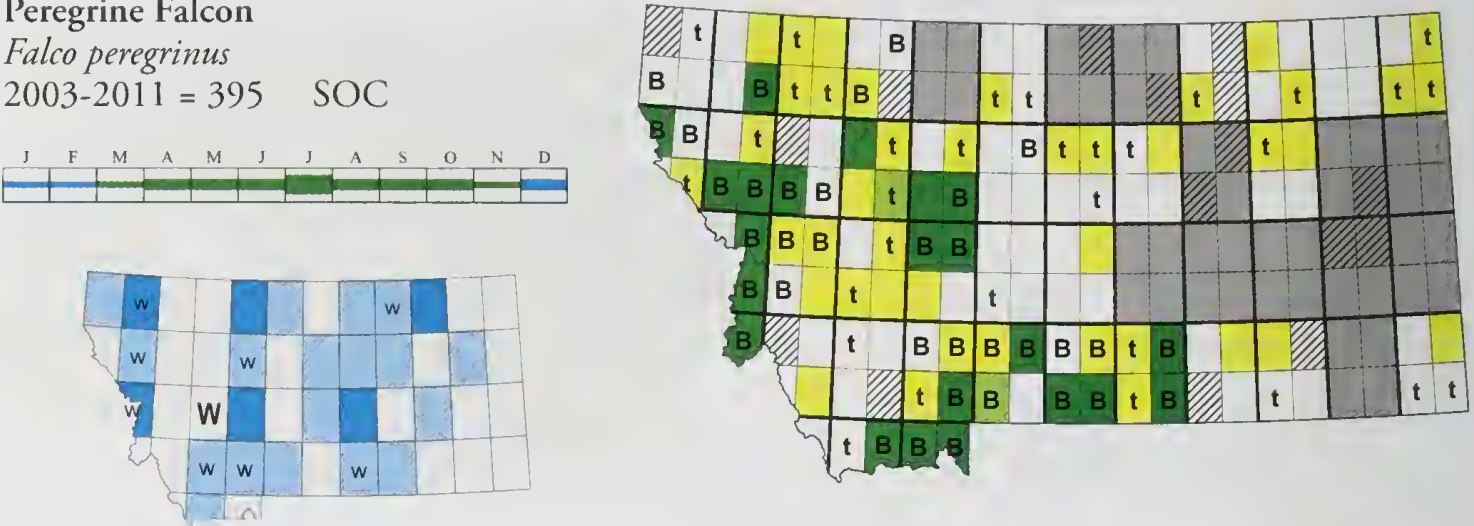

Prairie Falcon

Falco mexicanus

$2003-2011=496$
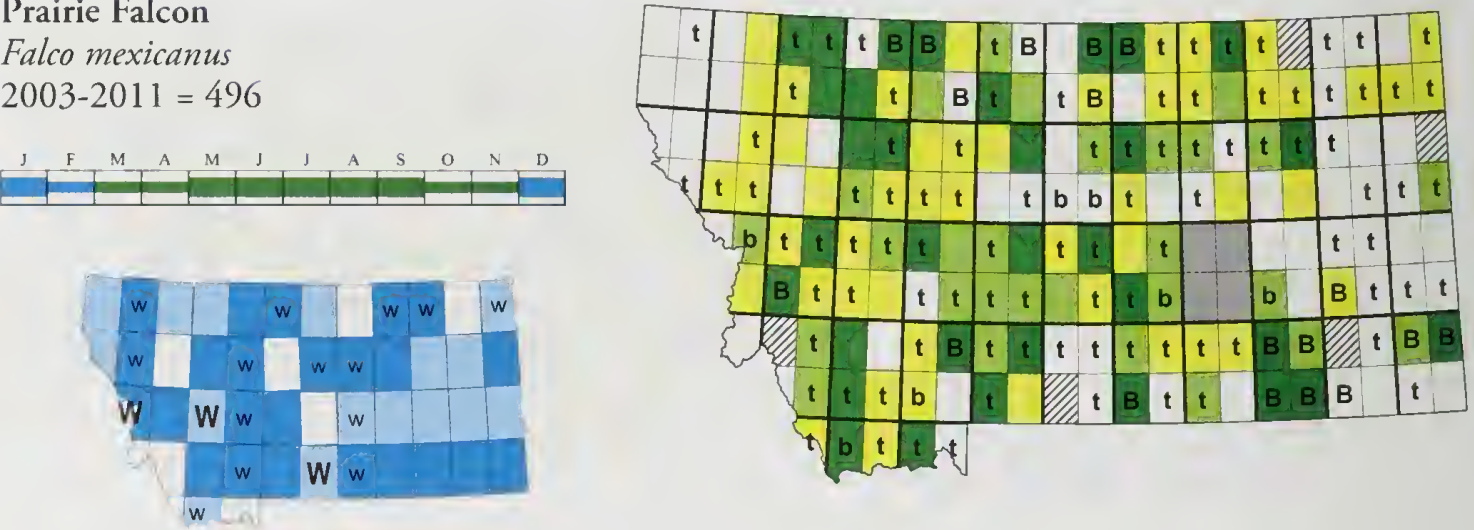

\section{GRUIFORMES Rallidae}

\section{Yellow Rail}

Coturnicops noveboracensis

2003-2011=3 SOC R2

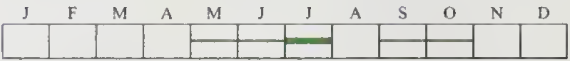

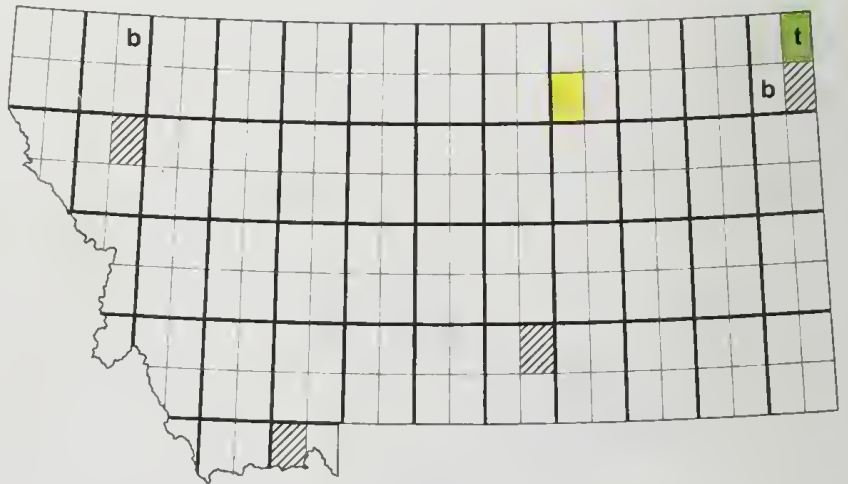




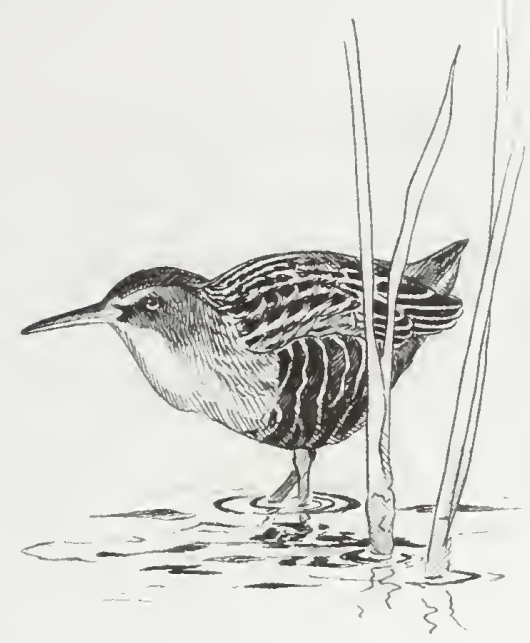

Virginia Rail

Rallus limicola

2003-2011 = 149 SI

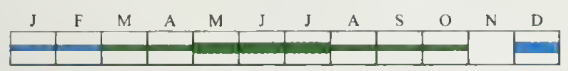
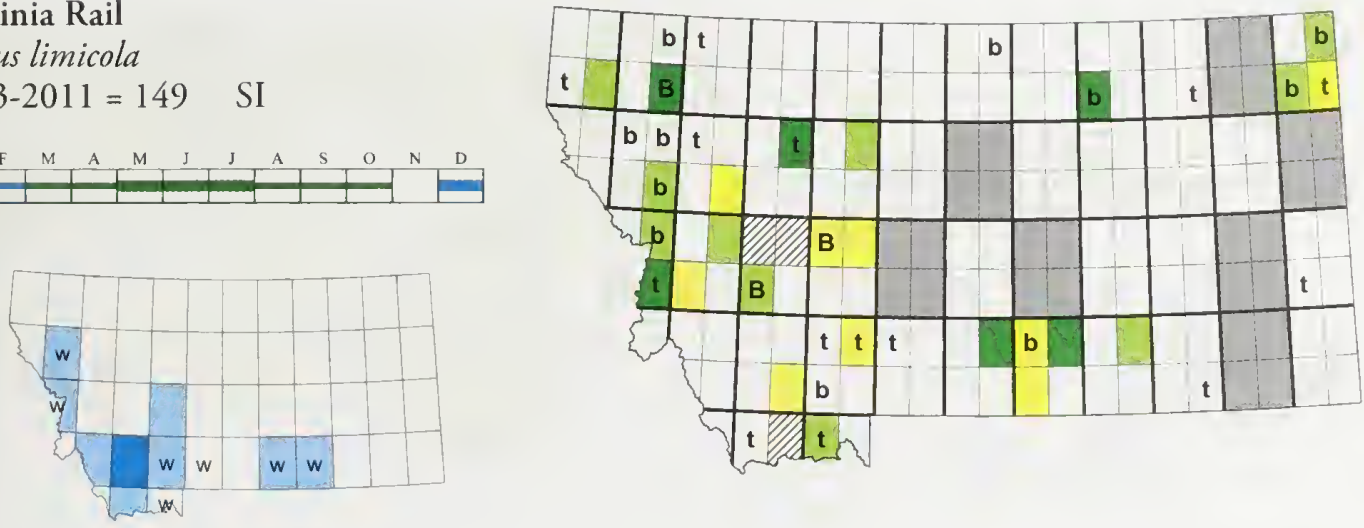

\section{Sora}

Porzana carolina

2003-2011 = 510
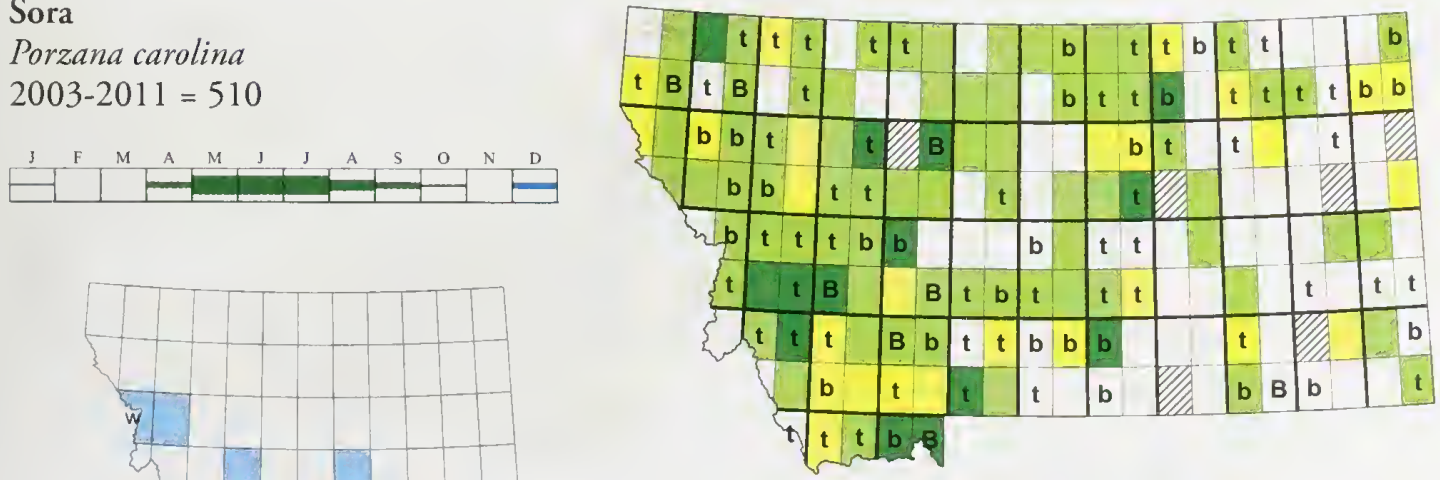
Common Gallinule

Gallinula galeata

2003-2011 = 0 R1

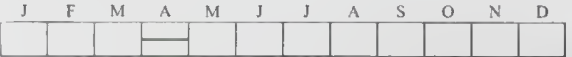

\section{American Coot}

Fulica americana

$2003-2011=1688$
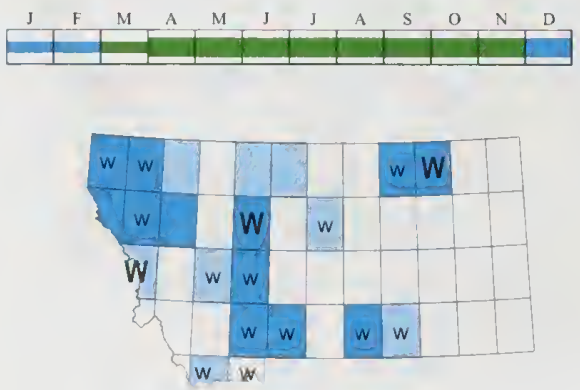

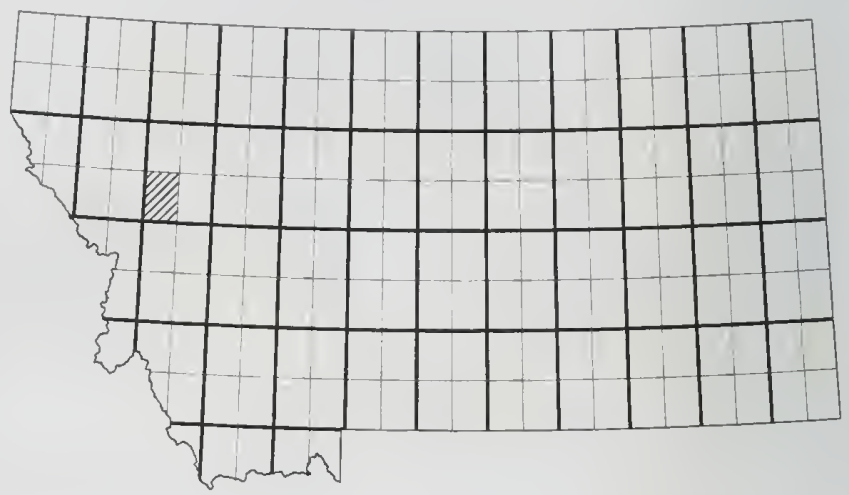

\section{Gruidae}

\section{Sandhill Crane}

Grus canadensis

2003-2011 = 1123
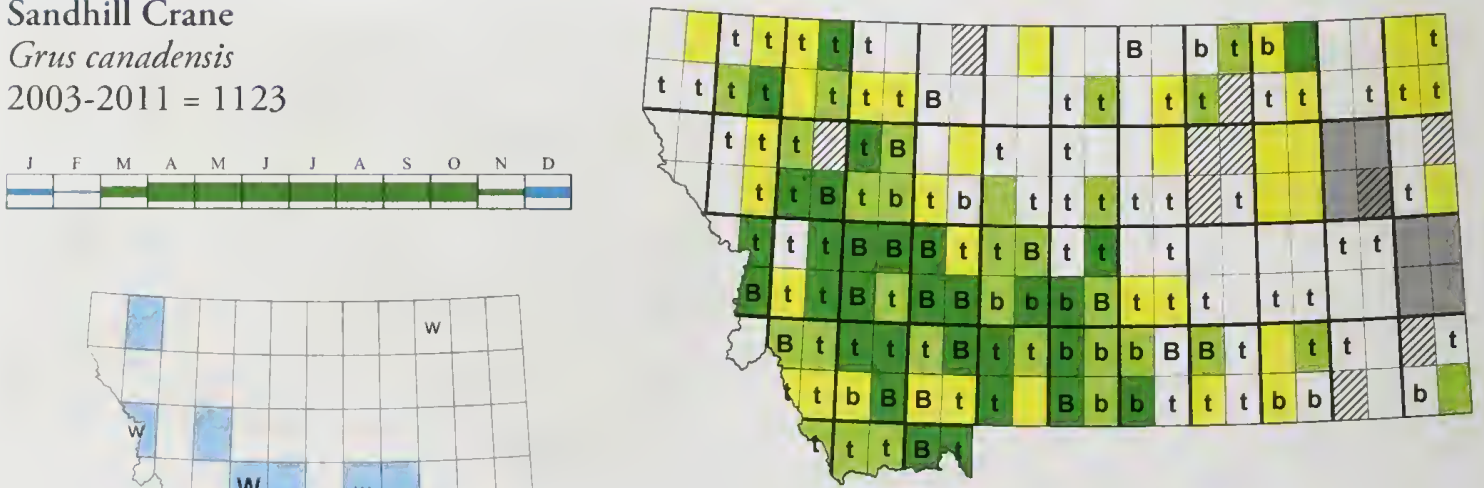
Whooping Crane

Grus americana

2003-2011 = $6 \quad \mathrm{E}$

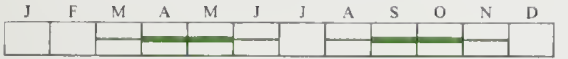
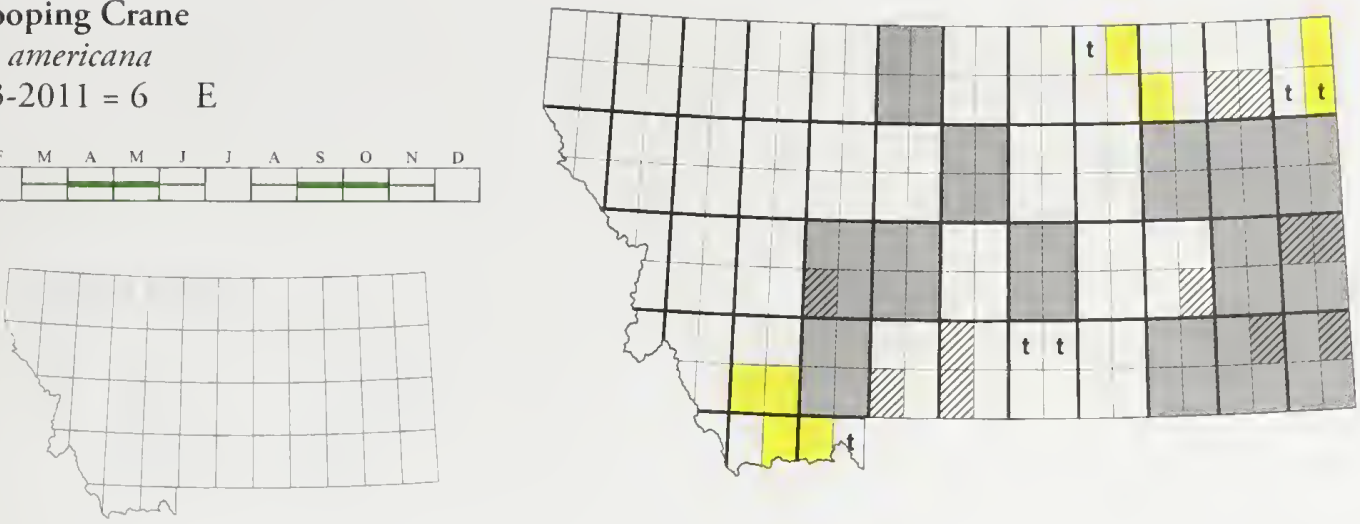

CHARADRIIFORMES Charadriidae

Black-bellied Plover

Pluvialis squatarola

2003-2011 = 82

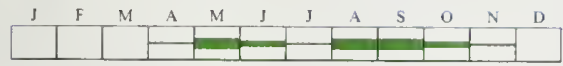

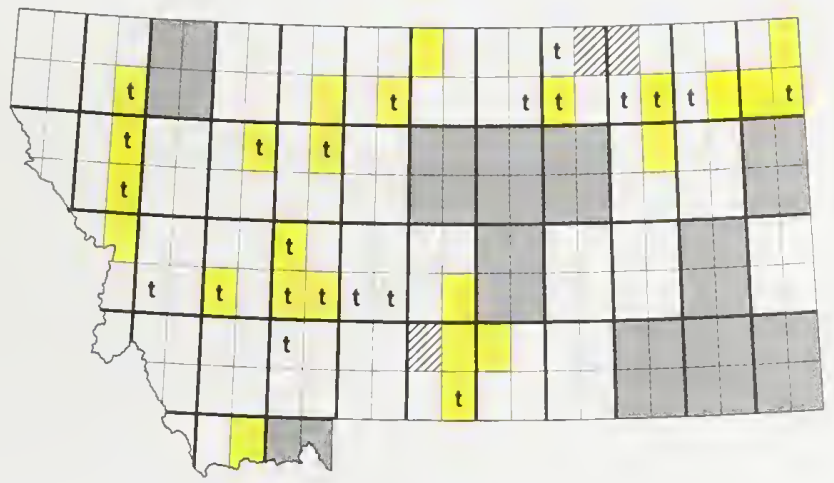

\section{American Golden-Plover}

Pluvialis dominica

2003-2011 = 30
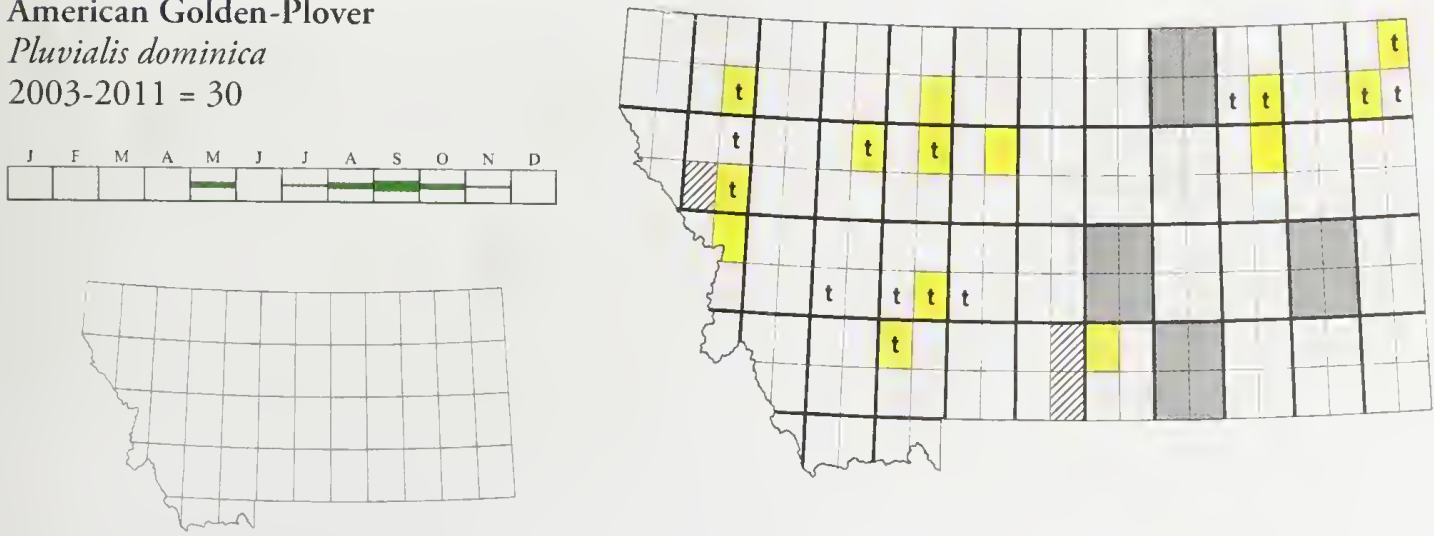
Snowy Plover

Charadrius nivosus

2003-2011 = 2 R1

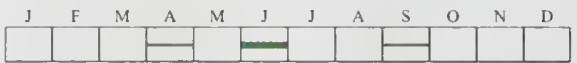
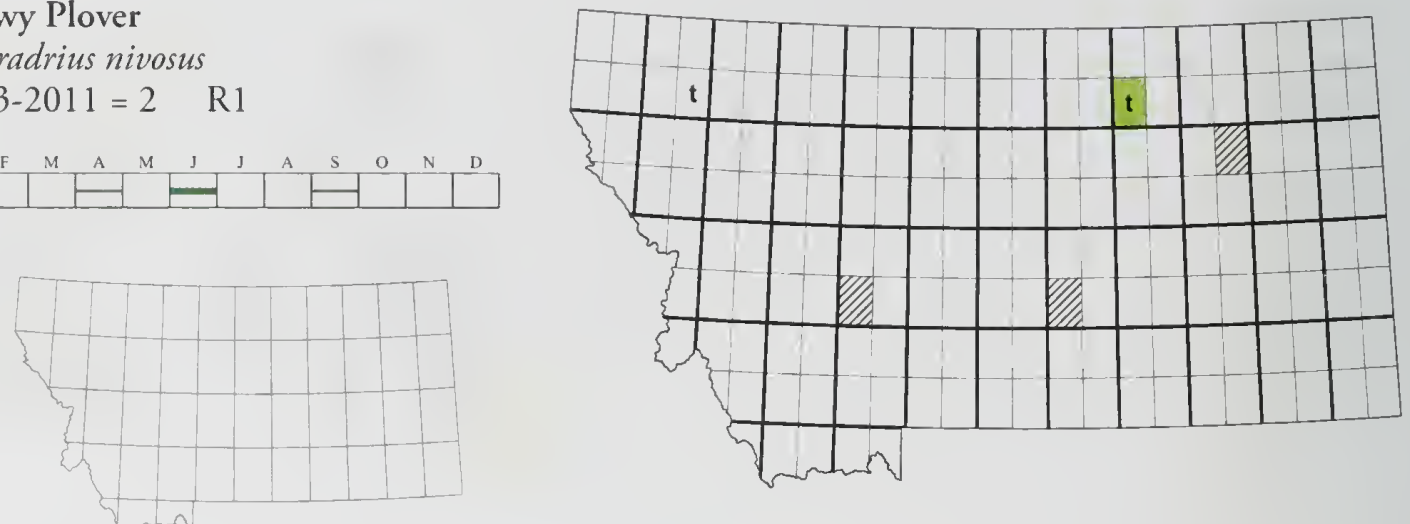

Semipalmated Plover

Charadrius semipalmatus

2003-2011 = 64
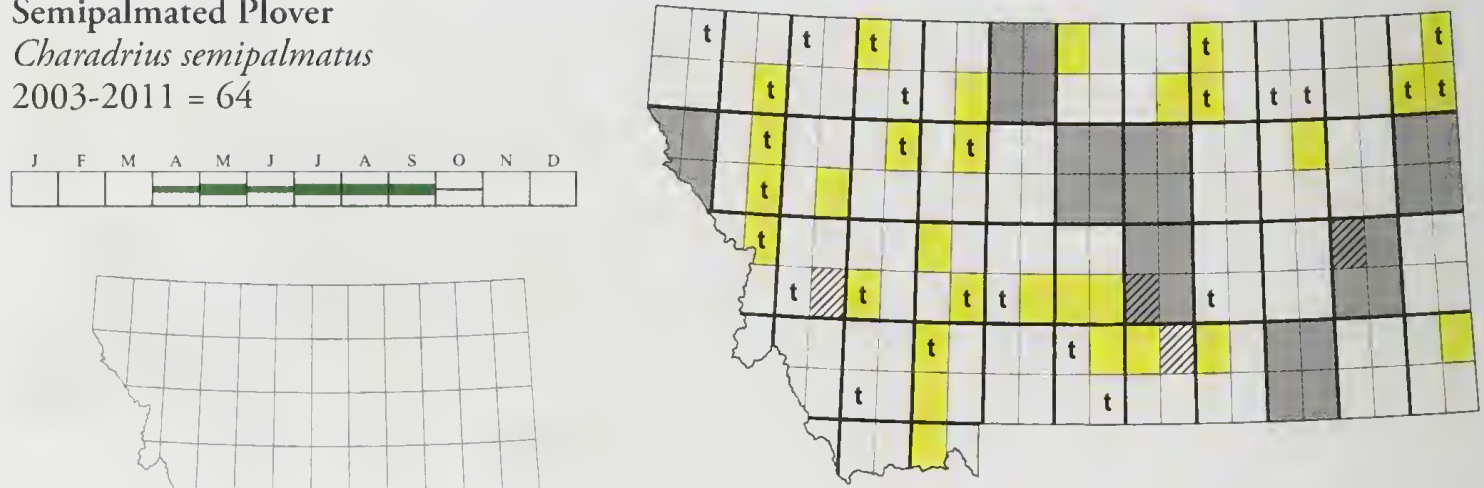

Piping Plover

Charadrius melodus

2003-2011 = 262 E
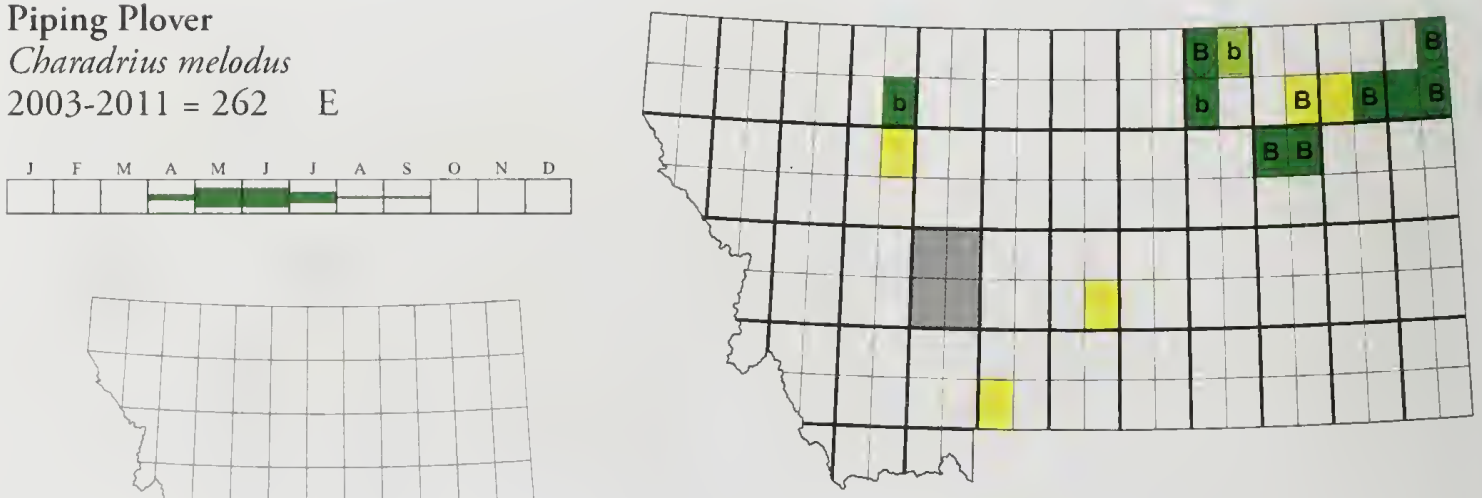
Killdeer

Charadrius vociferus

2003-2011 = 3564
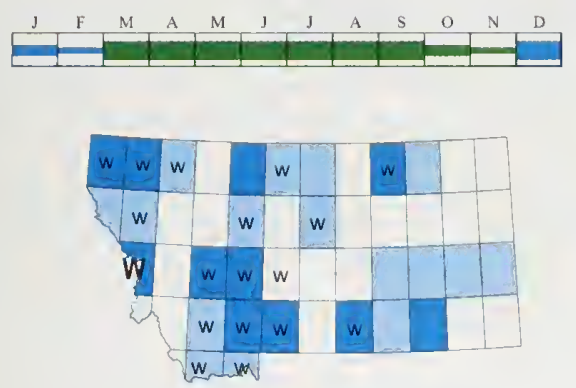

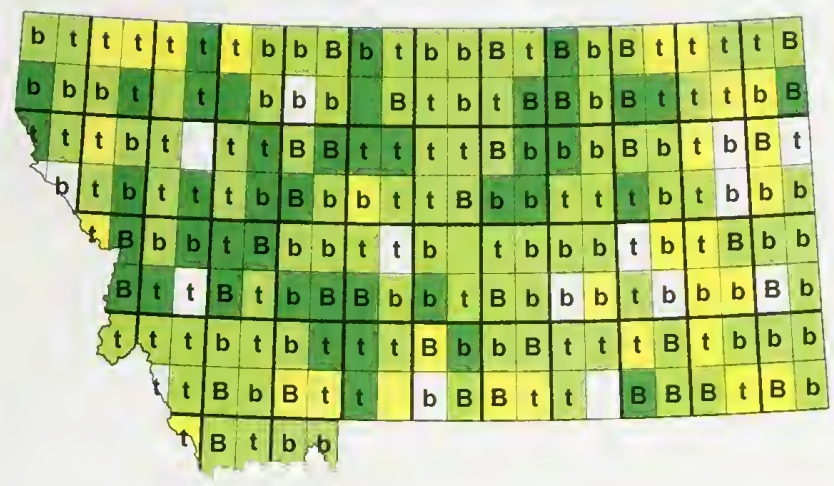

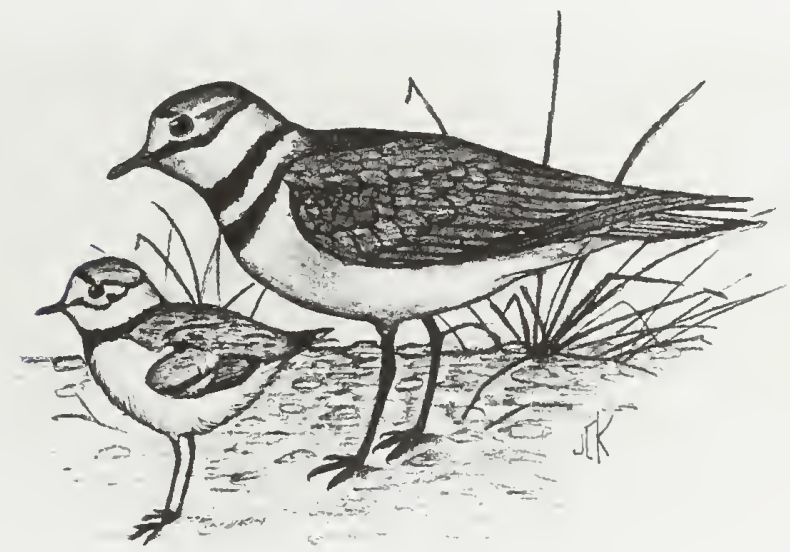

Mountain Plover

Charadrius montanus

2003-2011 = 1003 SOC
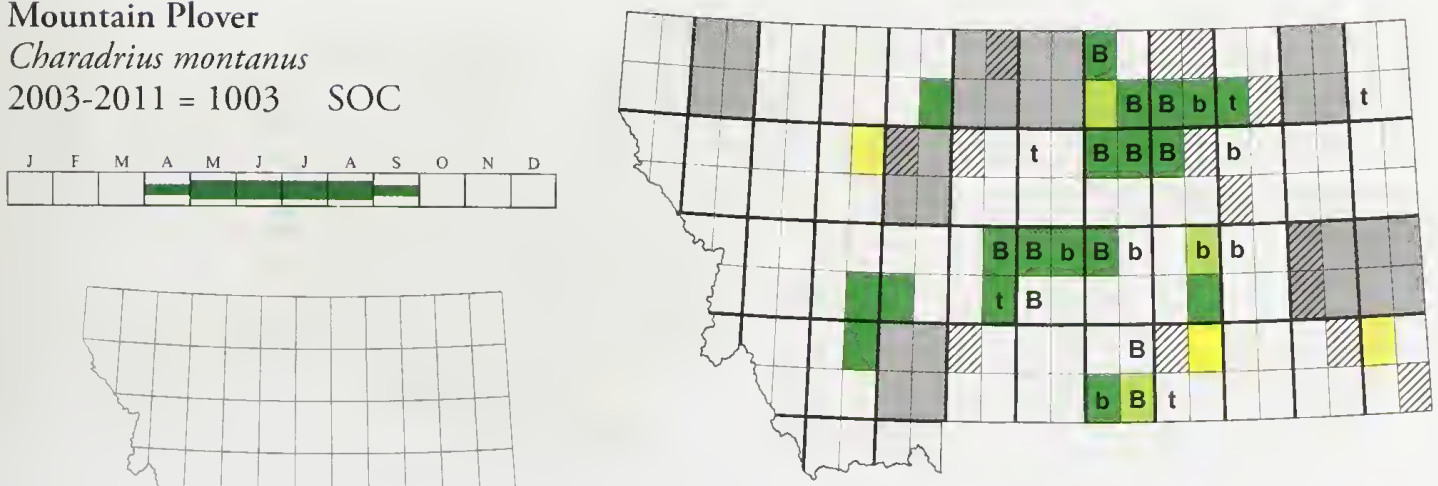
Black-necked Stilt

Himantopus mexicanus

$2003-2011=170 \quad$ SOC
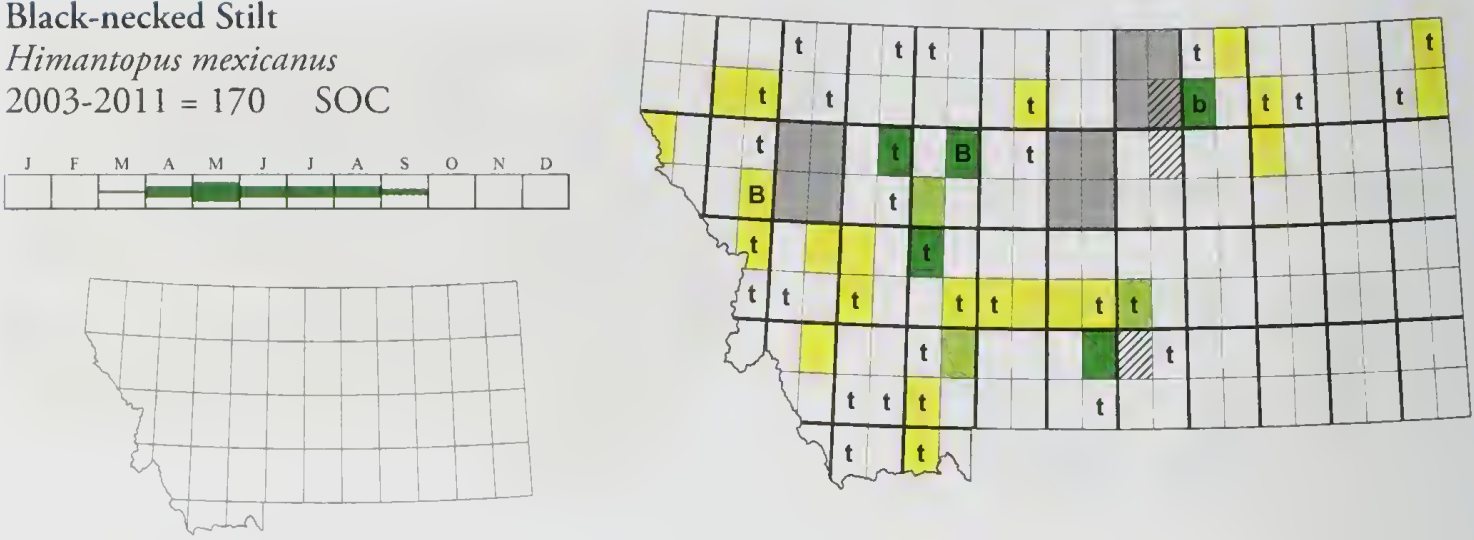

American Avocet

Recurvirostra americana

$2003-2011=638$
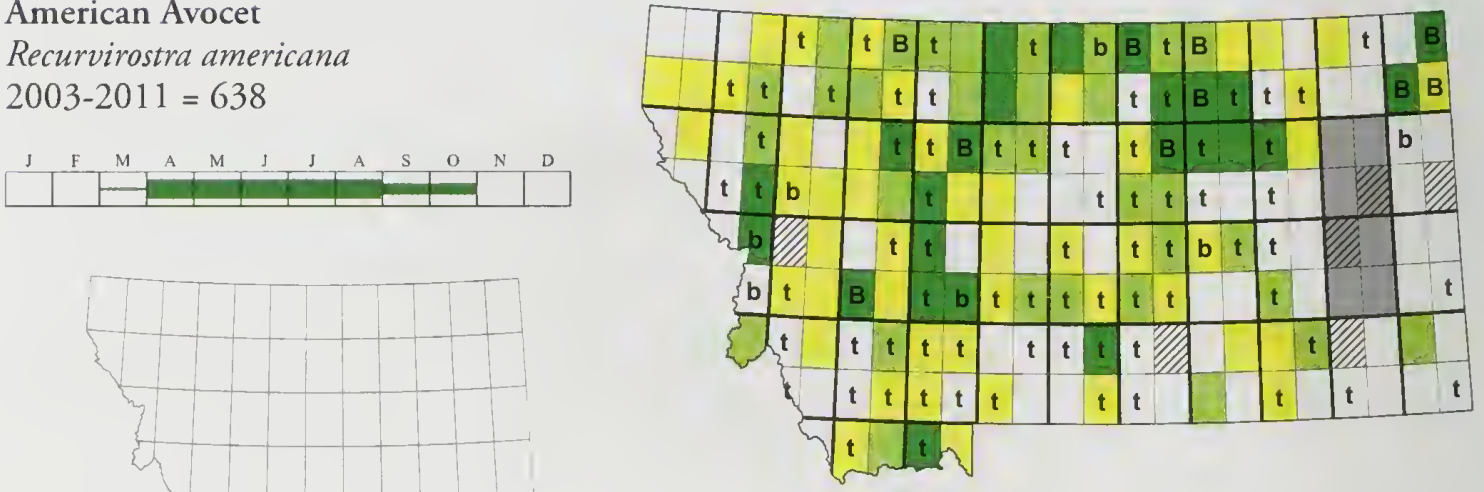

Scolopacidae

Spotted Sandpiper

Actitis macularius

2003-2011 = 1524
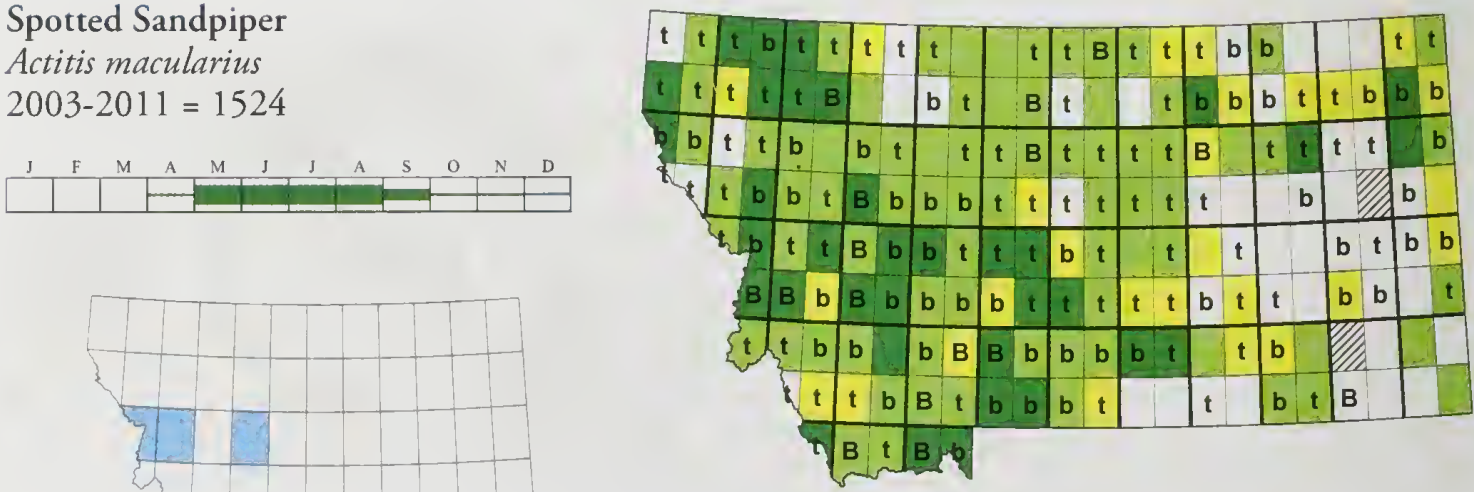
Solitary Sandpiper

Tringa solitaria

2003-2011 = 155
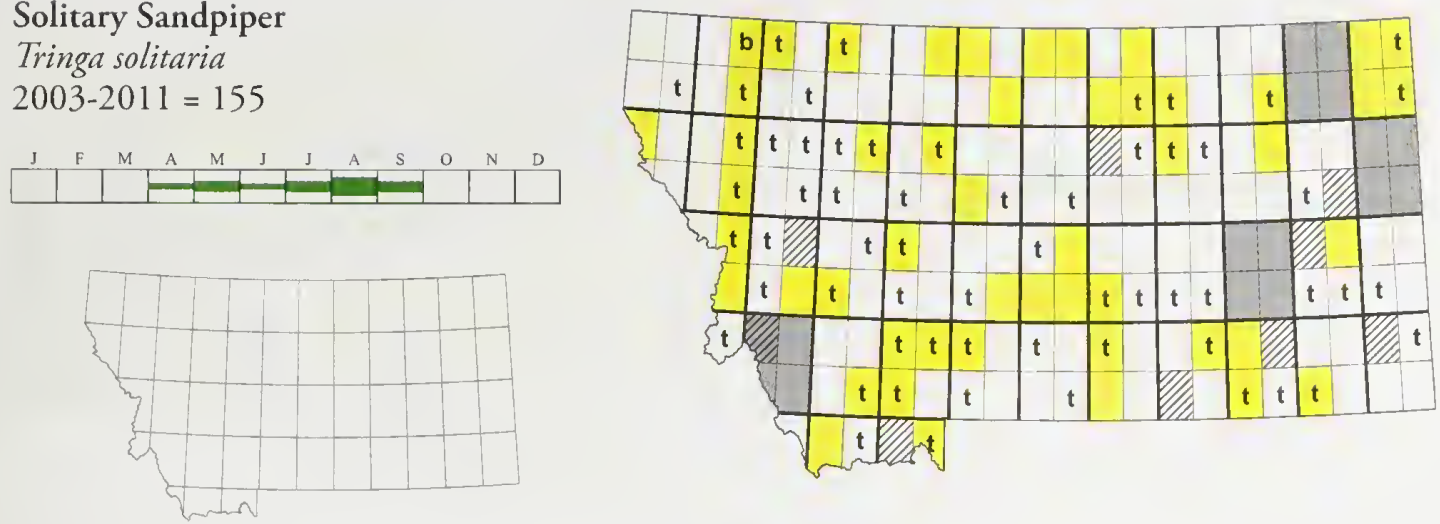

Greater Yellowlegs

Tringa melanoleuca

$2003-2011=289$
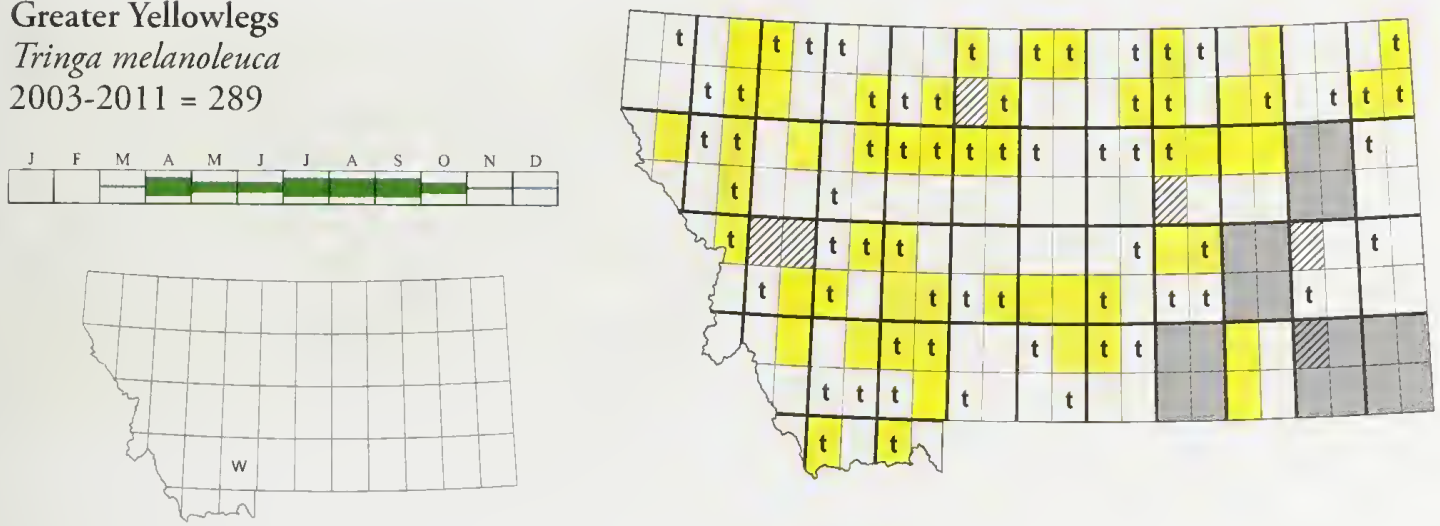

Willet

Tringa semipalmata

2003-2011 = 979
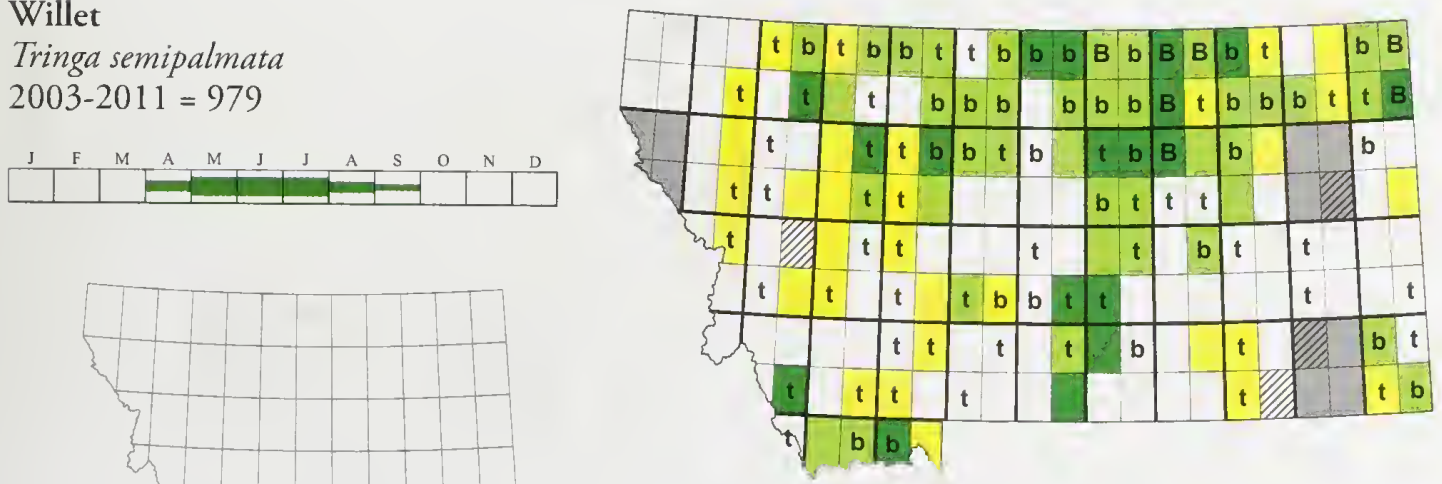
Lesser Yellowlegs

Tringa flavipes

$2003-2011=218$

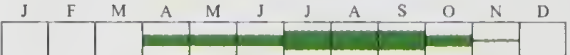

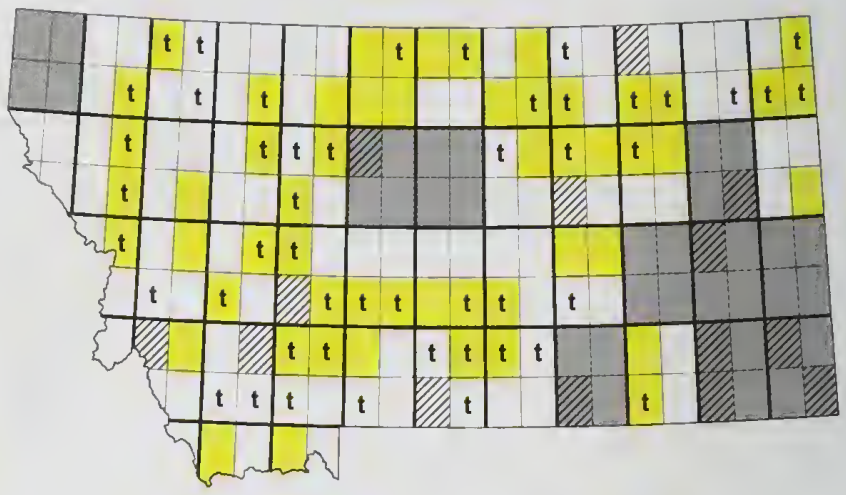

Upland Sandpiper

Bartramia longicauda

$2003-2011=970 \quad$ SI

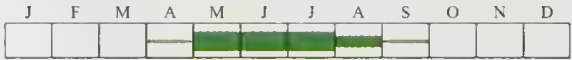

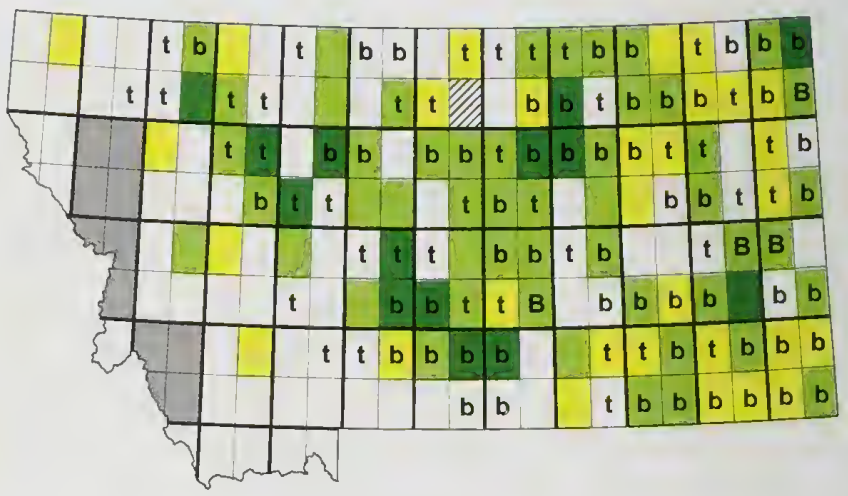

Whimbrel

Numenius phaeopus

2003-2011 = 13

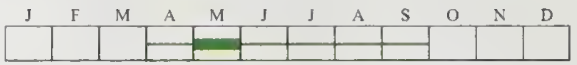

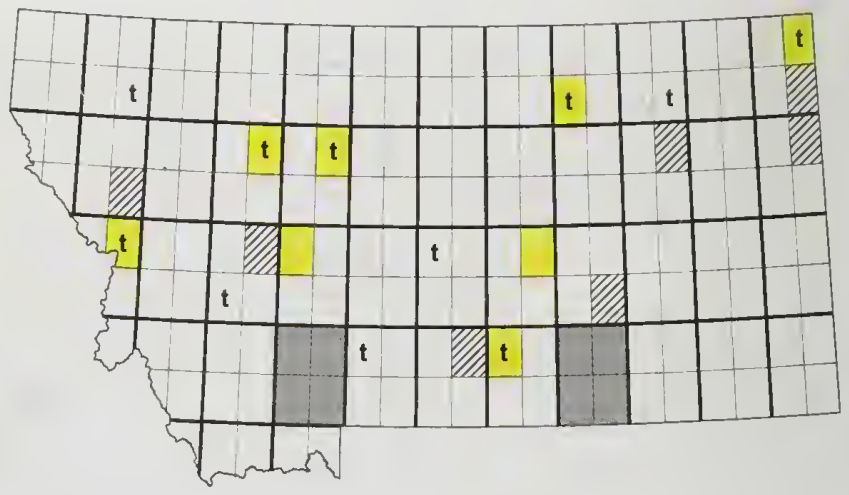




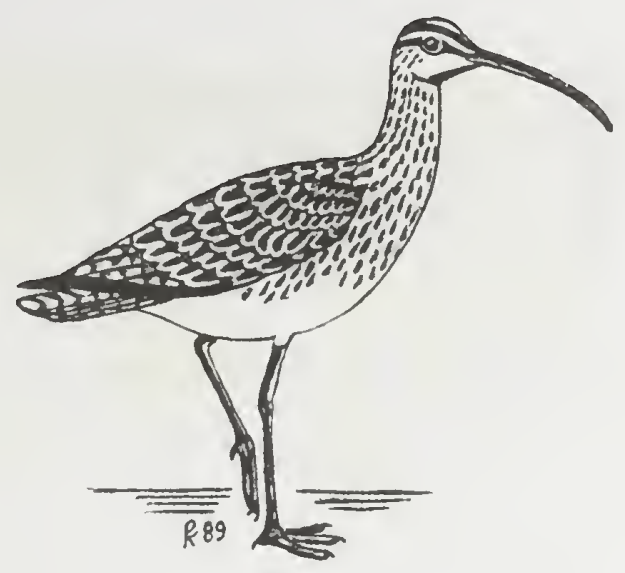

Long-billed Curlew

Numenius americanus

$2003-2011=1997$ SOC
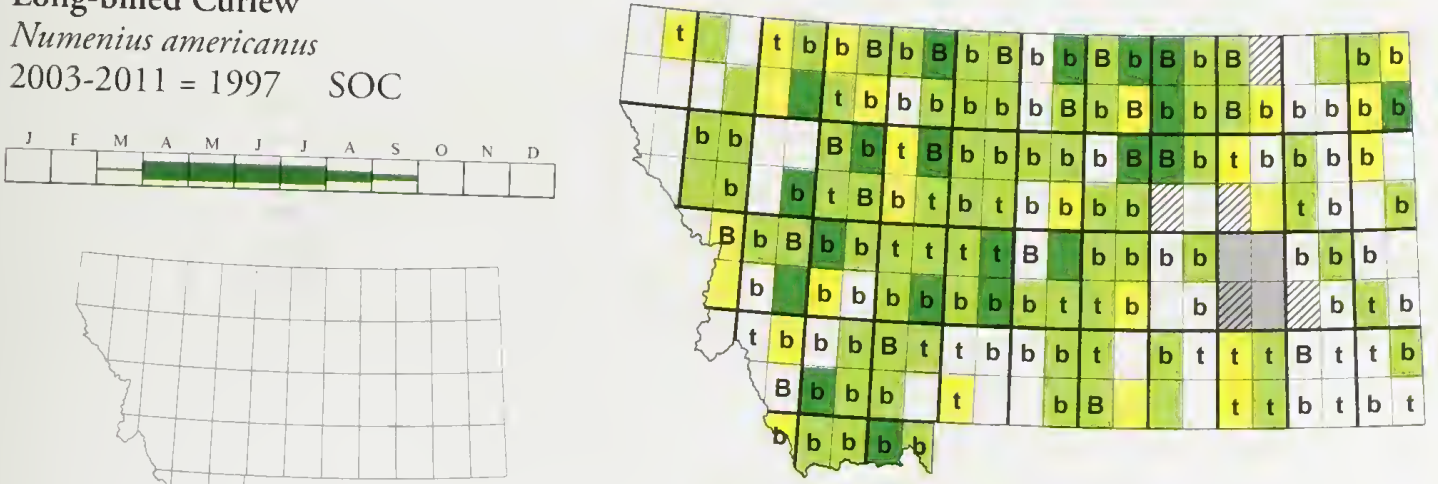

Hudsonian Godwit

Limosa haemastica

2003-2011 = 14
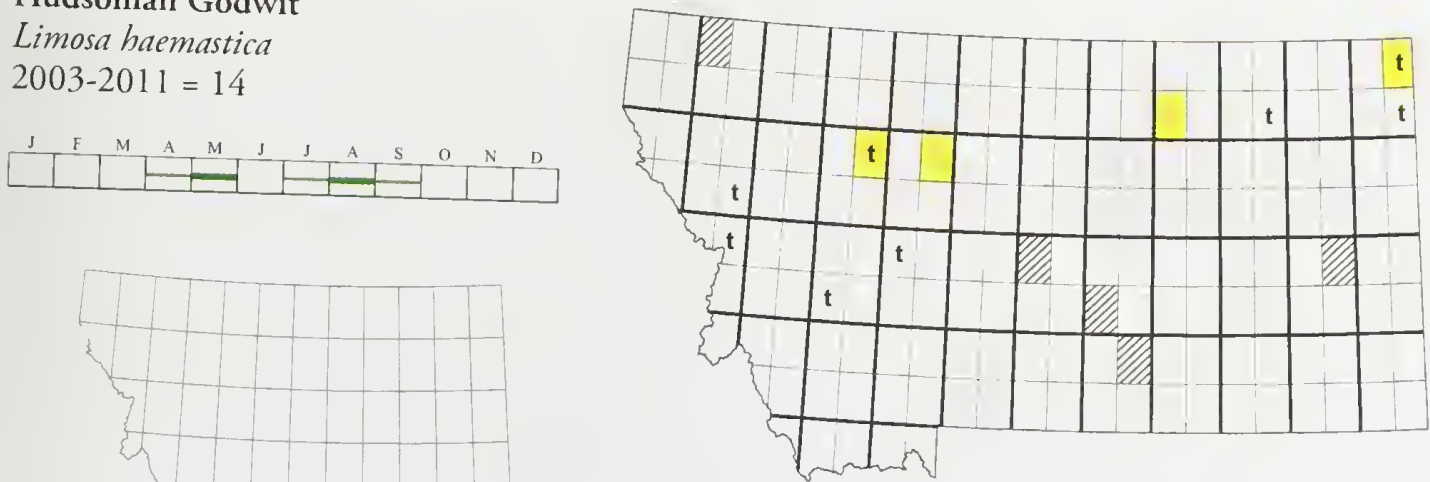
Marbled Godwit

Limosa fedoa

2003-2011 = 1351
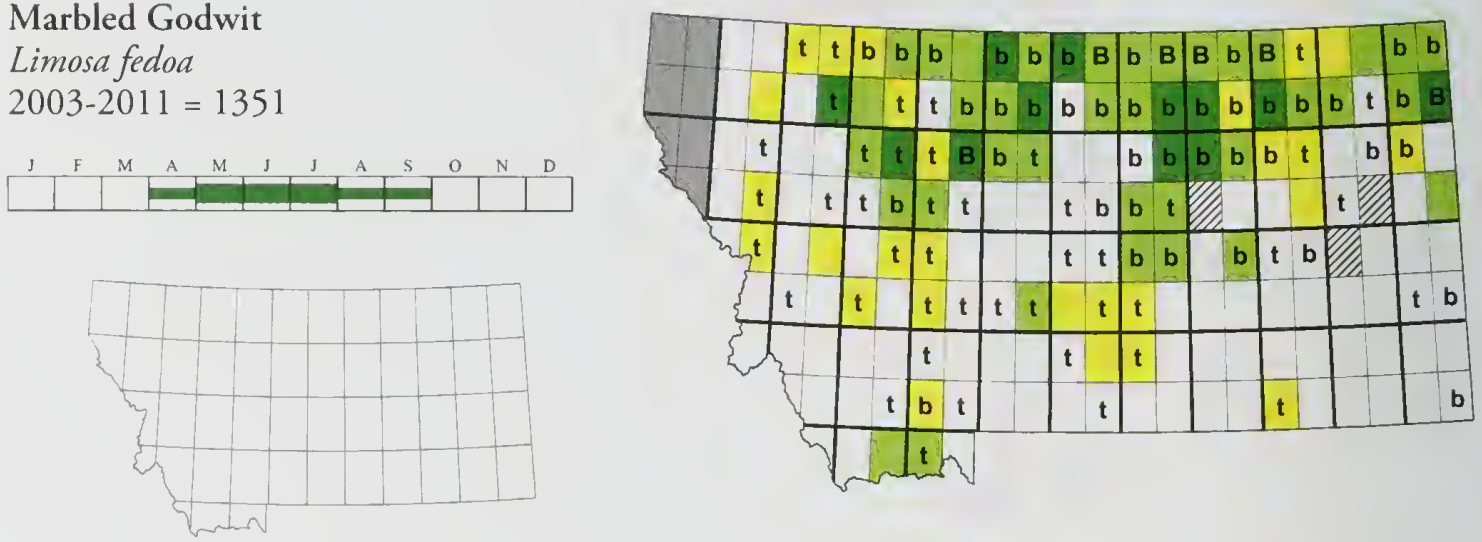

\section{Ruddy Turnstone}

Arenaria interpres

2003-2011 = 7
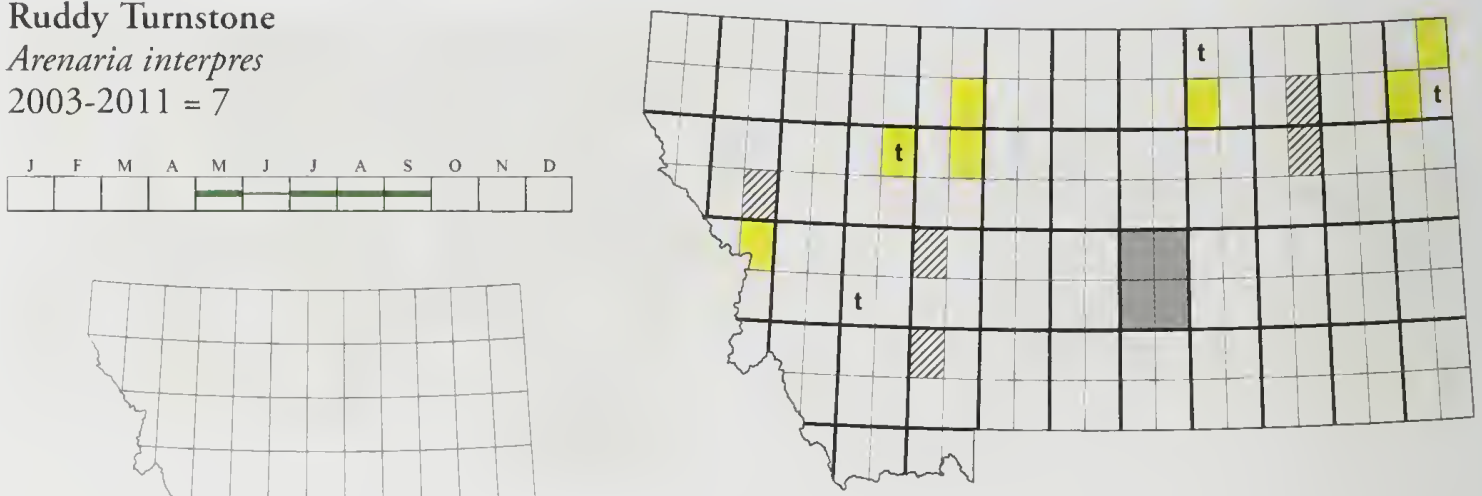

Black Turnstone

Arenaria melanocephala

2003-2011 = 0 R1

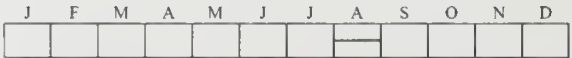

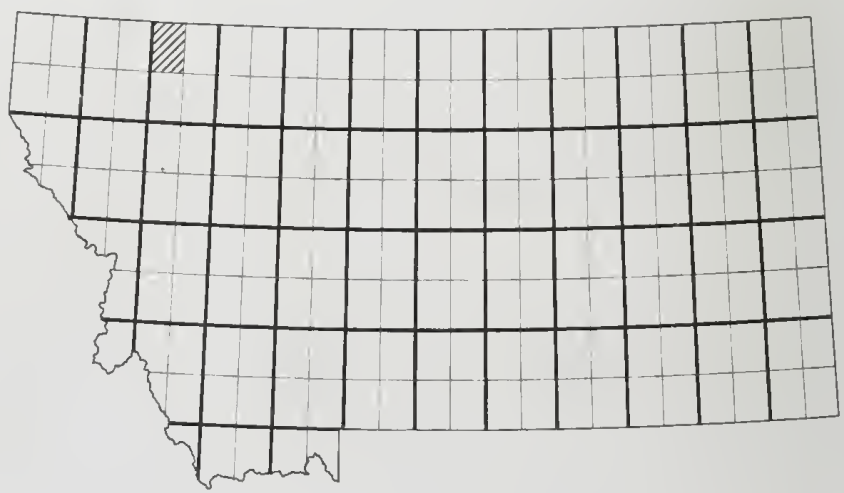




\section{Red Knot}

Calidris canutus

2003-2011 = 4
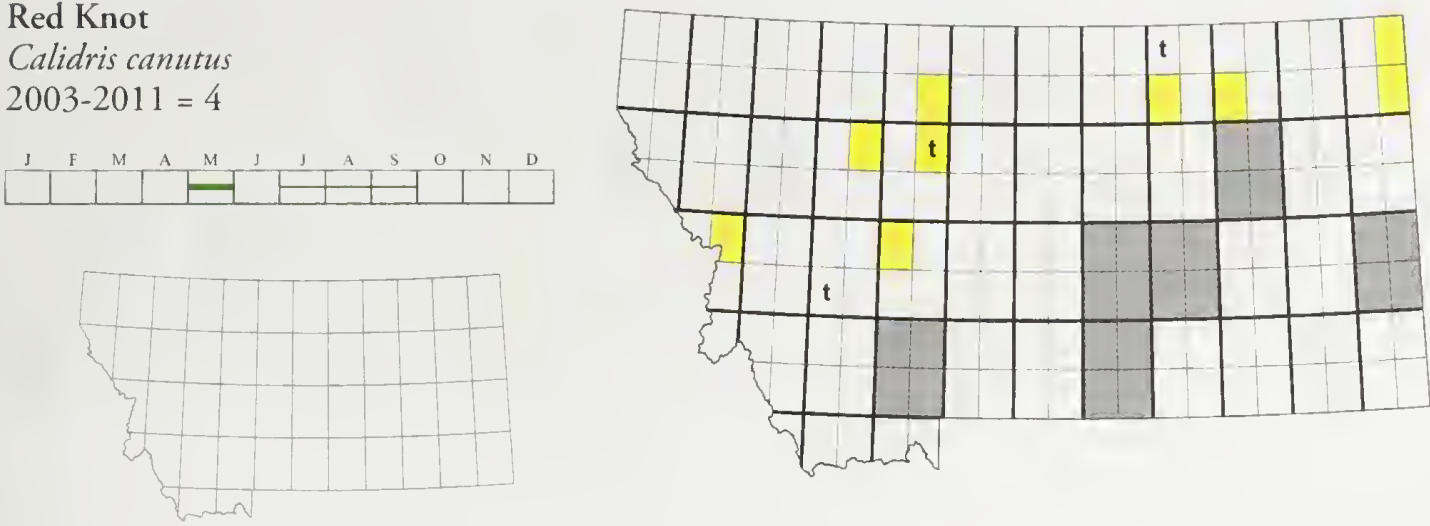

\section{Sanderling}

Calidris alba

2003-2011 = 72
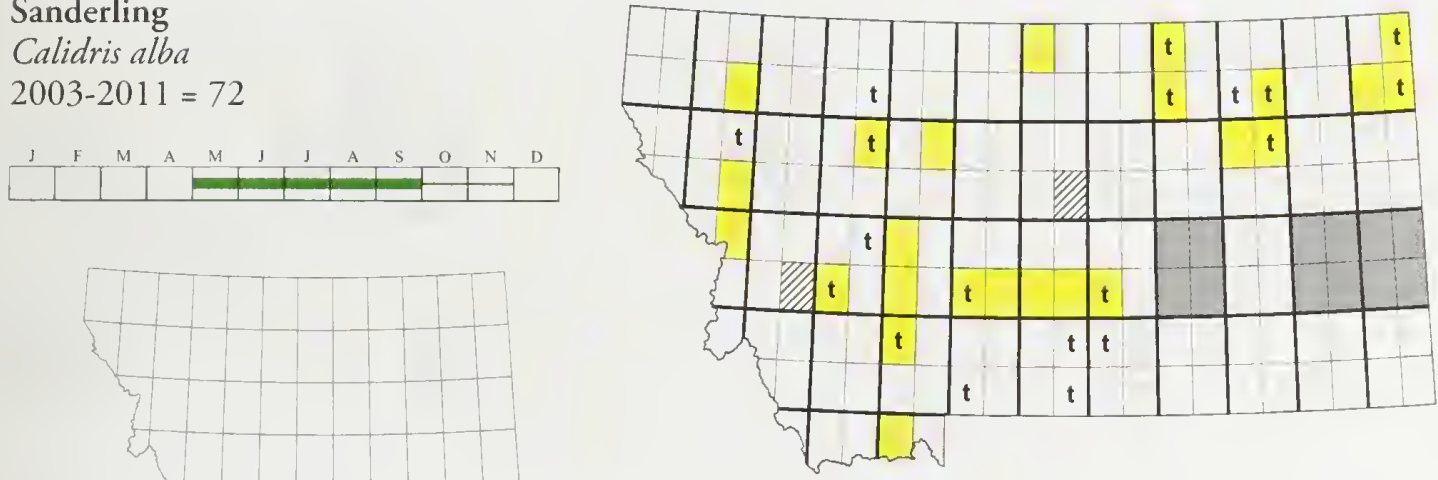

Semipalmated Sandpiper

Calidris pusilla

2003-2011 = 105
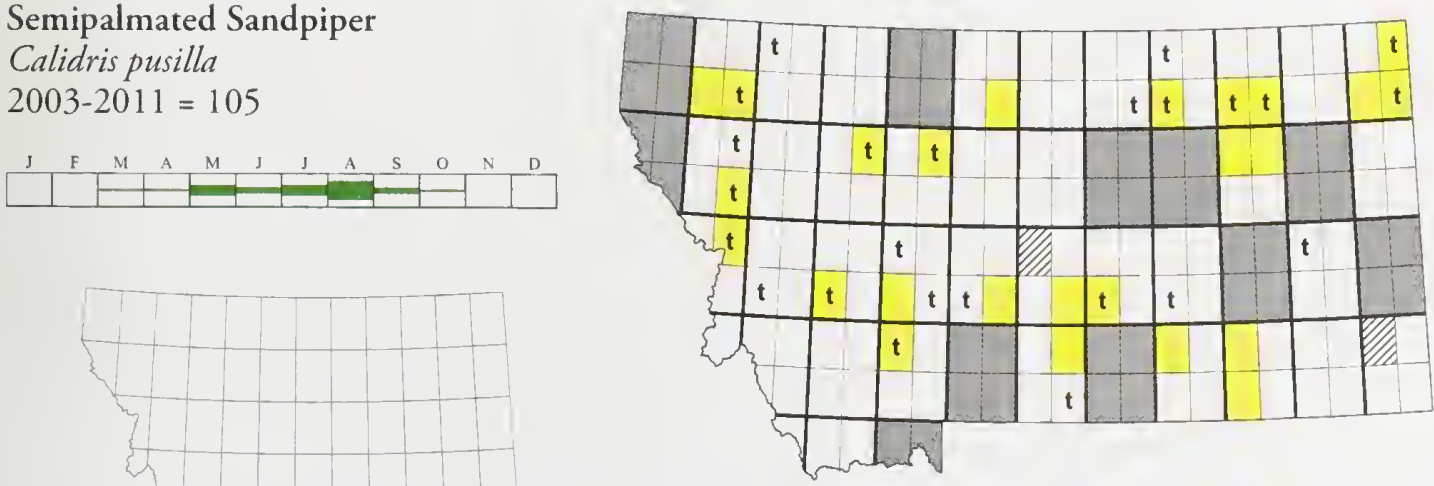
Western Sandpiper

Calidris mauri

2003-2011 = 61

\begin{tabular}{|l|l|l|l|l|l|l|l|l|l|l|l}
$\mathrm{S}$ & $\mathrm{F}$ & $\mathrm{M}$ & $\mathrm{A}$ & $\mathrm{M}$ & $\mathrm{J}$ & $\mathrm{J}$ & $\mathrm{A}$ & $\mathrm{S}$ & $\mathrm{O}$ & $\mathrm{N}$ & $\mathrm{D}$ \\
\hline & & & & & & & & & & & \\
\hline
\end{tabular}

\section{Least Sandpiper}

Calidris minutilla

2003-2011 = 97

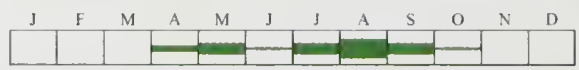

White-rumped Sandpiper

Calidris fuscicollis

2003-2011 = 16

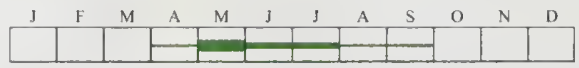

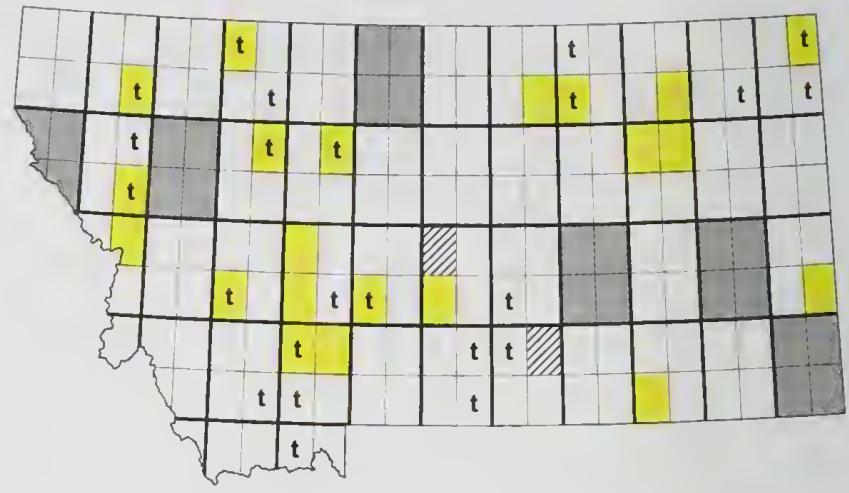


Baird's Sandpiper

Calidris bairdii

2003-2011 = 147
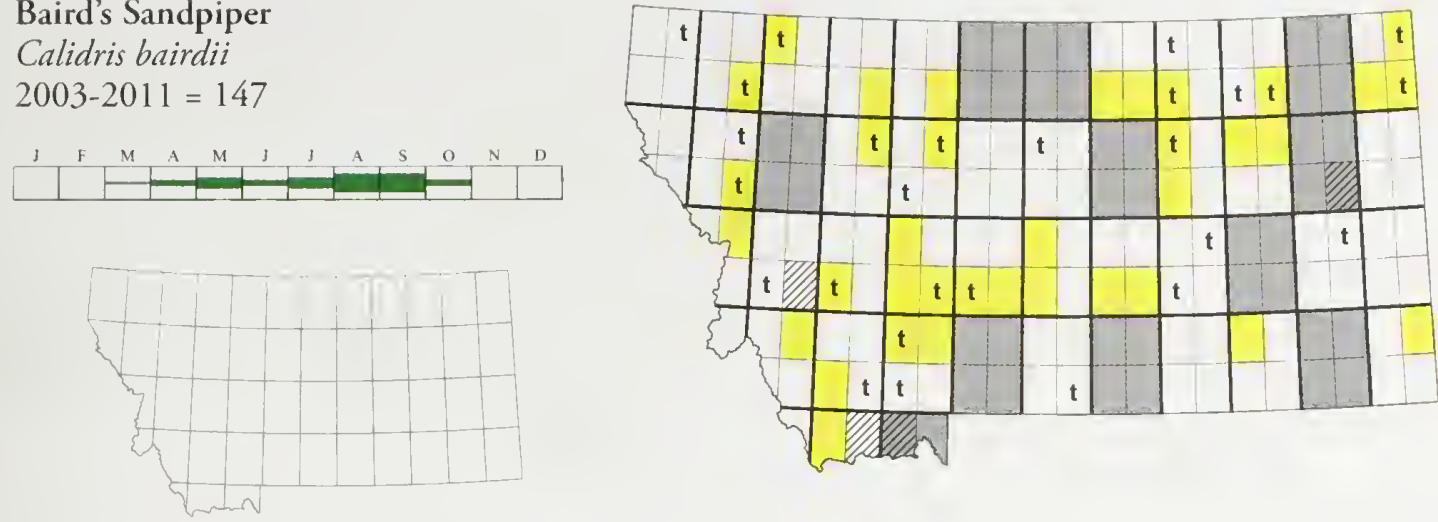

Pectoral Sandpiper

Calidris melanotos

2003-2011 = 80
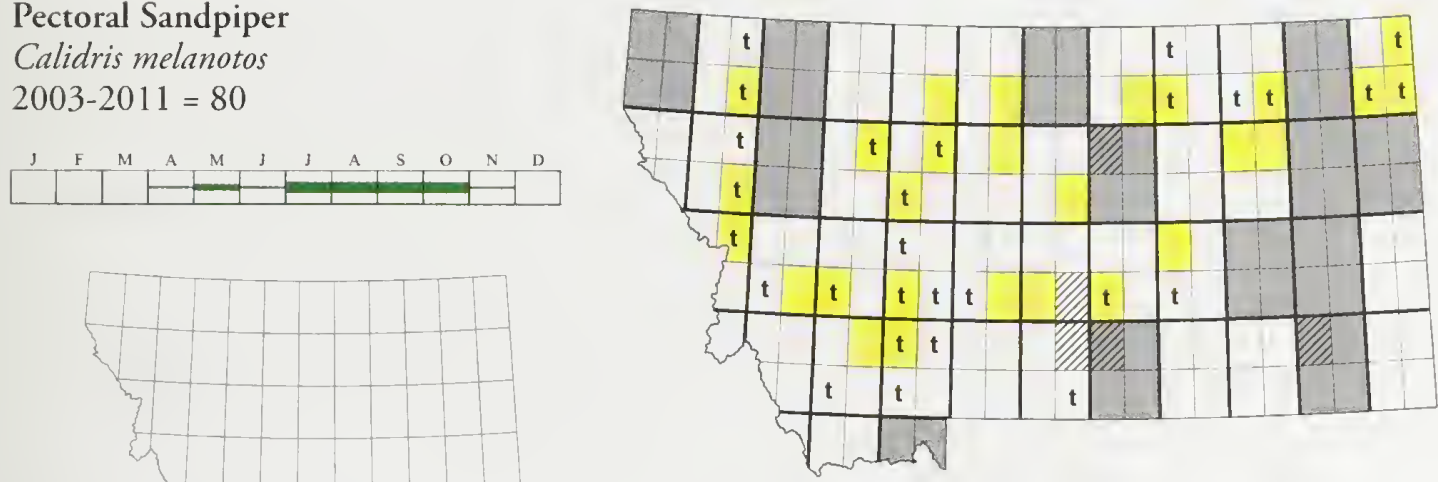

Sharp-tailed Sandpiper

Calidris acuminata

2003-2011 = 1 R1
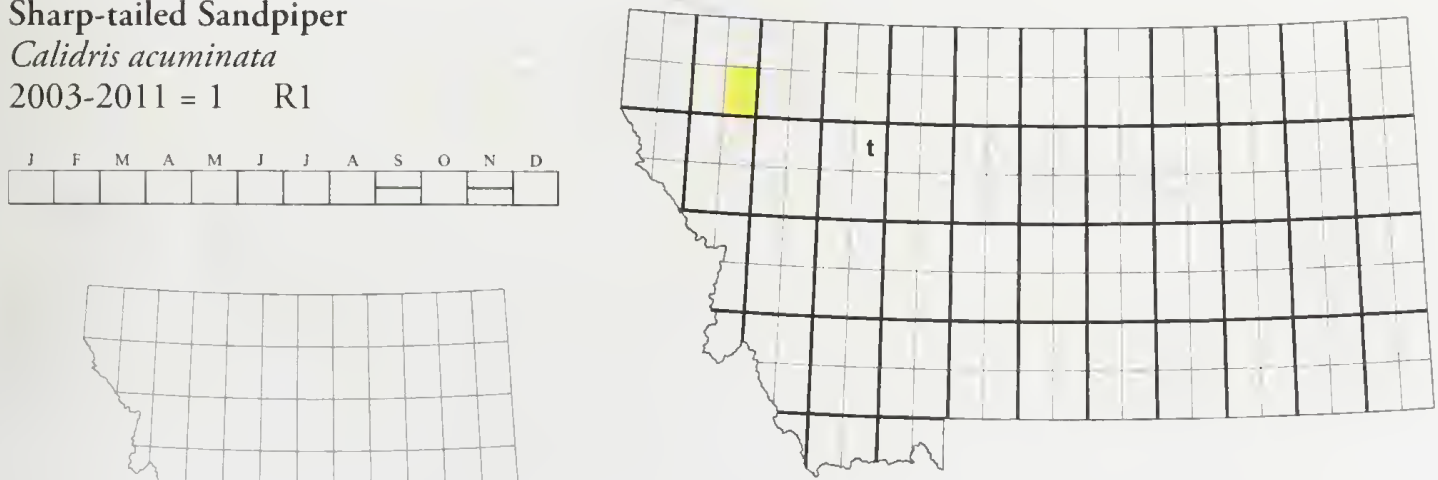
Dunlin

Calidris alpine

2003-2011 = 17
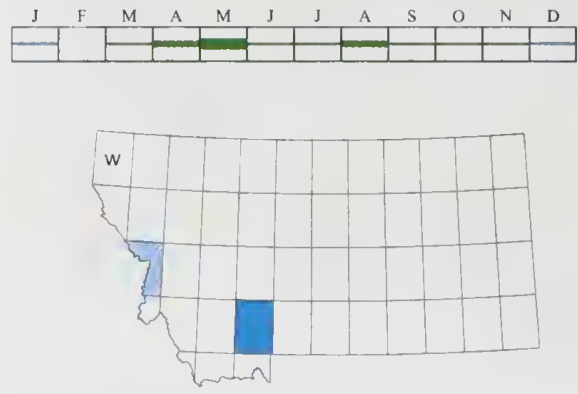

\section{Curlew Sandpiper}

Calidris ferruginea

2003-2011 = 2 R1

$\left.\square^{\mathrm{F}} \mathrm{M}^{\mathrm{M}} \mathrm{A}^{\mathrm{A}} \mathrm{M}^{\mathrm{M}} \mathrm{H}^{\mathrm{A}} \mathrm{A}^{\mathrm{A}} \mathrm{H}^{\mathrm{O}} \mathrm{H}^{\mathrm{N}}\right|^{\mathrm{D}}$
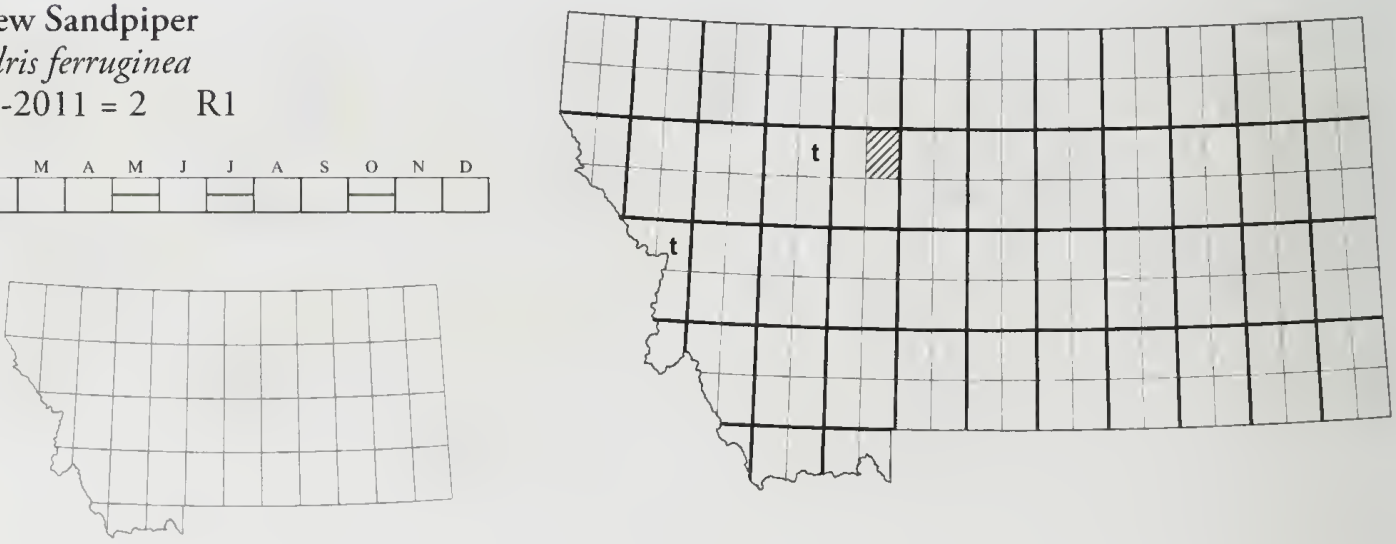

Stilt Sandpiper

Calidris himantopus

2003-2011 = 56
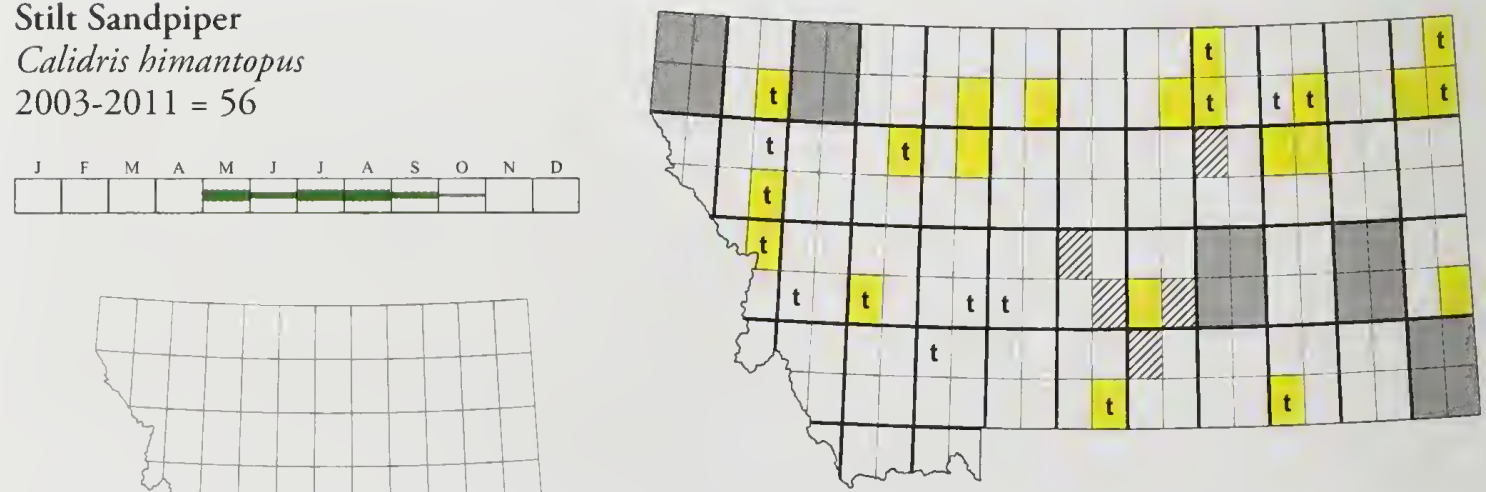
Buff-breasted Sandpiper

Tryngites subruficollis

2003-2011 = 10
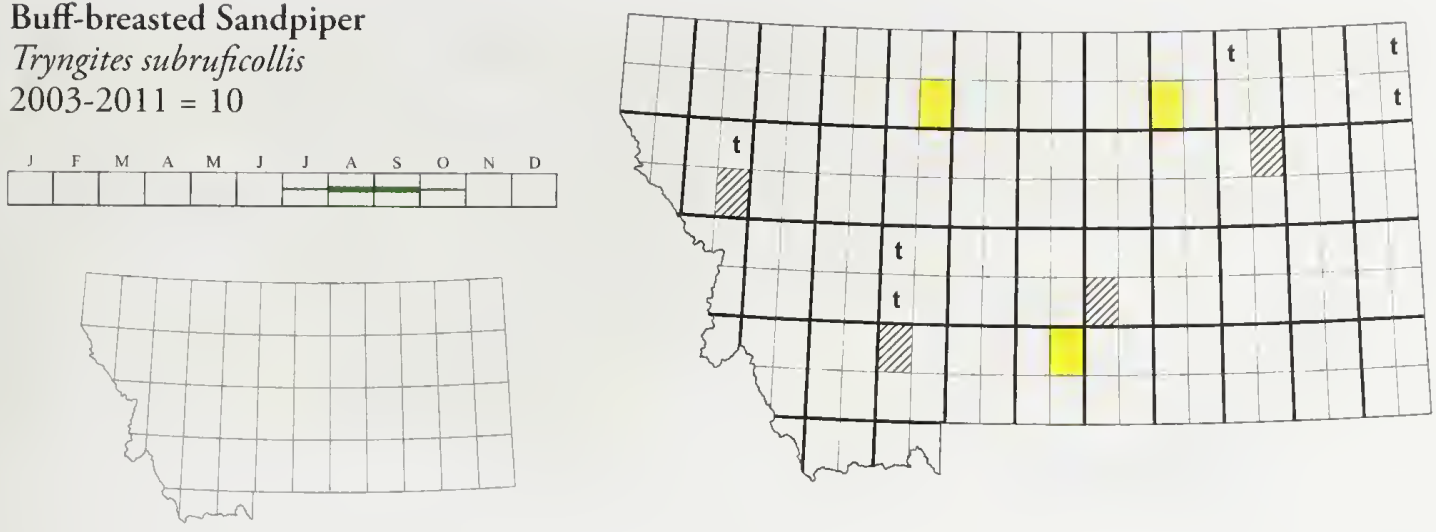

Short-billed Dowitcher

Limnodromus griseus

$2003-2011=29$
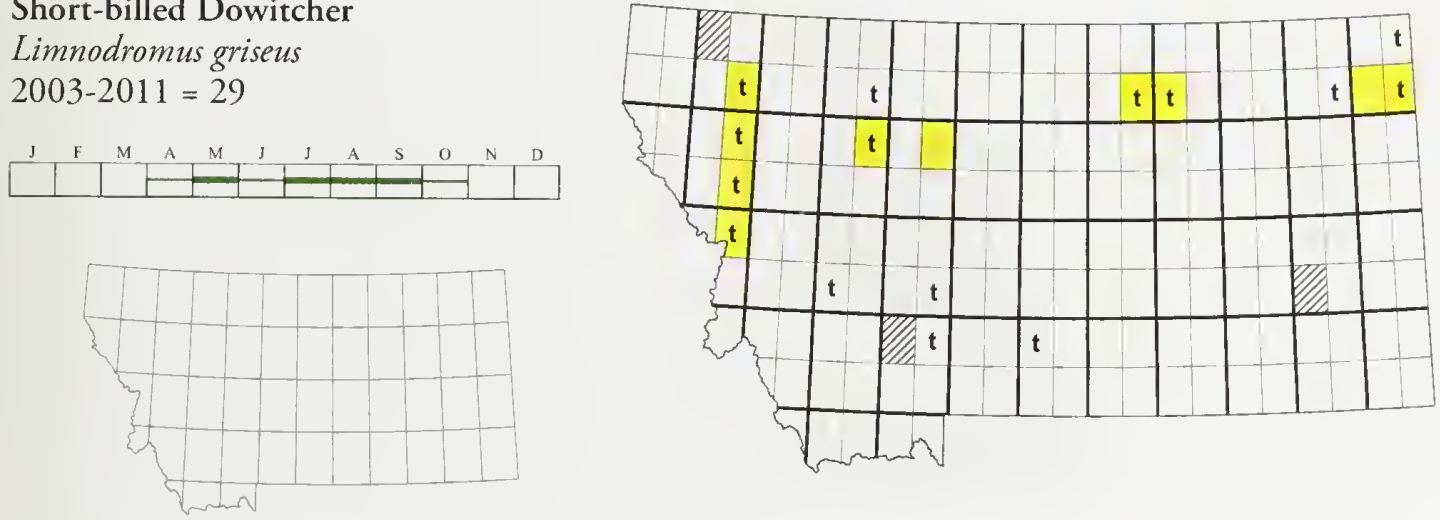

Long-billed Dowitcher

Limnodromus scolopaceus

2003-2011 $=178$

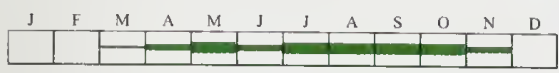

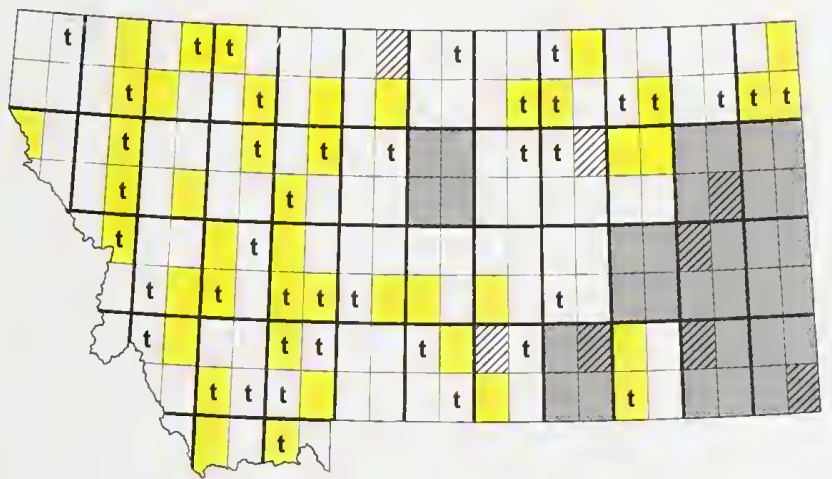


Wilson's Snipe

Gallinago delicata

2003-2011 = 2011
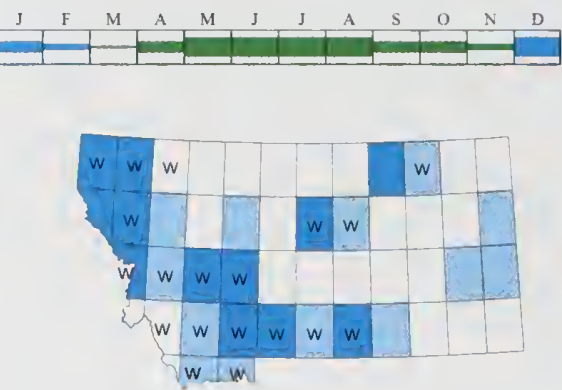

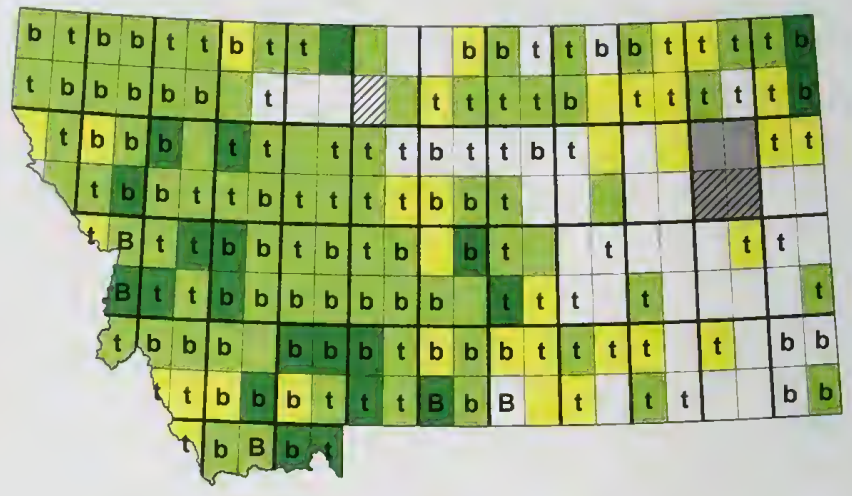

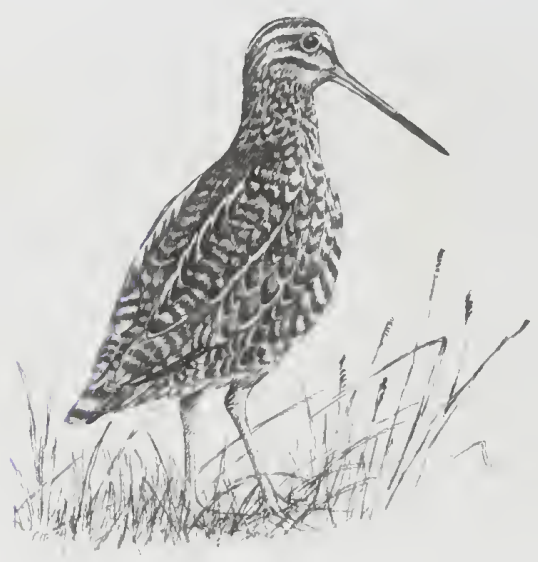

American Woodcock

Scolopax minor

2003-2011 = 1 R1

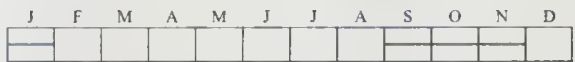

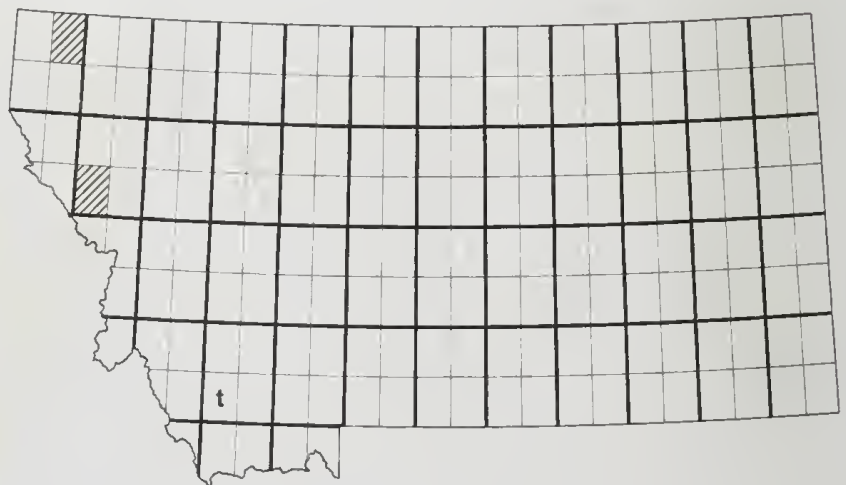


Wilson's Phalarope

Phalaropus tricolor

2003-2011 $=918$

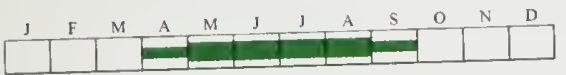

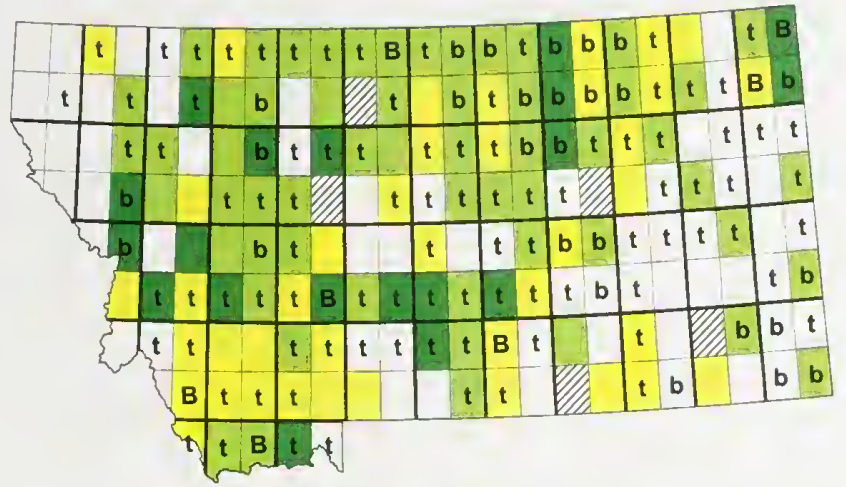

Red-necked Phalarope

Phalaropus lobatus

2003-2011 = 130

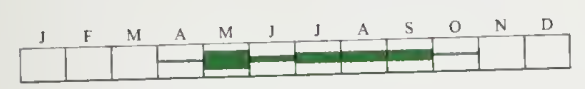

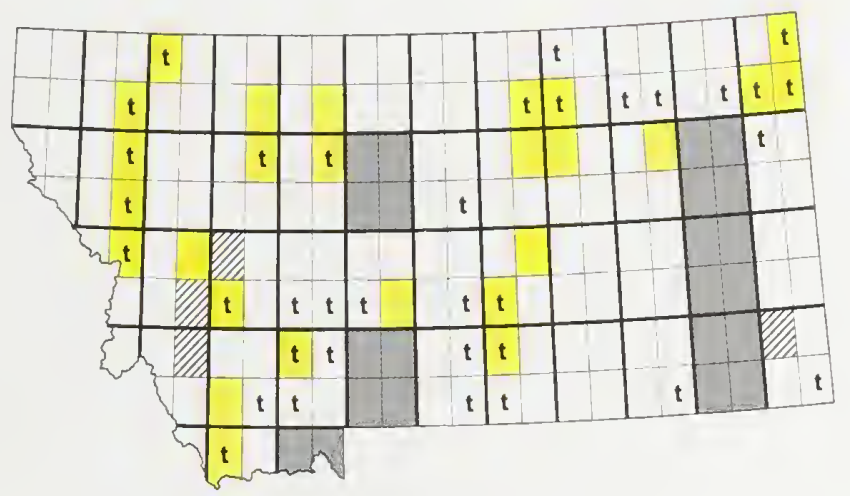

\section{Red Phalarope}

Phalaropus fulicarius

2003-2011=6 R1

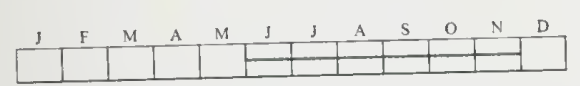

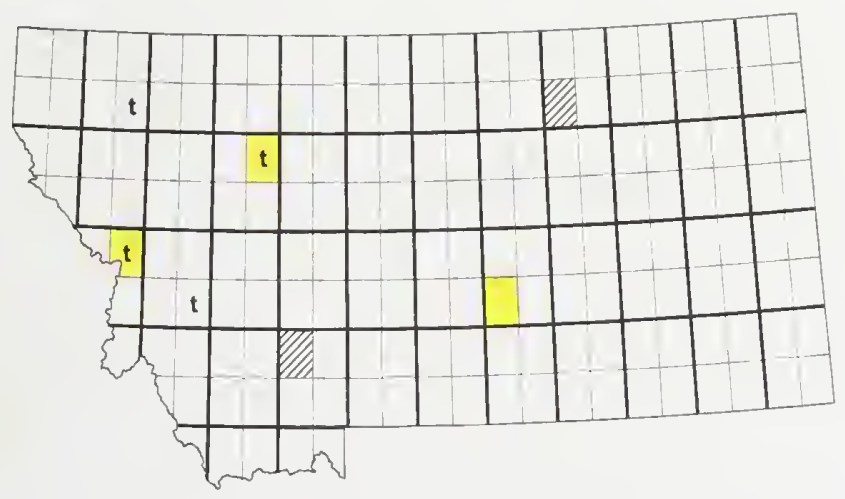


Black-legged Kittiwake

Rissa tridactyla

2003-2011 = $0 \quad$ R1

$\left.L^{\prime} I^{M} L^{A} H^{M} I^{\prime} I^{\prime} L^{A}\right|^{S} L^{O} H^{N}$
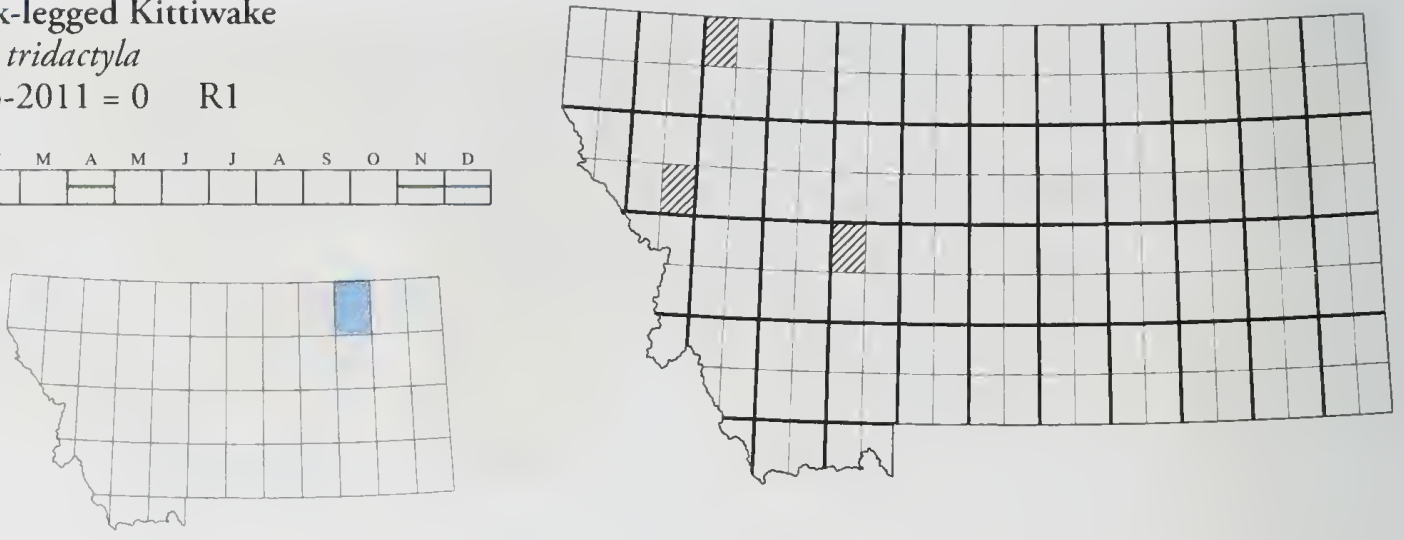

Sabine's Gull

Xema sabini

2003-2011 = 24
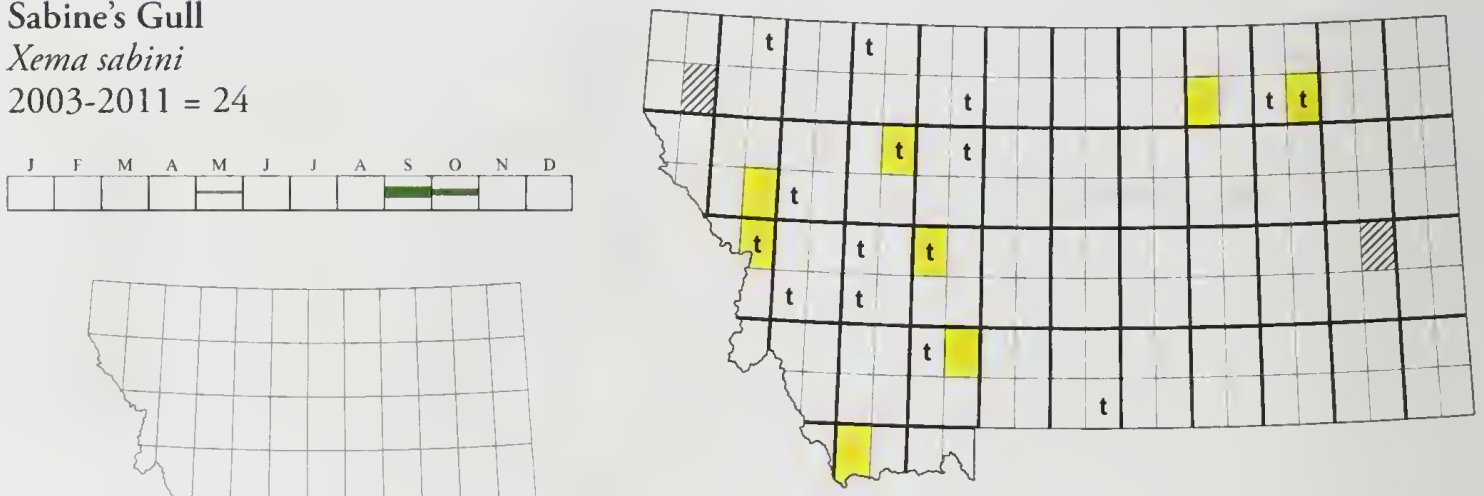

\section{Bonaparte's Gull}

Chroicocephalus philadelphia

2003-2011 = 84
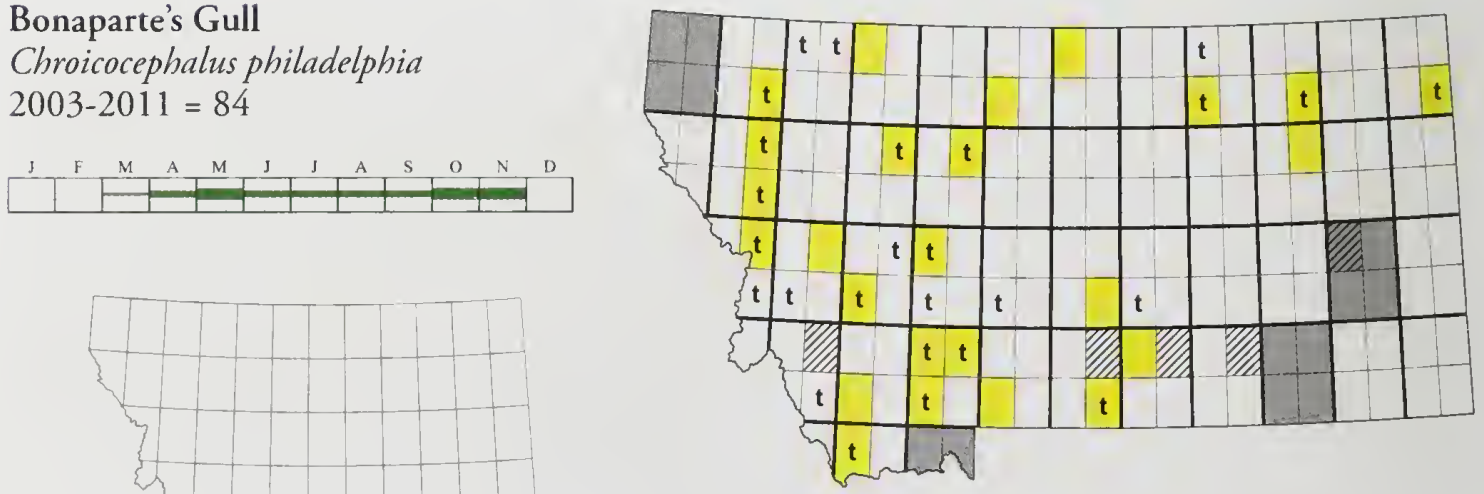


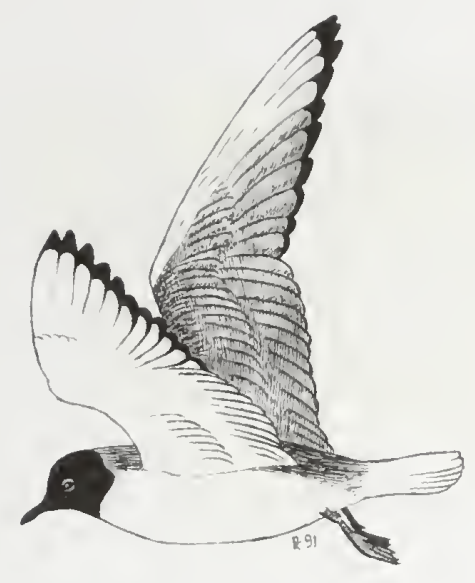

Little Gull

Hydrocoloeus minutus

2003-2011 = 0 R1

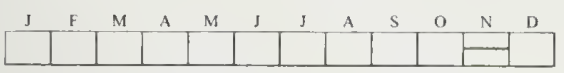
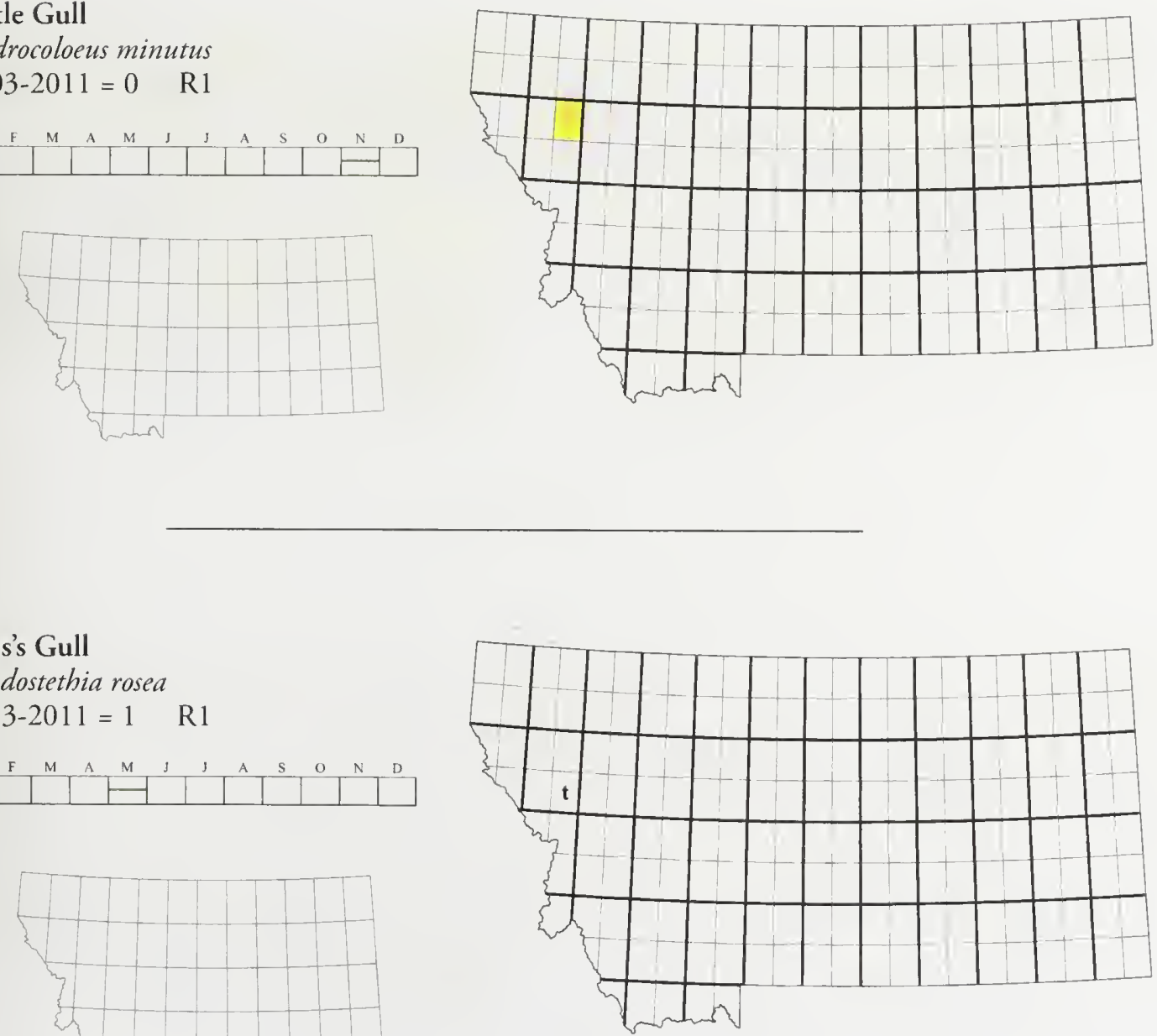

\section{Ross's Gull}

Rhodostethia rosea

2003-2011 = $1 \quad$ R1

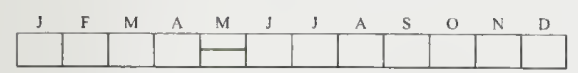

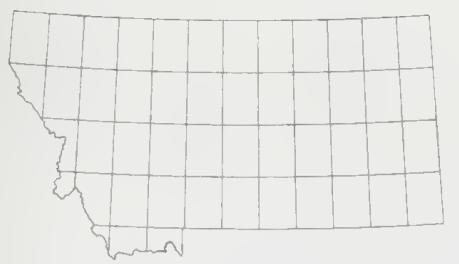


Laughing Gull

Leucophaeus atricilla

2003-2011 = 0 R1

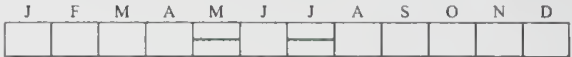
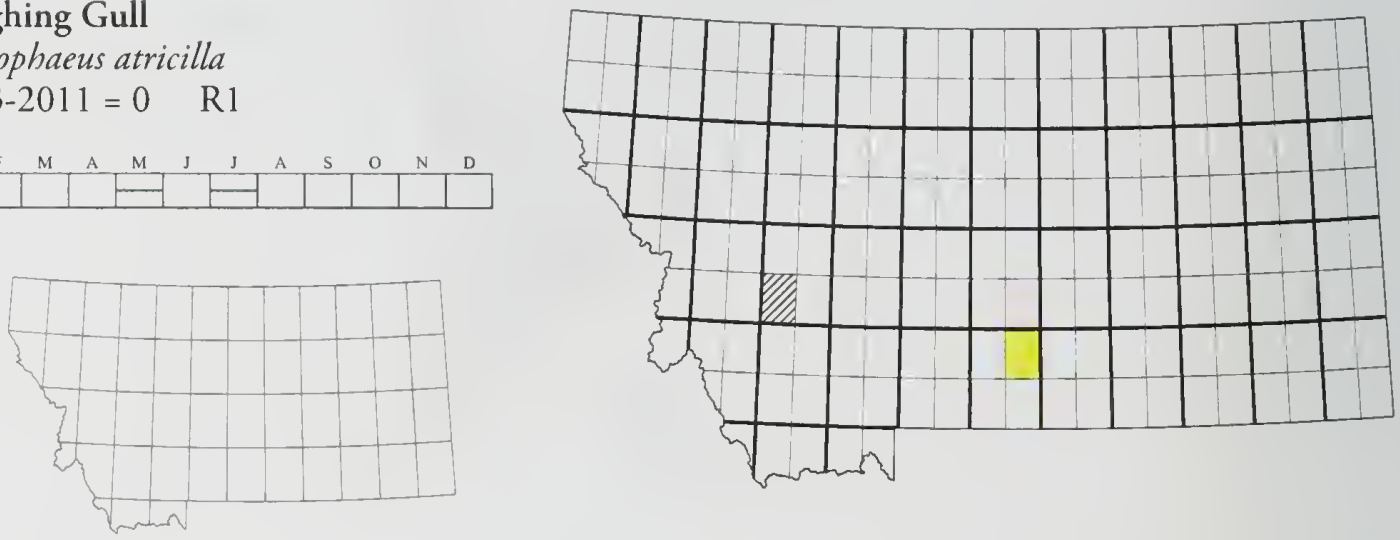

Franklin's Gull

Leucophaeus pipixcan

$2003-2011=416 \quad$ SOC

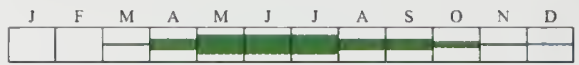

\section{Mew Gull}

Larus canus

$2003-2011=15$
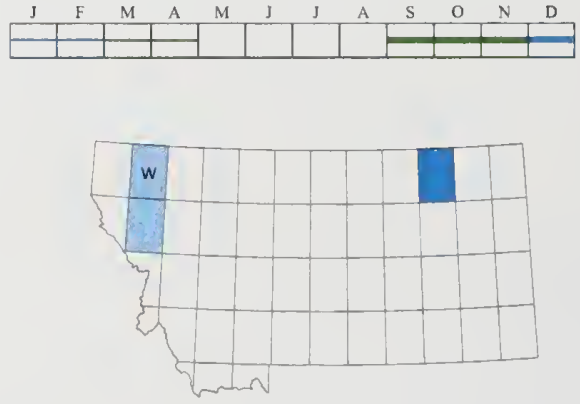
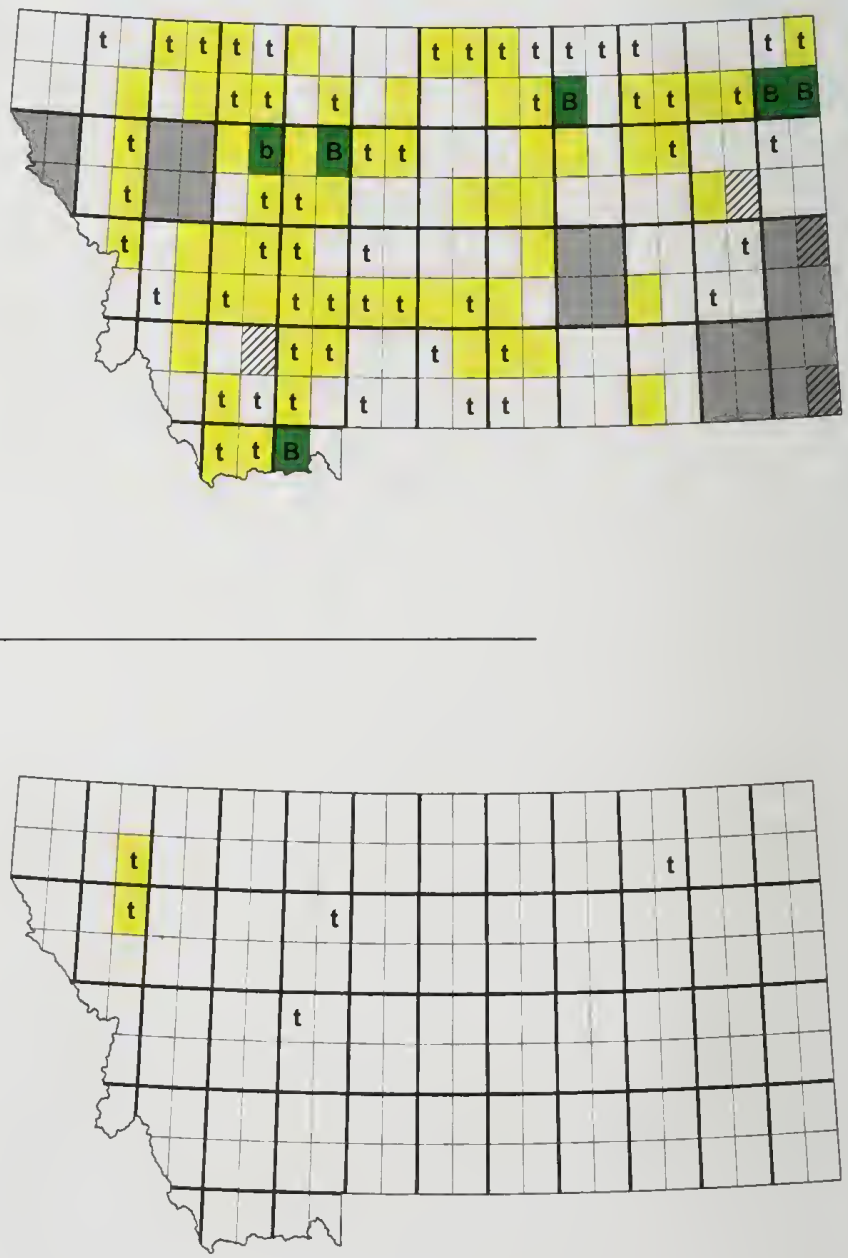
Ring-billed Gull

Larus delawarensis

2003-2011 = 1285
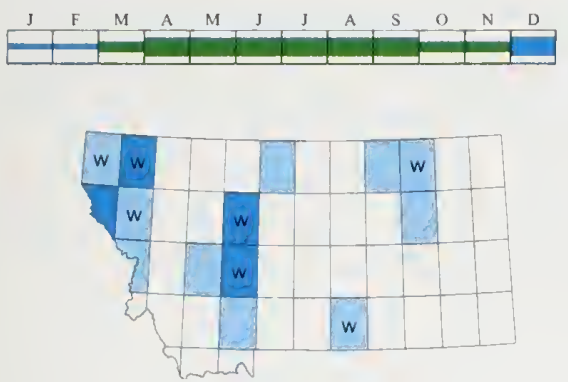

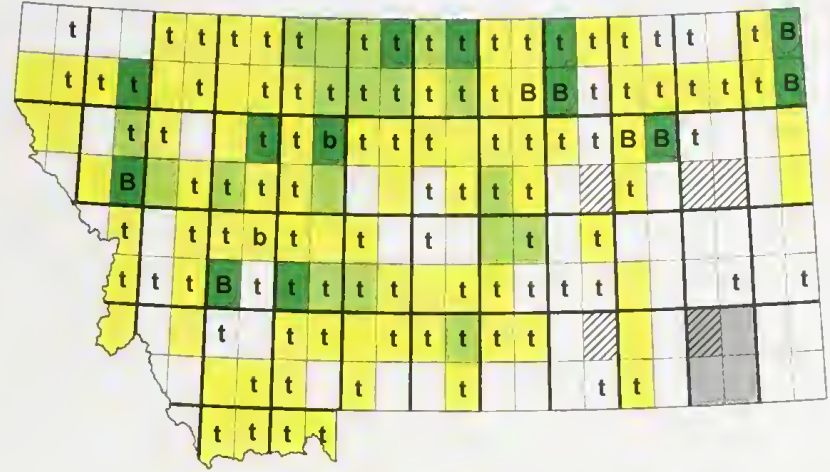

\section{California Gull}

Larus californicus

$2003-2011=667$
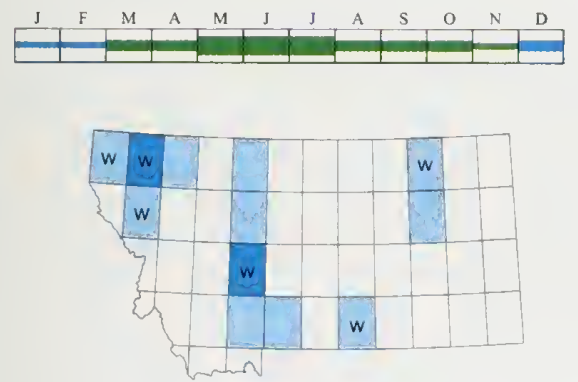

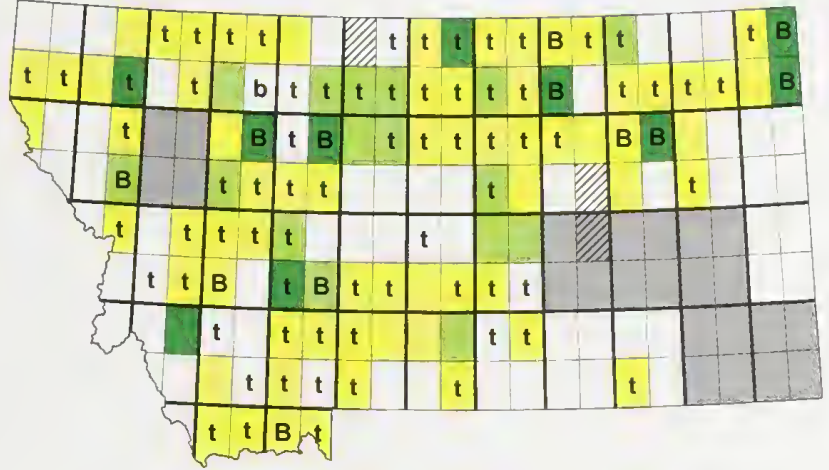

Herring Gull

Larus argentatus

$2003-2011=80$
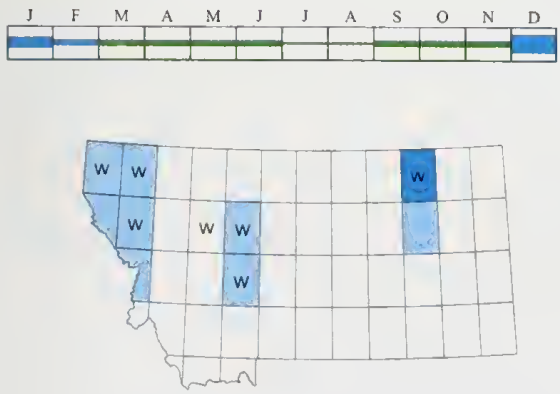

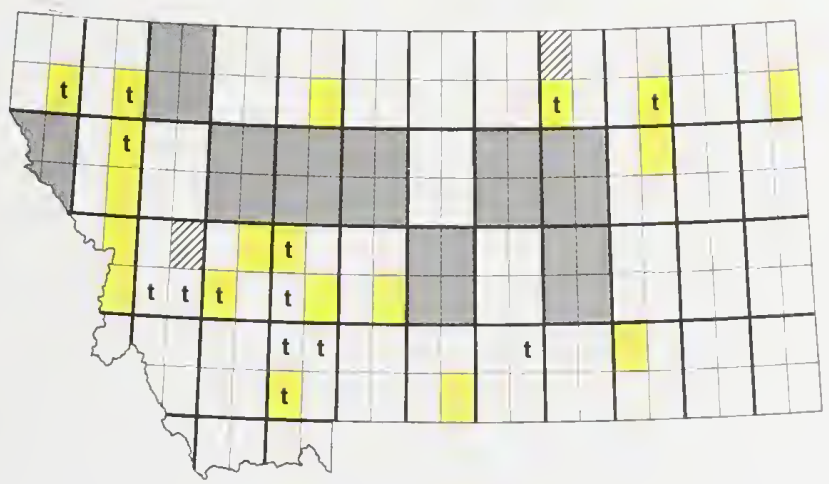


Thayer's Gull

Larus thayeri

2003-2011 = 34
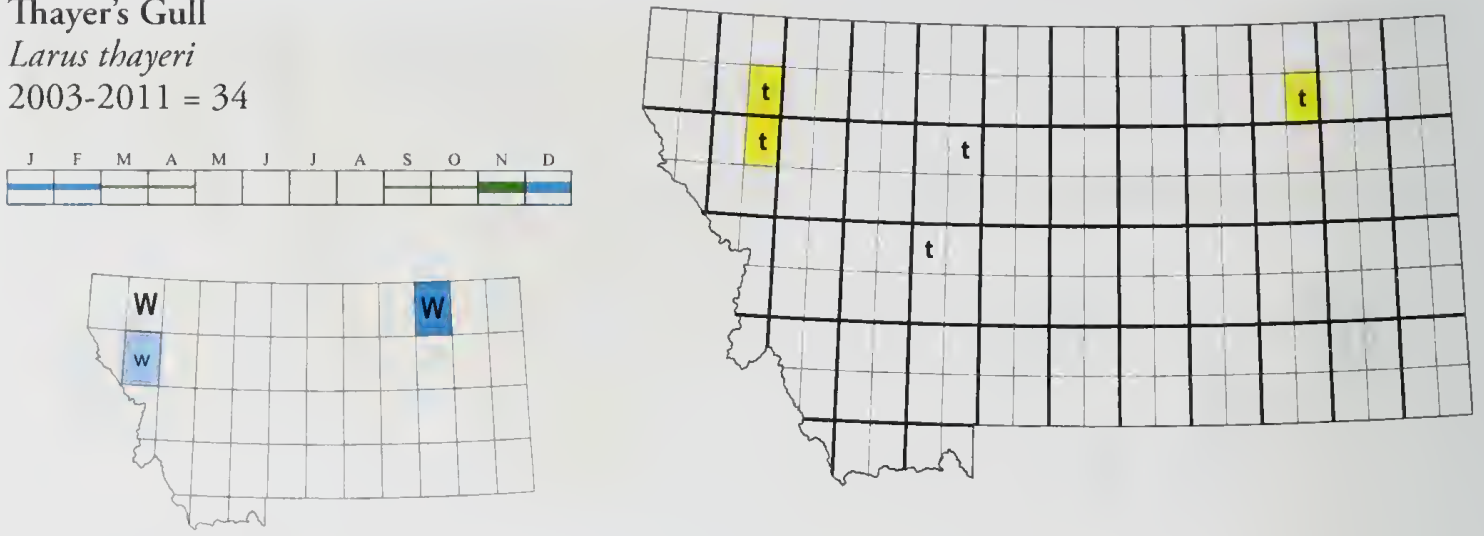

Iceland Gull

Larus glaucoides

$2003-2011=5 \quad R 1$

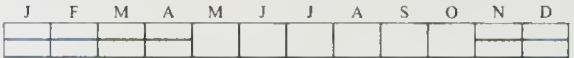
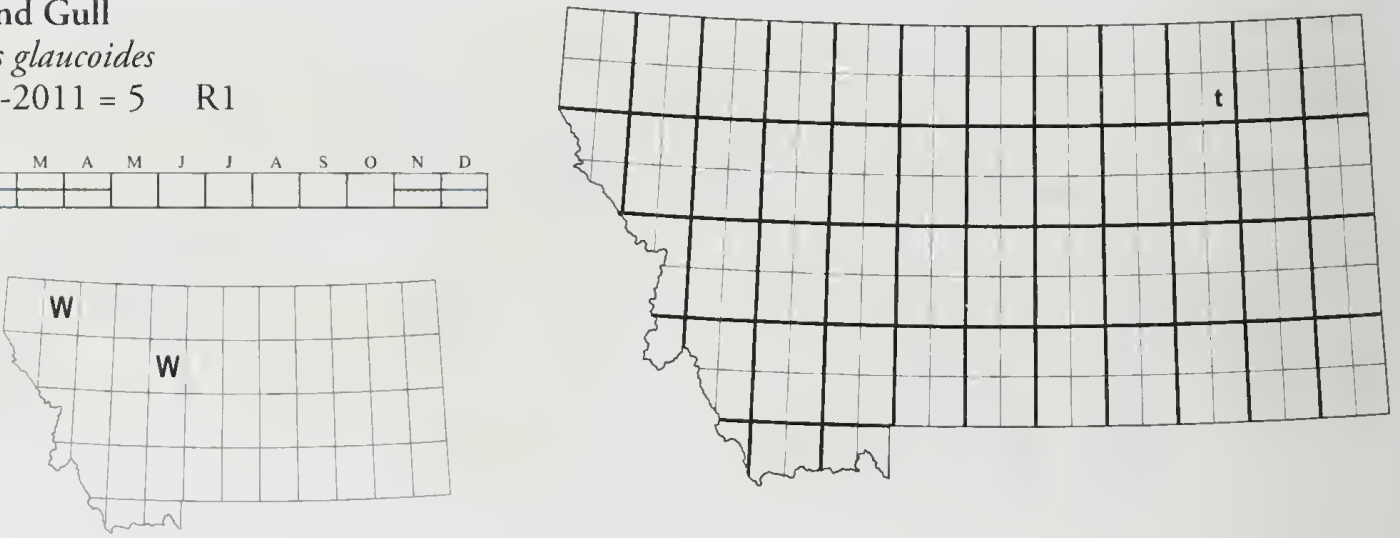

Lesser Black-backed Gull

Larus fuscus

2003-2011 = $6 \quad$ R 1

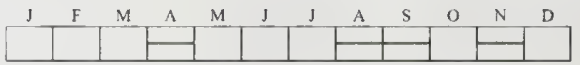

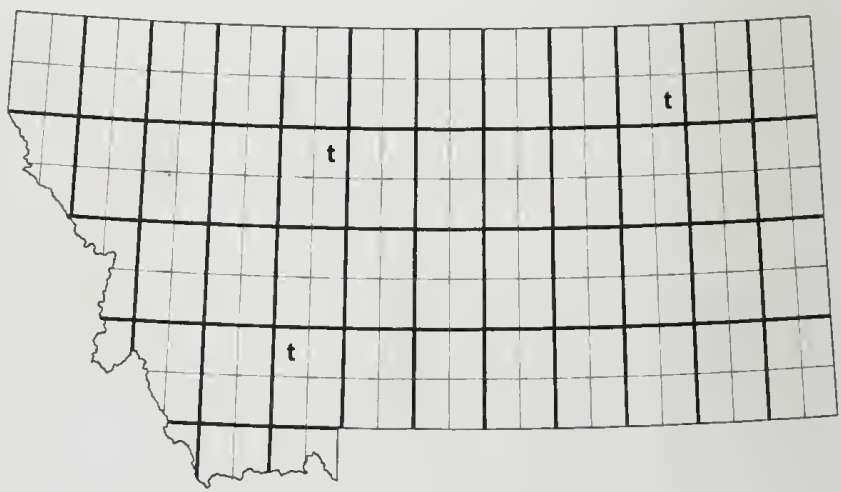


Glaucous-winged Gull

Larus glaucescens

2003-2011 = $8 \quad$ R2

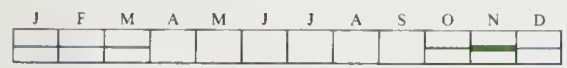
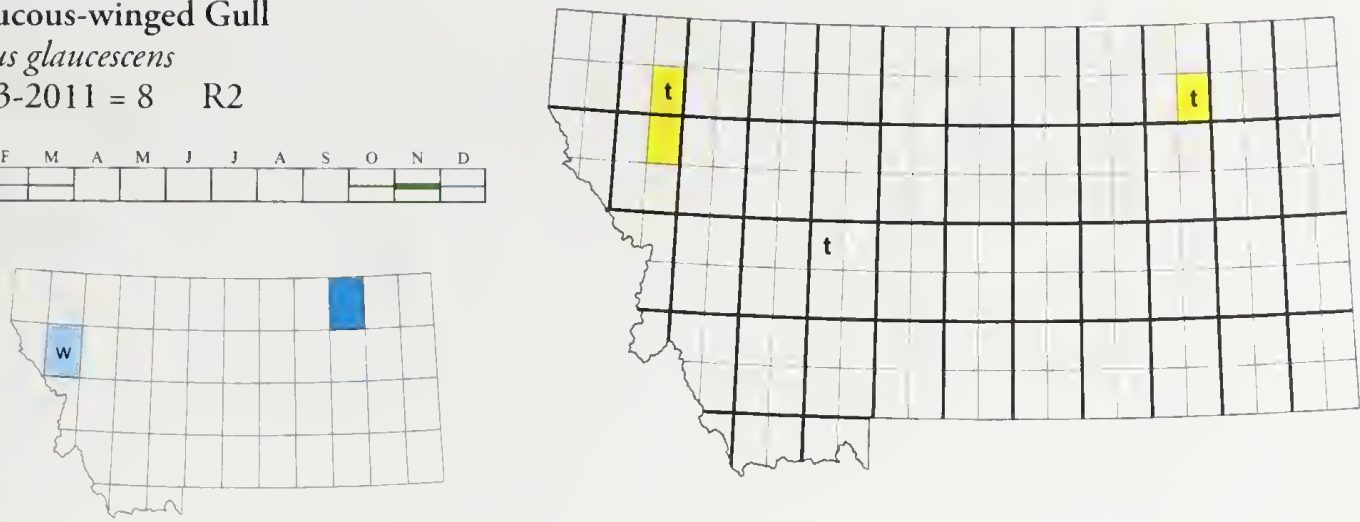

Glaucous Gull

Larus hyperboreus

2003-2011 = 24

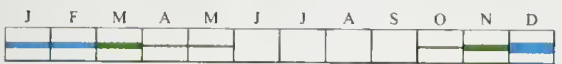
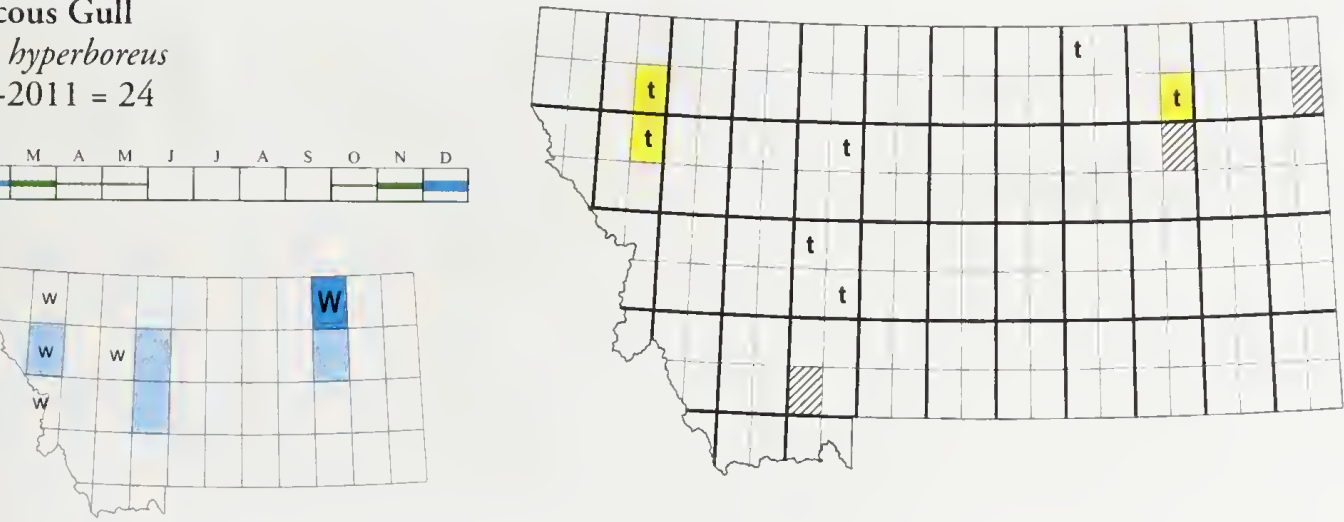

Great Black-backed Gull

Larus marinus

2003-2011 = $0 \quad \mathrm{R} 1$

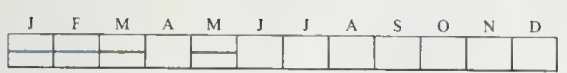

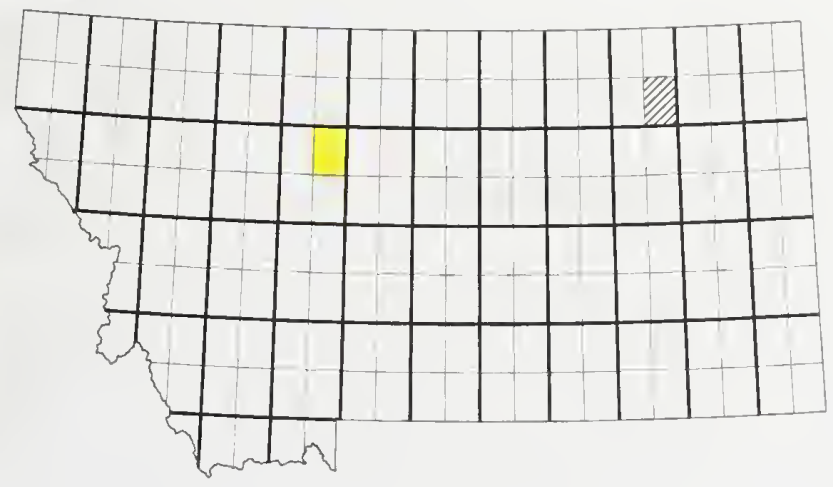


Least Tern

Sternula antillarum

2003-2011 = 219 E

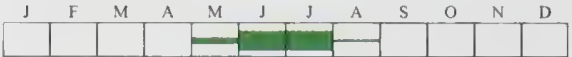
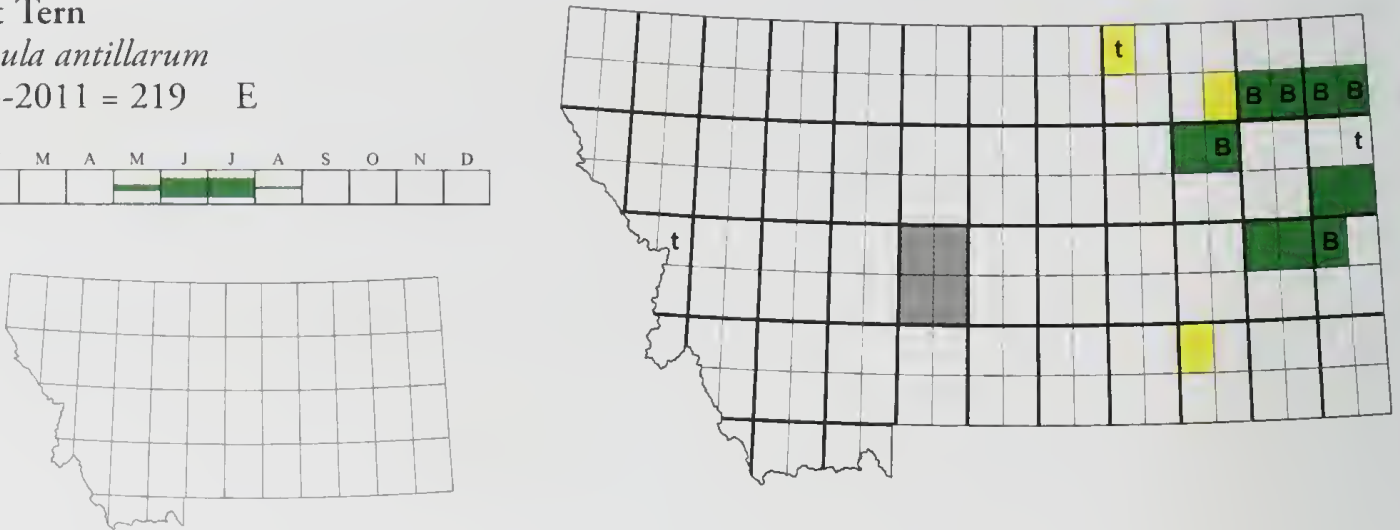

Caspian Tern

Hydroprogne caspia

$2003-2011=150 \quad$ SOC
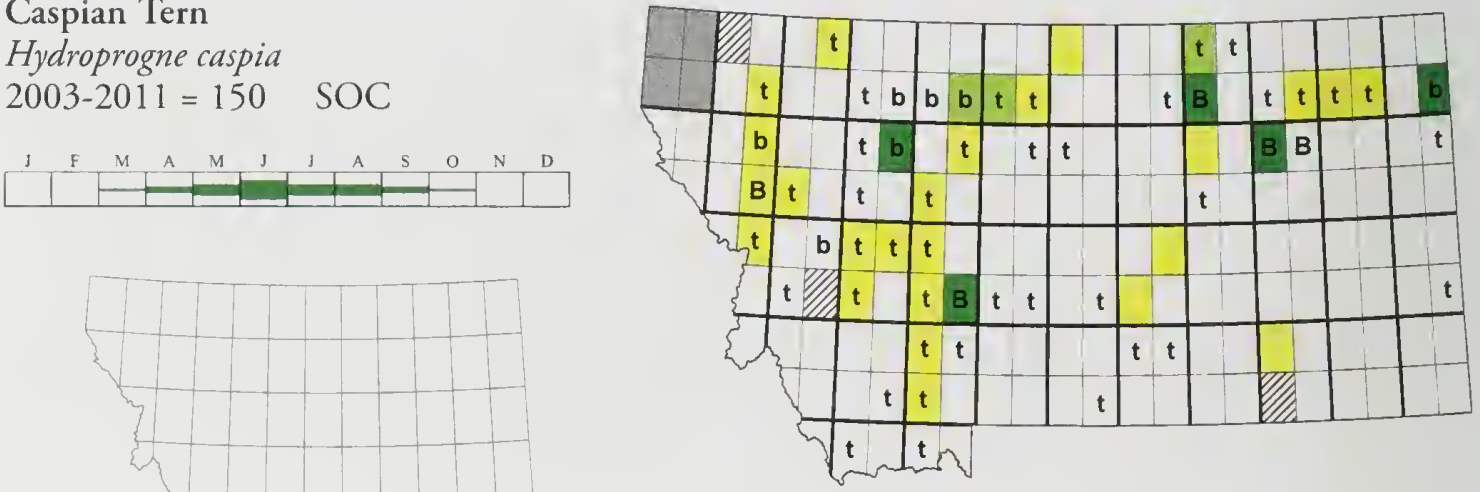

Black Tern

Chlidonias niger

$2003-2011=207 \quad$ SOC
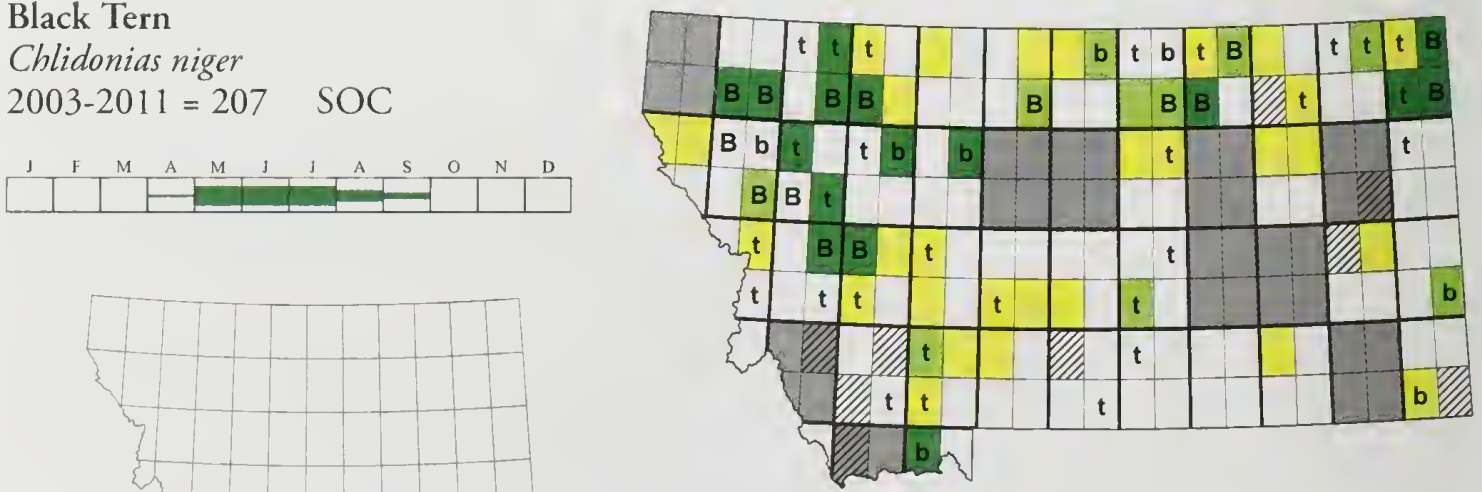
Common Tern

Sterna hirundo

2003-2011 = 157 SOC

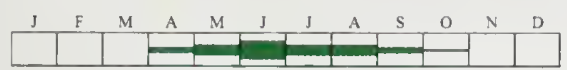
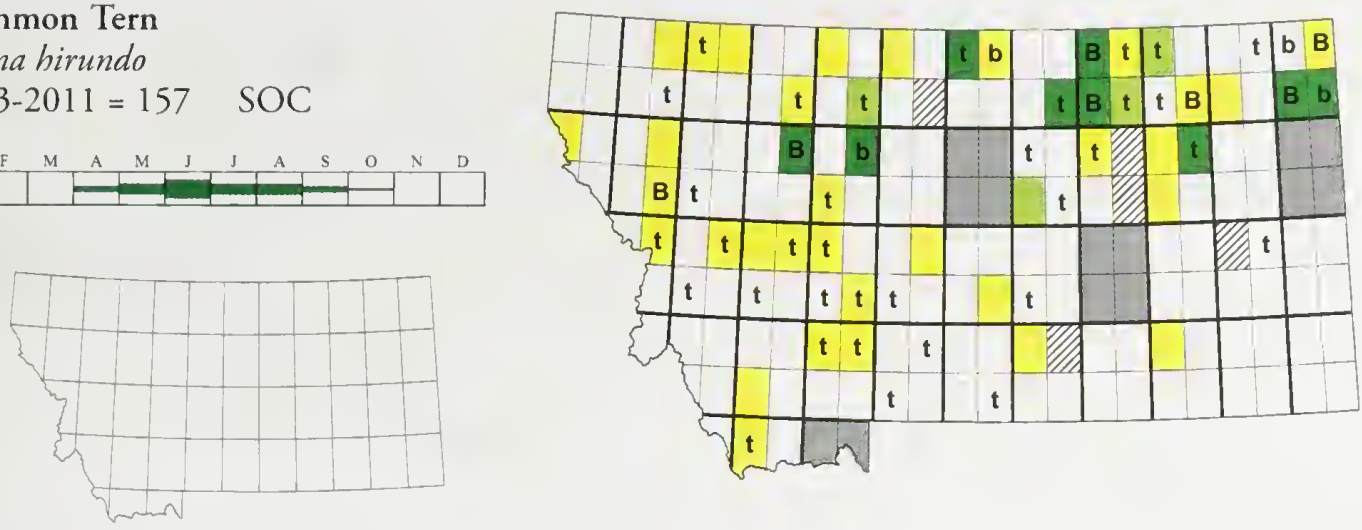
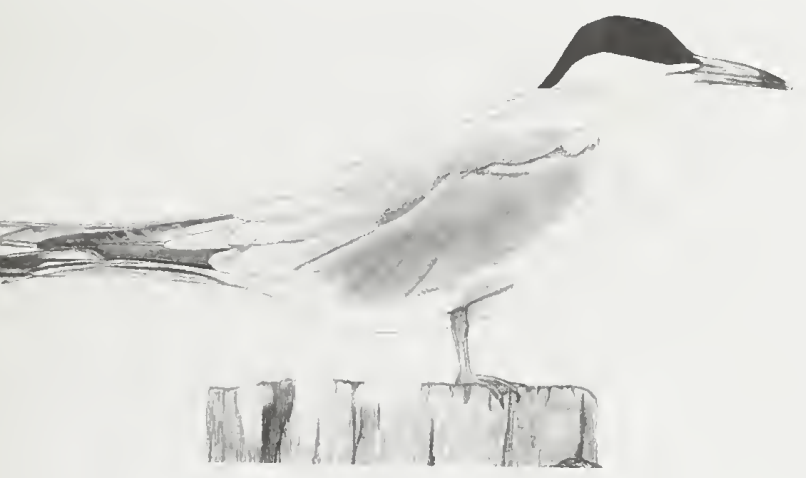

Arctic Tern

Sterna paradisaea

2003-2011 = 2 R1
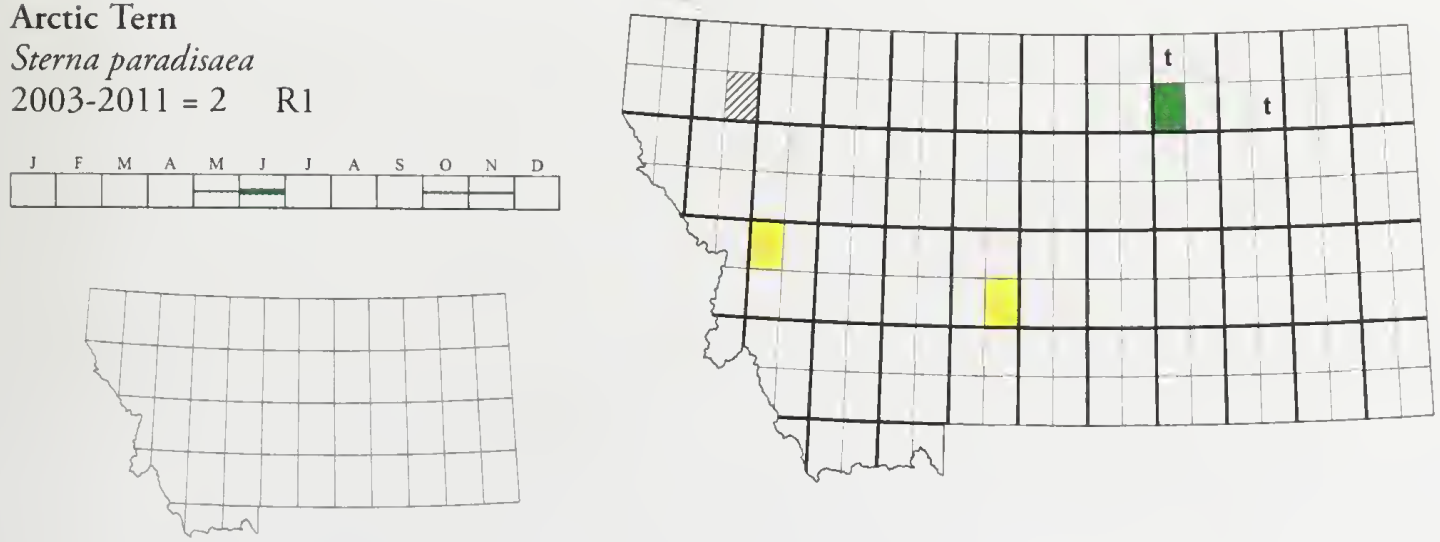
Forster's Tern

Sterna forsteri

$2003-2011=147 \quad$ SOC
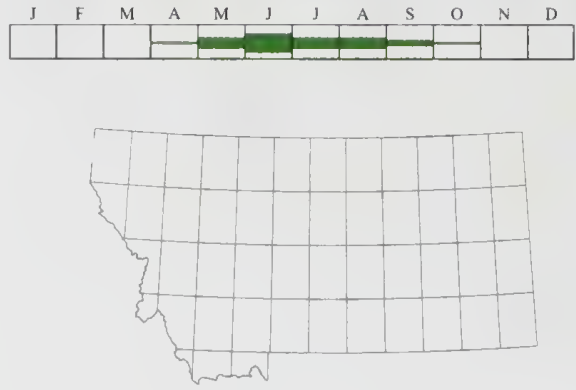

Stercorariidae

\section{Pomarine Jaeger}

Stercorarius pomarinus

2003-2011 = $0 \quad$ R1
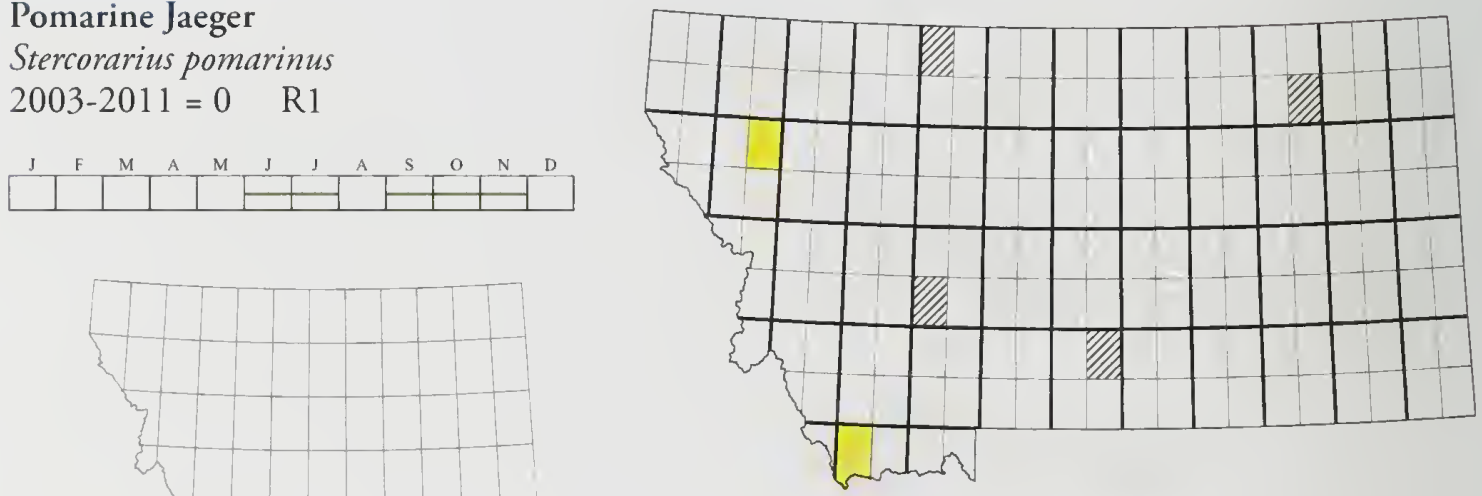

\section{Parasitic Jaeger}

Stercorarius parasiticus

2003-2011 = 6 R1
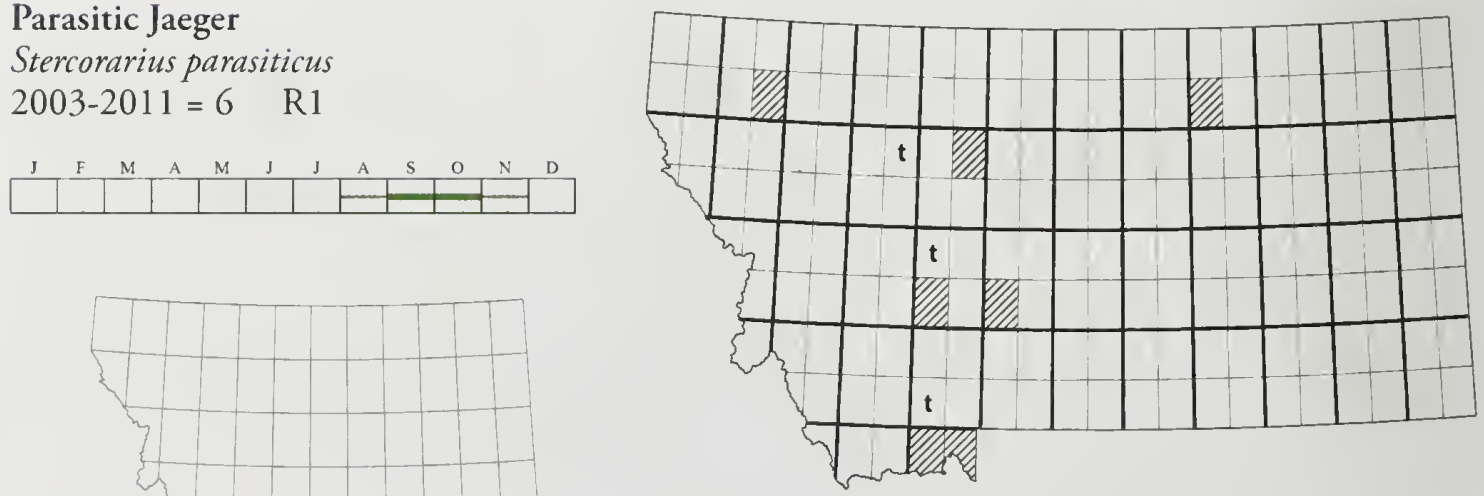
Long-tailed Jaeger

Stercorarius longicaudus

$2003-2011=3 \quad$ R1
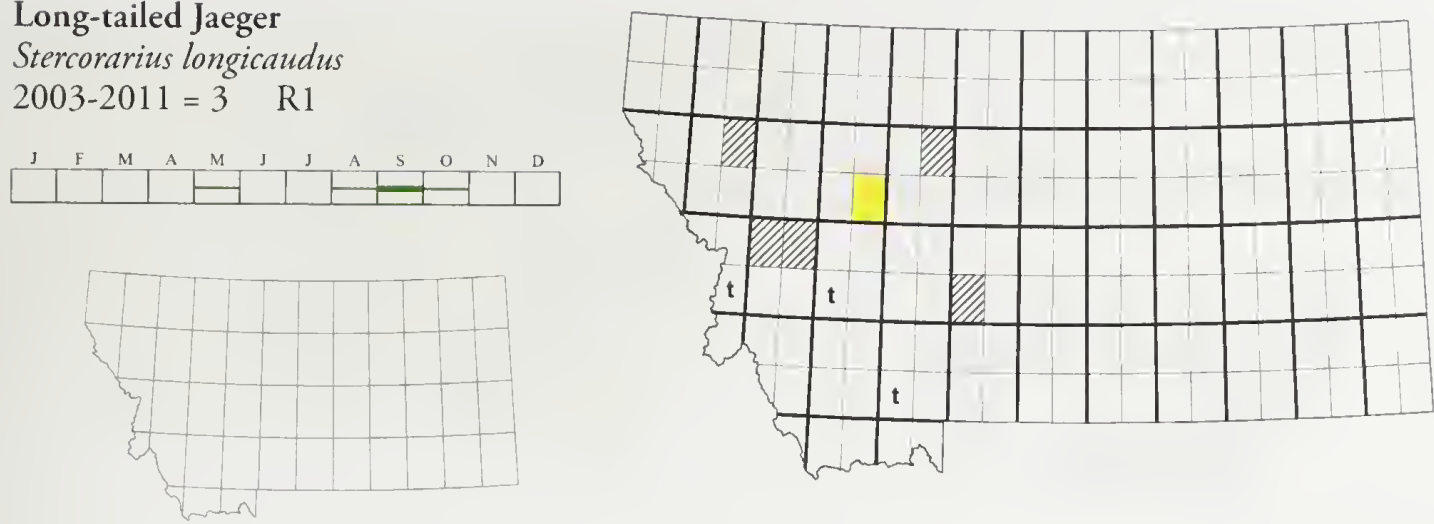

Alcidae

Long-billed Murrelet

Brachyramphus perdix

2003-2011 = 0 R1
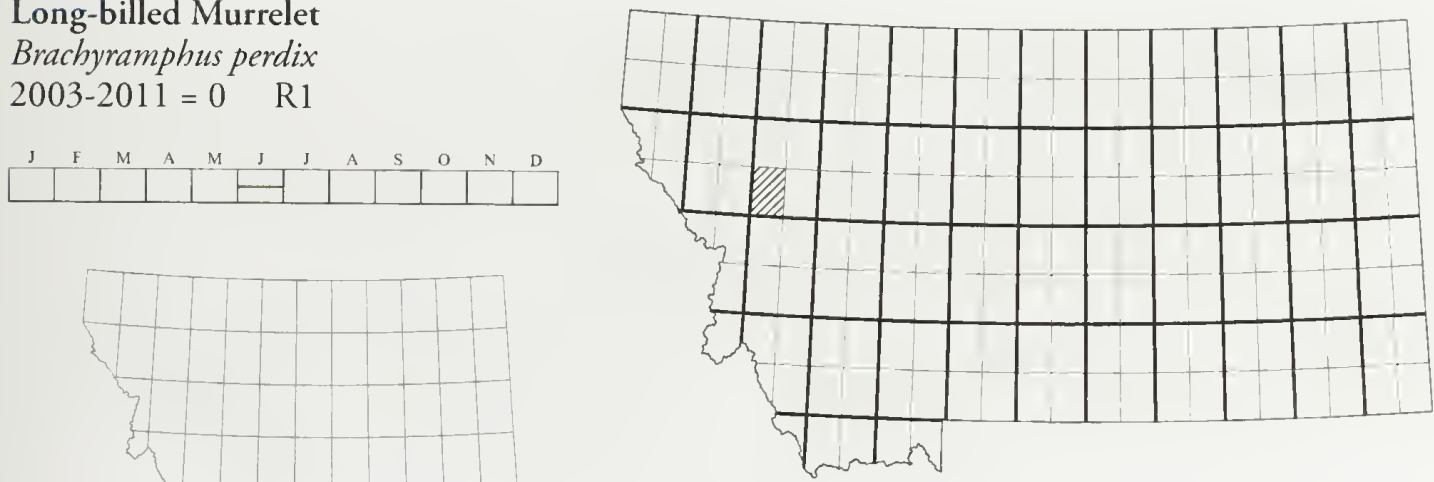

Ancient Murrelet

Synthliboramphus antiquus

2003-2011 = $2 \quad$ R1

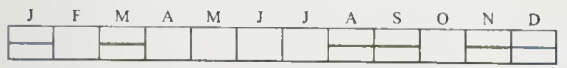

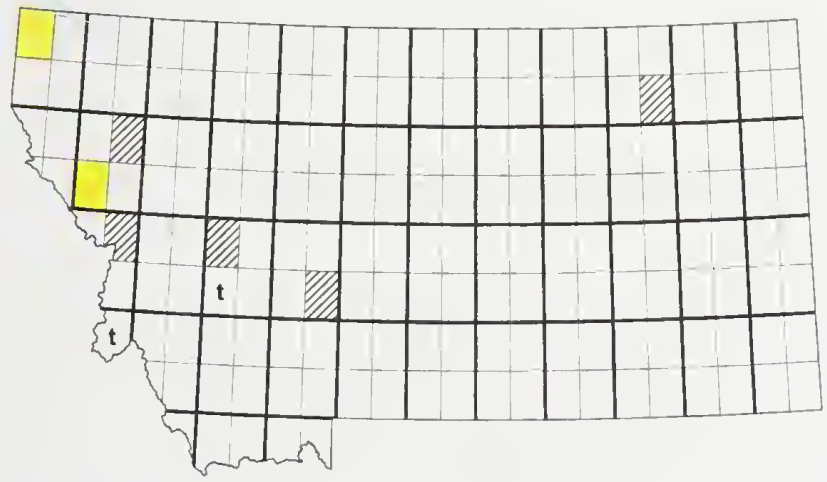




\section{Rock Pigeon}

\section{Columba livia}

2003-2011 = 1323
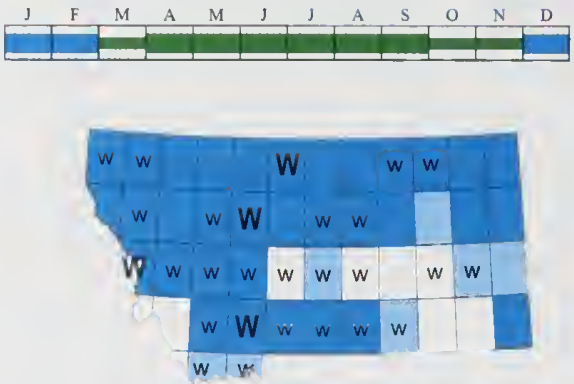

Band-tailed Pigeon

Patagioenas fasciata

$2003-2011=3$

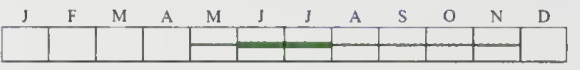

Eurasian Collared-Dove

Streptopelia decaocto

2003-2011 $=427$
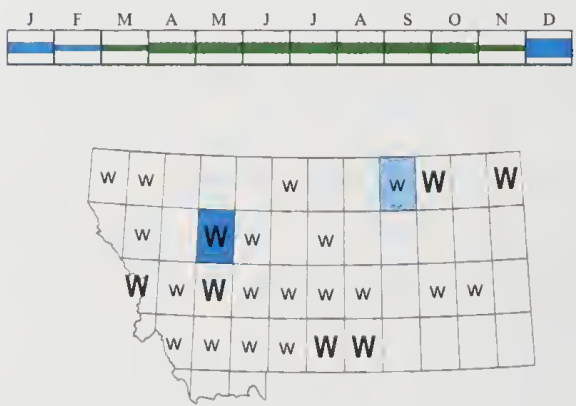
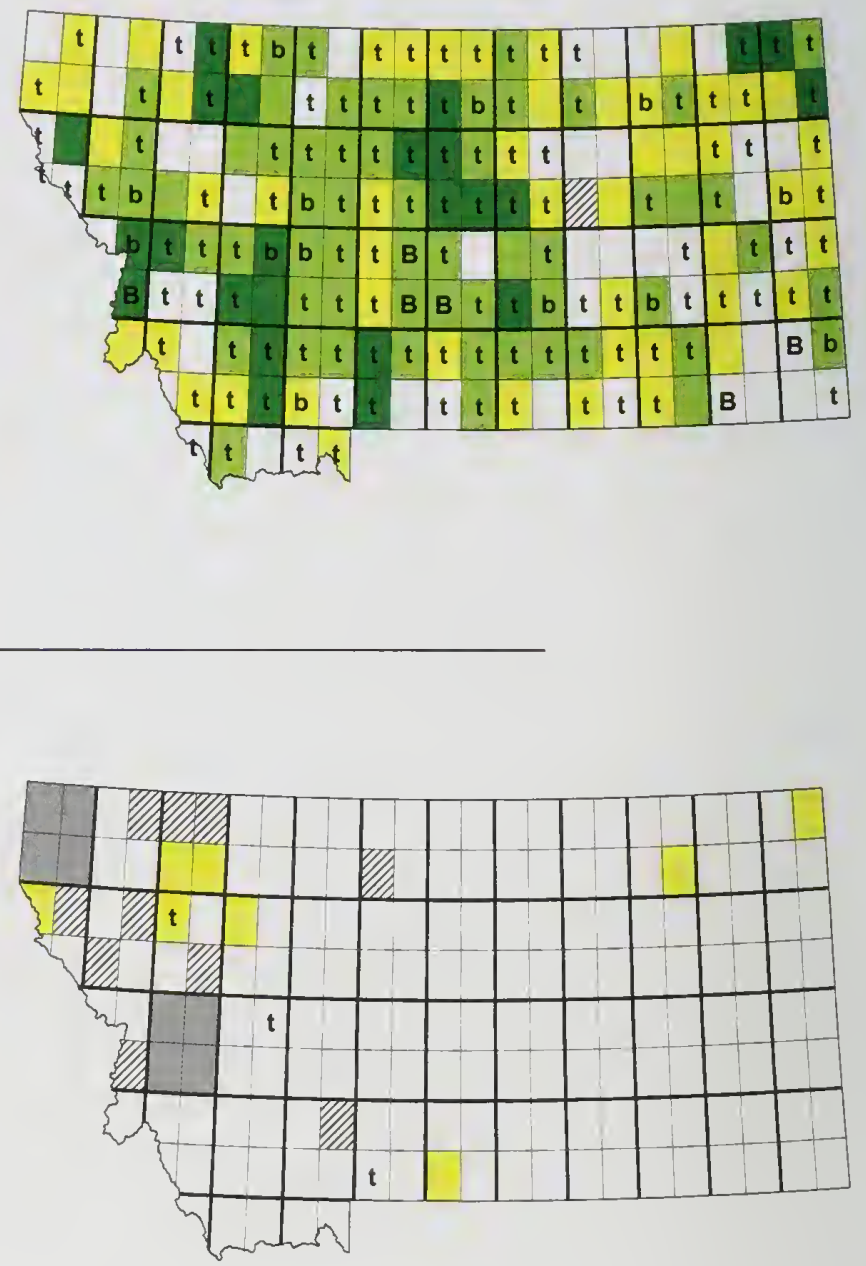

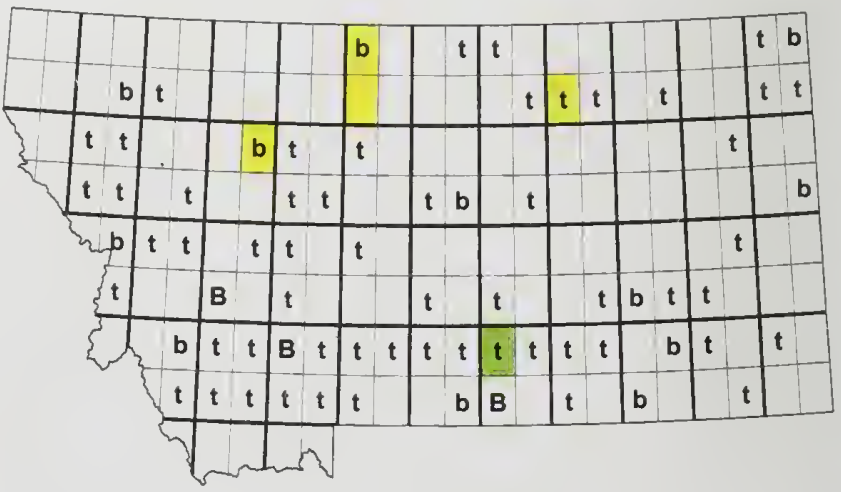


White-winged Dove

Zenaida asiatica

$2003-2011=16 \quad R 1$
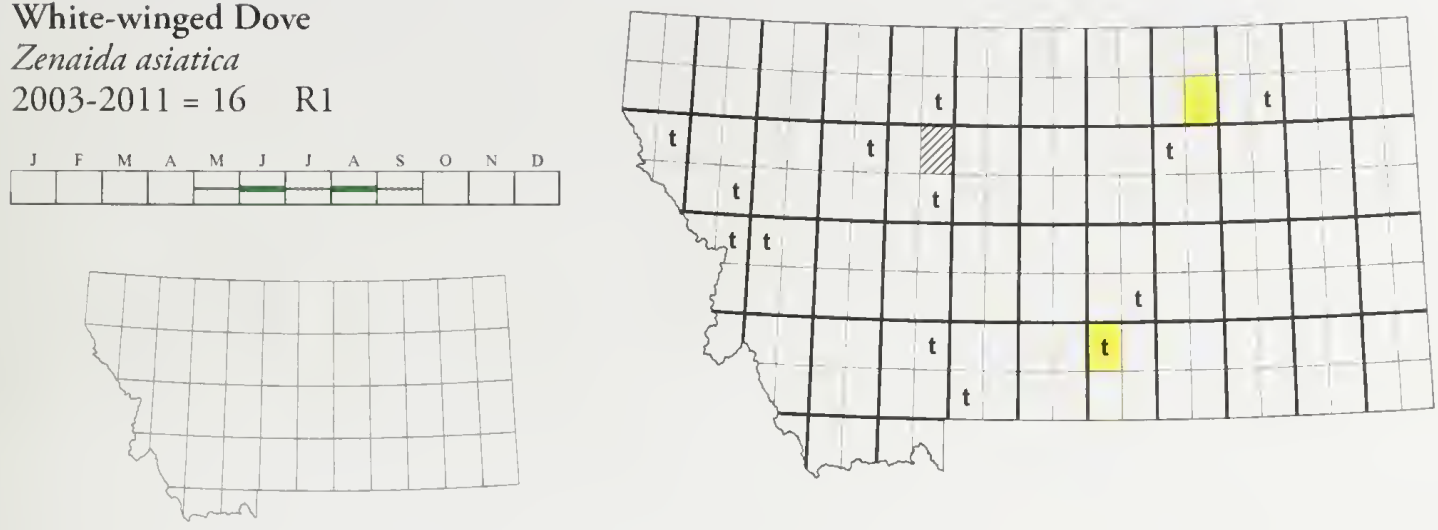

Mourning Dove

Zenaida macroura

2003-2011 = 6936
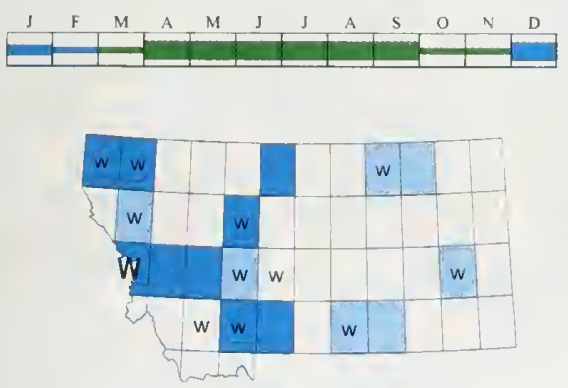

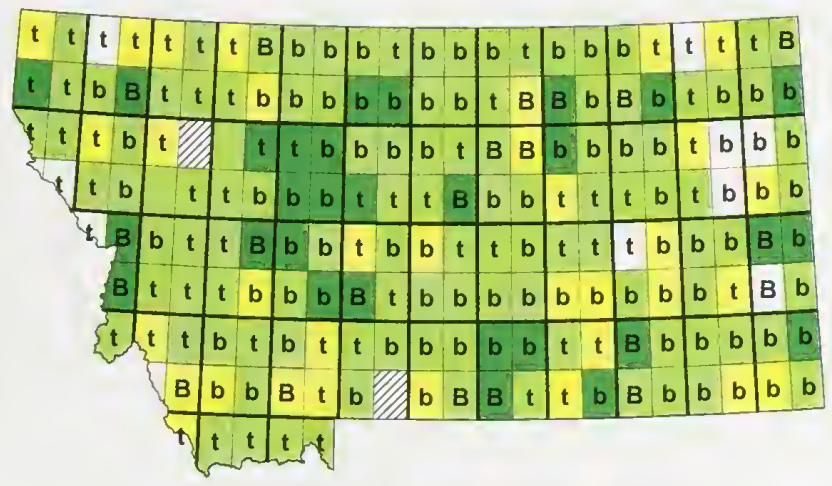

Inca Dove

Columbina inca

2003-2011 = $1 \quad$ R1

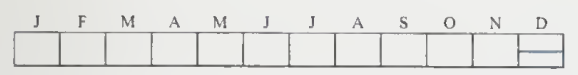

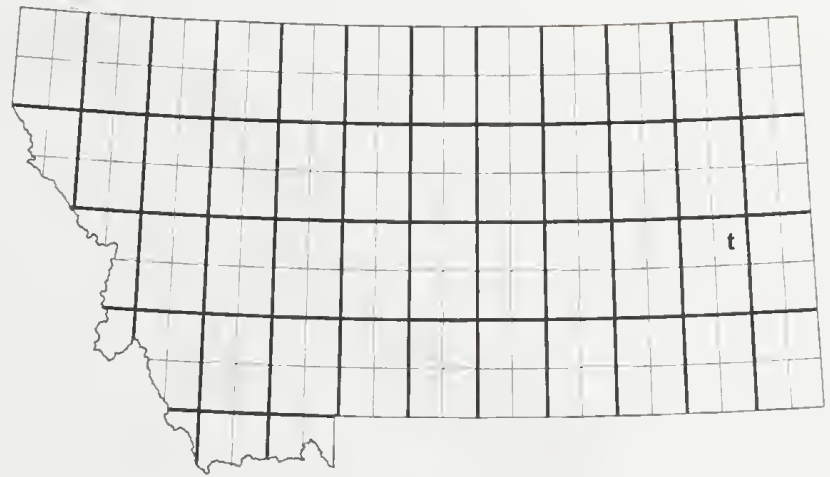


CUCULIFORMES Cuculidae

Yellow-billed Cuckoo

Coccyzus americanus

$2003-2011=1 \quad$ C $\quad$ R3
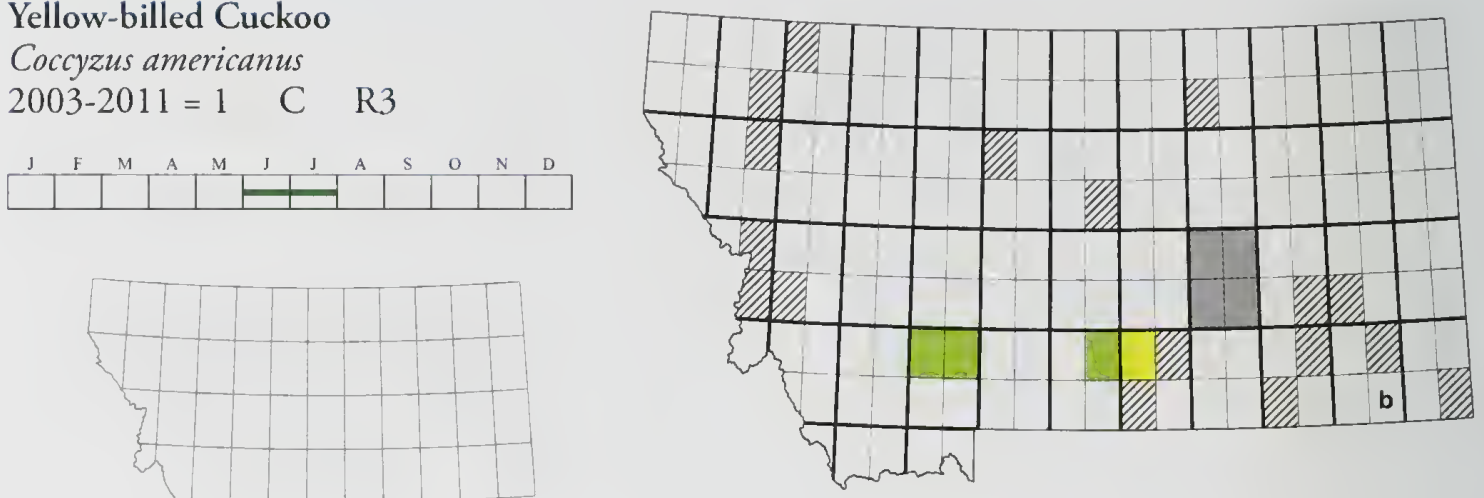

\section{Black-billed Cuckoo}

Coccyzus erythropthalmus

2003-2011 = 23 SOC
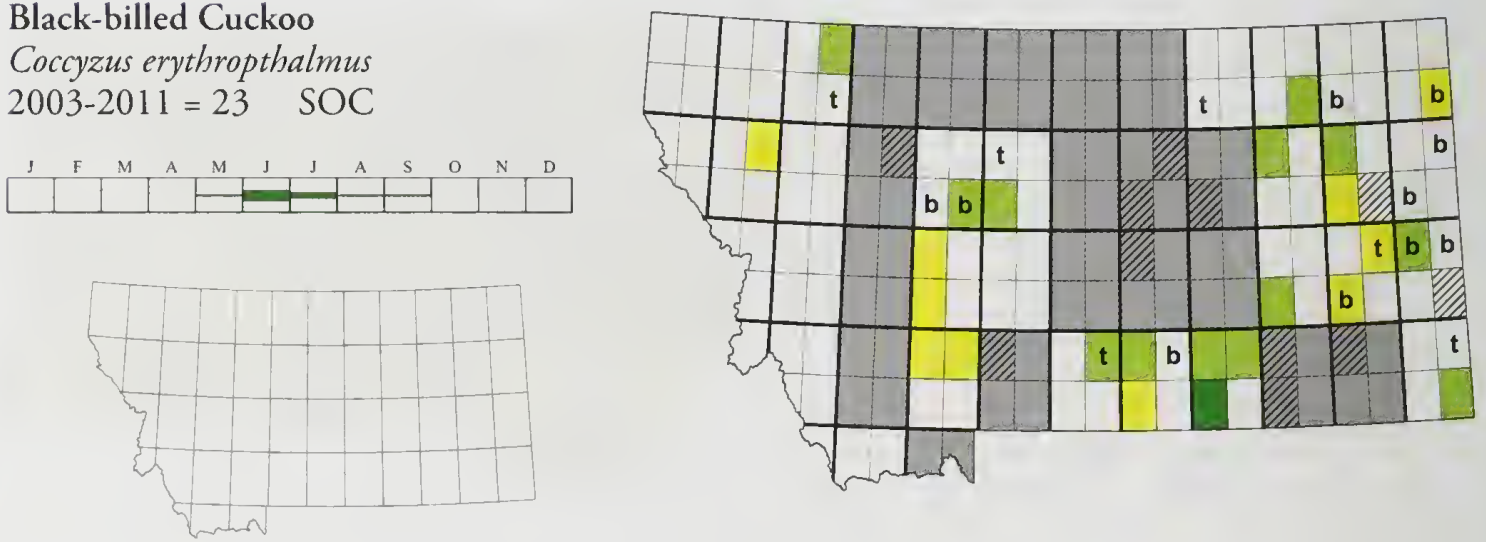
STRIGIFORMES Tytonidae

\section{Barn Owl}

Tyto alba

2003-2011 = 27
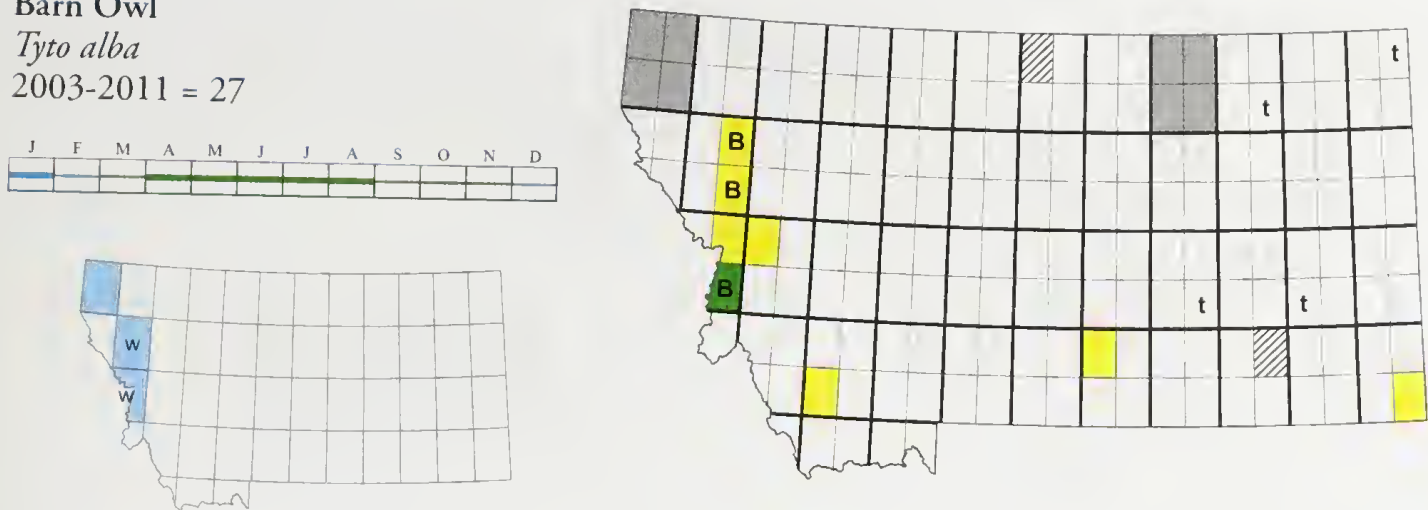

Strigidae

\section{Flammulated Owl}

Otus flammeolus

2003-2011 = 509 SOC
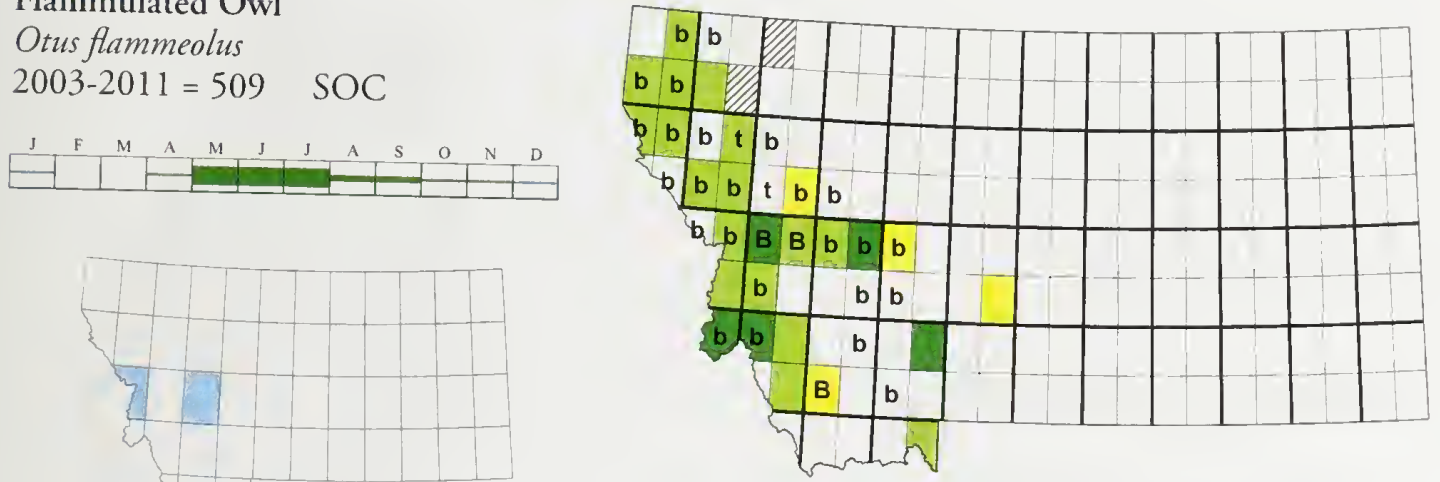

Western Screech-Owl

Megascops kennicottii

2003-2011 = 48 SI
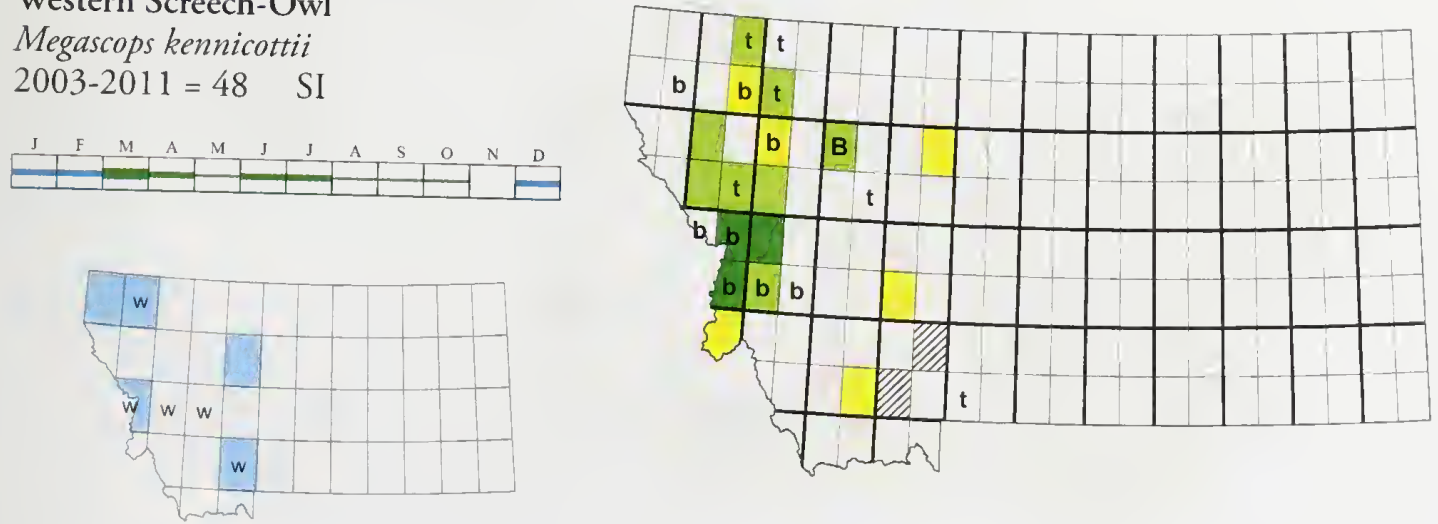
Eastern Screech-Owl

Megascops asio

$2003-2011=44 \quad$ SI
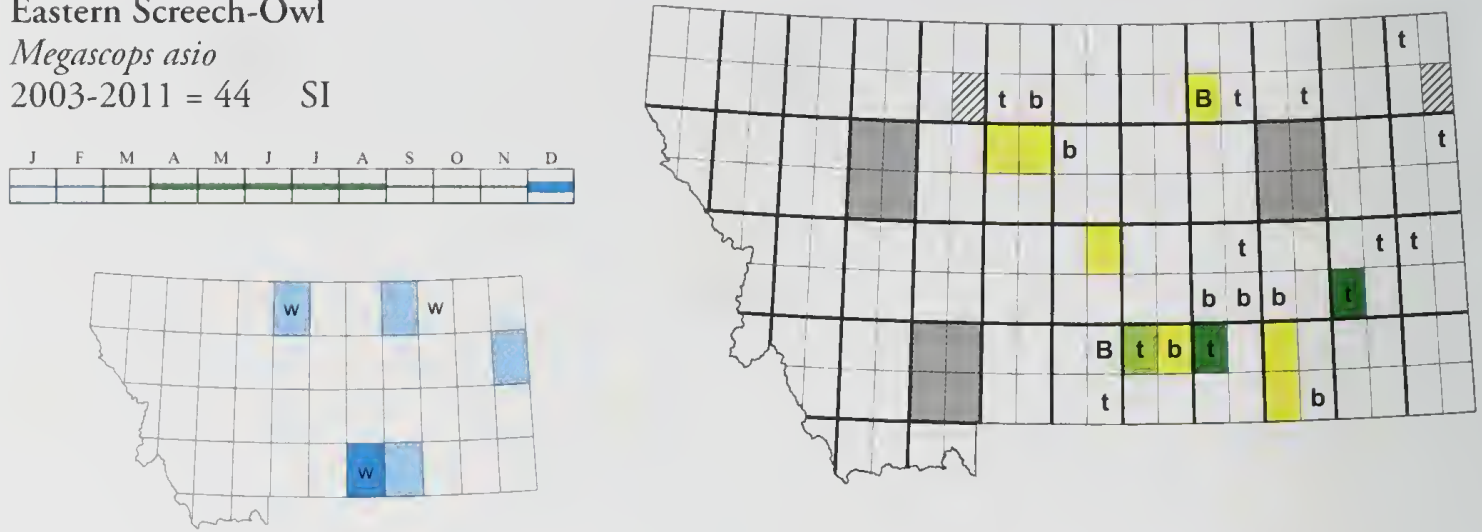

Great Horned Owl

Bubo virginianus

2003-2011 = 730
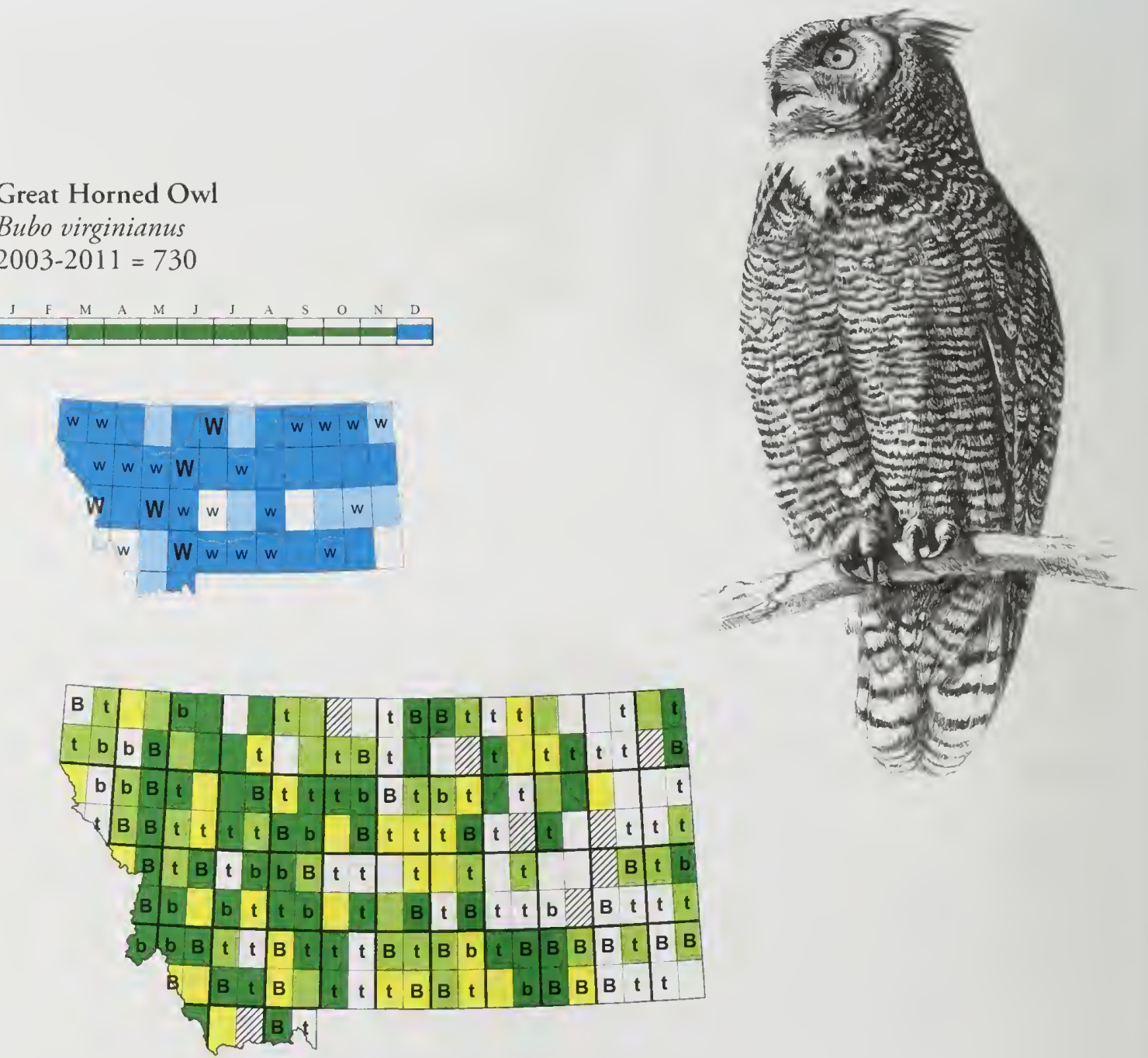
Snowy Owl

Bubo scandiacus

2003-2011 = 155
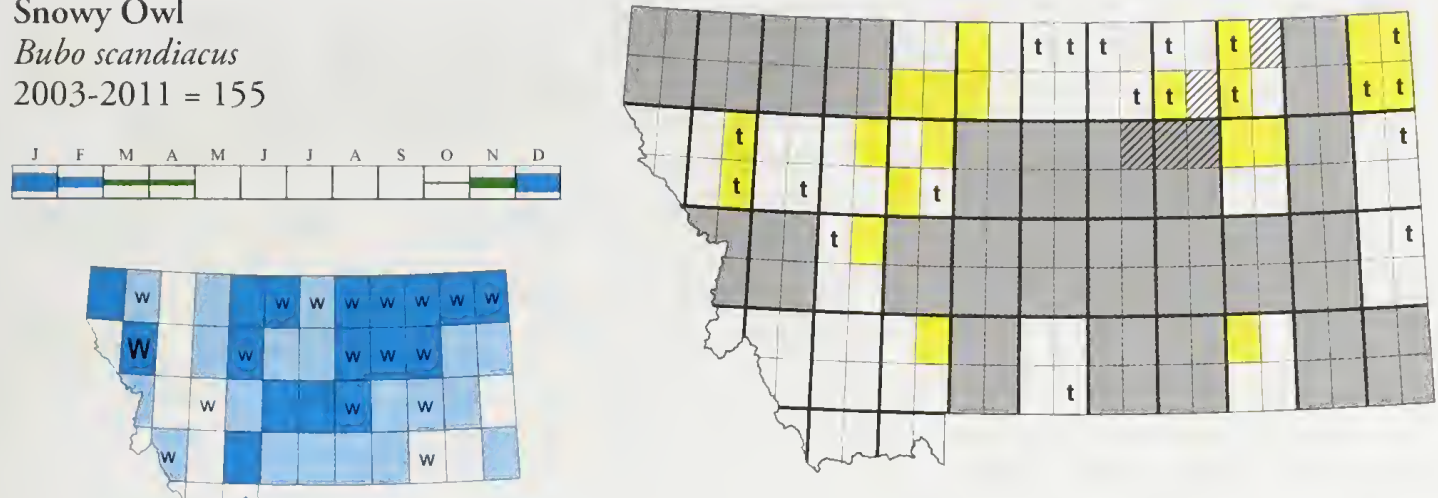

Northern Hawk Owl

Surnia ulula

2003-2011 = $38 \quad$ SOC

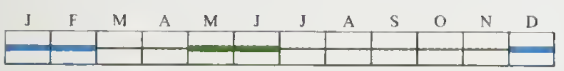
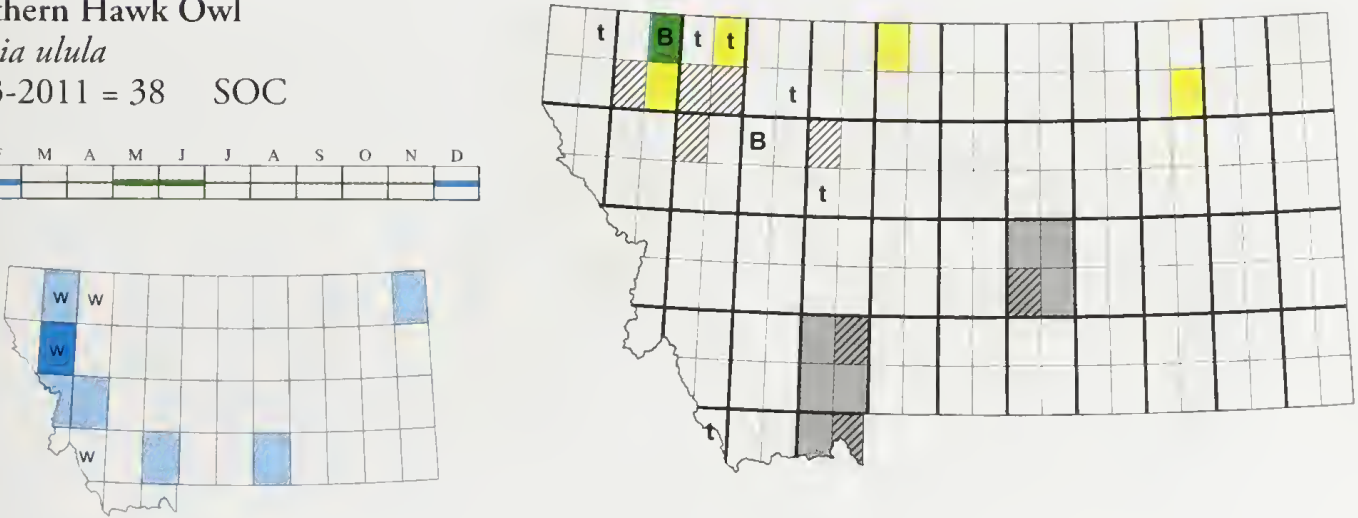

Northern Pygmy-Owl

Glaucidium gnoma

$2003-2011=171$
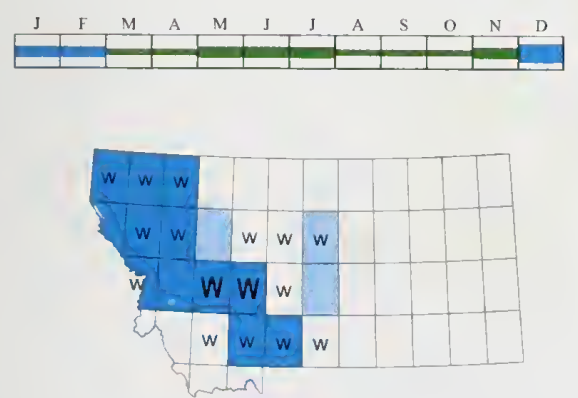

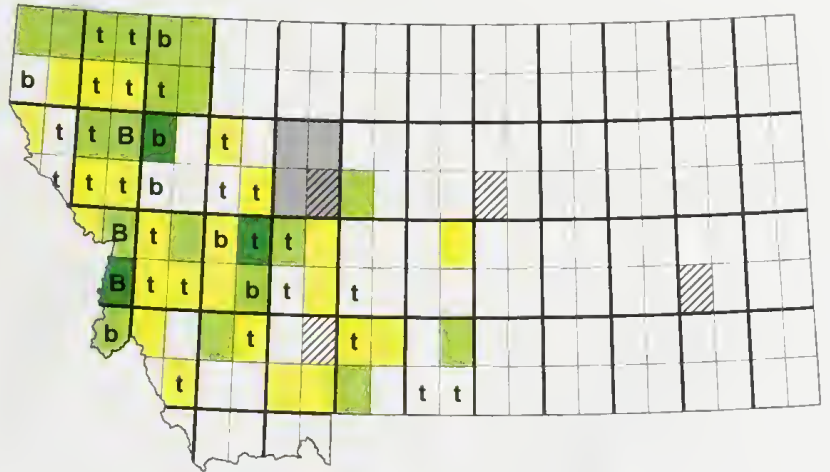


Burrowing Owl

Athene cunicularia

$2003-2011=522 \quad$ SOC
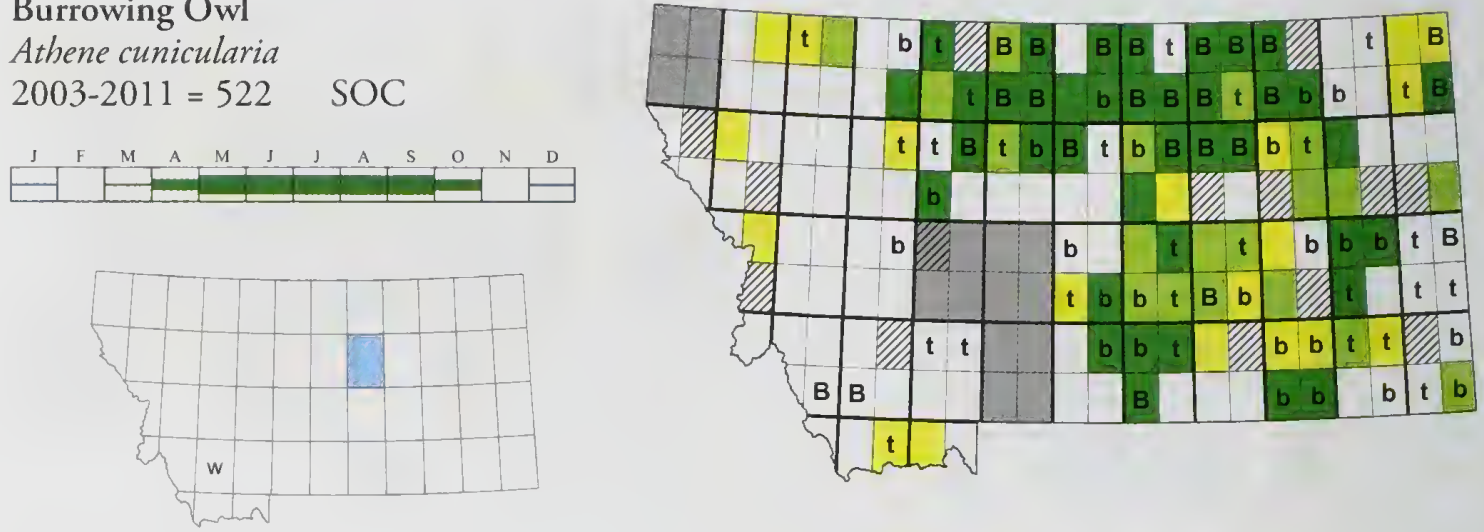

Barred Owl

Strix varia

2003-2011 = 81 SI
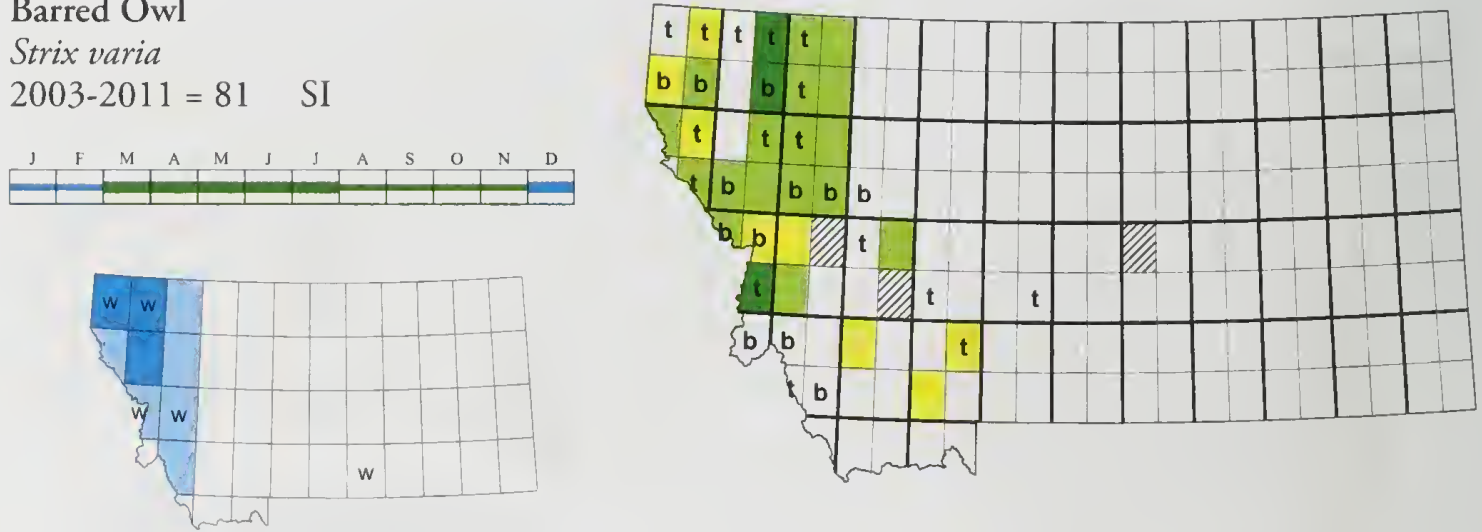

Great Gray Owl

Strix nebulosa

2003-2011 = $77 \quad$ SOC
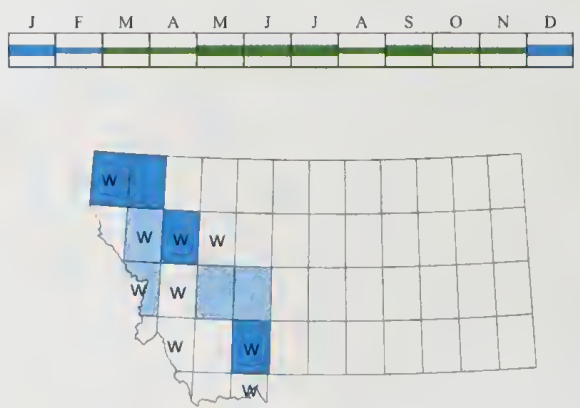
Long-eared Owl

Asio otus

$2003-2011=206$
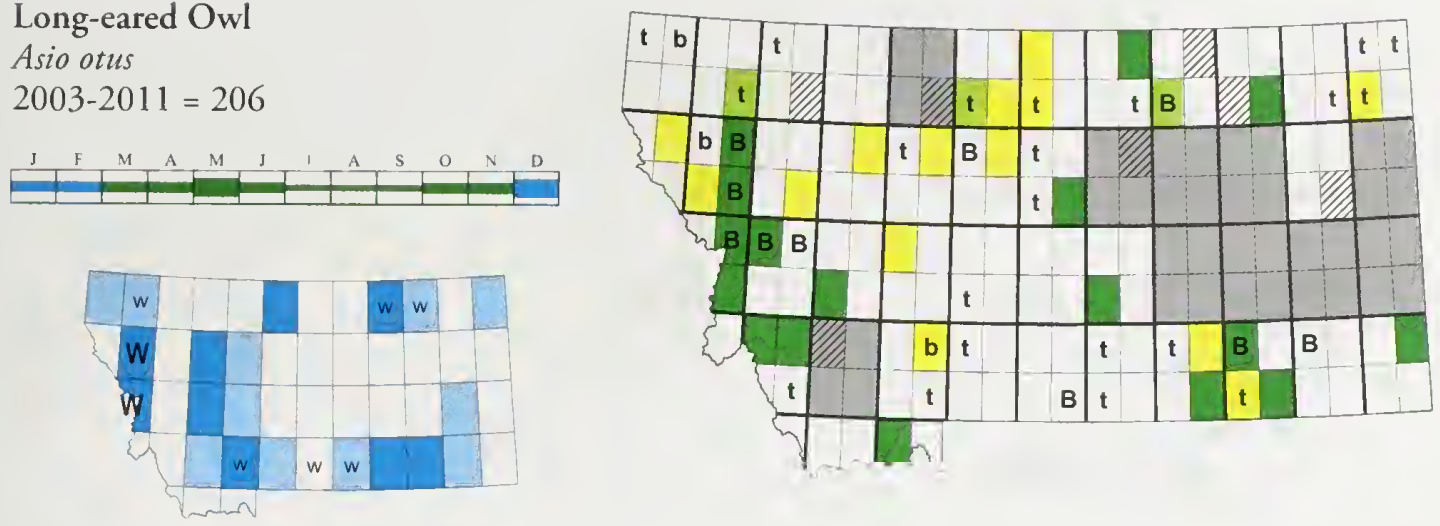

Short-eared Owl

Asio flammeus

$2003-2011=392 \quad$ SI
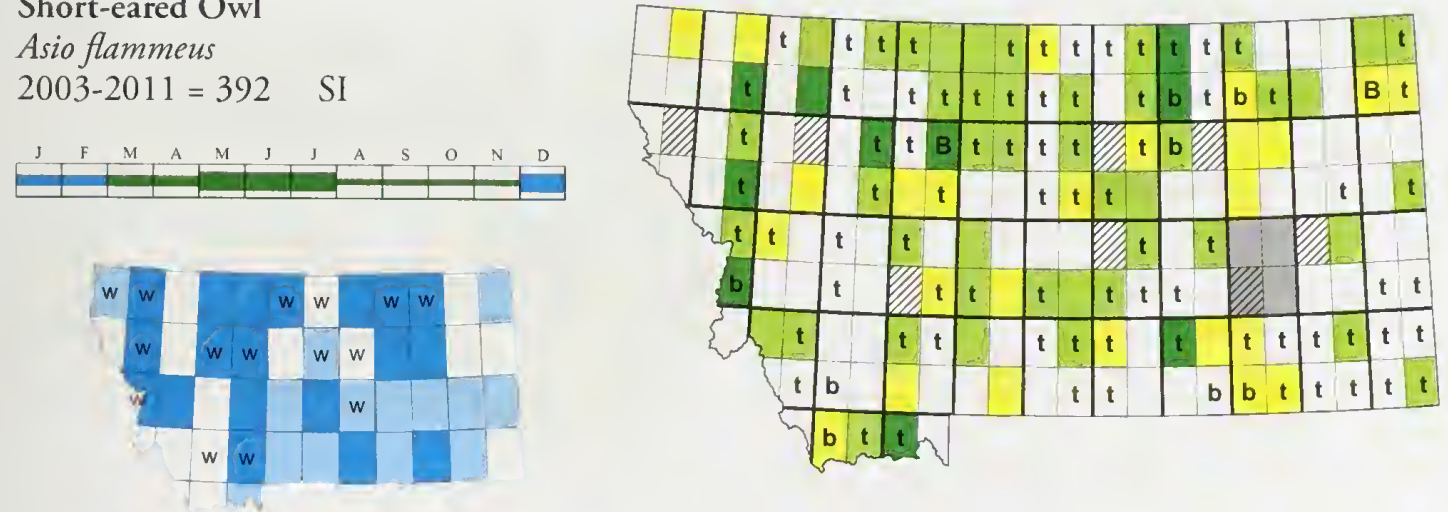

Boreal Owl

Aegolius funereus

$2003-2011=34 \quad$ SI
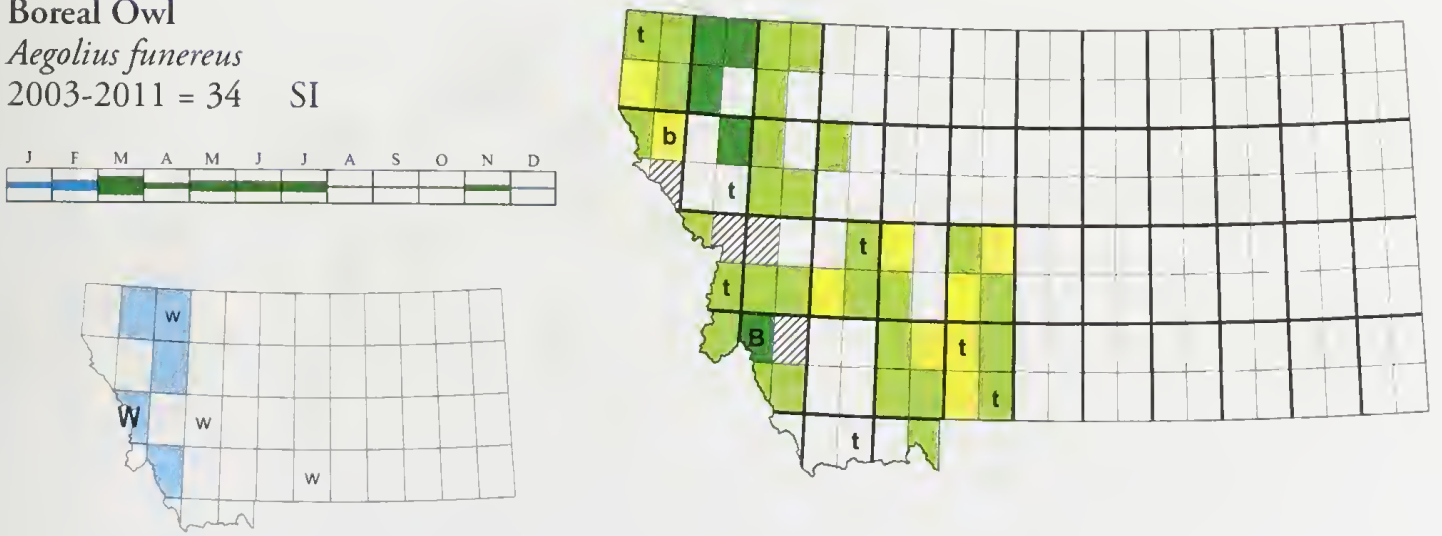
Northern Saw-whet Owl

Aegolius acadicus

2003-2011 = 128

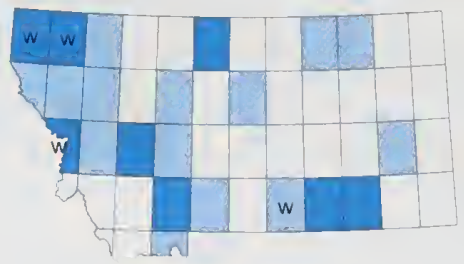

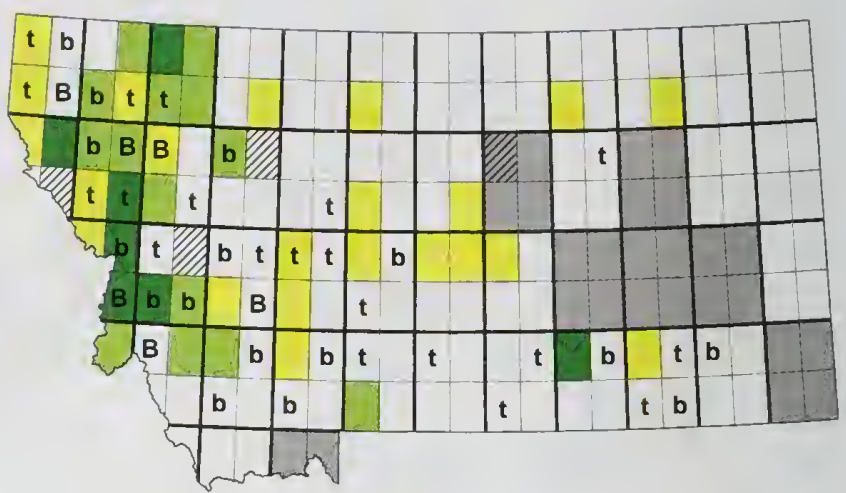

CAPRIMULGIFORMES Caprimulgidae

Common Nighthawk

Chordeiles minor

2003-2011 = 1367

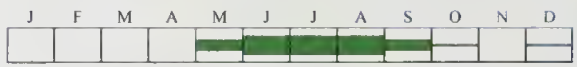

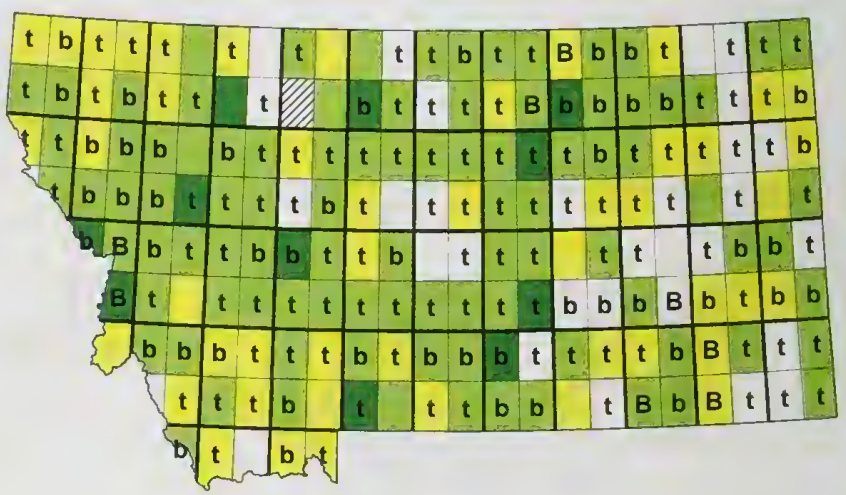

Common Poorwill

Phalaenoptilus nuttallii

2003-2011 = 81 SI

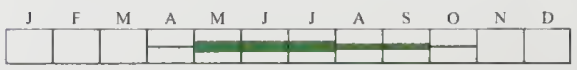

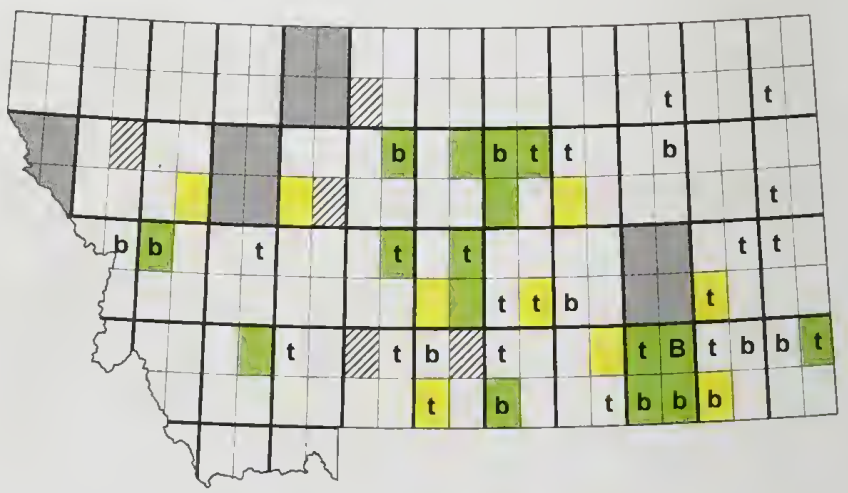


Whip-poor-will

Caprimulgus sp.

2003-2011 = 0 R1

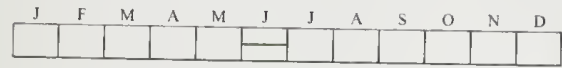

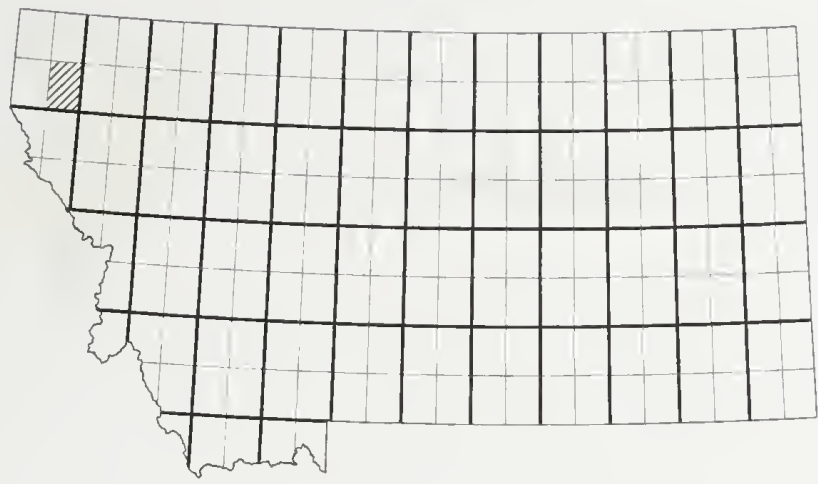

APODIFORMES Apodidae

\section{Black Swift}

Cypseloides niger

2003-2011 $=47$ SOC

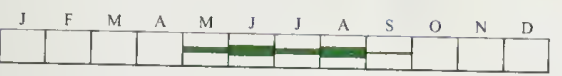

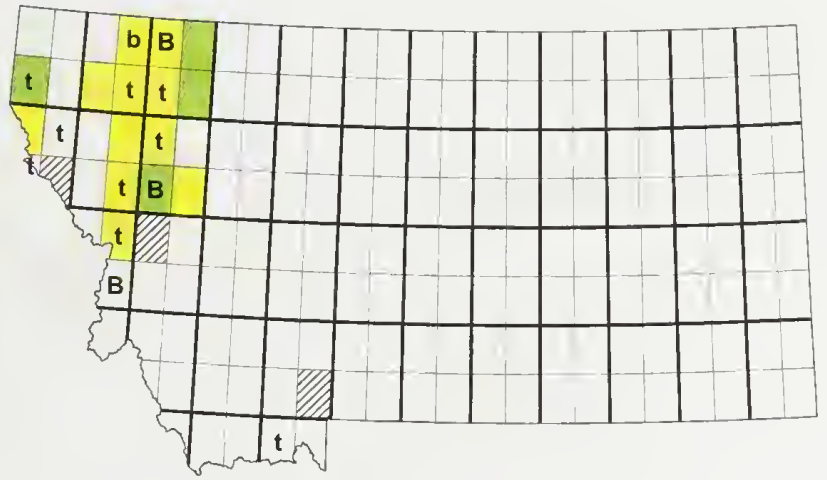

\section{Chimney Swift}

Chaetura pelagica

2003-2011 = 32 SI
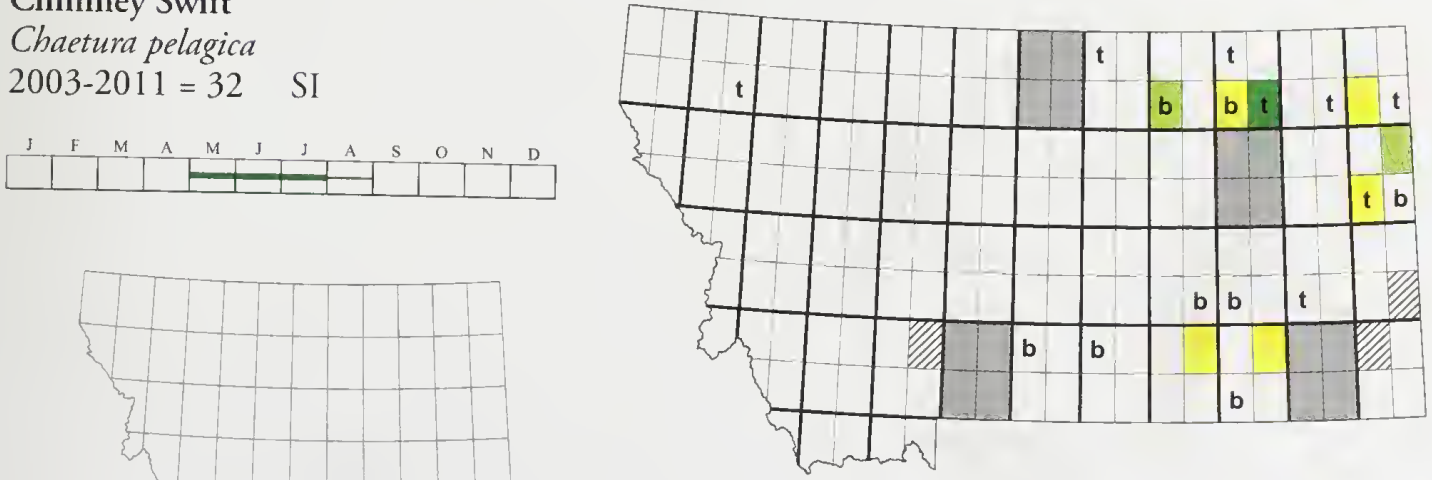
Vaux's Swift

Chaetura vauxi

2003-2011 = 149
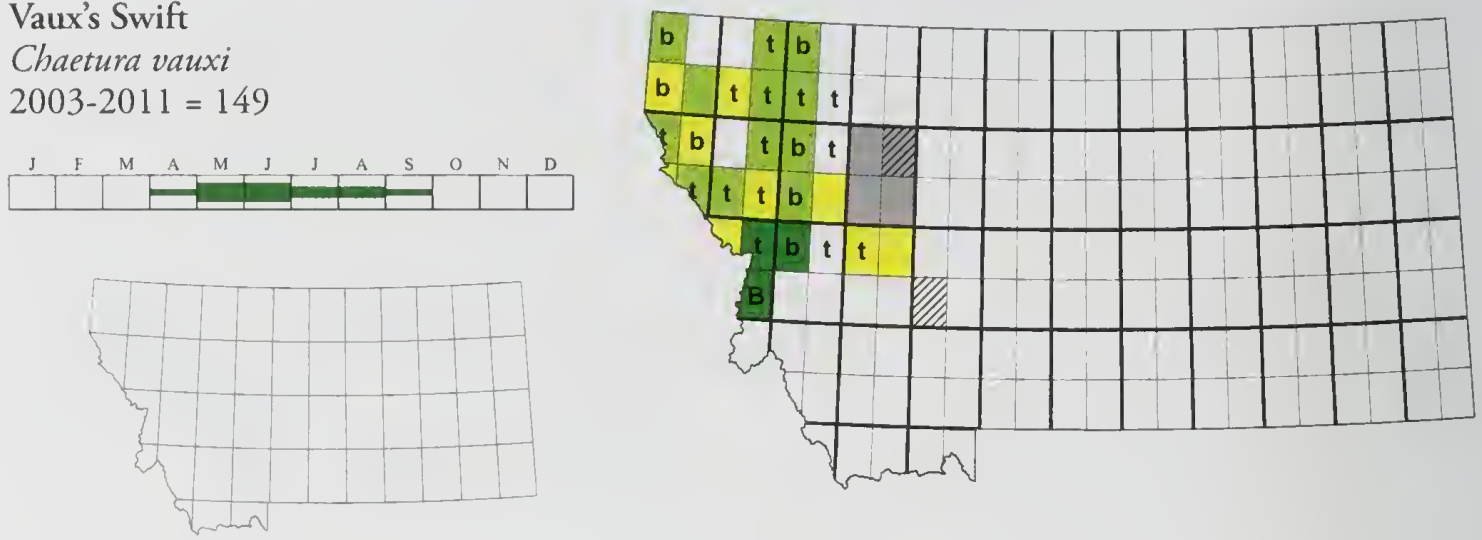

White-throated Swift

Aeronautes saxatalis

2003-2011 = 246
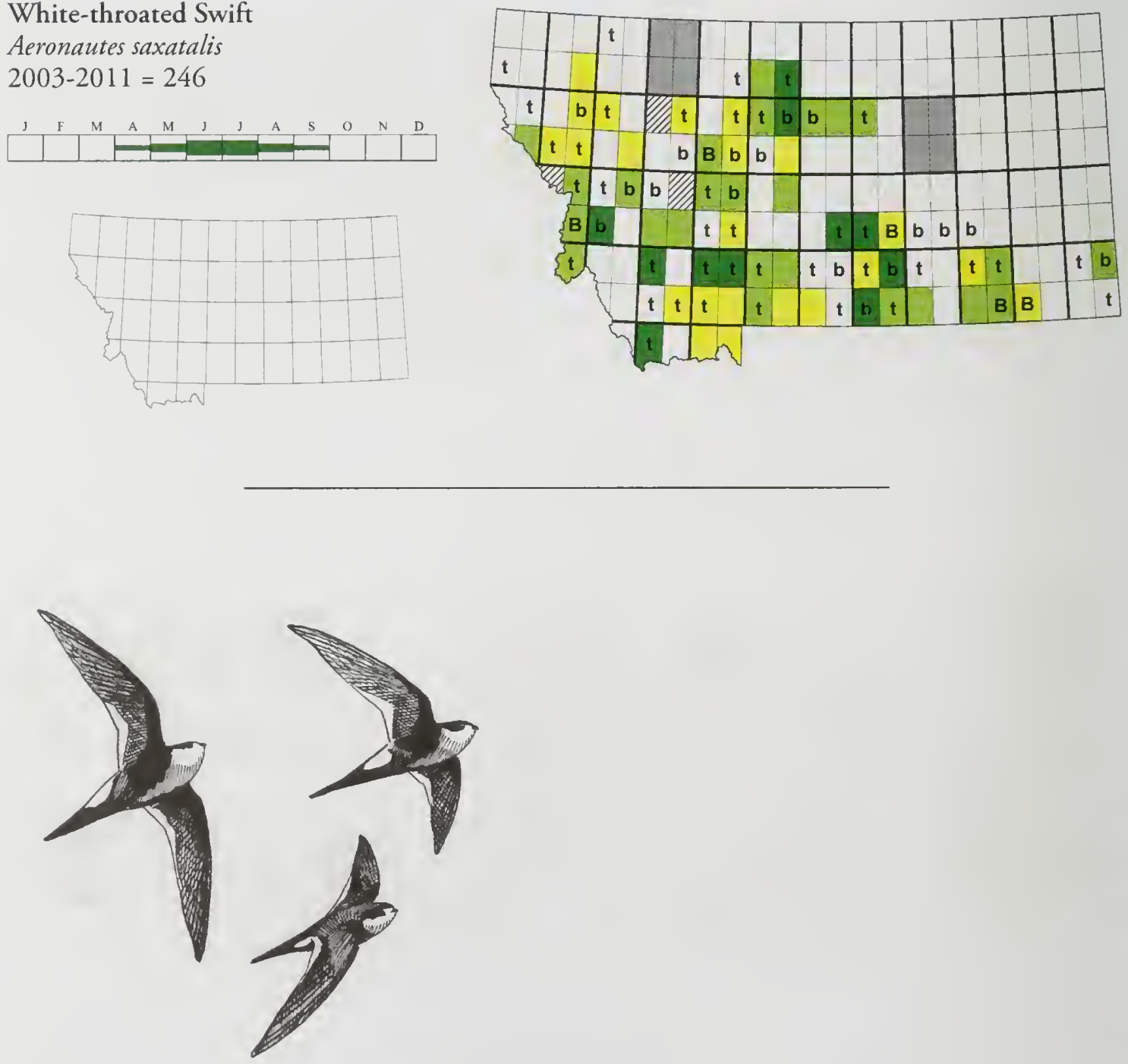
Trochilidae

Ruby-throated Hummingbird Archilochus colubris

2003-2011 = $18 \quad$ R2
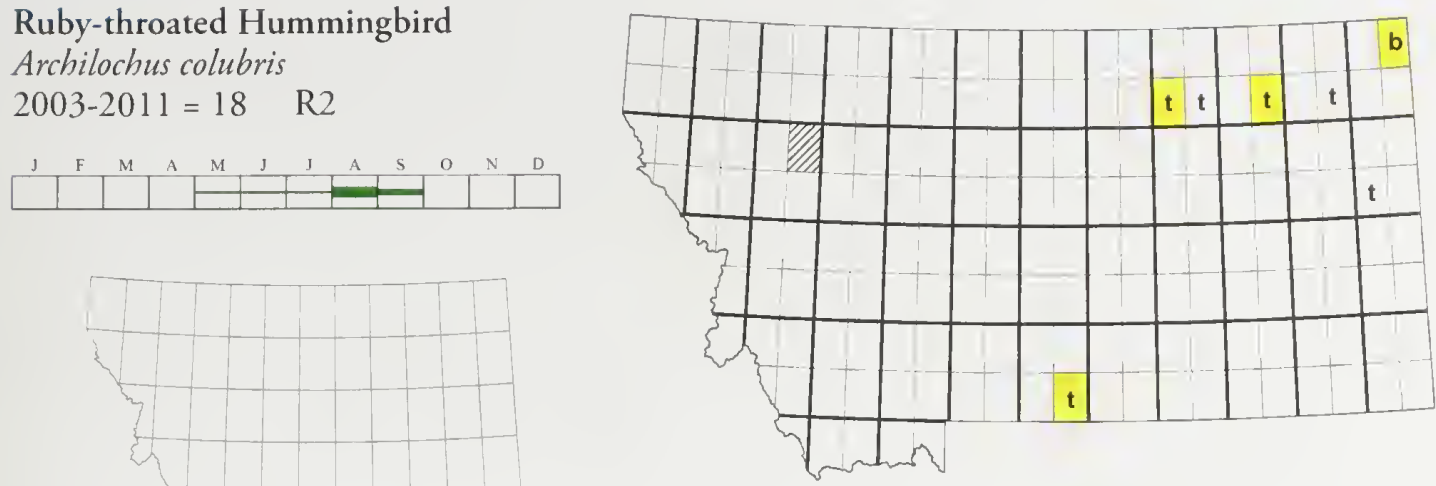

Black-chinned Hummingbird

Archilochus alexandri

2003-2011 = 160
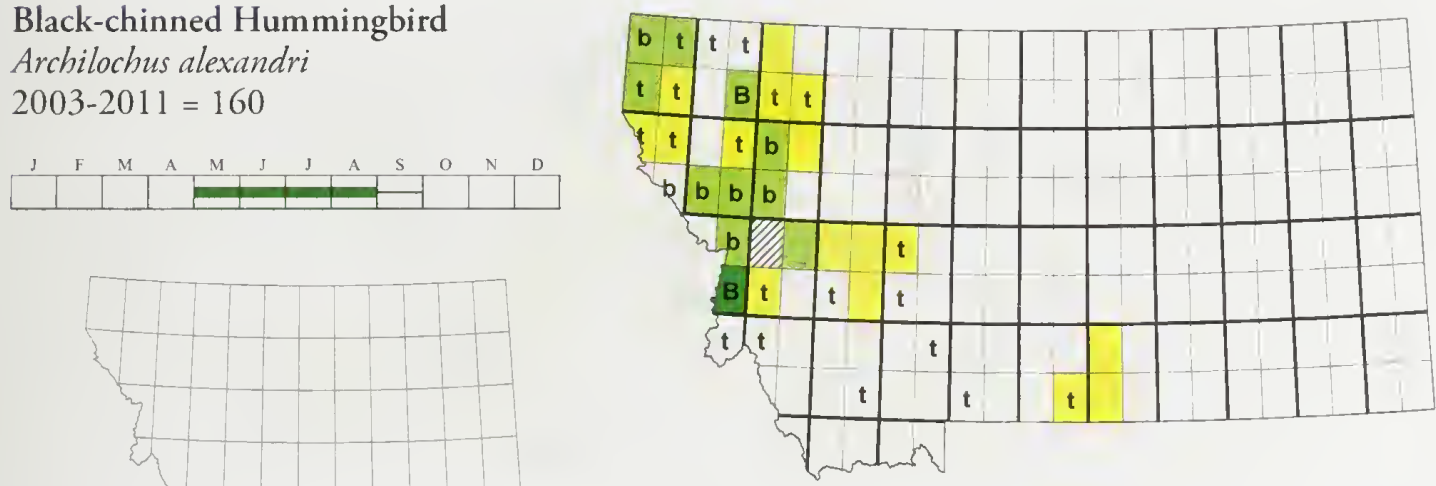

Anna's Hummingbird

Calypte anna

$2003-2011=10 \quad$ R2

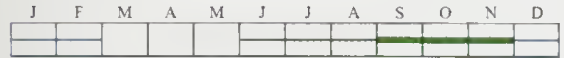

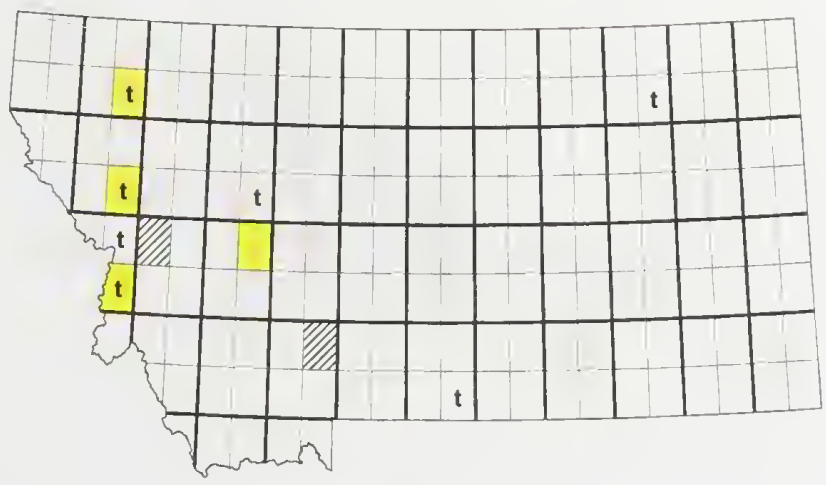


Costa's Hummingbird

Calypte costae

2003-2011 = 1 R1

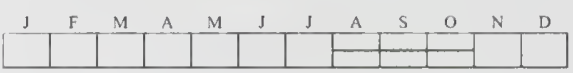
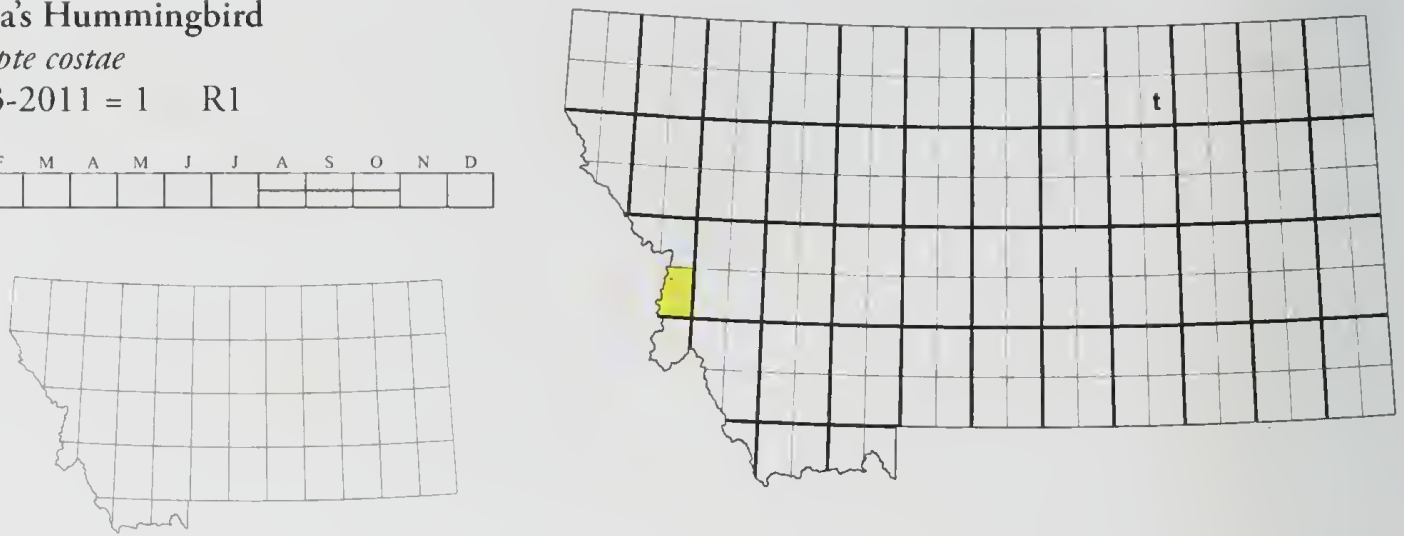

Calliope Hummingbird

Stellula calliope

2003-2011 = 458
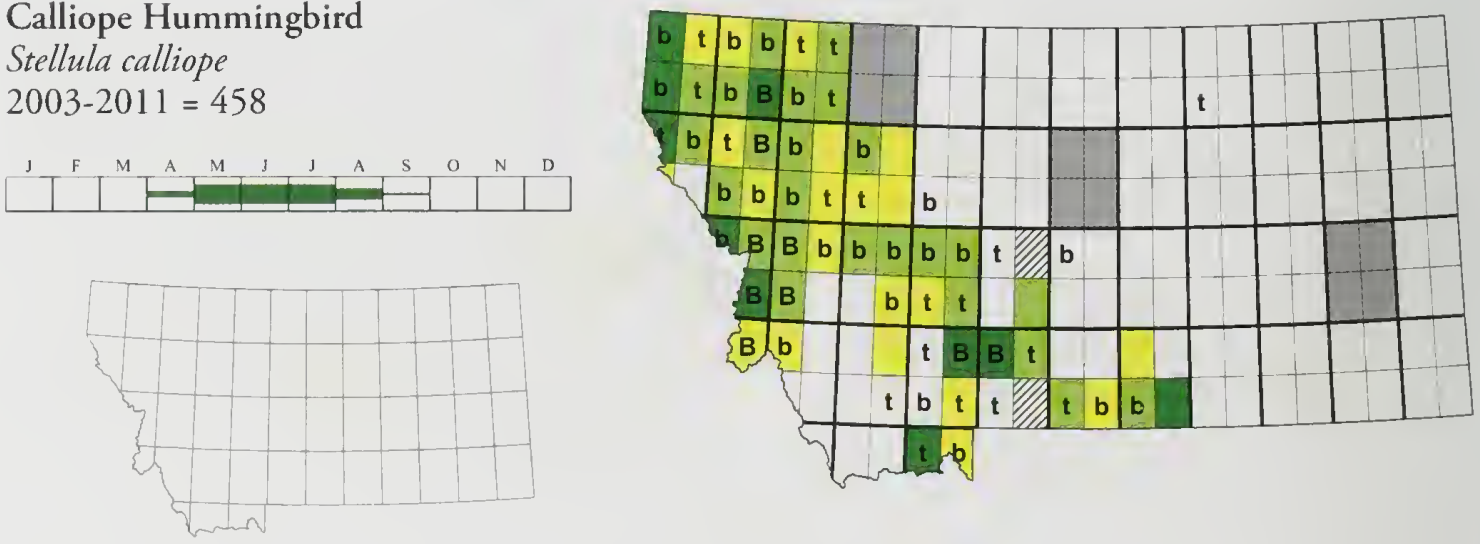
Broad-tailed Hummingbird

Selasphorus platycercus

$2003-2011=53 \quad$ SI
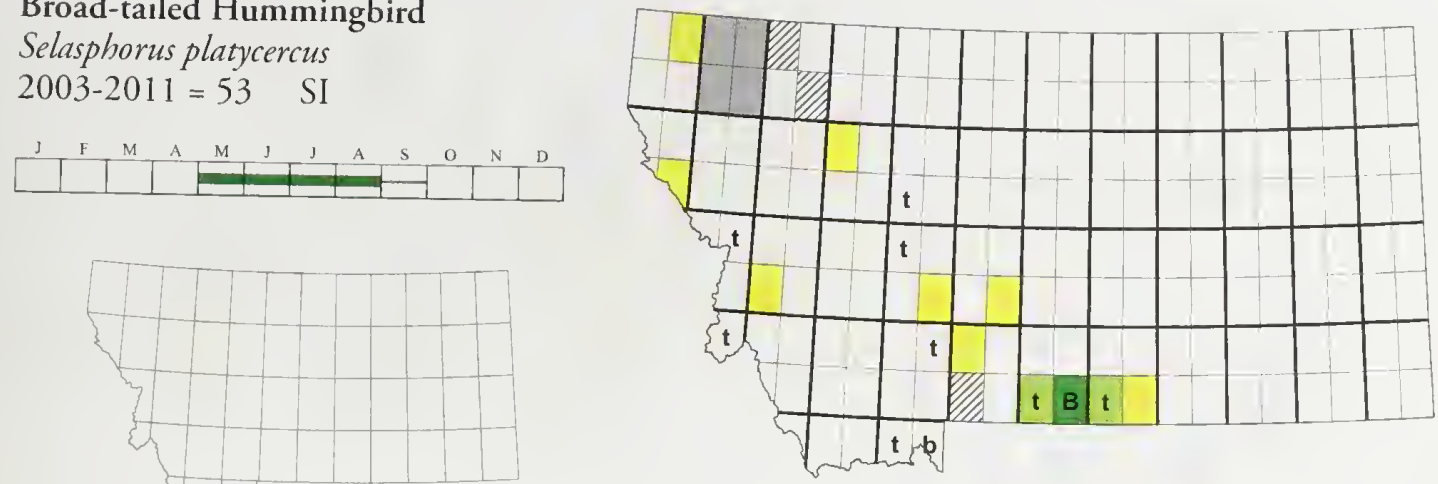

Rufous Hummingbird

Selasphorus rufus

$2003-2011=387 \quad$ SI
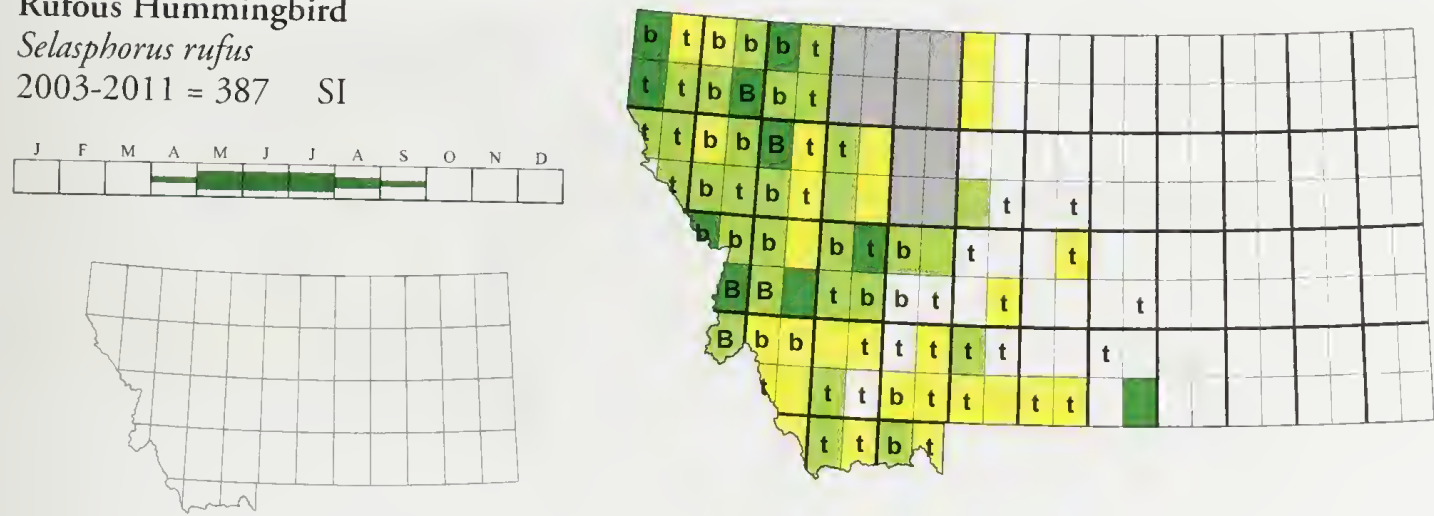

CORACIIFORMES Alcedinidae

Belted Kingfisher

Megaceryle alcyon

2003-2011 = 946
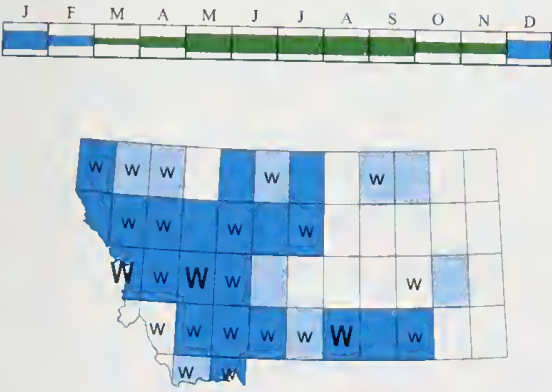

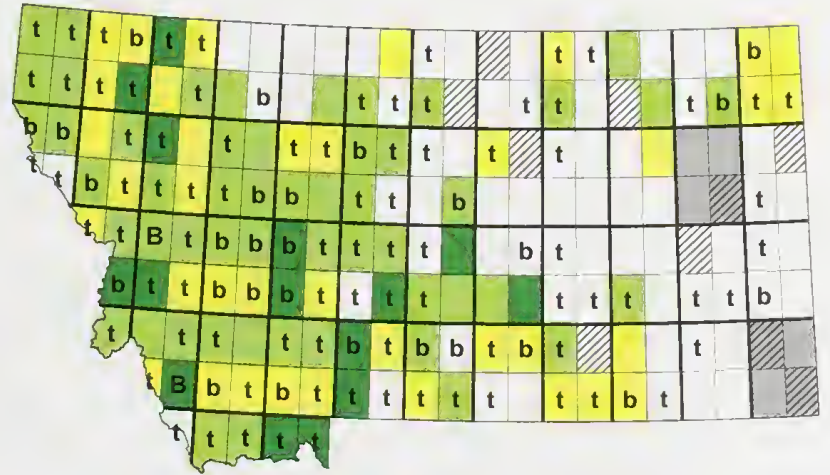


Lewis's Woodpecker

Melanerpes Lewis

2003-2011 $=175$ SOC
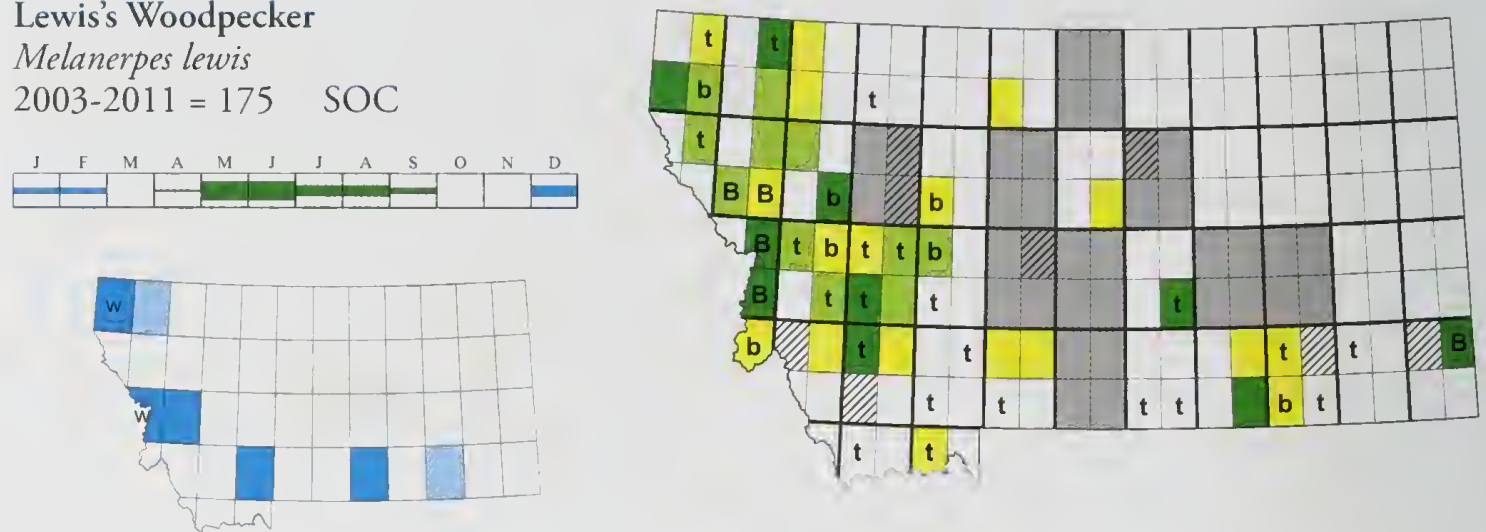

Red-headed Woodpecker

Melanerpes erythrocephalus

2003-2011 = 201 SOC

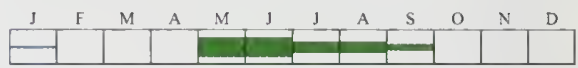

Red-bellied Woodpecker

Melanerpes carolinus

2003-2011 = $0 \quad$ R1

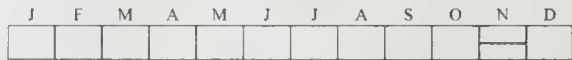
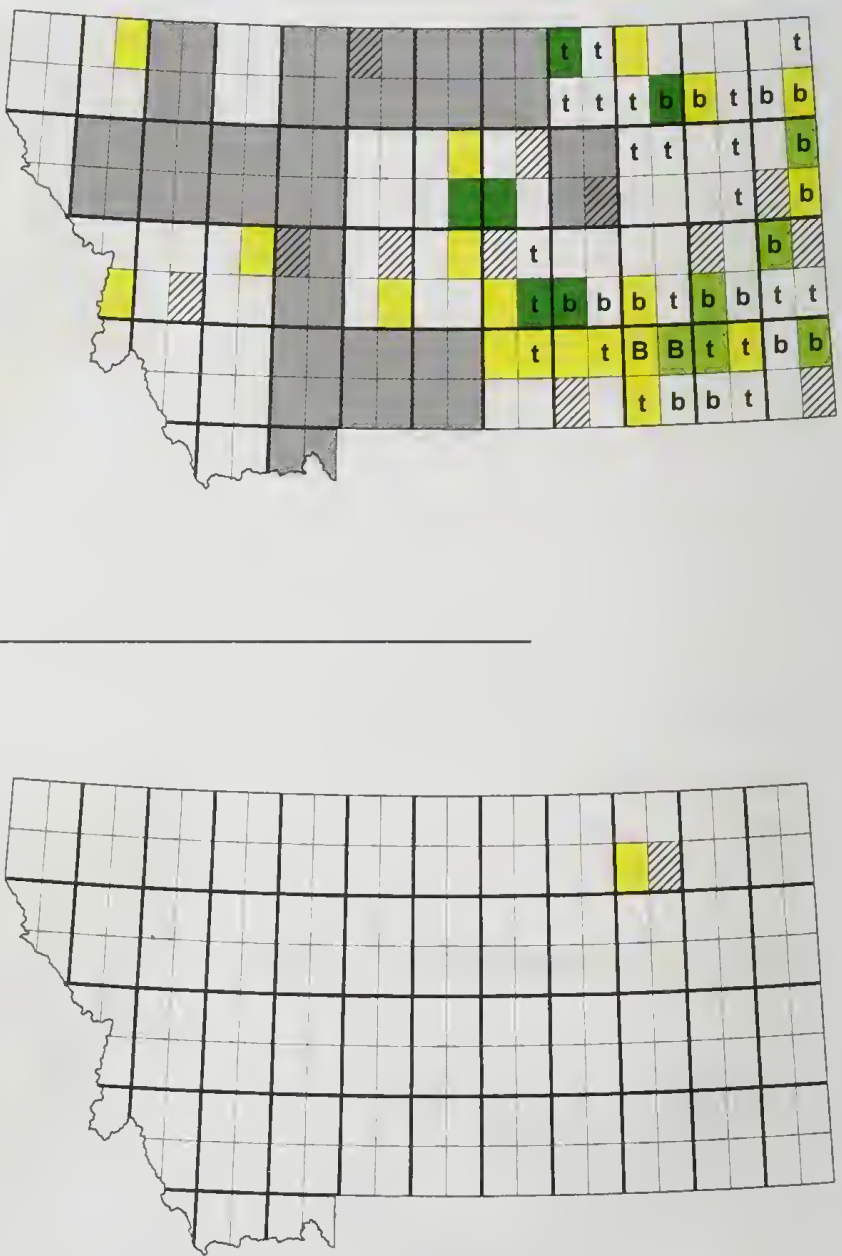
Williamson's Sapsucker

Sphyrapicus thyroideus

2003-2011 = 293

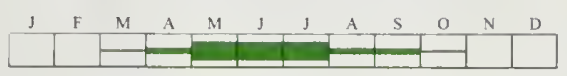

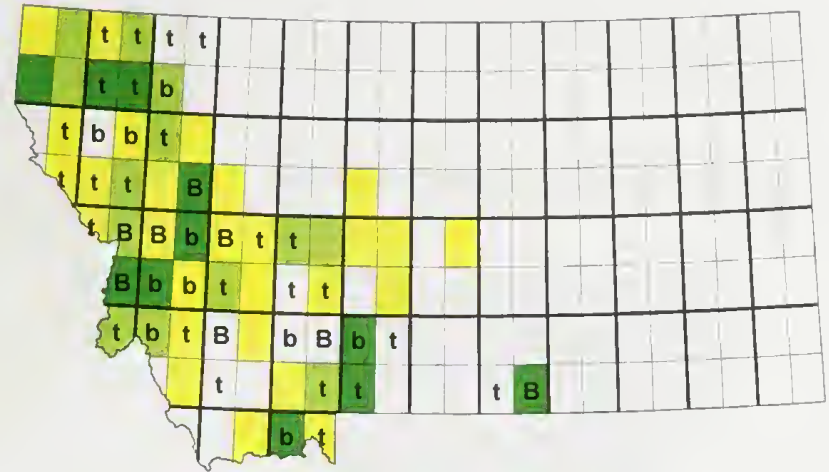

Yellow-bellied Sapsucker

Sphyrapicus varius

$2003-2011=10 \quad$ R2
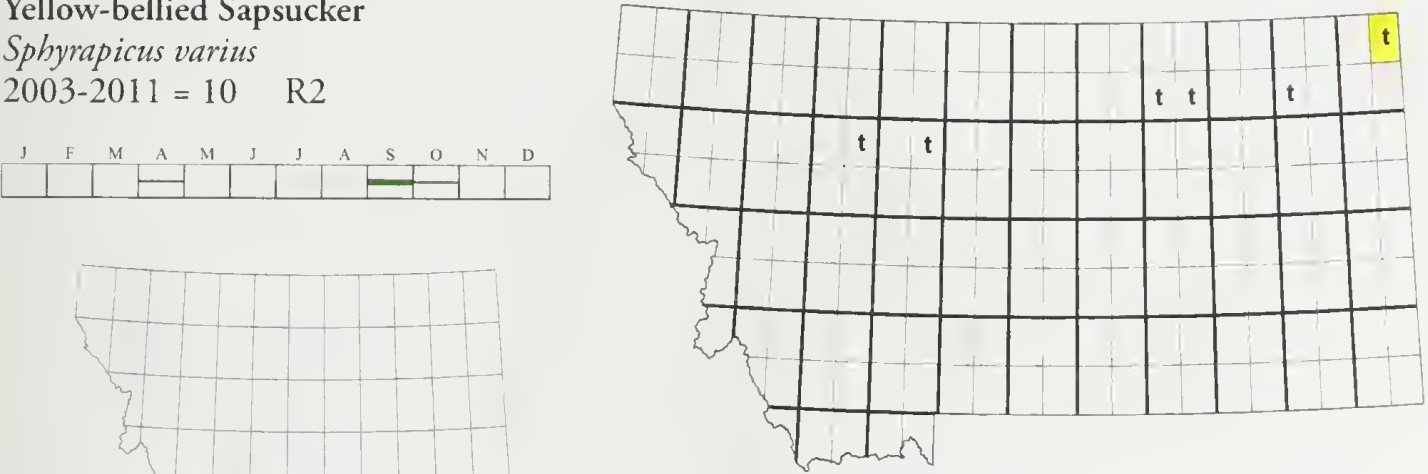

Red-naped Sapsucker

Sphyrapicus nuchalis

$2003-2011=1260$

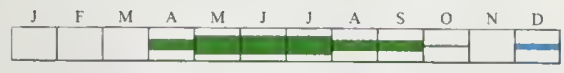

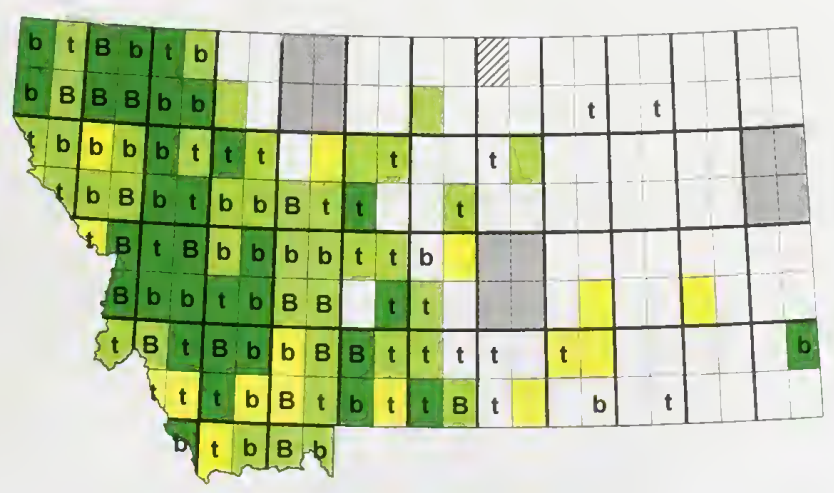


Downy Woodpecker

Picoides pubescens

2003-2011 = 1193

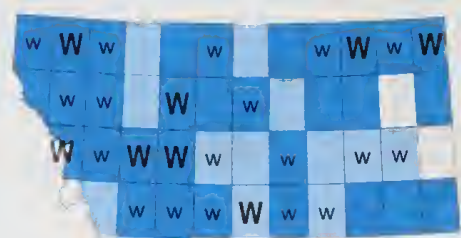

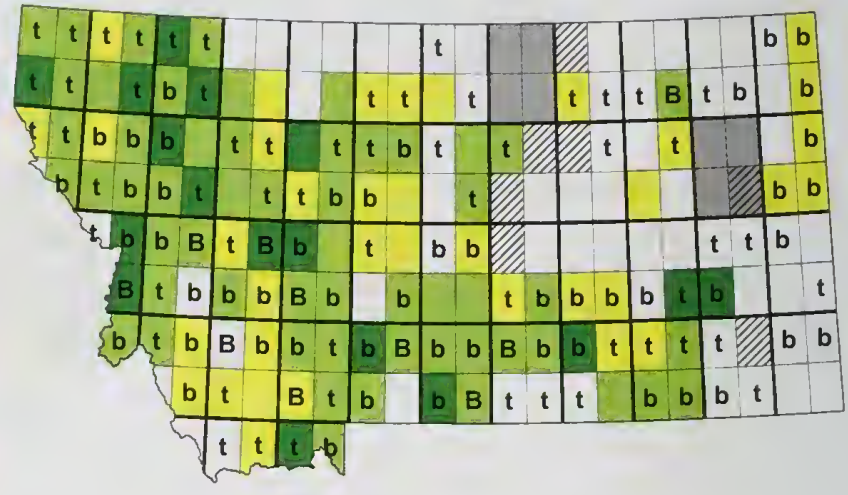

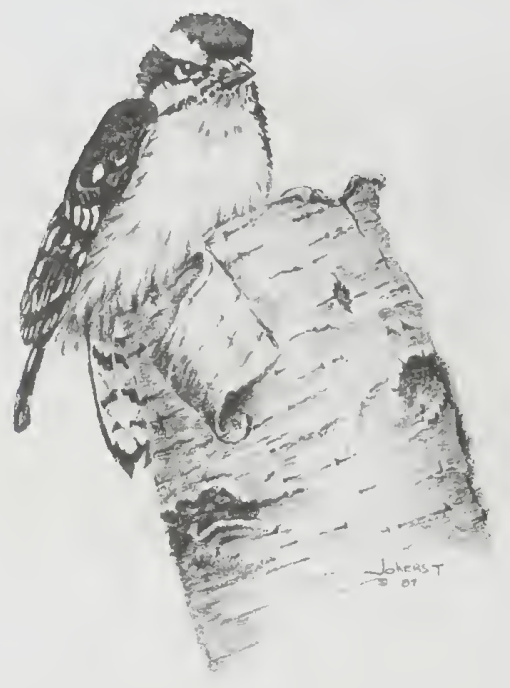

Hairy Woodpecker

Picoides villosus

2003-2011 = 1854
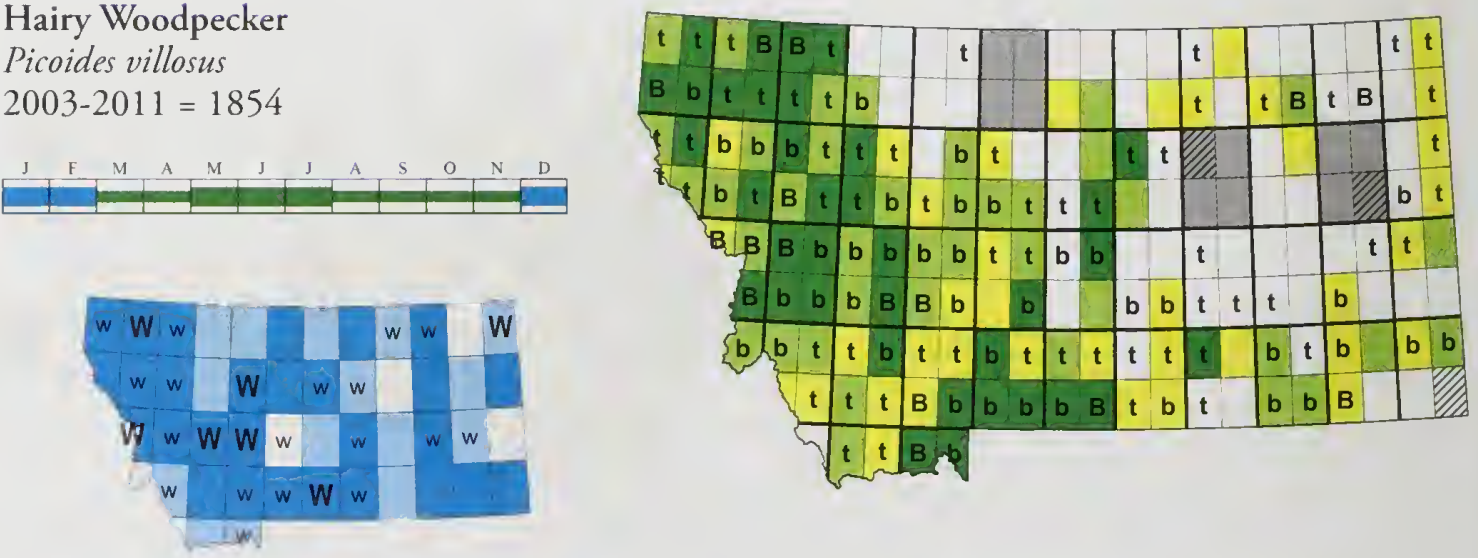
White-headed Woodpecker

Picoides albolarvatus

2003-2011 = 0 R1
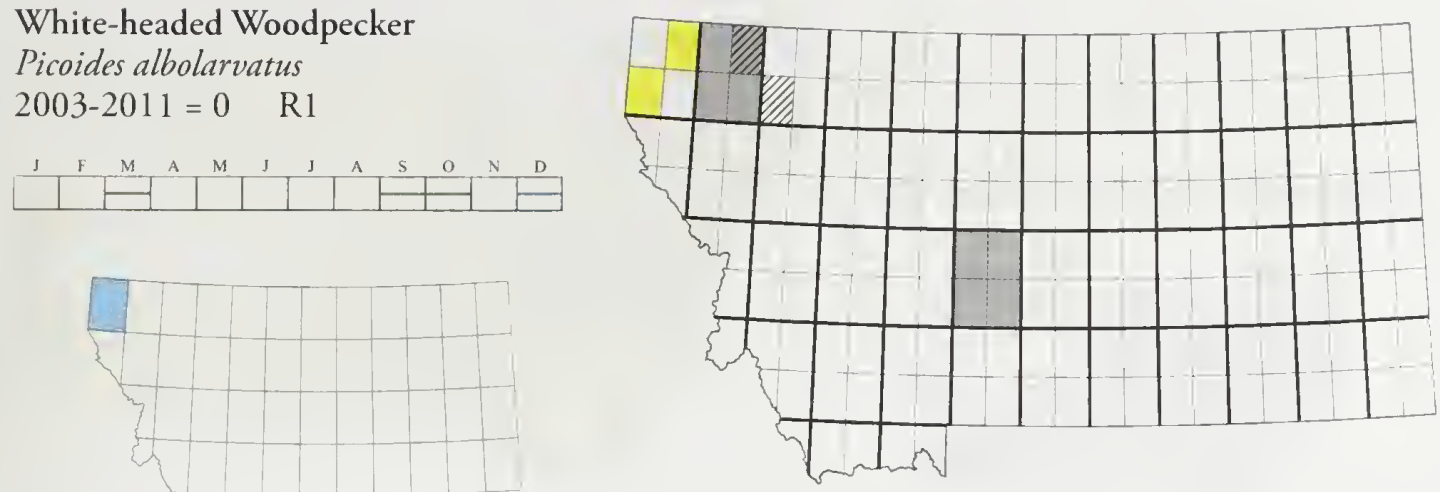

American Three-toed Woodpecker

Picoides dorsalis

2003-2011 = 449
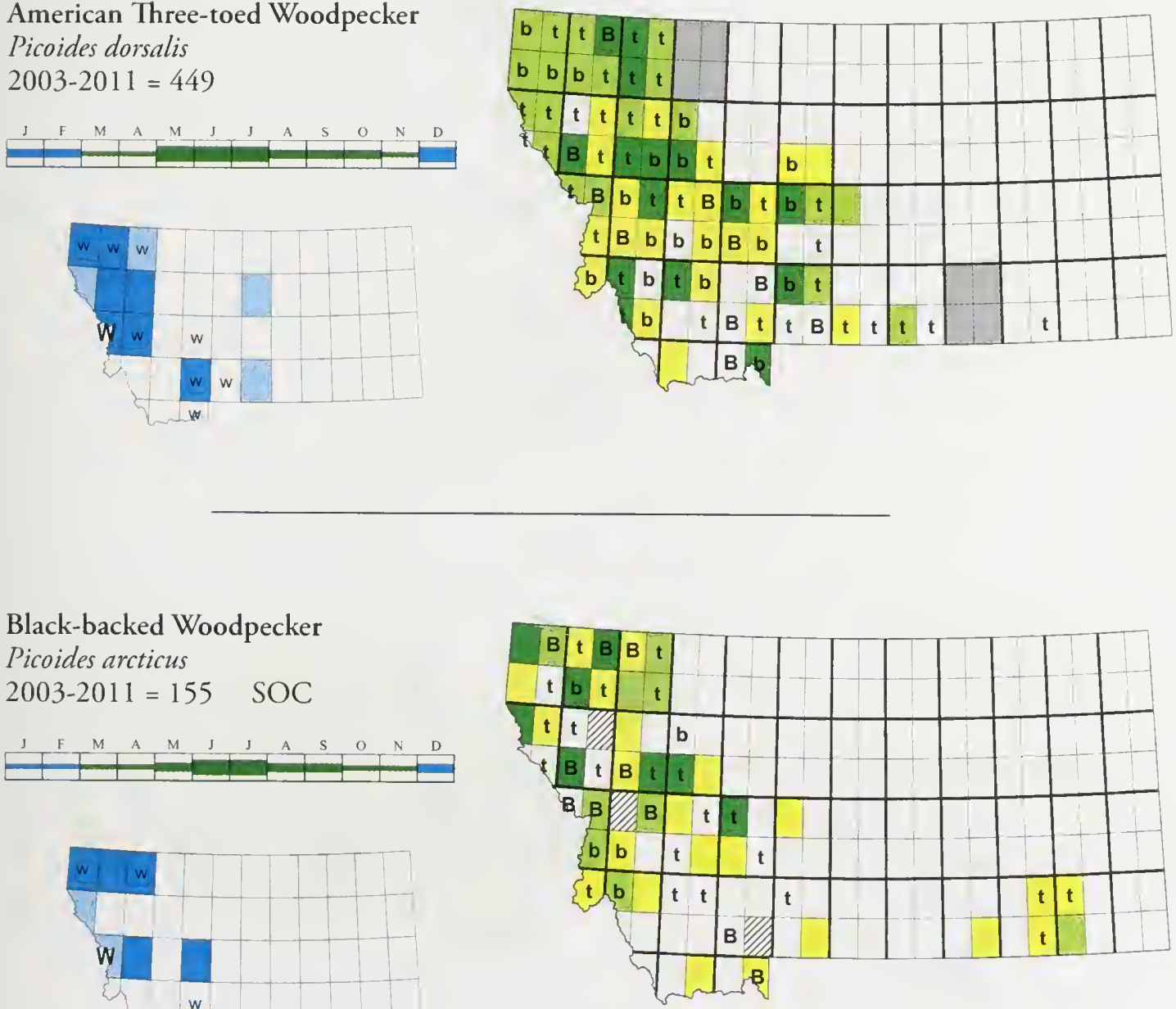

Black-backed Woodpecker

Picoides arcticus

2003-2011 = 155 SOC
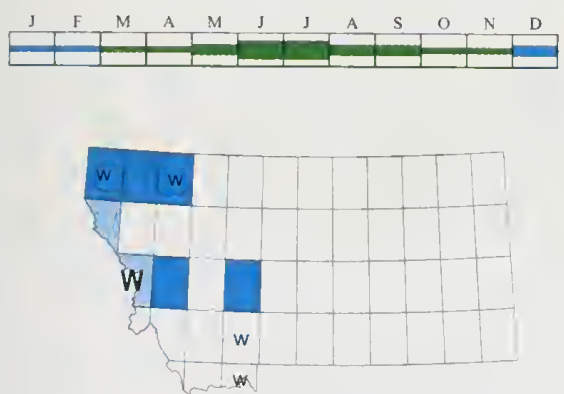
Northern Flicker

Colaptes auratus

2003-2011 = 4023
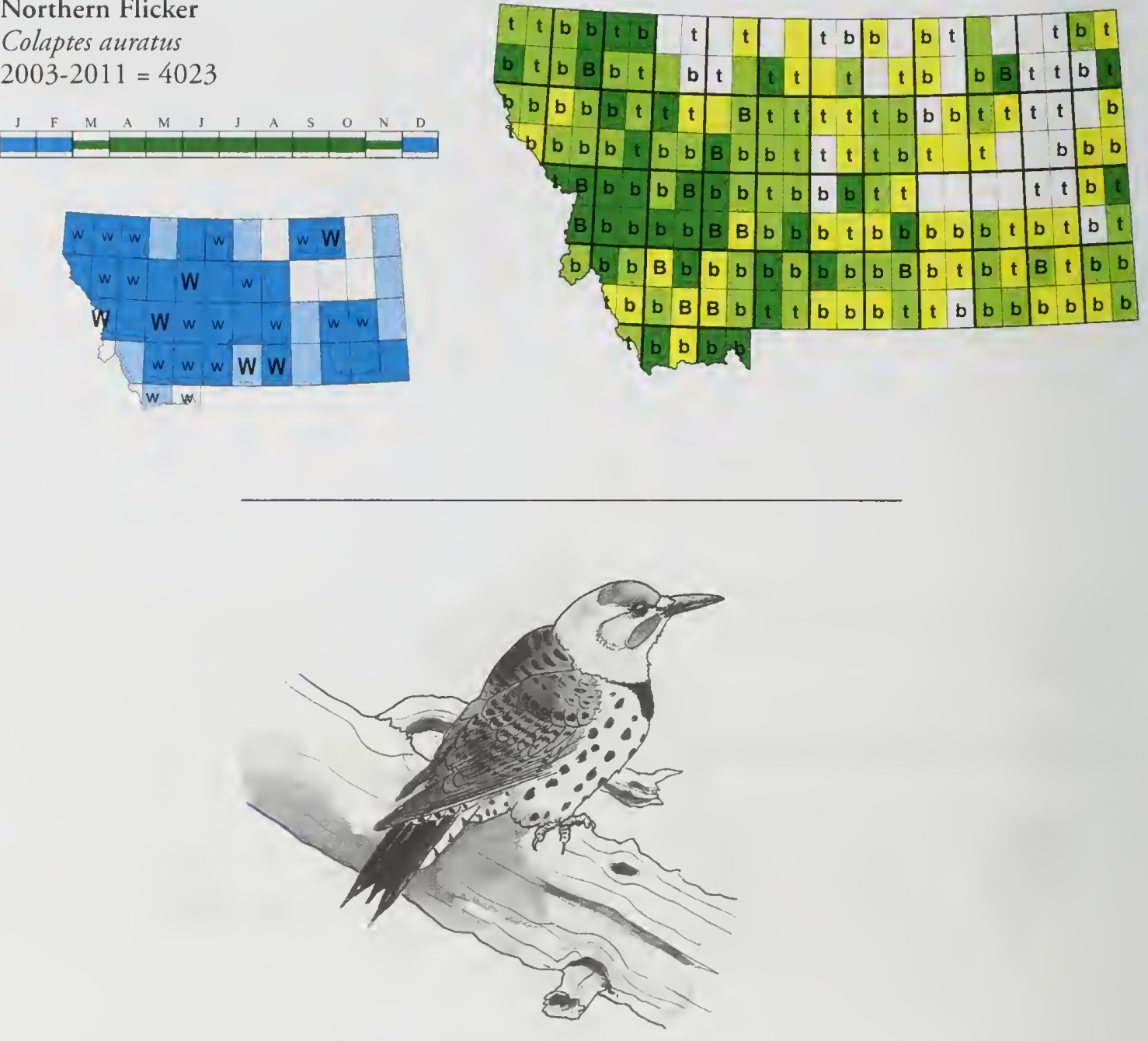

Red-shafted Flicker

Colaptes auratus cafer

2003-2011 = 1395

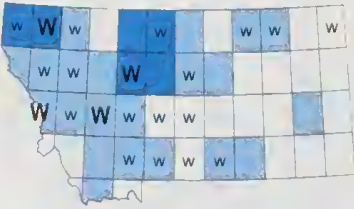


Yellow-shafted Flicker

Colaptes auratus auratus 2003-2011 = 84
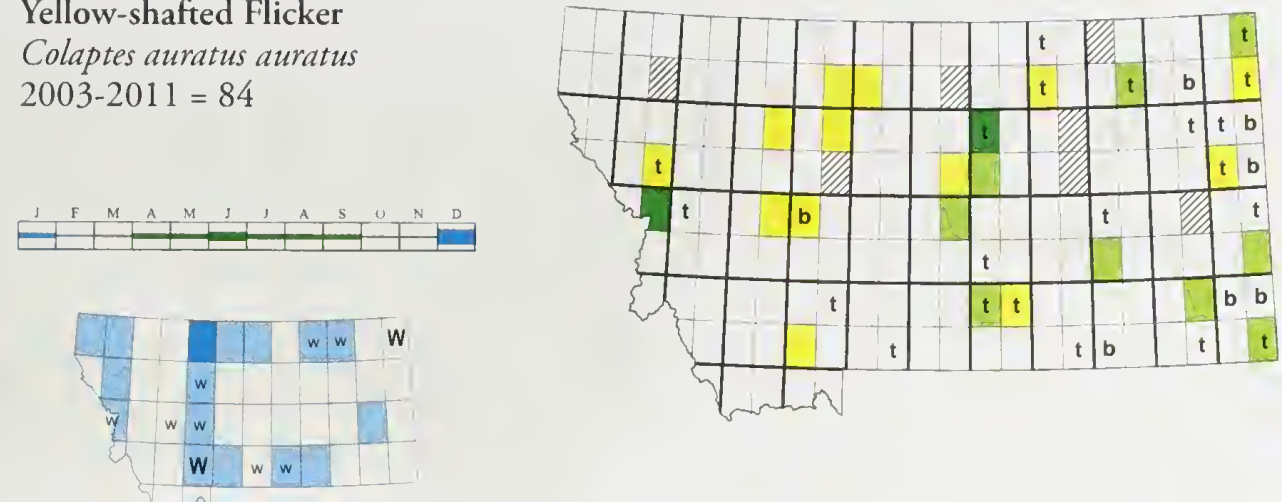

Pileated Woodpecker

Dryocopus pileatus

$2003-2011=855 \quad$ SOC
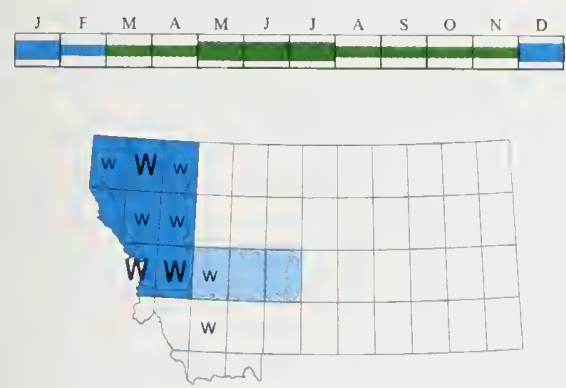

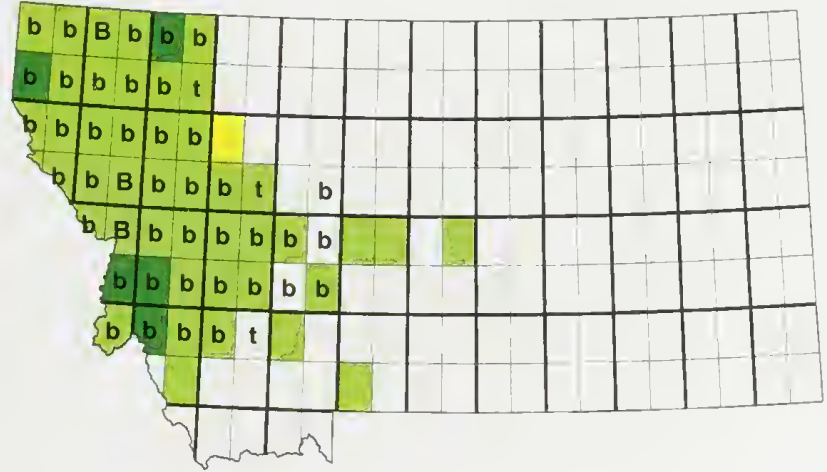

PASSERIFORMES Tyrannidae

Olive-sided Flycatcher

Contopus cooperi

2003-2011 = 1044

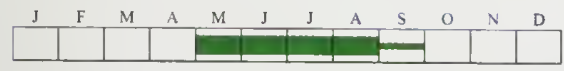

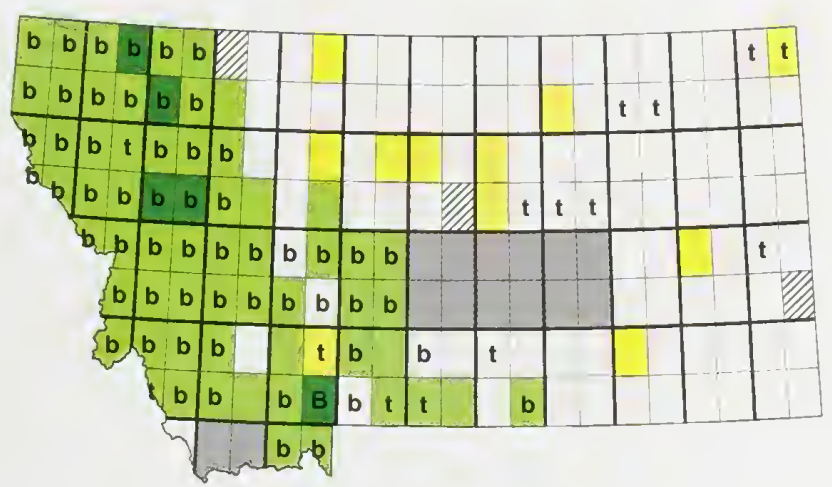


Western Wood-Pewee

Contopus sordidulus

$2003-2011=2756$

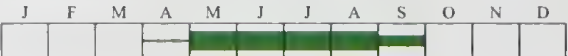

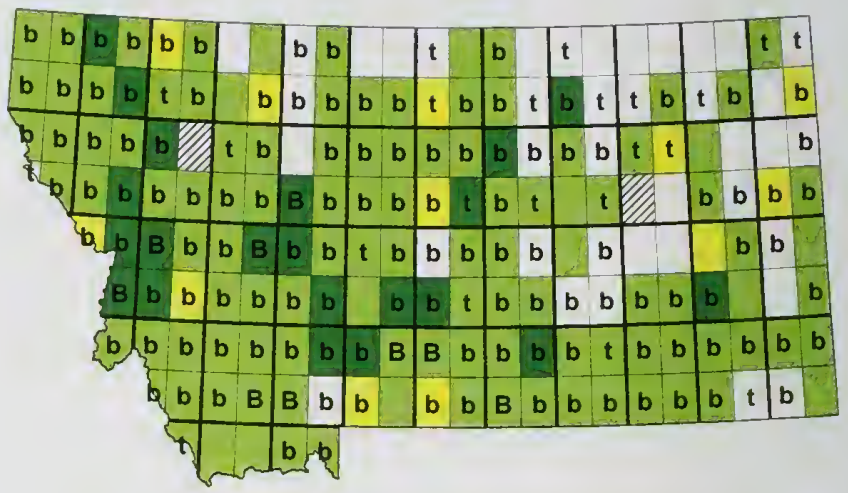

Eastern Wood-Pewee

Contopus virens

2003-2011 = 0 R1
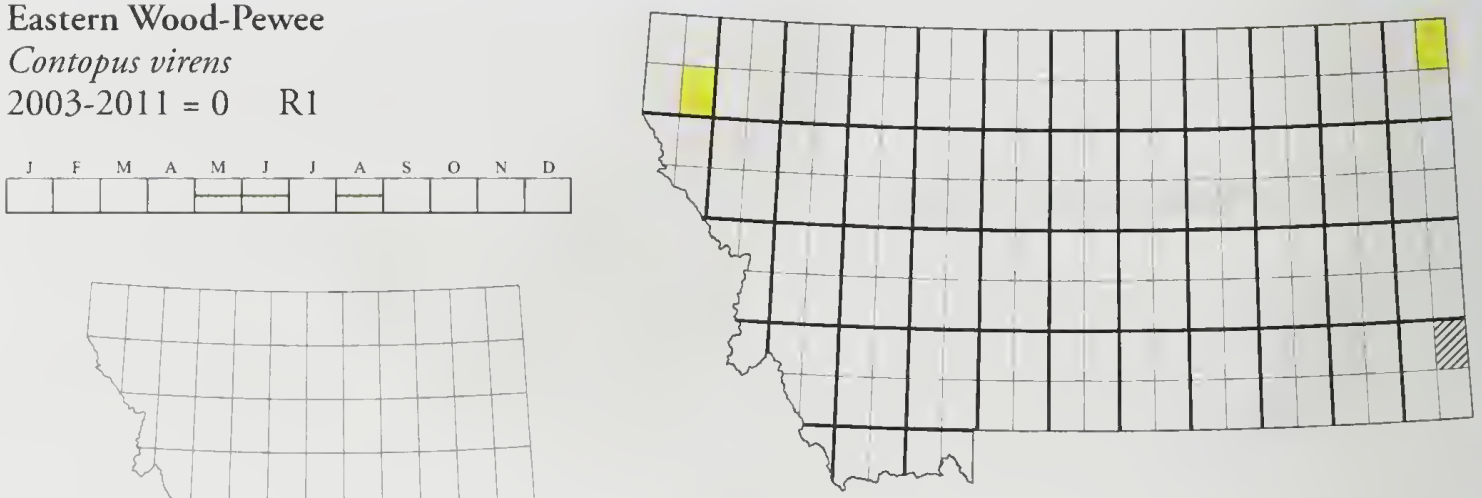

Yellow-bellied Flycatcher

Empidonax flaviventris

2003-2011 = $19 \quad$ R2

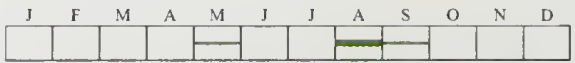

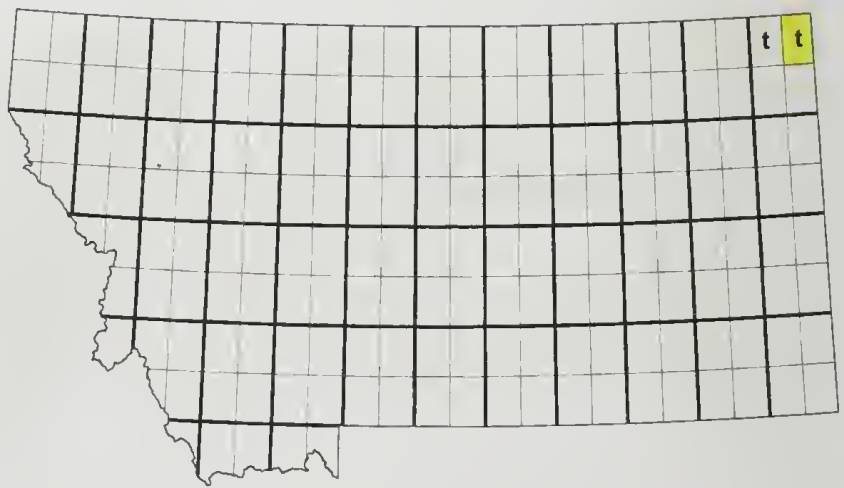


Alder Flycatcher

Empidonax alnorum

$2003-2011=23 \quad$ SOC

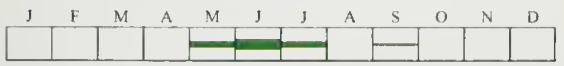

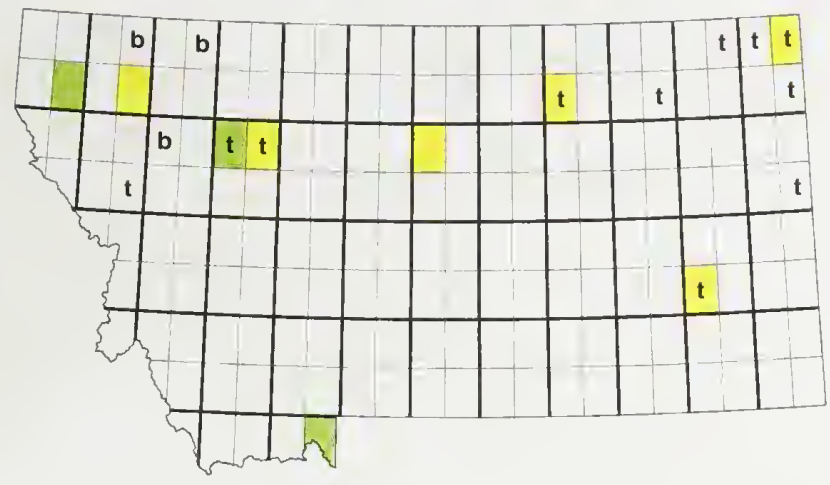

Willow Flycatcher

Empidonax traillii

2003-2011 = 1251

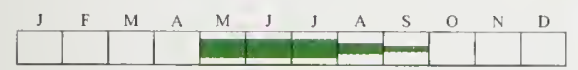

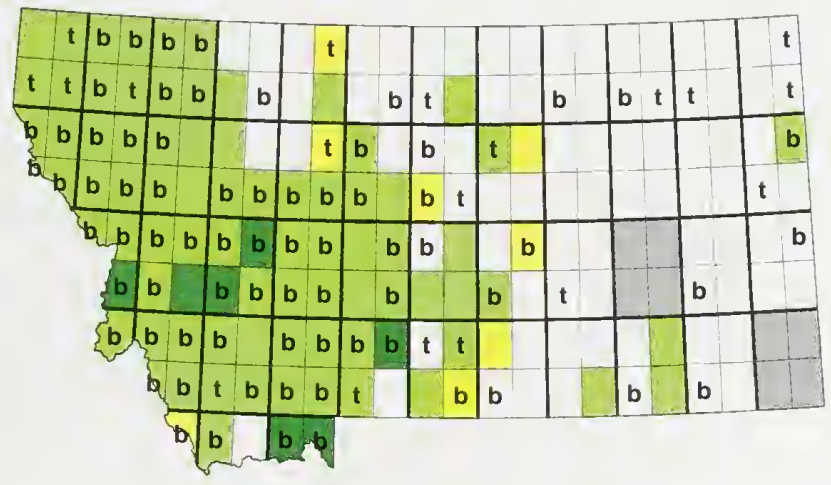

Least Flycatcher

Empidonax minimus

2003-2011 = 1314

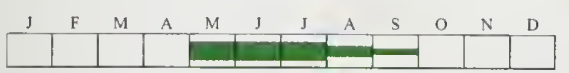

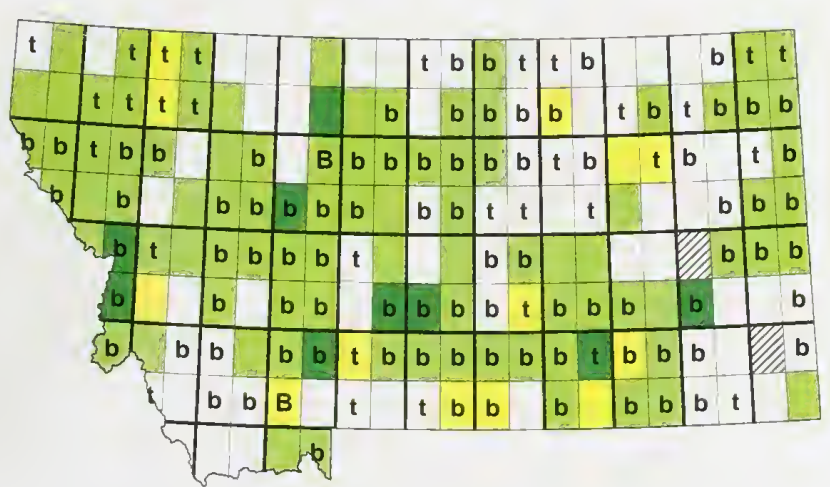


Hammond's Flycatcher

Empidonax hammondii

2003-2011 = 1762

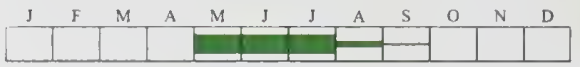

Gray Flycatcher

Empidonax wrightii

$2003-2011=38 \quad$ R2

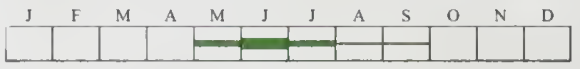

Dusky Flycatcher

Empidonax oberholseri

2003-2011 = 3275

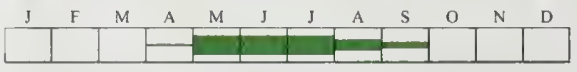

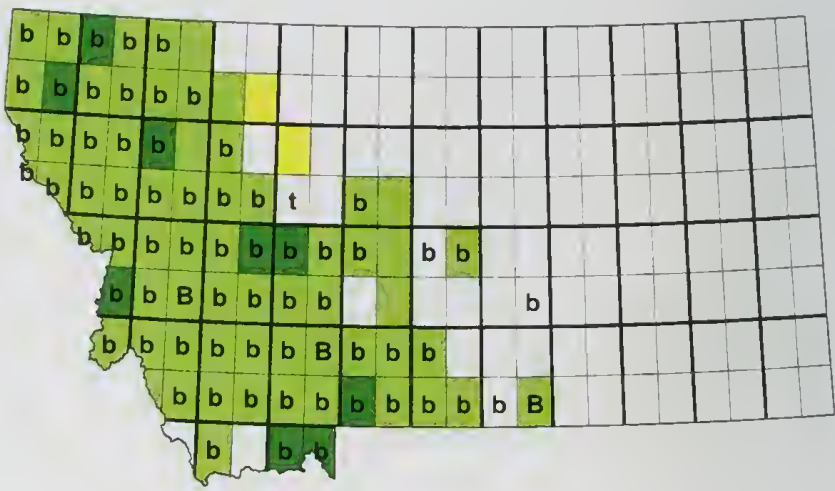

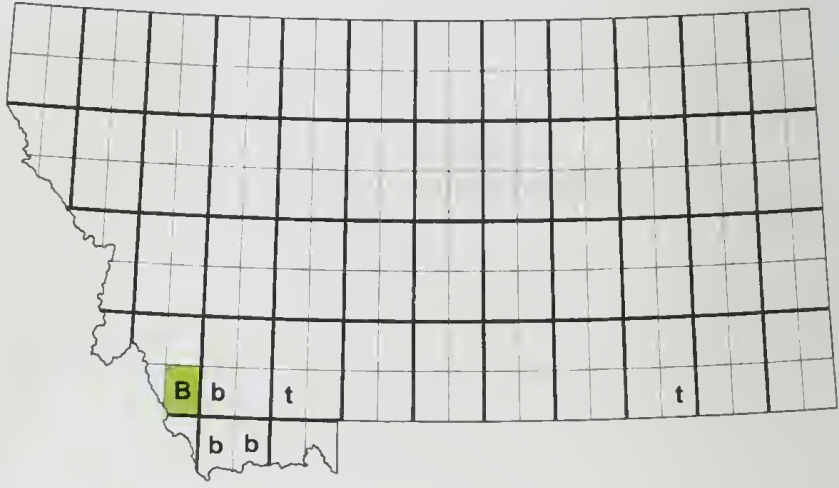

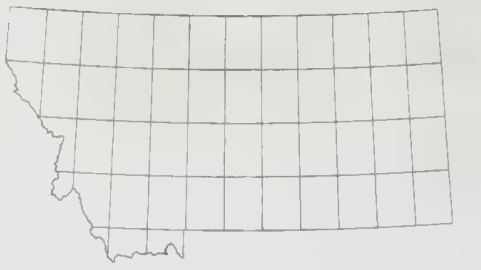

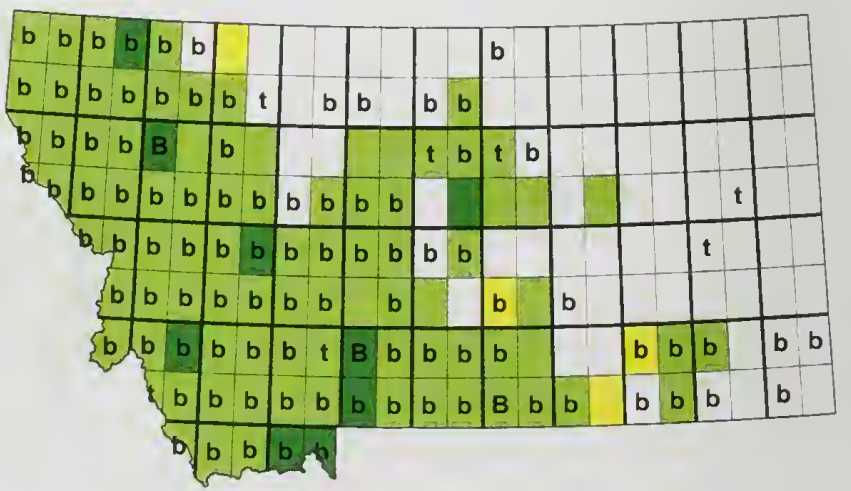


Cordilleran Flycatcher Empidonax occidentalis 2003-2011 = 349

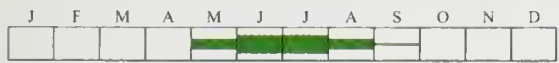

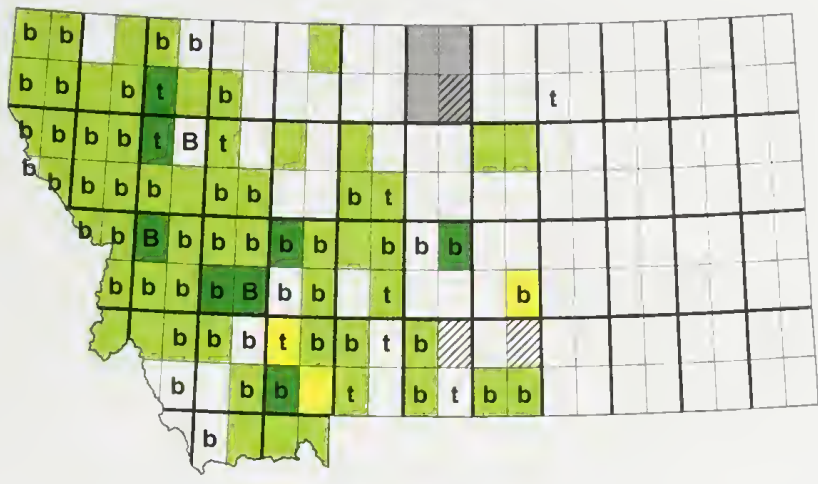

Eastern Phoebe

Sayornis phoebe

2003-2011 = $7 \quad$ R1

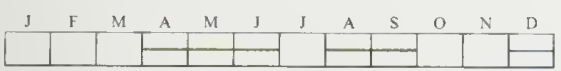

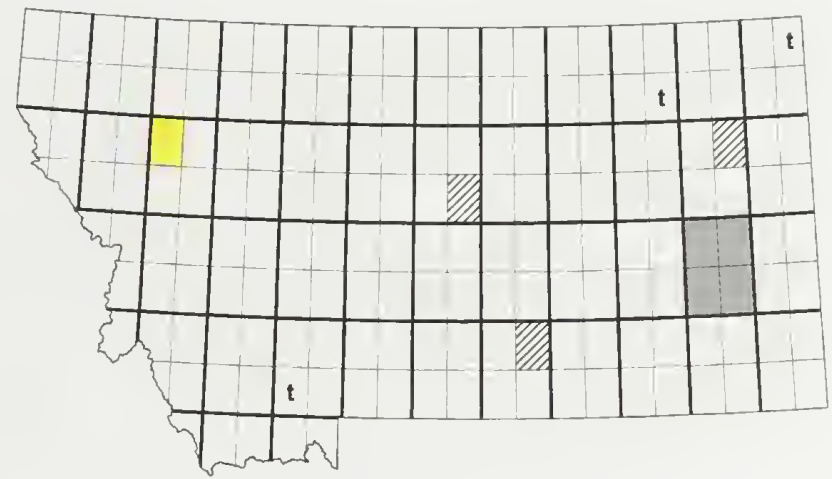

Say's Phoebe

Sayornis saya

2003-2011 = 514
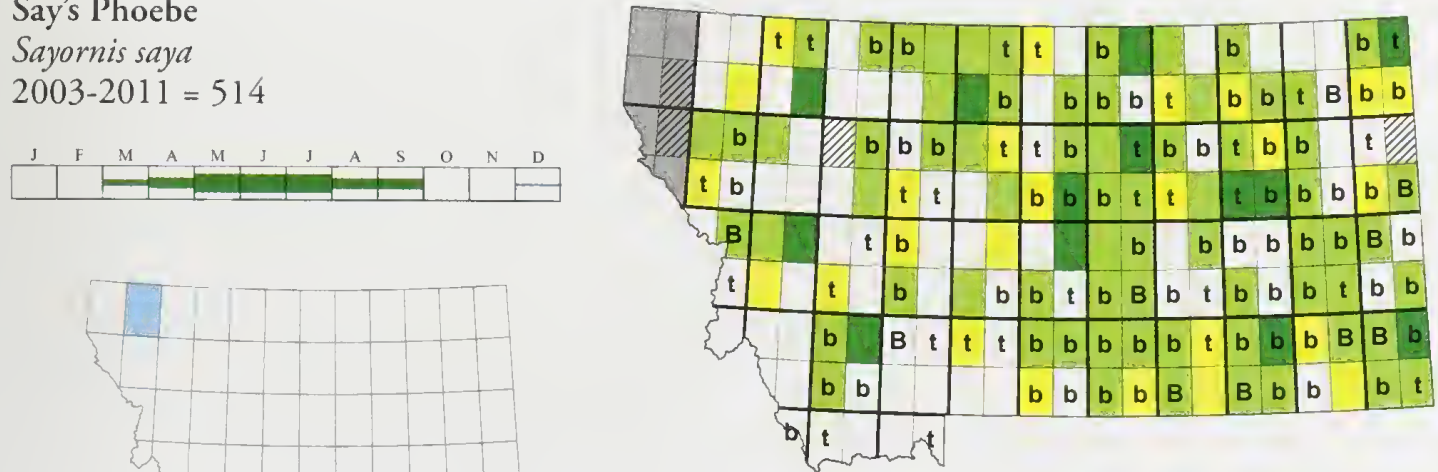
Vermilion Flycatcher

Pyrocephalus rubinus

2003-2011 = 0 R1

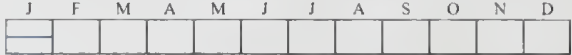
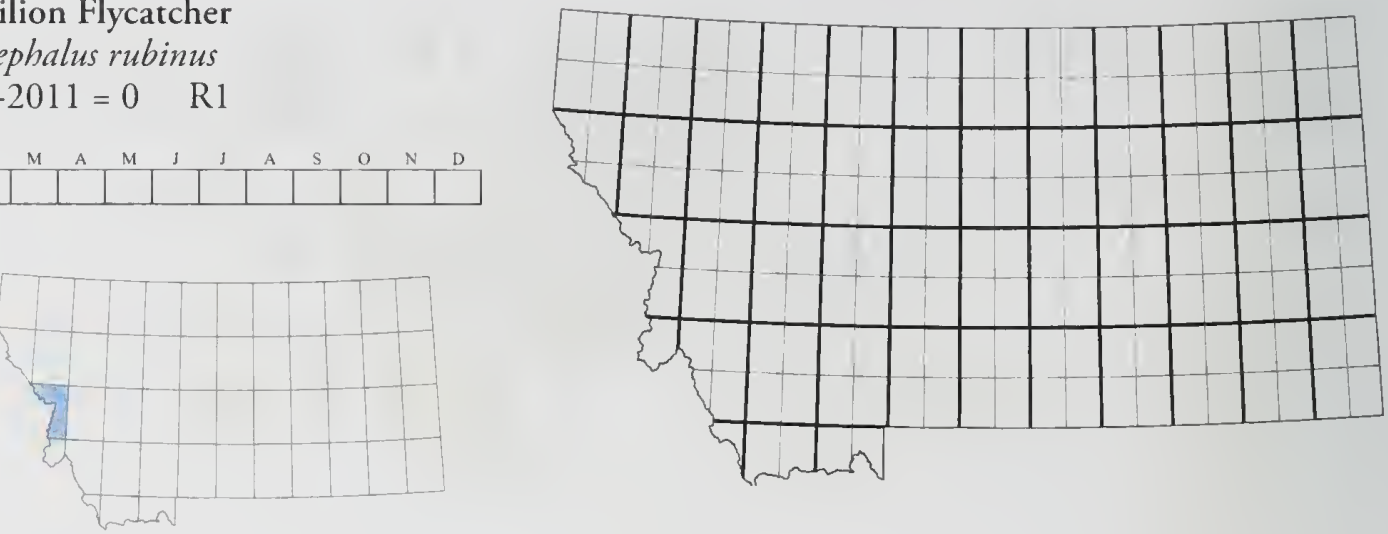

Ash-throated Flycatcher

Myiarchus cinerascens

2003-2011 = $3 \quad$ R1
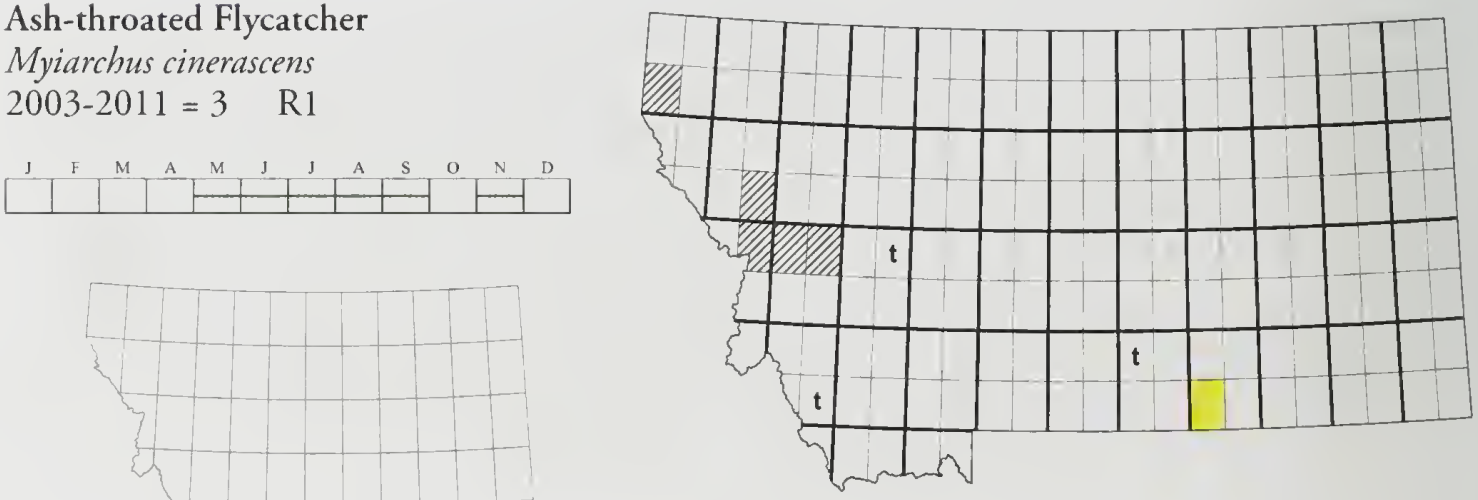

Great Crested Flycatcher

Myiarchus crinitus

2003-2011 = 6 R1
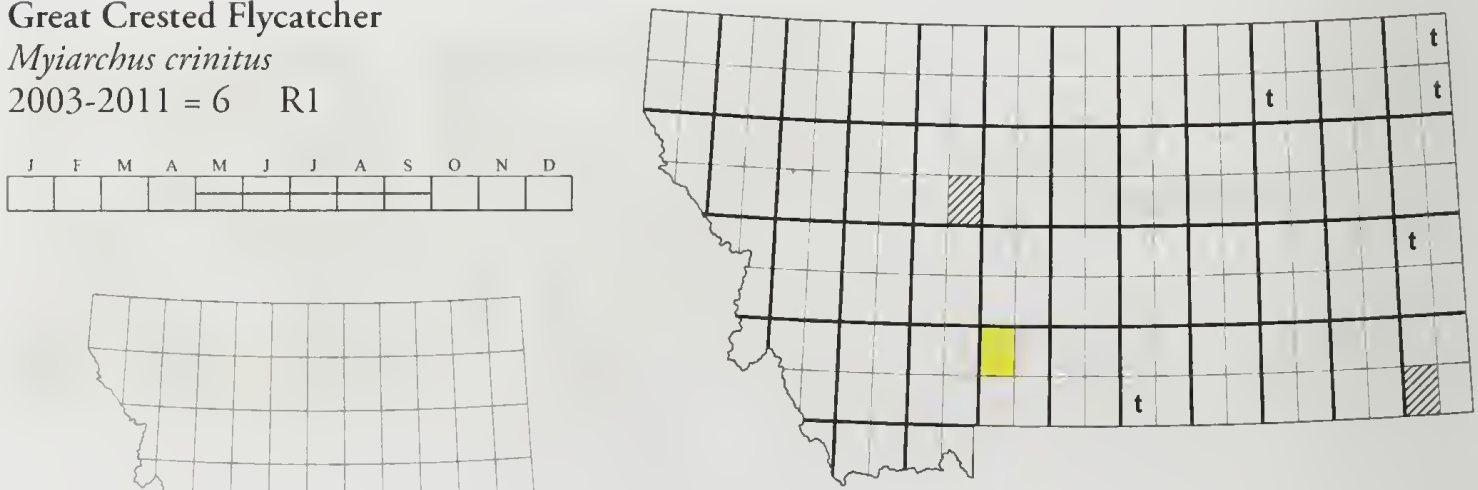
Cassin's Kingbird

Tyrannus vociferans

$2003-2011=115$ SI

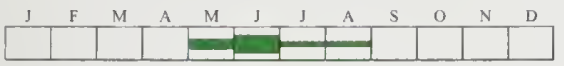

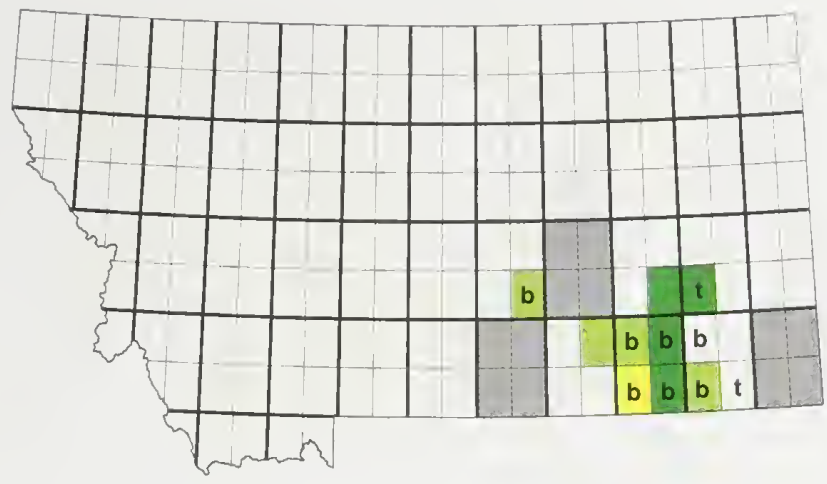

Western Kingbird

Tyrannus verticalis

2003-2011 = 1813

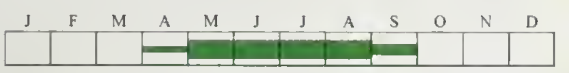

Eastern Kingbird

Tyrannus tyrannus

$2003-2011=3343$

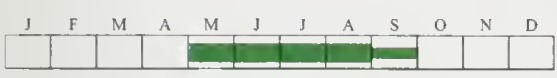

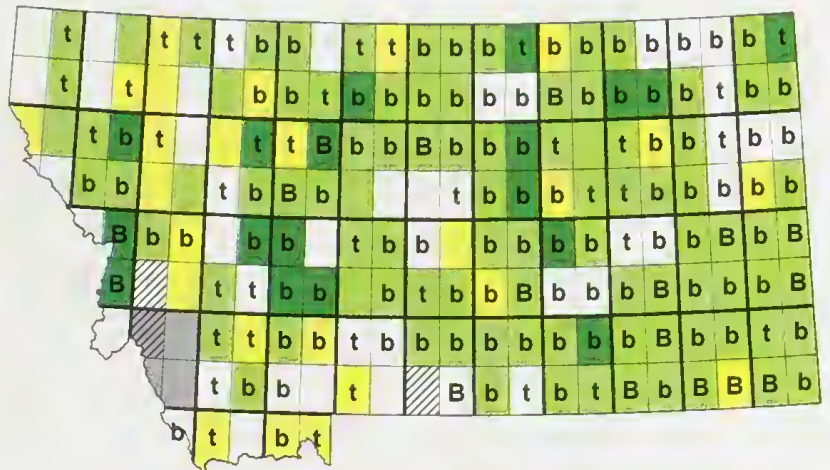


Scissor-tailed Flycatcher

Tyrannus forficatus

2003-2011 = 5 R1

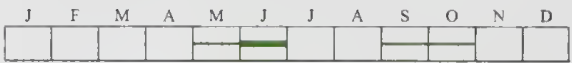

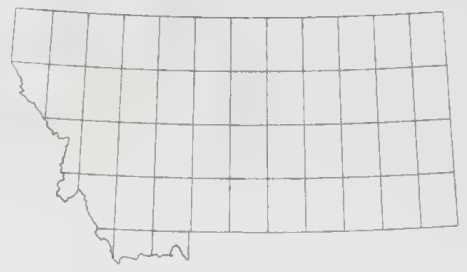

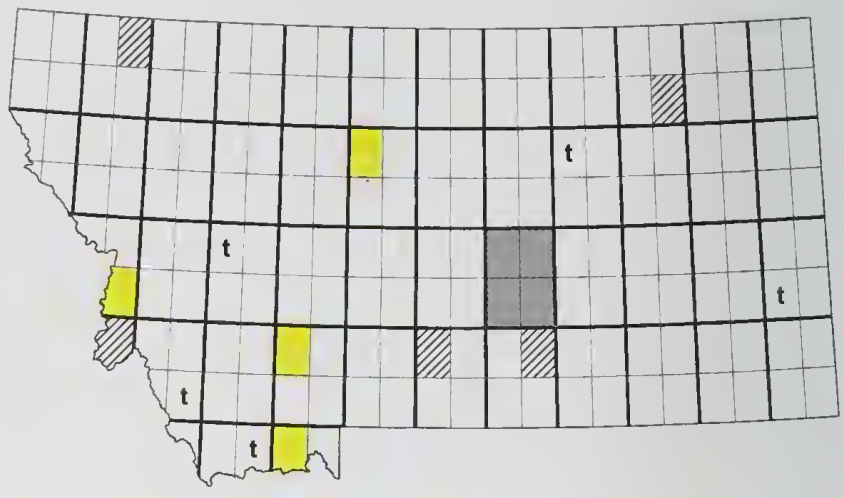

Laniidae

Loggerhead Shrike

Lanius ludovicianus

$2003-2011=508$ SOC
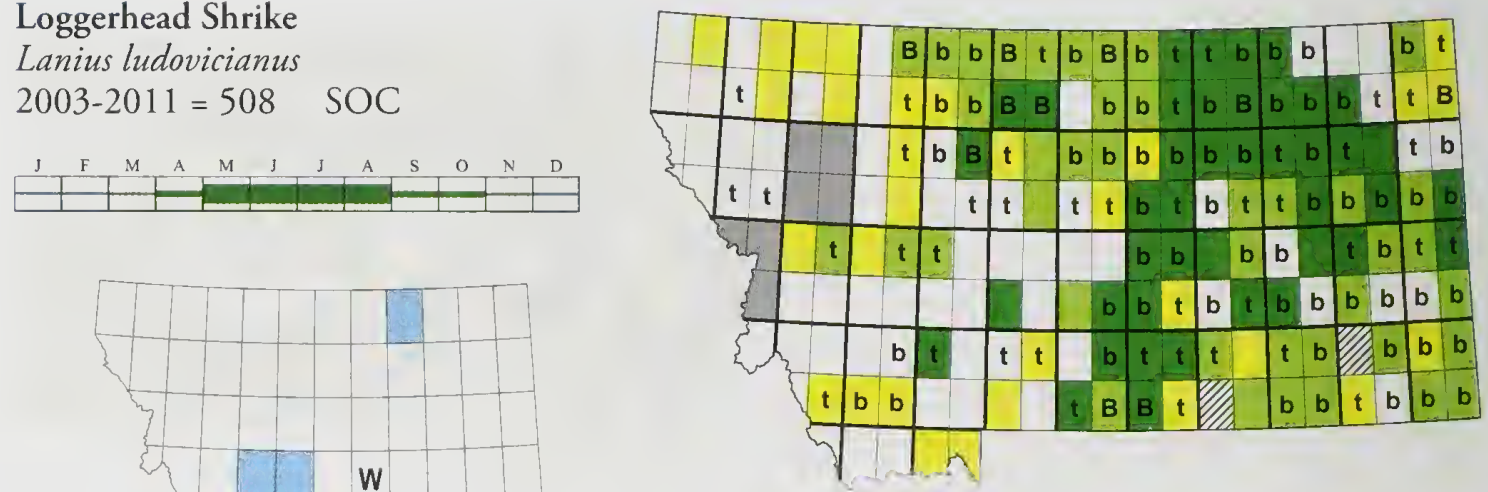

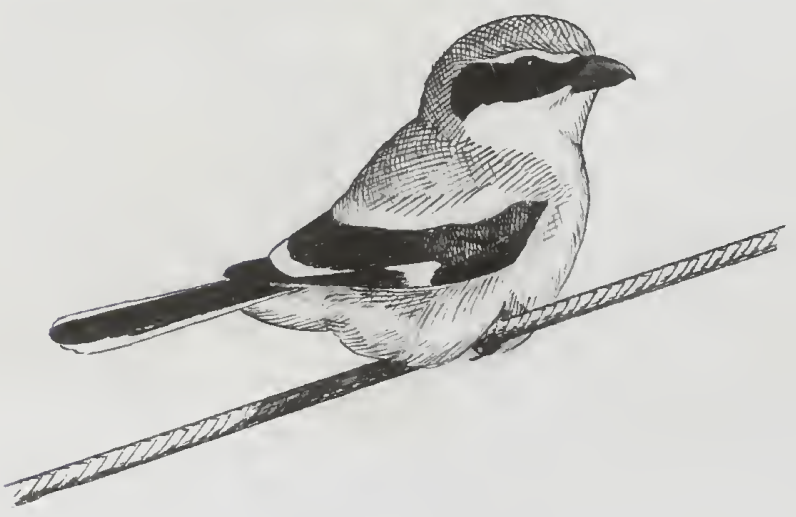


Northern Shrike

Lanius excubitor

2003-2011 = 360
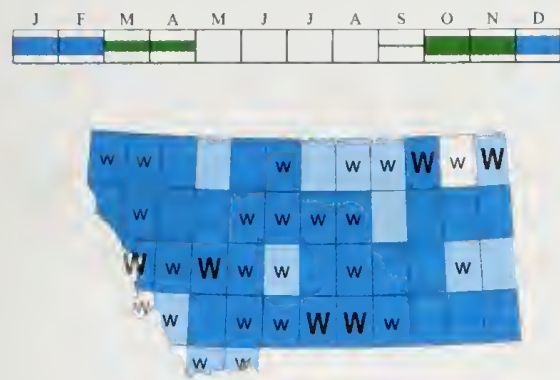

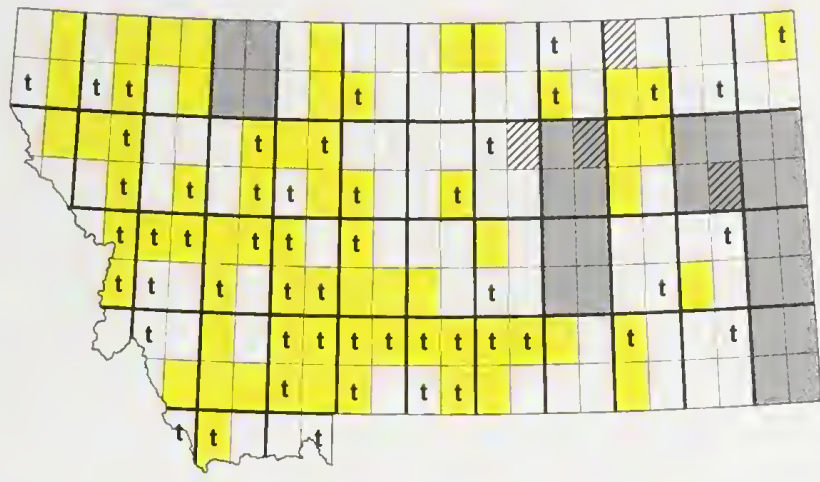

Vireonidae

White-eyed Vireo

Vireo griseus

2003-2011 = 0 R1

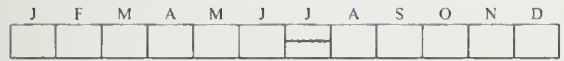

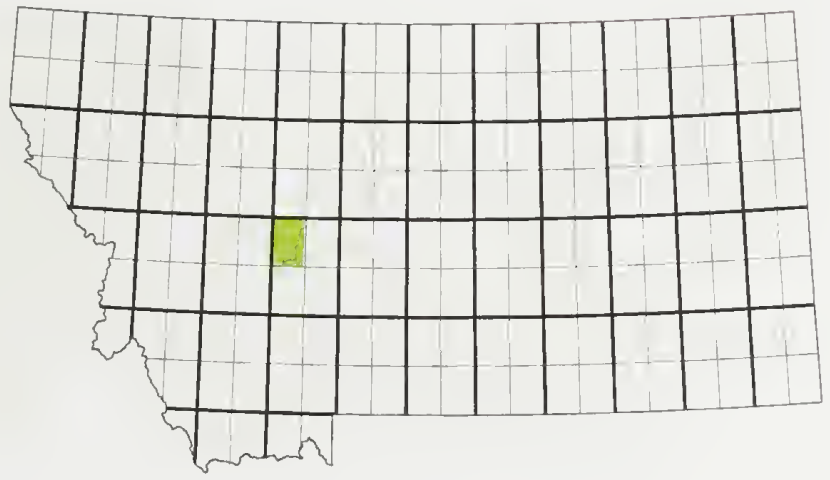

Yellow-throated Vireo

Vireo flavifrons

$2003-2011=4 \quad \mathrm{R} 1$
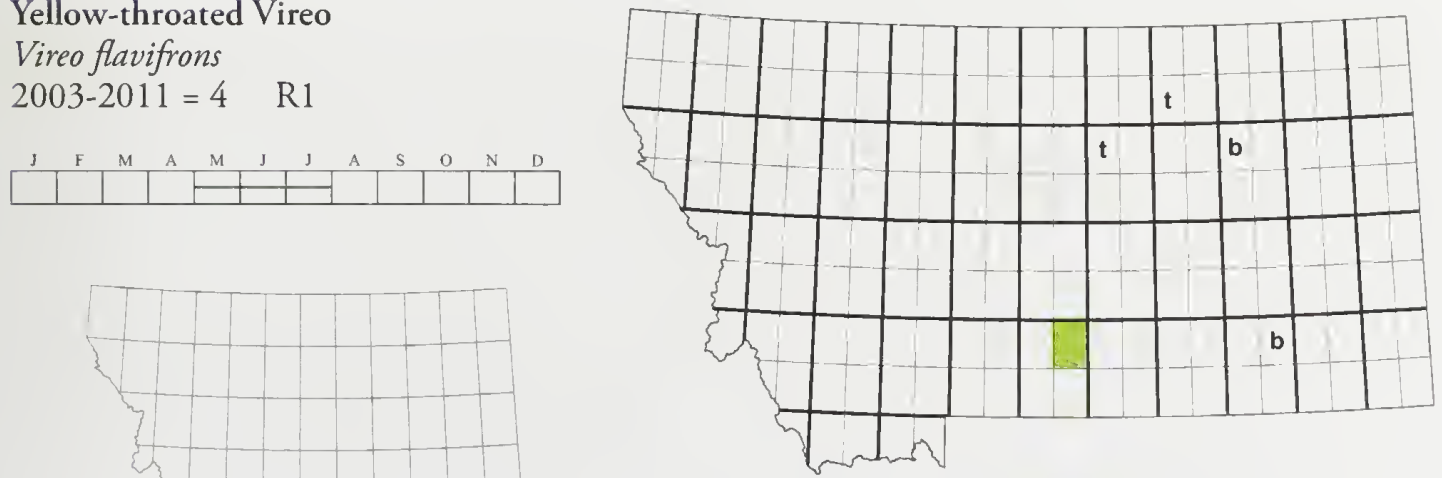
Plumbeous Vireo

Vireo plumbeus

$2003-2011=312$ SI

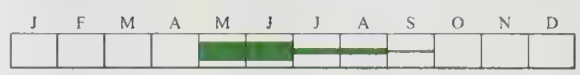

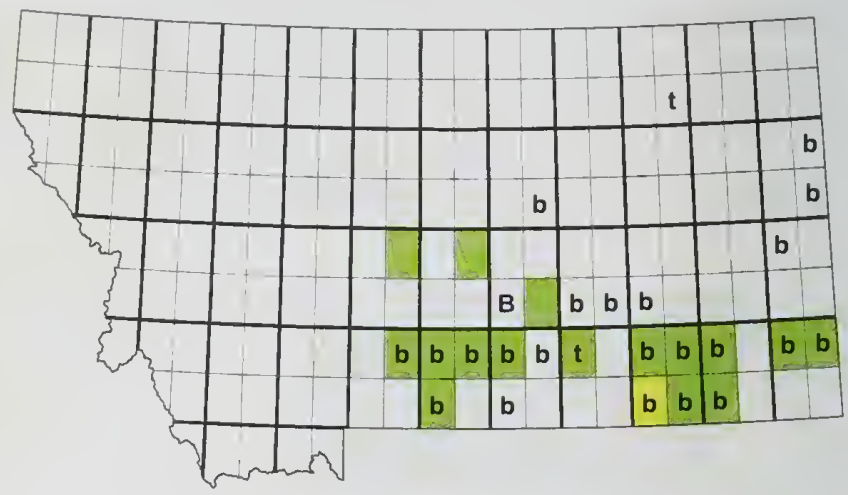

Cassin's Vireo

Vireo cassinii

2003-2011 = 1666
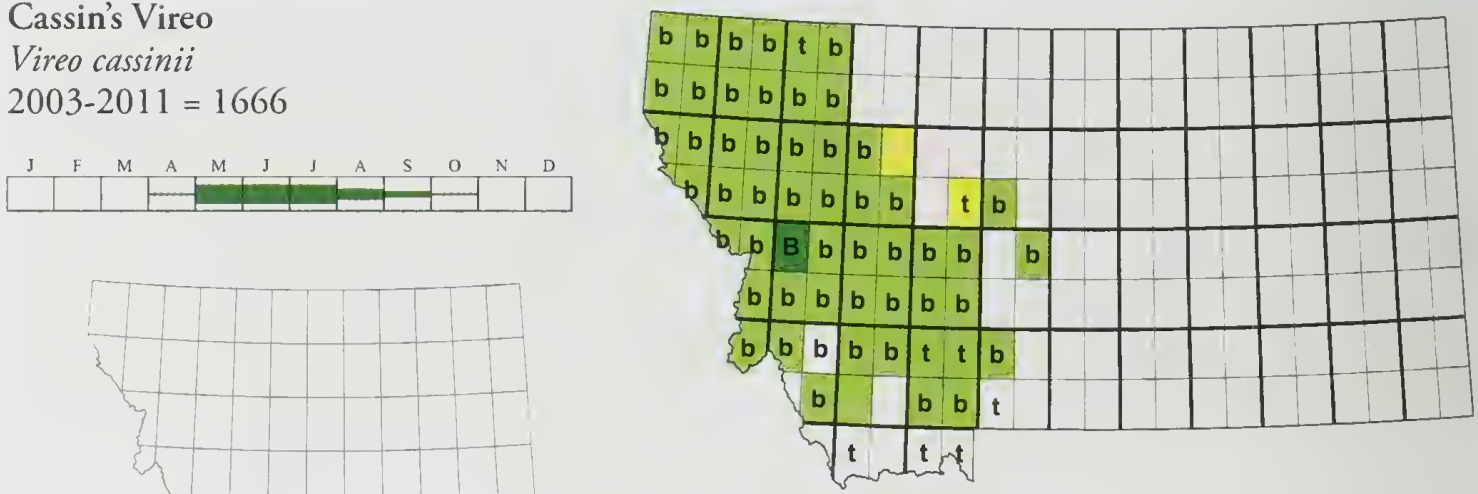

Blue-headed Vireo

Vireo solitarius

$2003-2011=13 \quad$ R2
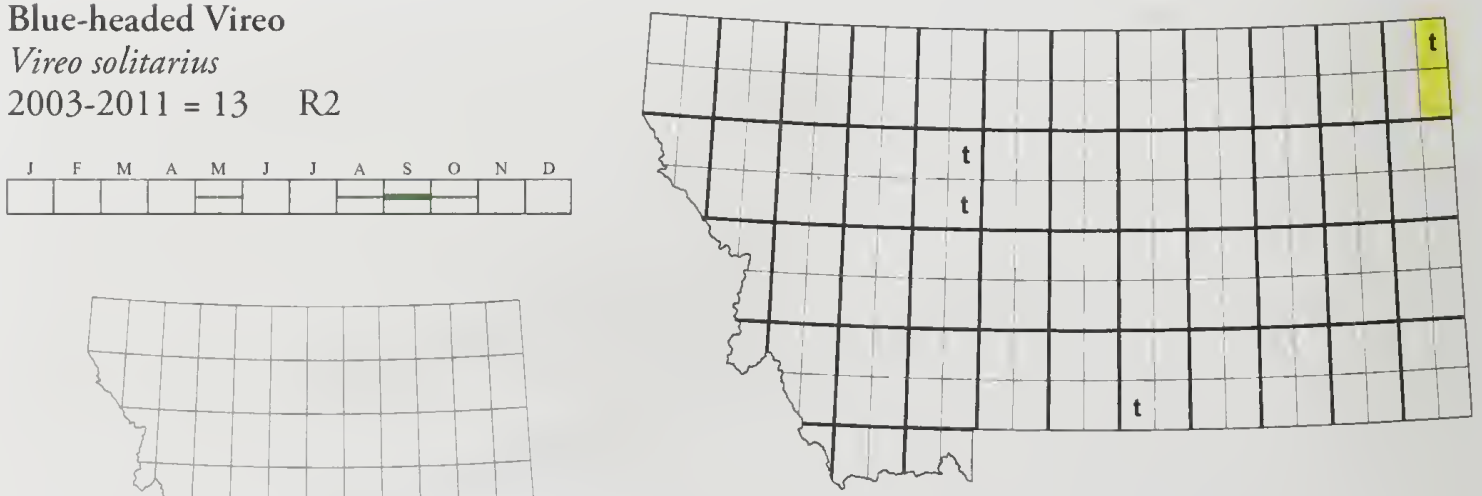
Solitary Vireo

Vireo solitarius

2003-2011 = 0
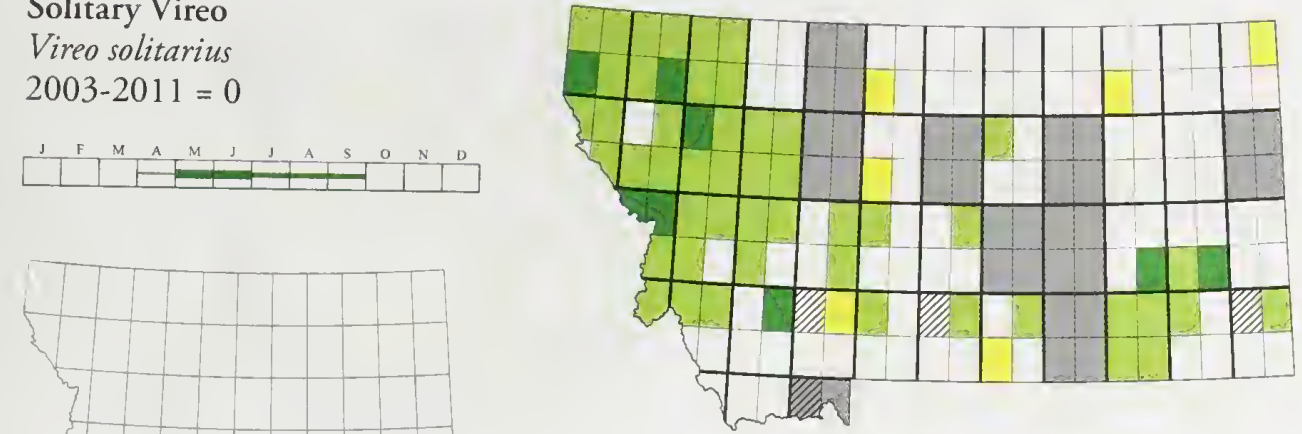

Warbling Vireo

Vireo gilvus

2003-2011 = 4918
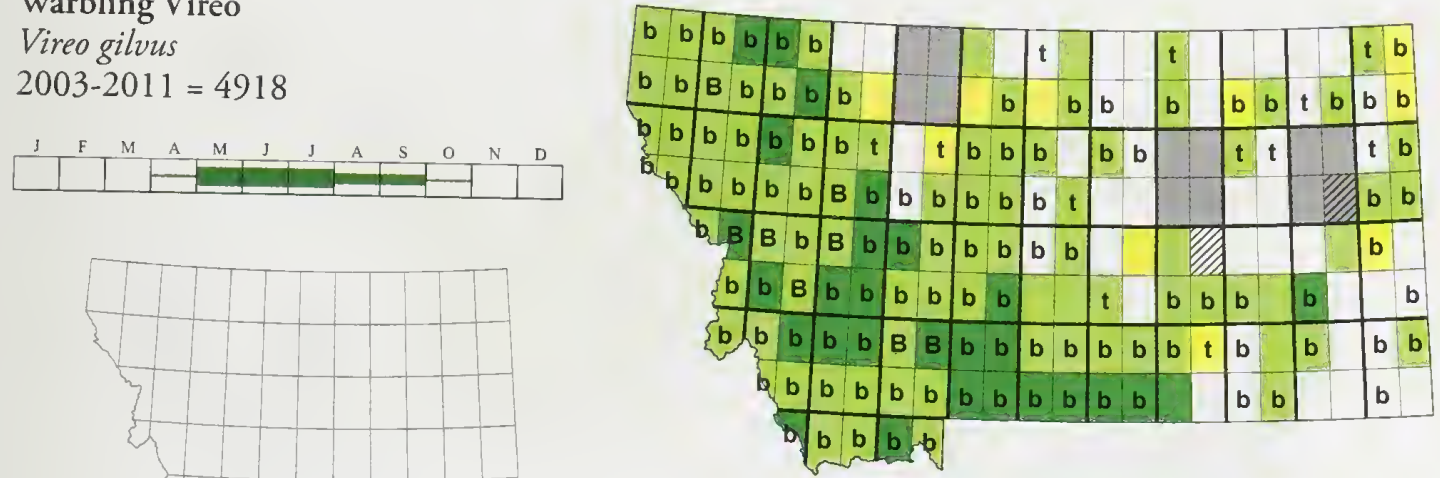

Philadelphia Vireo

Vireo philadelphicus

$2003-2011=13 \quad$ R2
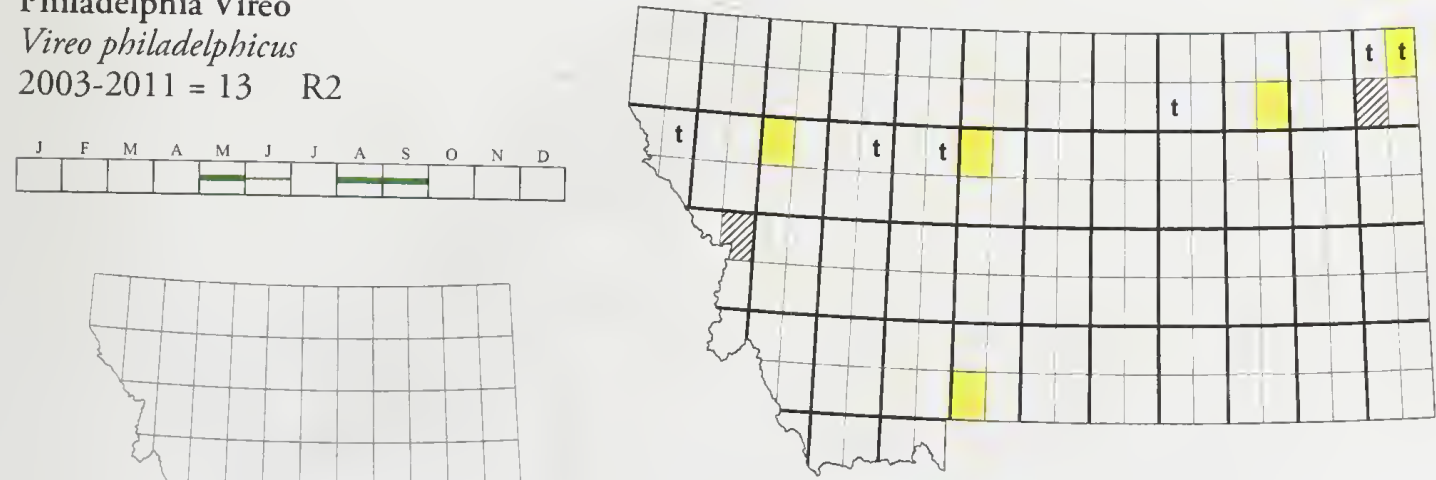
Red-eyed Vireo

Vireo olivaceus

2003-2011 $=258$

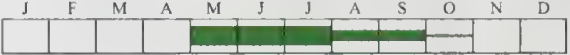

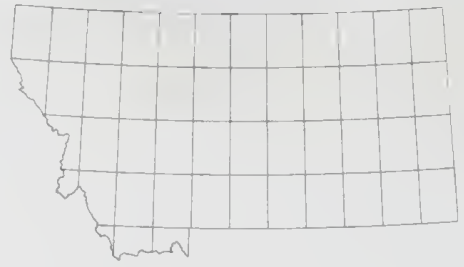

\section{Corvidae}

Gray Jay

Perisoreus canadensis

2003-2011 = 1033
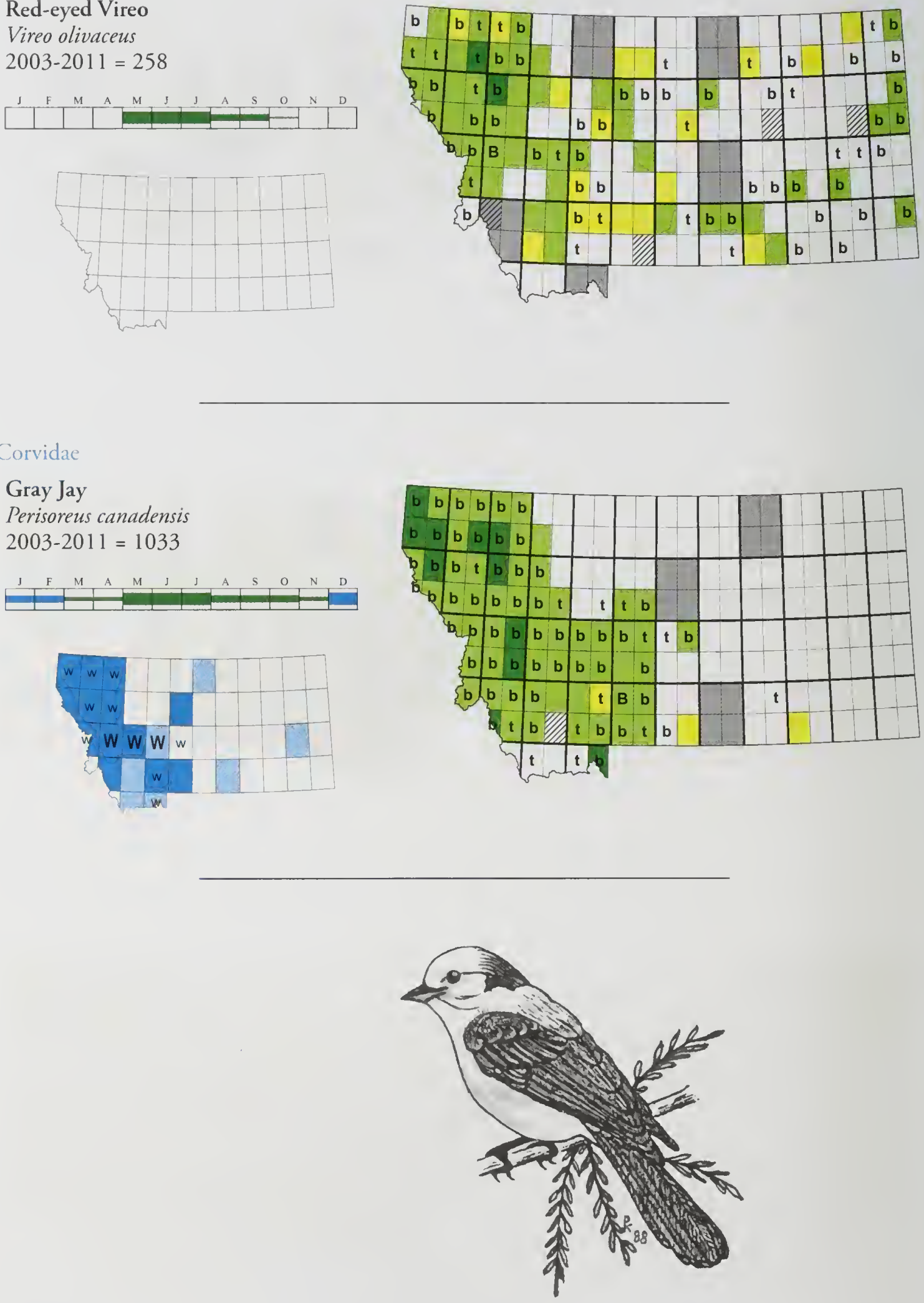
Pinyon Jay

Gymnorhinus cyanocephalus

$2003-2011=253 \quad$ SOC
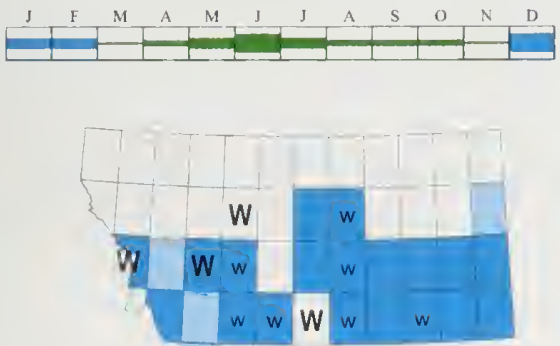

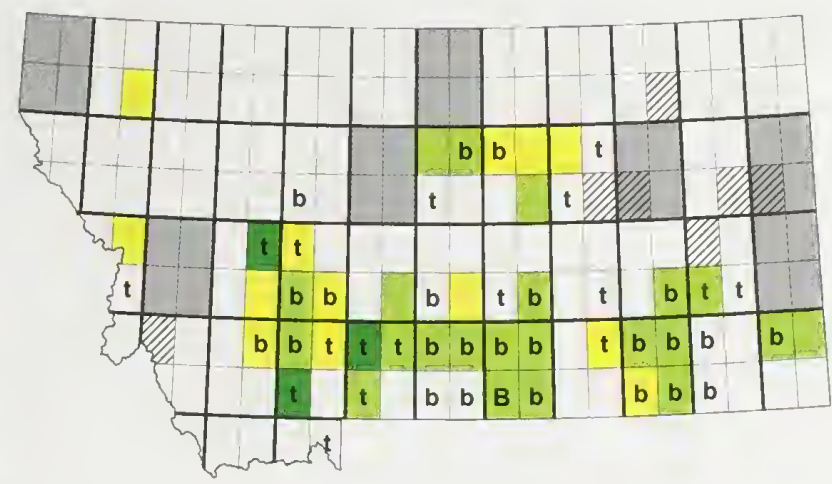

Steller's Jay

Cyanocitta stelleri

2003-2011 = 690
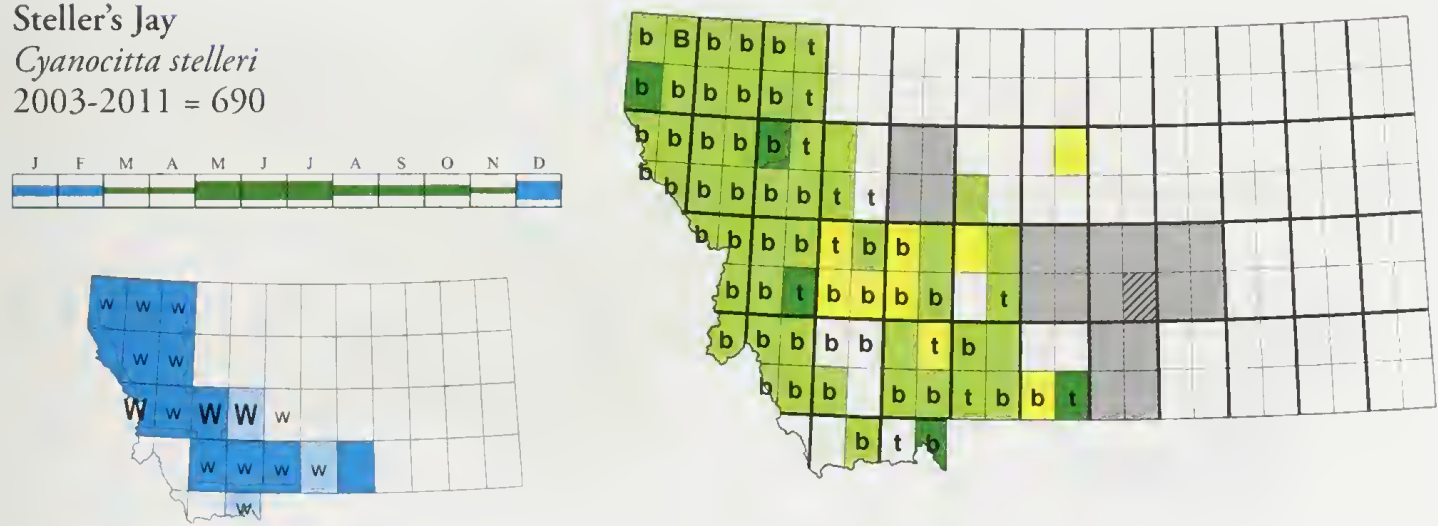

Blue Jay

Cyanocitta cristata

2003-2011 = 248
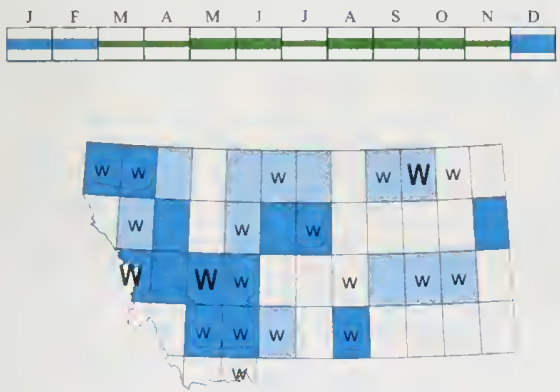
Western Scrub-Jay

Aphelocoma californica

2003-2011 = 1 R1

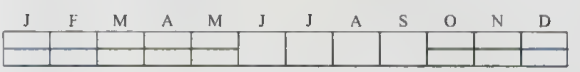
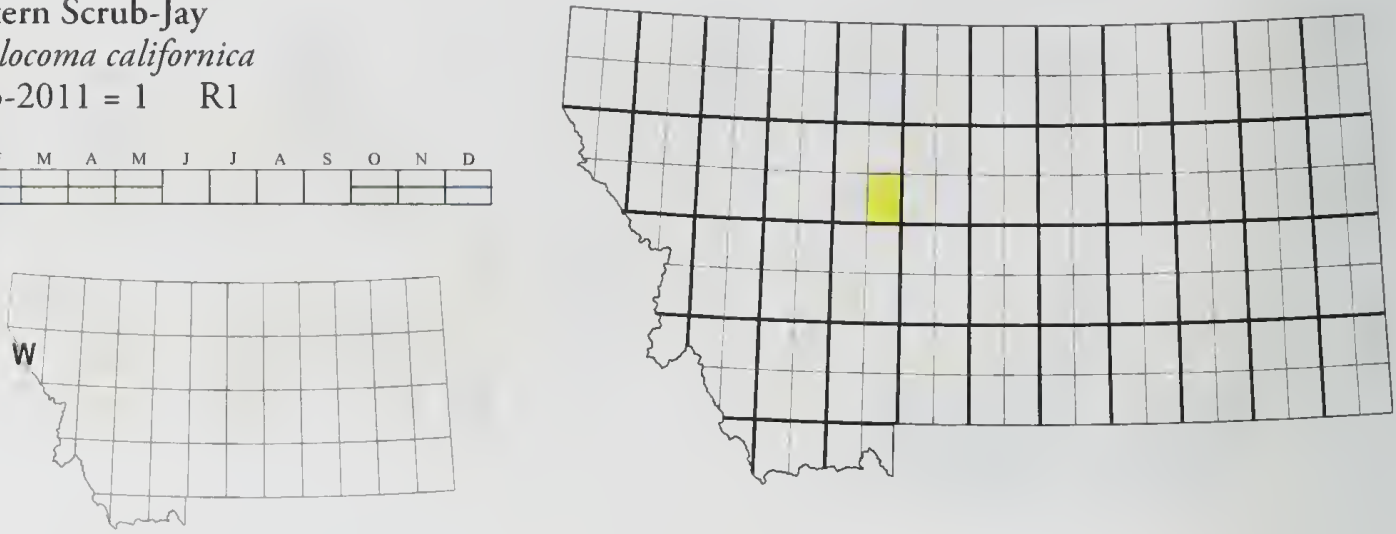

Clark's Nutcracker

Nucifraga columbiana

2003-2011 $=2107 \quad$ SOC
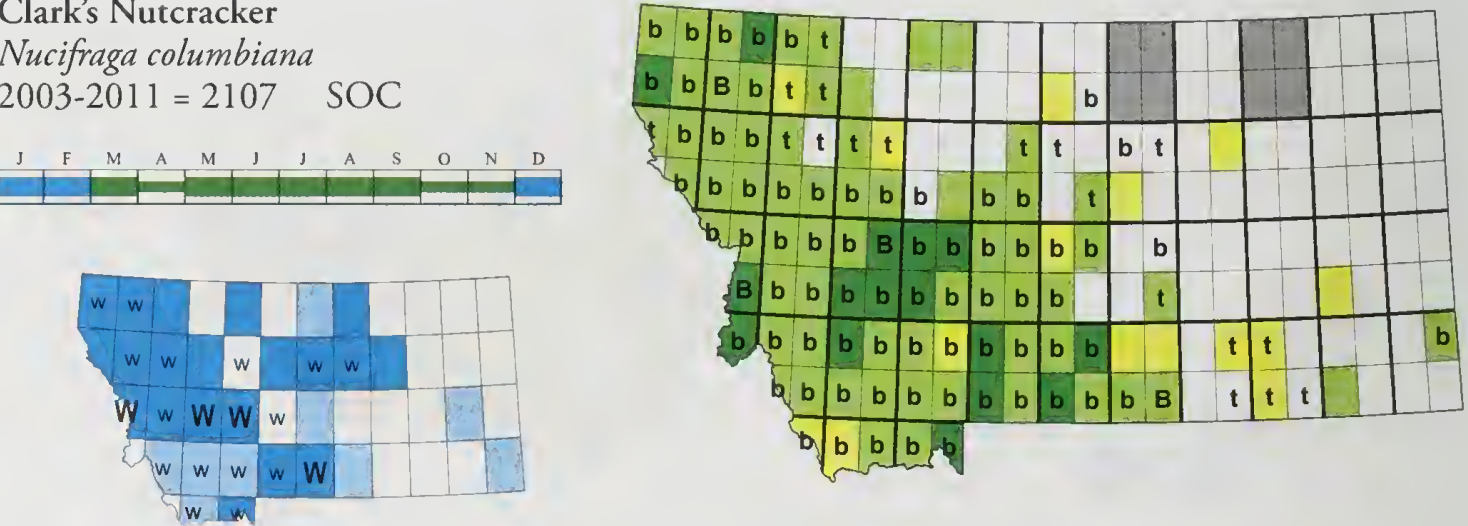

Black-billed Magpie

Pica hudsonia

2003-2011 = 4015
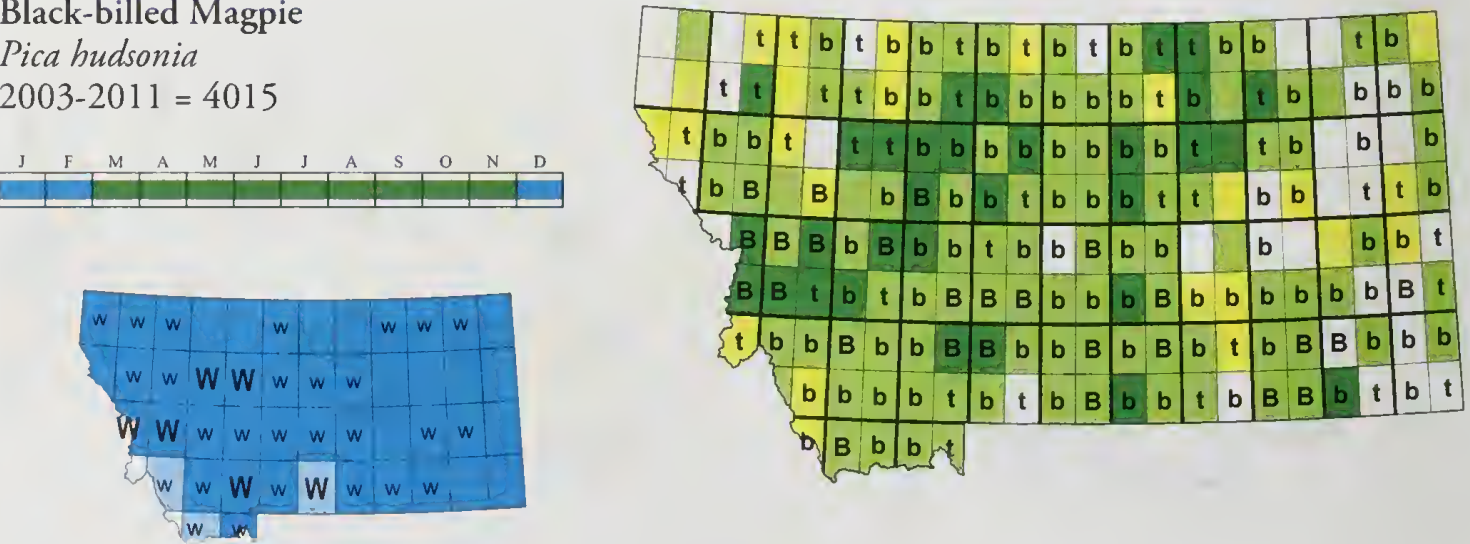
American Crow

Corvus brachyrhynchos

2003-2011 = 2924
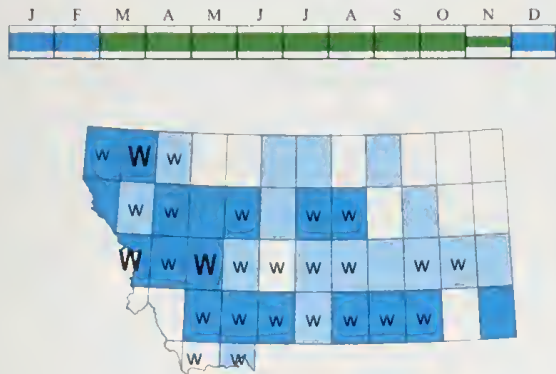

Common Raven

Corvus corax

$2003-2011=4479$
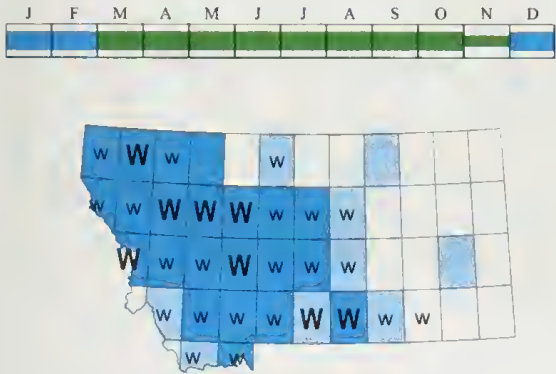
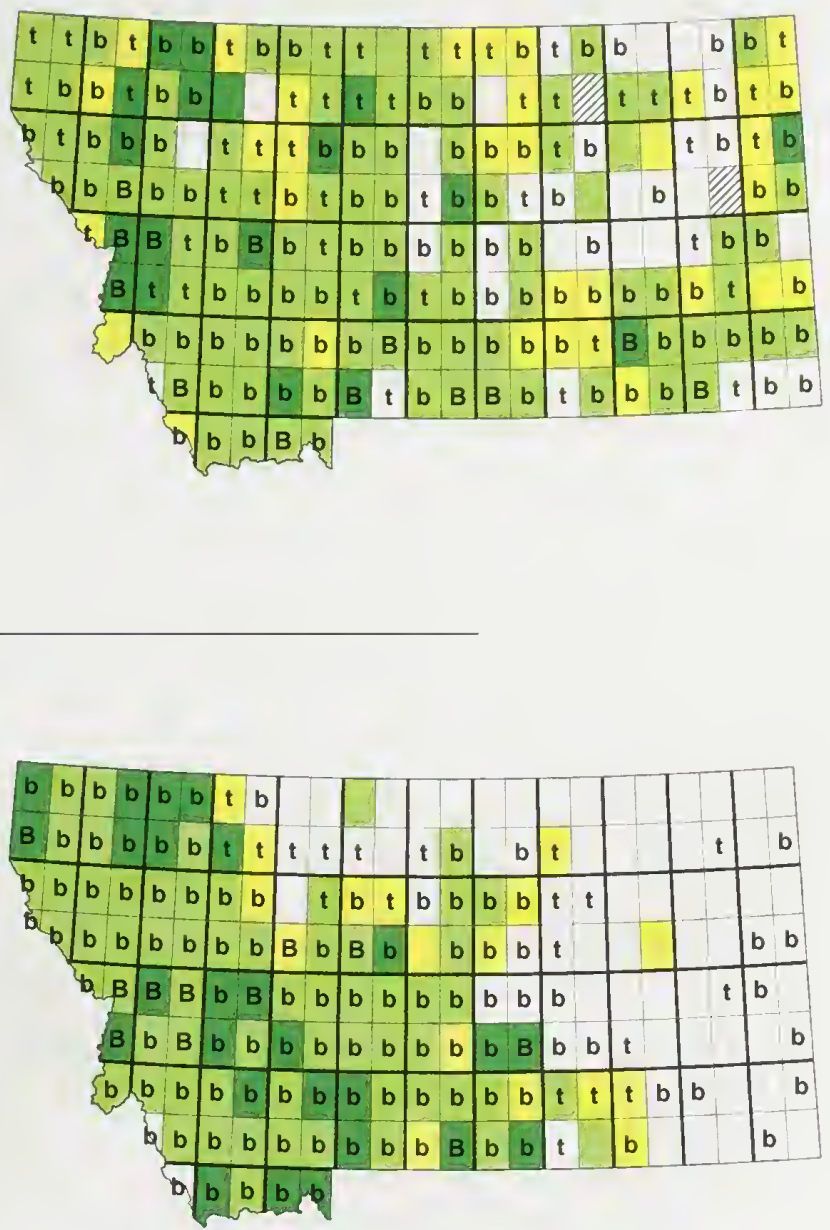

Alaudidae

Horned Lark

Eremophila alpestris

2003-2011 = 9017
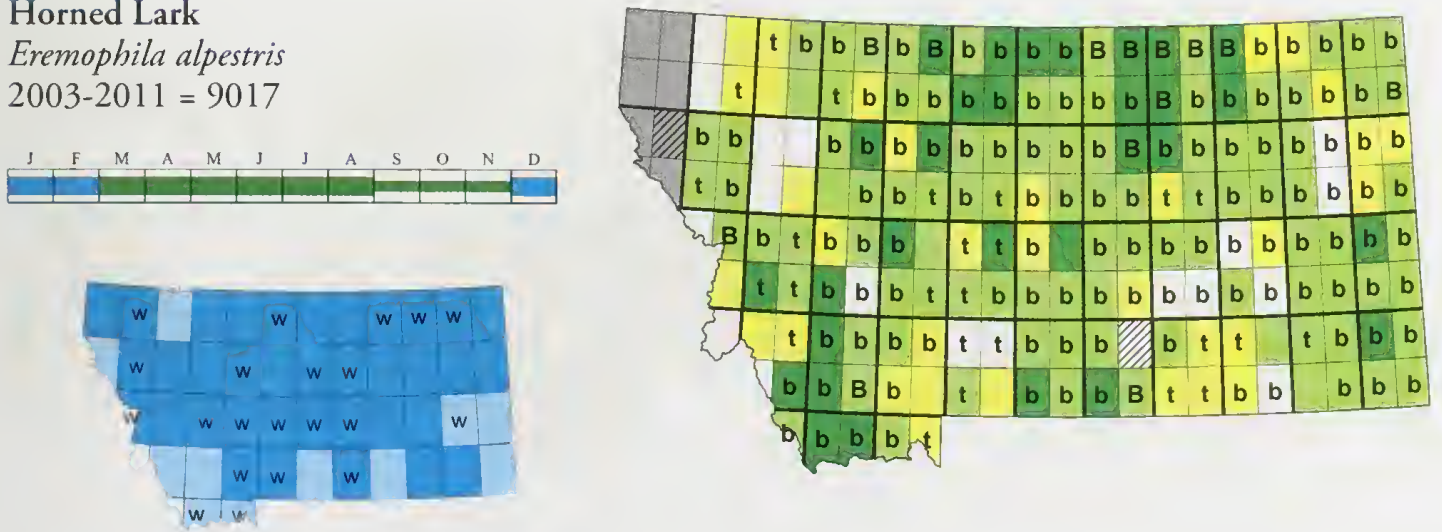
Hirundinidae

Purple Martin

Progne subis

2003-2011 = 15
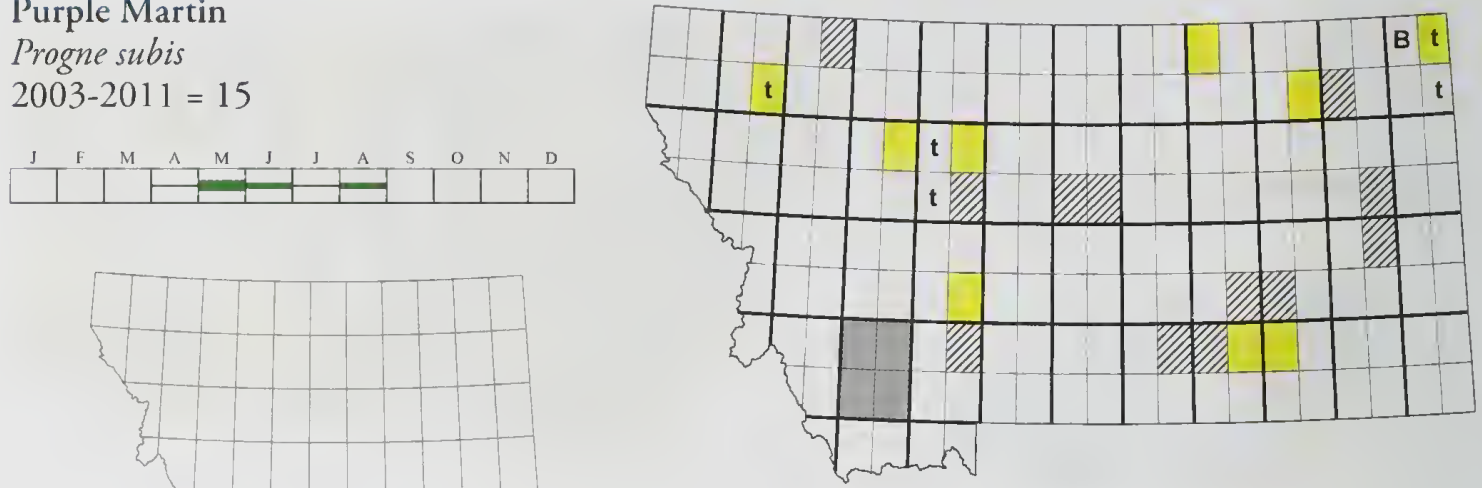

Tree Swallow

Tachycineta bicolor

2003-2011 = 2646

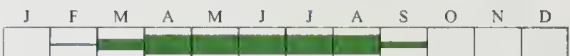

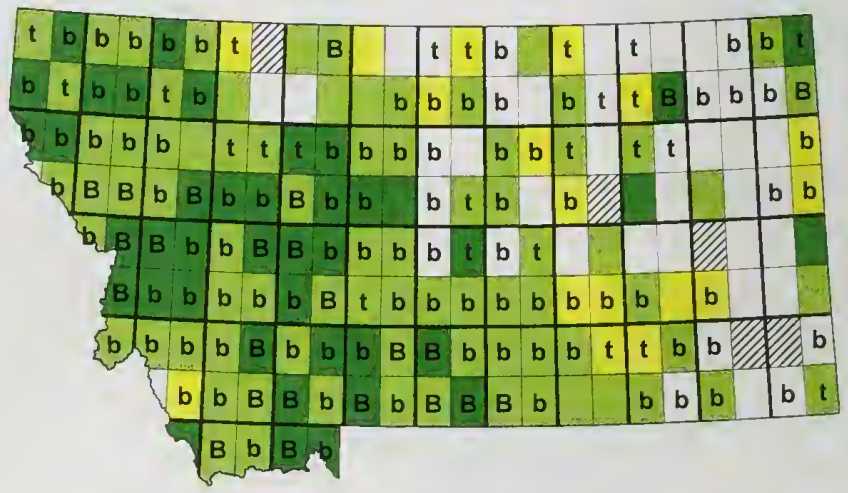

Violet-green Swallow

Tachycineta thalassina

$2003-2011=899$
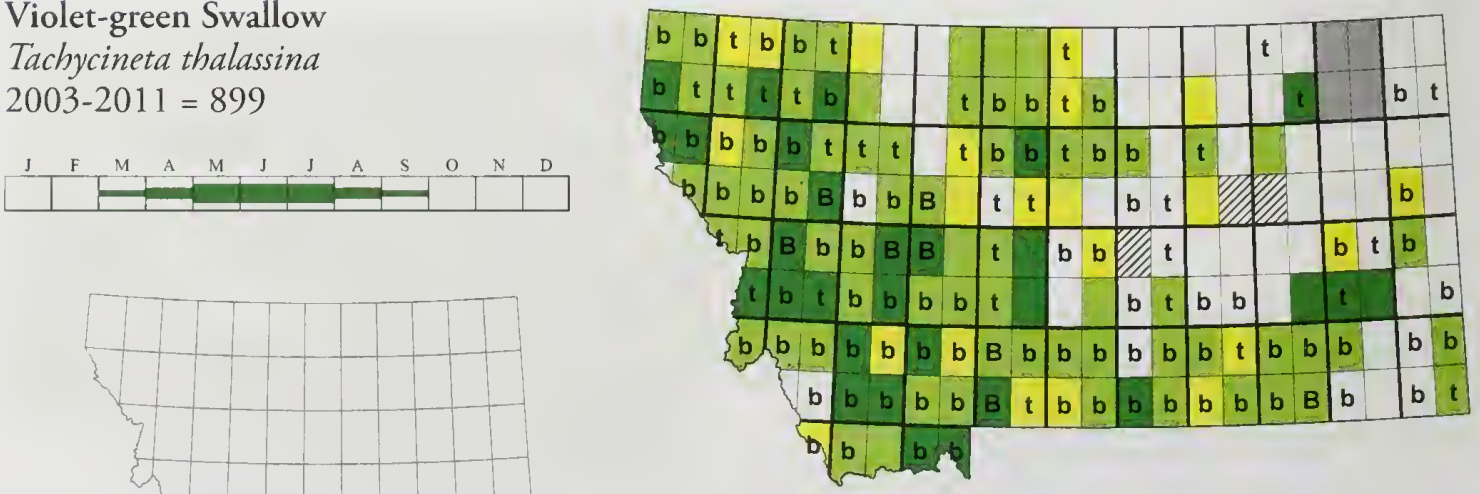
Northern Rough-winged Swallow Stelgidopteryx serripennis 2003-2011 = 688

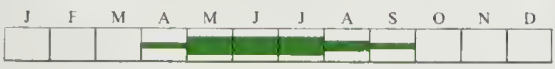

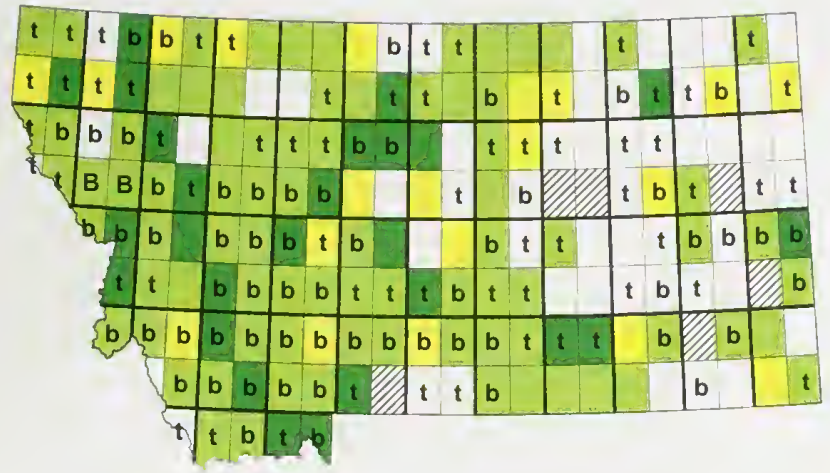

Bank Swallow

Riparia riparia

2003-2011 = 498

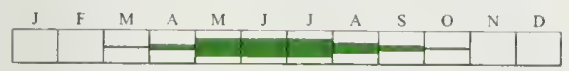

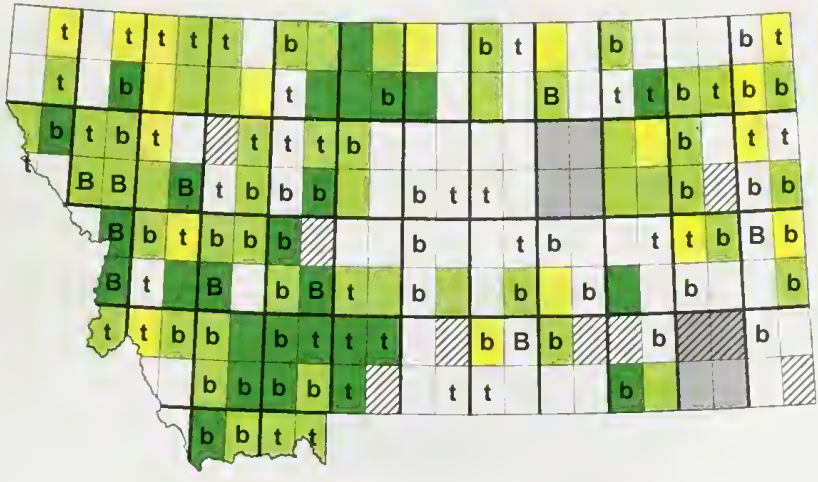

Cliff Swallow

Petrochelidon pyrrhonota

2003-2011 = 1518

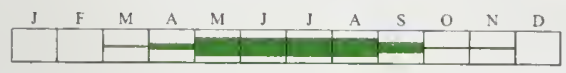

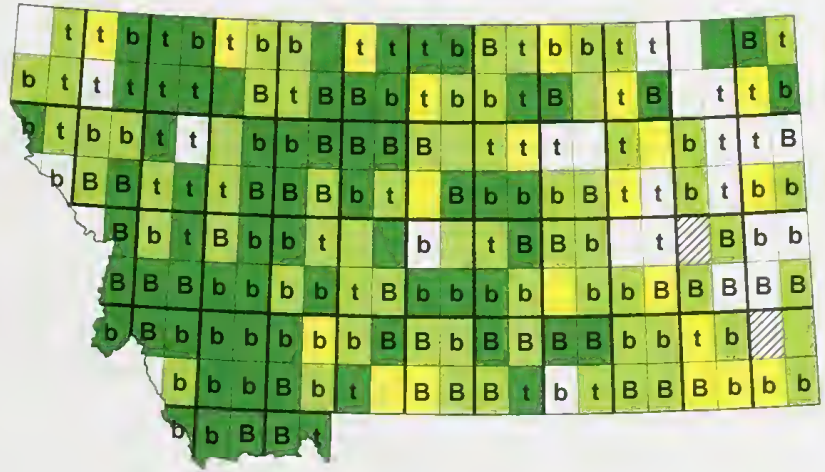


Barn Swallow

Hirundo rustica

2003-2011 = 1860

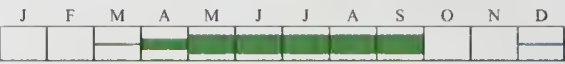

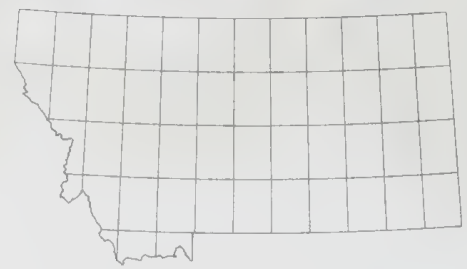

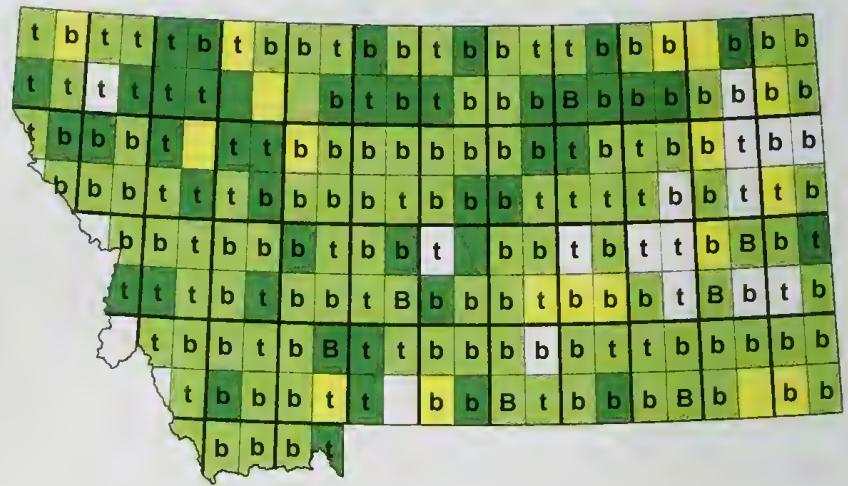

Paridae

Black-capped Chickadee

Poecile atricapillus

$2003-2011=4061$
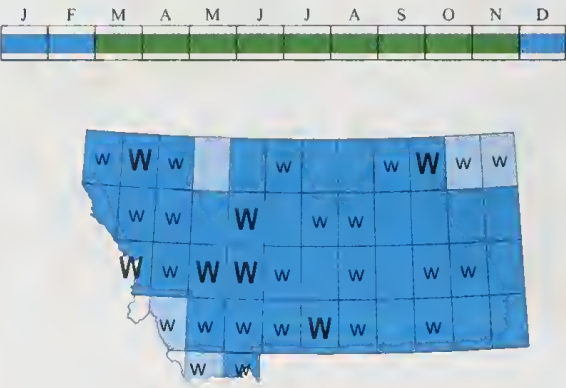

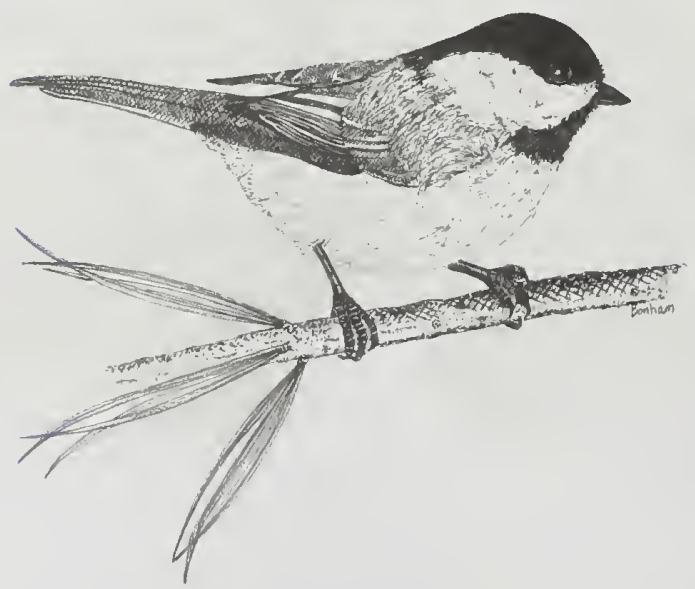


Mountain Chickadee

Poecile gambeli

2003-2011 = 5235
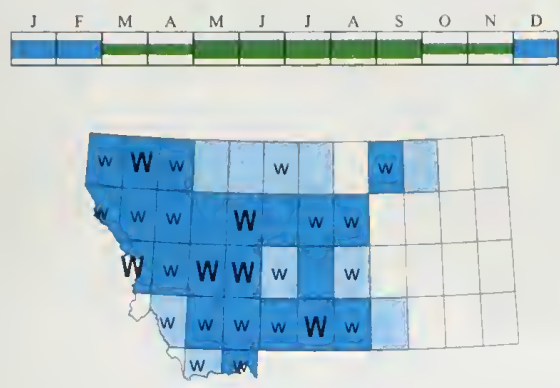

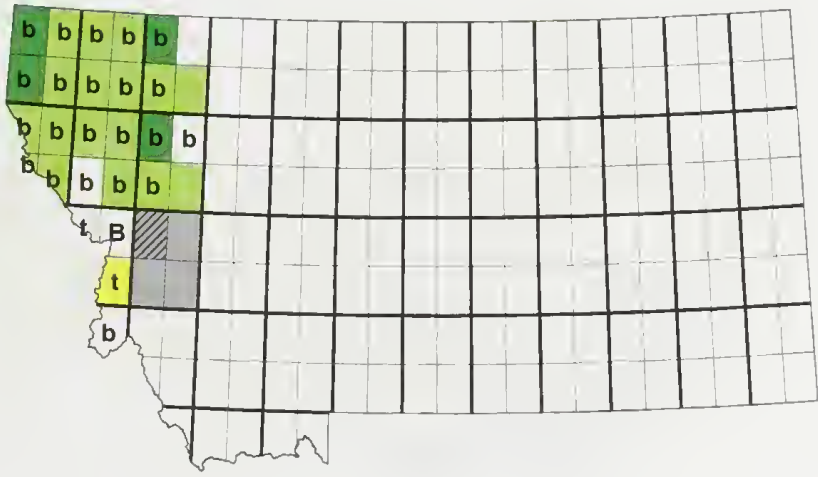

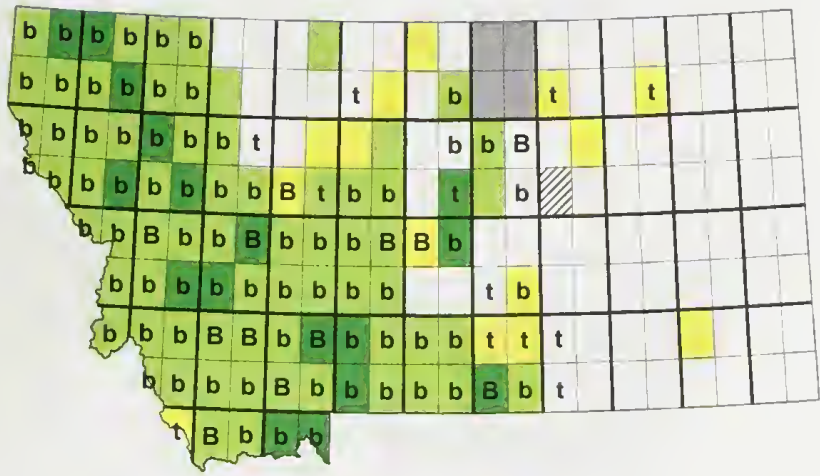

Chestnut-backed Chickadee

Poecile rufescens

2003-2011 = 328
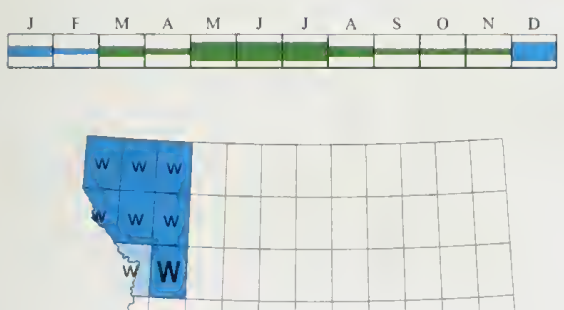
Red-breasted Nuthatch

Sitta canadensis

2003-2011 = 7281
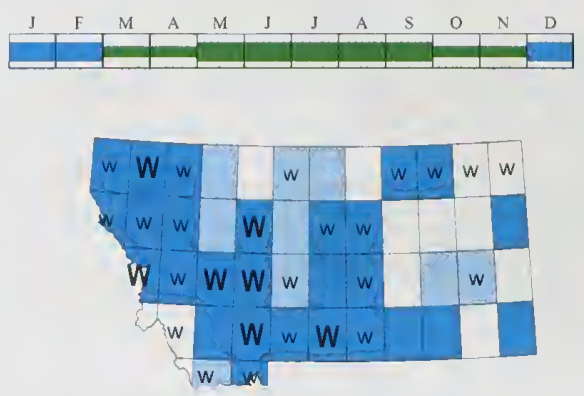

White-breasted Nuthatch

Sitta carolinensis

2003-2011 $=722$
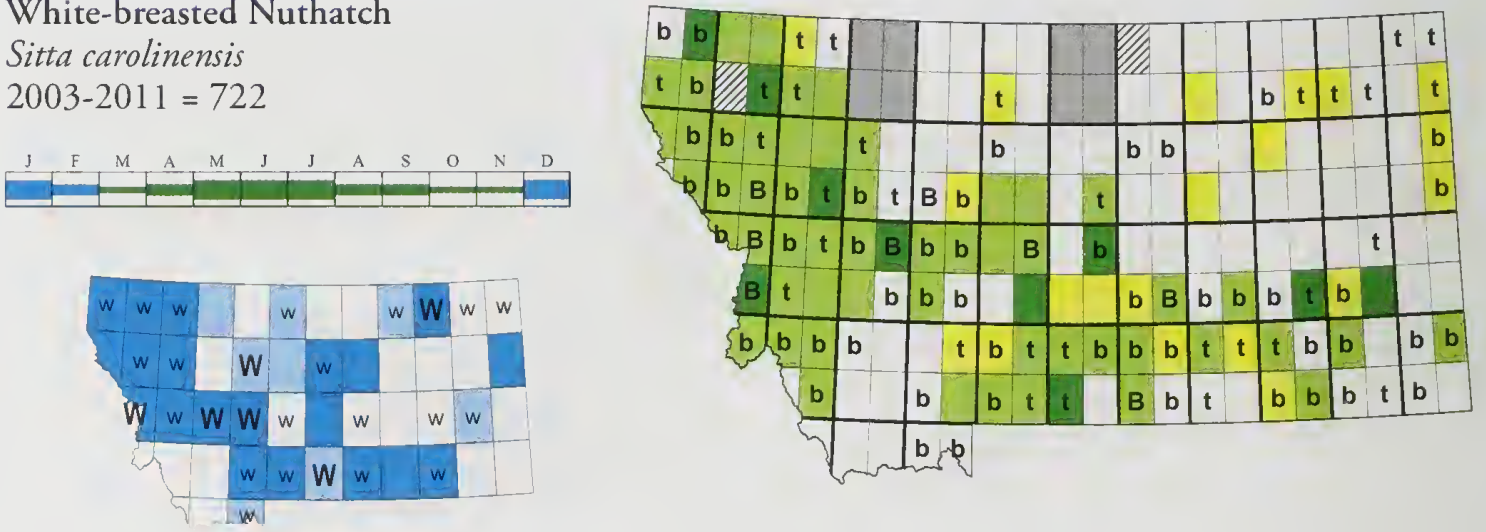

Pygmy Nuthatch

Sitta pygmaea

2003-2011 = 242
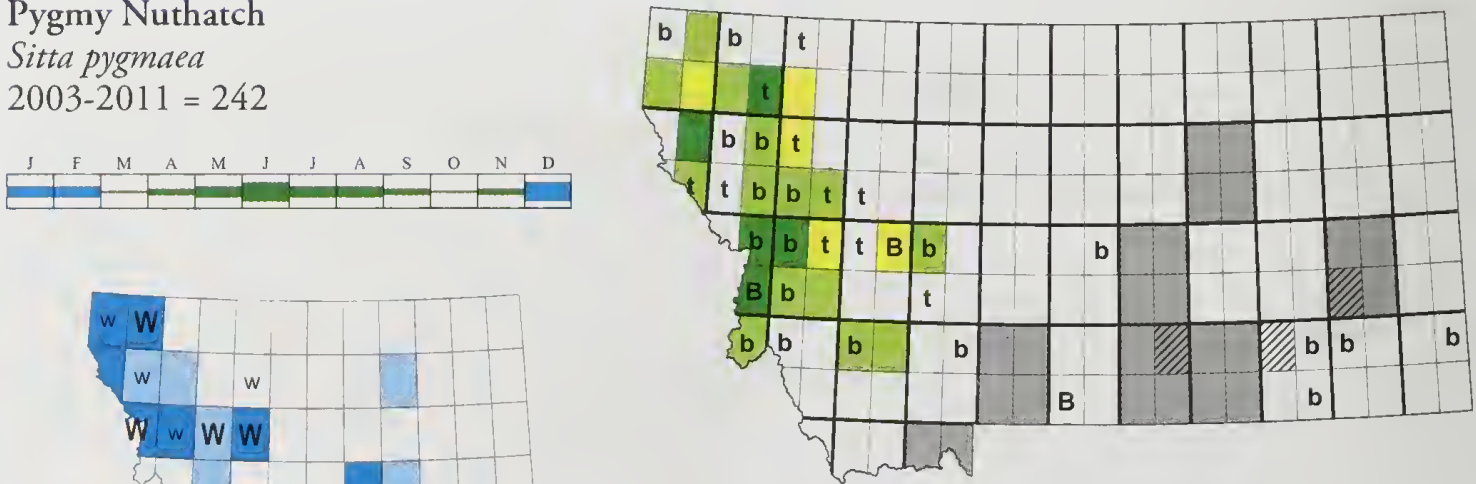
Certhiidae

\section{Brown Creeper}

Certhia americana

2003-2011 = 790 SOC
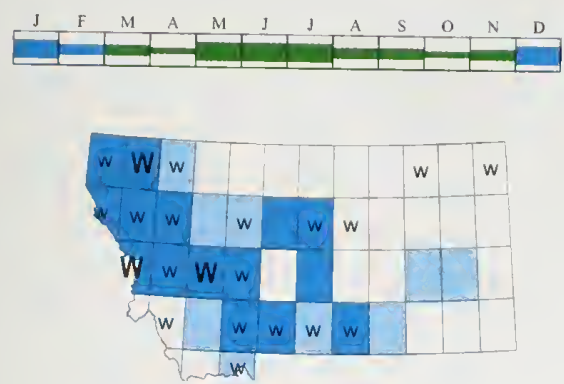

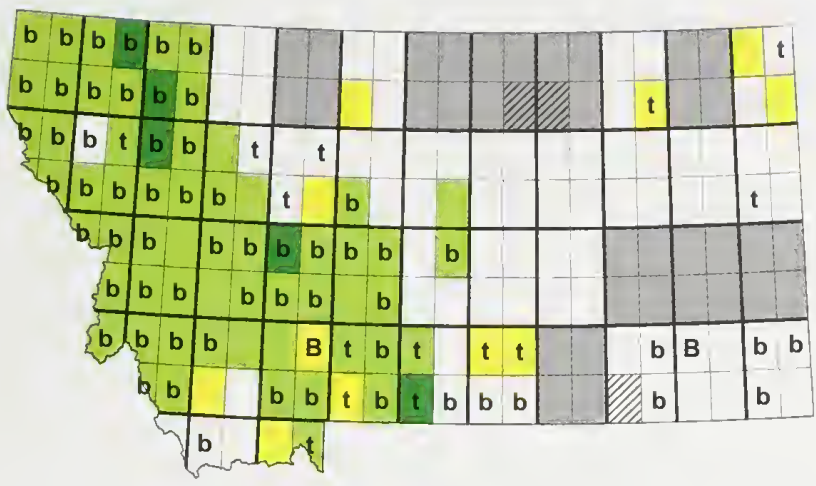

Troglodytidae

Rock Wren

Salpinctes obsoletus

2003-2011 = 1703

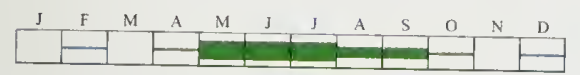

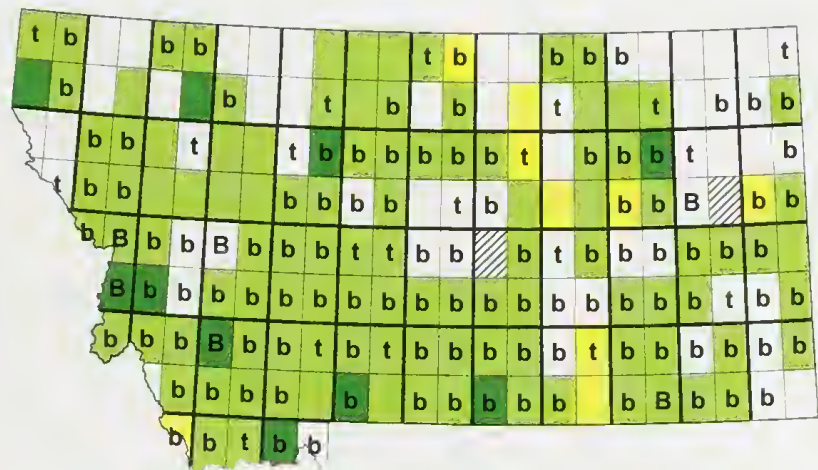

Canyon Wren

Catherpes mexicanus

$2003-2011=101$

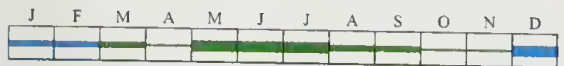

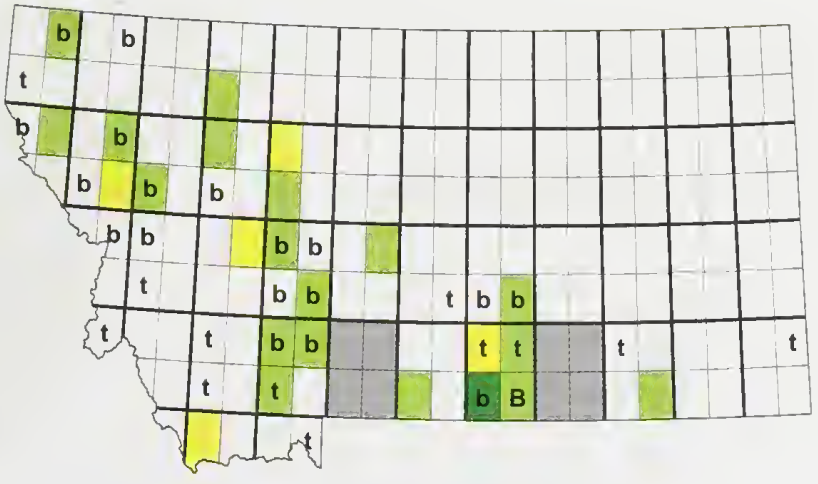


Carolina Wren

Thryothorus ludovicianus

2003-2011 = 1 R1

\begin{tabular}{|l|l|l|l|l|l|l|l|l|l|l|l|l|l}
\hline & $\mathrm{F}$ & $\mathrm{M}$ & $\mathrm{A}$ & $\mathrm{M}$ & $\mathrm{J}$ & $\mathrm{J}$ & $\mathrm{A}$ & $\mathrm{S}$ & $\mathrm{O}$ & $\mathrm{N}$ & $\mathrm{D}$ \\
\hline & & & & & & & & & & & & \\
\hline
\end{tabular}
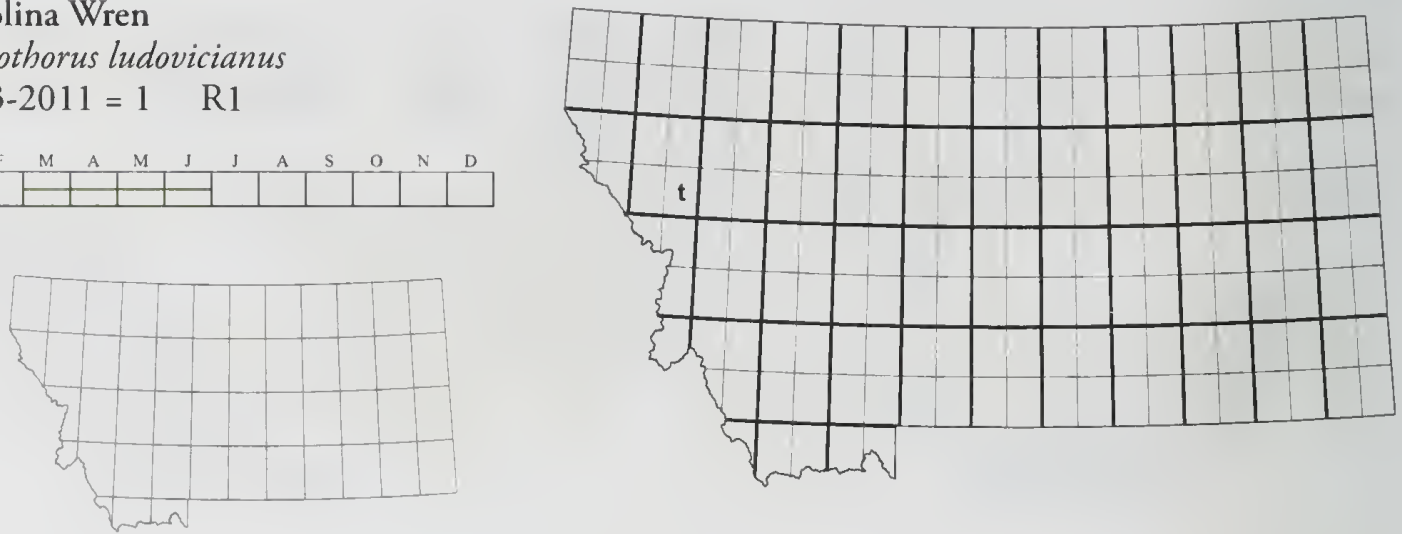

Bewick's Wren

Thryomanes bewickii

2003-2011 = 9 R1

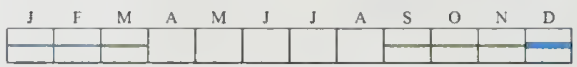
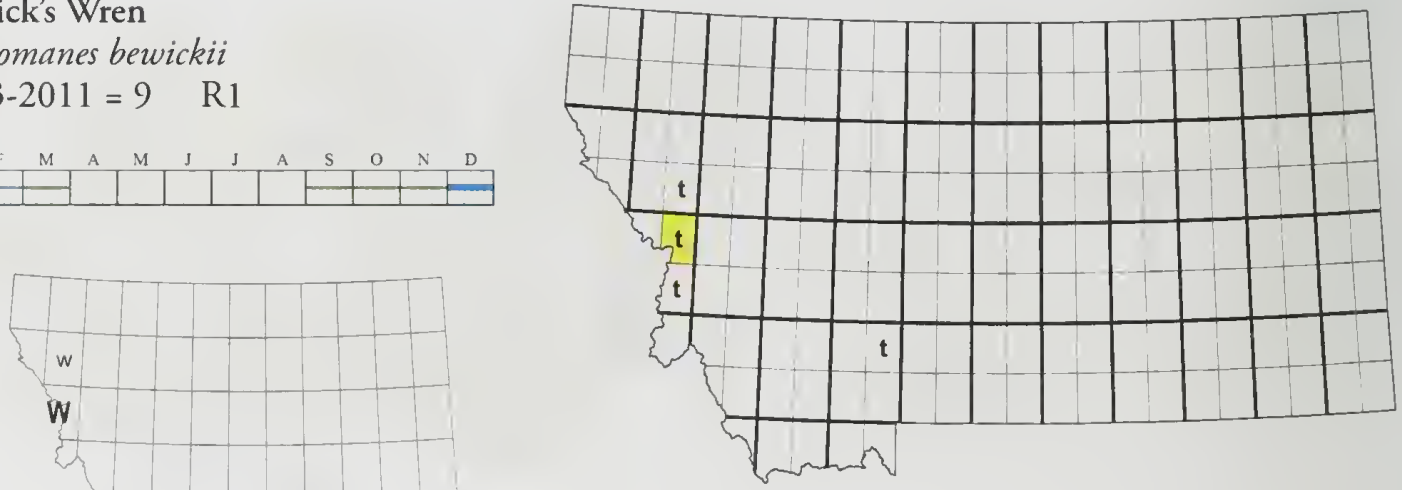

House Wren

Troglodytes aedon

$2003-2011=4255$
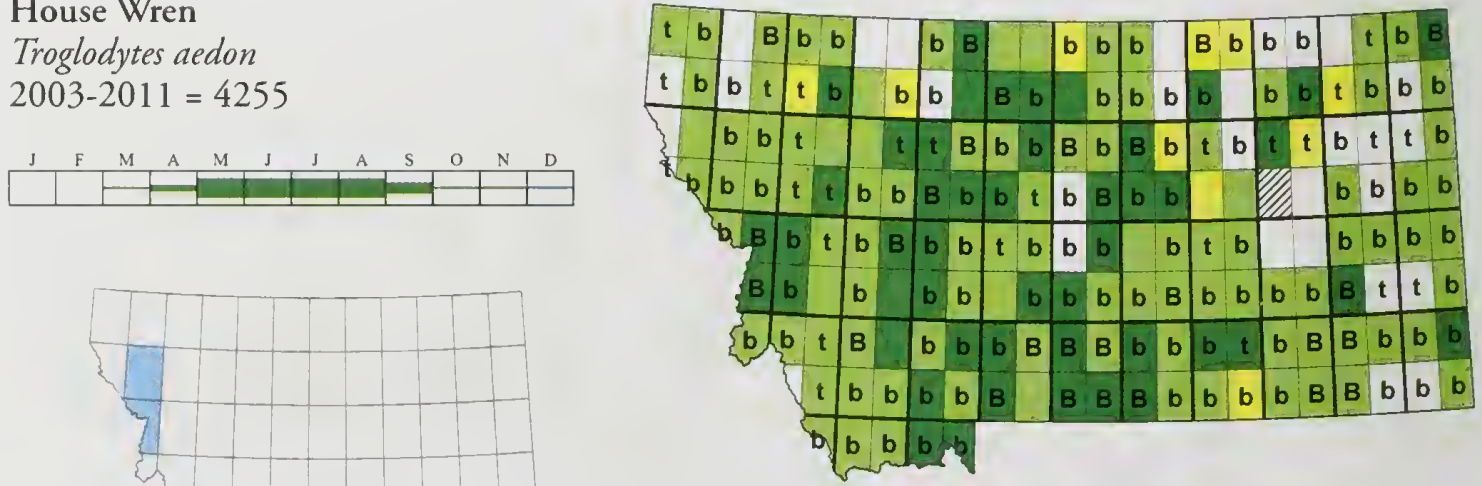


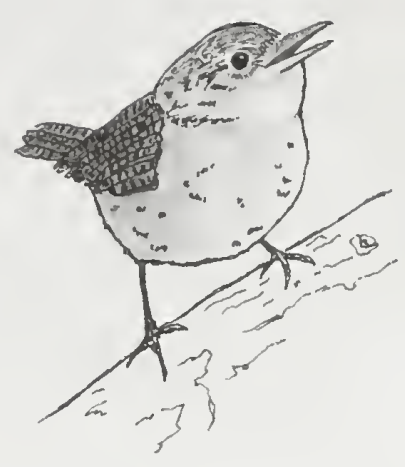

Pacific Wren

Troglodytes pacificus

$2003-2011=748 \quad$ SOC
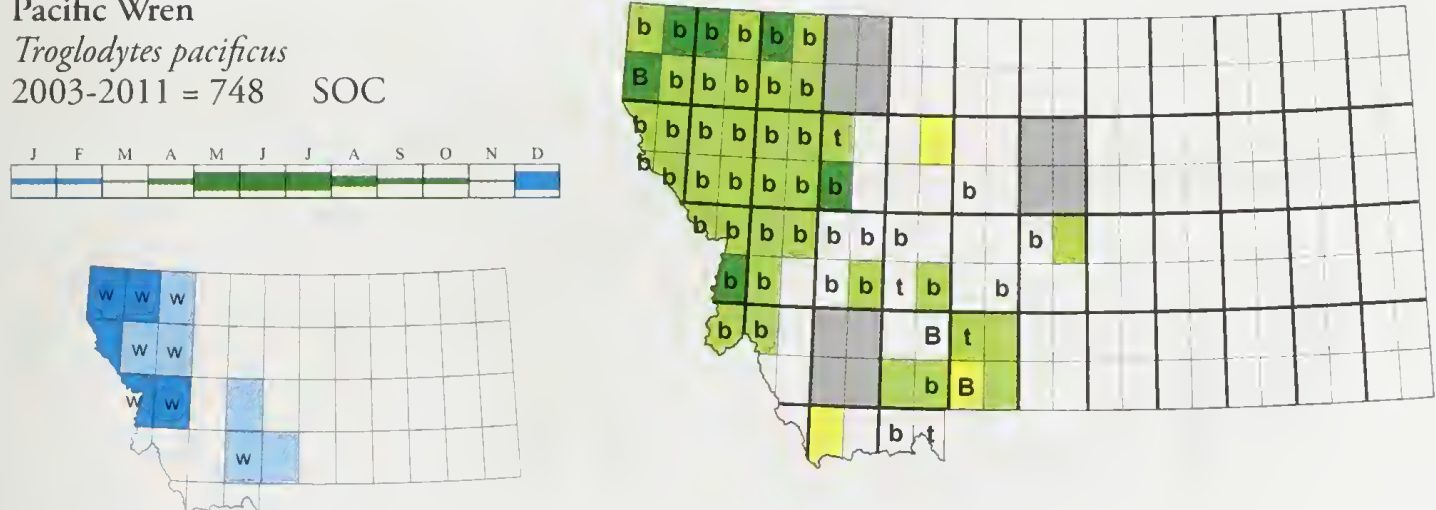

Winter Wren

Troglodytes troglodytes

2003-2011 = 1 R1
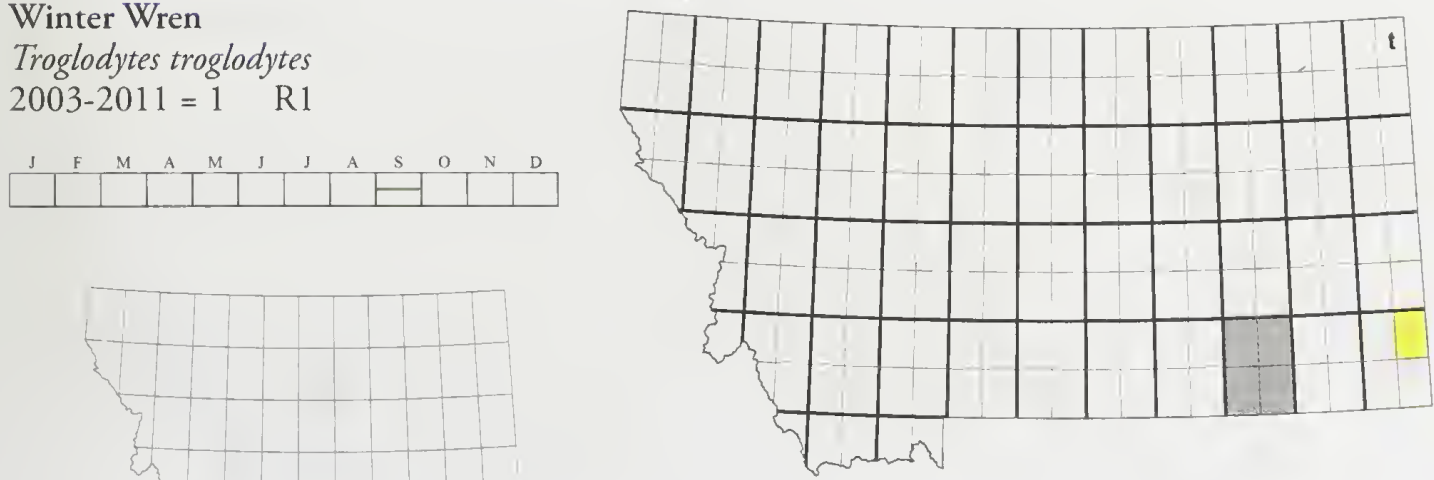
Sedge Wren

Cistothorus platensis

$2003-2011=12 \quad$ SOC R2

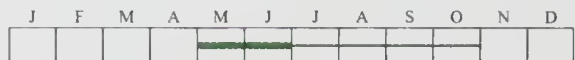
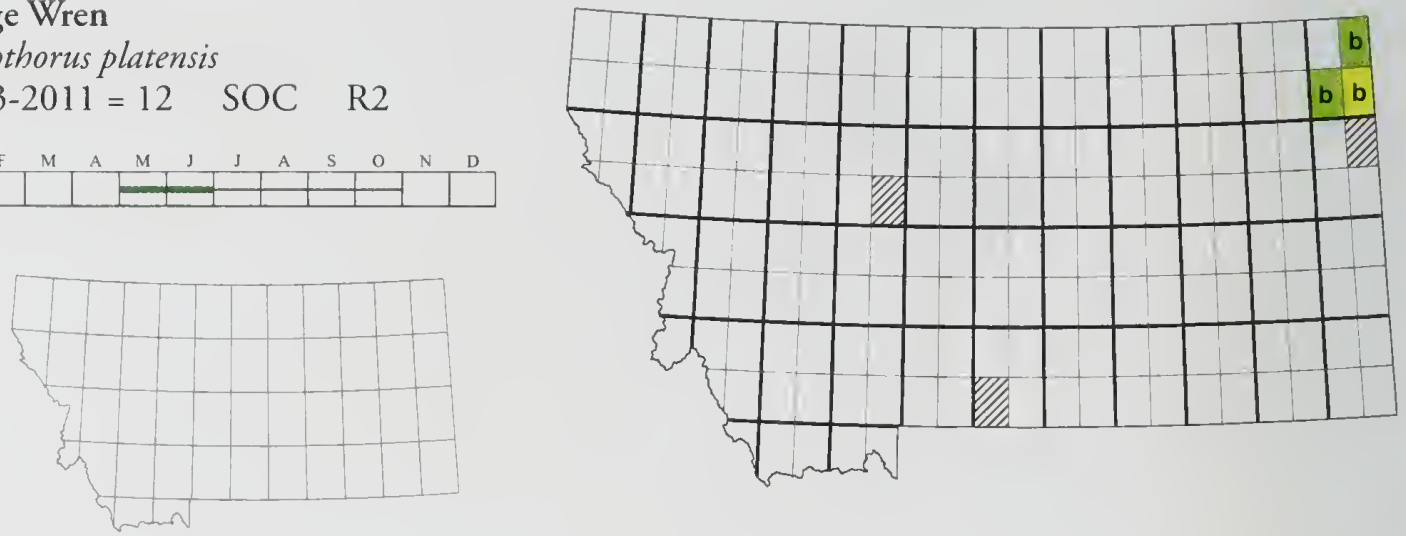

Marsh Wren

Cistothorus palustris

$2003-2011=686$
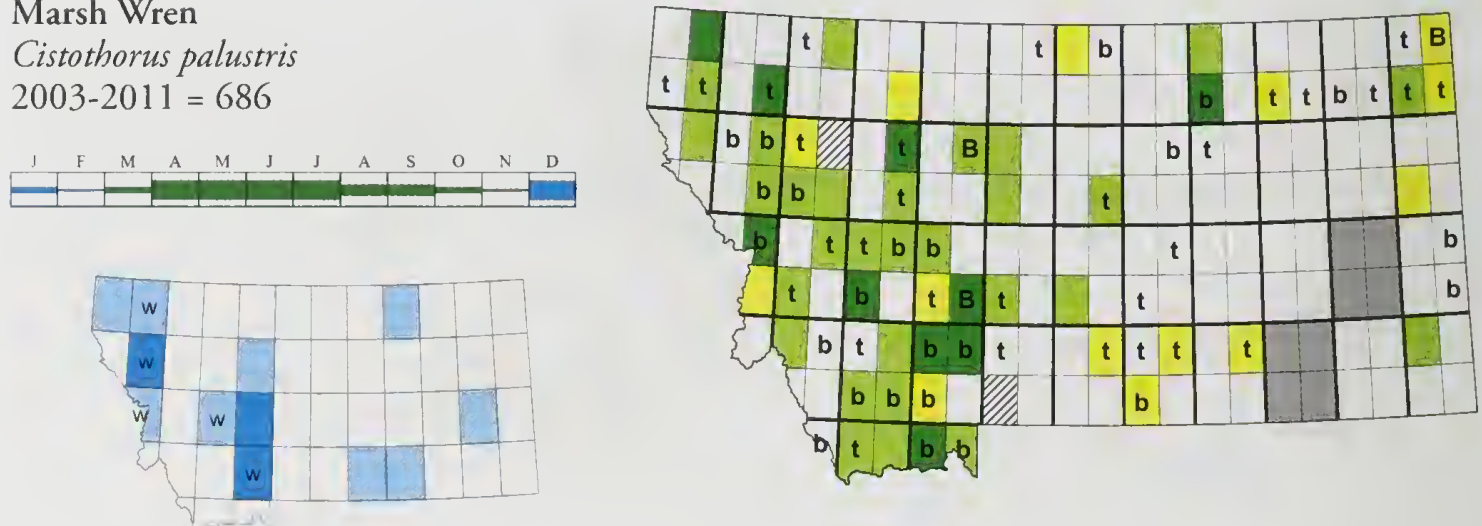

Polioptilidae

Blue-gray Gnatcatcher

Polioptila caerulea

2003-2011 = 20 SOC R2
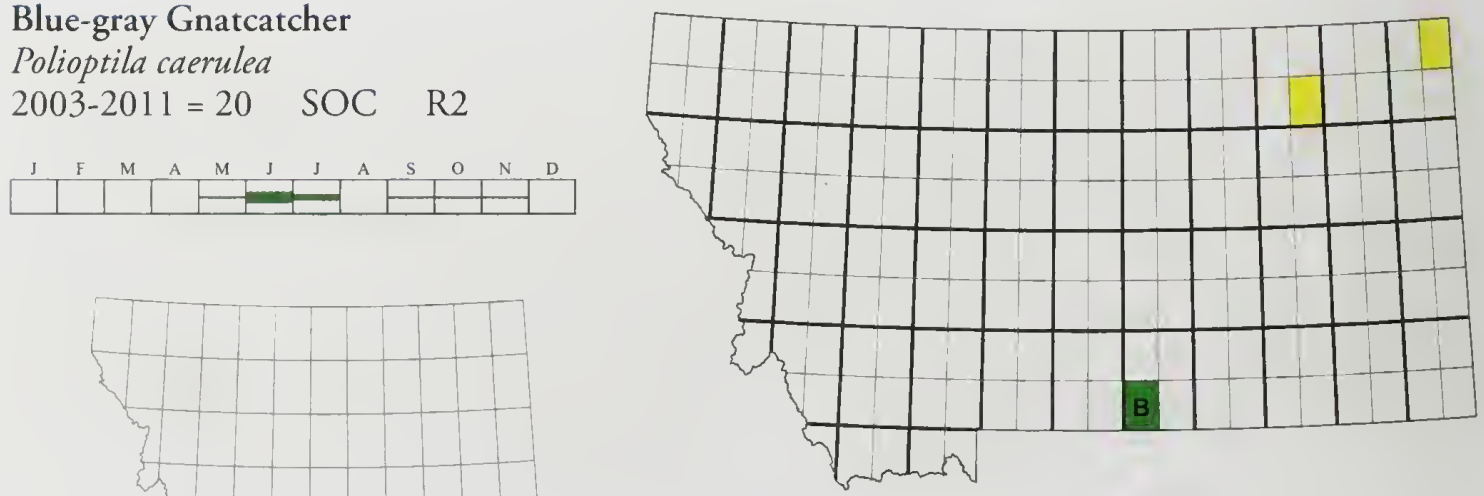


\section{Cinclidae}

American Dipper

Cinclus mexicanus

2003-2011 = 464
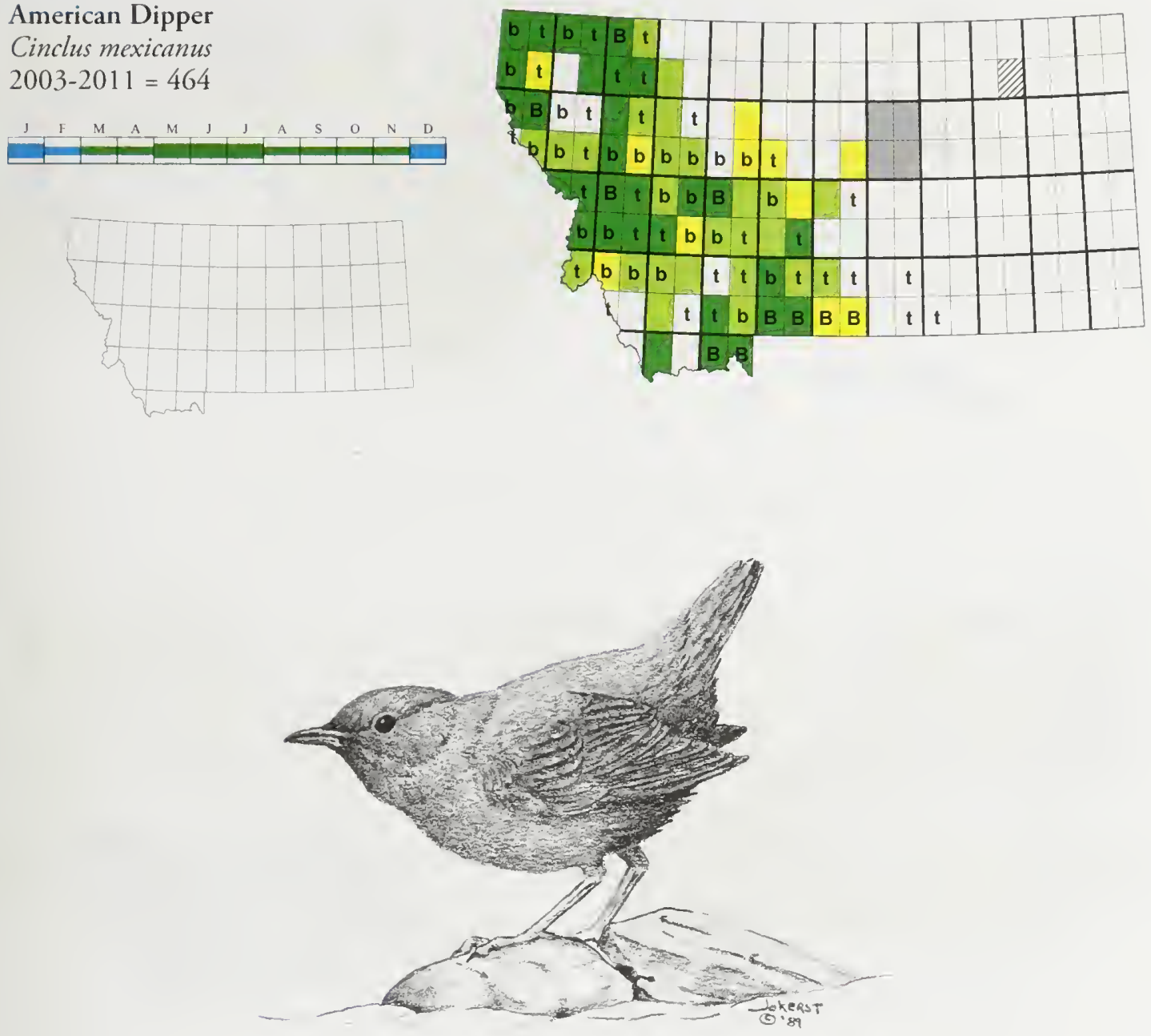

Regulidae

Golden-crowned Kinglet

Regulus satrapa

2003-2011 = 1494
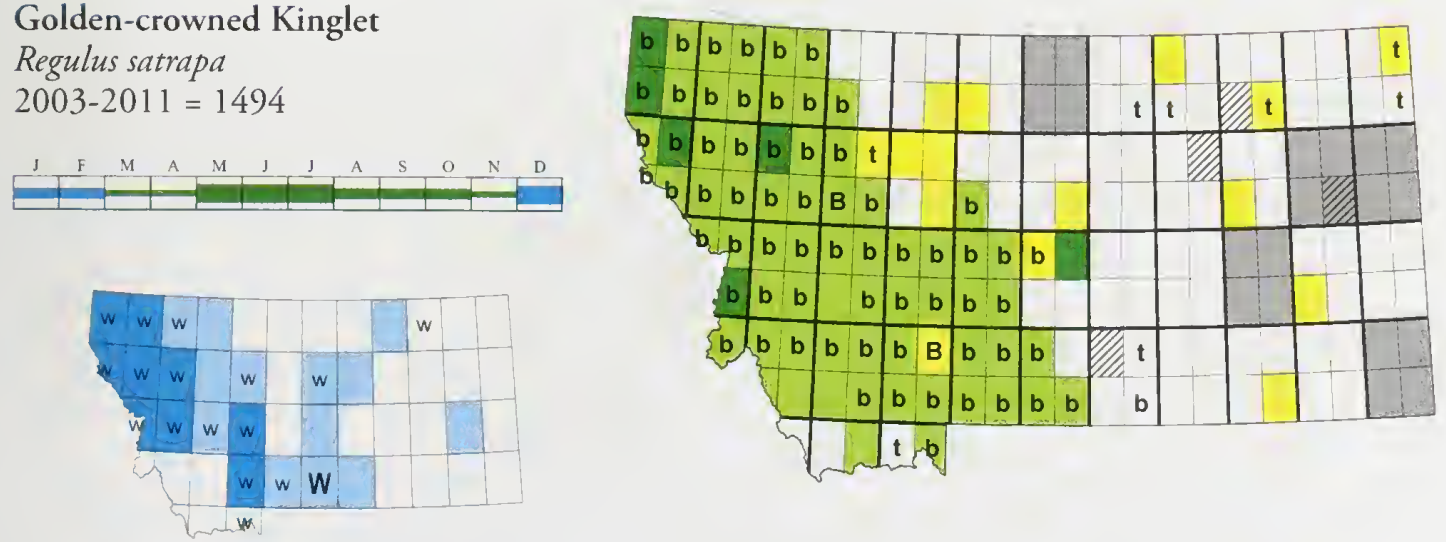
Ruby-crowned Kinglet

Regulus calendula

2003-2011 = 6419
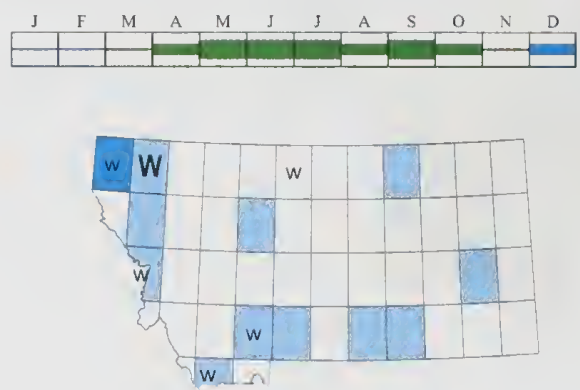

\section{Turdidae}

Eastern Bluebird

Sialia sialis

$2003-2011=58 \quad$ SI

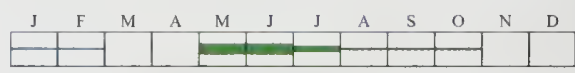
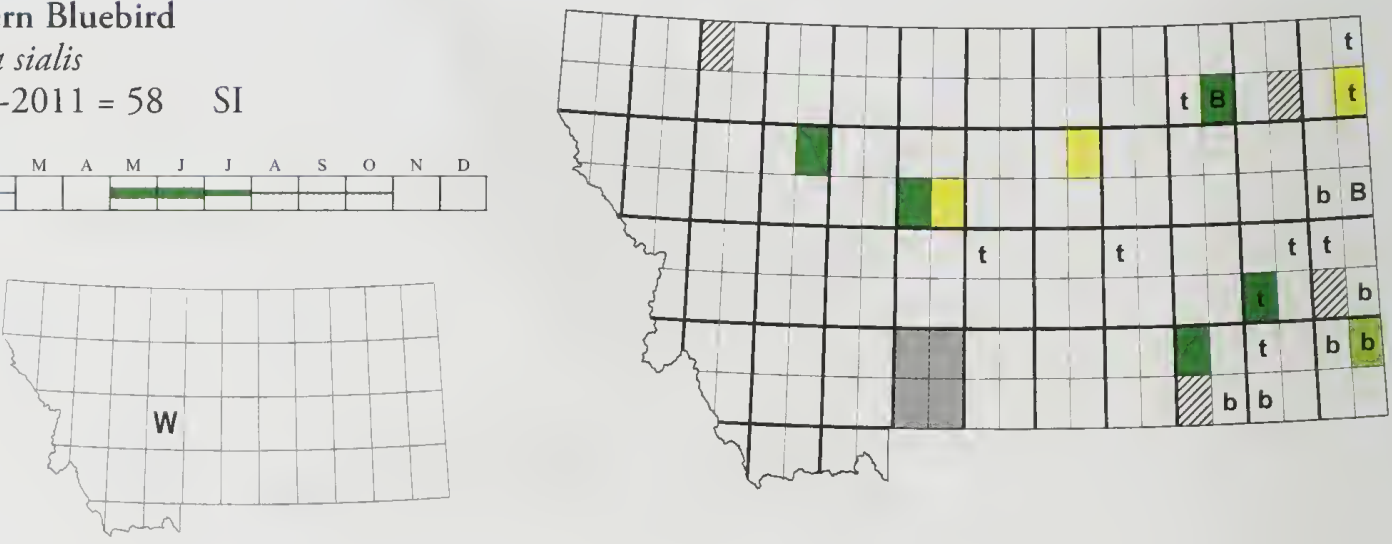

Western Bluebird

Sialia mexicana

2003-2011 = 128
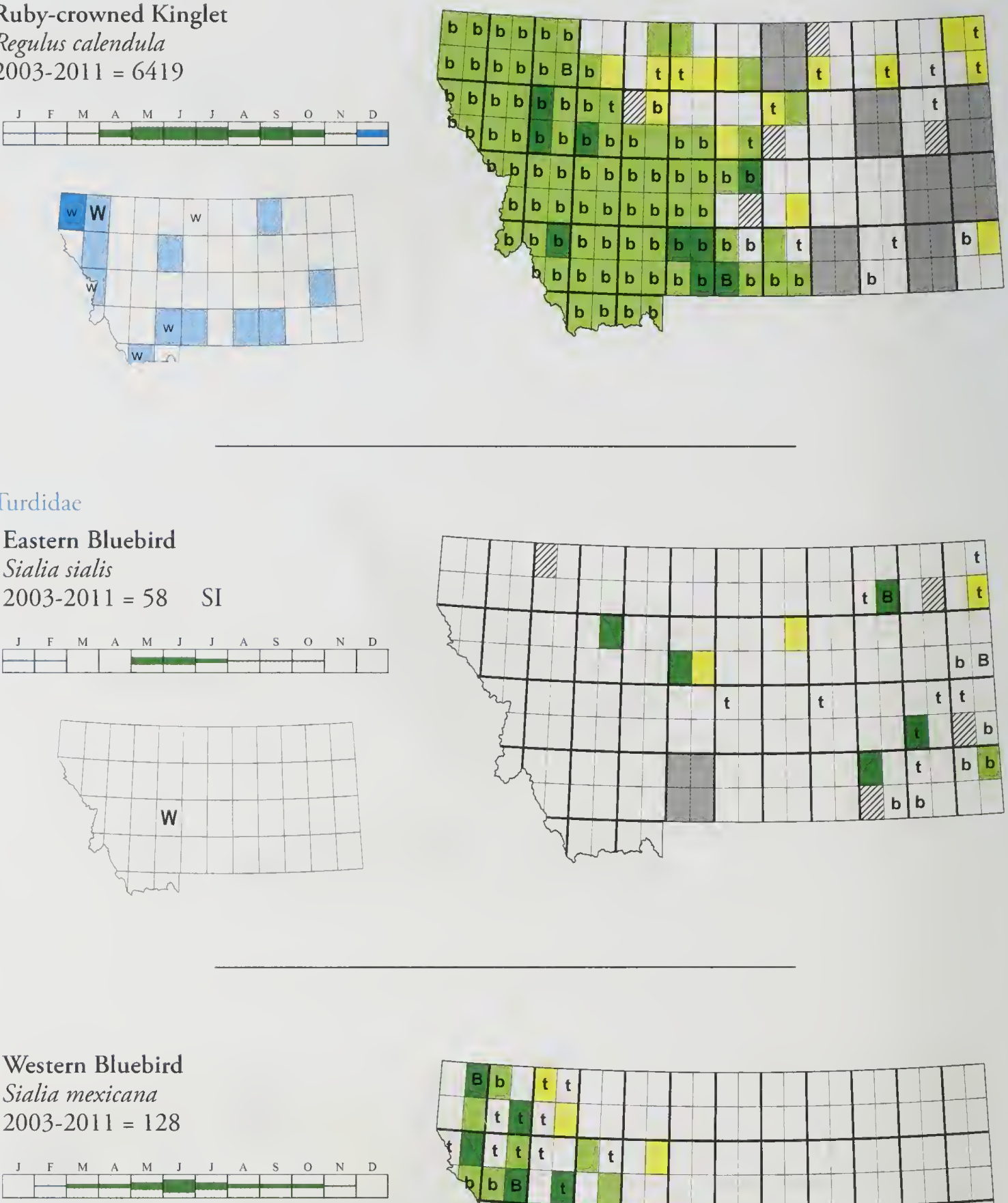
Mountain Bluebird

Sialia currucoides

2003-2011 = 2503
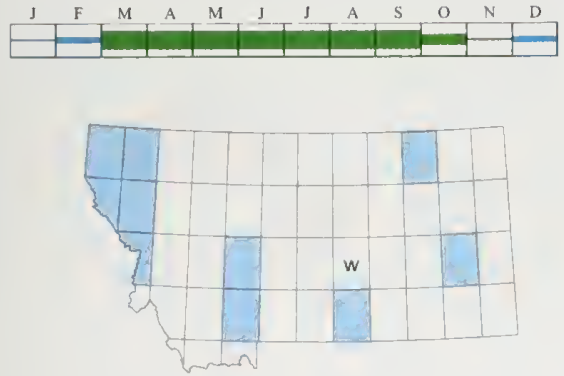

Townsend's Solitaire

Myadestes townsendi

2003-2011 = 2807
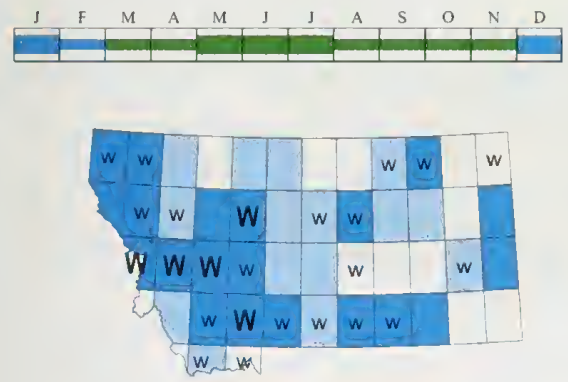

Veery

Catharus fuscescens

2003-2011 = 409 SOC

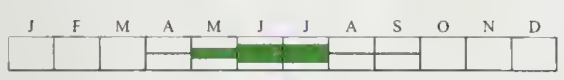

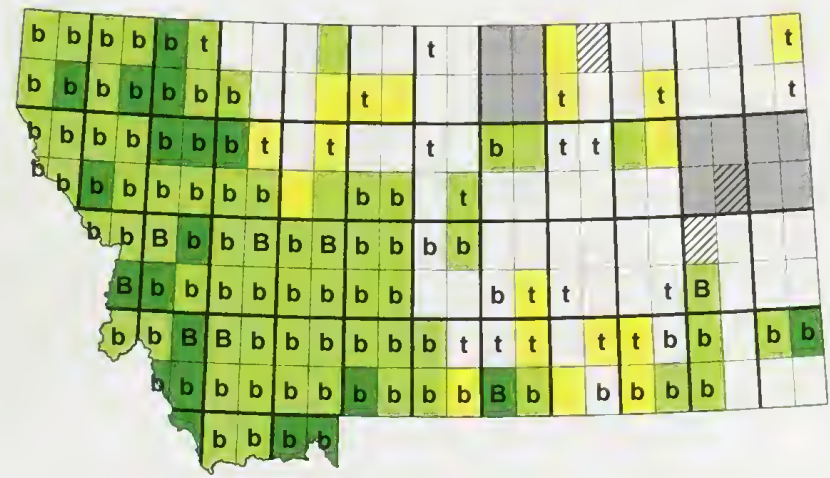

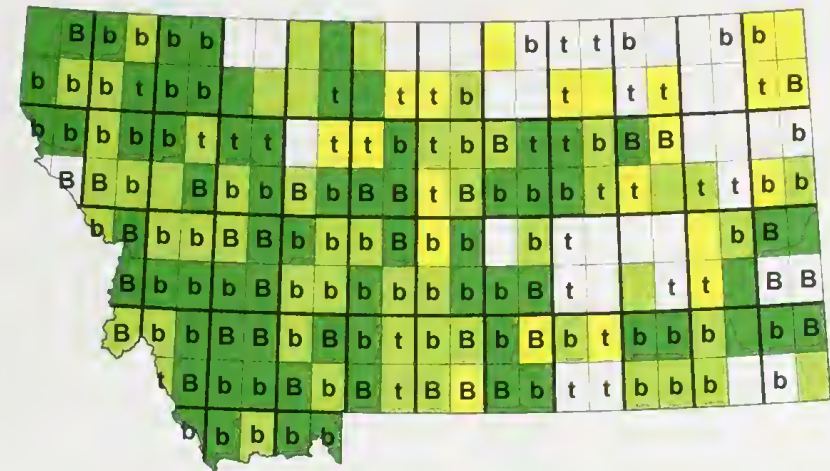


Gray-cheeked Thrush

Catharus minimus

$2003-2011=6 \quad$ R2
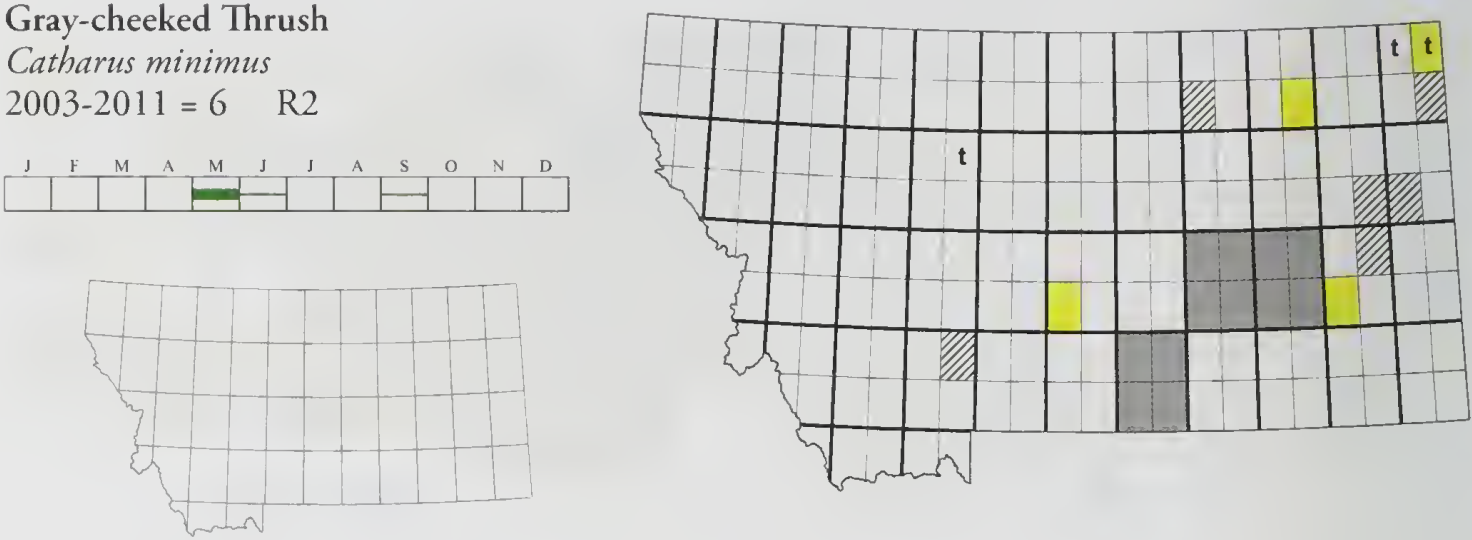

Swainson's Thrush

Catharus ustulatus

2003-2011 = 4787
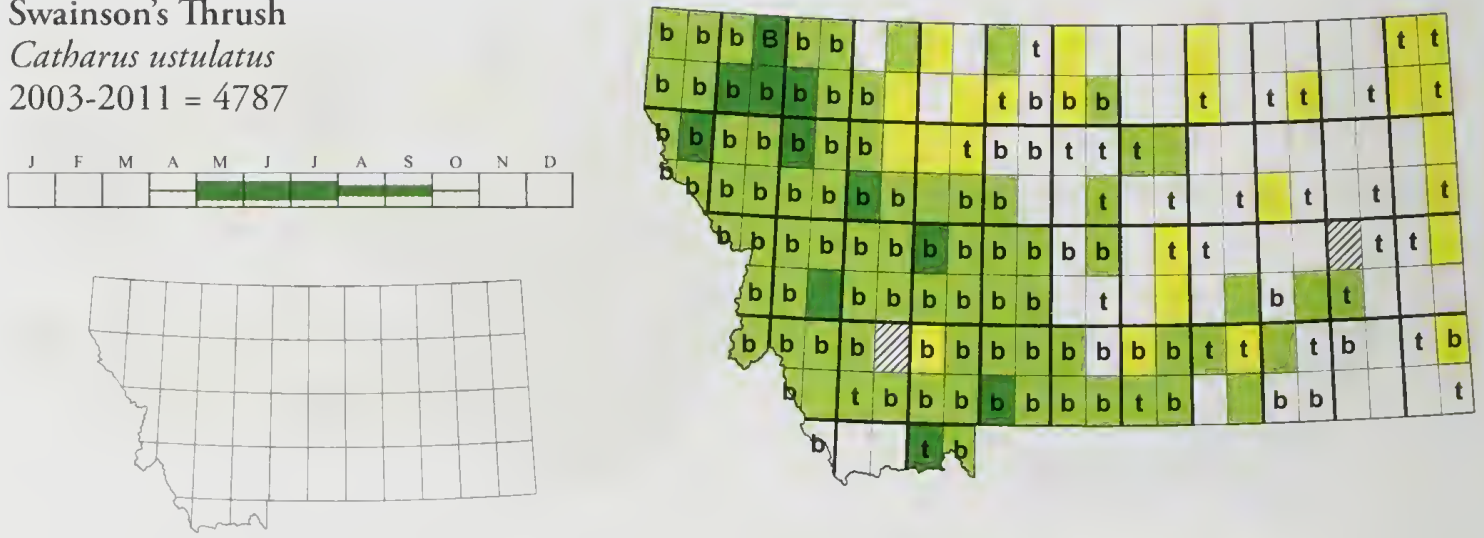

Hermit Thrush

Catharus guttatus

2003-2011 = 2206
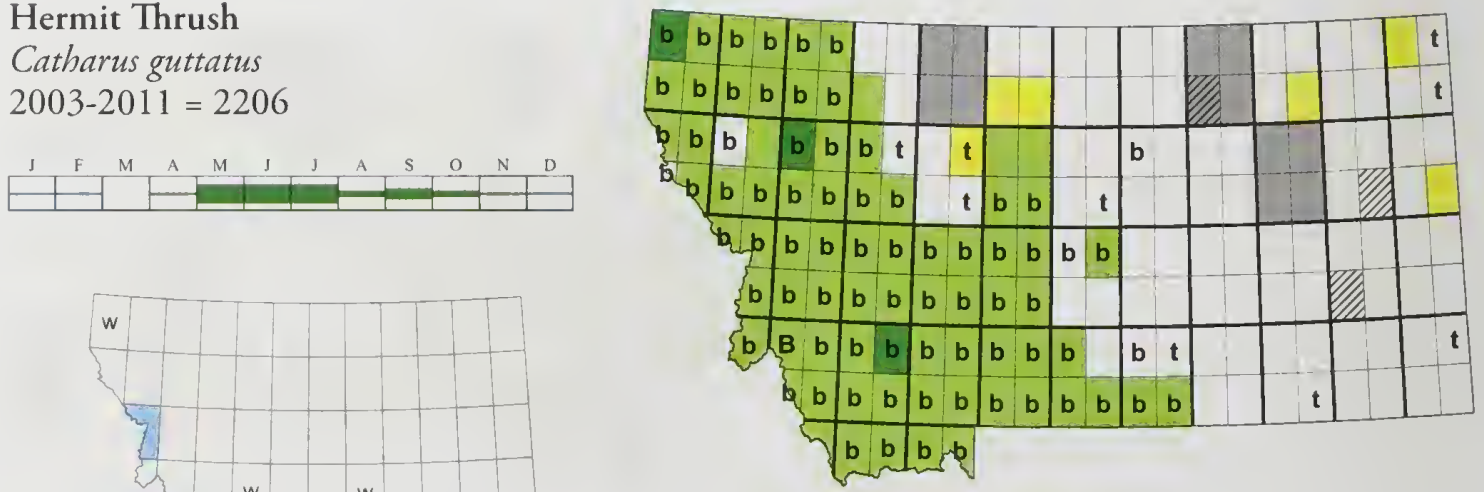
Wood Thrush

Hylocichla mustelina

2003-2011 = $4 \quad \mathrm{R} 1$
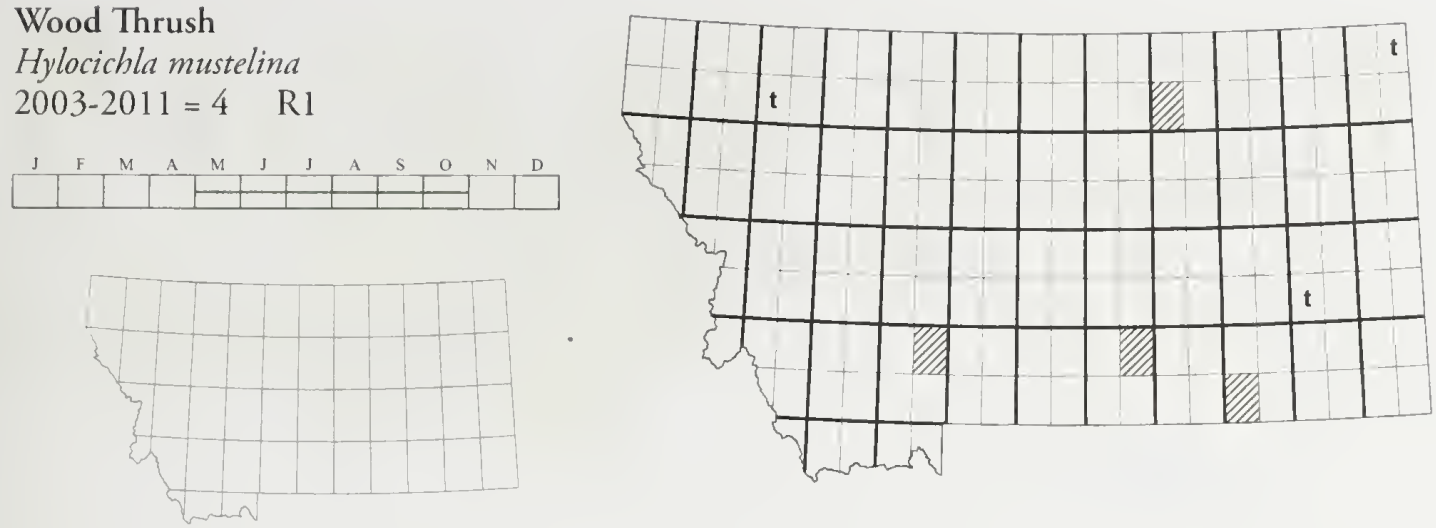

\section{American Robin}

Turdus migratorius

2003-2011 = 13537
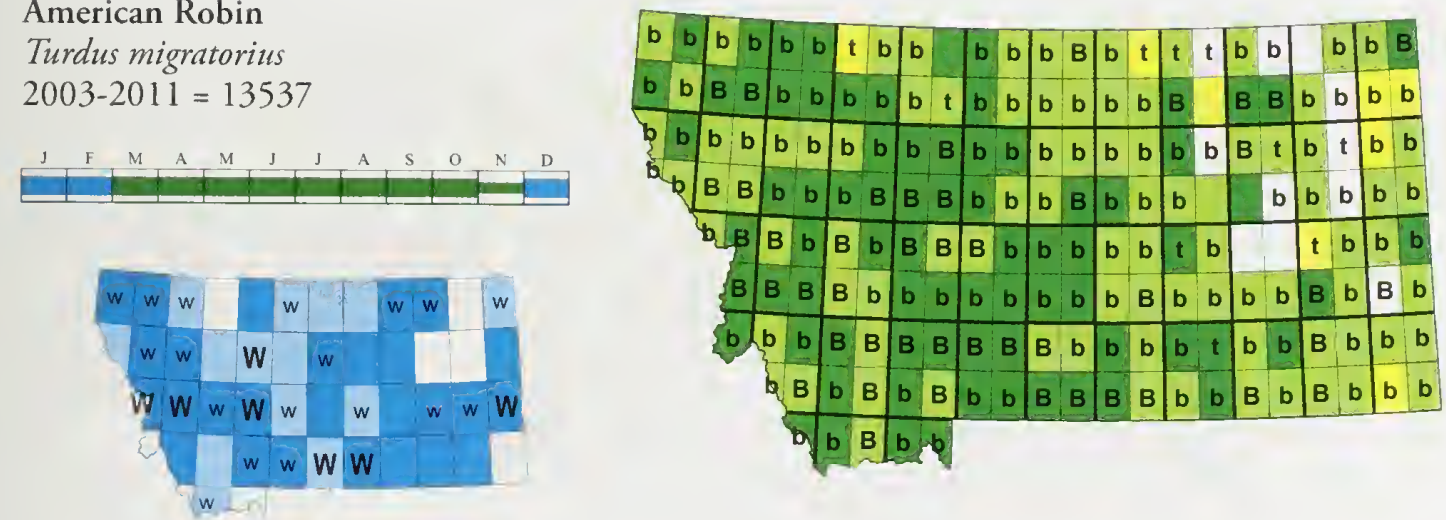

Varied Thrush

Ixoreus naevius

2003-2011 = 947 SOC
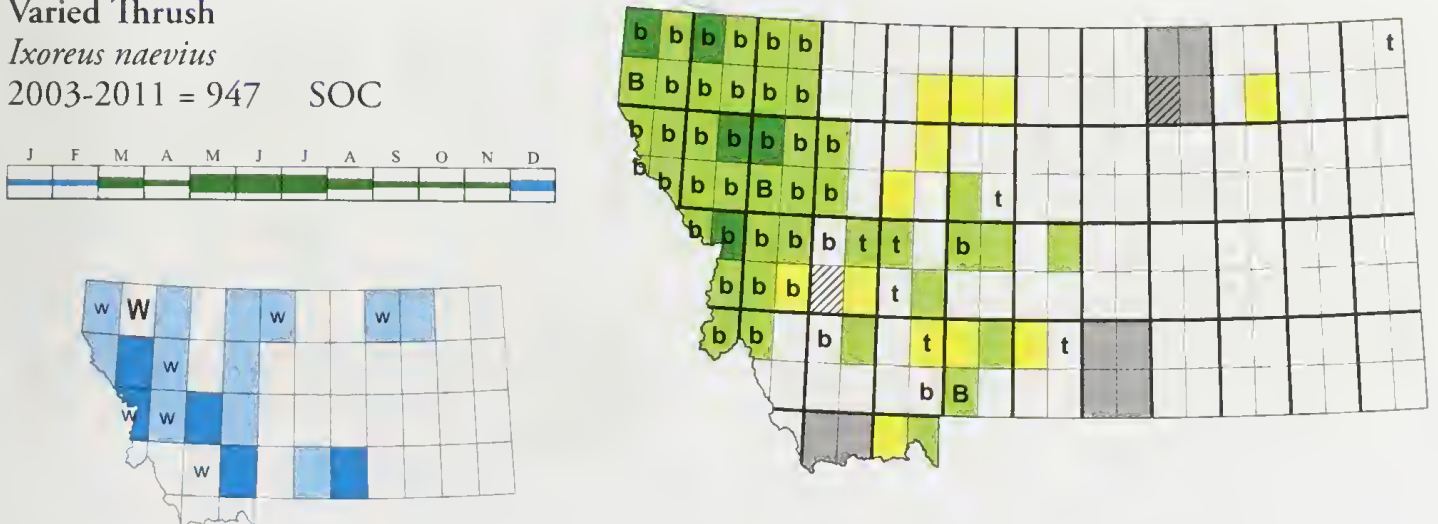
Mimidac

\section{Gray Catbird}

Dumetella carolinensis

2003-2011 = 1894
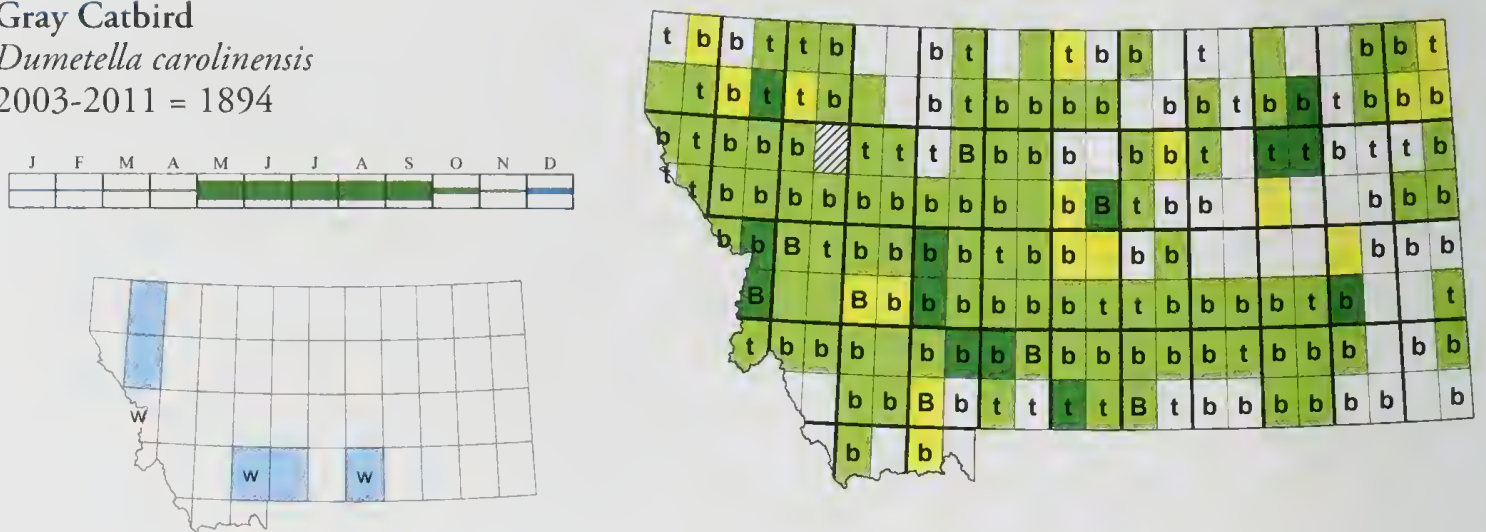

Northern Mockingbird

Mimus polyglottos

2003-2011 = 23

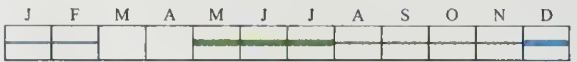
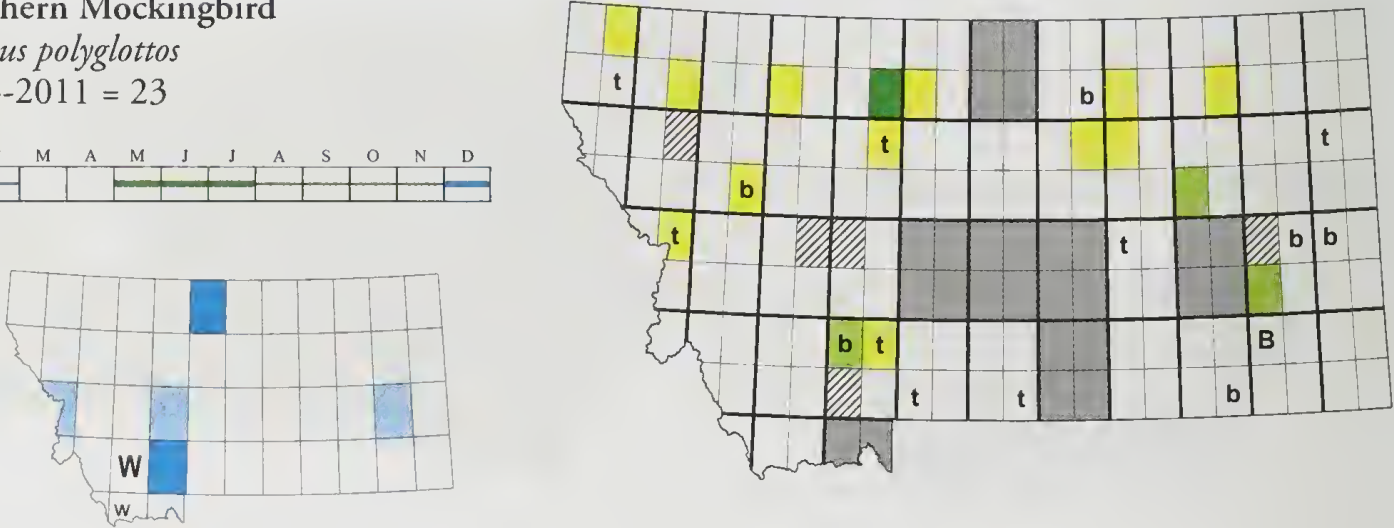

Sage Thrasher

Oreoscoptes montanus

$2003-2011=313 \quad$ SOC
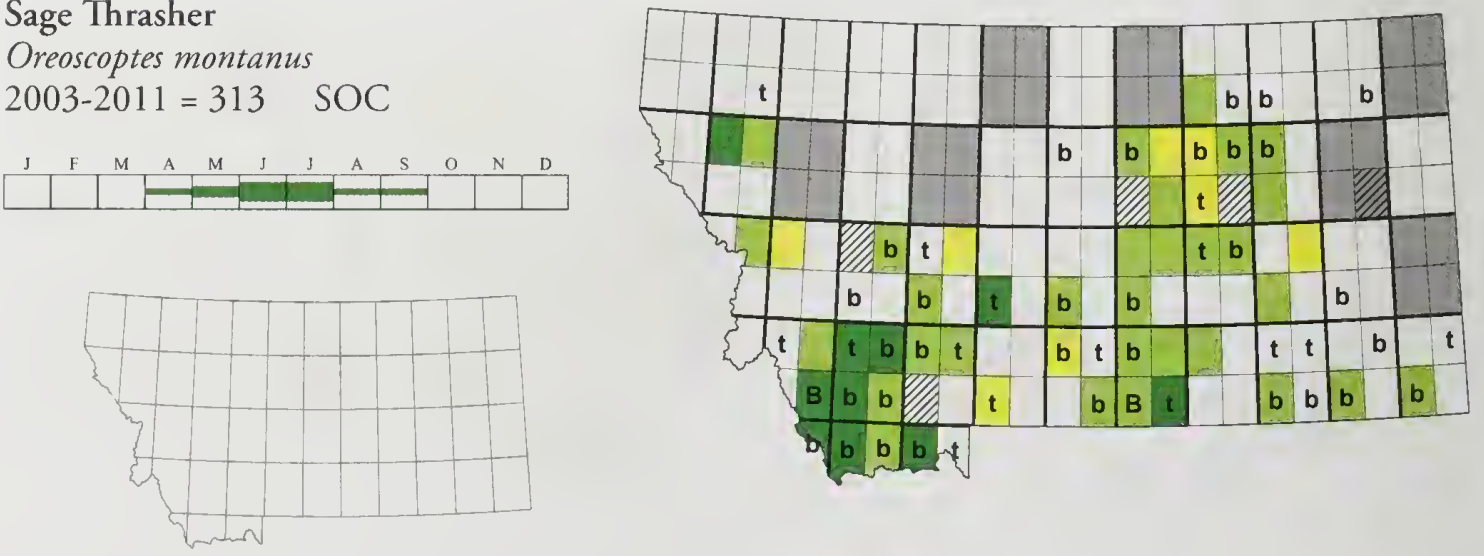
Brown Thrasher

Toxostoma rufum

2003-2011 = 403
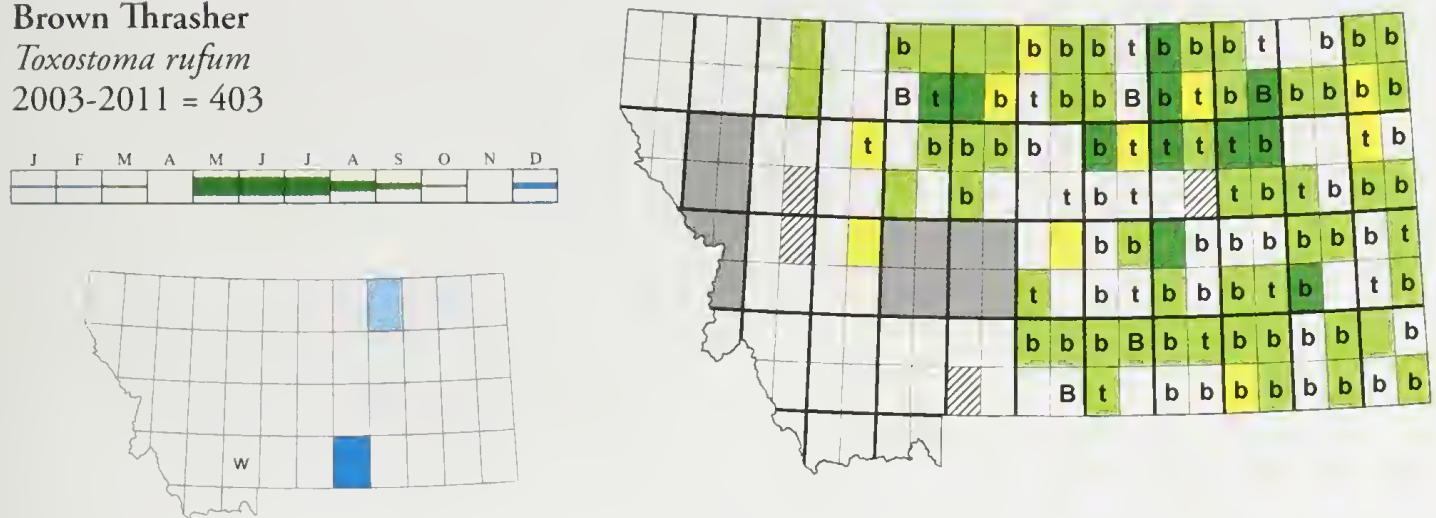

Curve-billed Thrasher

Toxostoma curvirostre

2003-2011 = 2 R1
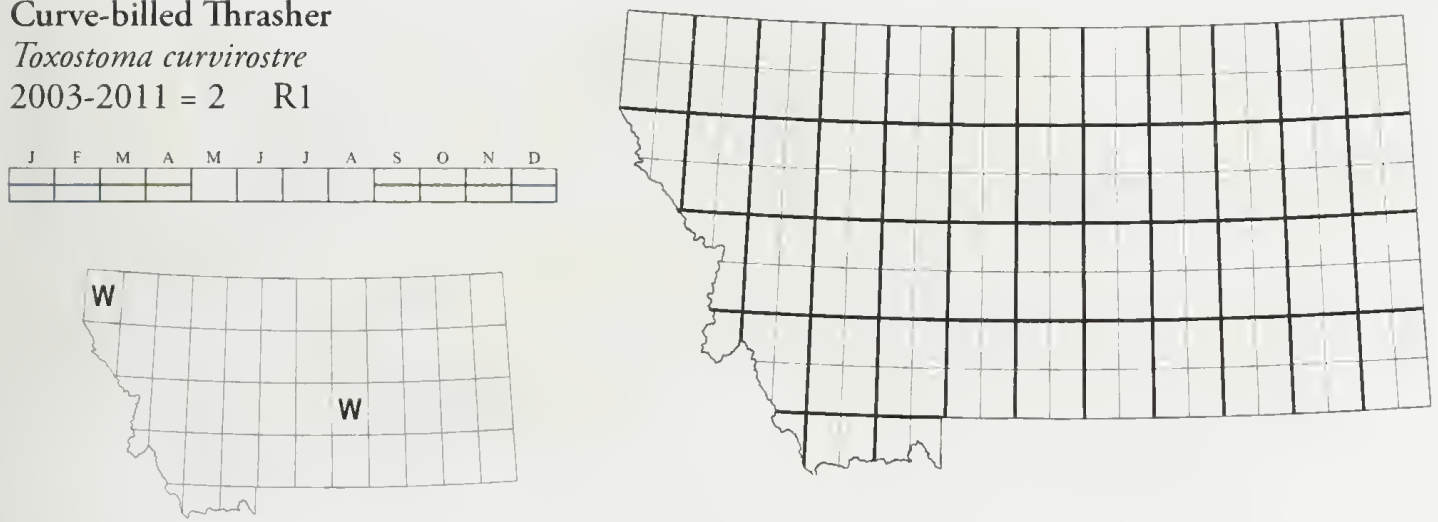

\section{Sturnidae}

European Starling

Sturnus vulgaris

2003-2011 = 3183
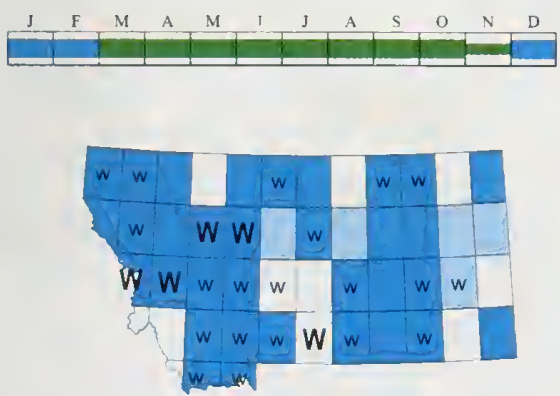
Prunellidae

Siberian Accentor

Prunella montanella

2003-2011 = 1 R1

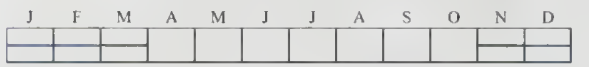

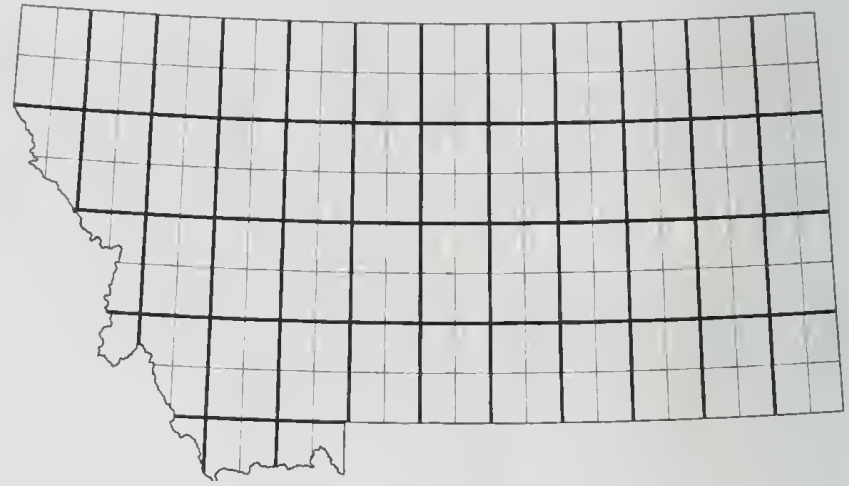

Motacillidae

\section{American Pipit}

Anthus rubescens

2003-2011 = 236
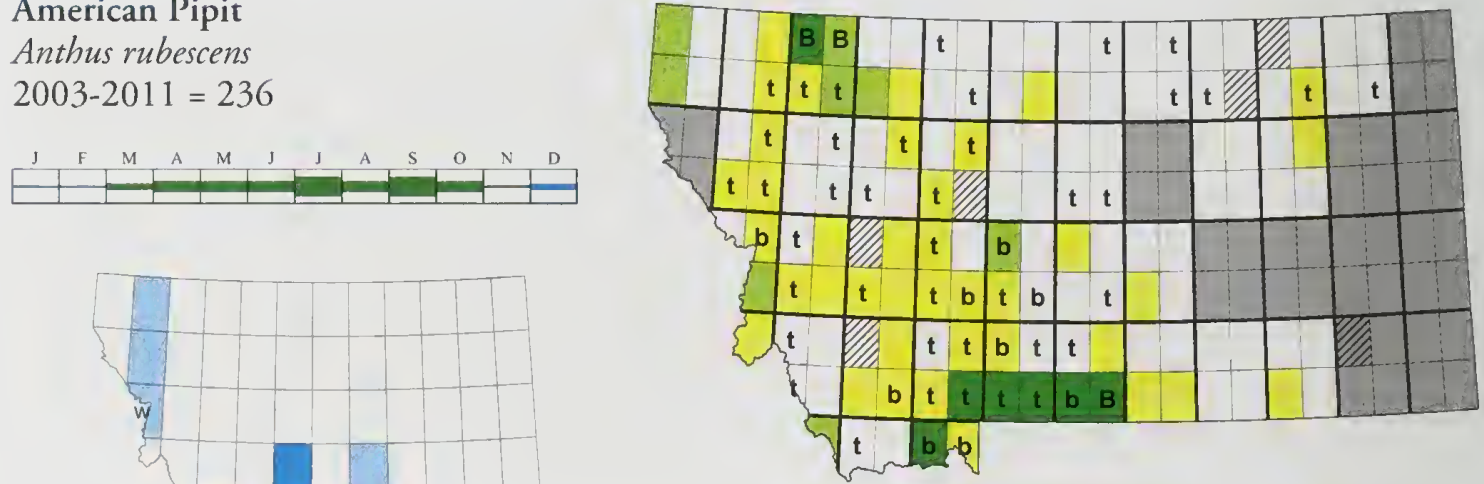

Sprague's Pipit

Anthus spragueii

2003-2011 = 2598 C
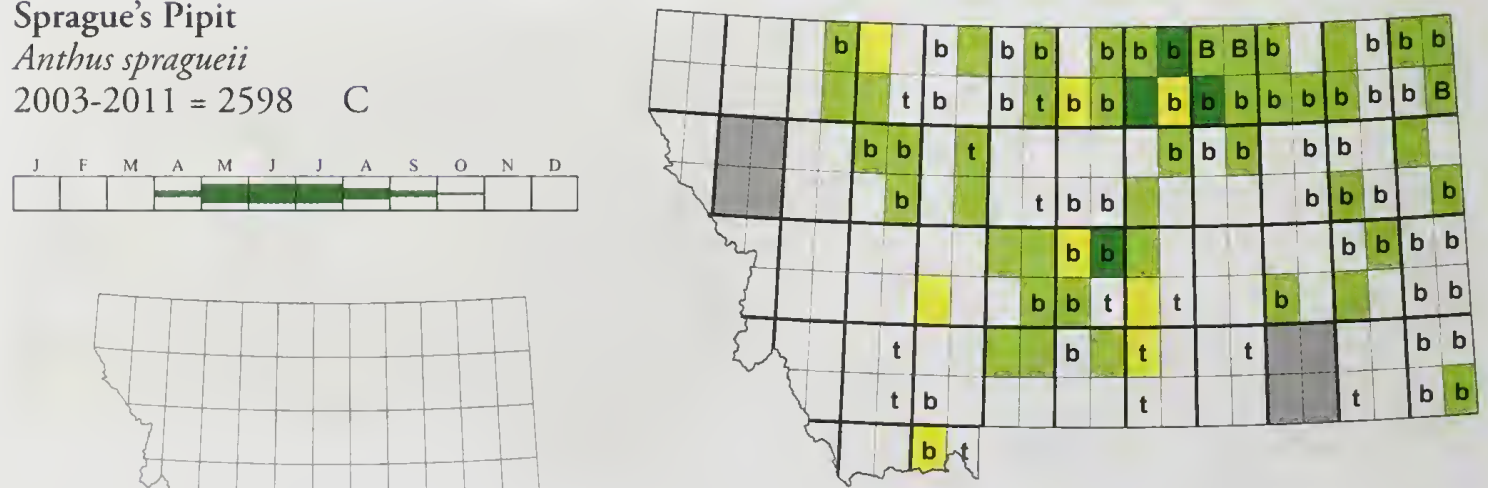
Bohemian Waxwing

Bombycilla garrulus

$2003-2011=288$

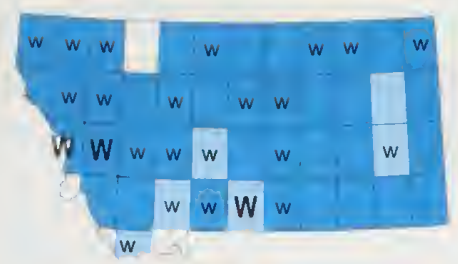

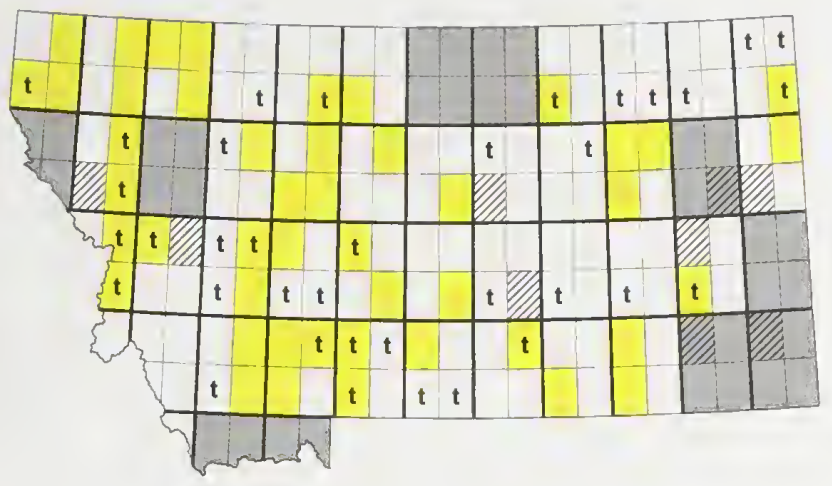

Cedar Waxwing

Bombycilla cedrorum

2003-2011 = 1982
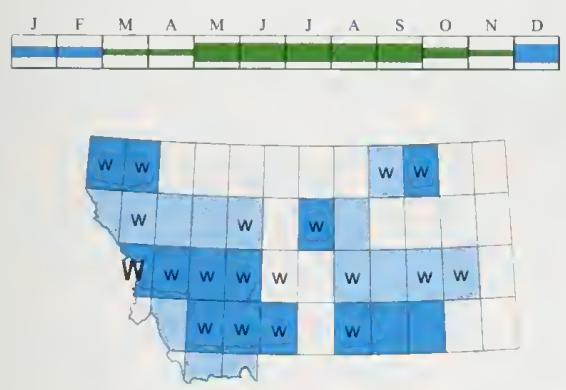

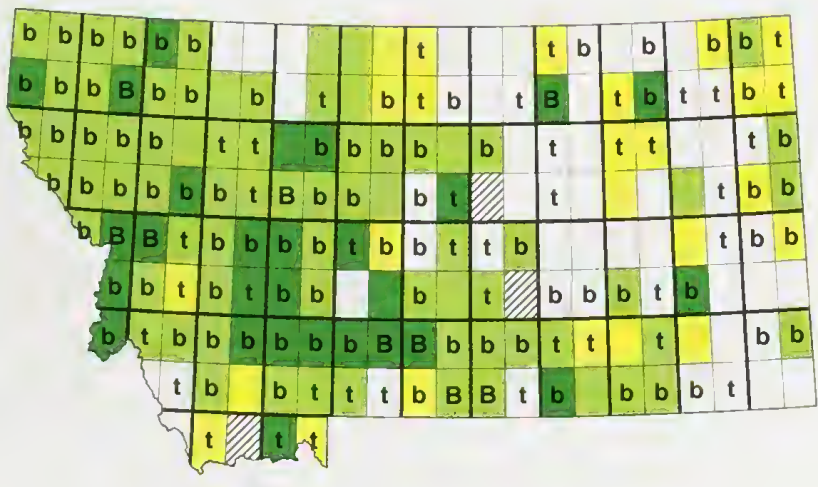

\section{Calcariidae}

\section{Lapland Longspur}

Calcarius lapponicus

2003-2011 = 39
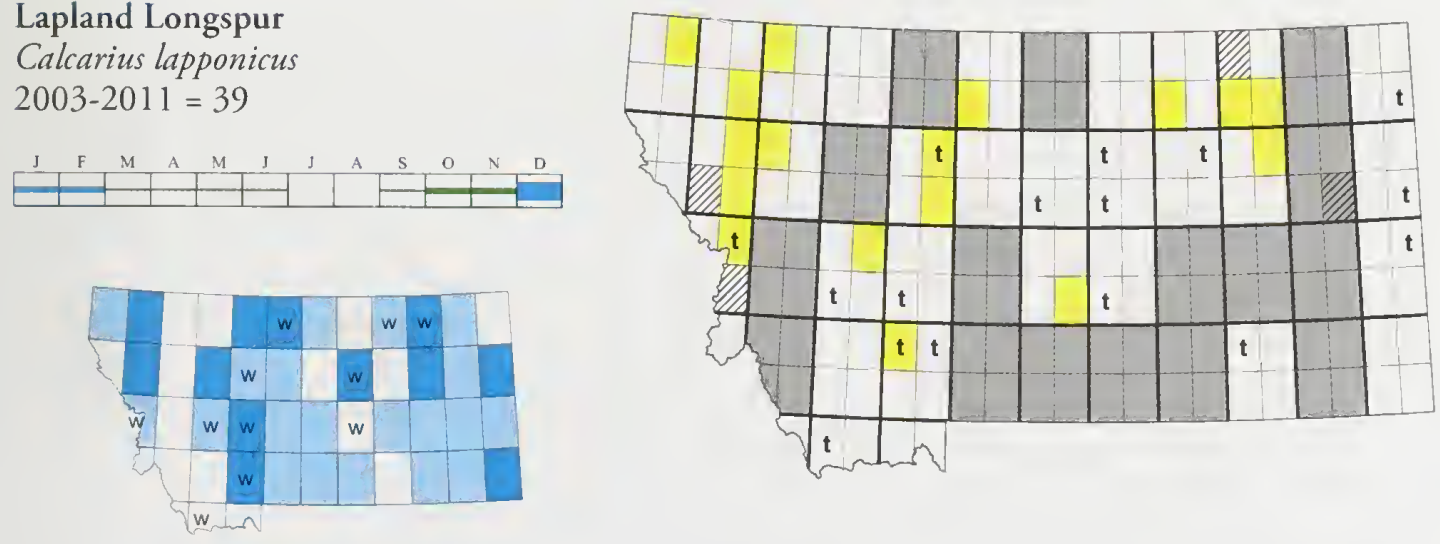
Chestnut-collared Longspur

Calcarius ornatus

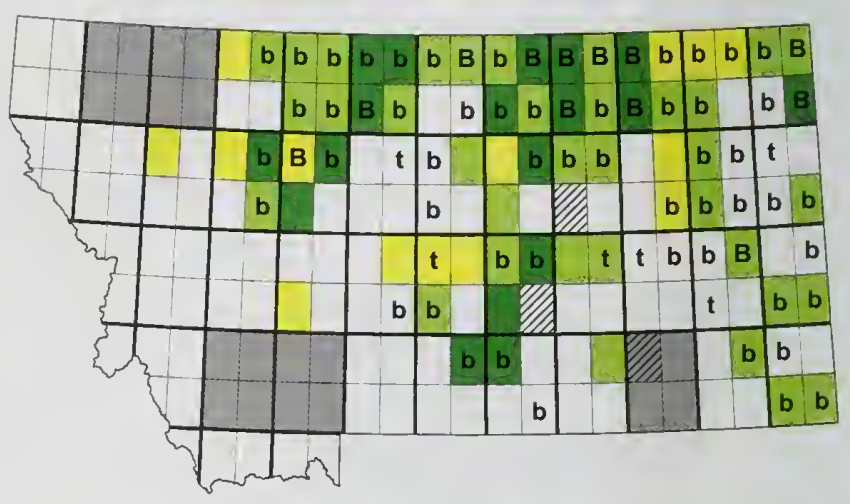

$2003-2011=4097 \quad$ SOC
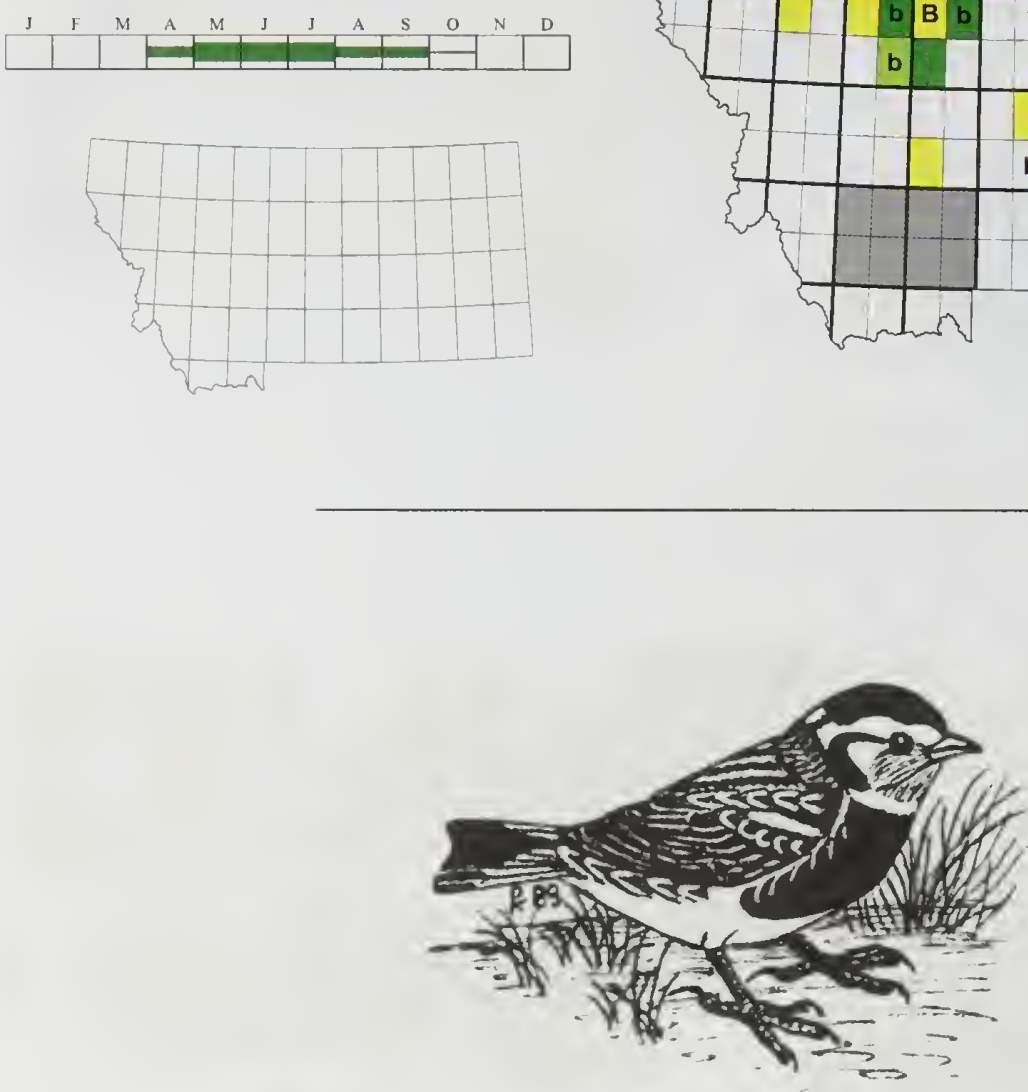

Smith's Longspur

Calcarius pictus

2003-2011 = 0 R1

\begin{tabular}{|l|l|l|l|l|l|l|l|l|l|l|l|}
\hline $\mathrm{J}$ & $\mathrm{F}$ & $\mathrm{M}$ & $\mathrm{A}$ & $\mathrm{M}$ & $\mathrm{J}$ & $\mathrm{J}$ & $\mathrm{A}$ & $\mathrm{S}$ & $\mathrm{O}$ & $\mathrm{N}$ & $\mathrm{D}$ \\
\hline & & & & & & & & & & & \\
\hline
\end{tabular}

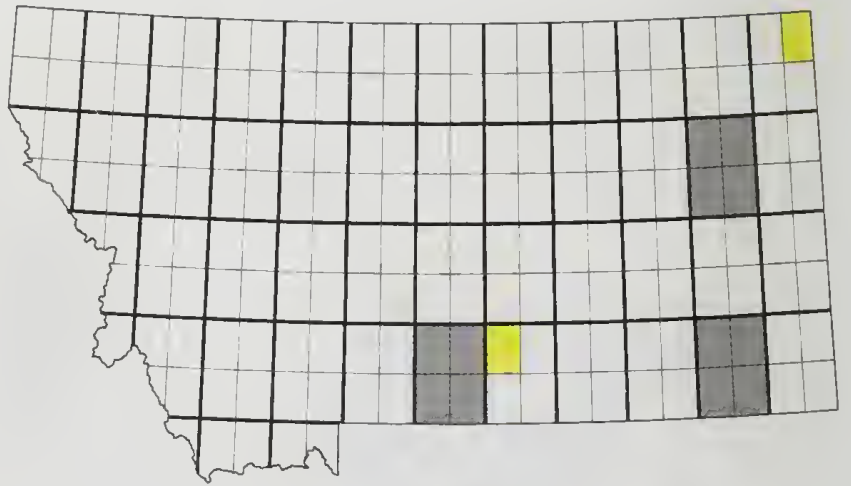


McCown's Longspur

Rhynchophanes mccownii

$2003-2011=1424$ SOC
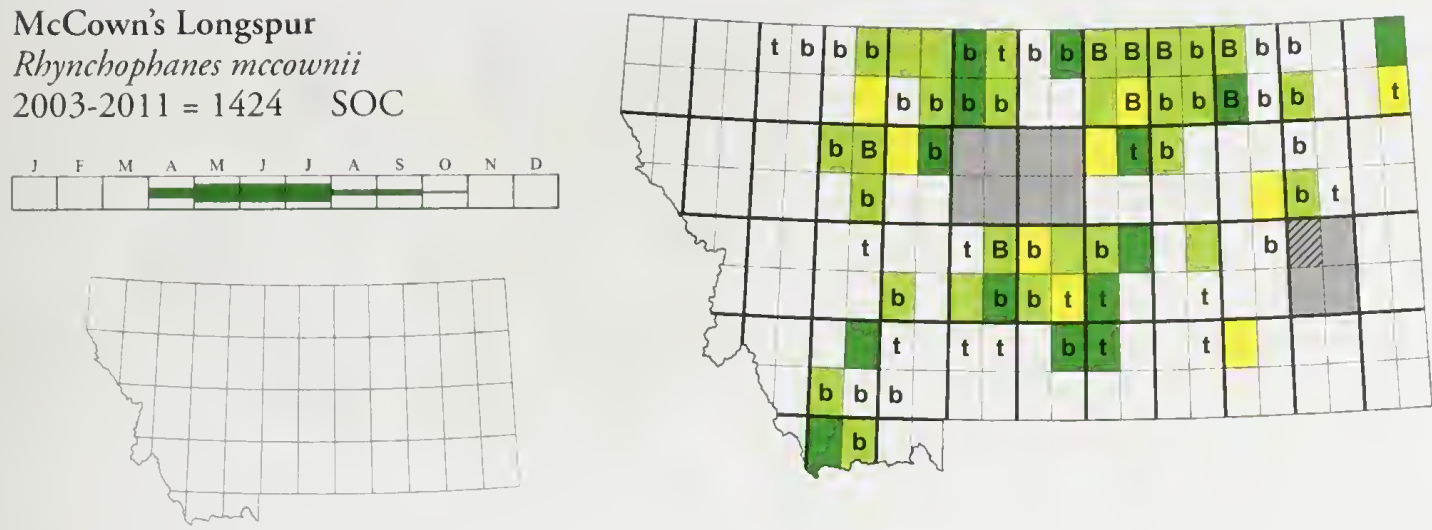

Snow Bunting

Plectrophenax nivalis

$2003-2011=101$
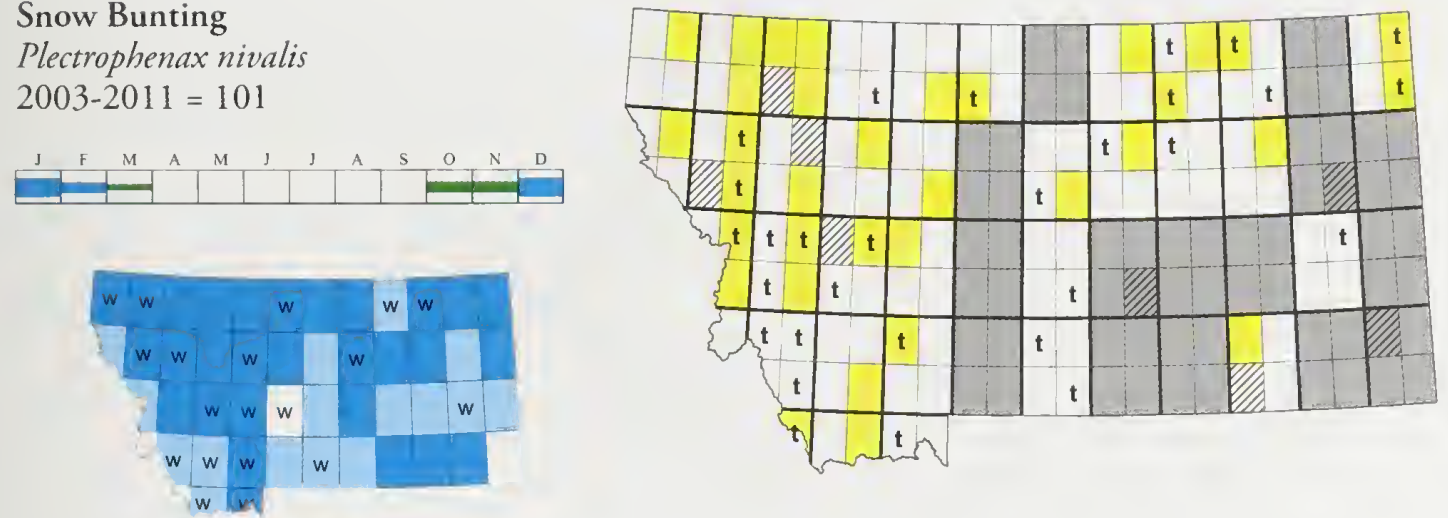

\section{Parulidae}

\section{Ovenbird}

Seiurus aurocapilla

2003-2011 = 514 SI
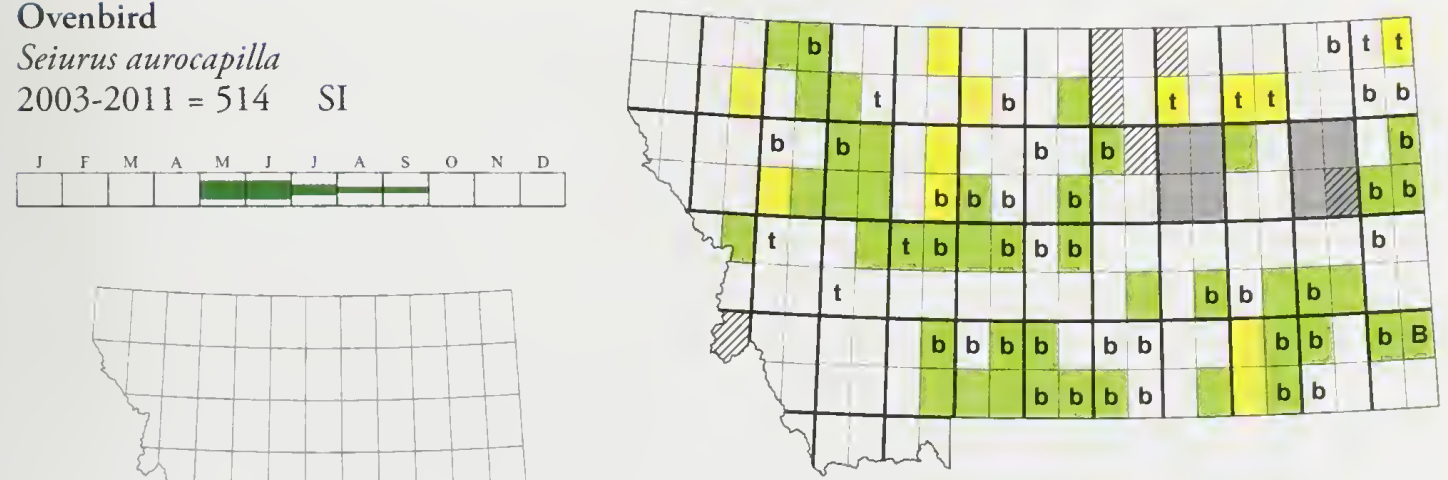
Northern Waterthrush

Parkesia noveboracensis

2003-2011 = 631

\begin{tabular}{|l|l|l|l|l|l|l|l|l|l|l|l|l|l}
$\mathrm{J}$ & $\mathrm{F}$ & $\mathrm{M}$ & $\mathrm{A}$ & $\mathrm{M}$ & $\mathrm{J}$ & $\mathrm{J}$ & $\mathrm{A}$ & $\mathrm{S}$ & $\mathrm{O}$ & $\mathrm{N}$ & $\mathrm{D}$ \\
\hline & & & & & & & & & & & \\
\hline
\end{tabular}
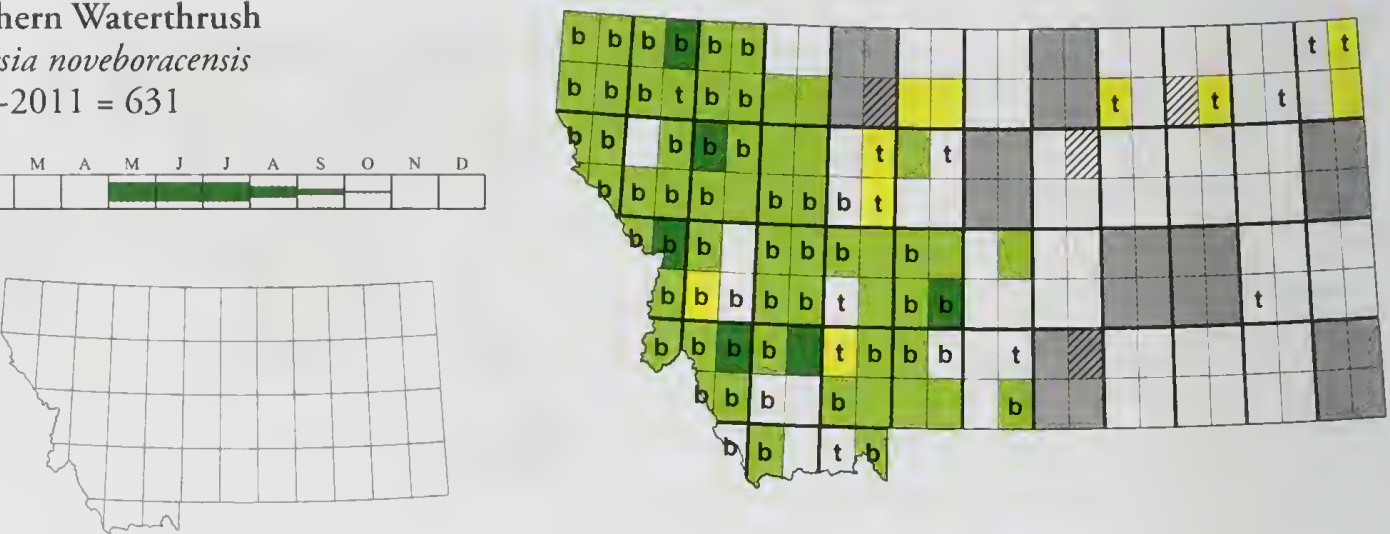

Golden-winged Warbler

Vermivora chrysoptera

2003-2011 = 2 RI

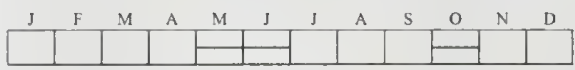
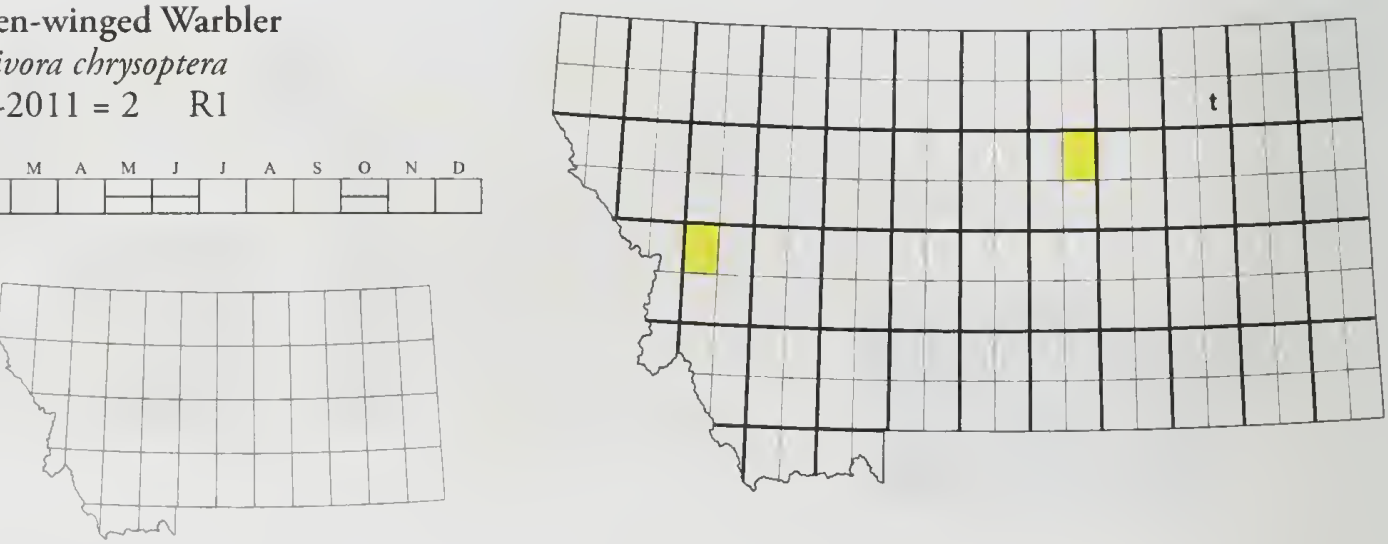

Blue-winged Warbler

Vermivora cyanoptera

$2003-2011=1 \quad R 1$

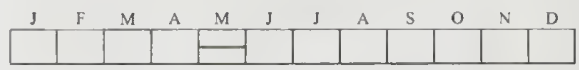

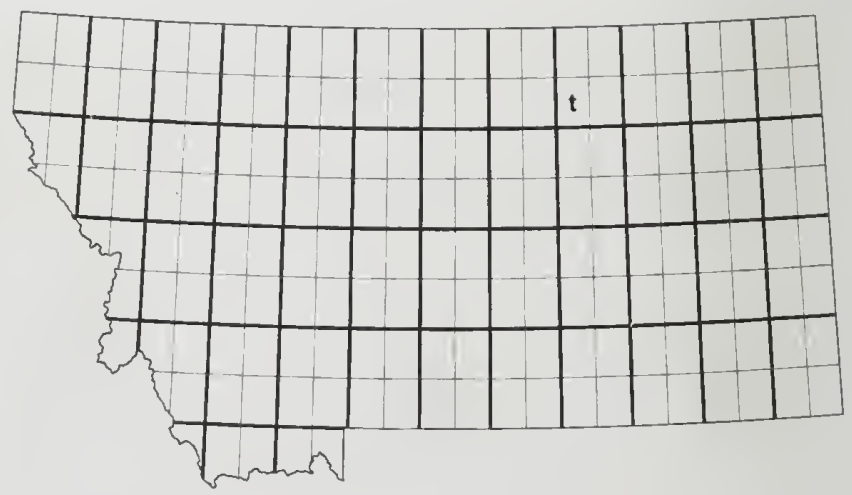


Black-and-white Warbler Mniotilta varia

2003-2011 = 68 SI
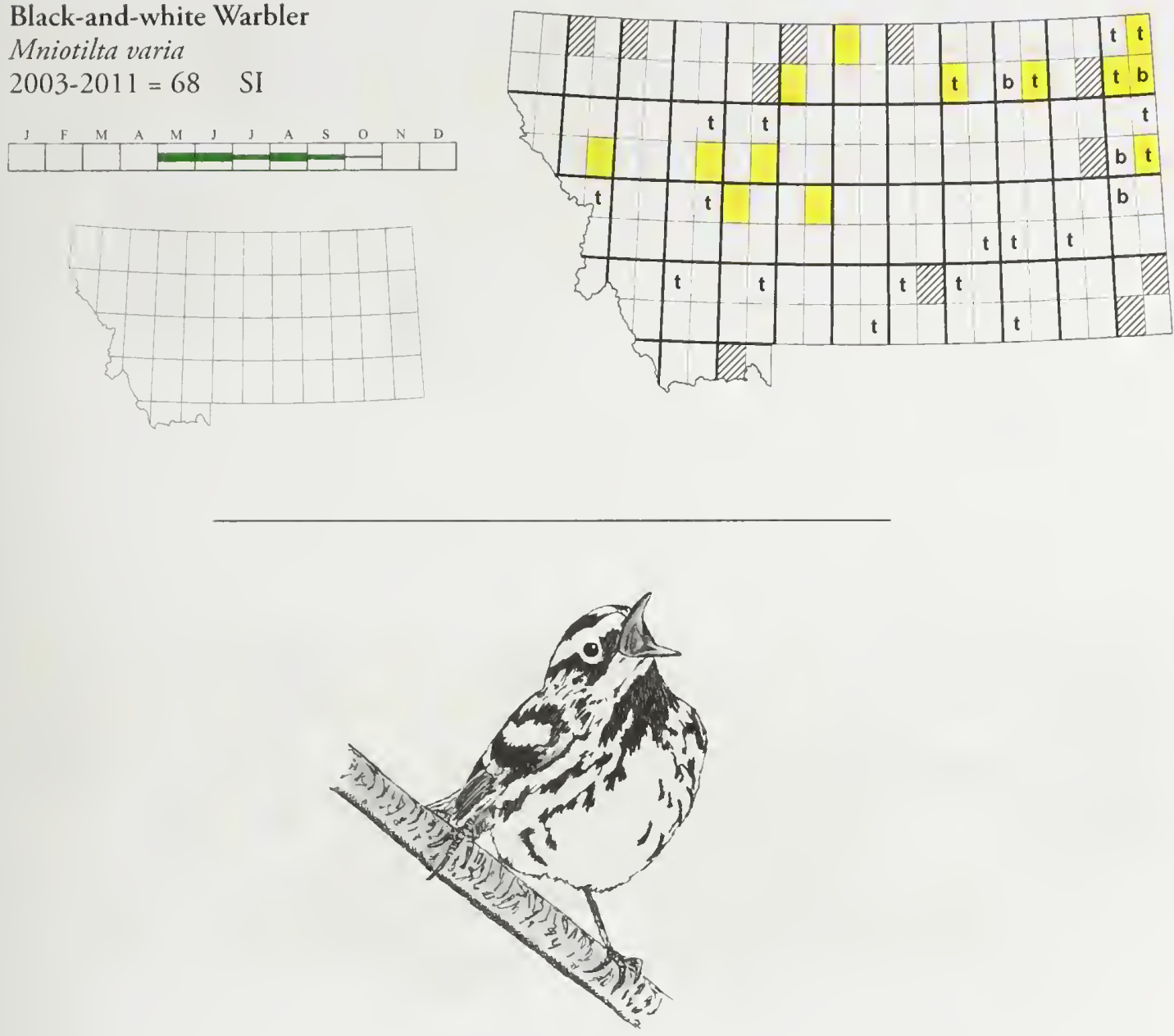

Prothonotary Warbler

Protonotaria citrea

2003-2011 = 1 R1
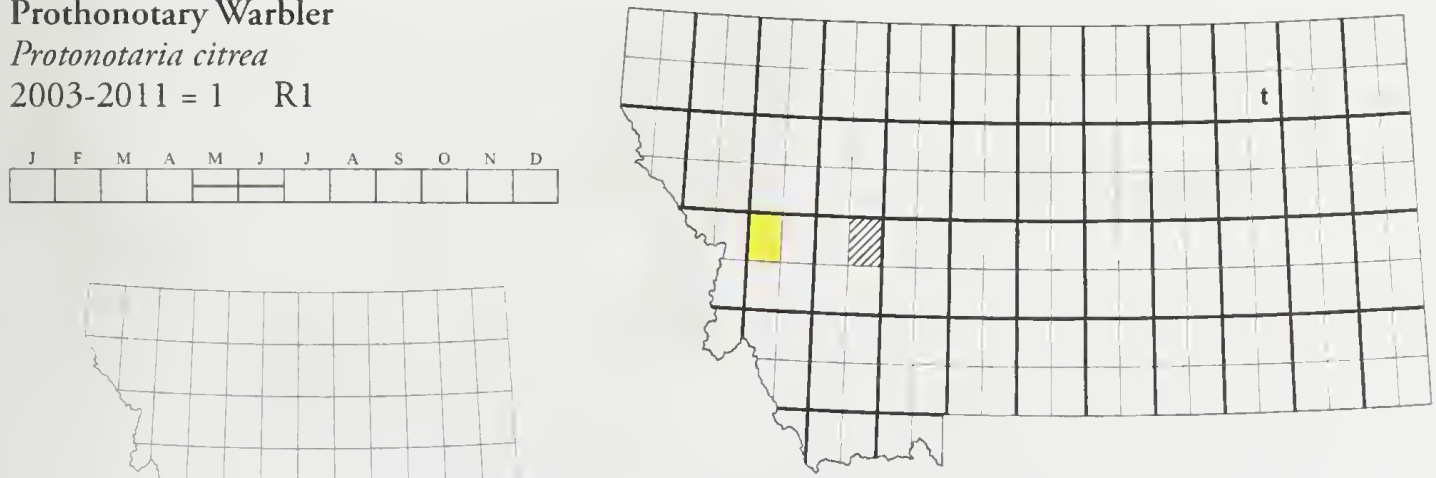
Tennessee Warbler

Oreothlypis peregrina

$2003-2011=59 \quad$ SI

\begin{tabular}{|l|l|l|l|l|l|l|l|l|l|l|l|}
$\mathrm{J}$ & \multicolumn{1}{|c}{$\mathrm{F}$} & $\mathrm{M}$ & $\mathrm{A}$ & $\mathrm{M}$ & $\mathrm{J}$ & $\mathrm{J}$ & $\mathrm{A}$ & $\mathrm{S}$ & $\mathrm{O}$ & $\mathrm{N}$ & $\mathrm{D}$ \\
\hline & & & & & & & & & & & \\
\hline
\end{tabular}
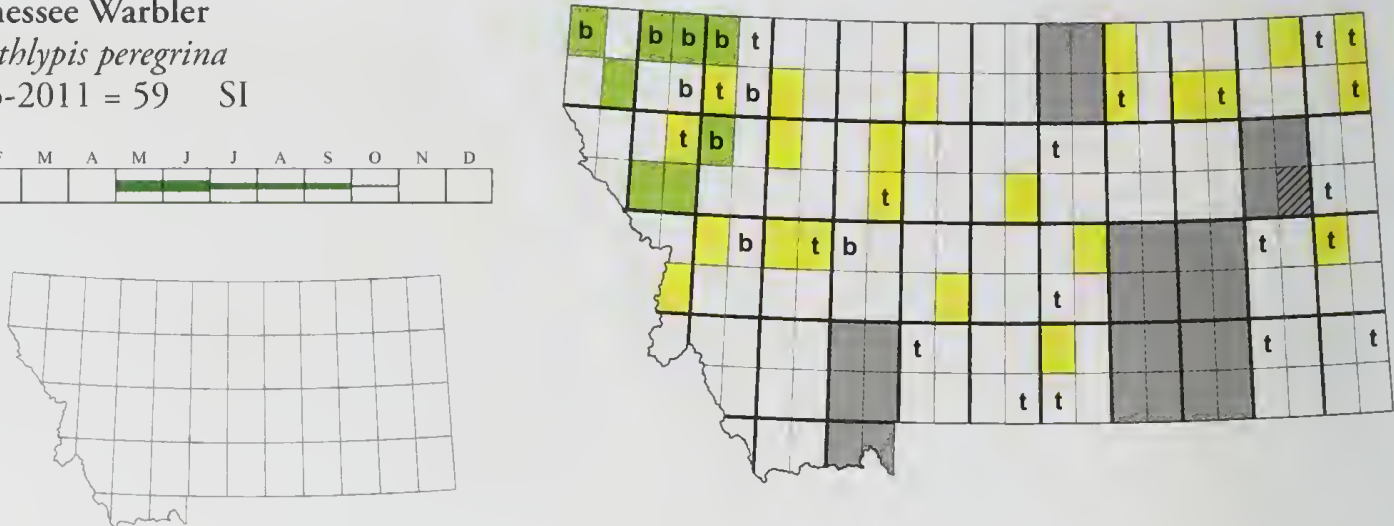

Orange-crowned Warbler

Oreothlypis celata

2003-2011 = 1168
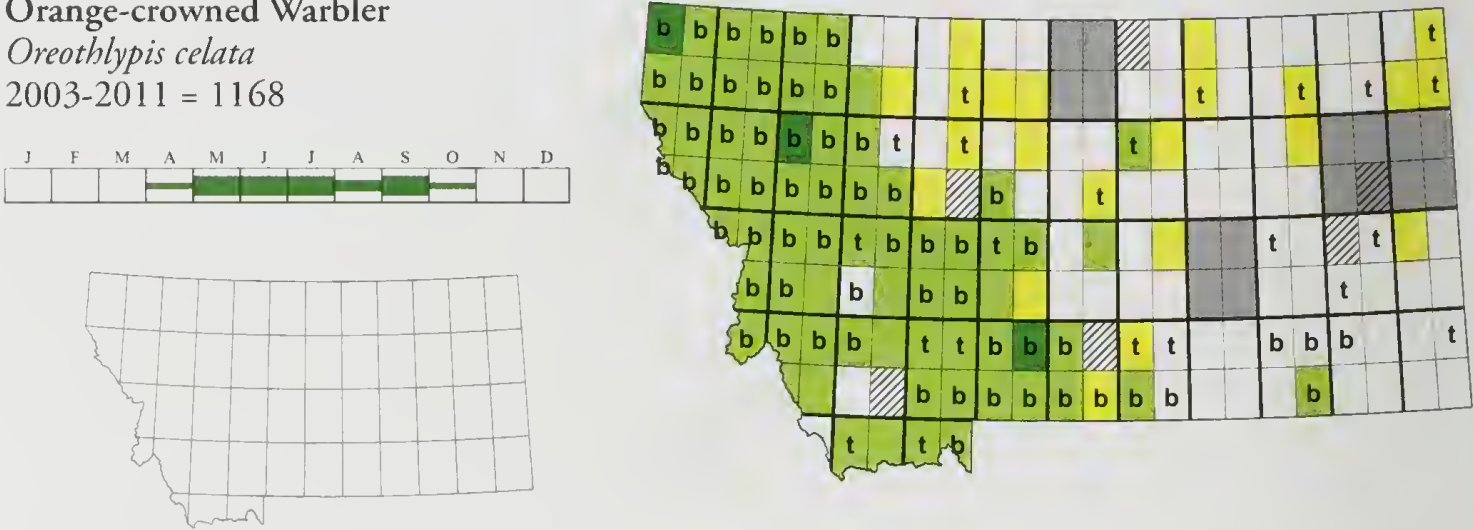

Nashville Warbler

Oreothlypis ruficapilla

2003-2011 = 146
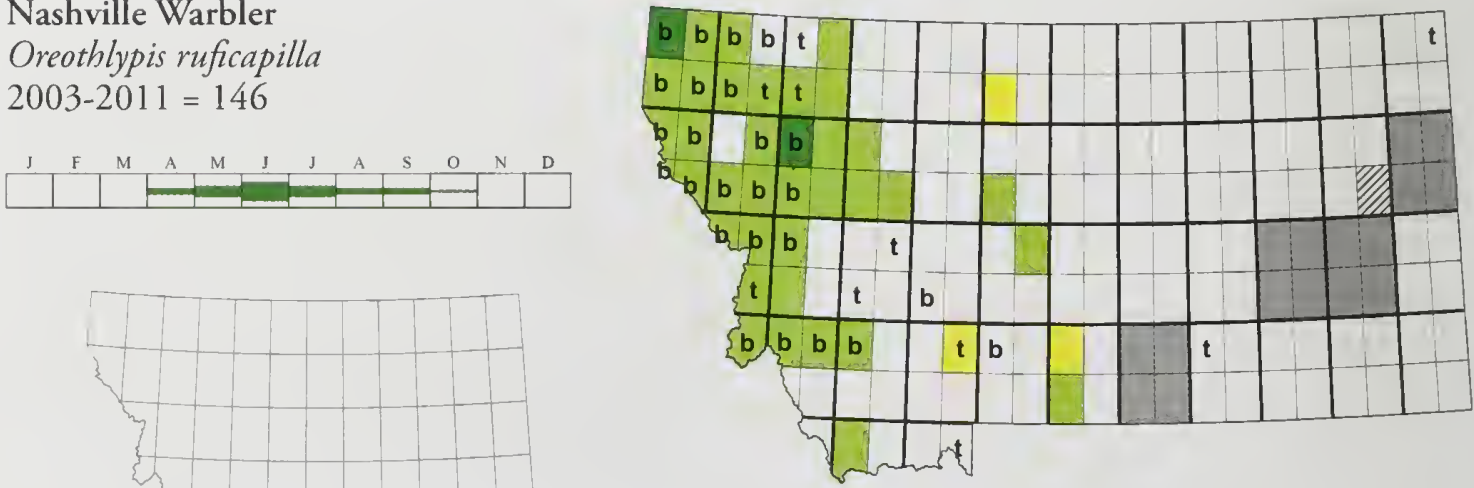
Connecticut Warbler

Oporornis agilis

2003-2011 = $4 \quad$ R1

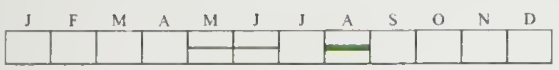
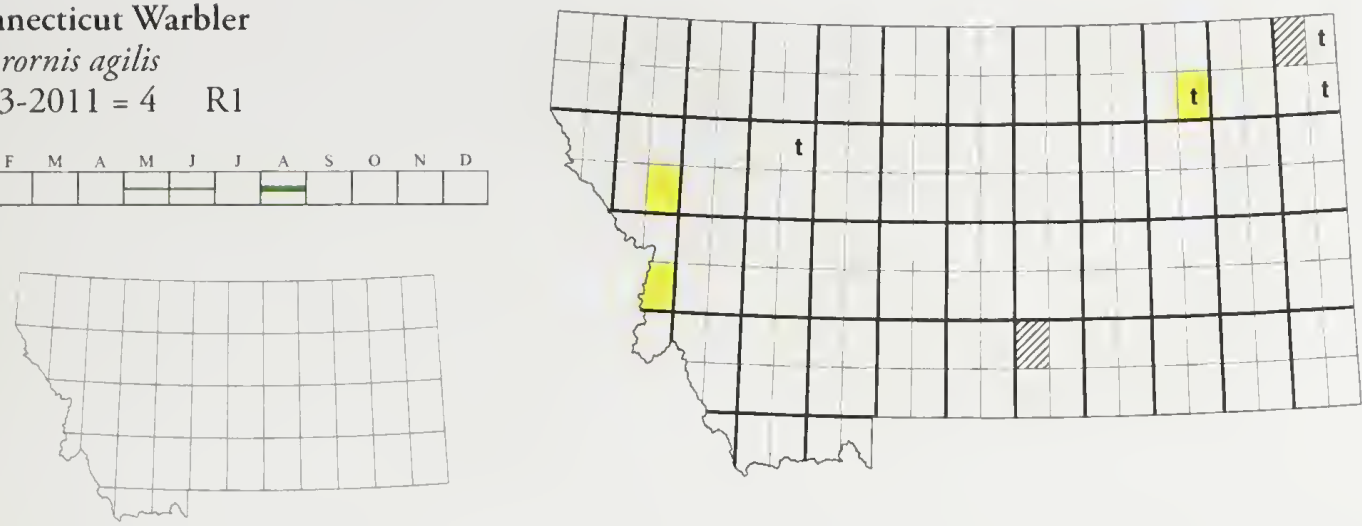

MacGillivray's Warbler

Geothlypis tolmiei

2003-2011 = 2981
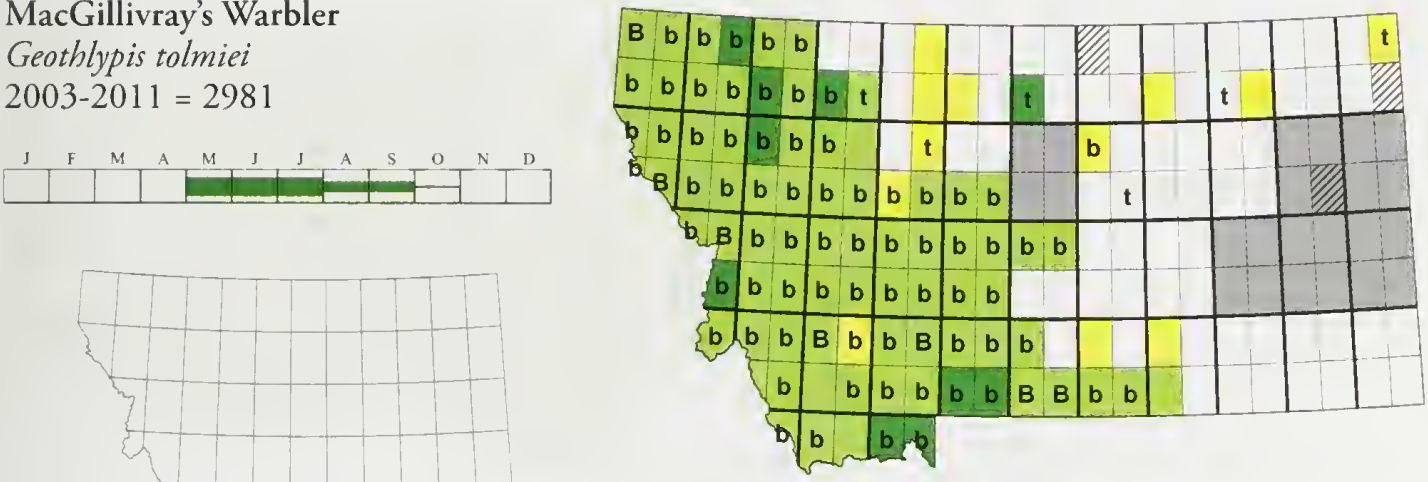

\section{Mourning Warbler}

Geothlypis philadelphia

2003-2011 = 24
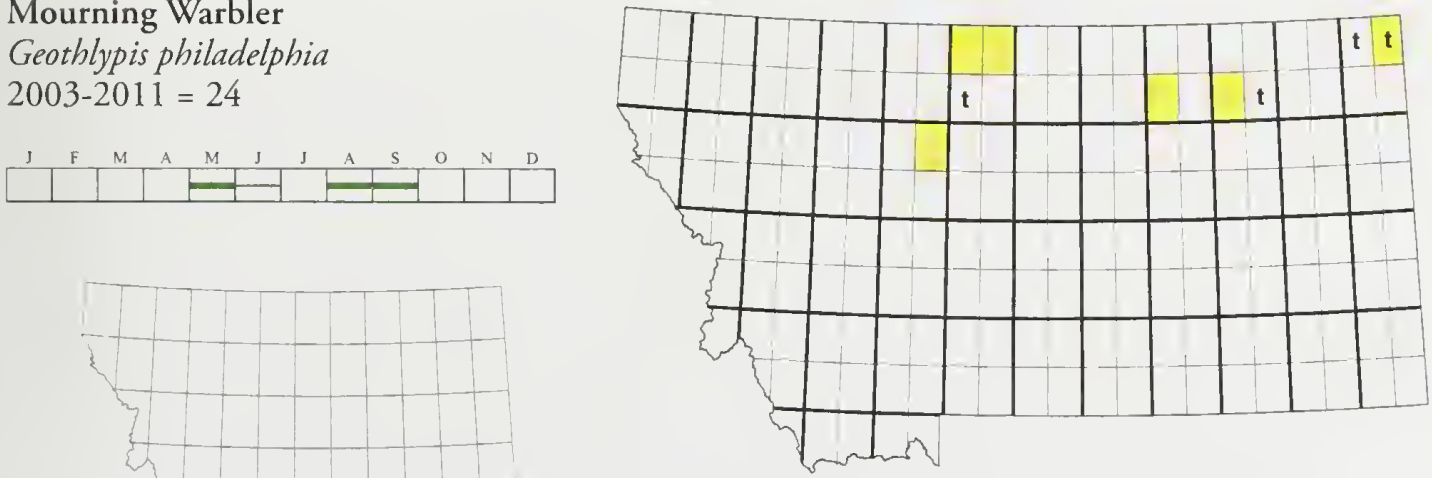
Kentucky Warbler

Geothlypis formosa

2003-2011 = 0 R1

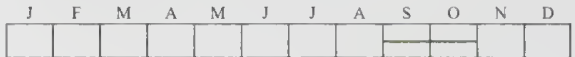

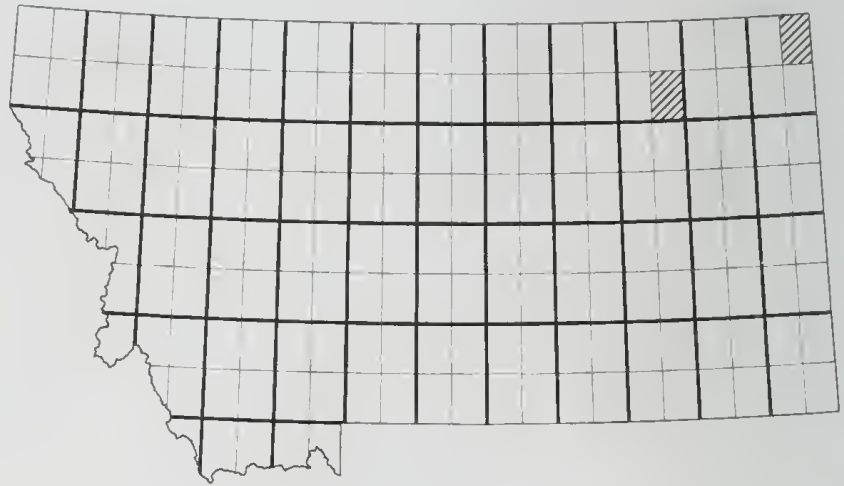

Common Yellowthroat

Geothlypis trichas

2003-2011 = 2058
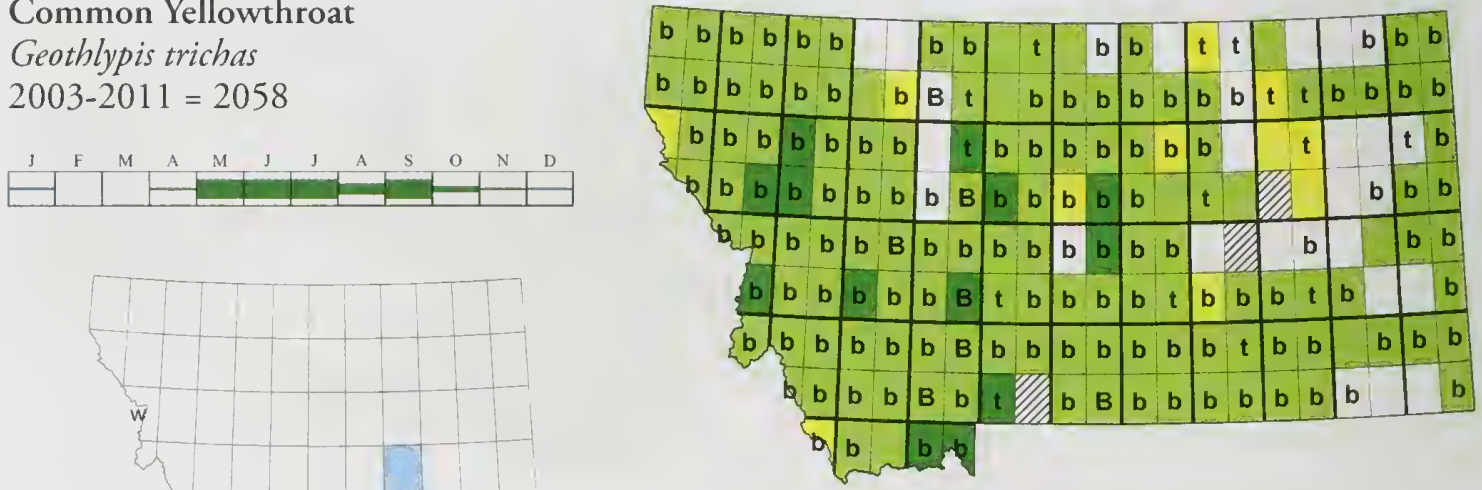

Hooded Warbler

Setophaga citrina

2003-2011 = 5 R1
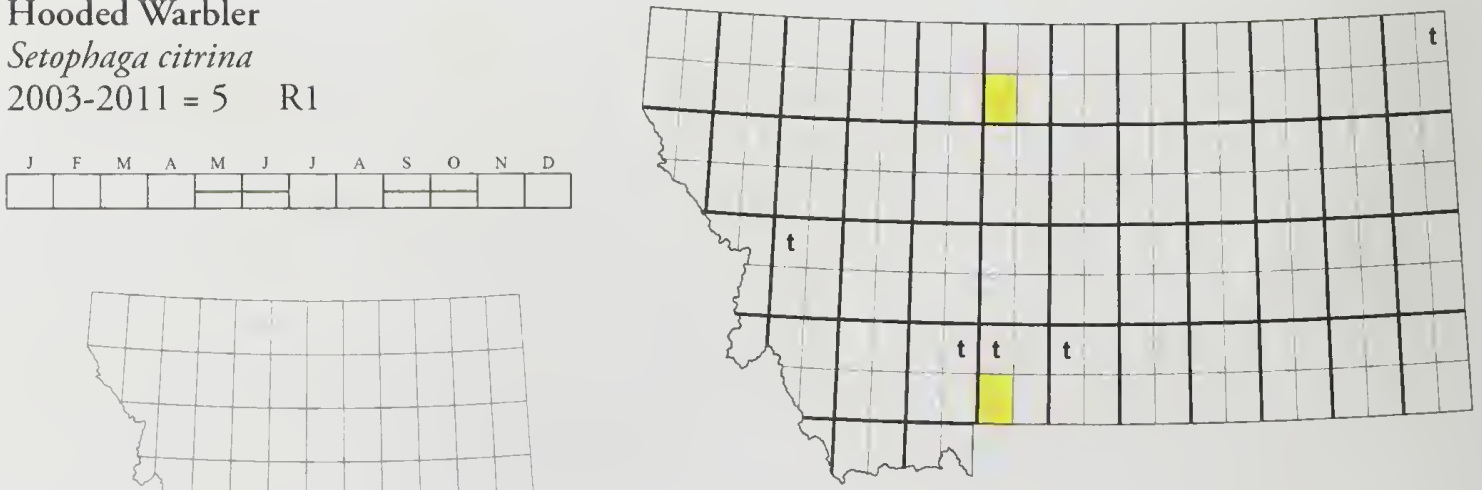
American Redstart

Setophaga ruticilla

2003-2011 = 644

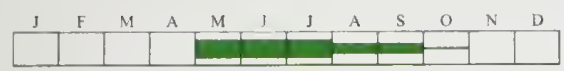

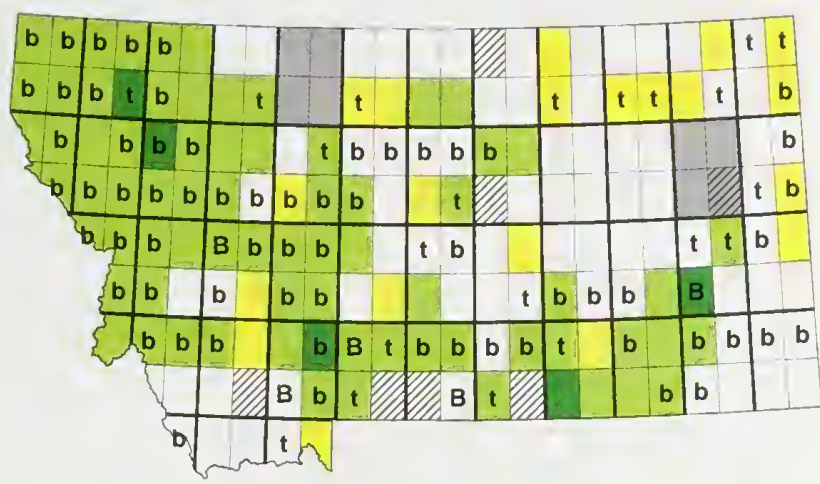

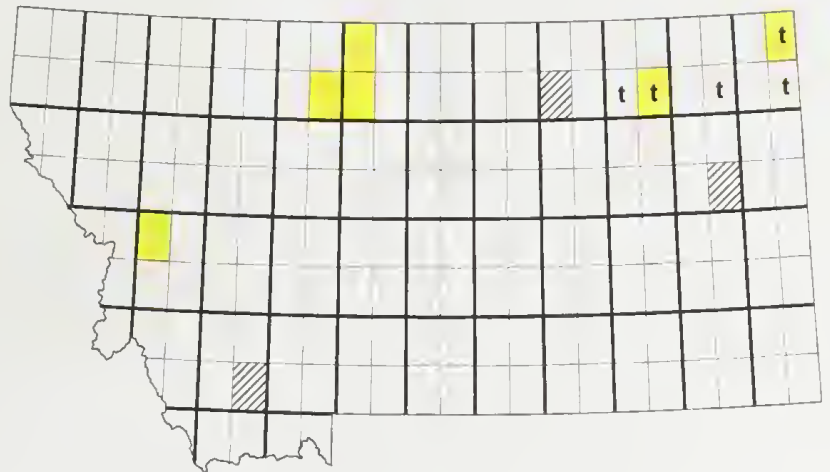

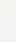

Cape May Warbler

Setophaga tigrina

2003-2011 = 10

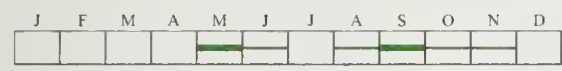


Magnolia Warbler

Setophaga magnolia

2003-2011 = 19

\begin{tabular}{|l|l|l|l|l|l|l|l|l|l|l|l|}
\hline $\mathrm{J}$ & $\mathrm{F}$ & $\mathrm{M}$ & $\mathrm{A}$ & $\mathrm{M}$ & $\mathrm{J}$ & $\mathrm{J}$ & $\mathrm{A}$ & $\mathrm{S}$ & $\mathrm{O}$ & $\mathrm{N}$ & $\mathrm{D}$ \\
& & & & & & & & & & & \\
\hline
\end{tabular}
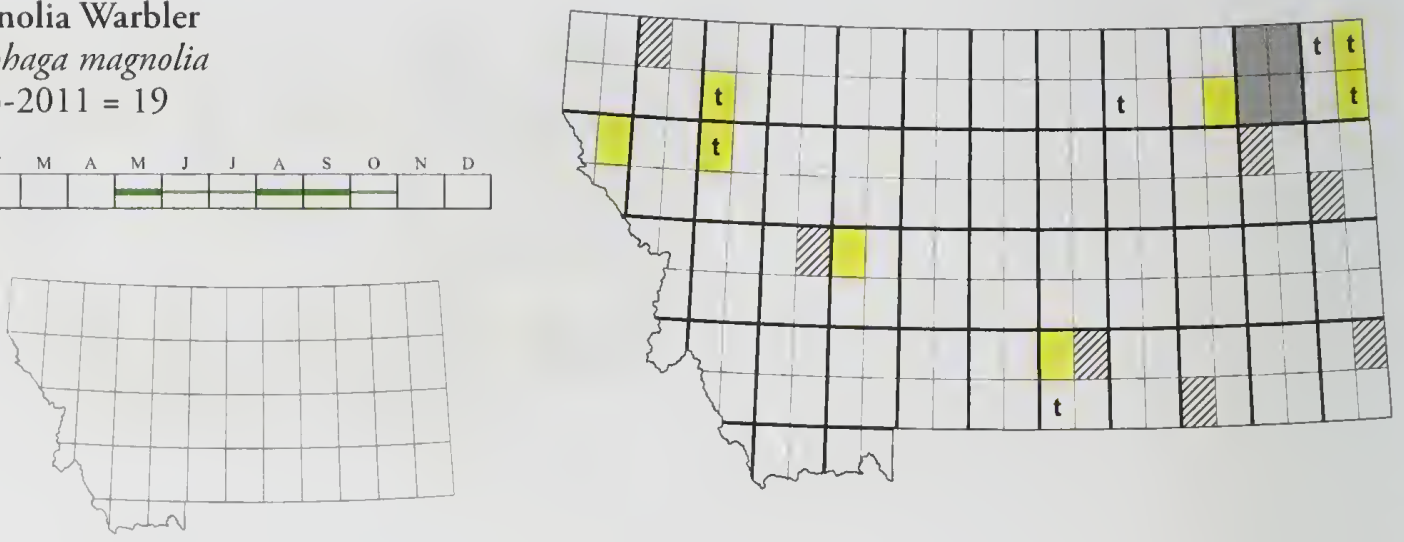

Bay-breasted Warbler

Setophaga castanea

2003-2011 = $6 \quad$ R2

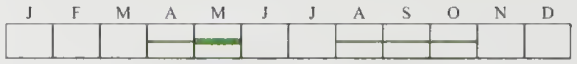

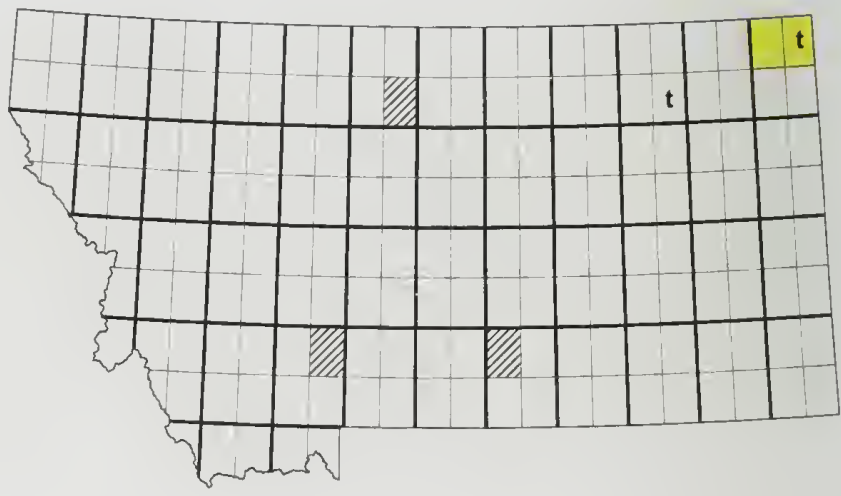

Blackburnian Warbler

Setophaga fusca

2003-2011 = $12 \quad$ R2
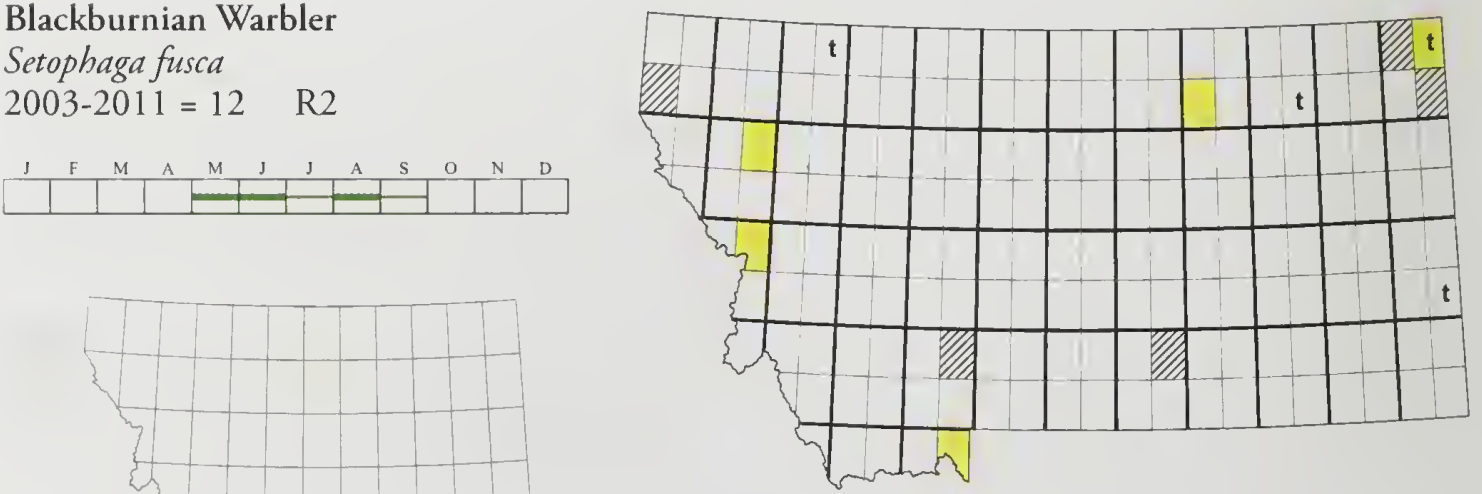
Yellow Warbler

Setophaga petechia

2003-2011 = 6481

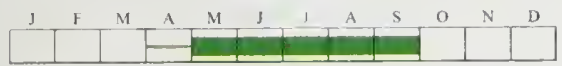
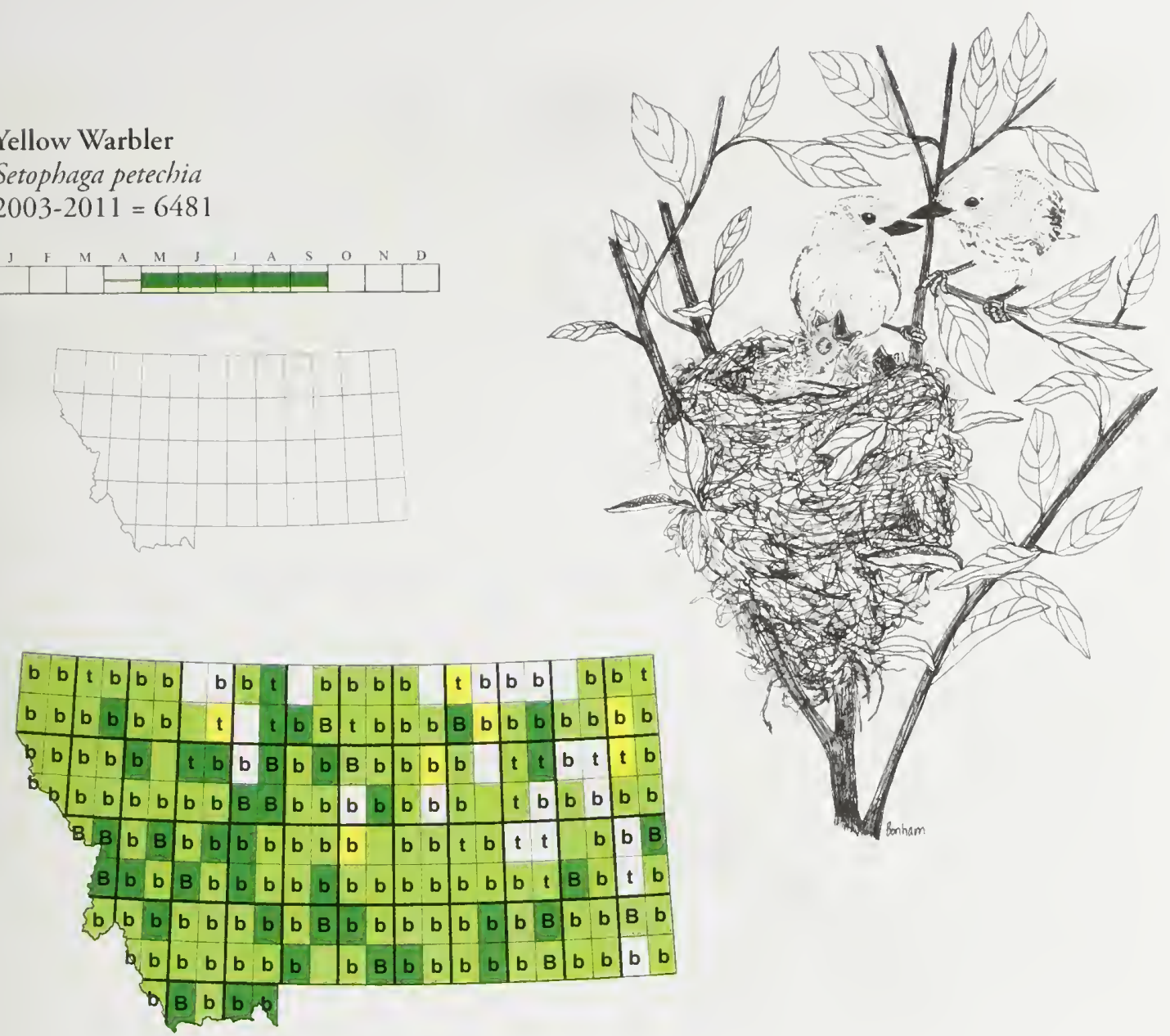

Chestnut-sided Warbler

Setophaga pensylvanica

2003-2011 = 7
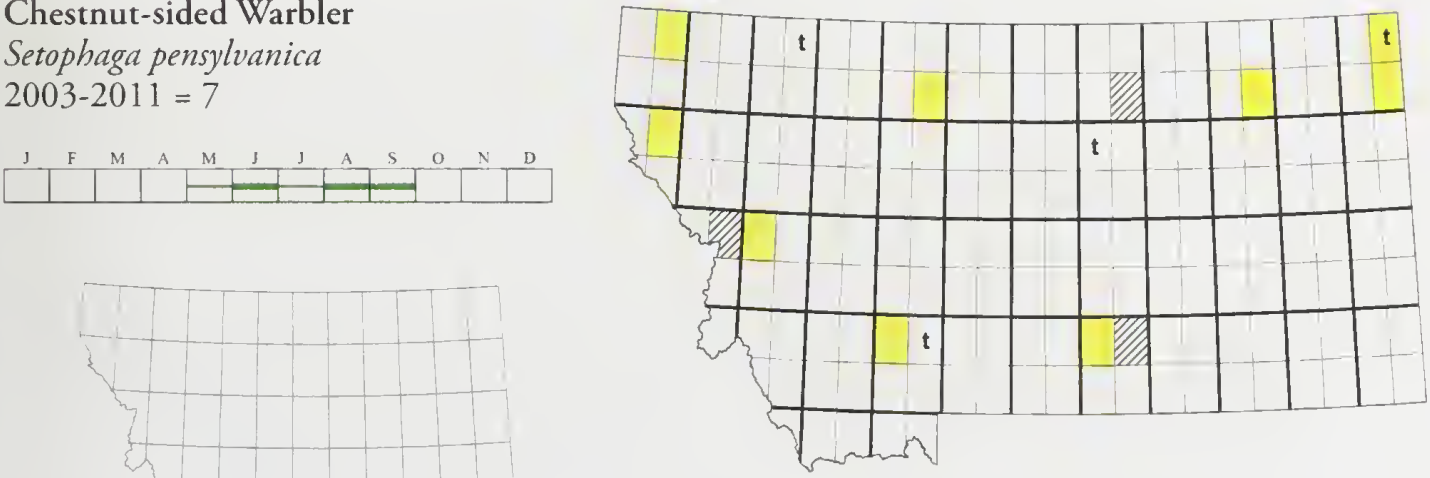
Blackpoll Warbler

Setophaga striata

$2003-2011=46$

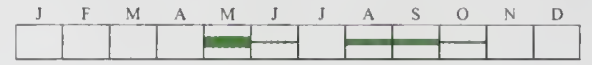
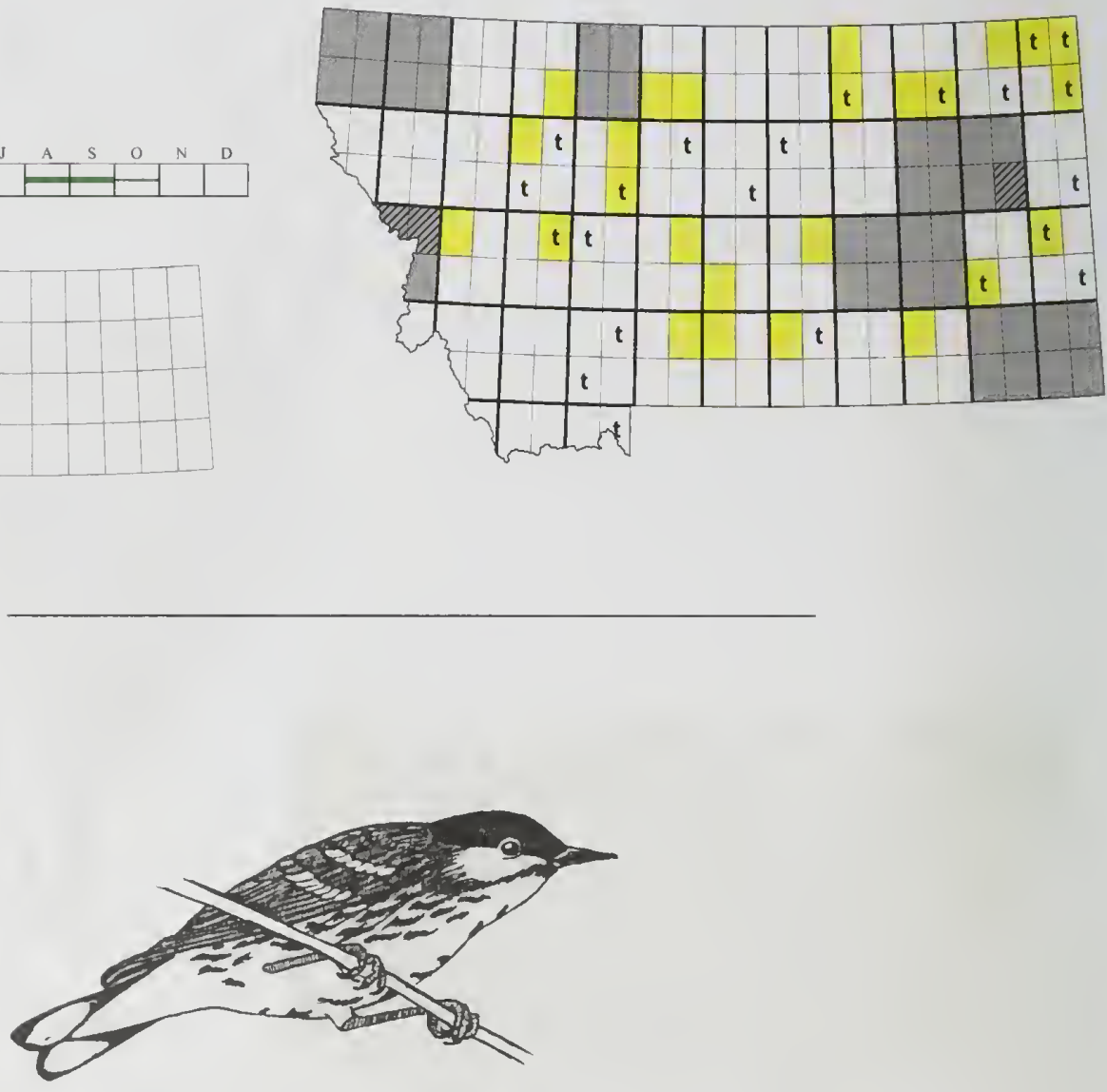

Black-throated Blue Warbler

Setophaga caerulescens

2003-2011 = 11

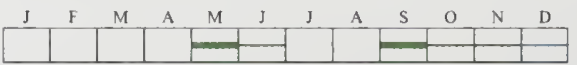

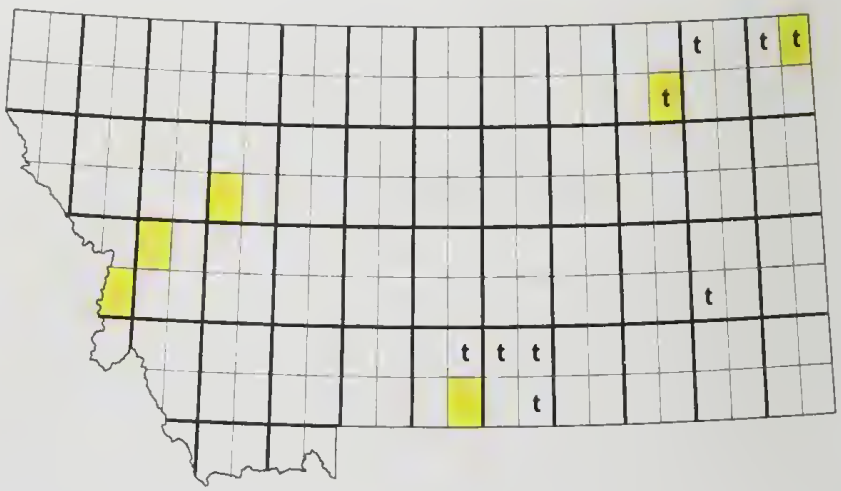


Palm Warbler

Setophaga palmarum

2003-2011 = 15
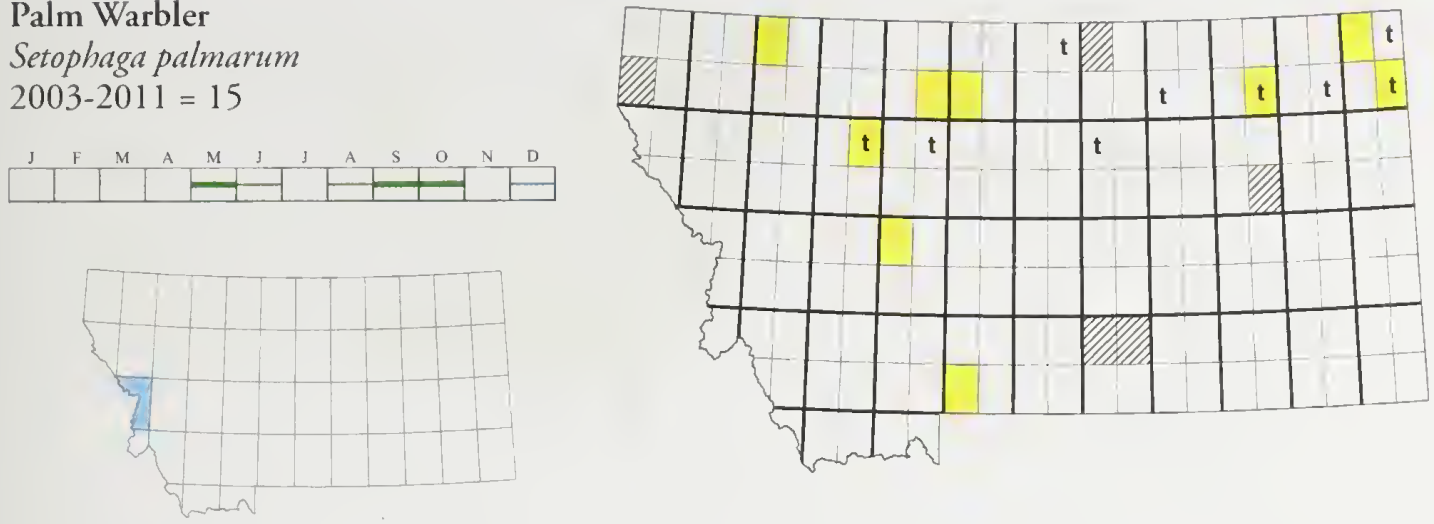

Pine Warbler

Setophaga pinus

2003-2011 = 3 R1
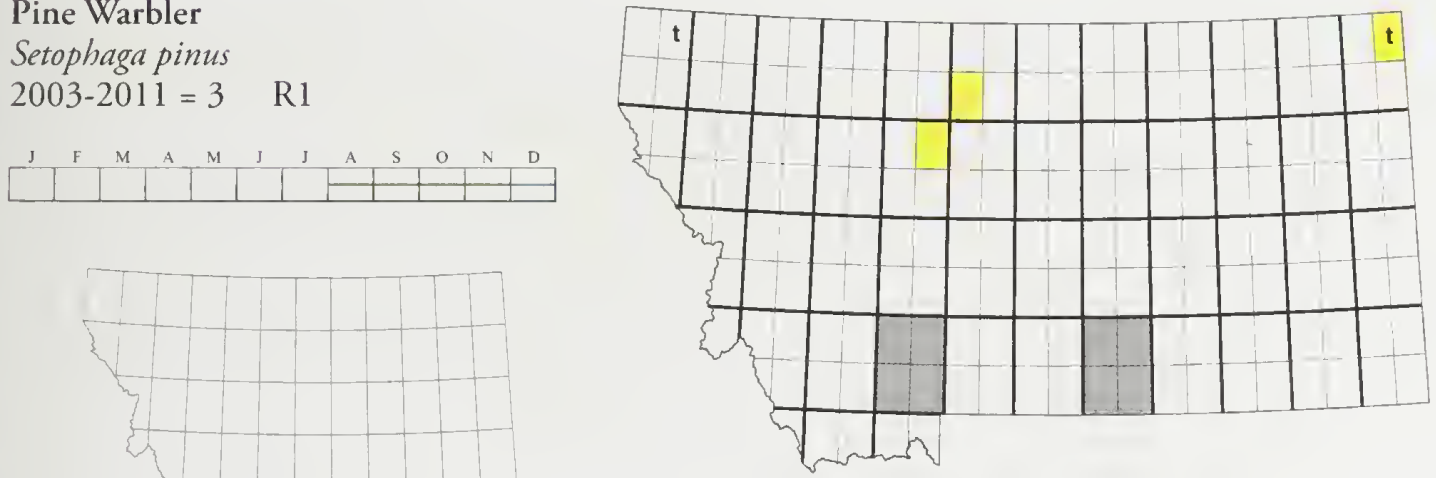

Yellow-rumped Warbler

Setophaga coronata

2003-2011 = 8192
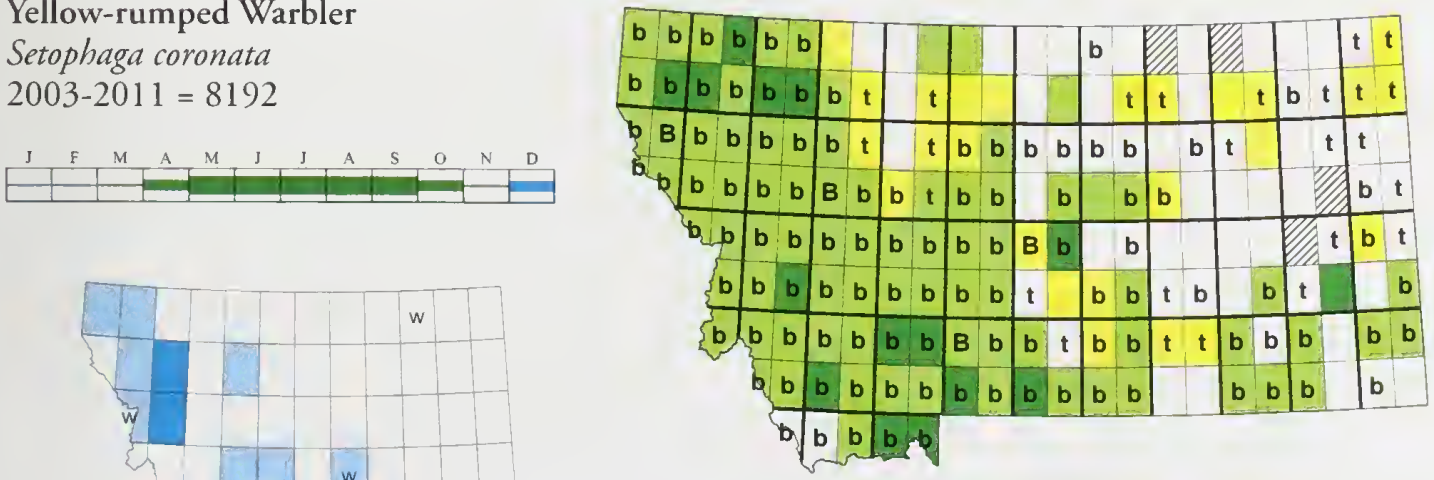
Audubon's Warbler

Setophaga coronata auduboni

2003-2011 = 2143

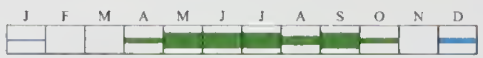

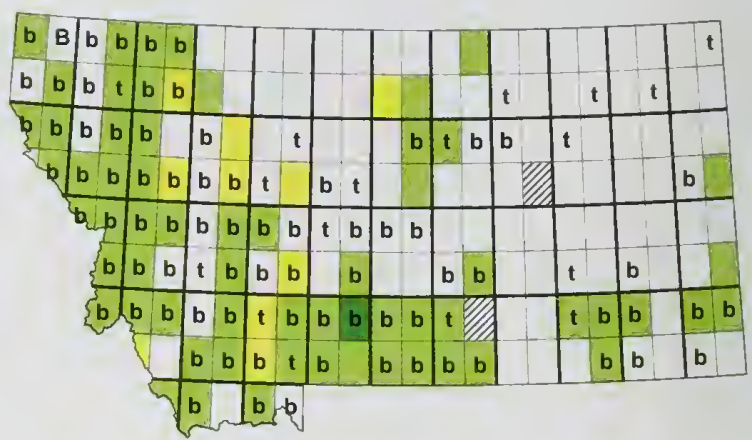

Myrtle Warbler

Setophaga coronata coronata

2003-2011 = 57
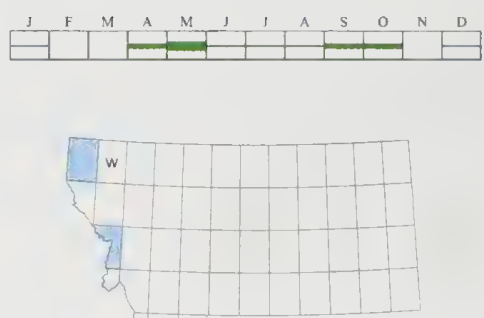

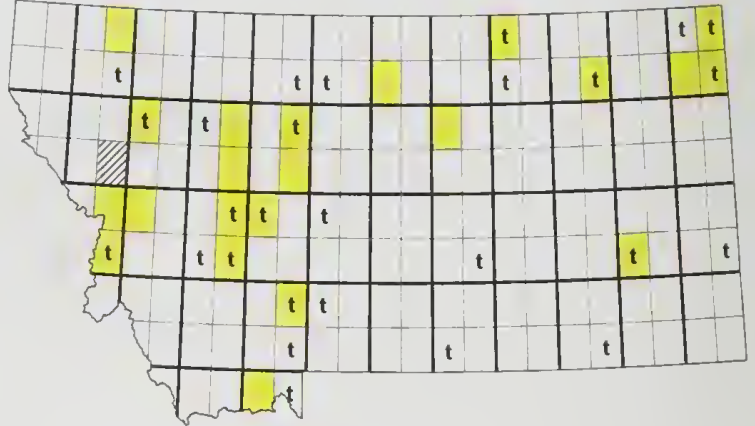

Yellow-throated Warbler

Setophaga dominica

2003-2011 = 2 R1

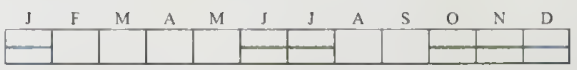

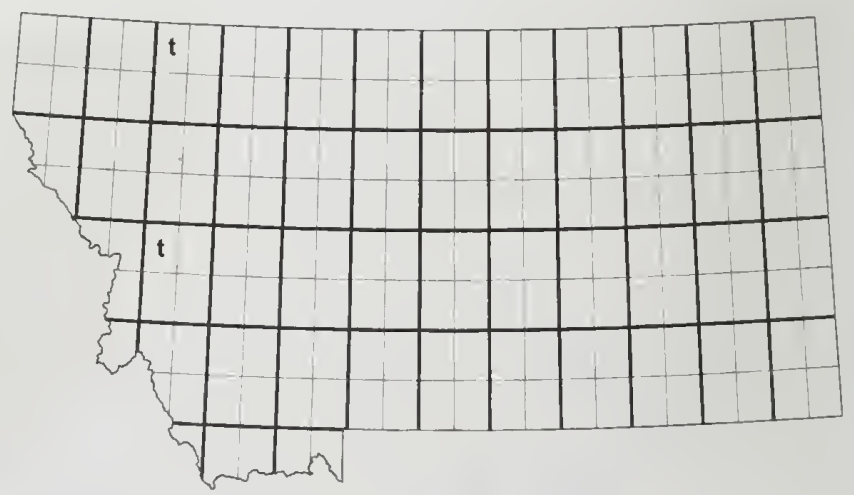


Prairie Warbler

Setophaga discolor

2003-2011 = 2 R1

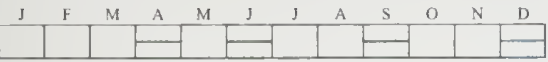

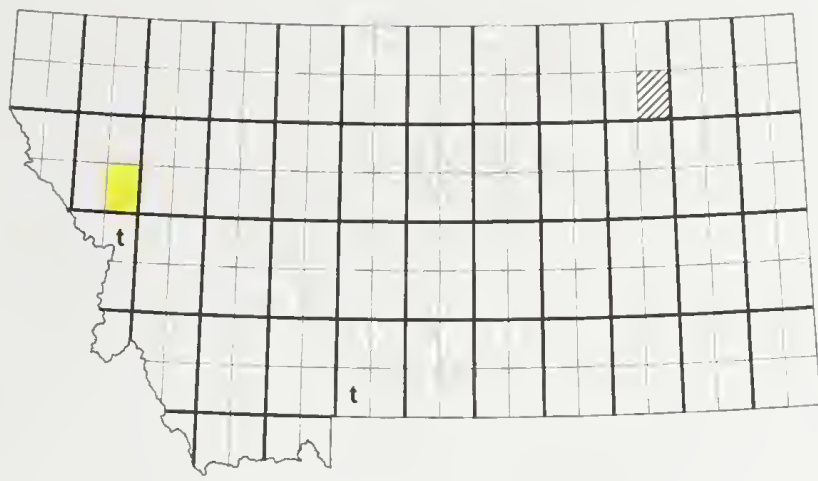

Black-throated Gray Warbler

Setophaga nigrescens

2003-2011 = $3 \quad \mathrm{R} 1$

$\left.\left.\left.\left.\square^{B} H^{M}{ }^{A} H^{M} L^{\prime} H^{\prime}\right|^{A}\right|^{S}\right|^{O}\right|^{N} H^{D}$

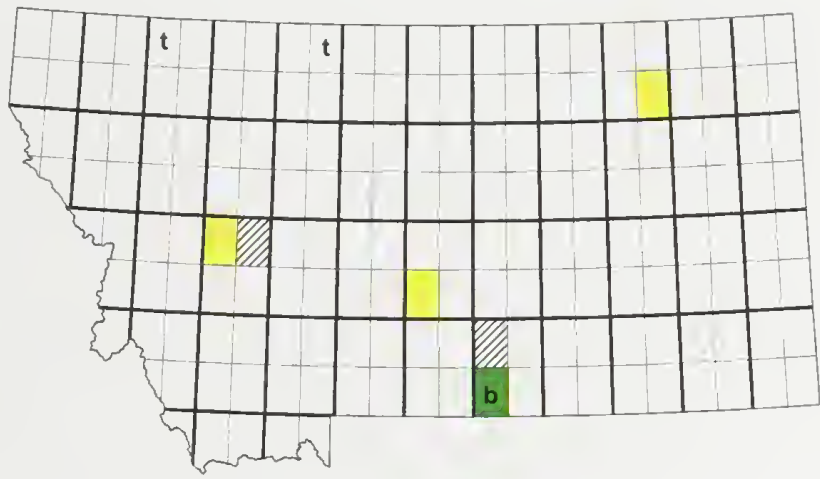

Townsend's Warbler

Setophaga townsendi

2003-2011 = 3118
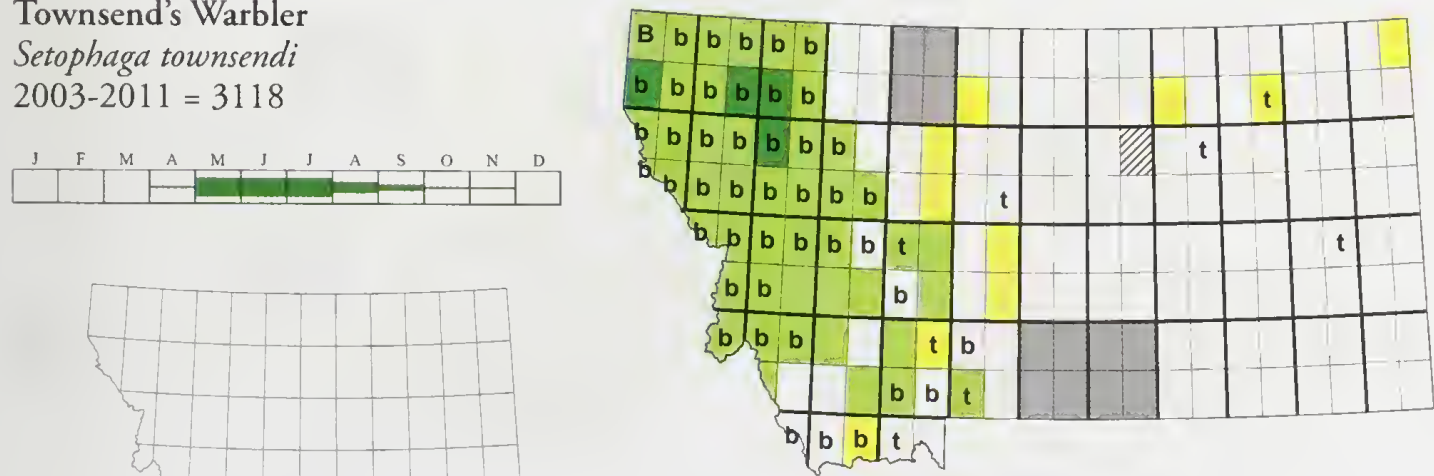
Black-throated Green Warbler

Setophaga virens

2003-2011 = 9 R2

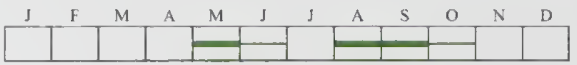
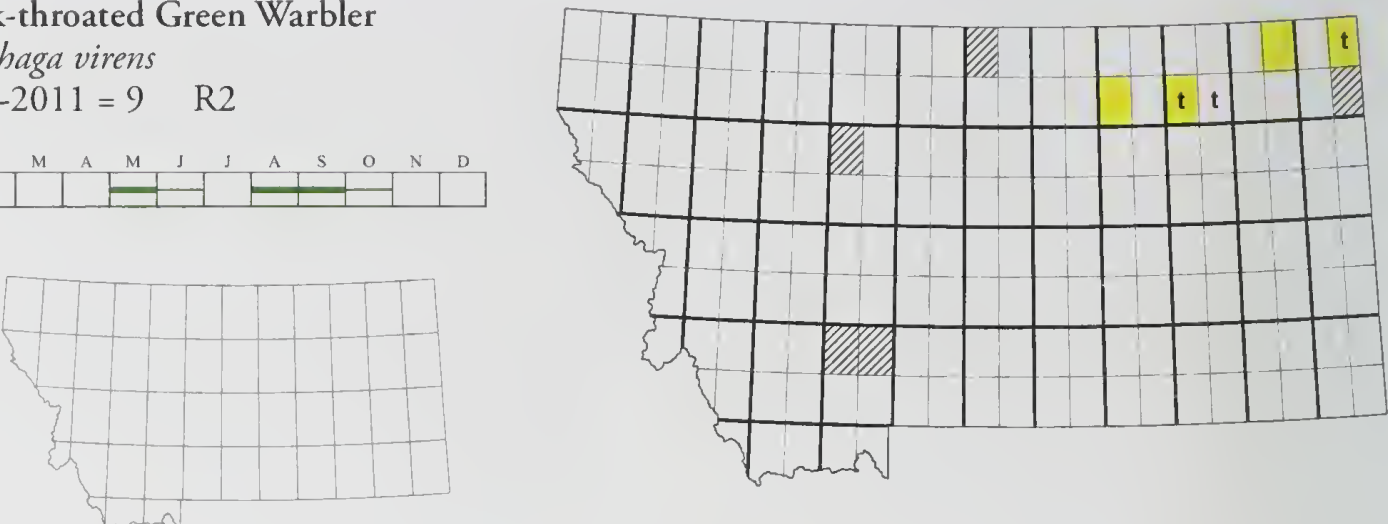

Canada Warbler

Cardellina canadensis

2003-2011 = 21

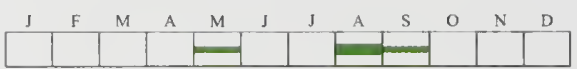

Wilson's Warbler

Cardellina pusilla

2003-2011 = 701
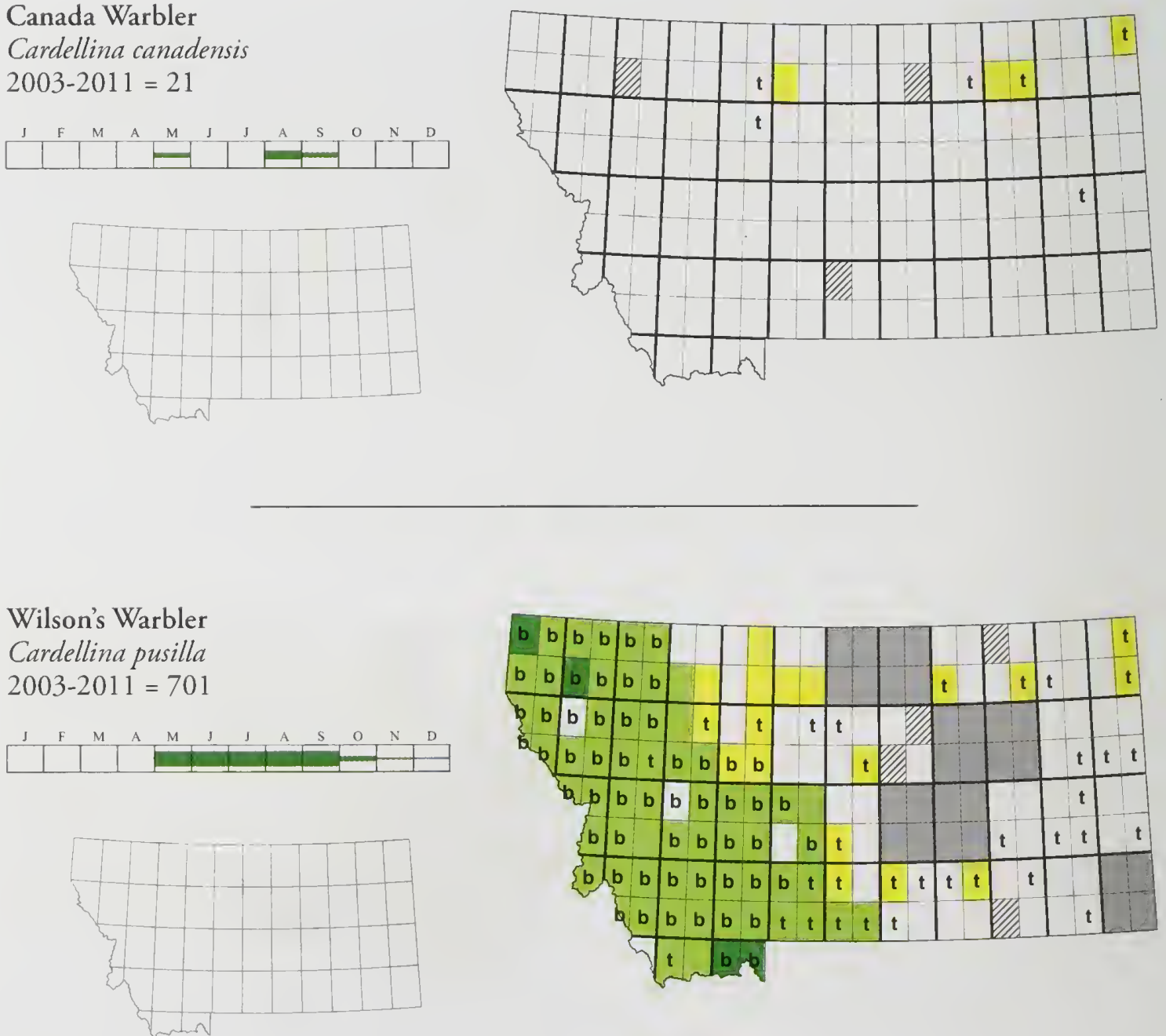

(4) 


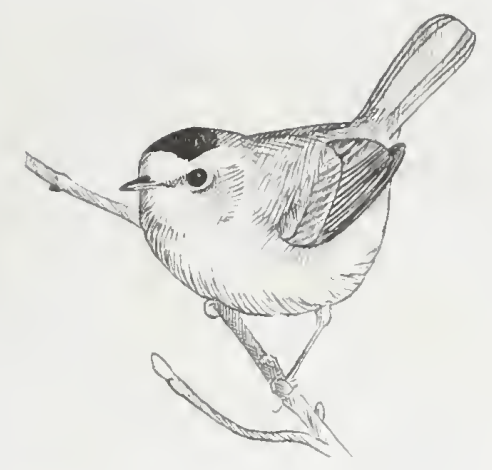

Painted Redstart

Myioborus pictus

2003-2011 = 0 Rl

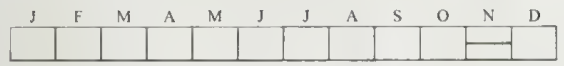

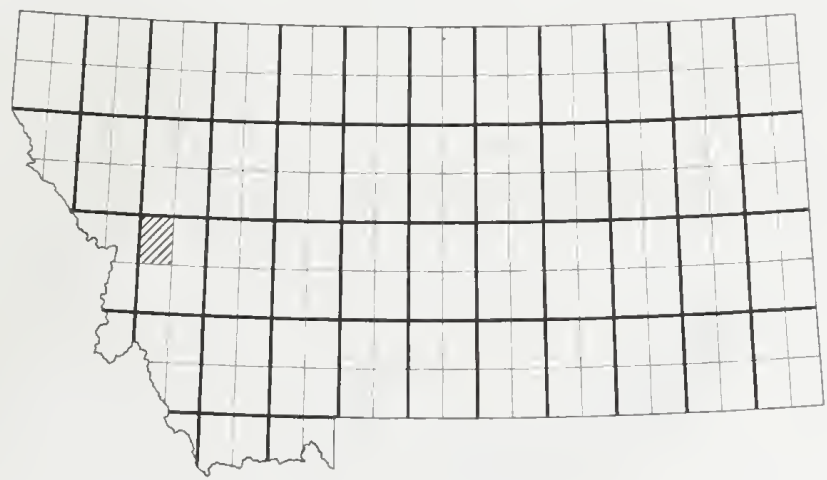

Yellow-breasted Chat

Icteria virens

2003-2011 = 696
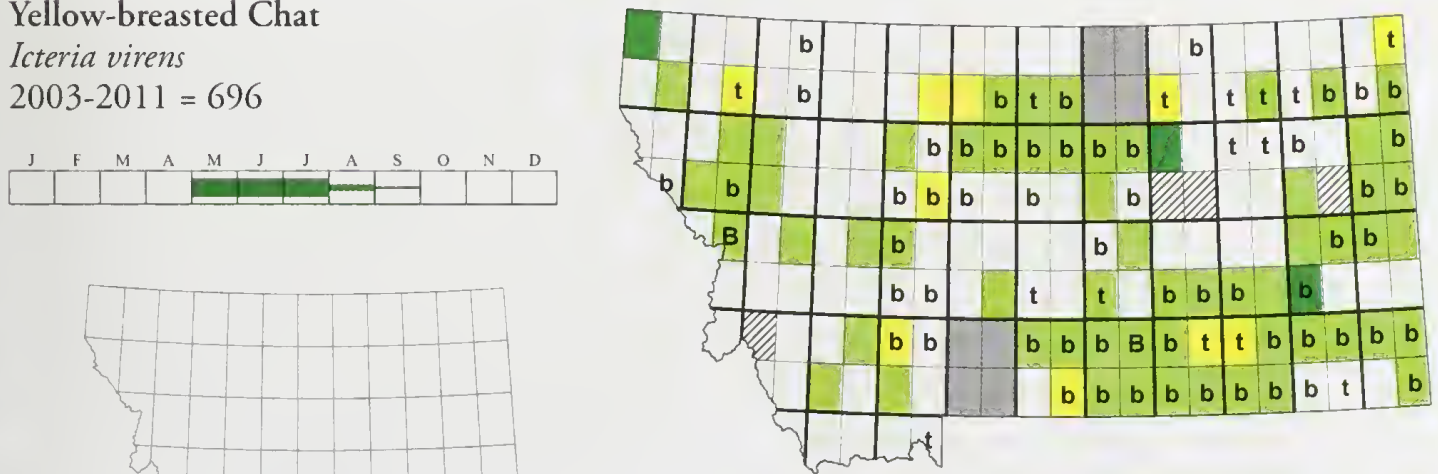
Emberizidae

Green-tailed Towhee

Pipilo chlorurus

$2003-2011=442 \quad$ SOC
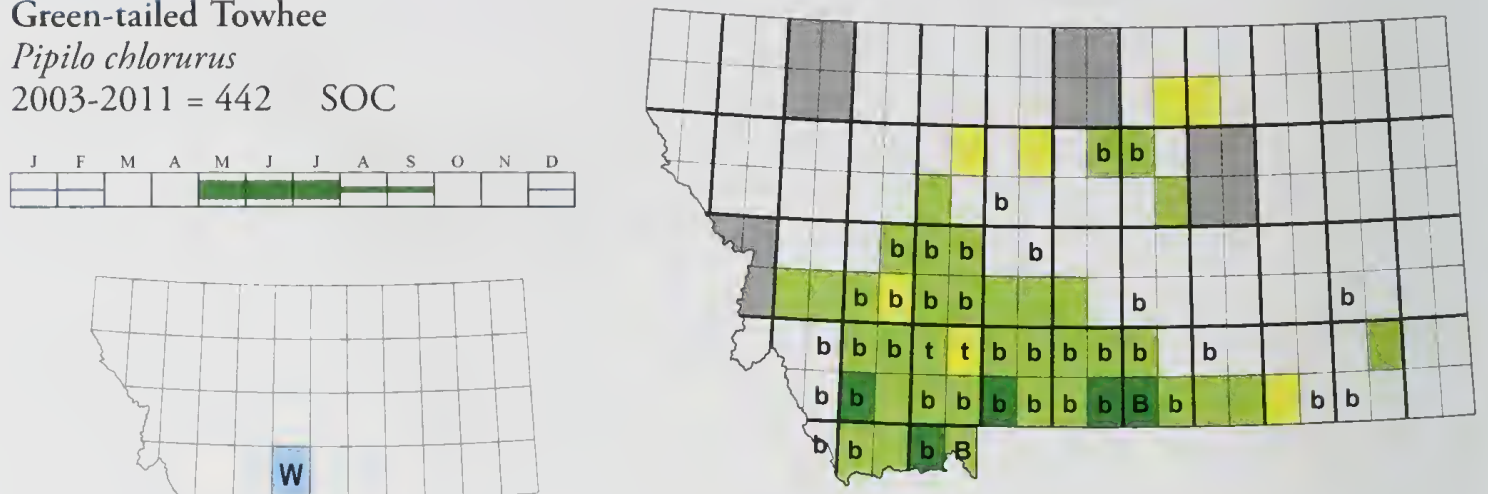

Spotted Towhee

Pipilo maculatus

2003-2011 = 2568
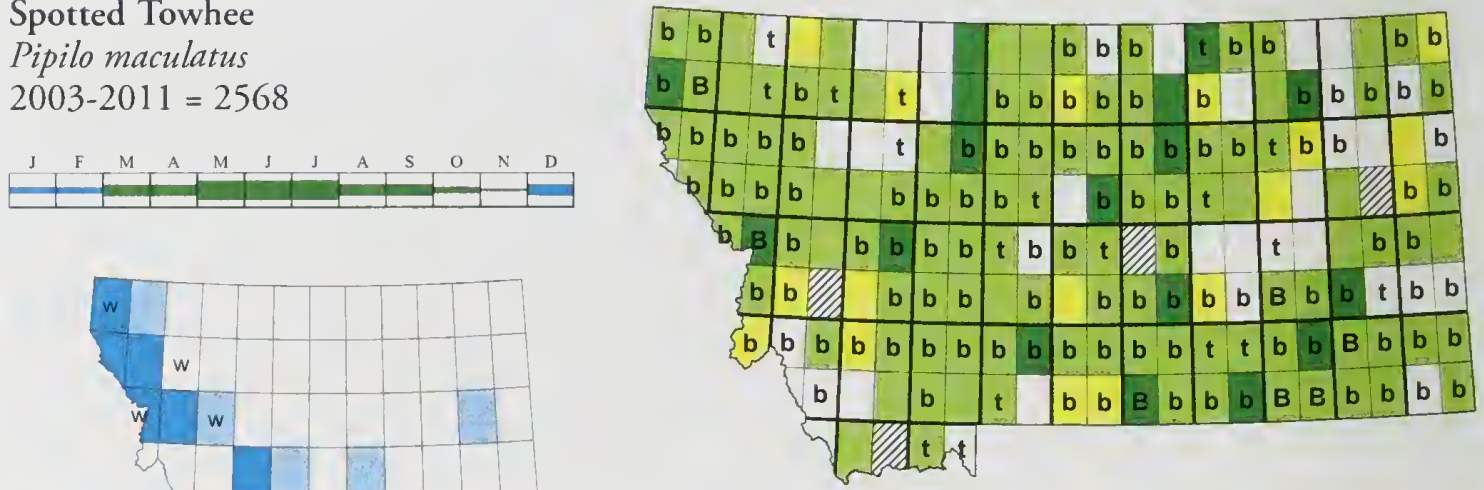

Eastern Towhee

Pipilo erythrophthalmus

2003-2011 = $1 \quad$ R1

\begin{tabular}{|l|l|l|l|l|l|l|l|l|l|}
\hline & $\mathrm{F}$ & $\mathrm{M}$ & $\mathrm{A}$ & $\mathrm{N}$ & $\mathrm{J}$ \\
\hline
\end{tabular}

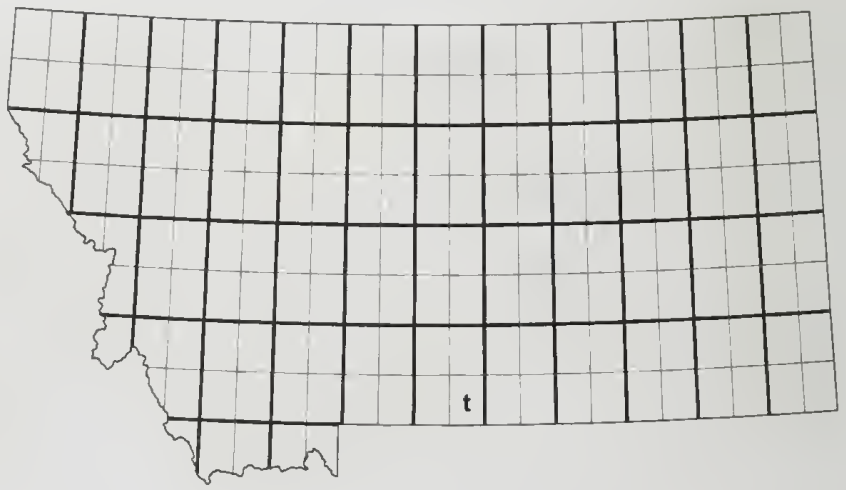


American Tree Sparrow

Spizella arborea

2003-2011 $=281$
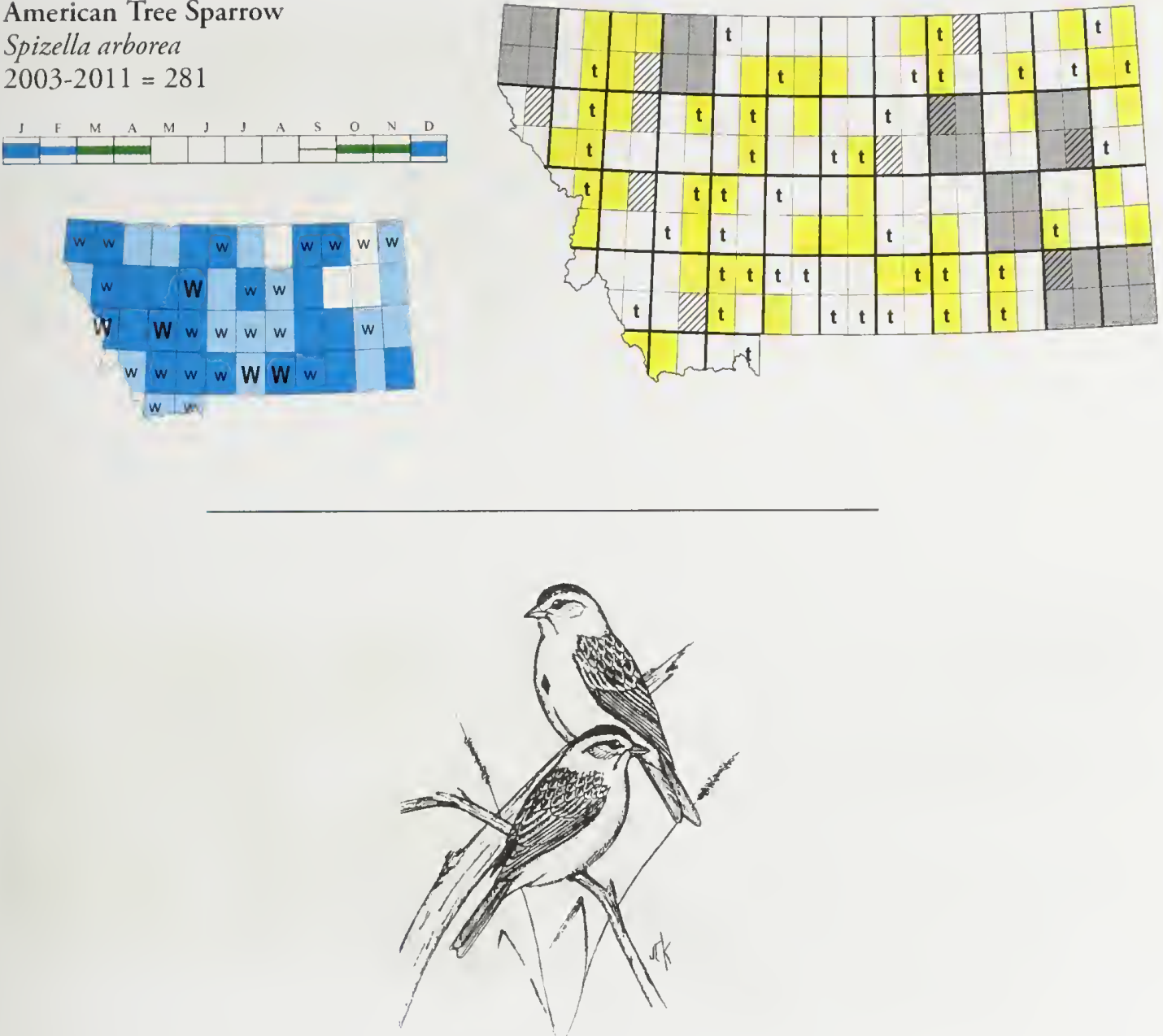

Chipping Sparrow

Spizella passerina

2003-2011 = 8067

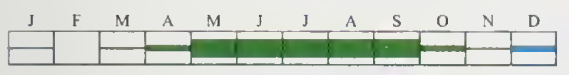

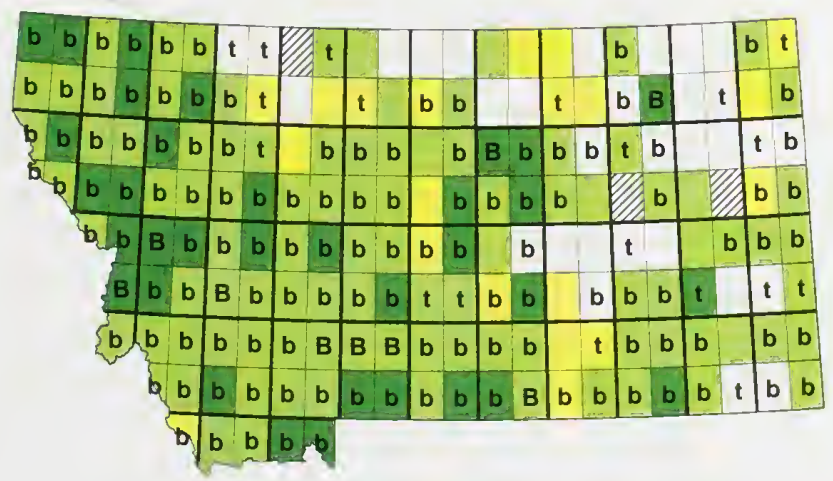


Clay-colored Sparrow

Spizella pallida

2003-2011 = 740
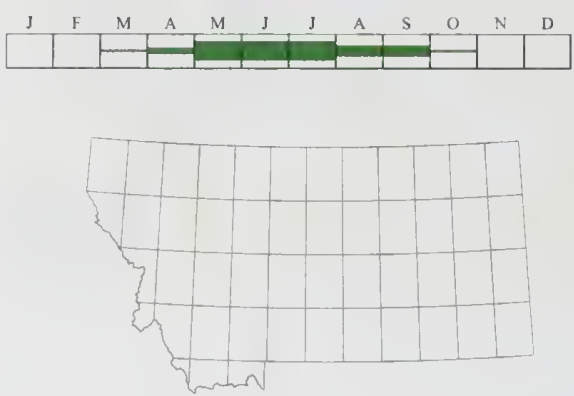

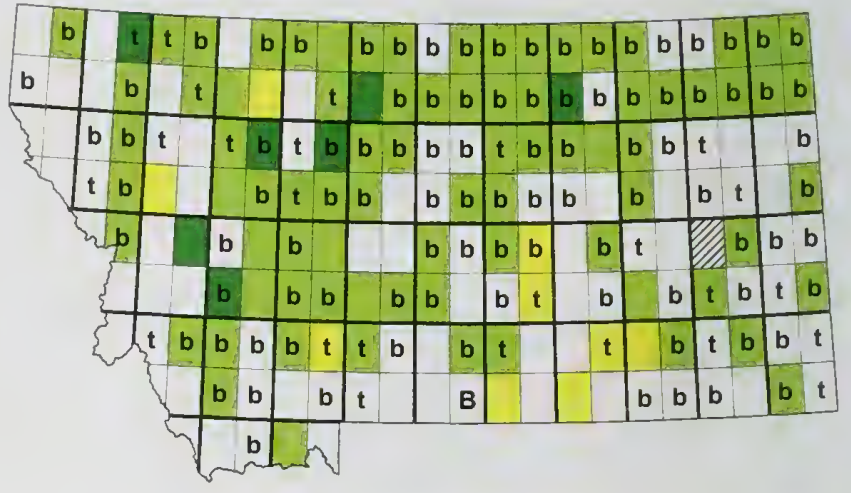

Brewer's Sparrow

Spizella breweri

$2003-2011=2658 \quad$ SOC

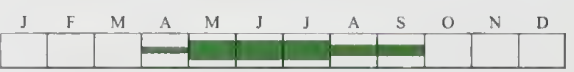

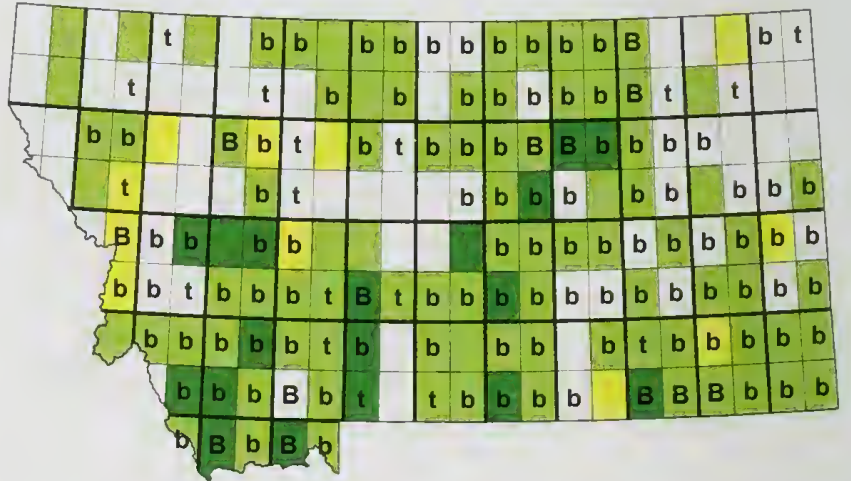

Timberline Sparrow

Spizella breweri taverneri 2003-2011 = 0
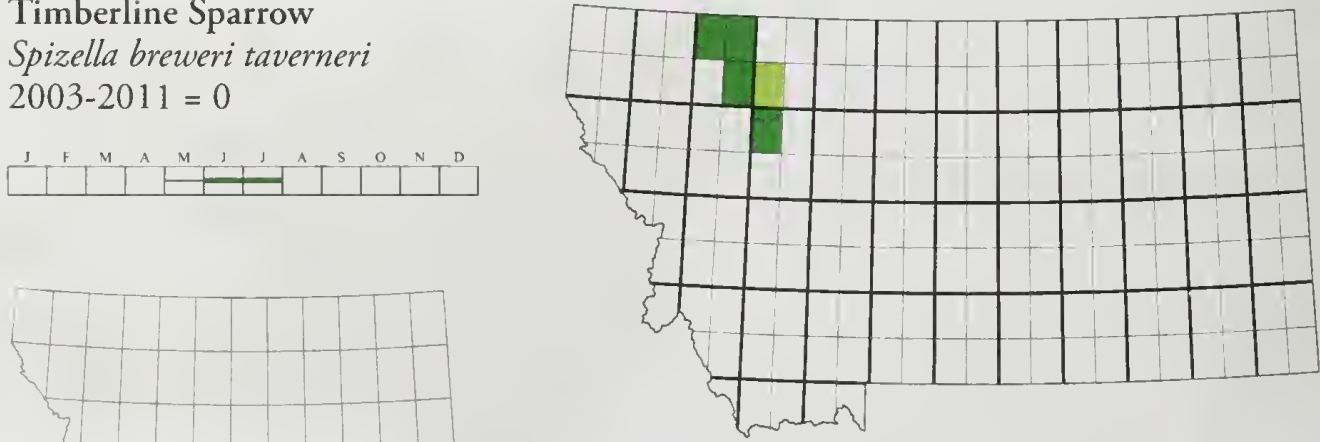
Field Sparrow

Spizella pusilla

2003-2011 = 518

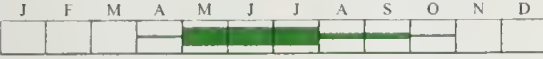
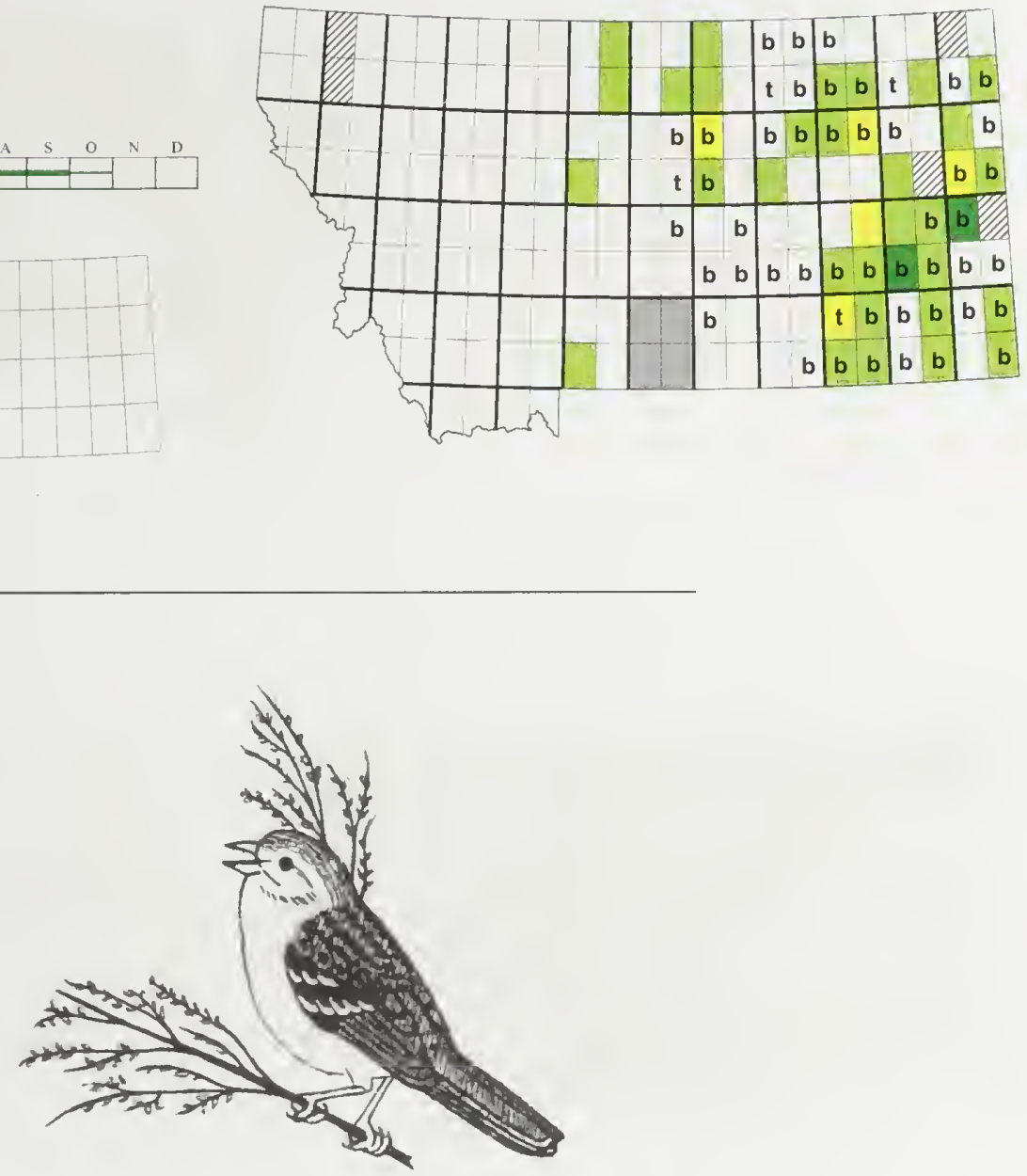

Vesper Sparrow

Pooecetes gramineus

2003-2011 = 9150
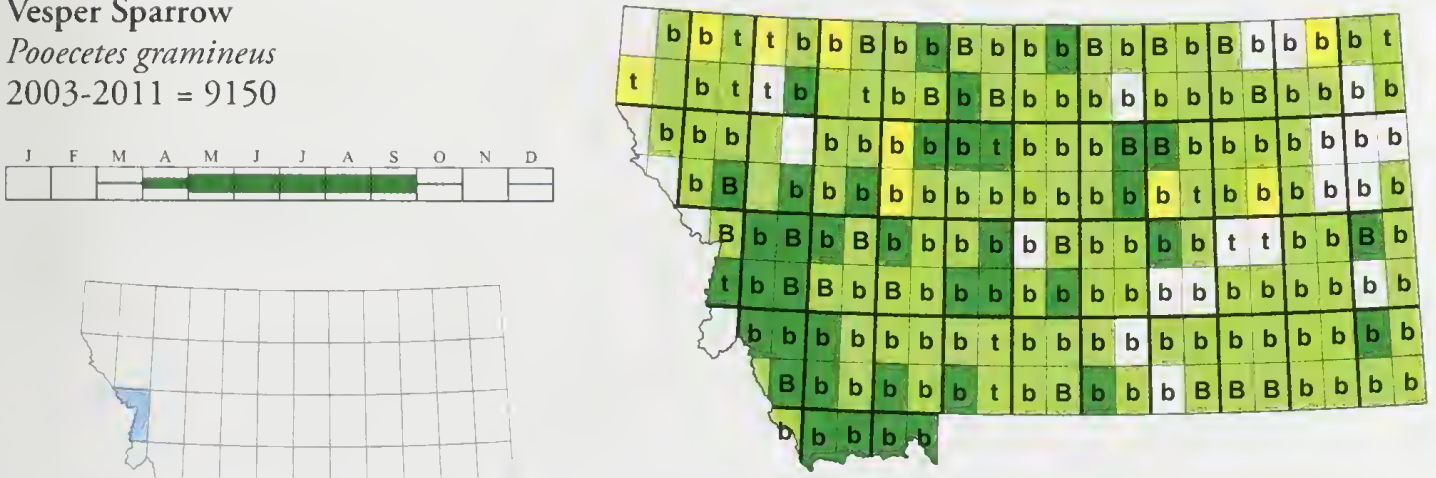
Lark Sparrow

Chondestes grammacus

2003-2011 = 2272

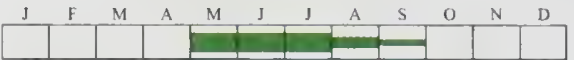
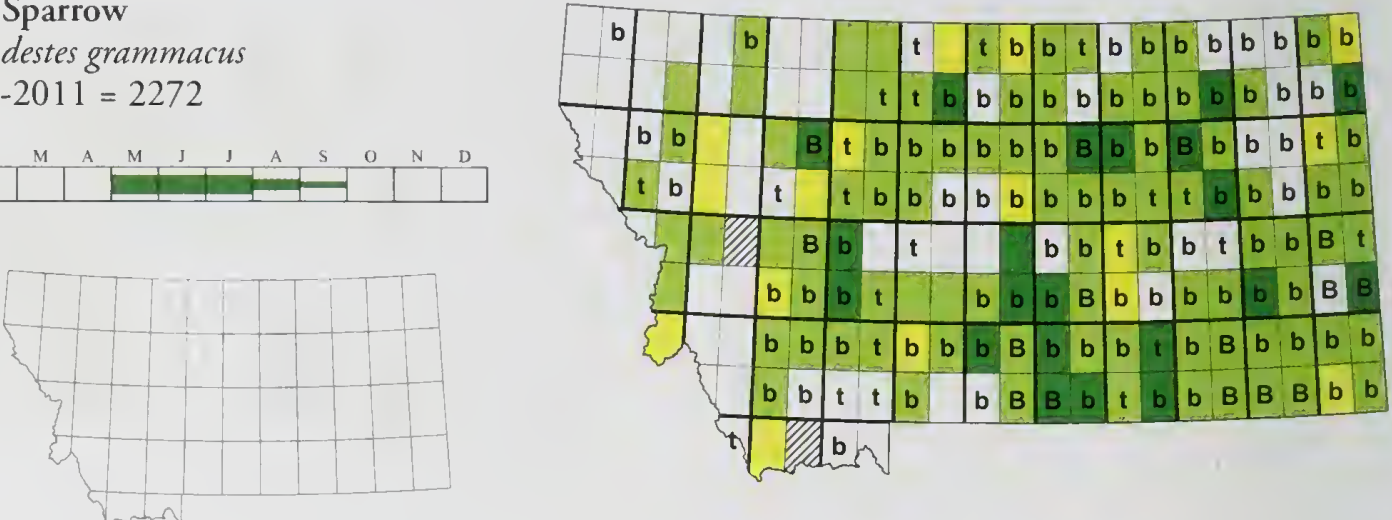

Black-throated Sparrow

Amphispiza bilineata

2003-2011 = $2 \quad$ R1

\begin{tabular}{|l|l|l|l}
$\mathrm{J}$ & $\mathrm{F}$ & $\mathrm{A}$ & $\mathrm{M}$ \\
\hline & & & $\mathrm{J}$ \\
\end{tabular}

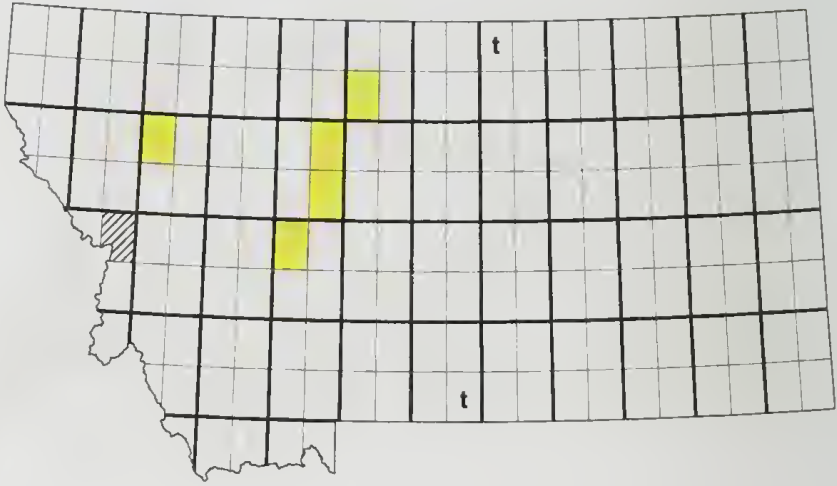

Sage Sparrow

Amphispiza belli

2003-2011=12 SOC R2
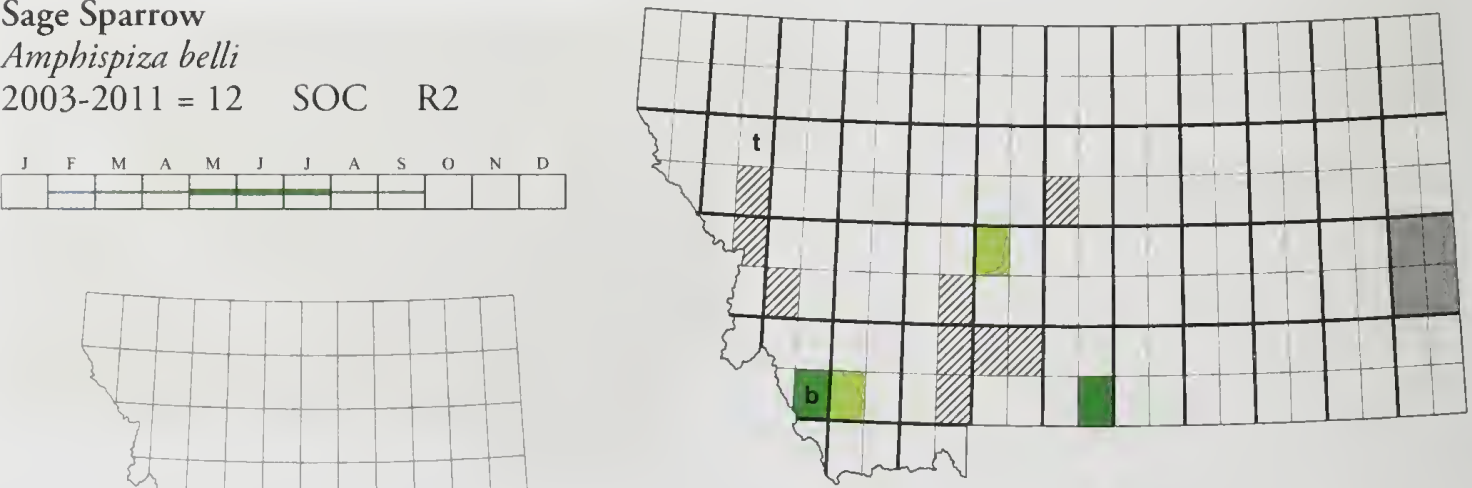
Lark Bunting

Calamospiza melanocorys

2003-2011 = 4554

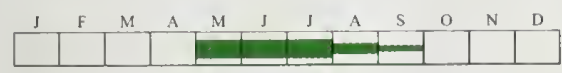

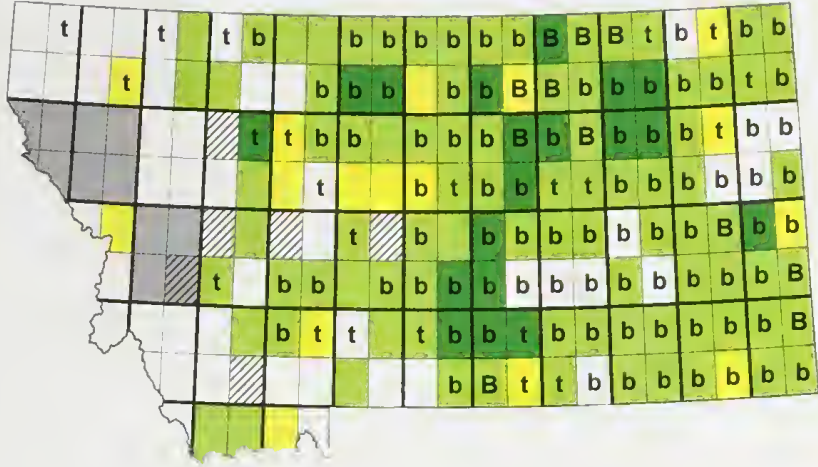

Savannah Sparrow

Passerculus sandwichensis

2003-2011 = 3693
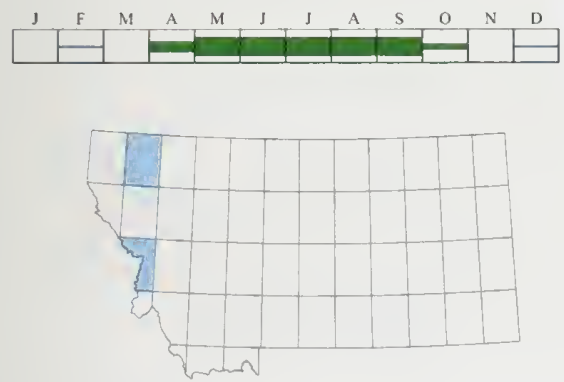

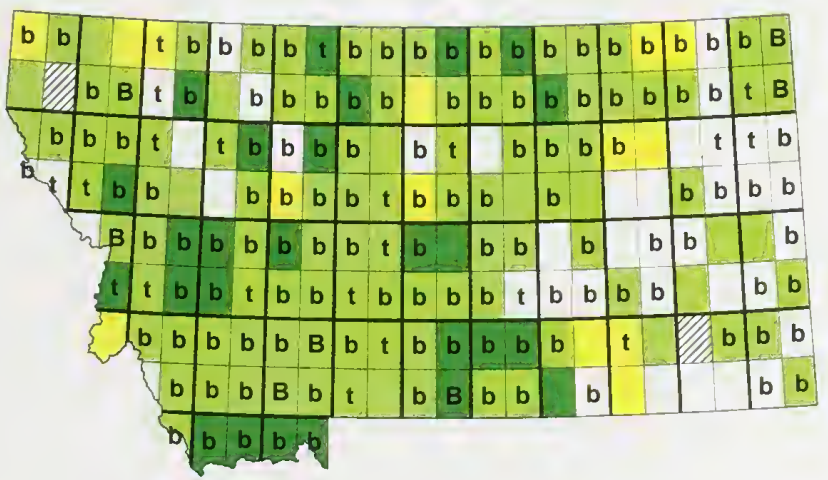

Grasshopper Sparrow

Ammodramus savannarum

2003-2011 = 2711 SOC

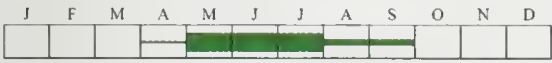

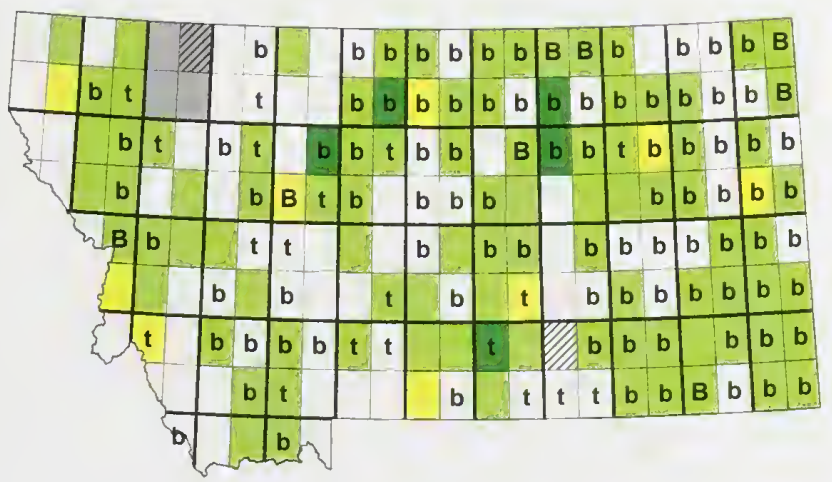


Baird's Sparrow

Ammodramus bairdii

$2003-2011=1820 \quad$ SOC

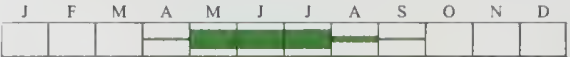
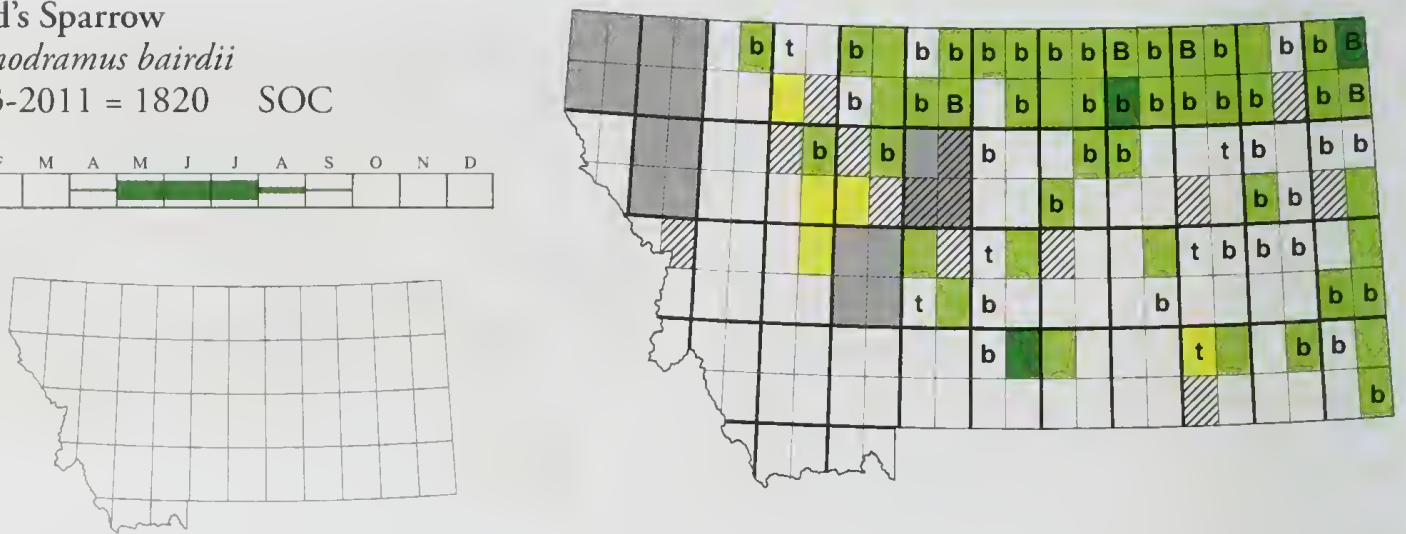

Le Conte's Sparrow

Ammodramus leconteii

$2003-2011=26 \quad$ SOC
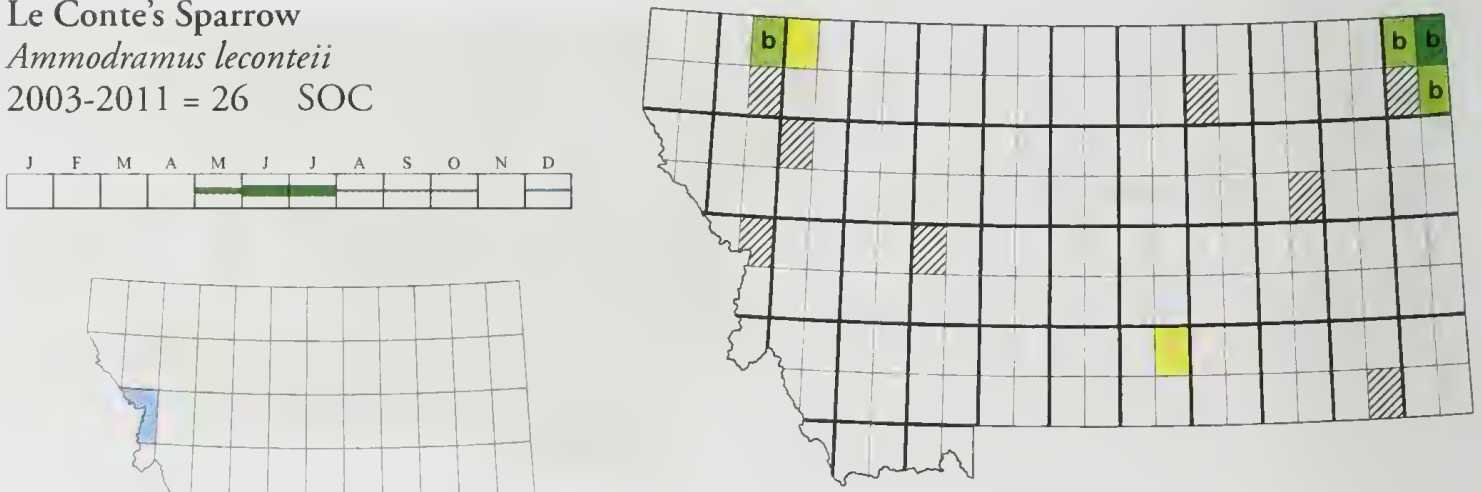

\section{Nelson's Sparrow}

Ammodramus nelsoni

2003-2011 $=107$ SOC
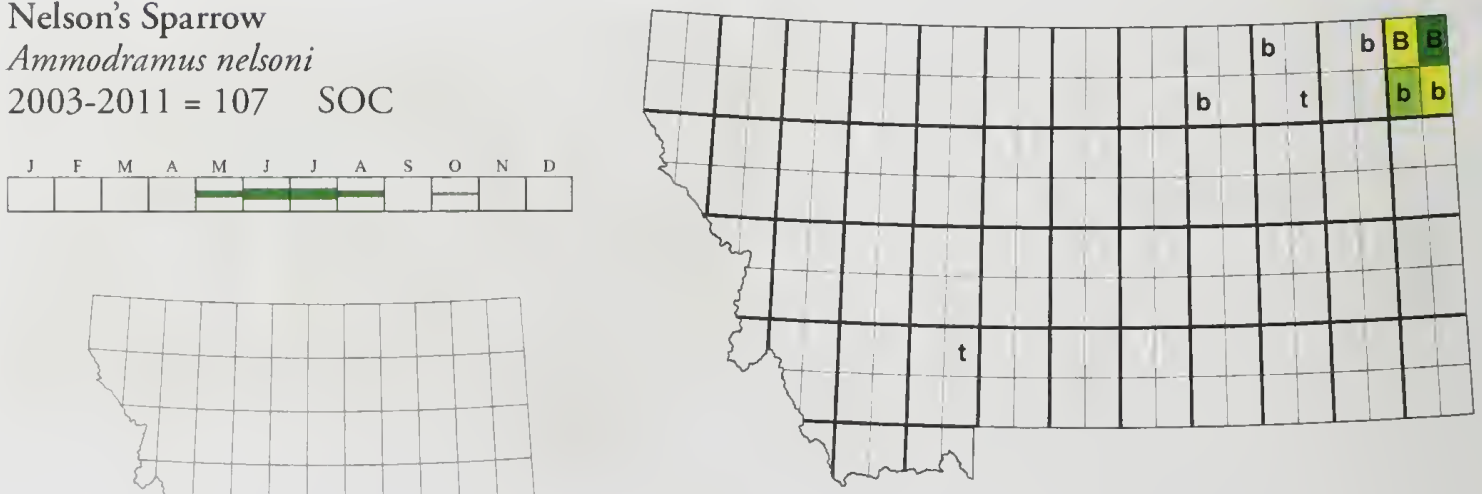
Fox Sparrow

Passerella iliaca

2003-2011 = 390
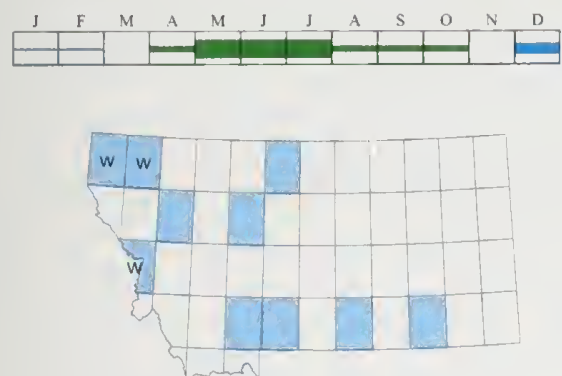

\section{Song Sparrow}

Melospiza melodia

2003-2011 = 4212
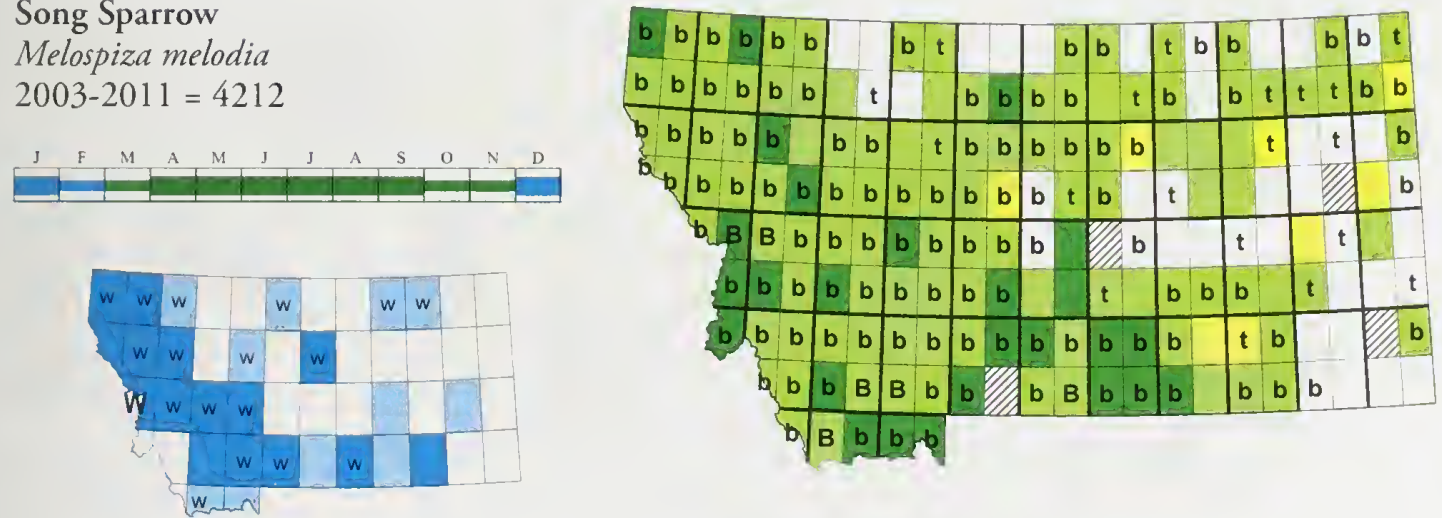

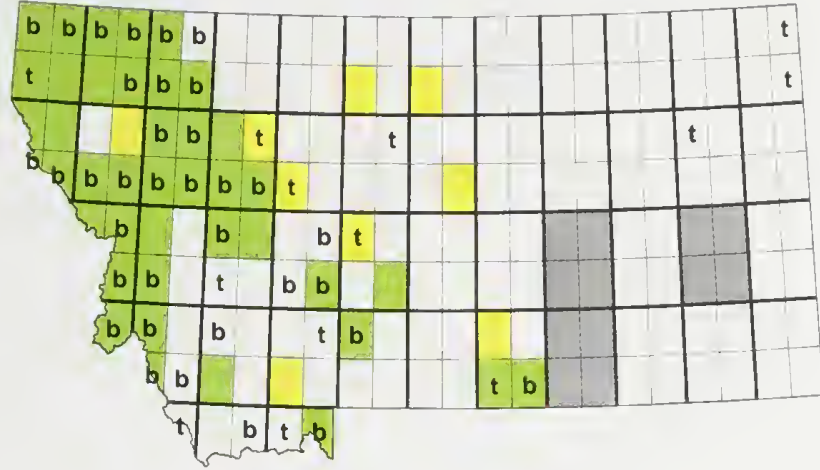

Lincoln's Sparrow

Melospiza lincolnii

2003-2011 = 1250
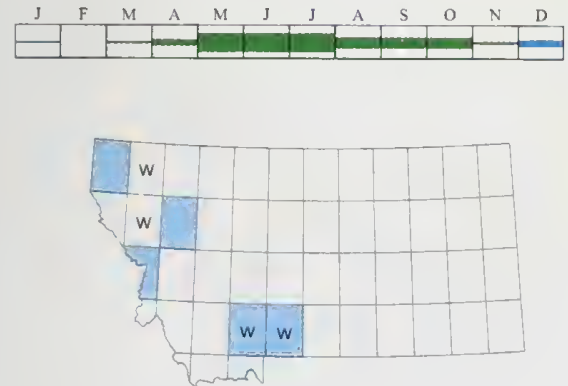
Swamp Sparrow

Melospiza georgiana

2003-2011 = 13

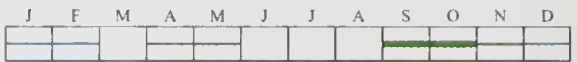
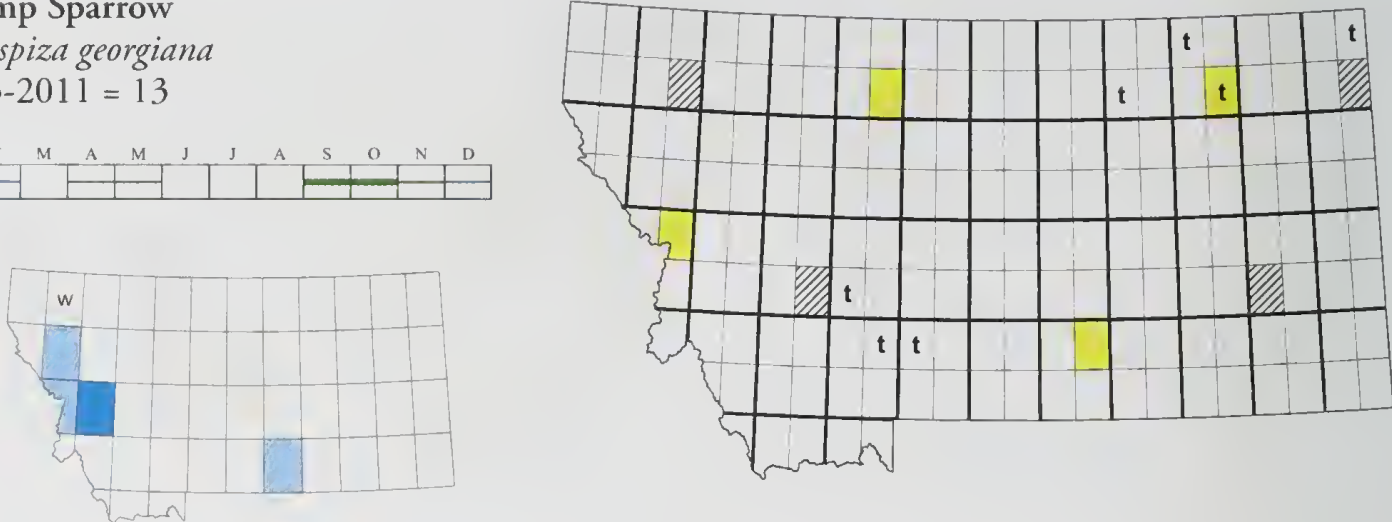

White-throated Sparrow

Zonotrichia albicollis

2003-2011 = 66
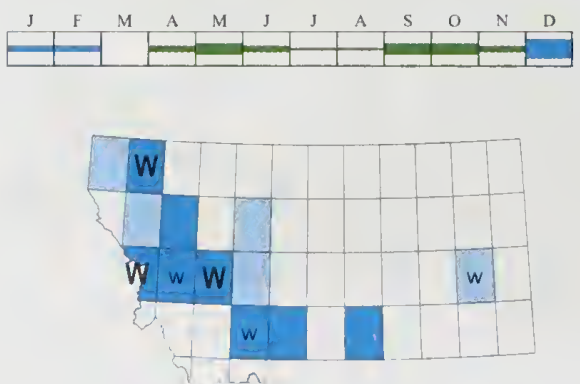

Harris's Sparrow

Zonotrichia querula

2003-2011 = 100
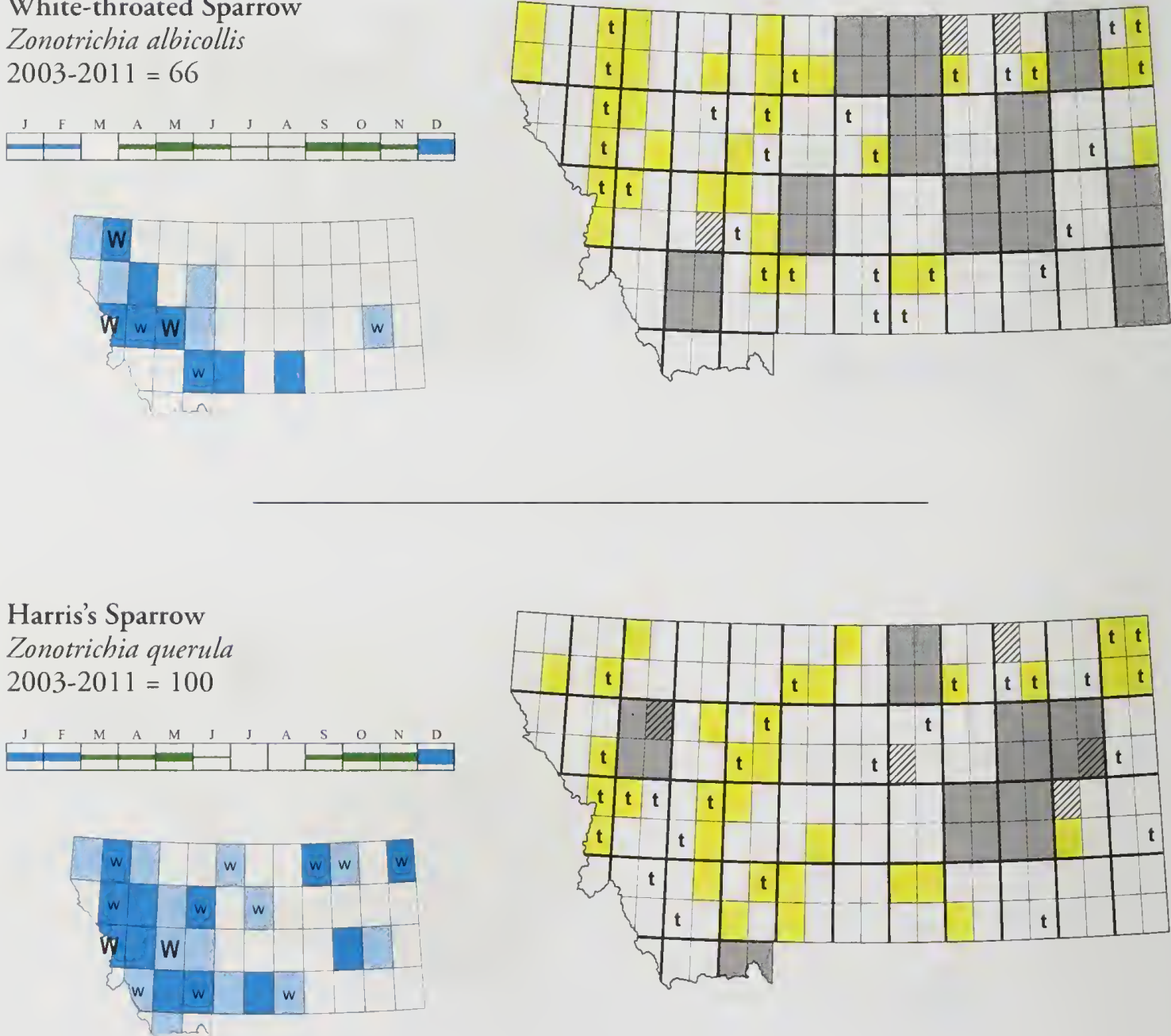
White-crowned Sparrow

Zonotrichia leucophrys

2003-2011 = 1534
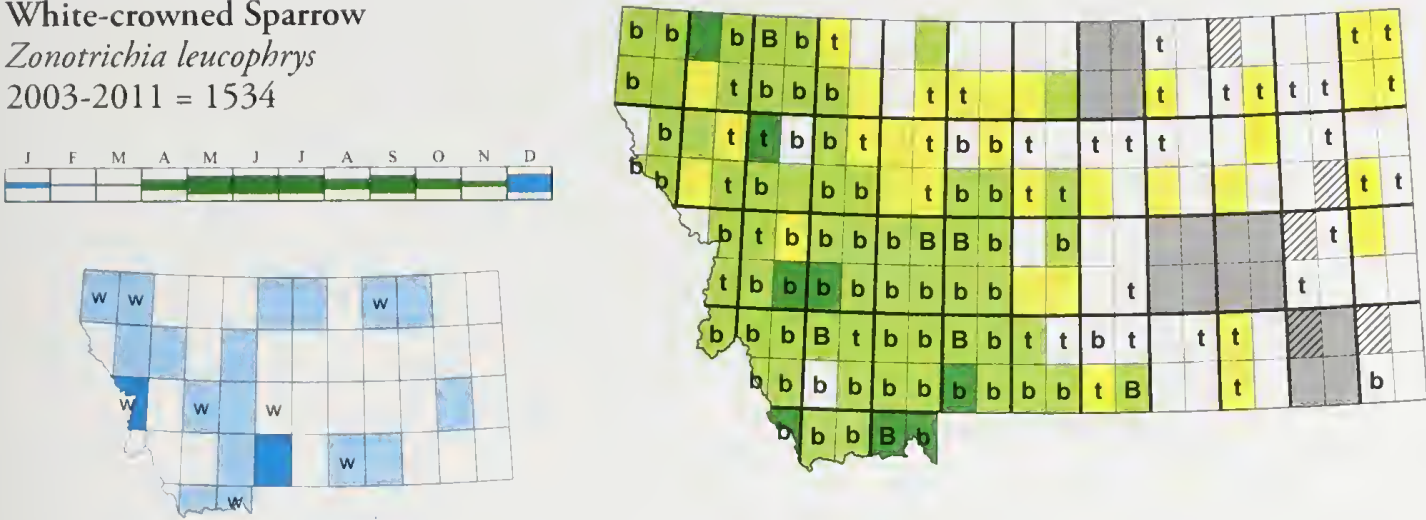

Golden-crowned Sparrow

Zonotrichia atricapilla

$2003-2011=4 \quad$ R 1
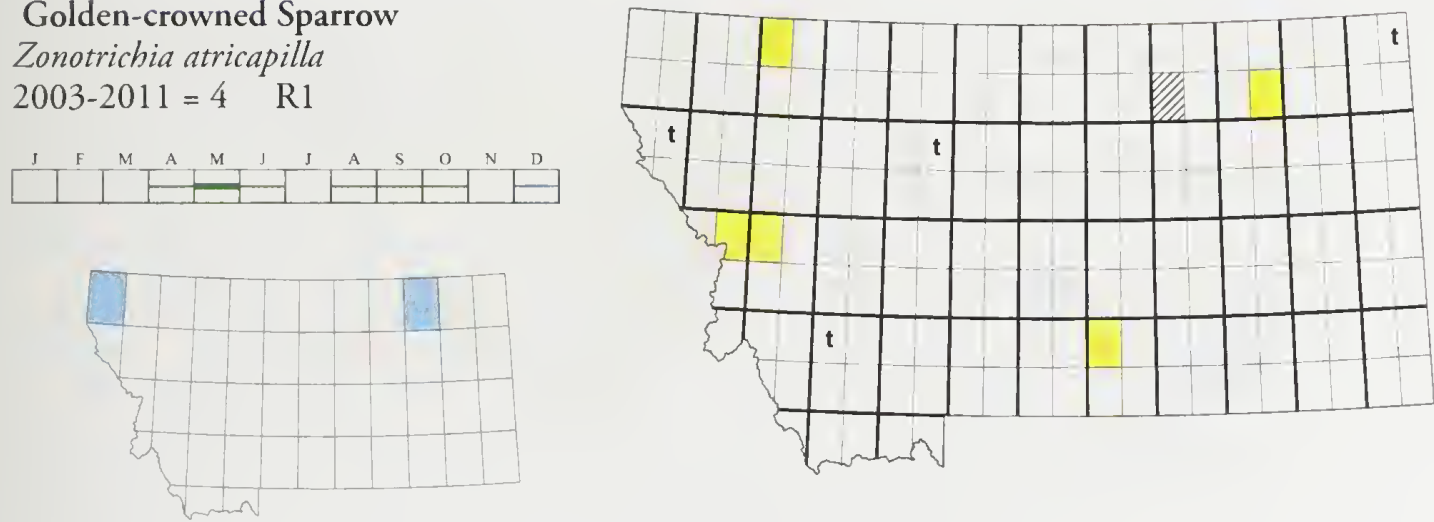

Dark-eyed Junco

Junco hyemalis

$2003-2011=7681$
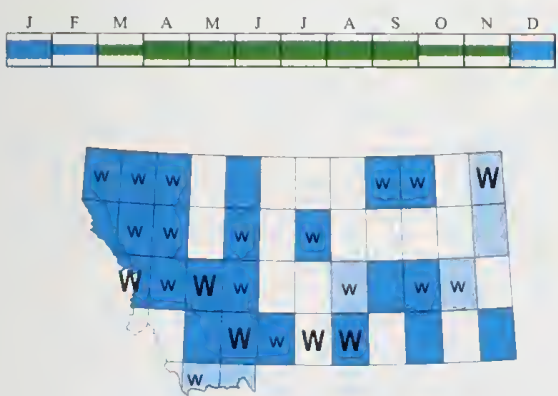

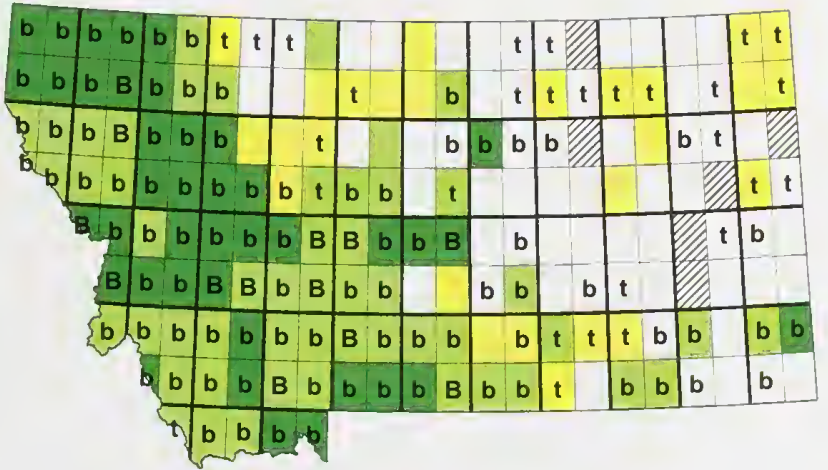


Slate-colored Junco

Junco hyemalis hyemalis / cismontanus

$2003-2011=93$
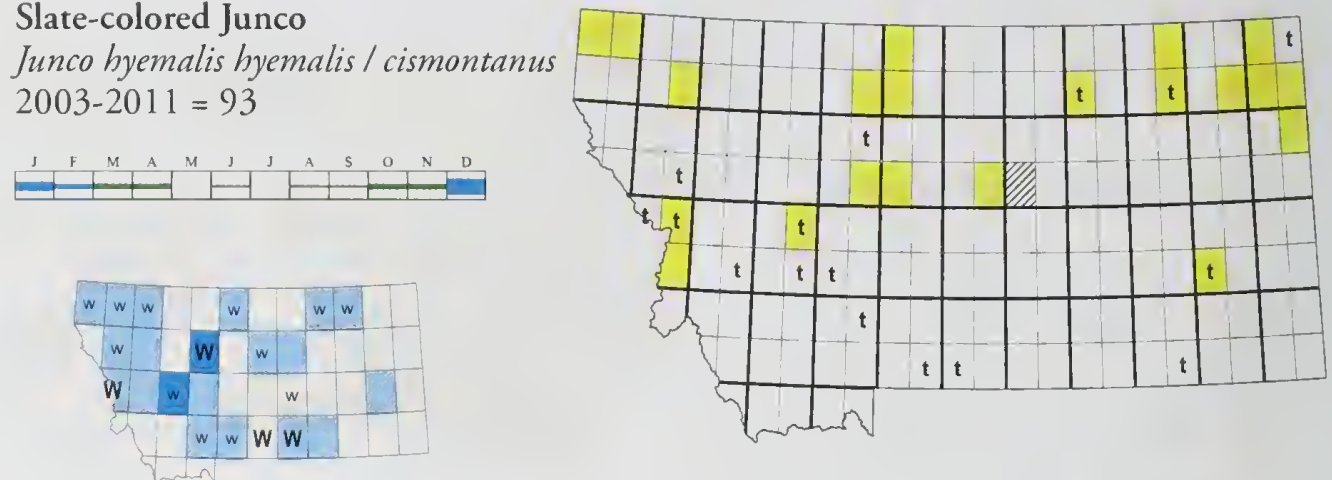

Oregon (Montana) Junco Junco hyemalis montanus 2003-2011 = 1429
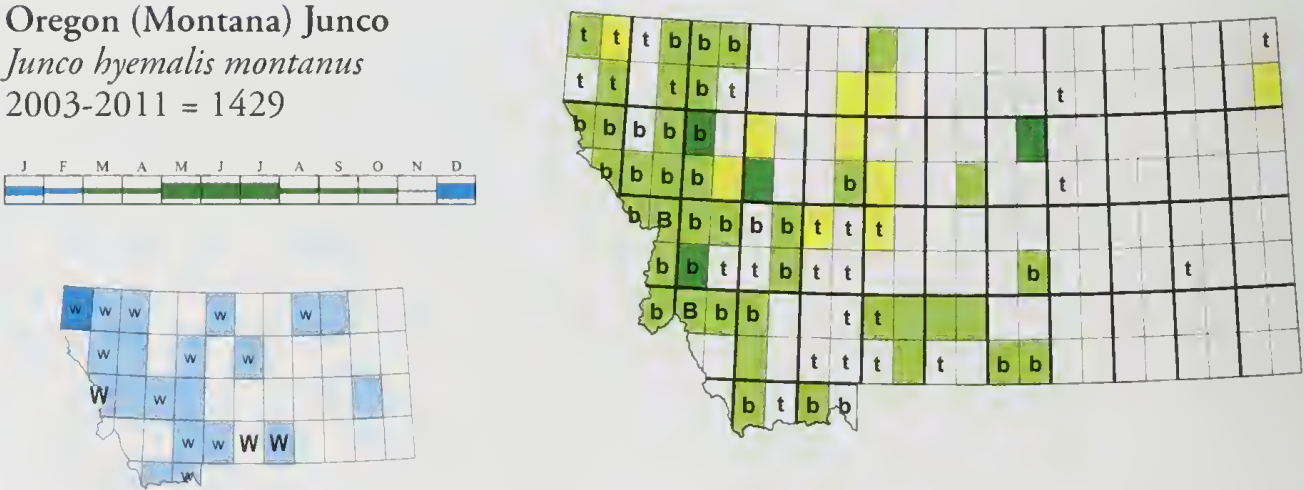

Pink-sided Junco

Junco hyemalis mearnsi

2003-2011 = 123
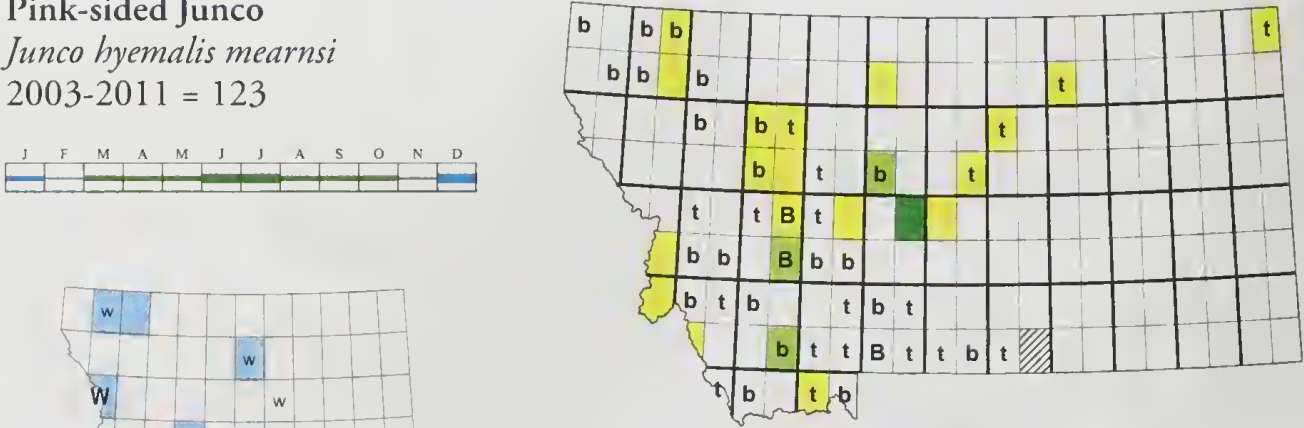
White-winged Junco Junco hyemalis aikeni $2003-2011=6$

\section{ث}

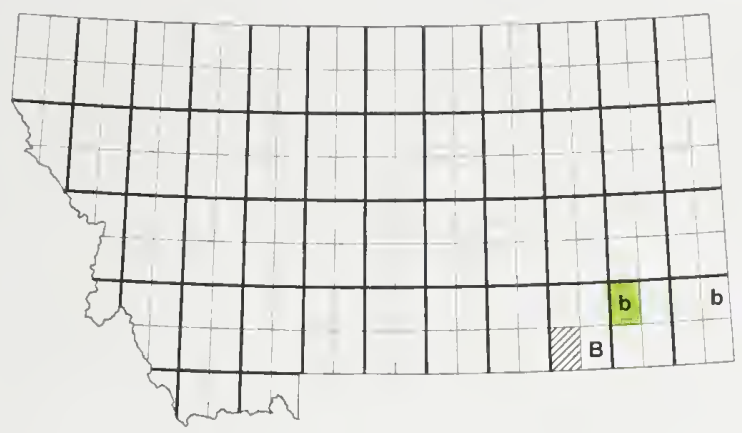

Gray-headed Junco

Junco hyemalis caniceps

2003-2011 = 1

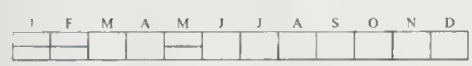

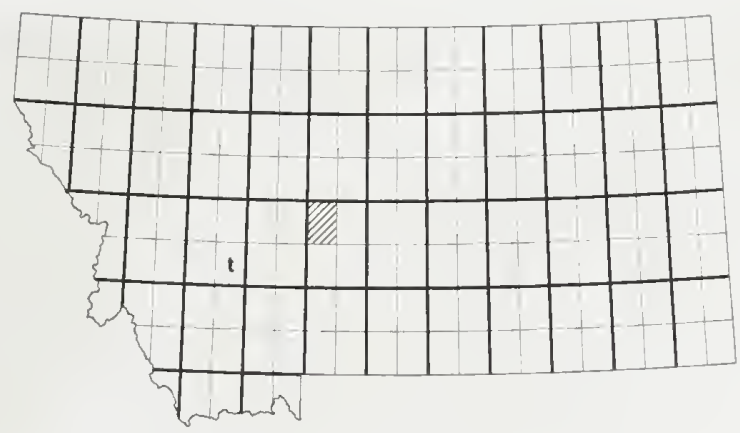

\section{Cardinalidae}

\section{Summer Tanager}

Piranga rubra

$2003-2011=2 \quad \mathrm{R} 1$
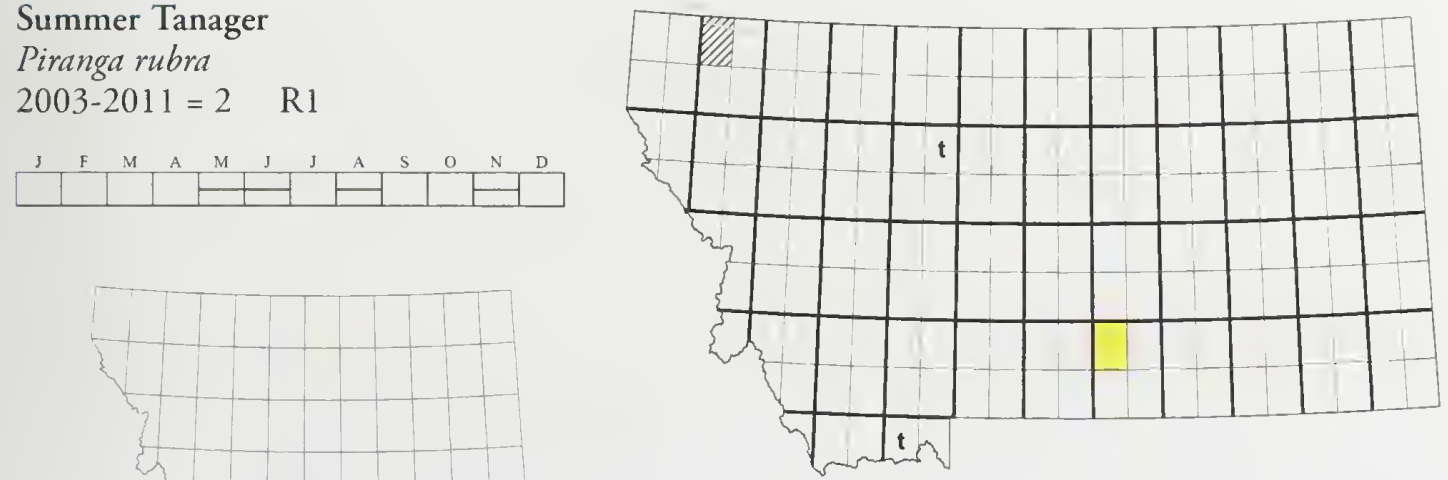
Scarlet Tanager

Piranga olivacea

2003-2011 = 3 R1

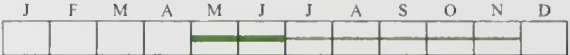
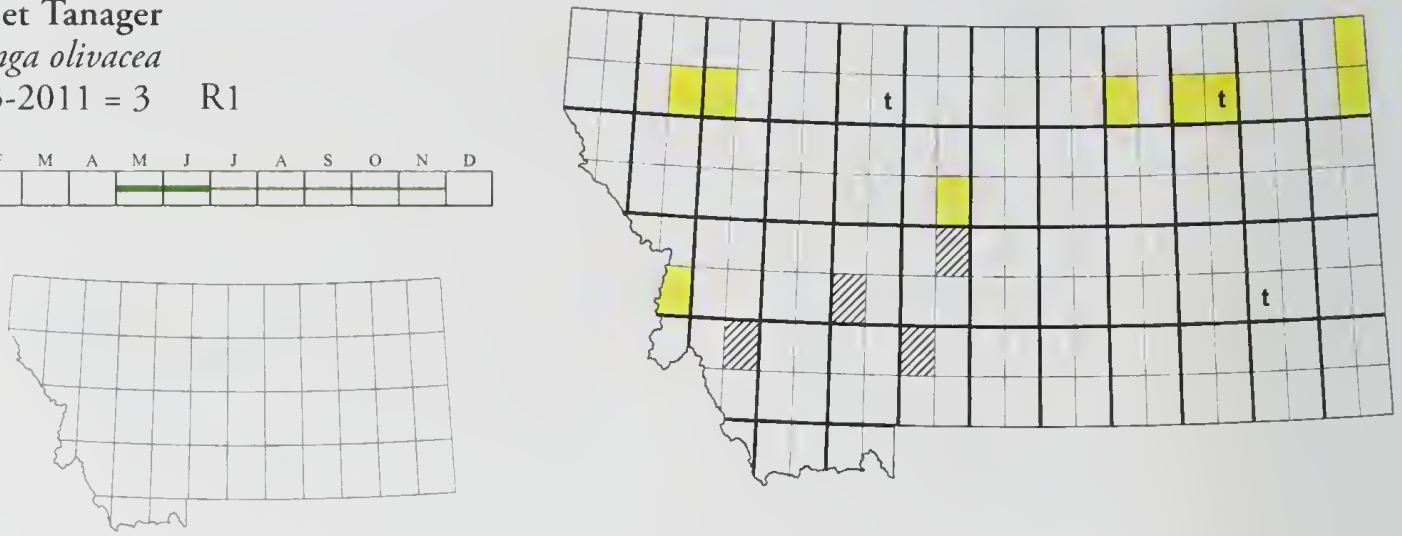

Western Tanager

Piranga ludoviciana

2003-2011 = 6109
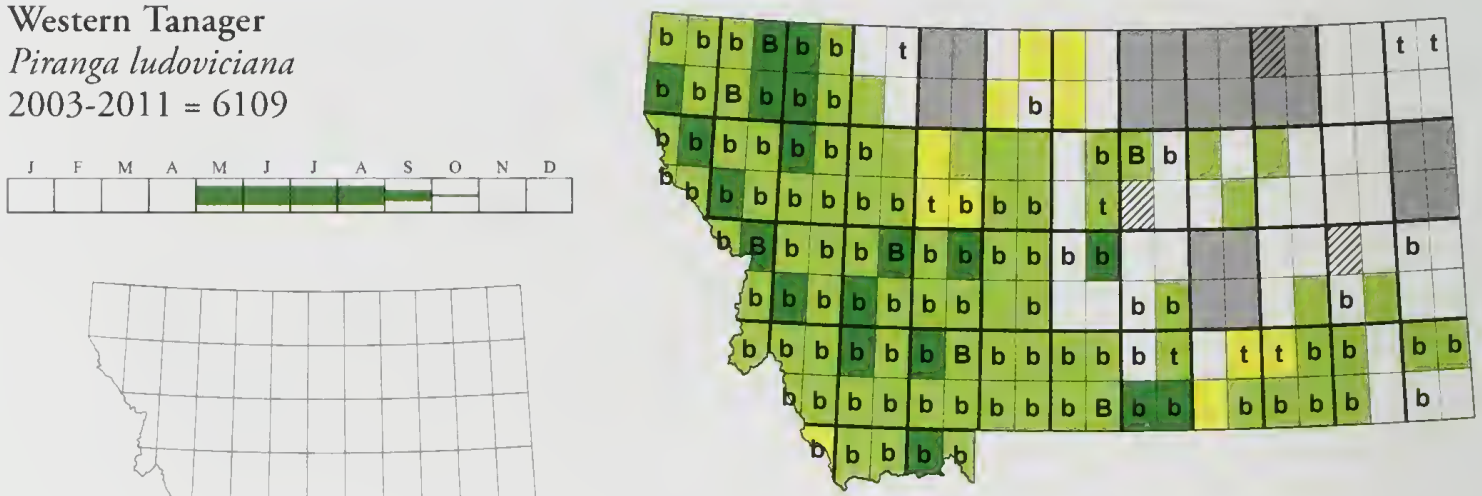

Northern Cardinal

Cardinalis cardinalis

2003-2011 = $6 \quad$ R1
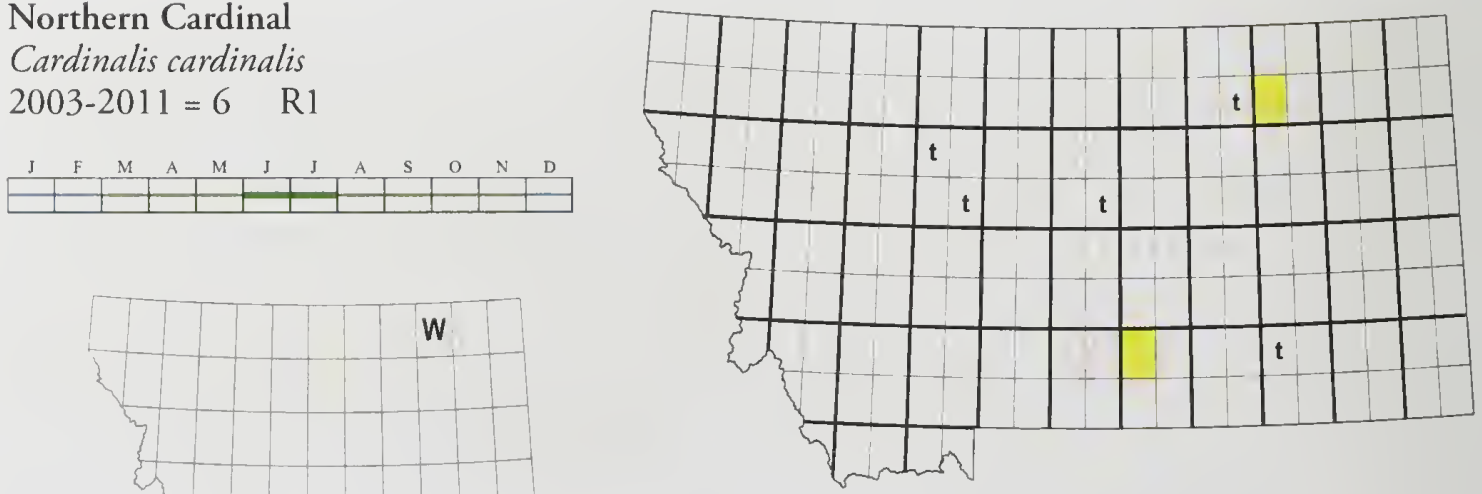
Pyrrhuloxia

Cardinalis sinuatus

2003-2011 = 0 R1

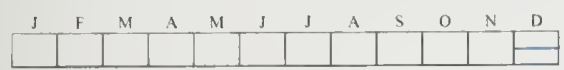

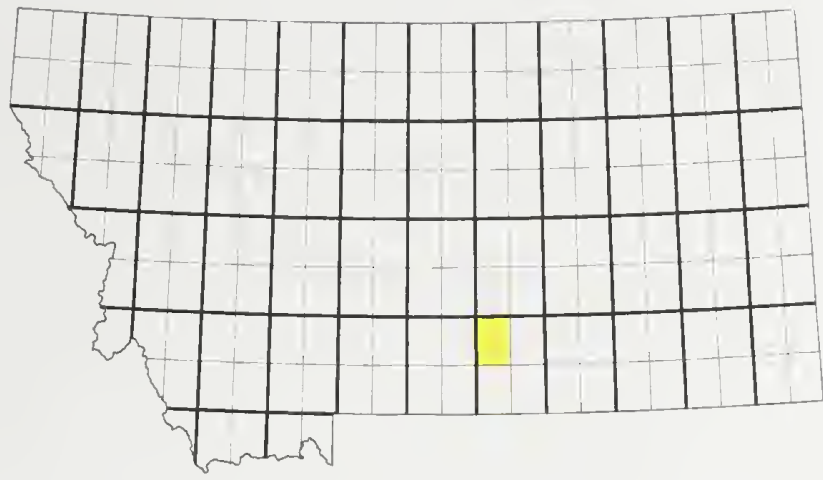

Rose-breasted Grosbeak

Pheucticus ludovicianus

2003-2011 = 61

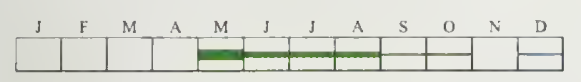

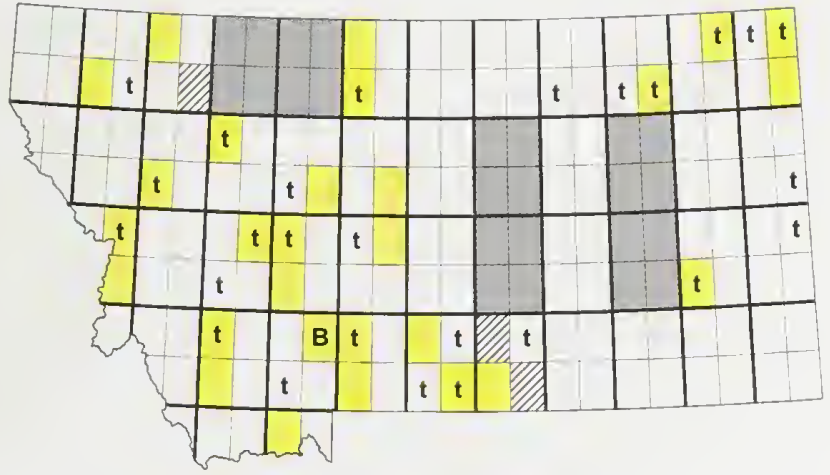

Black-headed Grosbeak

Pheucticus melanocephalus

2003-2011 = 1207

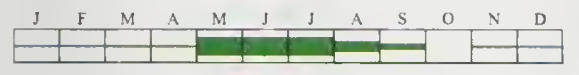

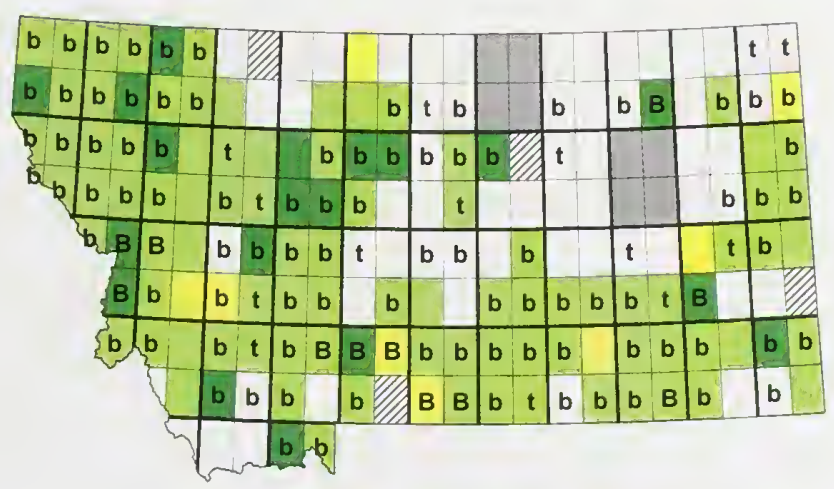


Blue Grosbeak

Passerina caerulea

2003-2011 = $7 \quad$ R2

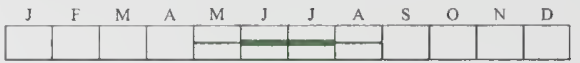

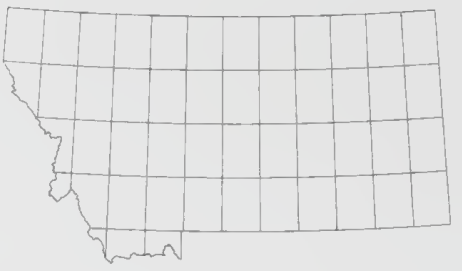

Lazuli Bunting

Passerina amoena

2003-2011 = 1677
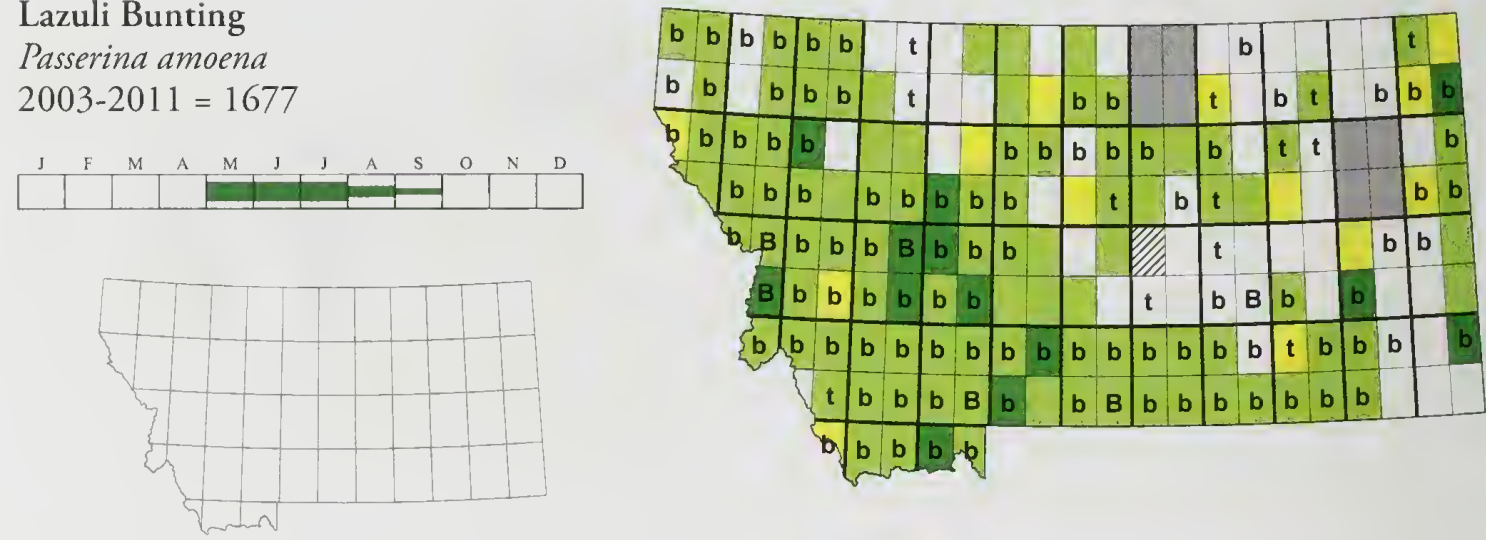

Indigo Bunting

Passerina cyanea

2003-2011 = 11
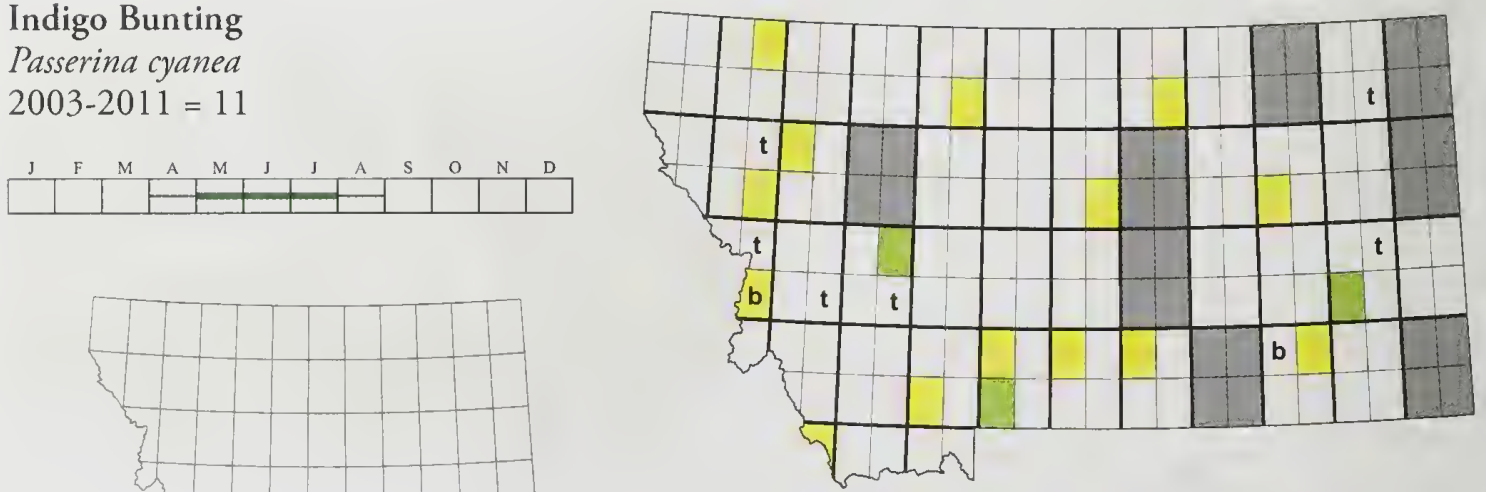


\section{Painted Bunting}

Passerina ciris

2003-2011 = 1 R1

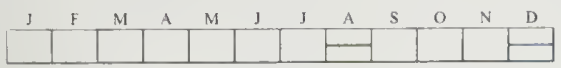

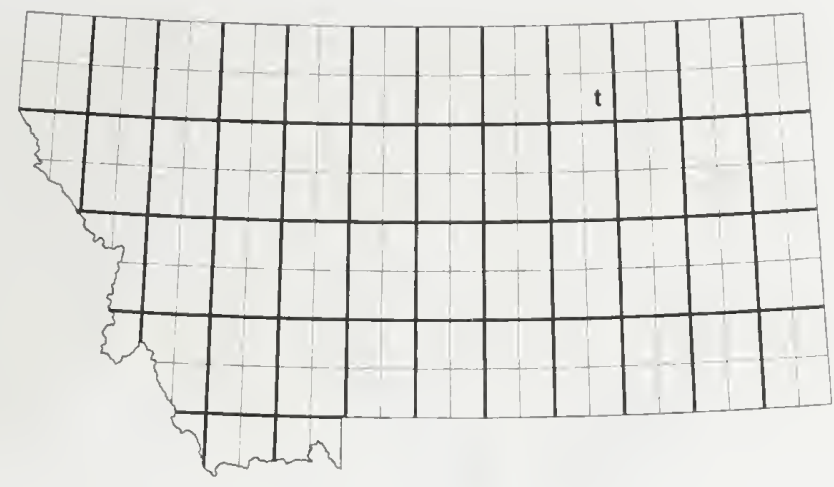

Dickcissel

Spiza americana

$2003-2011=44 \quad$ SI

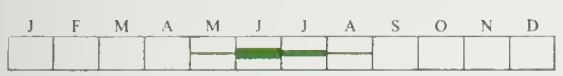

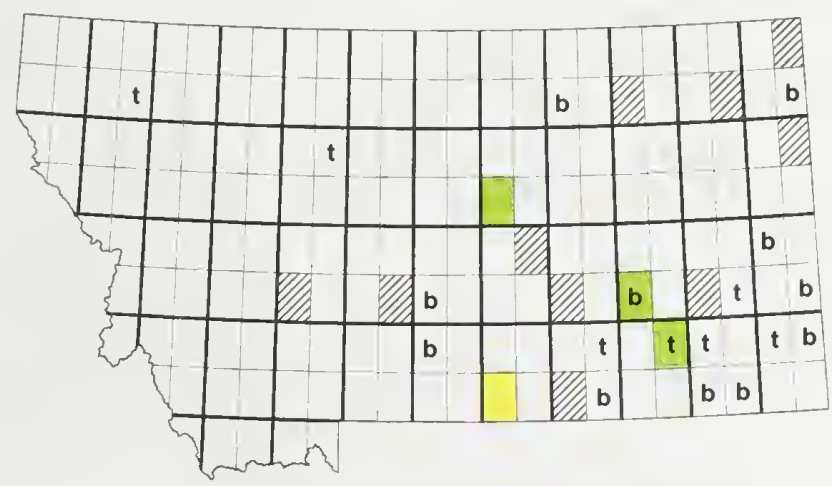

\section{Icteridae}

Bobolink

Dolichonyx oryzivorus

$2003-2011=588 \quad$ SOC
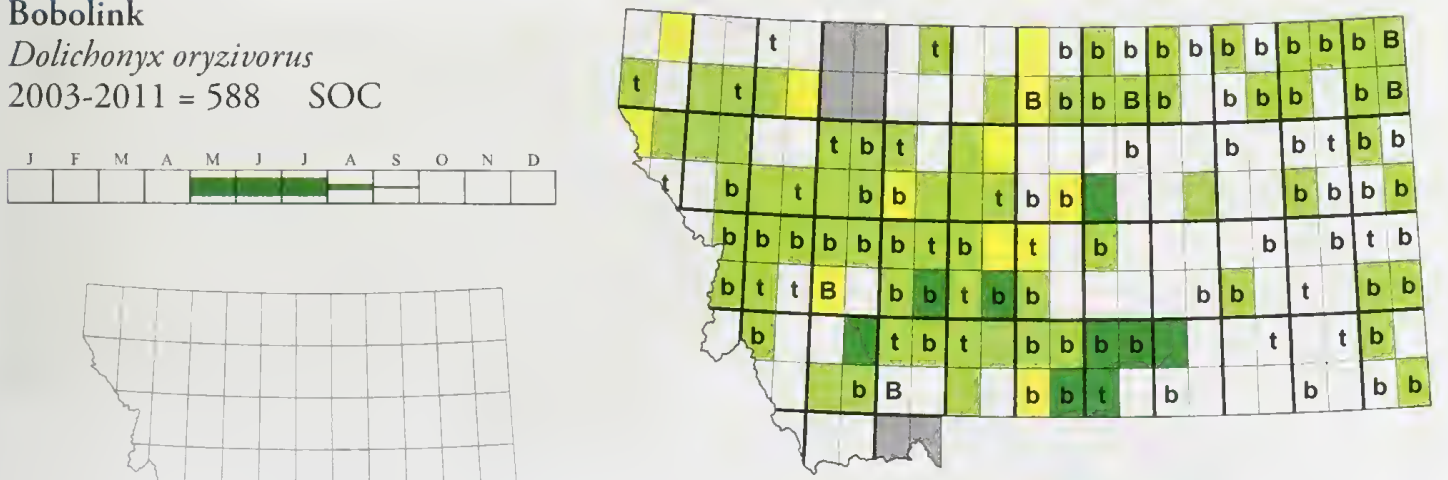
Red-winged Blackbird

Agelaius phoeniceus

2003-2011 = 5454
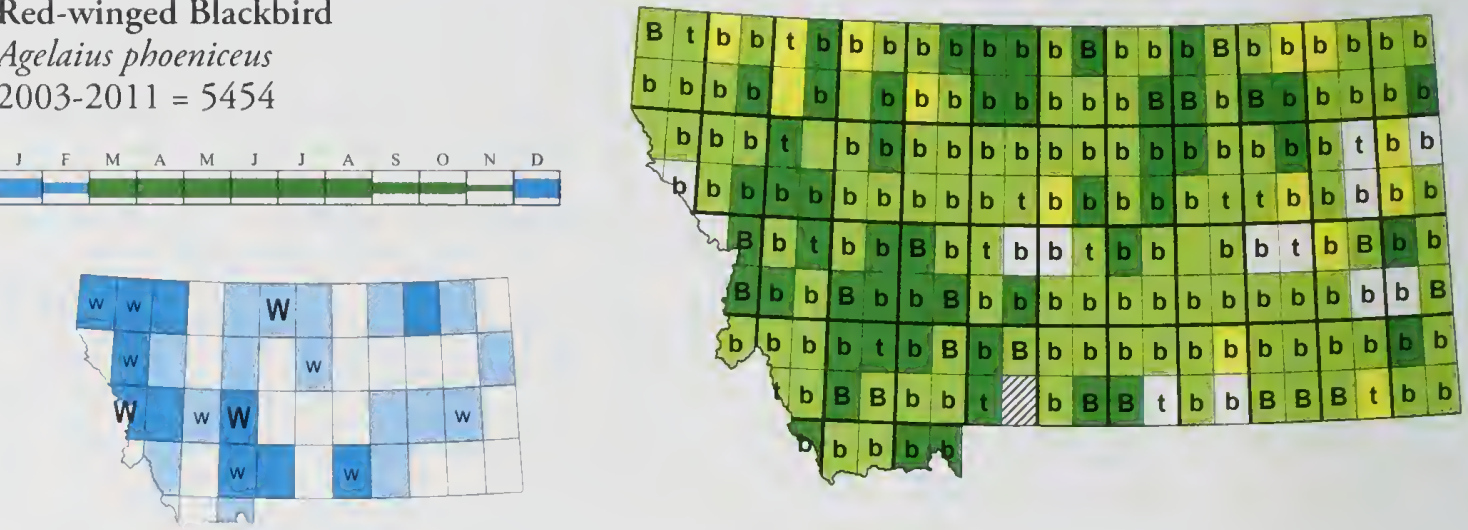

Eastern Meadowlark

Sturnella magna

2003-2011 = 1 R1

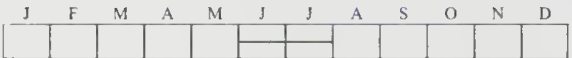

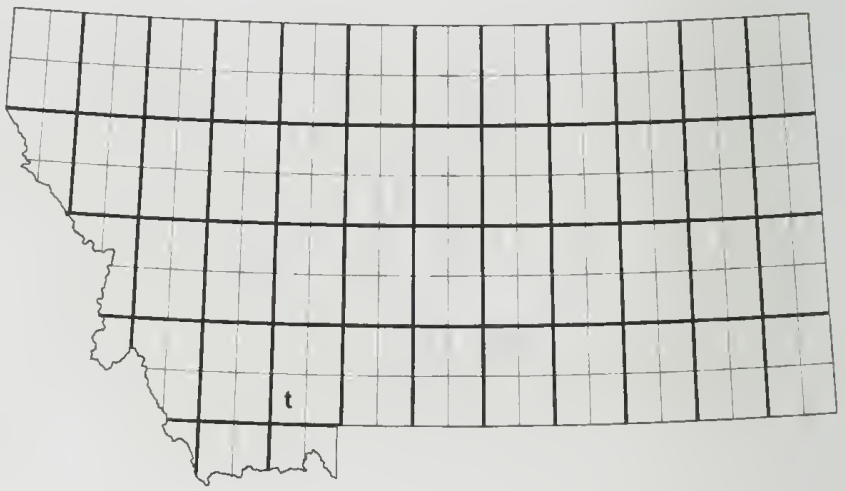

Western Meadowlark

Sturnella neglecta

2003-2011 = 17702
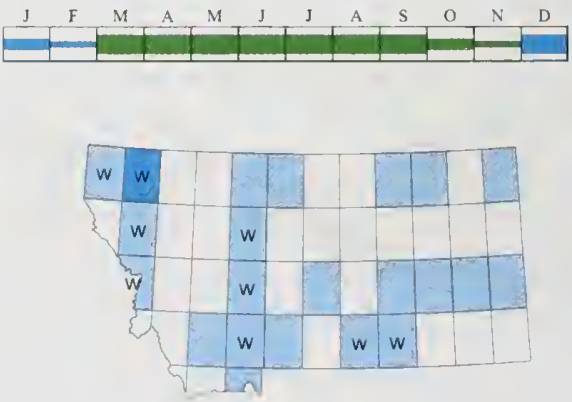

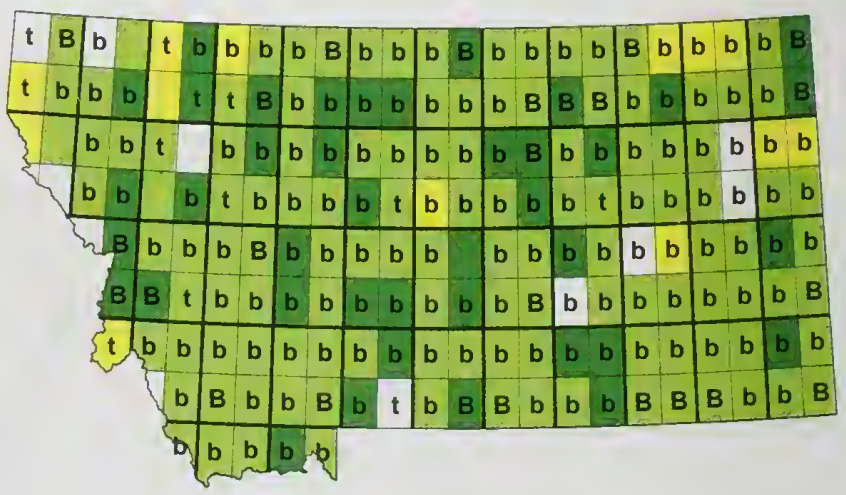


Yellow-headed Blackbird

Xanthocephalus xanthocephalus

2003-2011 = 1486

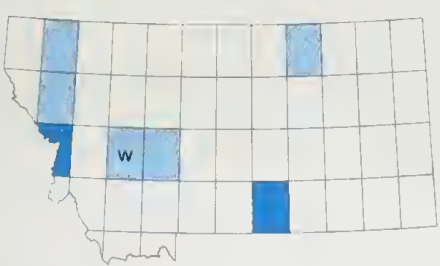

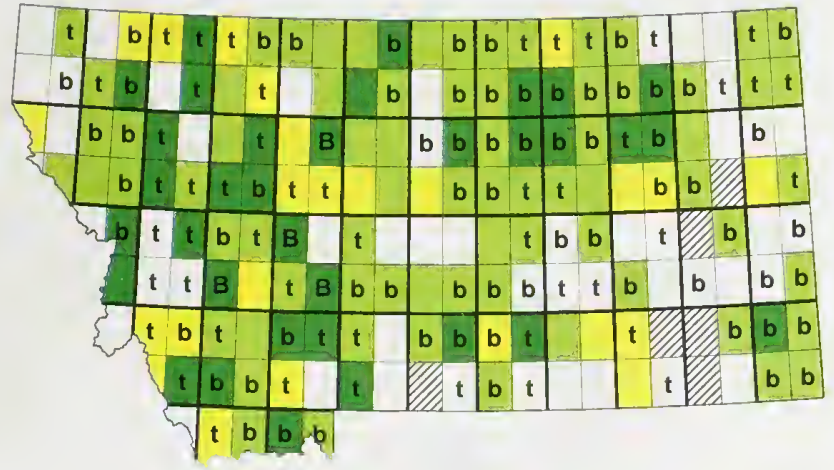

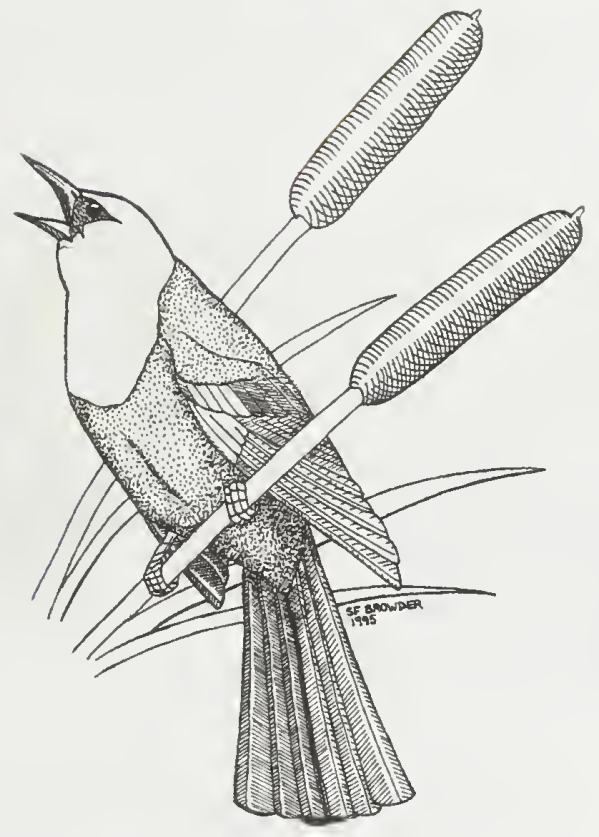

Rusty Blackbird

Euphagus carolinus

2003-2011 = 21
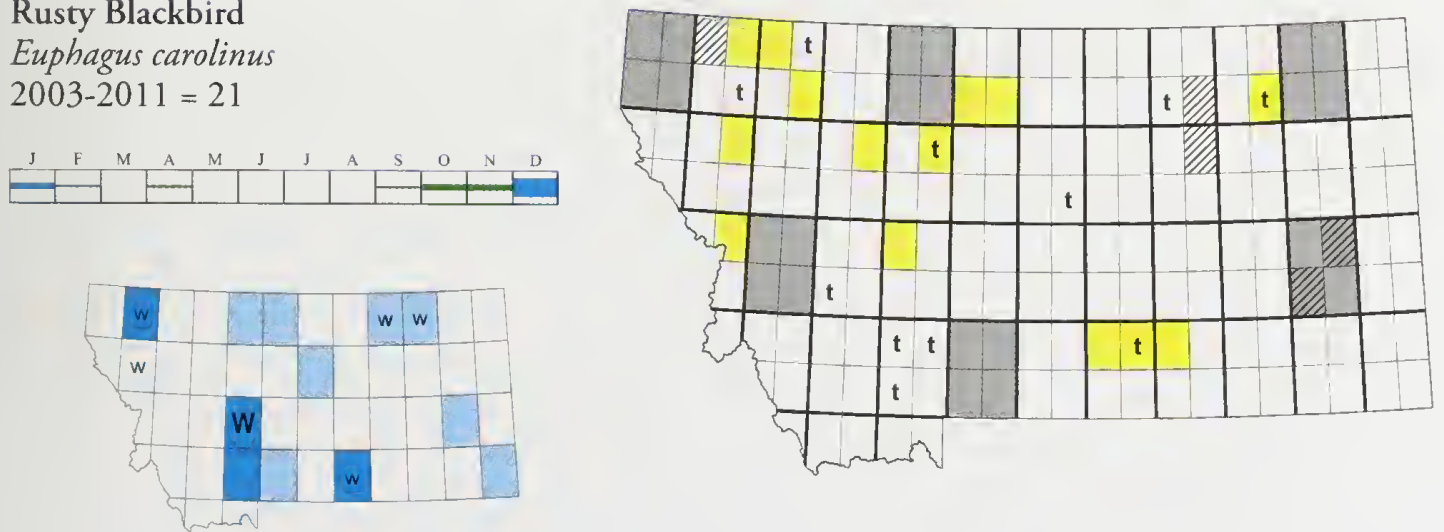
Brewer's Blackbird

Euphagus cyanocephalus

$2003-2011=3430$
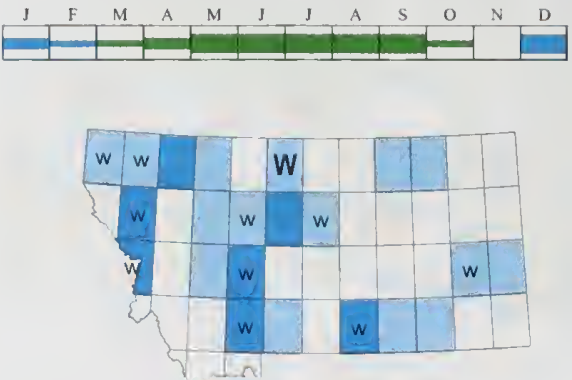

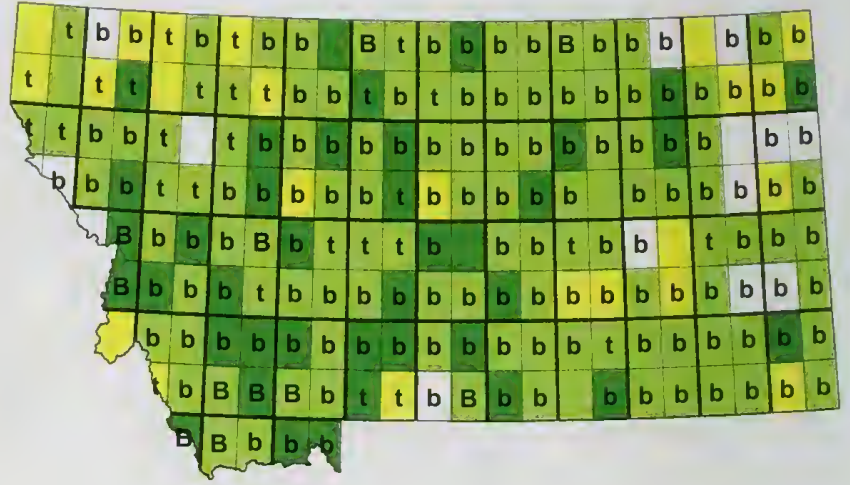

Common Grackle

Quiscalus quiscula

2003-2011 = 1091
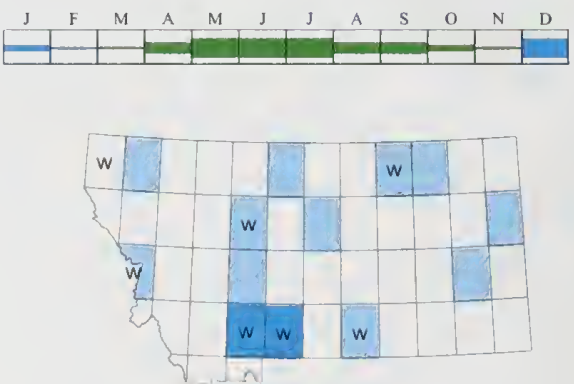

Great-tailed Grackle

Quiscalus mexicanus

2003-2011 = 1 R1

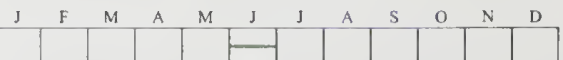

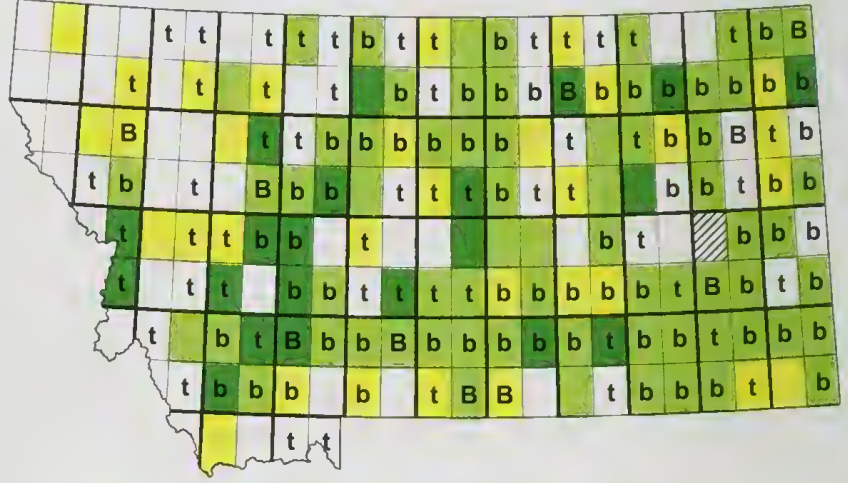

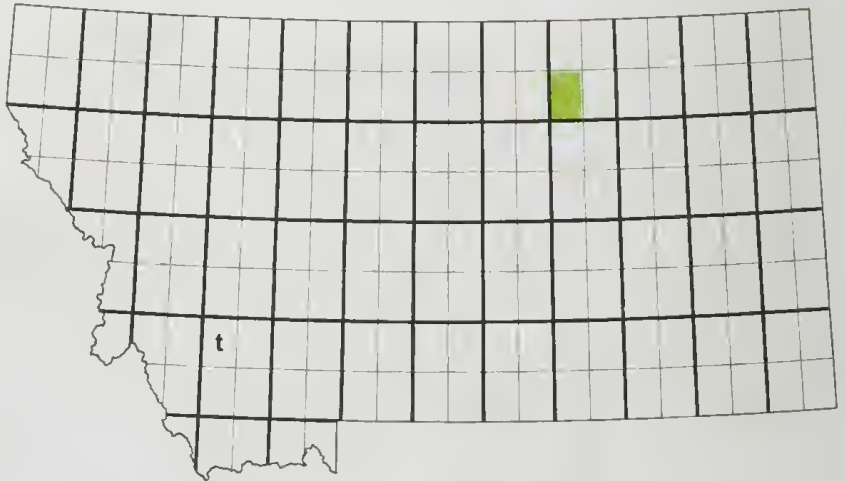


Brown-headed Cowbird

Molothrus ater

2003-2011 = 8264
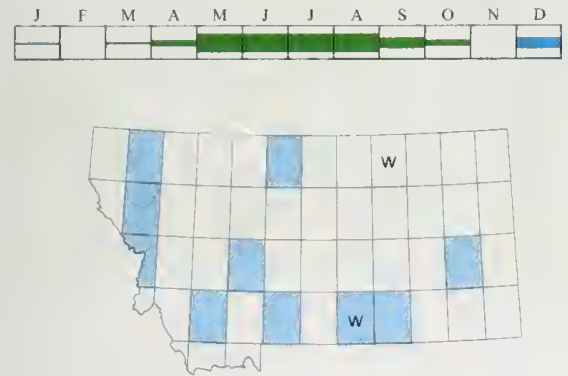

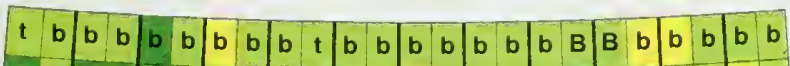
b $b$ b b

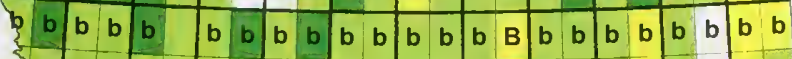
\begin{tabular}{llllllllllllllllllllllllll}
$b$ & $b$ & $b$ & $b$ & $b$ & $b$ & $b$ & $B$ & $b$ & $b$ & $b$ & $b$ & $b$ & $b$ & $b$ & $b$ & $t$ & $b$ & $b$ & $b$ & $b$ & $b$ & $b$ \\
\hline
\end{tabular} \begin{tabular}{lllllll|lllllllllllllll}
$b$ & $B$ & $B$ & $b$ & $B$ & $b$ & $b$ & $b$ & $b$ & $b$ & $b$ & $b$ & $b$ & $b$ & $b$ & $b$ & $b$ & $b$ & $b$ & $b$ & $B$ & $b$
\end{tabular} \begin{tabular}{llllllllllllllllllllllll}
$B$ & $b$ & $b$ & $b$ & $b$ & $b$ & $b$ & $b$ & $b$ & $b$ & $b$ & $b$ & $b$ & $b$ & $b$ & $b$ & $b$ & $b$ & $b$ & $b$ & $b$ \\
\hline
\end{tabular} $\left\{\begin{array}{lllllll|lllllllllllllllll}b & b & b & B & b & b & B & b & b & b & b & b & b & b & t & b & b & b & b & b & b \\ \hline\end{array}\right.$ \begin{tabular}{l|l|l|l|l|llllllllllllll}
$b$ & $b$ & $b$ & $b$ & $B$ & $b$ & $b$ & $B$ & $B$ & $B$ & $b$ & $b$ & $b$ & $B$ & $b$ & $b$ & $b$ & $b$ & $b$ \\
\hline$b$ & $b$ & $b$ & $b$ & $b$ & $b$ &
\end{tabular}

Orchard Oriole

Icterus spurius

$2003-2011=78$
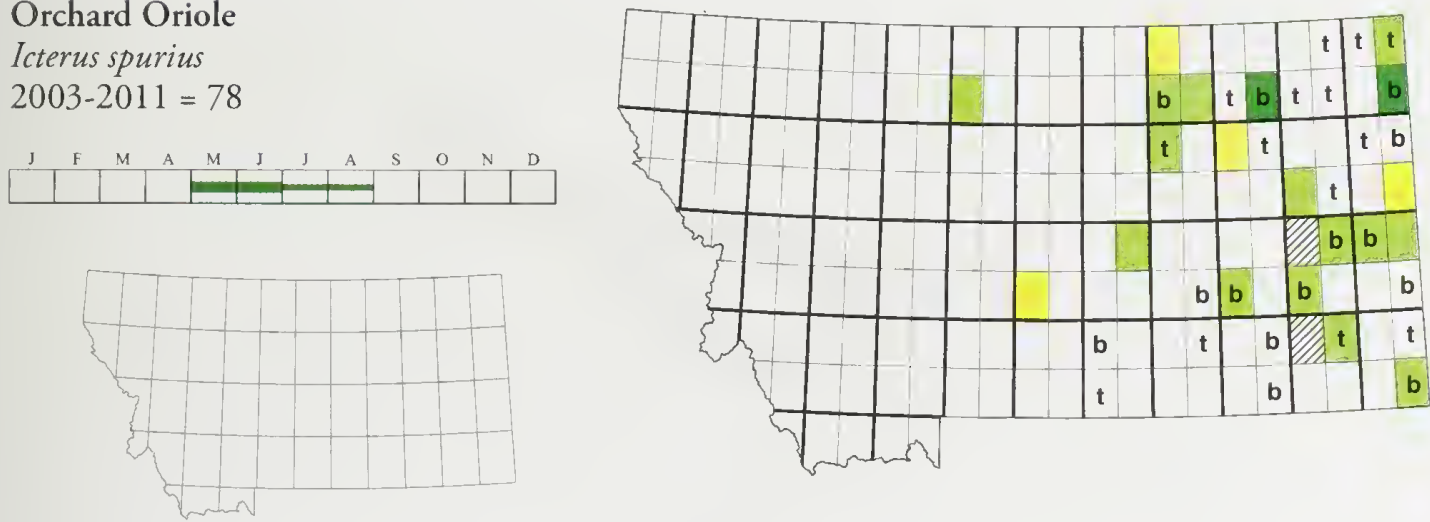

Hooded Oriole

Icterus cucullatus

2003-2011 = 0 R1

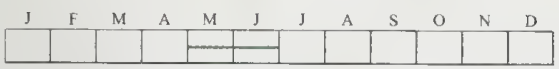
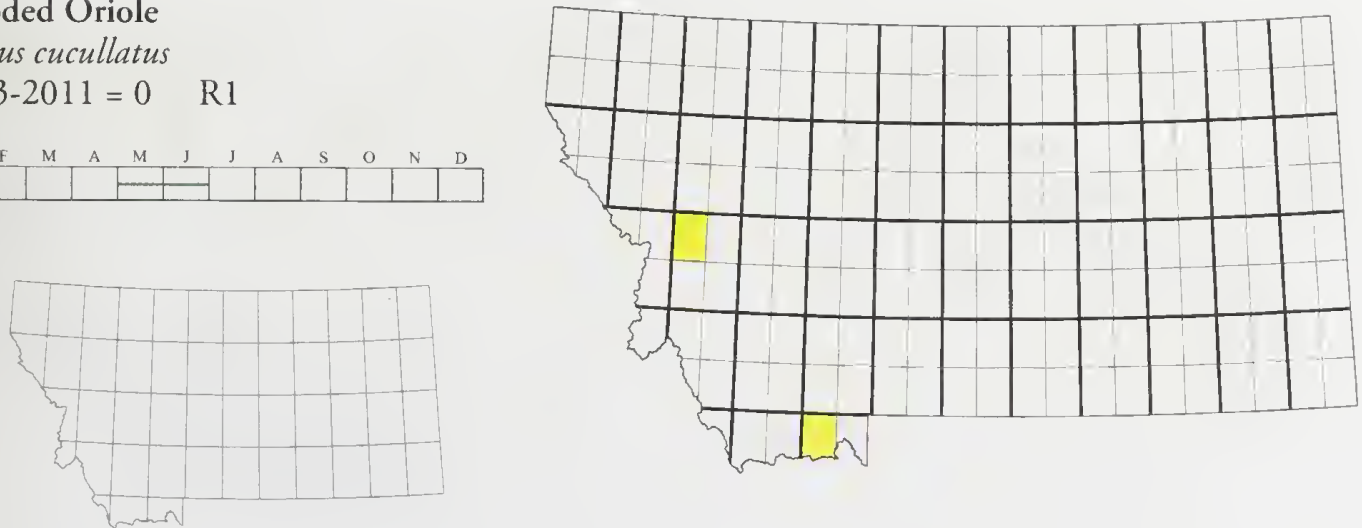
Bullock's Oriole

Icterus bullockii

2003-2011 = 1161

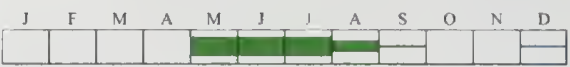
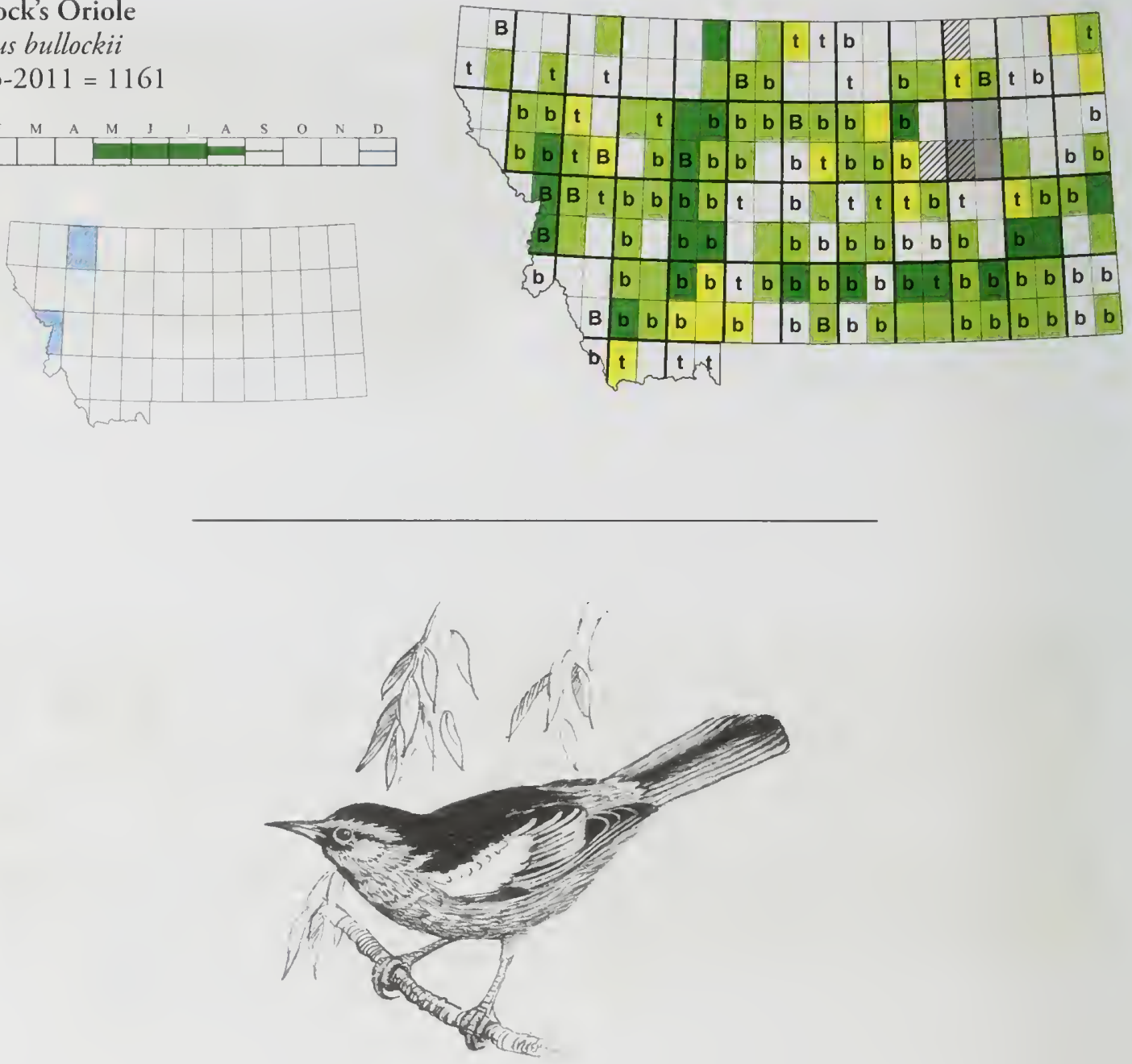

Baltimore Oriole Icterus galbula

2003-2011 = 114
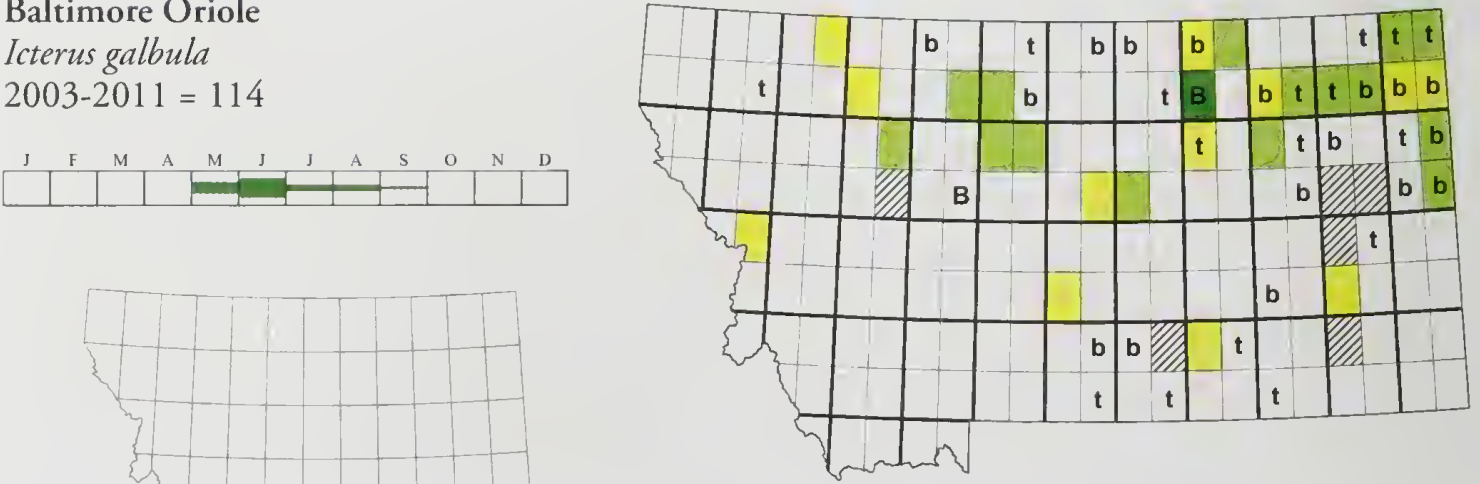
Northern Oriole

Icterus galbula

2003-2011 = 0
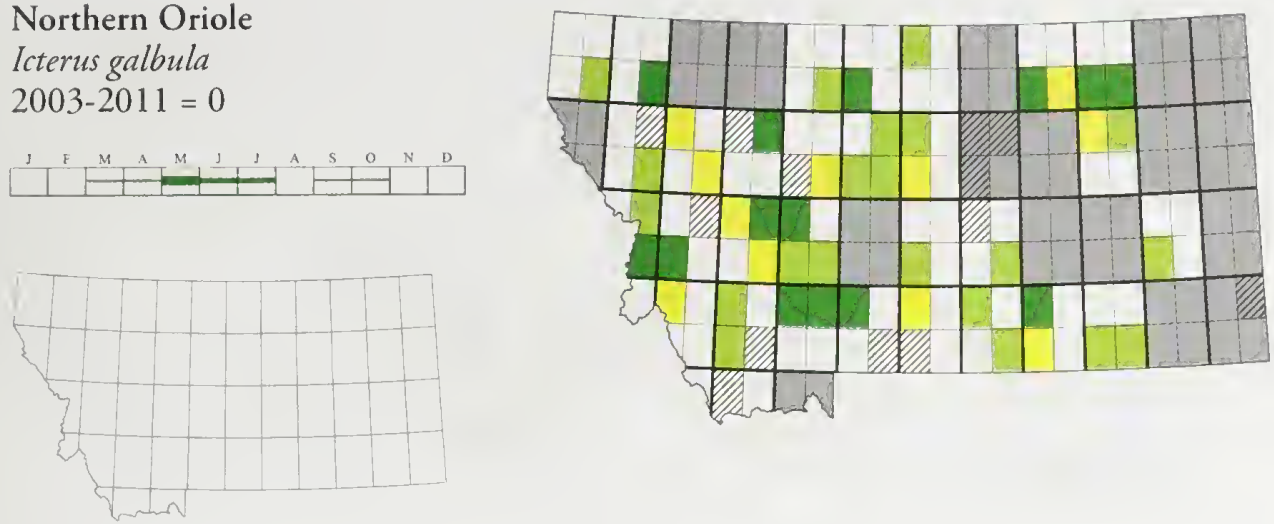

Fringillidae

\section{Brambling}

Fringilla montifringilla

2003-2011 = 1 R1
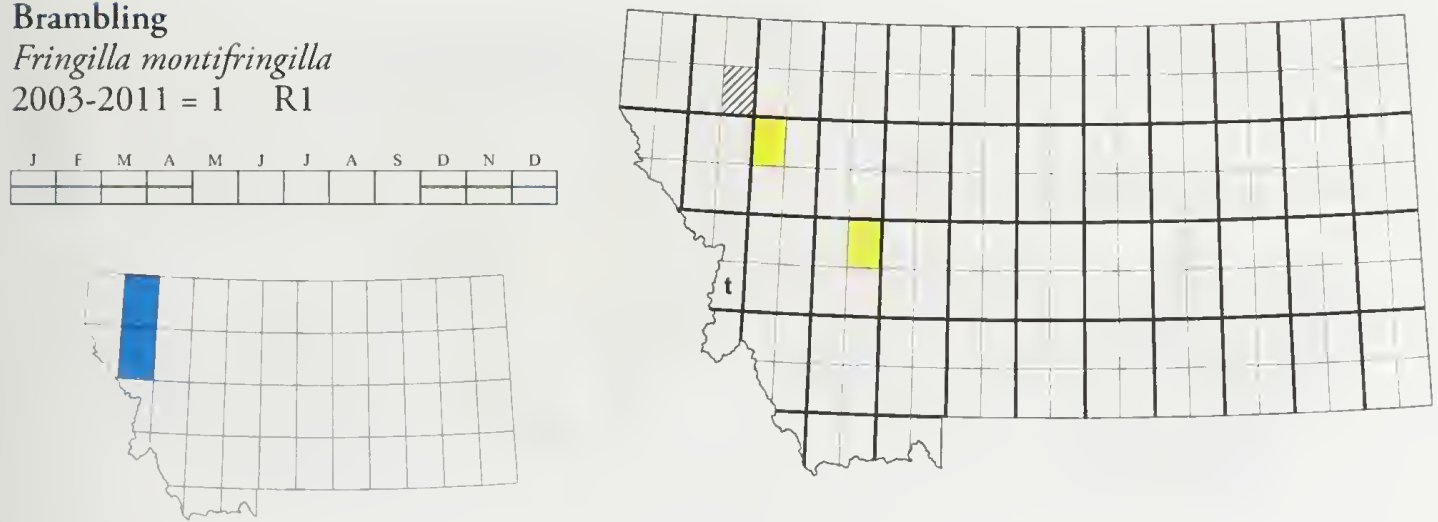

Gray-crowned Rosy-Finch

Leucosticte tephrocotis

$2003-2011=136$ SOC
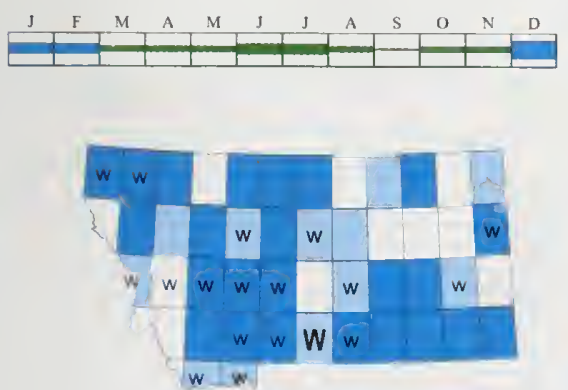

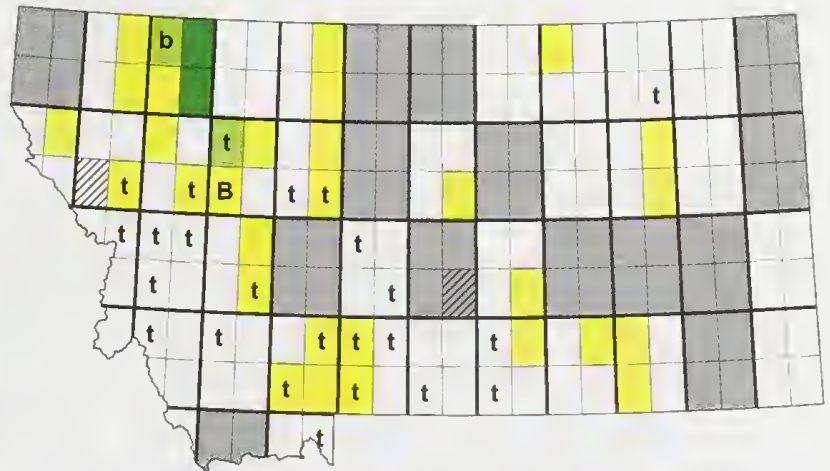


Black Rosy-Finch

Leucosticte atrata

$2003-2011=23 \quad$ SOC
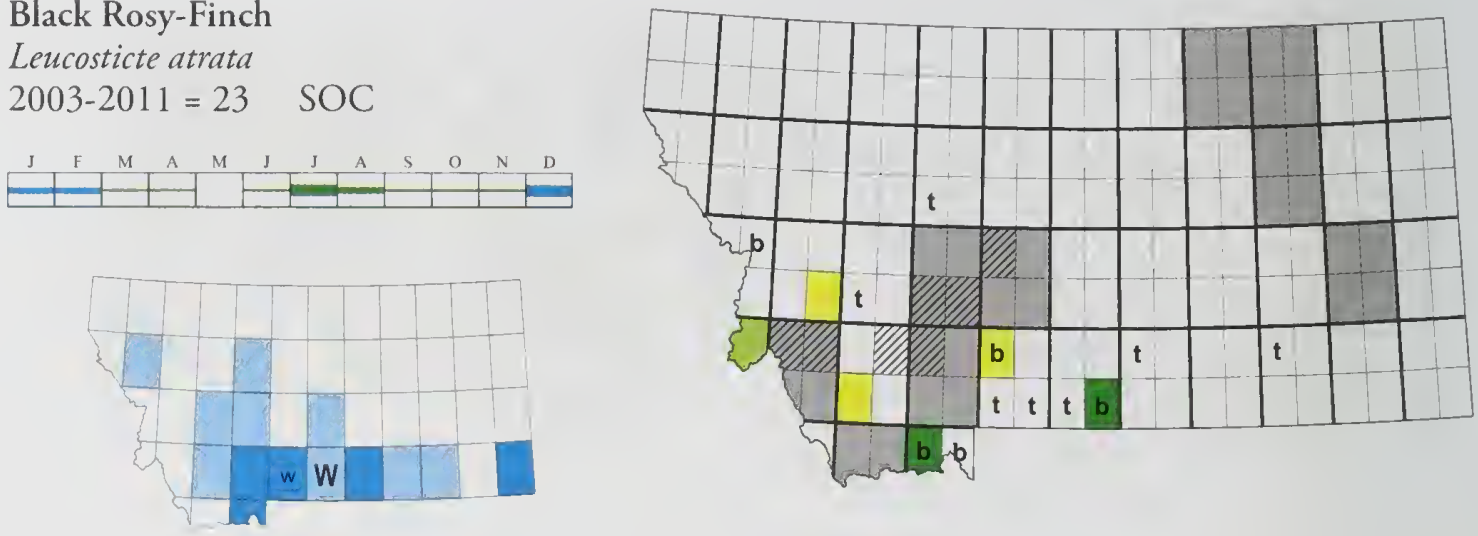

Pine Grosbeak

Pinicola enucleator

2003-2011 = 364
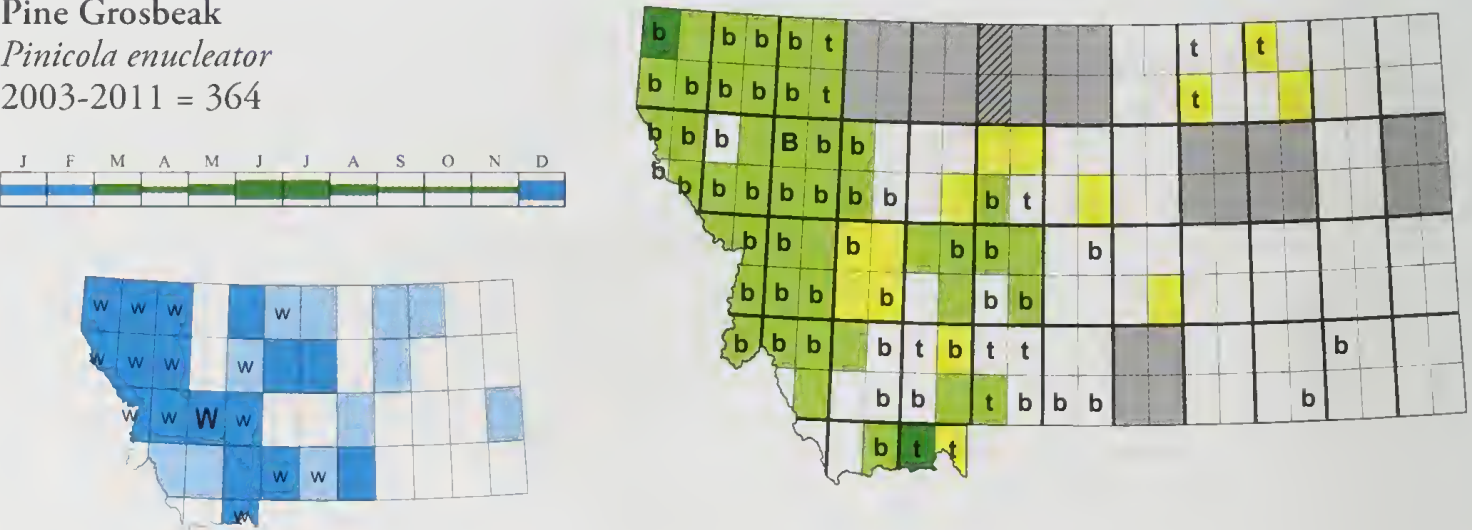

Purple Finch

Carpodacus purpureus

2003-2011 = 28
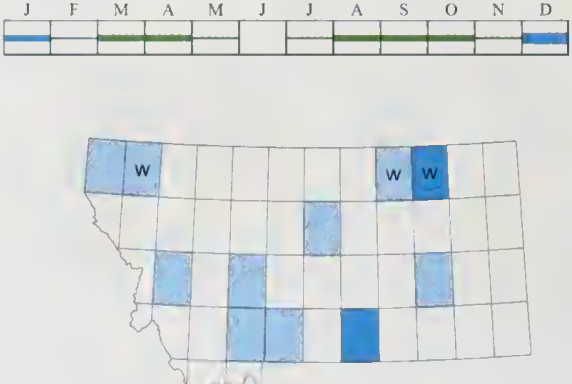
Cassin's Finch

Carpodacus cassinii

$2003-2011=794 \quad$ SOC
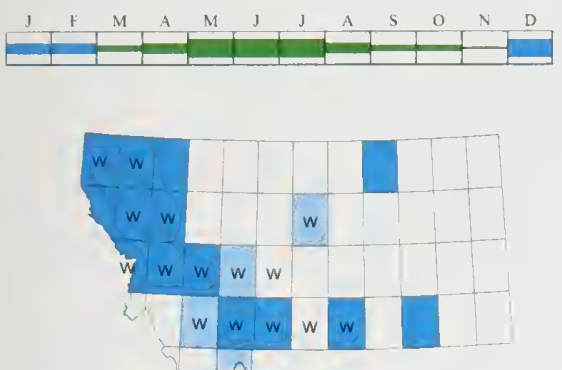

House Finch

Carpodacus mexicanus

2003-2011 = 1213
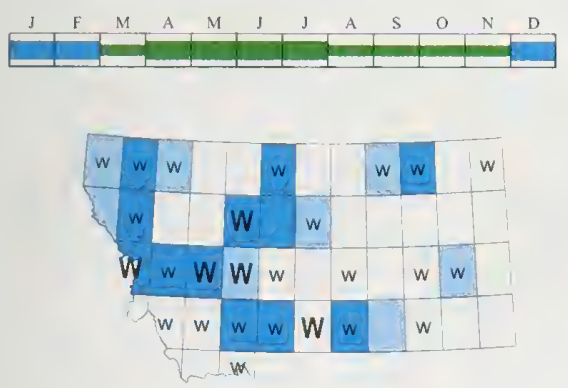

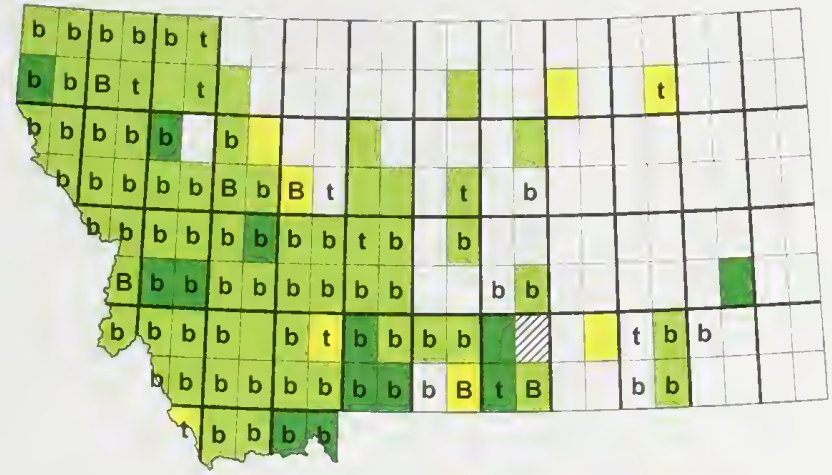

Red Crossbill

Loxia curvirostra

$2003-2011=2688$
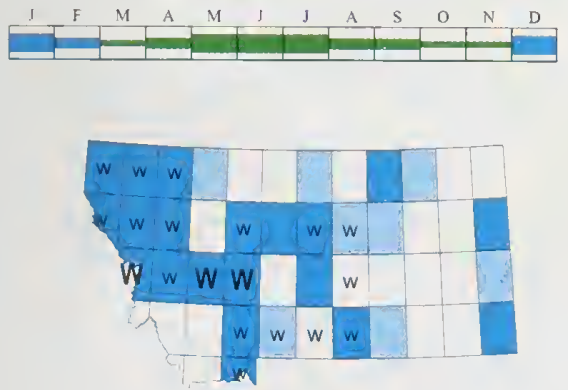
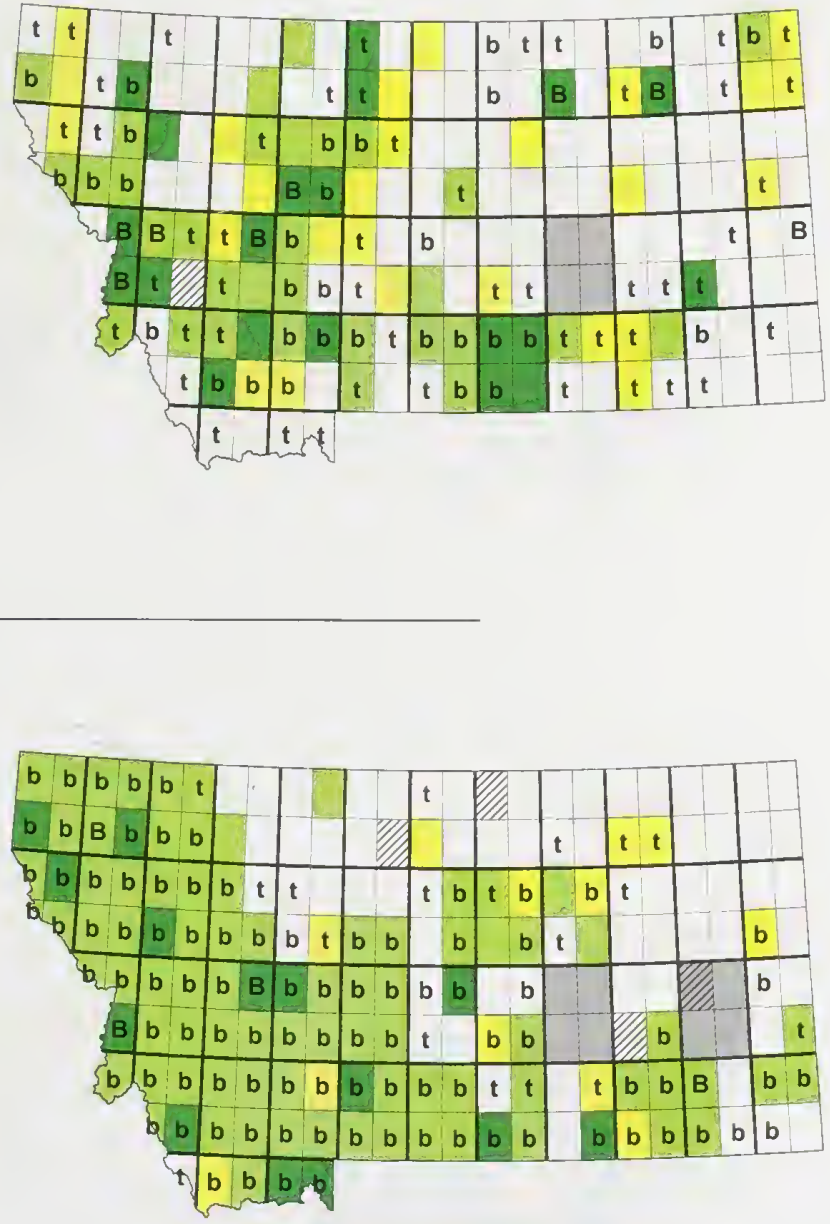


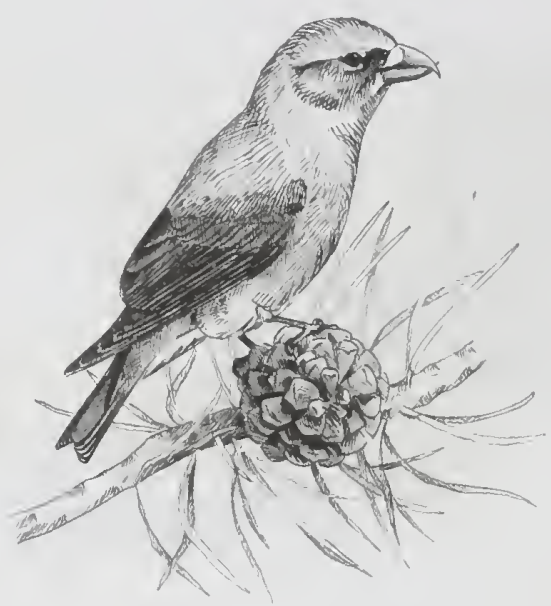

White-winged Crossbill

Loxia leucoptera

$2003-2011=84 \quad$ SI
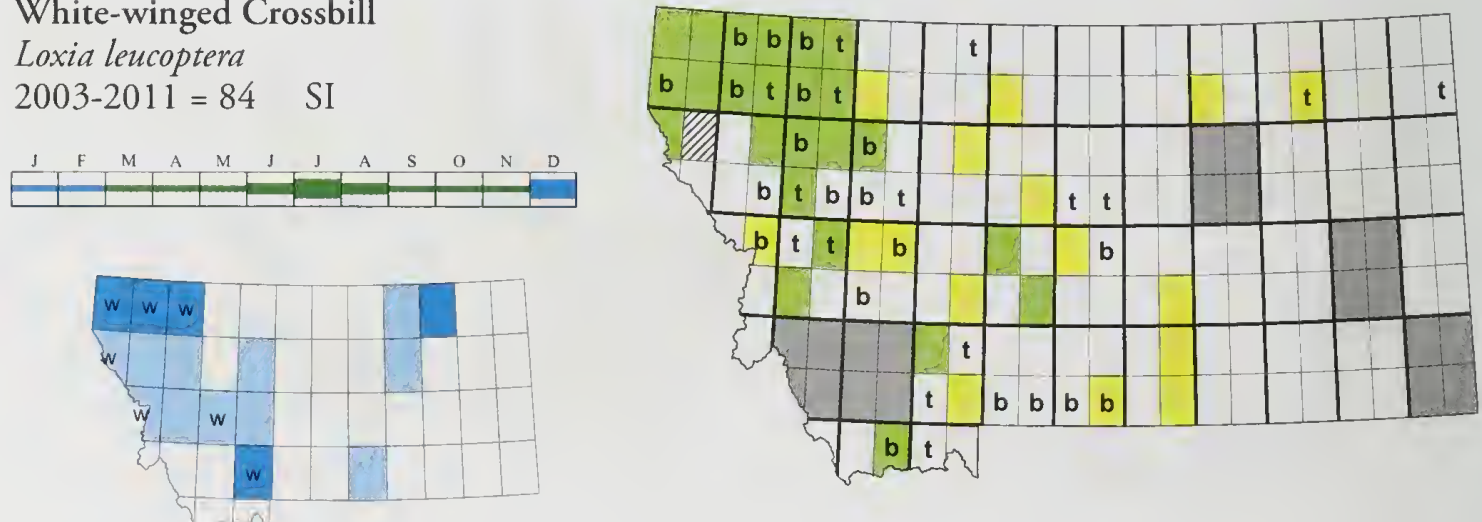

Common Redpoll

Acanthis flammea

2003-2011 = 190
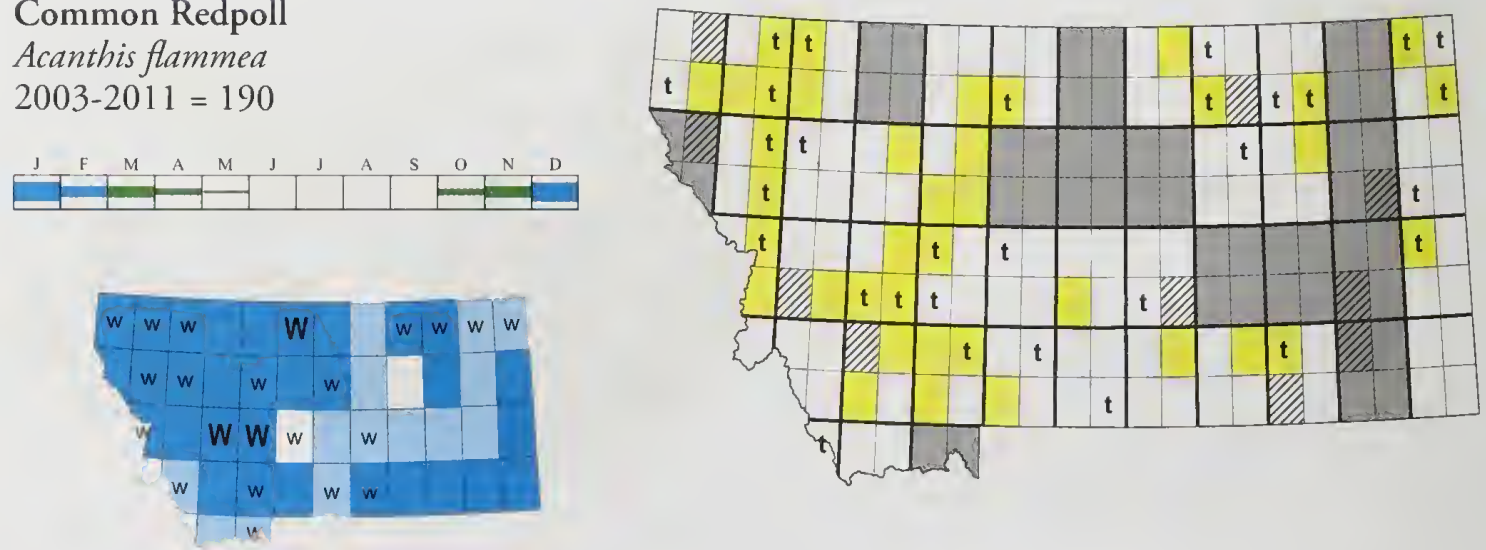
Hoary Redpoll

Acanthis hornemanni

2003-2011 = 11
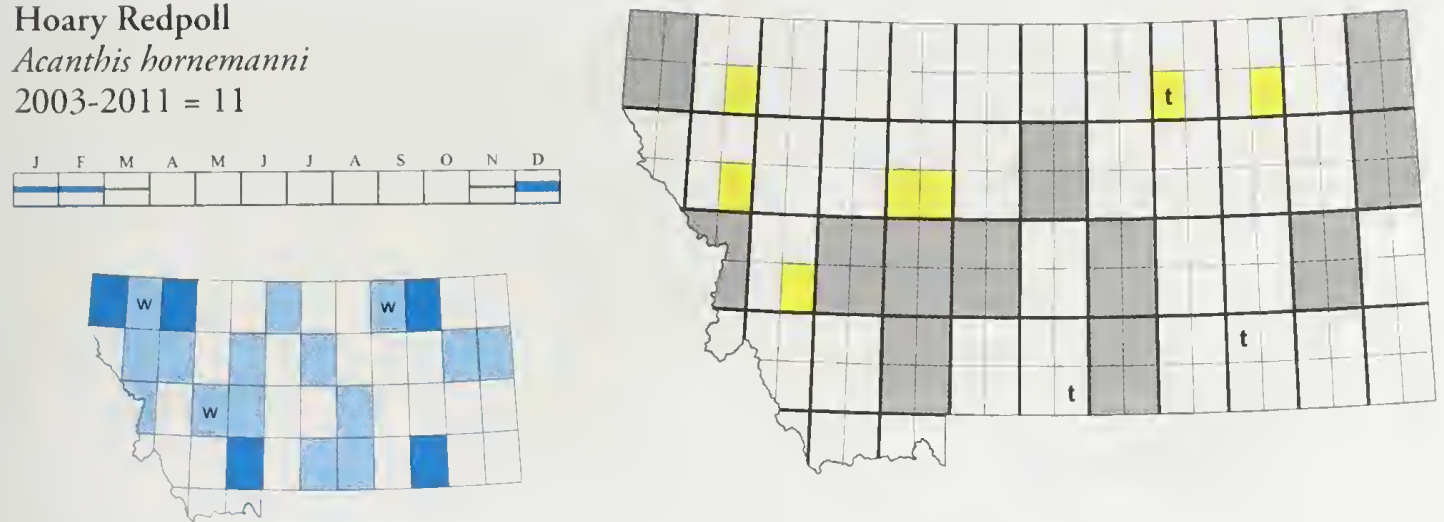

Pine Siskin

Spinus pinus

2003-2011 = 5533
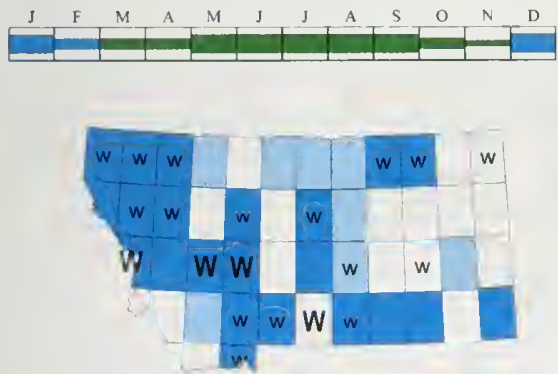

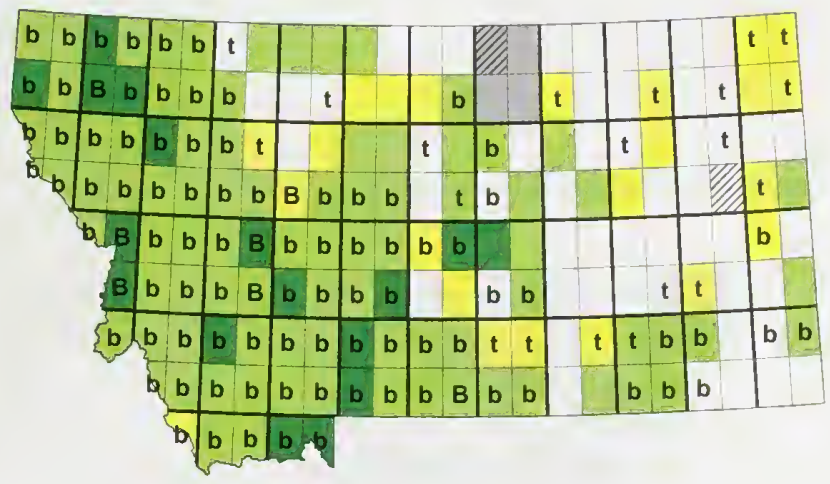

Lesser Goldfinch

Spinus psaltria

2003-2011 = 22

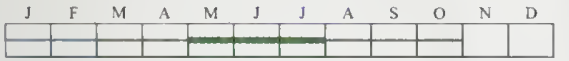

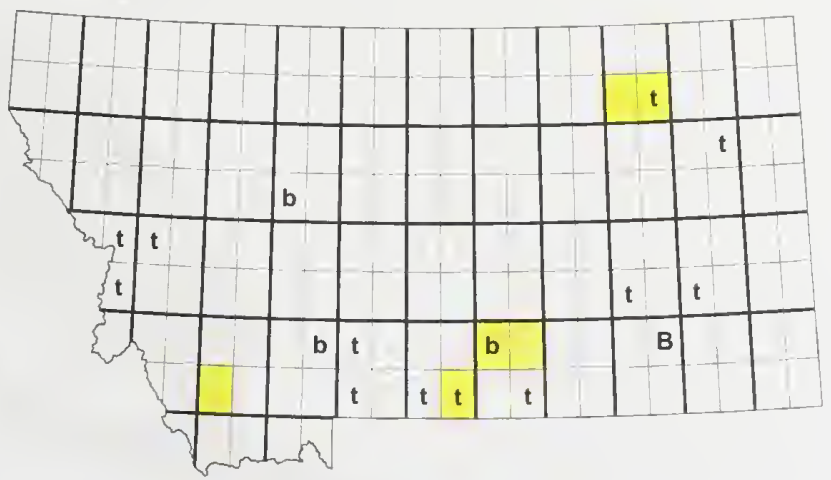


American Goldfinch

Spinus tristis

2003-2011 = 2476
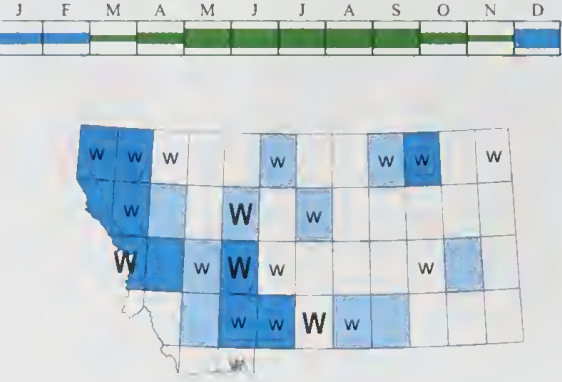

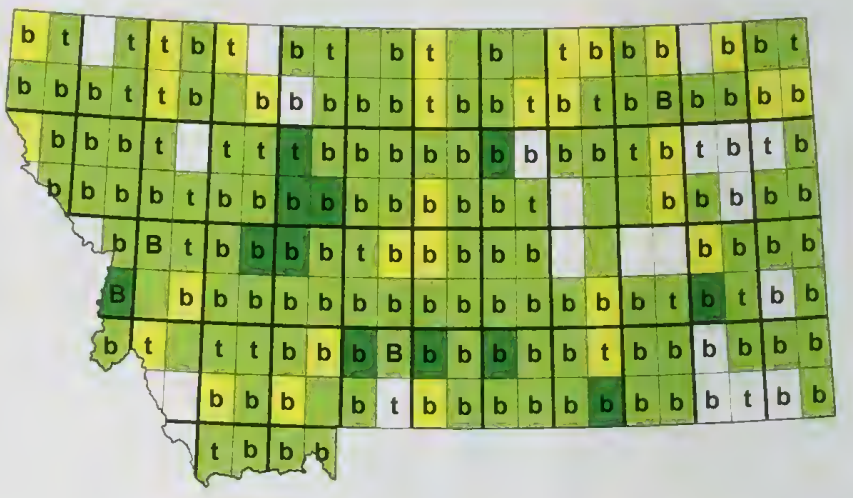

Evening Grosbeak

Coccothraustes vespertinus

$2003-2011=797 \quad$ SOC
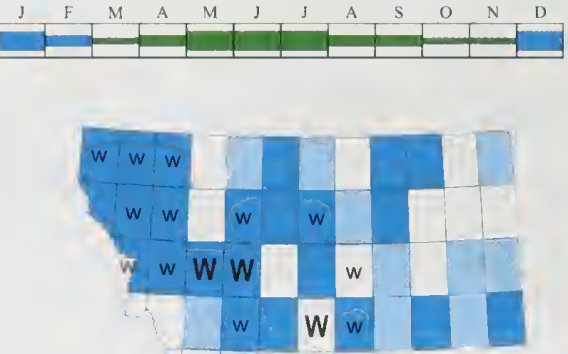

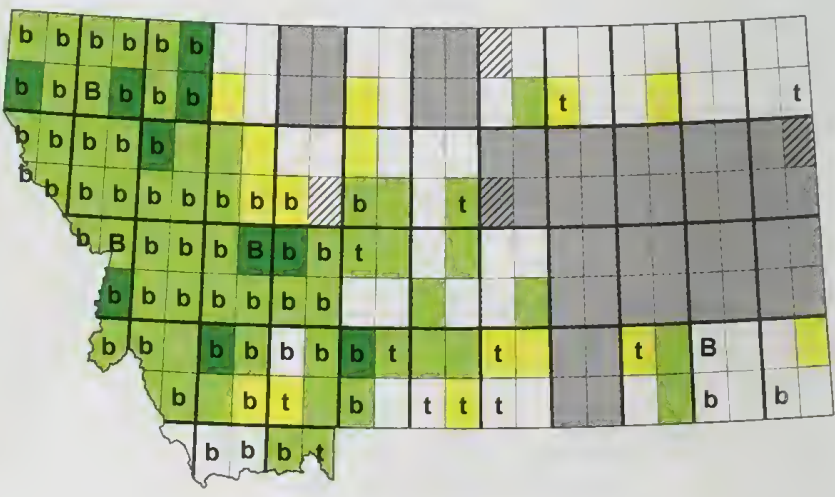

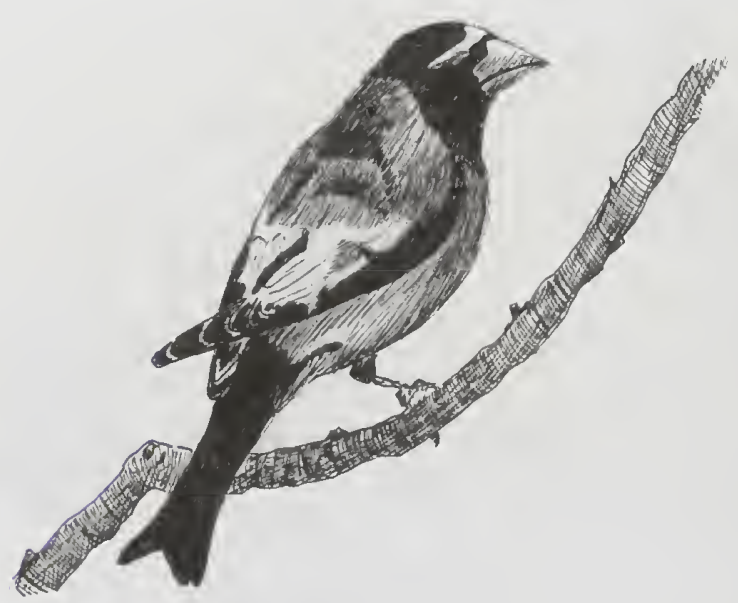


Passeridae

House Sparrow

Passer domesticus

2003-2011 = 1333

${ }^{\prime} \mathrm{F}^{\mathrm{M}} \mathrm{A} \mathrm{M}^{\prime} \mathrm{J}^{\mathrm{A}} \mathrm{S}^{\mathrm{O}} \mathrm{O}^{\mathrm{N}} \mathrm{D}$
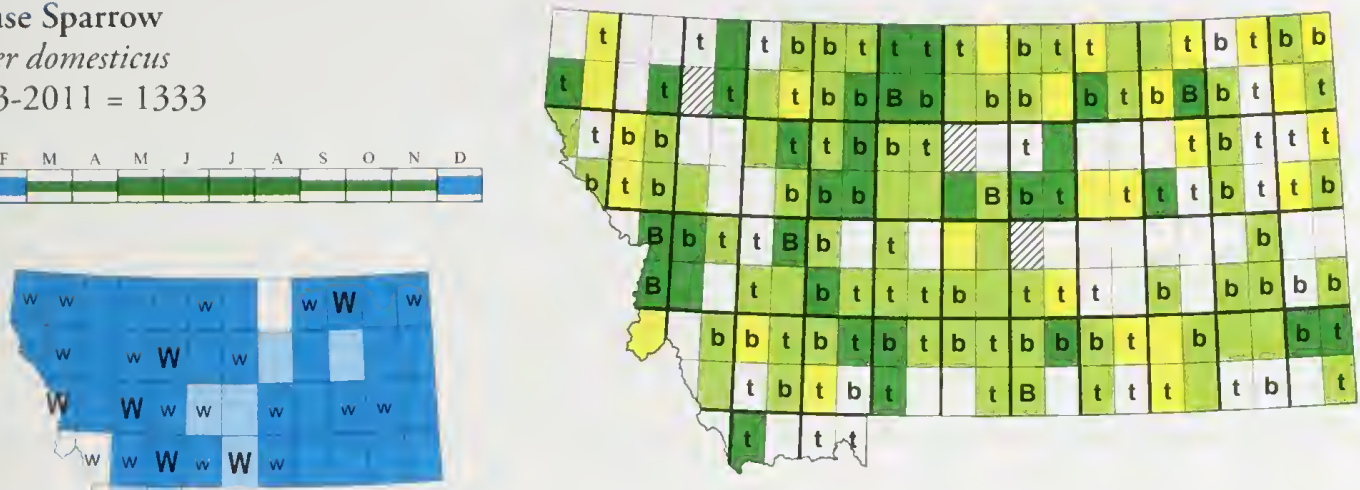


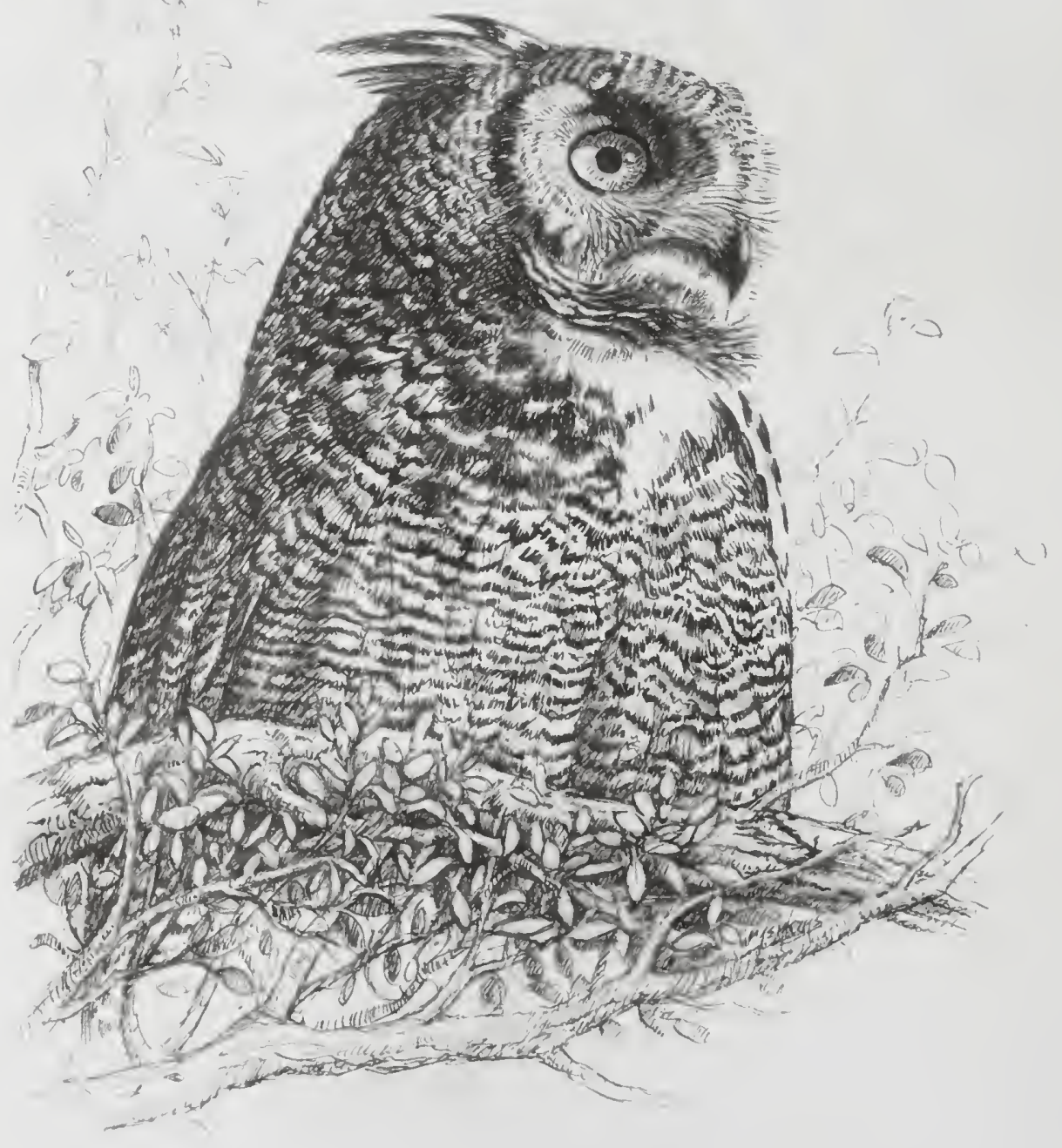




\section{Appendix A. Submission of Bird Records}

This section explains how to submit records to the Montana Bird Distribution (MBD) database, the information required for all observations, and an explanation of the extra information needed for Rare Birds.

\section{How to Submit Records}

The MBD Partnership strives to make numerous data-entry options available to promote the stewardship of birds and their habitats in Montana. Observation records entered via any of the routes below will eventually get into the Montana Bird Distribution database. Although there are pluses and minuses to each of these methods, there is no "best way" to submit records. Most of these methods can be found at the Montana Natural Heritage Program's website at: http://mtnhp.org/observations. asp.

\section{Online Record Submission Options:}

- Simple Web-based Animal Observation Form: http://mtnhp.org/observationform/. This simple web-based data entry tool is available for general entry of bird sightings. This tool can be used from any computer, with slower speed internet connections, and on all web browsers.

- Natural Heritage: MapViewer http://mtnlip.org/mapviewer/ and Natural Heritage Tracker http://mtnhp.org/tracker/. Both of these web applications can be used to view bird records via a dynamic map interface. Both applications also allow the user to see detailed information associated with individual records. The Natural Heritage MapViewer is based on newer technology, compatible with PCs and Macintosh computers, accessible from all web browsers, and compatible with slower internet connections. This application also allows users to generate bird lists for each QQLL. Observations can be entered in MapViewer under the "Generalized Observation" task option by clicking on the "Add an Animal Observation" link under the Tools menu. The Natural Heritage Tracker is based on older technology, is only compatible with PC computers and Internet Explorer, and requires a high speed internet connection; this application is intended to be phased out over the next few years. Observations can be added under the "Add Animal Observation" tab.

- eBird: https://ebird.org. Users can enter bird data through eBird, a cooperative project between the Cornell Lab of Ornithology and the National Audubon Society. eBird is an internet-based checklist program that allows birders to post sightings, keep track of their bird lists, manage their personal records, and explore bird observations being reported by other birders. It also has a variety of informative reports available. eBird data will be incorporated into the MBD database at approximately annual intervals.

The eBird and MBD databases capture similar information. However, there are some important points to consider on whether one should be using eBird or entering observations into the MBD database:

a. Whichever system is used, observations will be more valuable if precise coordinates or reference to a prominent map feature are given, in addition to information on the observed breeding status and relevant comments on what the bird was doing and what habitats it was using. 
b. The MBD database is not meant for (1) reporting daily or multiple entries of backyard birds or (2) field trip bird lists within a season. Instead, if using the MBD database, this information should be collapsed to the highest status $(\mathrm{B}, \mathrm{b}, \mathrm{t}, \mathrm{W}, \mathrm{w})$ within the season observed. If an observer is interested in entering detailed entries for backyard or local trips then eBird is the better database to use.

c. The MBD database strives to document only a single record of a particular bird at a particular location on a particular day. This goal is especially important for "rare" species. If multiple people within a bird outing or group want to submit observations toward their personal bird list, eBird is the more appropriate venue.

d. The MBD database is focused on gathering observations that include exact location information. Precise location information is especially important for breeding records because this information is commonly used in management and conservation efforts. For breeding observations to influence conservation projects and activities, it is especially important to precisely map each breeding bird record (i.e., do not generalize the location in a large geographic area such as "Fort Peck Lake"; instead, document where on Fort Peck Lake the bird was observed). Similarly, lists of birds for large areas - such as a county, National Park, or Wildlife Refuge - would be more appropriately entered into eBird.

\section{Other Submission Options:}

- MBD Sighting Excel Spreadsheet: http://mtnhp.org/docs/MBD Sighting Form.xls. This form can be downloaded from the Natural Heritage Program website; it is the preferred method for receiving large numbers of bird observation records. Submissions using this format can be added to the main database easily.

- Adopt a QQLL Spreadsheet: http://www.mtaudubon.org/birds/distributionQQLL. A simple Excel spreadsheet is available for individuals who want to focus their observations to a specific QQLL. Developed as part of an effort to have better coverage of bird records for local areas across the state, this form should be submitted to Montana Audubon.

- Submit written records: In order to keep data entry costs and errors at a minimum, we prefer that observers enter their own records using one of the record submission options listed above. However, if an observer selects to submit handwritten records, copies of the form found in Appendix B can be filled in and mailed to: Montana Bird Distribution, Montana Audubon, P. O. Box 595 Helena, MT 59624.

\section{Information Required for All Sightings}

The MBD database strives to include only records that are accurate and complete. To be included in the database, every record submitted must have the following information:

- BIRD SPECIES (either common or scientific name). Only include observations where the species identification is certain. Comments on numbers of individuals observed, behavior, and habitat are encouraged, but are not required.

- DATE of observation. Dates are critical for knowing when birds arrive, breed, overwinter, or migrate through Montana. They also give us a tool for verifying records. For example, a breeding record for a warbler in March would not be accepted without substantial documentation. Consequently, records without dates are not accepted. Date ranges may 
be submitted for a list of bird species observed at one locality over time (e.g., a backyard bird list for the summer, or a bird list for a QQLL over a weekend of birding).

- LOCATION of observation. A locality description is required (i.e., southeast corner of Wild Horse Lake; $2.0 \mathrm{~km}$ northwest of Ear Mountain at headwaters of South Fork Willow Creek; Hwy 287 at Badger Pass; Pumpkin Creek 400 meters west of Volborg; QQLL $19 \mathrm{~A} 1$; etc.). For finer resolution in determining locations or QQLL boundaries, please refer to the maps found at the back of this book; U.S. Forest Service, Bureau of Land Management, or U.S. Geological Survey maps; or the Montana Atlas and Gazetteer (DeLorme 2011). We encourage the use of map coordinates (preferably latitude and longitude in decimal degrees), but they are not required.

- $\quad$ STATUS: $\mathrm{B}, \mathrm{b}, \mathrm{t}, \mathrm{W}, \mathrm{w}$ (see definitions on page 10 and 11 ). Observations submitted with a status of "B" or "W" must be accompanied by comments documenting why the designation was used. For backyard bird lists or for areas where an observer documents birds repeatedly during a season, we request submission of only the highest status observed during that season ("B" higher than " $b$ " higher than " $t$ " and "W" higher than "w").

- OBSERVER NAME and CONTACT INFORMATION. This information allows the observation to be credited to the observer and allows the observer to be contacted should more information be needed.

Because of the volume of data being managed and the limited time available for scrutiny of individual submissions, efforts to provide complete and accurate information are greatly appreciated!

\section{Reporting Rare Birds}

The Montana Bird Records Committee (MBRC) was established in 1991 to review rare and unusual bird sightings. For an officially "Rare" species, a written report must be reviewed and accepted by the MBRC before the record can be entered into the MBD database and included in this publication. MBRC membership, bylaws, and minutes can be found at http://mtaudubon.org/birds/report.html.

As of 2012, there were 104 species on the Official State Bird List scarce enough (generally fewer than 20 records) to require a Rare Bird Report be submitted for any sighting. There are notable location (QLL) exceptions for a small number of additional species (Appendix C). Species not previously documented in the state also need documentation. These birds will be added to the State List (Appendix M) only if the MBRC accepts written accounts from two independent observers, or if the record is verified by a photograph or specimen.

For individuals submitting a rare bird sighting, the observer should describe only what was actually witnessed. Seldom is it possible to observe all details of a bird's appearance or behavior. The report should be completed as soon as possible following the observation, while details are fresh. Although the main emphasis should focus on describing the species under consideration, it is also important to note how similar species were eliminated. Photographs assist greatly in determining species identification and should be included with the report whenever possible.

The Rare Bird Report Form is available in Appendix D and can be downloaded at http://mtaudubon. org/birds/report.html. Reports should be sent electronically (preferred) to the email link provided on the MBRC website, or printed and mailed to: Montana Bird Distribution, Montana Audubon, P.O. Box 595, Helena, MT 59624. 
Appendix B. Bird Observation Report Form

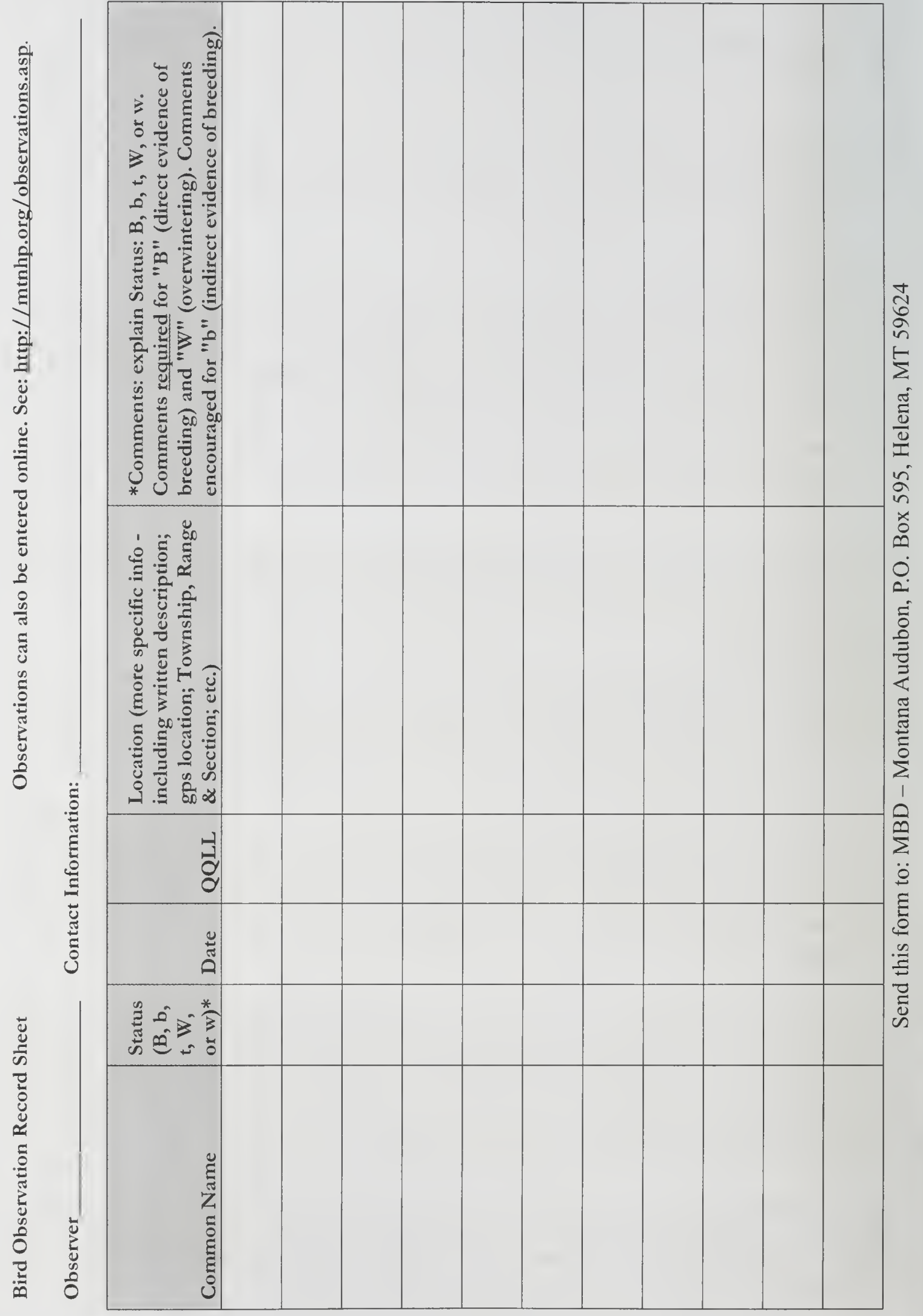




\section{Appendix C. Rare Birds}

The list below identifies 104 species rare enough in Montana to require full documentation of any reported observations in the state. A species is generally considered rare if it has been recorded fewer than 20 times in the state. Most of the species on this list have been reported less than 20 times in the state and are considered rare throughout Montana (R1); a few species are regionally rare within the state (R2); and one species, Yellow-billed Cuckoo (R3), is considered rare due to its apparent decline in the state. Although more than 20 observations have been recorded, the infrequent documentation of this species over the past twenty five years warrants continued status.

The Montana Bird Records Committee reviews reports of observations of rare species before their inclusion in Montana Bird Distribution. Any species not previously seen in the state will only be added to the state list if the committee accepts independent documentation from two or more skilled observers, or if verified by a photograph or specimen.

The following species are considered rare throughout Montana unless otherwise marked (R2).

Brant

Cackling Goose R2

Garganey

Tufted Duck

Black Scoter

Willow Ptarmigan

Greater Prairie-Chicken

Red-throated Loon

Yellow-billed Loon

Manx Shearwater

Wood Stork

Least Bittern

Little Blue Heron

Green Heron

Yellow-crowned Night-Heron

Glossy Ibis

White-tailed Kite

Mississippi Kite

Red-shouldered Hawk

Crested Caracara

Yellow Rail R2

Common Gallinule

Snowy Plover

Black Turnstone

Sharp-tailed Sandpiper

Curlew Sandpiper

American Woodcock

Red Phalarope

Black-legged Kittiwake

Little Gull

Ross's Gull

Laughing Gull

Iceland Gull

Lesser Black-backed Gull

Glaucous-winged Gull R2
Great Black-backed Gull

Arctic Tern

Pomarine Jaeger

Parasitic Jaeger

Long-tailed Jaeger

Long-billed Murrelet

Ancient Murrelet

White-winged Dove

Inca Dove

Yellow-billed Cuckoo R3

Whip-poor-will

Ruby-throated Hummingbird R2

Anna's Hummingbird R2

Costa's Hummingbird

Red-bellied Woodpecker

Yellow-bellied Sapsucker R2

White-headed Woodpecker

Eastern Wood-Pewee

Yellow-bellied Flycatcher R2

Gray Flycatcher R2

Eastern Phoebe

Vermilion Flycatcher

Ash-throated Flycatcher

Great Crested Flycatcher

Scissor-tailed Flycatcher

White-eyed Vireo

Yellow-throated Vireo

Blue-headed Vireo R2

Philadelphia Vireo R2

Western Scrub-Jay

Carolina Wren

Bewick's Wren

Winter Wren

Sedge Wren R2

Blue-gray Gnatcatcher R2
Gray-cheeked Thrush R2

Wood Thrush

Curve-billed Thrasher

Siberian Accentor

Smith's Longspur

Golden-winged Warbler

Blue-winged Warbler

Prothonotary Warbler

Connecticut Warbler

Kentucky Warbler

Hooded Warbler

Northern Parula

Bay-breasted Warbler R2

Blackburnian Warbler R2

Pine Warbler

Yellow-throated Warbler

Prairie Warbler

Black-throated Gray Warbler

Black-throated Green Warbler R2

Painted Redstart

Eastern Towhee

Black-throated Sparrow

Sage Sparrow R2

Golden-crowned Sparrow

Summer Tanager

Scarlet Tanager

Northern Cardinal

Pyrrhuloxia

Blue Grosbeak R2

Painted Bunting

Eastern Meadowlark

Great-tailed Grackle

Hooded Oriole

Brambling 
The gray shading on the maps below indicate where no Rare Bird Report Form is necessary for observations of R2 species.

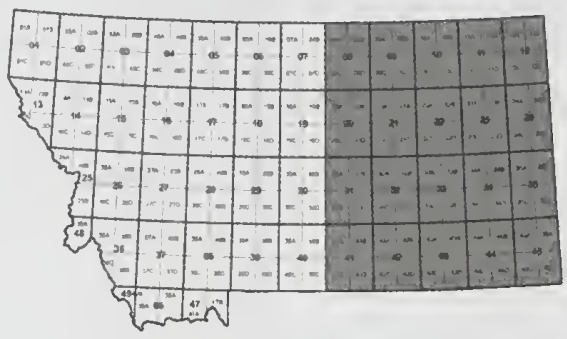

Cackling Goose

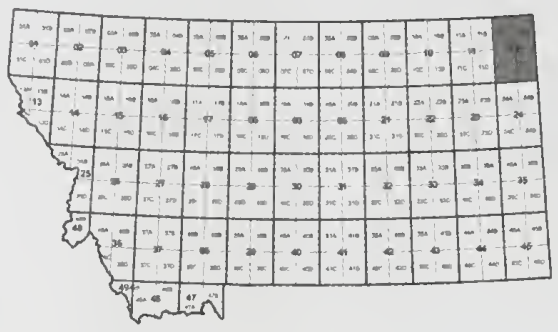

Yellow Rail, Yellow-bellied Flycatcher, Blueheaded Vireo, Sedge Wren

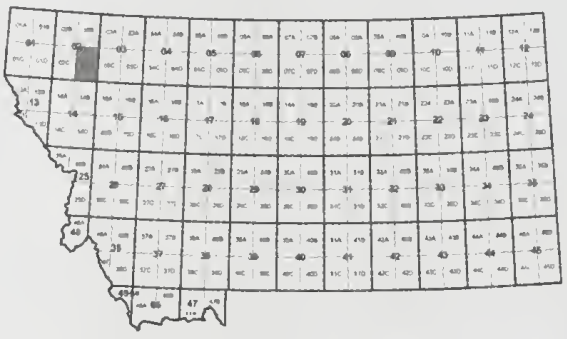

Glaucous-winged Gull

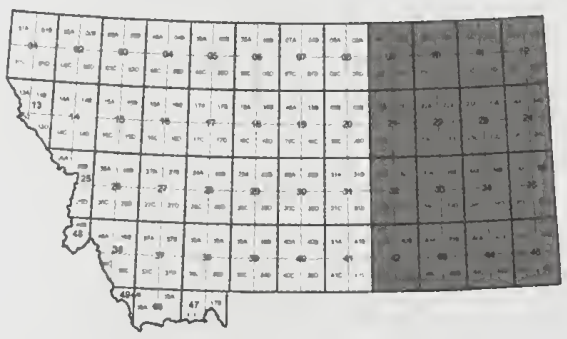

Ruby-throated Hummingbird, Philadelphia Vireo, Gray-cheeked Thrush, Black-rhroated Green Warbler

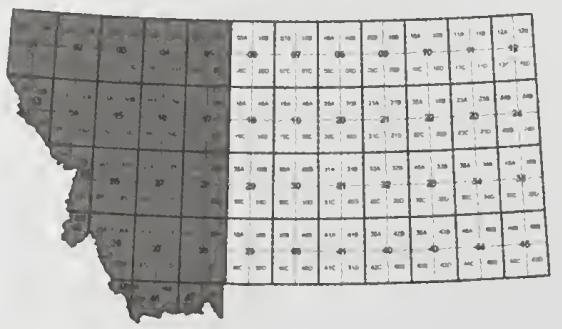

Anna's Hummingbird

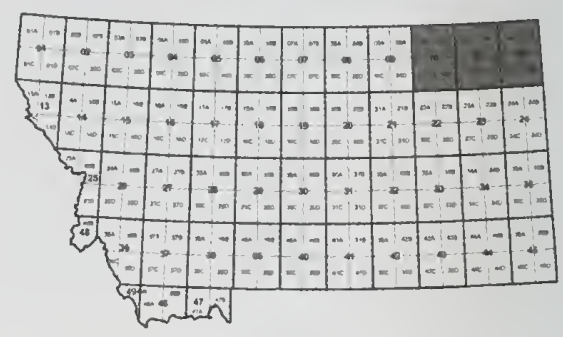

Yellow-bellied Saspsucker, Bay-breasted Warbler

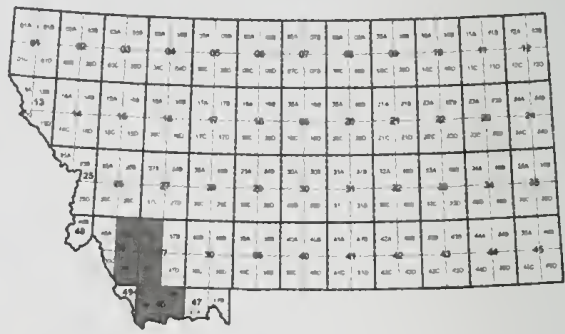

Gray Flycatcher

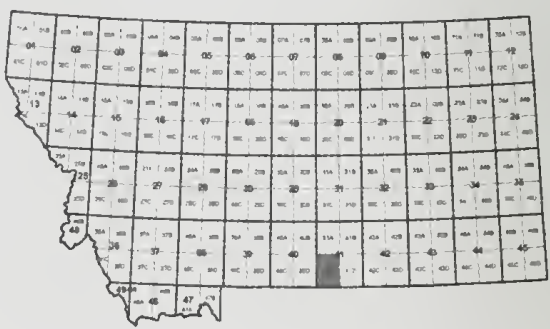

Blue-gray Gnatcatcher

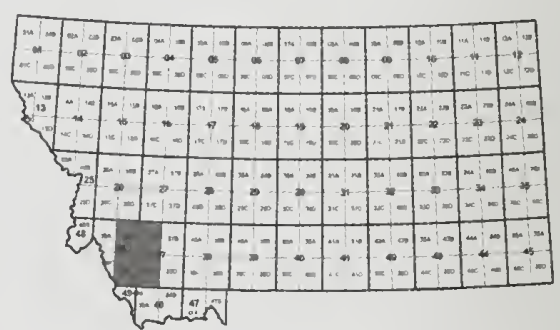

Sage Sparrow

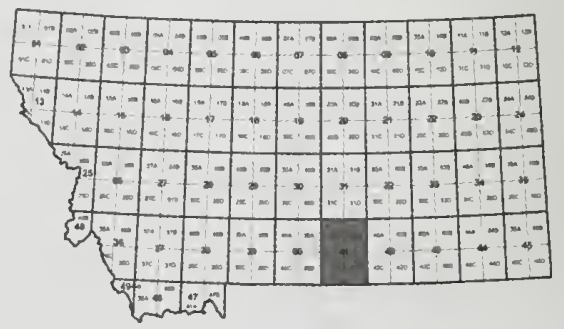

Blue Grosbeak 


\section{Appendix D. Montana Rare Bird Report Form \\ MONTANA RARE BIRD REPORT FORM}

Species:

Date:

Photo taken / enclosed?:

Other observers:

QLL:

Specific location:

Latitude / Longitude:

Describe the habitat:

Plumage:

Quality and direction of light (if applicable):

Distance from bird:

Duration of observation:

Previous experience with species:

This report was written (from memory, field notes, at time of sighting, etc.):

Notes:

Describe the bird. Record only field marks and behaviors you actually saw. State size (compared with other nearby known or common birds). Record relevant information such as color, body shape, bill, legs, voice, behavior, number of individuals, sex, etc.:

Discus how similar species were eliminated:

This document can be found online at http://mtaudubon.org/birds/report.html

Send electronically to mtauduboncamtadubon.org or by mail to Montana Bird Records Committee, Montana Audubon, P.O. Box 595, Helena, MT 59624 USA.
Observer:

Address:

Phone:

E-mail:

Specimen taken?:

Nearest town:
Sex / age:

Optics used: 


\section{Appendix E. Threatened (T), Endangered (E), Candidate (C), other Species of Concern (SOC), and Species of Interest (SI)}

Listed below are species identified as Animal Species of Concern by the Montana Natural Heritage Program and Montana Department of Fish, Wildlife \& Parks. They include (1) species federally listed under the Endangered Species Act as Endangered, Threatened, or Candidates for federal listing, and (2) U.S. Forest Service Sensitive Species, Bureau of Land Management Special Status Species, or other Species of Concern as identified by the Montana Animal Species of Concern Committee. A third category, termed Special Interest, includes species for which supplemental information is requested; generally, these are species with unique breeding habits that warrant further study, whose status and distribution are poorly known in Montana, or have special protection under federal law. These lists are subject to change. For current information, contact the U.S. Fish \& Wildlife Service, U.S. Forest Service, Bureau of Land Management, or Montana Natural Heritage Program (http://mtnhp.org).

Birds listed in this appendix are identified in this publication by a Status code below their names on the pages with their associated maps: Threatened (T), Endangered (E), Candidate (C), other Species of Concern (SOC), and Special Interest (SI) species.

The precise location for any record of species on these lists is particularly important. Therefore, a detailed description of location, habitat, number of individuals, and behavior should accompany reports for all of the birds in this appendix, and is particularly important for reporting evidence of nesting. Observations can be entered online at the Montana Natural Heritage Program website or reported on the form found in Appendix B and submitted by mail to the Montana Natural Heritage Program, P. O. Box 201800, 1515 East Sixth Avenue, Helena, MT 59620-1800.

\section{Federally Listed Species of Concern}

Greater Sage-Grouse - Candidate

Whooping Crane - Endangered

Least Tern - Endangered

Piping Plover - Threatened

Yellow-billed Cuckoo - Candidate

(west of Continental Divide)

Sprague's Pipit - Candidate

\section{Other Species of Concern}

Trumpeter Swan

Harlequin Duck

White-tailed Ptarmigan

Sharp-tailed Grouse

Common Loon

Horned Grebe

Clark's Grebe

American White Pelican

American Bittern

Great Blue Heron

Black-crowned Night-Heron

White-faced Ibis

Northern Goshawk
Ferruginous Hawk

Golden Eagle

Peregrine Falcon

Yellow Rail

Mountain Plover

Black-necked Stilt

Long-billed Curlew

Franklin's Gull

Caspian Tern

Black Tern

Common Tern

Forster's Tern

Black-billed Cuckoo
Flammulated Owl

Burrowing Owl

Northern Hawk Owl

Great Gray Owl

Black Swift

Lewis's Woodpecker

Red-headed Woodpecker

Black-backed Woodpecker

Pileated Woodpecker

Alder Flycatcher

Loggerhead Shrike

Pinyon Jay

Clark's Nutcracker 
Boreal Chickadee

Brown Creeper

Pacific Wren

Sedge Wren

Blue-gray Gnatcatcher

Veery

Varied Thrush

Sage Thrasher

\section{Species of Interest (SI)}

Eared Grebe

Barrow's Goldeneye

Hooded Merganser

Bald Eagle

Virginia Rail

Short-eared Owl

Western Screech-Owl

Eastern Screech-Owl
Chestnut-collared Longspur

McCown's Longspur

Green-tailed Towhee

Brewer's Sparrow

Sage Sparrow

Baird's Sparrow

Le Conte's Sparrow

Nelson's Sparrow

Boreal Owl

Common Poorwill

Chimney Swift

Broad-tailed Hummingbird

Rufous Hummingbird

Cassin's Kingbird

Plumbeous Vireo

Eastern Bluebird
Bobolink

Gray-crowned Rosy-Finch

Black Rosy-Finch

Cassin's Finch

Evening Grosbeak
Ovenbird

Black-and-white Warbler

Tennessee Warbler

Dickcissel

White-winged Crossbill

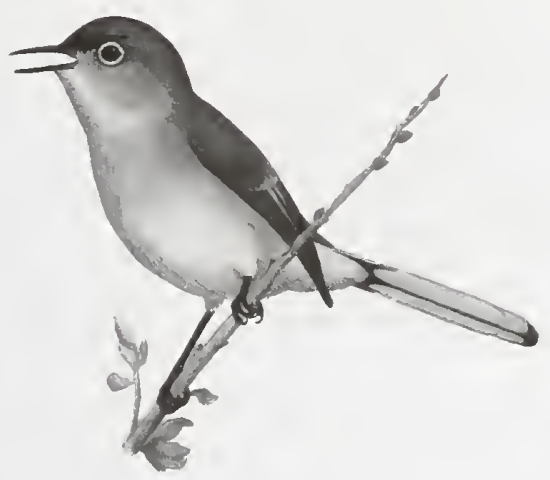




\section{Appendix F. Subspecies}

As more research and genetic evidence emerges, the potential for taxonomic splits of one or more of the species listed in this Appendix becomes likely. If any of these subspecies are elevated to species status in the future, observations including information on breeding habits and location of these birds will become necessary. This situation can be illustrated through the examples of the Winter Wren and the Whip-poor-will, two recent taxonomic splits with relevance in Montana. The Winter Wren was recently split into three species (Eurasian Wren, Winter Wren, and Pacific Wren); Montana has both North American species. The Winter Wren is a rare migrant to the eastern part of the state, whereas the Pacific Wren is a regular breeder and year-round resident in the western half of the state. Separating existing records for this species was relatively easy, due to habitat comments and their apparent disjunct ranges. Conversely, the Whip-poor-will, a rare bird in Montana, was split into two species: Eastern Whip-poor-will and Mexican Whip-poor-will. Montana's only documented observation of this species did not contain enough information to determine which of the newly named species was observed (which is why the "lumped" name Whip-poor-will, Caprimulgus sp., occurs in this edition).

Where information is available, maps displaying both species and subspecies distribution for the birds listed in this Appendix are included in this publication. Bird distribution maps were created for each subspecies to encourage birdwatchers to submit future records at this taxonomic level. No maps appear for the three subspecies of Fox Sparrow due to a lack of data. However, they may be included in future editions.

Species for which information is requested at the subspecies, race, or form level:

\section{Green-winged Teal}

Eurasian Green-winged Teal

\section{Northern Flicker}

Red-shafted Flicker

Yellow-shafted Flicker

\section{Brewer's Sparrow}

Timberline Sparrow

\section{Fox Sparrow}

Sooty (Pacific) Fox Sparrow

Slate-colored (Interior West) Fox Sparrow

Red (Taiga) Fox Sparrow

\section{Dark-eyed Junco}

Slate-colored Junco

Oregon (Montana) Junco

Pink-sided Junco

White-winged Junco

Gray-headed Junco

\section{Yellow-rumped Warbler}

Myrtle Warbler

Audubon's Warbler 


\section{Appendix G. Introduced Species}

The following species are not native to Montana and were either introduced into or colonized the state on their own after being introduced elsewhere in North America. The Wild Turkey is the only species on the following list native to other regions of the continent. Because these species have bred in the wild and persist in Montana, they are included on the official list of birds for the state. Distribution maps for these species appear in this book. We stress that the introduction of new bird species is regulated in Montana because it may pose a threat to native wildlife, human health, and/or public safety.

$\begin{array}{ll}\text { Mute Swan } & \text { Ring-necked Pheasant } \\ \text { Chukar } & \text { Wild Turkey } \\ \text { Gray Partridge } & \text { Rock Pigeon }\end{array}$

\section{Eurasian Collared-Dove European Starling House Sparrow}

The Cattle Egret is not identified as introduced in Montana as its presence in North America is considered a natural expansion of its native range. Originally from subtropical and tropical Africa and Asia, the Cattle Egret colonized northeastern South America in the late 1870's. It arrived in the United States by the early 1940's, and was first documented in Montana in May 1974 in the Bitterroot Valley (QLL 25D).

Individuals of the following species are not native to Montana but, at times, do occur in the state. Their initial presence resulted from the release and/or escape of captive-raised birds; in some cases these releases are ongoing. Some may breed in the wild and survive for multiple years (e.g., California Quail), but they have not maintained wild populations long enough to conclude that they persist naturally in the state. Therefore, they do not appear on the official list of birds of Montana, and maps on their distribution do not appear in this book.

\section{California Quail Northern Bobwhite Ringed Turtle-Dove}

The Northern Bobwhite appeared in previous editions of Montana Bird Distribution, but no maps for this species were included in the 6 th edition nor in this edition. Northern Bobwhites were widely transplanted in Montana during the latter part of the 19th century and became established for some time. All of these birds died out many years ago, and we believe all recent records are from birds released or escaped from captivity. We are not aware of a single case where escaped bobwhites have persisted for the 10 years regarded as minimal for an established occurrence (Wright 1996).

The status of California Quail in Montana has been a point of on-going debate. Their continued and increasing presence in parts of the state, especially the Bitterroot Valley, has raised the question of whether this species should be added to the official list of Montana birds. Although many observers have documented this species successfully breeding, as well as persisting through wintering months (Christmas Bird Count data), the continued release of captive birds into the region prevents any quantitative assessment of their status. The Montana Bird Records Committee, the organization responsible for the official checklist of birds of Montana, voted in 2011 against adding the California Quail to the official state bird list. The Committee will continue to monitor the status of the California Quail in the state through collection of relevant observations, annual datasets, and research efforts. 


\section{Appendix H. Hypothetical Records}

All reports of species new to Montana must be reviewed and accepted by the Montana Bird Records Committee (MBRC) in order to be included in the MBD database. The MBRC requires at least one of the following for approval of first state records: a specimen, a definitive photograph, or independent documentation by two or more skilled observers.

"Hypothetical records" are for observations of species for which we have convincing reports in Montana, but which have not been added to the official state list by MBRC or by previous compilers of Montana Bird Distribution because of specific, identified reasons. The species listed below are placed on the hypothetical records list for the reasons noted. Other species have been reported, but details were insufficient to warrant inclusion here.

Common Crane (Grus grus): QLL 12B, 1999. Single-observer sighting with convincing details.

Pacific Golden-Plover (Pluvialis fulva): QLL 09C, 2001. Single-observer sighting with convincing details.

Red-breasted Sapsucker (Sphyrapicus ruber): QLL 27B, 1984. Single-observer sighting with convincing details.

Bell's Vireo (Vireo bellii): QLL 24B, 1995 and QLL 12B, 2007. Both observations with convincing details, but both were single-observer sightings.

Phainopepla (Phainopepla nitens): QLL 03A, 1962. Carried over from previous editions. Original observer interviewed by P.D. Skaar in 1971, resulting in Skaar's placement of species on hypothetical list. Information insufficient for review under current criteria established by the MBRC.

Virginia's Warbler (Oreothlypis virginiae): QLL 41C, 2005. Single-observer sighting with convincing details.

Hermit Warbler (Setophaga occidentalis): LL 38, 2005. Convincing details provided, but hybrid origin could not be ruled out.

Cassin's Sparrow (Pencaea cassinii): QLL 29C, 1999. Sight record with convincing details, but independent documentation from two observers absent.

Since the 6th edition of Montana Bird Distribution was published, the following species have been removed from the hypothetical list and placed on the official list:

Glossy Ibis: QLL 16B, 05/2002; first documented state record.

Iceland Gull: QLL 17B, 12/2005; first documented state record.

Long-billed Murrelet: 15C, 06/1985; accepted by Wright (1997). Previously presented as Marbled Murrelet. Record reviewed and accepted by MBRC as Long-billed Murrelet.

Blue-winged Warbler: QLL 09C, 05/2006; first documented state record.

Painted Bunting: QLL 41A, 12/1999; report submitted and accepted by MBRC in 2003. 


\section{Appendix I. Extinct Species}

The Passenger Pigeon is the only species recorded in Montana known to be extinct. The last reported specimen of this species was a "young" bird collected on 23 August 1874 near Waterton Lake in Glacier National Park (Latilong 4) by Elliott Coues. This specimen is still in the rare bird display at the U.S. National Museum. Grinnell (1876) reported "small companies in July [1875] along the Missouri River bottom, where it was doubtless breeding" between the mouth of Yellowstone River and Carroll (townsite 38 miles above mouth of Musselshell River) on the Missouri River. Earlier records showed the Passenger Pigeon inhabiting latilongs $3,4,5,7,11,12,17,24,26$, and 41 .

\section{Appendix J. A Summary of Bird Record Information by Latilong}

The following maps (Figure 3 and 4) use the latilong system to summarize the information stored in the Montana Bird Distribution database in two different ways. Both maps generally reflect bird locations, but they also reflect where our contributors are watching birds and submitting records.

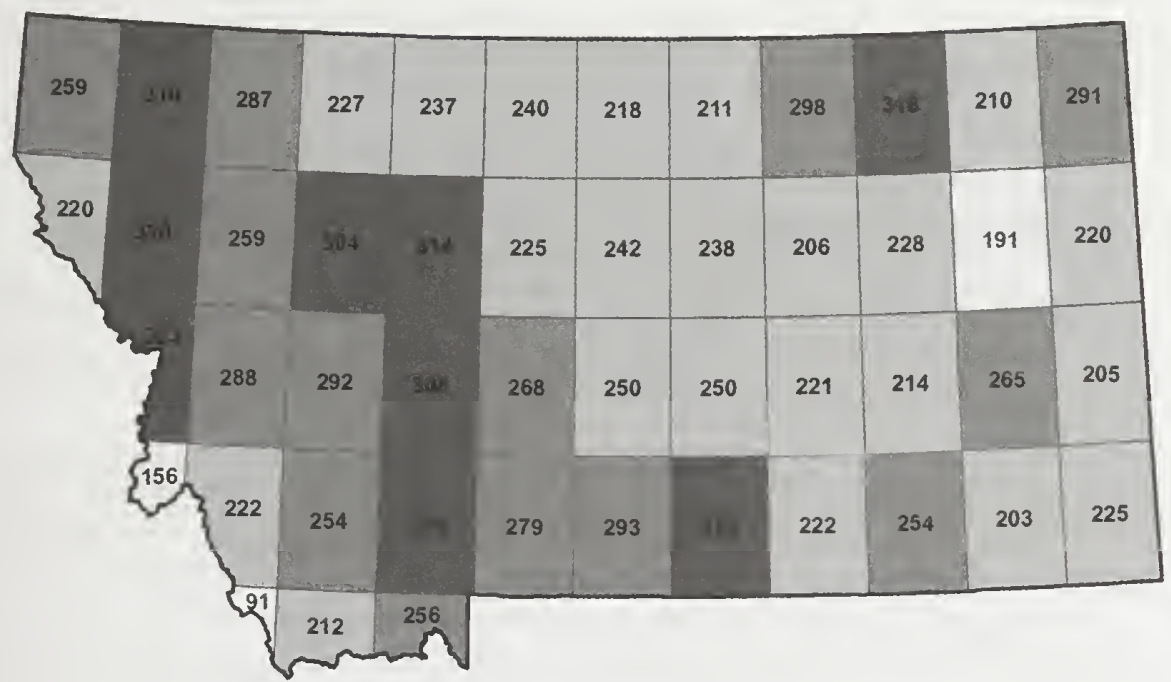

Figure 3. Species Richness by Latilong. The map shows the total number of bird species that have been reported in each latilong. Shading represents general species richness for each latilong; the center number is the total number of species recorded in that latilong (out of the statewide total of 427). 


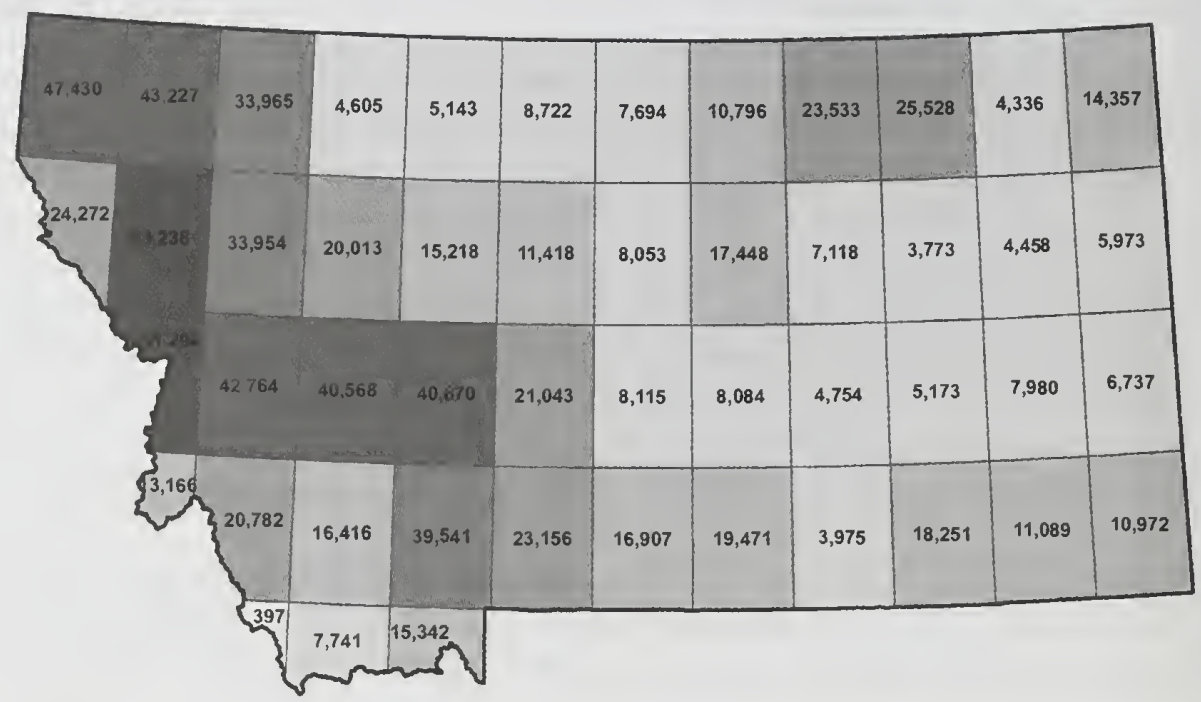

Figure 4. Total Records by Latilong. The map shows the total number of bird records for all species reported for each latilong. Shading represents general observation numbers reported for each latilong; the center number is the total number of records for that latilong. This figure can alert birders to areas of the state that are poorly documented.

\section{Appendix K. Why Bird Distribution Information is Important}

Bird records in the MBD database directly help bird conservation in Montana. Submission of current bird observations ensures that Montana continues to have reliable, objective information about the status and distribution of bird species. This information has many uses, including:

Informing Resource Development Decisions. Resource managers and developers benefit significantly from information on sensitive bird species and their habitats. This information is used by the private and public sectors for planning timber sales, subdivisions, utility and pipeline corridors, weed control efforts, wind farm siting, oil and gas extraction, mines, highway construction, and more. Providing easy access to this information saves untold dollars, reduces conflicts, and decreases delays in decisionmaking. Knowing where and when sensitive species are present helps developers avoid needless impacts and protect habitat in order to maintain healthy bird populations.

Identifying Species of Concern. Keeping accurate records in the MBD database on the state's most vulnerable bird species aids resource managers, decision-makers, conservationists, and businesses. This information is used by Montana Fish, Wildlife \& Parks and the Montana Natural Heritage Program to maintain the Montana Animal Species of Concern list. This list contains native Montana animals that are considered to be "at risk" due to declining population trends, threats to their habitats, and/or restricted distribution (Table 1). This list also identifies animals for which additional data are needed before an accurate status assessment can be made. As of April 2012, there were 66 bird Species of Concern.

Assisting with Stewardship of Species of Concern. Understanding our vulnerable species and their habitats also improves the success of resource management and conservation efforts. Although designation as a Species of Concern (Appendix E) provides no regulatory protection, these designations allow resource managers and decision-makers to make proactive decisions regarding species conservation and data collection priorities. Keeping records on the status of species in one location provides everyone 
with a single well recognized and comprehensive source of this information. It also can help with stewardship efforts to avoid additional declines in bird populations and/or the listing/de-listing of species under the Endangered Species Act.

Monitoring Distribution Trends Over Time. Reported bird observations are extremely important to understanding the distribution and status of individual species over time. For example, these data have allowed us to track the expansion of Eurasian Collared-Doves, the potential expansion of species like the Lesser Goldfinch, or potential range contractions in Yellow-billed and Black-billed Cuckoos. As examples, maps for the Eurasian Collared Dove (Figure 5) and Yellow-billed Cuckoo (Figure 6) are provided.

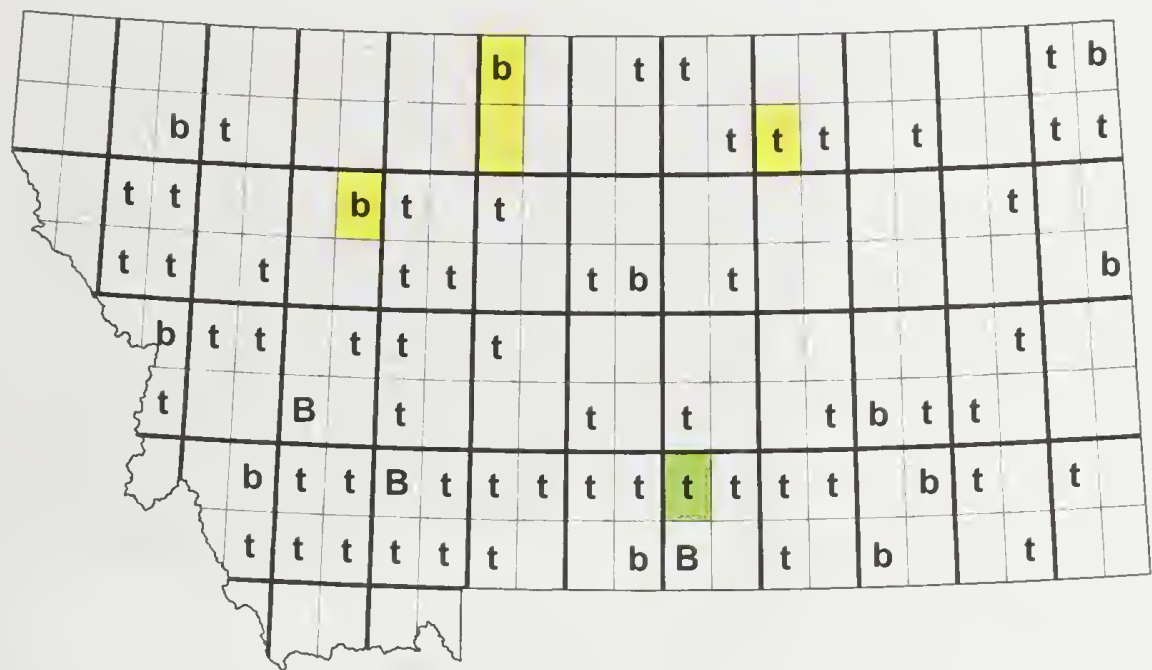

Figure 5. Map showing the expansion of Eurasian Collared-Dove distribution in the state. The first Montana record for this species occurred in July 1997; this species is now observed throughout the state and breeding has also been documented.

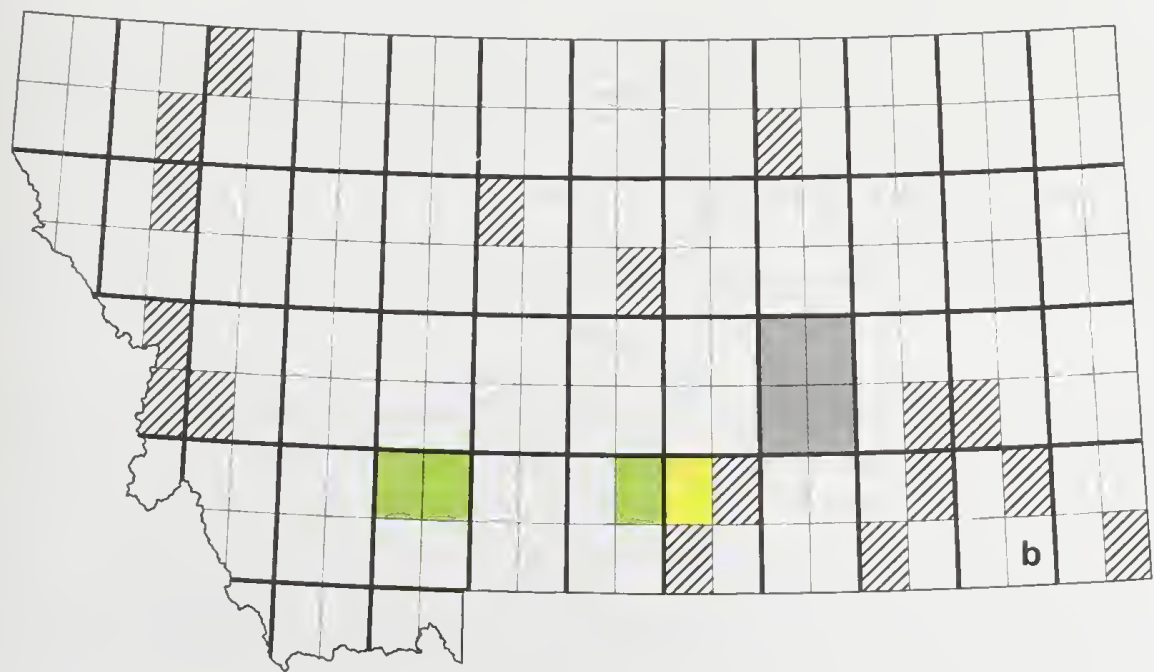

Figure 6. Map showing potential contraction of Yellow-billed Cuckoo distribution in the state. These birds were reported annually in the 1980's; between 2002 and 2012 , the only record reported occurred in July 2011. 
Documenting Changes in Migration Patterns Over Time. Reporting spring and fall arrival dates for migratory birds, as well as overwintering records, will help Montanans understand how species are responding to climate change. This information can then be used to assist with long-term conservation efforts of our bird species in a warming world.

Identifying Important Habitats for Regional Planning. Bird records with detailed habitat information and/or those that are precisely mapped can be used to create models that predict habitat suitability for species. As an example, the map in Figure 7 predicts where the best habitat for Sprague's Pipit can be found in the state. Habitat maps such as this are being used by a variety of resource management agencies in regional planning efforts for the benefit of bird Species of Concern.

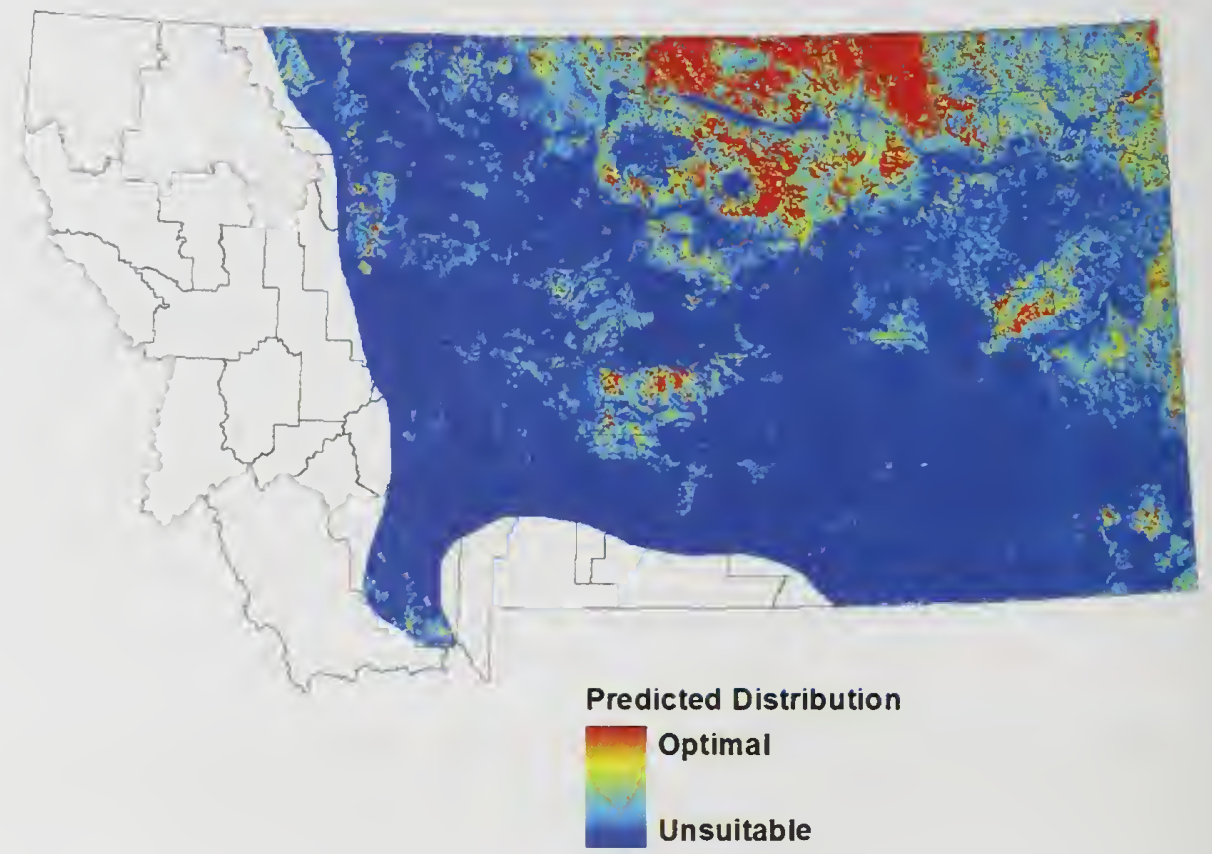

Figure 7. Predicted habitat suitability map for Sprague's Pipit.

Providing Natural History Information on Birds to the Public. The Montana Field Guide summarizes the biology, distribution, status, habitat use, and other natural history information for Montana's birds, as well as other animals, plants, and habitat types. This web-based resource is used by natural resource professionals, K-12 and university students, the general public from throughout Montana, and around the world. The Montana Field Guide is a cooperative project between the Montana Natural Heritage Program and Montana Fish, Wildlife \& Parks and can be found at http://fieldguide. mt.gov/.

Providing Important Bird Information for Habitat Protection Efforts. Table 1 classifies Montana birds by optimal bird habitat, noting the number of Species of Concern found in each major habitat type. Derived from the MBD database, this information helps provide the basis for management decisions at the local and state level. Understanding habitat and species associations can aid efforts to protect habitats representing a disproportionate amount of critical habitat for Montana's bird species. For example, while wetland and riparian habitats make up approximately $4 \%$ of Montana's land base; they provide optimal habitat for 102 (39\%) of the state's breeding birds, 51 (72\%) of the state's passthrough migrants, and 31 (49\%) of the state's Species of Concern. 
Table 1. Optimal habitats for Montana's 336 bird species that are knon'n to breed, migrate through, or oveminter in the state. Species of Concern are identified. Ninety one rare species, which bave been seen in Montana fewer than 20 times, are excluded from this table.

\begin{tabular}{|c|c|c|c|c|c|c|}
\hline $\begin{array}{l}\text { Optimal } \\
\text { Habitat } \\
\text { Type }\end{array}$ & $\begin{array}{l}\text { Total } \\
\text { Birds } \\
\end{array}$ & $\begin{array}{l}\text { Breeding } \\
\text { Birds } \\
\text { (Percent) }\end{array}$ & $\begin{array}{c}\text { Migrant } \\
\text { Birds }\end{array}$ & $\begin{array}{c}\text { Species of } \\
\text { Concern } \\
\text { (SOC) } \\
\text { (Percent) }\end{array}$ & $\begin{array}{c}\text { Percent } \\
\text { SOC in } \\
\text { this } \\
\text { habitat }\end{array}$ & $\begin{array}{l}\text { Species of Concern (SOC). NOTE: } \\
\text { underlined/bolded birds are threatened (T), } \\
\text { endangered (E), or candidate (C) species under } \\
\text { the Endangered Species Act. }\end{array}$ \\
\hline Alpine & 4 & $\begin{array}{c}3 \\
(1.2 \%)\end{array}$ & 1 & $\begin{array}{c}3 \\
(4.5 \%)\end{array}$ & $75.0 \%$ & $\begin{array}{l}\text { White-tailed Ptamigan, Gray-crowned Rosy- } \\
\text { Finch, Black Rosy-Finch }\end{array}$ \\
\hline Cliff & 5 & $\begin{array}{c}5 \\
(1.9 \%) \\
\end{array}$ & 0 & $\begin{array}{c}1 \\
(1.5 \%)\end{array}$ & $20.0 \%$ & Peregrine Falcon \\
\hline Grasslands & 29 & $\begin{array}{c}29 \\
(11.2 \%)\end{array}$ & & $\begin{array}{c}9 \\
(13.6 \%)\end{array}$ & $31.0 \%$ & $\begin{array}{l}\text { Golden Eagle, Mountain Plover, Long-billed } \\
\text { Curlew, Burrowing Owl, Sprague's Pipit (C), } \\
\text { Chestnut-collared Longspur, McCown's Longspur, } \\
\text { Baird's Sparrow, Bobolink }\end{array}$ \\
\hline Forest & 85 & $\begin{array}{c}73 \\
(28.1 \%)\end{array}$ & 13 & $\begin{array}{c}14 \\
(21.2 \%)\end{array}$ & $16.5 \%$ & $\begin{array}{l}\text { Northern Goshawk, Flammulated Owl, Northern } \\
\text { Hawk Owl, Great Gray Owl, Black-backed } \\
\text { Woodpecker, Pileated Woodpecker, Pinyon Jay, } \\
\text { Clark's Nutcracker, Boreal Chickadee, Brown } \\
\text { Creeper, Pacific Wren, Varied Thrush, Cassin's } \\
\text { Finch, Evening Grosbeak }\end{array}$ \\
\hline $\begin{array}{l}\text { Shrub and } \\
\text { forest }\end{array}$ & 12 & $\begin{array}{c}8 \\
(3.1 \%) \\
\end{array}$ & 4 & $\begin{array}{c}1 \\
(1.5 \%) \\
\end{array}$ & $8.3 \%$ & Green-tailed Towhee \\
\hline $\begin{array}{l}\text { Sagebrush/ } \\
\text { shrub }\end{array}$ & 21 & $\begin{array}{c}18 \\
(6.9 \%)\end{array}$ & 3 & $\begin{array}{c}8 \\
(12.1 \%)\end{array}$ & $38.1 \%$ & $\begin{array}{l}\text { Greater Sage-Grouse (C), Sharp-tailed Grouse, } \\
\text { Fernuginous Hawk, Loggerhead Shrike, Blue-gray } \\
\text { Gnatcatcher, Sage Thrasher, Brewer's Sparrow, } \\
\text { Sage Sparrow }\end{array}$ \\
\hline $\begin{array}{l}\text { Wetland/ } \\
\text { riparian }\end{array}$ & 153 & $\begin{array}{c}102 \\
(39.2 \%)\end{array}$ & 51 & $\begin{array}{c}30 \\
(45.5 \%)\end{array}$ & $19.6 \%$ & $\begin{array}{l}\text { Trumpeter Swan, Harlequin Duck, Common } \\
\text { Loon, Horned Grebe, Clark's Grebe, American } \\
\text { White Pelican, American Bittern, Great Blue } \\
\text { Heron, Black-crowned Night-Heron, White-faced } \\
\text { Ibis, Yellow Rail, Whooping Crane (E), Piping } \\
\text { Plover (T), Black-necked Stilt, Franklin's Gull, } \\
\text { Least Tern (E), Caspian Tern, Black Tern, } \\
\text { Common Tern, Forster's Tern, Yellow-billed } \\
\text { Cuckoo (C), Black-billed Cuckoo, Black Swift, } \\
\text { Lewis's Woodpecker, Red-headed Woodpecker, } \\
\text { Alder Flycatcher, Sedge Wren, Veery, LeConte's } \\
\text { Sparrow, Nelson's Sparrow }\end{array}$ \\
\hline $\begin{array}{l}\text { Shrub and } \\
\text { riparian }\end{array}$ & 2 & $\begin{array}{c}2 \\
(0.8 \%) \\
\end{array}$ & & & $0 \%$ & \\
\hline Generalists & 15 & $\begin{array}{c}13 \\
(5.0 \%) \\
\end{array}$ & 2 & & $0 \%$ & \\
\hline $\begin{array}{l}\text { Open } \\
\text { habitat }\end{array}$ & 6 & $\begin{array}{c}5 \\
(1.9 \%) \\
\end{array}$ & 3 & & $0 \%$ & \\
\hline $\begin{array}{l}\text { Woody } \\
\text { draws }\end{array}$ & 4 & $\begin{array}{c}2 \\
(0.8 \%) \\
\end{array}$ & & & $0 \%$ & \\
\hline TOTAL & 336 & 260 & 77 & 66 & & \\
\hline
\end{tabular}




\section{Appendix L. Contributors to this Edition}

More than 10,000 records

Steve Gniadek, Paul Hendricks, Harriet Marble, and Gary Swant

From 5000 to 9999 records

Charles Carlson, Coburn L. Currier, Georgia Frazier, Brett Gullett, Wayne Harper, Denver Holt, Susan Lenard, Mike Lesnik, David Lockman, Chris Paige, Dwain Prellwitz, Susan Reel, and Jim Sparks

\section{0 to 4999 records}

Ellie and Larry Akins, John Ashley, Eric Atkinson, Bobby Baker, Lewis Barnett, Clifton Barry, Dwight Bergeron, Sharon Browder, John Carlson, Daniel Casey, Phyllis Casperson, Lynn Clark, Mary CroweCostello, Jill Davies, Gordon Dicus, Steve Dinsmore, Kristi DuBois, Kirk Eakin, Deborah Goslin, John Grensten, Ed Harper, S.W. Haywood, Kathryn Hicks, Chris Hoff, Pamela Hunt, Mitch Hurt, S.T. Hyland, Radd Icenoggle, Barbara Jaquith, Danielle Anne Jones, Nate Kohler, Larry Langstaff, David Laufenberg, Janene Lichtenberg, Elizabeth Madden, Jeff Marks, Stephen Martin, Bryce Maxell, Tracy McCreery, Jennifer Nagy, Ted Nordhagen, Barb Pitman, Caleb Putnam, Eric Rasmussen, Ryan Rauscher, Jeremy Roberts, James Rogers, William Roney, Scott Ross, Don Sasse, Don Skaar, Henning Stabins, Kate Stone, Jim Story, Karen Stutzman, Daniel Sullivan, Terry Toppins, Dale Tribby, Ramona Kay Turnbull, Kent Undlin, Lynn Verlanic, Virginia Vincent, Jim Waldo, David Waller, Jeffrey Warren, Wayne Weber, Robin Wolcott, and Stephanie Wood

\section{0 to 999 records}

Dan Bachen, Ellen Baker, Jason Bivens, Edward Boyd, Bob Bramblett, James Brown, Durel Carstensen, Rab Cummings, David DeSante, Ronan Donovan, Debra Elwood, David Ely, Robert Eng, Gina Gahagan, Stephanie Gillin, John Gobeille, Pat Grantham, Kristina Gunderson, Andrew Guttenberg, Nick Hajdukovich, Philip Henson, Elizabeth Hill, Judy Hoy, Joe Johnson, Jon King, Frederick Klasner, Kathleen Knaphus, Craig Knowles, Marisa Lipsey, Lisa Lister, Bill Long, Robert Lubbers, Curtis Lund, Terry McEneaney, Jay McGowan, Kris McLean, Guy McWethy, Janet Millard, Heather Nenninger, Matthew Ocko, Patricia O'Connor, Tonja Opperman, Ellison Orcutt, John Prange, Adam Quinn, Beverly Skinner, Douglas Stotz, Brian Sullivan, Jay Sumner, James Swanson, Ted J. Torgerson, Mike Vennard, Bernard Volet, Dean Waltee, Susan Weller, Michelle Williams, Melissa Wolfe, Richard Yates, and Jock Young

\section{0 to 499 records}

Jan Allen, Michael Andersen, John Anderson, Renner Anderson, Kirk Andrews, Nick and Paula Anich, Frederick Atwood, M. Barrick, Ned and Gigi Batchelder, Dale Becker, Mikael Behrens, Katie Benzel, Jeff Berglund, J. Berner, Jim Berry, Steve Betchkal, Ashley Beyer, Michael Bowen, Mike Boyce, Alan Bragg, Julie Breeden, Aaron Brees, Lou Bruno, Christopher Burney, Hunter Burningham, Geoff Butcher, Nancy Butkovich, Rory Cameron, Richard Cannings, Russell Cannings, Jay Carlisle, Steve Carson, Bruce Carter, Linda Chittum, Carolee Colter, Shilo Comeau, Anne and Kenneth Cook, Carey Cooper, Jacob Cooper, Charlotte Corkran, Patricia Corry, Joshua Covill, Charles Crawford, Mark Crawford, Mark Cress, Bob Crowley, Chad Cyrus, Kelsey Dalton, Steven Dawes, Andre Desrochers, Dave Dickson, Mac R. Donofrio, Arnie Dood, Paul Drescher, Steve Drilling, James Dunn, Peter Dunwiddie, Christopher Eliot, Chris Elphick, Laura Elze, Paulette Epple, Roger Evans, 
Erin Fairbank, Patrick Farmer, Vanessa Fields, John Finnegan, Mike Fisher, John Fitzpatrick, Dennis Flath, Jenny Flesch, Robert Foppe, Tolline Gallagher, Rob Gappert, Maureen Geiger, Barbara Geller, Mel Goff, Daniel Gomez, Andrew Grainger, Bob Green, Jim Greene, Dale Gunderson, Richard Guthrie, Margaret Hahr, Nate Hall, Cliff Halverson, Joe Hanfman, Janet Hardin, Rita Harding, Christopher Harris, Lou Ann Harris, Bob Haynes, Stan Heath, Charles Hedeen, Bernie Hildebrand, Beth Hill, Shannon Hilty, Barton Hoag, Ray Hodson, Robert Hoffmann, Alex Hughes, Heather Hundt, Richard Hutto, Andrew Jakes, Ashley Jensen, August Johnson, Peggy Joki, Cedron Jones, Janice Jorgensen, Thomas Kallmeyer, Adam Kent, Craig Kesselheim, Jack Kirkley, Ethan Kistler, Marvin Krook, Mari Krumwiede, Nancy LaFramboise, Scott Lang, David Lautenbach, Debbie Leick, Jeremy Linden, Kurt Lindsay, Tim Ludwick, Peter Lundberg, Laura Lundby, Geof Lynn, Susan Maclin, Mary Anne Marjamaa, Bob Martinka, William Matthews, Terence McClelland, Andrew McGann, John McKeever, Matt McKim-Louder, Poody McLaughlin, Adam Messer, Annie Meyer, Craig Miller, Mark Mizak, Jim Moore, Karen Moulder, Jennifer Muscha, Lewis Myers, Susan Newell, Dan Nickerson, Johan Nilsson, Tim O’Brien, Marty O’Malley, John Parker, Marcia Parker, Grace Pearsall, Jody Peters, Dick Porter, Paul Prappas, Charles Pregler, Bill Pulliam, Christie Quarles, Barbara Quinlan, Brett Quiring, Michael Rabenberg, Bill Rainey, Larry Rau, Richard Reading, Monte Reder, Tom Reed, James Reichel, Van Remsen, Marco Restani, Kenneth Rieker, Wendy Rihner, Bob Ringler, Greg Risdahl, Ralph Rogers, Forrest Rowland, Jennifer Rycenga, Thomas Sanders, Karla Sartor, Kari Sawyer, Scott Schuette, Sandy Schull, Mary Schvetz, Michael Schwitters, Kimberly Sellers, Nathan Senner, David Shea, Maria Shepherd, Steve Sherman, Kate Shick, Neil Simpson, James Sipiora, Winter Smith, Ty Smucker, John Spencer, Leslie Starr, Andy Stepniewski, Monty Sullins, Brian Sullivan, Mark Sullivan, Shannon Swanson, Pete Swigert, Alex Taft, Lance Tanino, Bryan Tarbox, Graham Taylor, Steve Taylor, Fred Tilly, Steve Ting, Patrick Toomey, Neil Travis, Mark Traxler, Wayne Tree, Van Truan, C. Vince, Martha Vogt, Ben Wagner, Brett Walker, Larry Weeks, Phil Wegener, Herbert Weigl, Hillary Welch, Jackie Weller, Harold Wentland, George West, Chelsey Whenham, Stu and Rynel Wickend, Tate Wilcox, Aubrey Williams, Jason Wilmot, Rob Wingard, Steve Winger, Linda Winnie, Chris Wright, David Wrobleski, Ben Young, Sarah Young, Kathi and J. Kamps Younger, James Zelenak, and Michael Zielinski

\section{1 to 99 records}

More than two thousand individuals contributed from 1 to 99 records; each of these is of value. Space constraints prevent listing every name here, but we extend our THANKS to everyone for contributing their observations and assisting with improving our understanding of bird distributions in Montana.

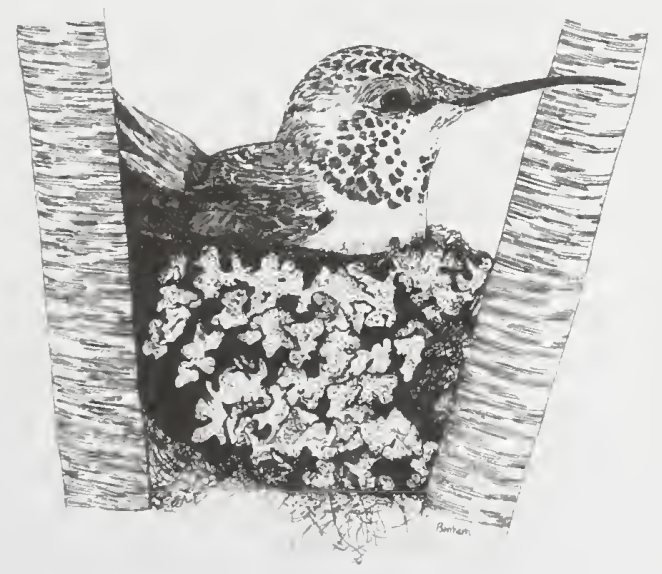


Greater White-fronted Goose

— Snow Goose

_ Ross's Goose

_ Cackling Goose*

_ Canada Goose

- Brant*

Mute Swan

_ Trumpeter Swan

- Tundra Swan

- Wood Duck

_ Gadwall

_ Eurasian Wigeon

_ American Wigeon

- American Black Duck

Mallard

_ Blue-winged Teal

Cinnamon Teal

- Northern Shoveler

Northern Pintail

_ Garganey*

_ Green-winged Teal

- Canvasback

— Redhead

— Ring-necked Duck

— Tufted Duck*

_ Greater Scaup

- Lesser Scaup

_ Harlequin Duck

_ Surf Scoter

— White-winged Scoter

_ Black Scoter*

— Long-tailed Duck

— Bufflehead

_ Common Goldeneye

_ Barrow's Goldeneye

_ Hooded Merganser

- Common Merganser

— Red-breasted Merganser

— Ruddy Duck

- Chukar

— Gray Partridge

_ Ring-necked Pheasant

— Ruffed Grouse

— Greater Sage-Grouse

- Spruce Grouse

— Willow Ptarmigan*

_ White-tailed Ptarmigan

— Dusky Grouse

- Sharp-tailed Grouse

- Greater Prairie-Chicken

— Wild Turkey

_ Red-throated Loon*

- Pacific Loon

- Common Loon

_ Yellow-billed Loon*

_ Pied-billed Grebe

_ Horned Grebe
_ Red-necked Grebe

_ Eared Grebe

- Western Grebe

_ Clark's Grebe

_ Manx Shearwater*

— Wood Stork*

— Double-crested Cormorant

- American White Pelican

_ American Bittern

Least Bittern*

_ Great Blue Heron

_ Great Egret

— Snowy Egret

_ Little Blue Heron*

— Cattle Egret

_ Green Heron*

_ Black-crowned Night-Heron

— Yellow-crowned Night-Heron*

_ Glossy Ibis*

White-faced Ibis

_ Turkey Vulture

— Osprey

White-tailed Kite*

_ Mississippi Kite*

_ Bald Eagle

Northern Harrier

Sharp-shinned Hawk

Cooper's Hawk

— Northern Goshawk

_ Red-shouldered Hawk*

_ Broad-winged Hawk

Swainson's Hawk

Red-tailed Hawk

_ Ferruginous Hawk

Rough-legged Hawk

Golden Eagle

Crested Caracara*

American Kestrel

Merlin

Gyrfalcon

— Peregrine Falcon

Prairie Falcon

Yellow Rail*

_ Virginia Rail

- Sora

_ Common Gallinule*

American Coot

Sandhill Crane

Whooping Crane

Black-bellied Plover

American Golden-Plover

Snowy Plover*

Semipalmated Plover

Piping Plover

Killdeer

Mountain Plover

Black-necked Stilt
American Avocet

Spotted Sandpiper

Solitary Sandpiper

Greater Yellowlegs

Willet

Lesser Yellowlegs

Upland Sandpiper

Whimbrel

Long-billed Curlew

Hudsonian Godwit

Marbled Godwit

Ruddy Turnstone

Black Turnstone*

Red Knot

Sanderling

Semipalmated Sandpiper

Western Sandpiper

Least Sandpiper

White-rumped Sandpiper

Baird's Sandpiper

Pectoral Sandpiper

Sharp-tailed Sandpiper*

Dunlin

Curlew Sandpiper*

Stilt Sandpiper

Buff-breasted Sandpiper

Short-billed Dowitcher

Long-billed Dowitcher

Wilson's Snipe

American Woodcock*

Wilson's Phalarope

Red-necked Phalarope

Red Phalarope*

Black-legged Kitriwake*

Sabine's Gull

Bonaparte's Gull

Little Gull*

_ Ross's Gull*

— Laughing Gull*

— Franklin's Gull

Mew Gull

Ring-billed Gull

California Gull

Herring Gull

— Thayer's Gull

Iceland Gull*

_ Lesser Black-backed Gull*

_ Glaucous-winged Gull*

_ Glaucous Gull

_ Great Black-backed Gull*

- Least Tern

_ Caspian Tern

— Black Tern

— Common Tern

_ Arctic Tern*

— Forster's Tern

_ Pomarine Jaeger* 
Parasitic Jaeger ${ }^{*}$

Long-tailed Jaeger*

Long-billed Murrelet*

Ancient Murrelet*

Rock Pigeon

Band-tailed Pigeon

Eurasian Collared-Dove

White-winged Dove*

Mourning Dove

Inca Dove*

Yellow-billed Cuckoo*

Black-billed Cuckoo

Barn Owl

Flammulated Owl

Western Screech-Owl

Eastern Screech-Owl

Great Horned Owl

Snowy Owl

Northern Hawk Owl

_ Northern Pygmy-Owl

_ Burrowing Owl

Barred Owl

Great Gray Owl

_ Long-eared Owl

_ Short-eared Owl

_ Boreal Owl

Northern Saw-whet Owl

Common Nighthawk

Common Poorwill

Whip-poor-will*

Black Swift

Chimney Swift

Vaux's Swift

White-throated Swift

Ruby-throated Hummingbird*

_ Black-chinned Hummingbird

Anna's Hummingbird*

Costa's Hummingbird*

Calliope Hummingbird

_ Broad-tailed Hummingbird

_ Rufous Hummingbird

— Belted Kingfisher

Lewis's Woodpecker

_ Red-headed Woodpecker

_ Red-bellied Woodpecker*

-Williamson's Sapsucker

_ Yellow-bellied Sapsucker*

_ Red-naped Sapsucker

_ Downy Woodpecker

_ Hairy Woodipecker

_ White-headed Woodpecker*

_ American Three-toed Woodpecker

Black-backed Woodpecker

— Northern Flicker

_ Pileated Woodpecker

_ Olive-sided Flycatcher

_ Western Wood-Pewee

_ Eastern Wood-Pewee*

_ Yellow-bellied Flycatcher ${ }^{*}$
Alder Flycatcher

Willow Flycatcher

_ Least Flycatcher

— Hammond's Flycatcher

Gray Flycatcher*

Dusky Flycatcher

Cordilleran Flycatcher

Eastern Phoebe*

Say's Phoebe

Vermilion Flycatcher*

_ Ash-throated Flycatcher*

_ Great Crested Flycatcher*

Cassin's Kingbird

Western Kingbird

Eastern Kingbird

Scissor-tailed Flycatcher*

Loggerhead Shrike

Northern Shrike

White-eyed Vireo*

Yellow-throated Vireo*

Plumbeous Vireo

Cassin's Vireo

Blue-headed Vireo*

Warbling Vireo

Philadelphia Vireo*

Red-eyed Vireo

Gray Jay

Pinyon Jay

Steller's Jay

Blue Jay

Western Scrub-Jay*

- Clark's Nutcracker

_ Black-billed Magpie

- American Crow

Common Raven

Horned Lark

Purple Martin

- Tree Swallow

_ Violet-green Swallow

_ Northern Rough-winged Swallow Bank Swallow

_ Cliff Swa!low

Barn Swallow

_ Black-capped Chickadee

- Mountain Chickadee

_ Chestnut-backed Chickadee

- Boreal Chickadee

_ Red-breasted Nuthatch

_. White-breasted Nuthatch

_ Pygmy Nuthatch

_ Brown Creeper

_ Rock Wren

- Canyon Wren

_ Carolina Wren*

— Bewick's Wren*

_ House Wren

_ Pacific Wren

— Winter Wren*

— Sedge Wren*
Marsh Wren

Blue-gray Gnatcatcher*

American Dipper

_ Golden-crowned Kinglet

Ruby-crowned Kinglet

Eastern Bluebird

Western Bluebird

- Mountain Bluebird

— Townsend's Solitaire

Veery

_ Gray-cheeked Thrush*

Swainson's Thrush

_ Hermit Thrush

_ Wood Thrush*

- American Robin

_ Varied Thrush

_ Gray Catbird

— Northern Mockingbird

- Sage Thrasher

- Brown Thrasher

_ Curve-billed Thrasher*

European Starling

Siberian Accentor*

- American Pipit

Sprague's Pipit

- Bohemian Waxwing

- Cedar Waxwing

_ Lapland Longspur

_ Chestnut-collared Longspur

Smith's Longspur*

— McCown's Longspur

_ Snow Bunting

_ Ovenbird

_ Northern Waterthrush

_ Golden-winged Warbler*

_ Blue-winged Warbler*

— Black-and-white Warbler

— Prothonotary Warbler*

— Tennessee Warbler

_ Orange-crowned Warbler

Nashville Warbler

_ Connecticut Warbler*

- MacGillivray's Warbler

_ Mourning Warbler

_ Kentucky Warbler*

— Common Yellowthroat

_ Hooded Warbler*

American Redstart

_ Cape May Warbler

- Northern Parula*

_ Magnolia Warbler

_ Bay-breasted Warbler*

- Blackburnian Warbler*

_ Yellow Warbler

- Chestnut-sided Warbler

_ Blackpoll Warbler

_ Black-throated Blue Warbler

_ Palm Warbler

— Pine Warbler* 
Yellow-rumped Warbler

Yellow-throated Warbler*

Prairie Warbler*

Black-throated Gray Warbler*

Townsend's Warbler

Black-throated Green Warbler*

Canada Warbler

Wilson's Warbler

_ Painted Redstart* Yellow-breasted Chat

Green-tailed Towhee

- Spotted Towhee

_ Eastern Towhee*

_ American Tree Sparrow

_ Chipping Sparrow

_. Clay-colored Sparrow

_ Brewer's Sparrow

_ Field Sparrow

_ Vesper Sparrow

_ Lark Sparrow

_ Black-throated Sparrow*

_ Sage Sparrow*

_ Lark Bunting

_ Savannah Sparrow

_ Grasshopper Sparrow

_ Baird's Sparrow

_ Le Conte's Sparrow
_ Nelson's Sparrow

Fox Sparrow

_ Song Sparrow

_ Lincoln's Sparrow

Swamp Sparrow

White-throated Sparrow

_ Harris's Sparrow

White-crowned Sparrow

_ Golden-crowned Sparrow*

Dark-eyed Junco

_ Summer Tanager*

_ Scarlet Tanager*

_ Western Tanager

_ Northern Cardinal*

_ Pyrrhuloxia*

Rose-breasted Grosbeak

_ Black-headed Grosbeak

_ Blue Grosbeak*

_ Lazuli Bunting

_ Indigo Bunting

_ Painted Bunting*

_ Dickcissel

Bobolink

_ Red-winged Blackbird

Eastern Meadowlark*

— Western Meadowlark

— Yellow-headed Blackbird
Rusty Blackbird

_ Brewer's Blackbird

Common Grackle

_ Great-tailed Grackle*

_ Brown-headed Cowbird

- Orchard Oriole

_ Hooded Oriole*

_ Bullock's Oriole

_ Baltimore Oriole

_ Brambling*

_ Gray-crowned Rosy-Finch

Black Rosy-Finch

Pine Grosbeak

Purple Finch

Cassin's Finch

House Finch

Red Crossbill

White-winged Crossbill

Common Redpoll

Hoary Redpoll

Pine Siskin

Lesser Goldfinch

American Goldfinch

Evening Grosbeak

House Sparrow

* Rare species in Montana

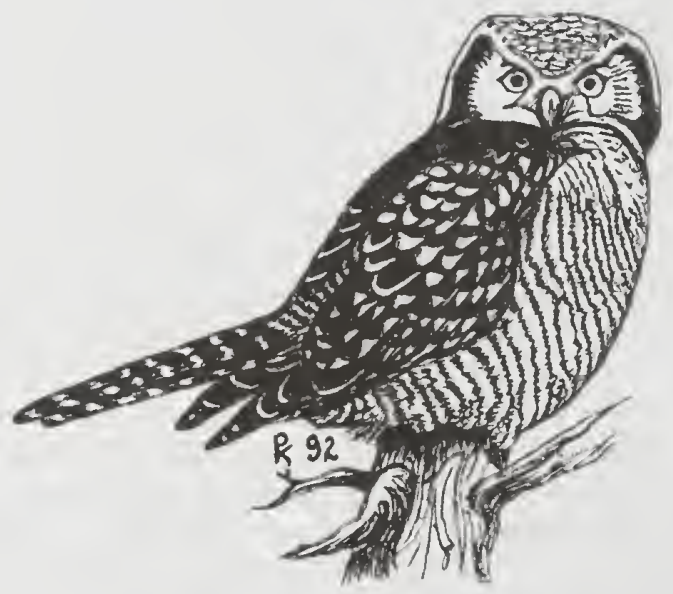




\section{References}

American Ornithologists' Union (AOU). 1998. Check-list of North American Birds, 7th ed. American Ornithologists' Union, Washington, D.C.

AOU. 2003. Forty-fourth supplement to the American Ornithologists' Union Check-list of North American birds. Auk 119:923-931.

AOU. 2007. Forty-eighth supplement to the American Ornithologists' Union Check-list of North American birds. Auk 124:1109-1115.

AOU. 2008. Forty-ninth supplement to the American Ornithologists' Union Check-list of North American birds. Auk 125:758-768.

AOU. 2009. Fiftieth supplement to the American Ornithologists' Union Check-list of North American birds. Auk 126:705-714.

AOU. 2010. Fifty-first supplement to the American Ornithologists' Union Check-list of North American birds. Auk 127:726-744.

AOU. 2011. Fifty-second supplement to the American Ornithologists' Union Check-list of North American birds. Auk 128:600-613.

Bergeron, D., C. Jones, D.L. Genter, and D. Sullivan. 1992. P.D. Skaar's Montana Bird Distribution, 4th Edition. Special Publication No. 2. Montana Natural Heritage Program, Helena, MT.

DeLorme. 2011. Montana Atlas \& Gazetteer, 8th Edition. DeLorme Publishing, Yarmouth, ME.

Lenard, S., J. Carlson, J. Ellis, C. Jones, and C. Tilly. 2003. P.D. Skaar's Montana Bird Distribution, 6th Edition. Montana Audubon, Helena, MT.

Montana Bird Distribution Committee. 1996. P.D. Skaar's Montana Bird Distribution, 5th Edition. Special Publication No. 3. Montana Natural Heritage Program, Helena, MT.

Saunders, A.A. 1921. A distributional list of the birds of Montana: With notes on the migration and nesting of the better known species. Pacific Coast Avifauna No. 14.

Skaar, D., D. Flath, and L.S. Thompson. 1985. Montana Bird Distribution, 3rd Edition. Montana Academy of Sciences, Monograph No. 3.

Skaar, P.D. 1969. Birds of the Bozeman Latilong. Published by author, Bozeman, MT.

Skaar, P.D. 1975. Montana Bird Distribution, 1st Edition. Published by author, Bozeman, MT.

Skaar, P.D. 1980. Montana Bird Distribution, 2nd Edition. Published by author, Bozeman, MT.

Wright, P. L. 1996. Status of rare birds in Montana, with comments on known hybrids. Northwestern Naturalist 77:57-85. 


\section{Index}

A

Accentor, Siberian, 126

Avocet, American, 54

B

Bittern

American, 36

Least, 36

Blackbird

Brewer's, 162

Red-winged, 160

Rusty, 161

Yellow-headed, 161

Bluebird

Eastern, 120

Mountain, 121

Western, 120

Bobolink, 159

Brambling, 165

Brant, 14

Bufflehead, 24

Bunting

Indigo, 158

Lark, 149

Lazuli, 158

Painted, 159

Snow, 129

C

Canvasback, 21

Caracara, Crested, 46

Cardinal, Northern, 156

Catbird, Gray, 124

Chat, Yellow-breasted, 143

Chickadee

Black-capped, 112

Boreal, 113

Chestnut-backed, 113

Mountain, 113

Chukar, 27

Collared-Dove, Eurasian, 76

Coot, American, 50

Cormorant, Double-crested, 35

Cowbird, Brown-headed, 163

Crane

Sandhill, 50

Whooping, 51

Creeper, Brown, 115

Crossbill

Red, 167

White-winged, 168
Crow, American, 109

Cuckoo

Black-billed, 78

Yellow-billed, 78

Curlew, Long-billed, 57

D

Dickcissel, 159

Dipper, American, 119

Dove

Inca, 77

Mourning, 77

White-winged, 77

Dowitcher

Long-billed, 63

Short-billed, 63

Duck

American Black, 17

Harlequin, 23

Long-tailed, 24

Ring-necked, 21

Ruddy, 26

Tufted, 22

Wood, 16

Dunlin, 62

E

Eagle

Bald, 42

Golden, 46

Egret

Cattle, 38

Great, 37

Snowy, 38

F

Falcon

Peregrine, 48

Prairie, 48

Finch

Cassin's, 167

House, 167

Purple, 166

Flicker

Northern, 94

Red-shafted, 94

Yellow-shafted, 95

Flycatcher

Alder, 97

Ash-throated, 100

Cordilleran, 99
Dusky, 98

Gray, 98

Great Crested, 100

Hammond's, 98

Least, 97

Olive-sided, 95

Scissor-tailed, 102

Vermilion, 100

Willow, 97

Yellow-bellied, 96

G

Gadwall, 16

Gallinule, Common, 50

Garganey, 19

Gnatcatcher, Blue-gray, 118

Godwit

Hudsonian, 57

Marbled, 58

Goldeneye

Barrow's, 25

Common, 25

Golden-Plover

American, 51

Goldfinch

American, 170

Lesser, 169

Goose

Cackling, 14

Canada, 14

Greater White-fronted, 13

Ross's, 13

Snow, 13

Goshawk, Northern, 44

Grackle

Common, 162

Great-tailed, 162

Grebe

Clark's, 34

Eared, 34

Horned, 33

Pied-billed, 33

Red-necked, 33

Western, 34

Grosbeak

Black-headed, 157

Blue, 158

Evening, 170

Pine, 166

Rose-breasted, 157 
Grouse

Dusky, 29

Ruffed, 28

Sharp-tailed, 30

Spruce, 28

Gull

Bonaparte's, 66

California, 69

Franklin's, 68

Glaucous, 71

Glaucous-winged, 71

Great Black-backed, 71

Herring, 69

Iceland, 70

Laughing, 68

Lesser Black-backed, 70

Little, 67

Mew, 68

Ring-billed, 69

Ross's, 67

Sabine's, 66

Thayer's, 70

Gyrfalcon, 47

$\mathrm{H}$

Harrier, Northern, 42

Hawk

Broad-winged, 44

Cooper's, 43

Ferruginous, 45

Red-shouldered, 44

Red-tailed, 45

Rough-legged, 46

Sharp-shinned, 43

Swainson's, 45

Heron

Great Blue, 37

Green, 39

Little Blue, 38

Hummingbird

Anna's, 87

Black-chinned, 87

Broad-tailed, 89

Calliope, 88

Costa’s, 88

Ruby-throated, 87

Rufous, 89

$$
\text { I }
$$

Ibis

Glossy, 40

White-faced, 40
J

Jaeger

Long-tailed, 75

Parasitic, 74

Pomarine, 74

Jay

Blue, 107

Gray, 106

Pinyon, 107

Steller's, 107

Junco

Dark-eyed, 153

Gray-headed, 155

Oregon (Montana), 154

Pink-sided, 154

Slate-colored, 154

White-winged, 155

K

Kestrel, American, 47

Killdeer, 53

Kingbird

Cassin's, 101

Eastern, 101

Western, 101

Kingfisher, Belted, 89

Kinglet

Golden-crowned, 119

Ruby-crowned, 120

Kite

Mississippi, 42

White-tailed, 41

Kittiwake, Black-legged, 66

Knot, Red, 59

L

Lark, Horned, 109

Longspur

Chestnut-collared, 128

Lapland, 127

McCown's, 129

Smith's, 128

Loon

Common, 32

Pacific, 31

Red-throated, 31

Yellow-billed, 32

M

Magpie, Black-billed, 108

Mallard, 18

Martin, Purple, 110
Meadowlark

Eastern, 160

Western, 160

Merganser

Common, 26

Hooded, 25

Red-breasted, 26

Merlin, 47

Mockingbird, Northern, 124

Murrelet

Ancient, 75

Long-billed, 75

N

Nighthawk, Common, 84

Night-Heron

Black-crowned, 39

Yellow-crowned, 39

Nutcracker, Clark's, 108

Nuthatch

Pygmy, 114

Red-breasted, 114

White-breasted, 114

O

Oriole

Baltimore, 164

Bullock's, 164

Hooded, 163

Northern, 165

Orchard, 163

Osprey, 41

Ovenbird, 129

Owl

Barn, 79

Barred, 82

Boreal, 83

Burrowing, 82

Flammulated, 79

Great Gray, 82

Great Horned, 80

Long-eared, 83

Northern Hawk, 81

Northern Saw-whet, 84

Short-eared, 83

Snowy, 80

P

Partridge, Gray, 27

Parula, Northern, 135

Pelican, American White, 36

Phalarope

Red, 65 
Red-necked, 65

Wilson's, 65

Pheasant, Ring-necked, 27

Phoebe

Eastern, 99

Say's, 99

Pigeon

Band-tailed, 76

Rock, 76

Pintail, Northern, 19

Pipit

American, 126

Sprague's, 126

Plover

Black-bellied, 51

Mountain, 53

Piping, 52

Semipalmated, 52

Snowy, 52

Poorwill, Common, 84

Prairie-Chicken, Greater, 30

Ptarmigan

White-tailed, 29

Willow, 29

Pygmy-Owl, Northern, 81

Pyrrhuloxia, 157

\section{$\mathrm{R}$}

Rail

Virginia, 49

Yellow, 48

Raven, Common, 109

Redhead, 21

Redpoll

Common, 168

Hoary, 169

Redstart

American, 135

Painted, 143

Robin, American, 123

Rosy-Finch

Black, 166

Gray-crowned, 165

$S$

Sage-Grouse, Greater, 28

Sanderling, 59

Sandpiper

Baird's, 61

Buff-breasted, 63

Curlew, 62

Least, 60

Pectoral, 61
Semipalmated, 59

Sharp-tailed, 61

Solitary, 55

Spotted, 54

Stilt, 62

Upland, 56

Western, 60

White-rumped, 60

Sapsucker

Red-naped, 91

Williamson's, 91

Yellow-bellied, 91

Scaup

Greater, 22

Lesser, 22

Scoter

Black, 24

Surf, 23

White-winged, 23

Screech-Owl

Eastern, 80

Western, 79

Scrub-Jay, Western, 108

Shearwater, Manx, 35

Shoveler, Northern, 19

Shrike

Loggerhead, 102

Northern, 103

Siskin, Pine, 169

Snipe, Wilson's, 64

Solitaire, Townsend's, 121

Sora, 49

Sparrow

American Tree, 145

Baird's, 150

Black-throated, 148

Brewer's, 146

Timberline, 146

Chipping, 145

Clay-colored, 146

Field, 147

Fox, 151

Golden-crowned, 153

Grasshopper, 149

Harris's, 152

House, 171

Lark, 148

Le Conte's, 150

Lincoln's, 151

Nelson's, 150

Sage, 148

Savannah, 149

Song, 151
Swamp, 152

Vesper, 147

White-crowned, 153

White-throated, 152

Starling, European, 125

Stilt, Black-necked, 54

Stork, Wood, 35

Swallow

Bank, 111

Barn, 112

Cliff, 111

Northern Rough-winged, 111

Tree, 110

Violet-green, 110

Swan

Mute, 15

Trumpeter, 15

Tundra, 16

Swift

Black, 85

Chimney, 85

Vaux's, 86

White-throated, 86

$\mathrm{T}$

Tanager

Scarlet, 156

Summer, 155

Western, 156

Teal

Blue-winged, 18

Cinnamon, 18

Green-winged, 20 Tern

Eurasian Green-winged, 20

Arctic, 73

Black, 72

Caspian, 72

Common, 73

Forster's, 74

Least, 72

Thrasher

Brown, 125

Curve-billed, 125

Sage, 124

Thrush

Gray-cheeked, 122

Hermit, 122

Swainson's, 122

Varied, 123

Wood, 123 
Towhee

Eastern, 144

Green-tailed, 144

Spotted, 144

Turkey, Wild, 31

Turnstone

Black, 58

Ruddy, 58

$\mathrm{V}$

Veery, 121

Vireo

Blue-headed, 104

Cassin's, 104

Philadelphia, 105

Plumbeous, 104

Red-eyed, 106

Solitary, 105

Warbling, 105

White-eyed, 103

Yellow-throated, 103

Vulture, Turkey, 40

W

Warbler

Bay-breasted, 136

Black-and-white, 131

Blackburnian, 136

Blackpoll, 138

Black-throated Blue, 138

Black-throated Gray, 141

Black-throated Green, 142

Blue-winged, 130

Canada, 142

Cape May, 135

Chestnut-sided, 137

Connecticut, 133

Golden-winged, 130

Hooded, 134

Kentucky, 134

MacGillivray's, 133

Magnolia, 136

Mourning, 133

Nashville, 132

Orange-crowned, 132

Palm, 139

Pine, 139

Prairie, 141

Prothonotary, 131

Tennessee, 132

Townsend's, 141

Wilson's, 142

Yellow, 137
Yellow-rumped, 139

Audubon's, 140

Myrtle, 140

Yellow-throated, 140

Waterthrush, Northern, 130

Waxwing

Bohemian, 127

Cedar, 127

Whimbrel, 56

Whip-poor-will, 85

Wigeon

American, 17

Eurasian, 17

Willet, 55

Woodcock, American, 64

Woodpecker

American Three-toed, 93

Black-backed, 93

Downy, 92

Hairy, 92

Lewis's, 90

Pileated, 95

Red-bellied, 90

Red-headed, 90

White-headed, 93

Wood-Pewee

Eastern, 96

Western, 96

Wren

Bewick's, 116

Canyon, 115

Carolina, 116

House, 116

Marsh, 118

Pacific, 117

Rock, 115

Sedge, 118

Winter, 117

Y
Yellowlegs
Greater, 55
Lesser, 56
Yellowthroat, Common, 134

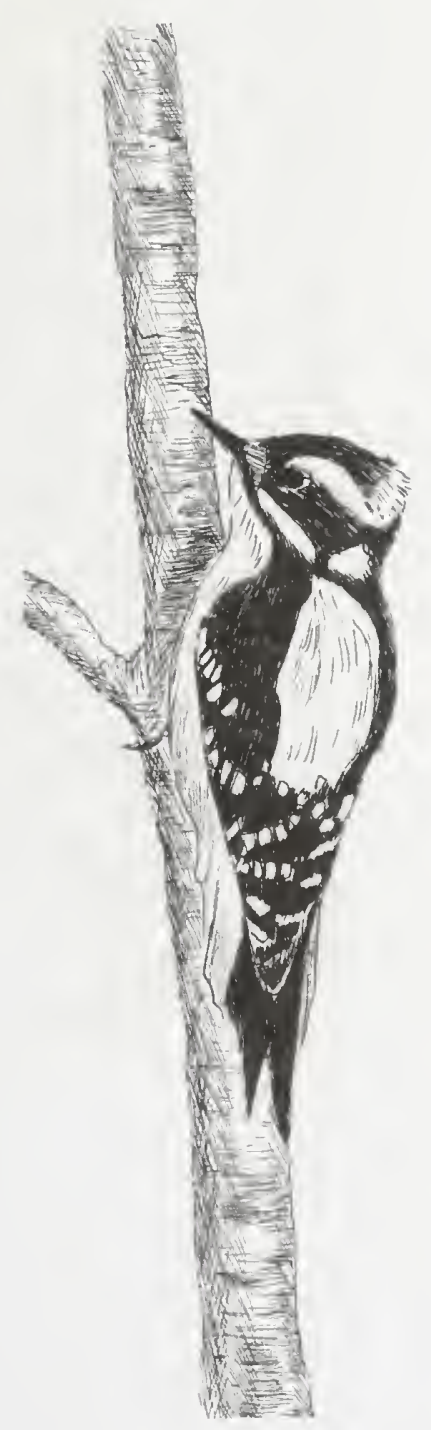




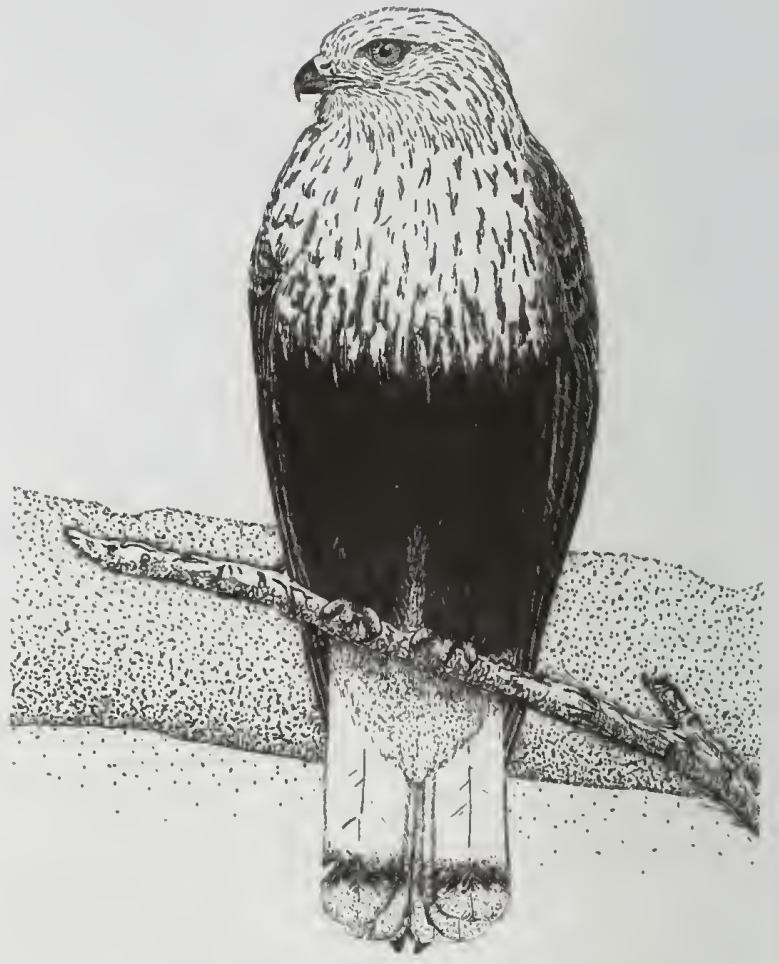


Travel Maps

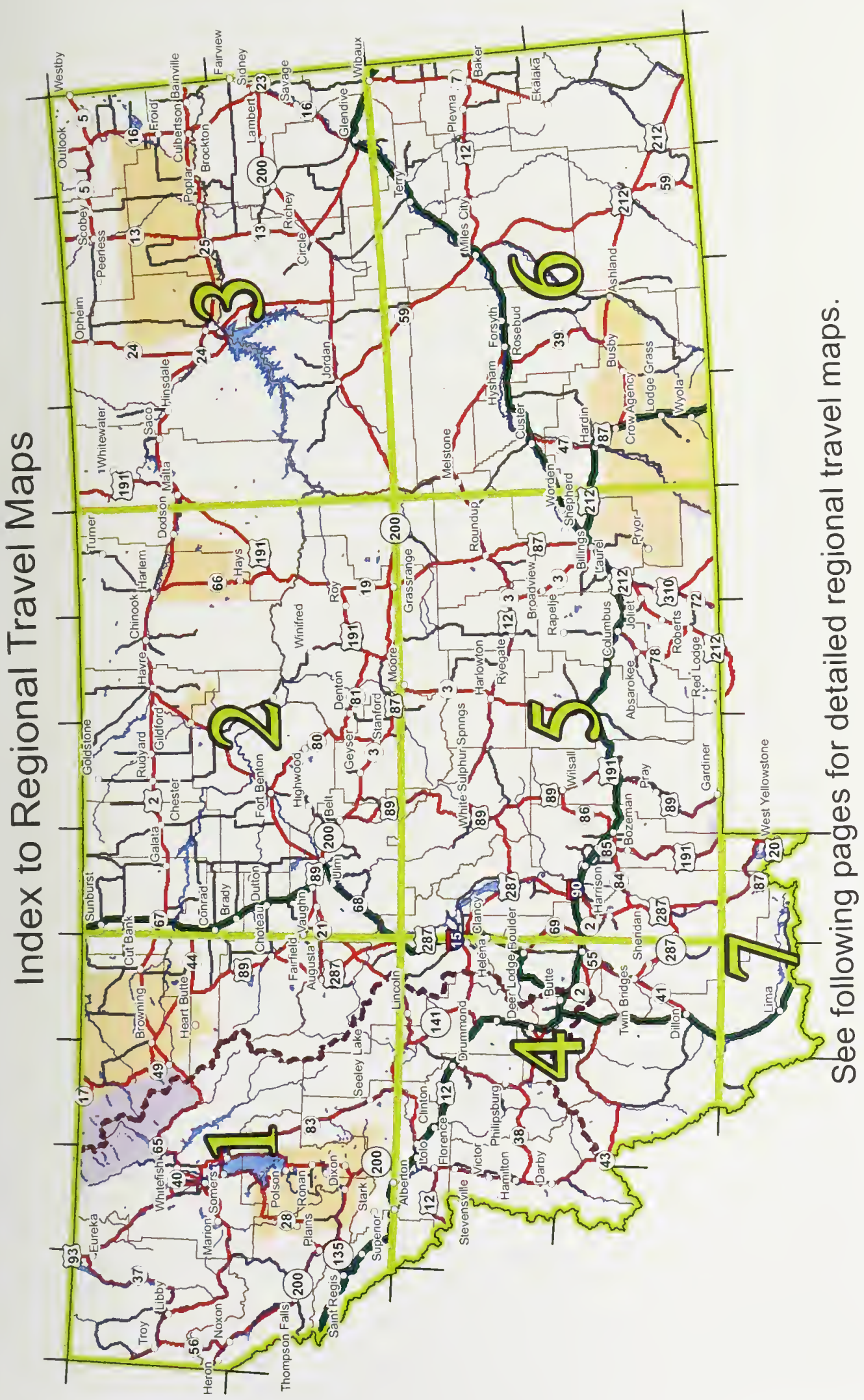




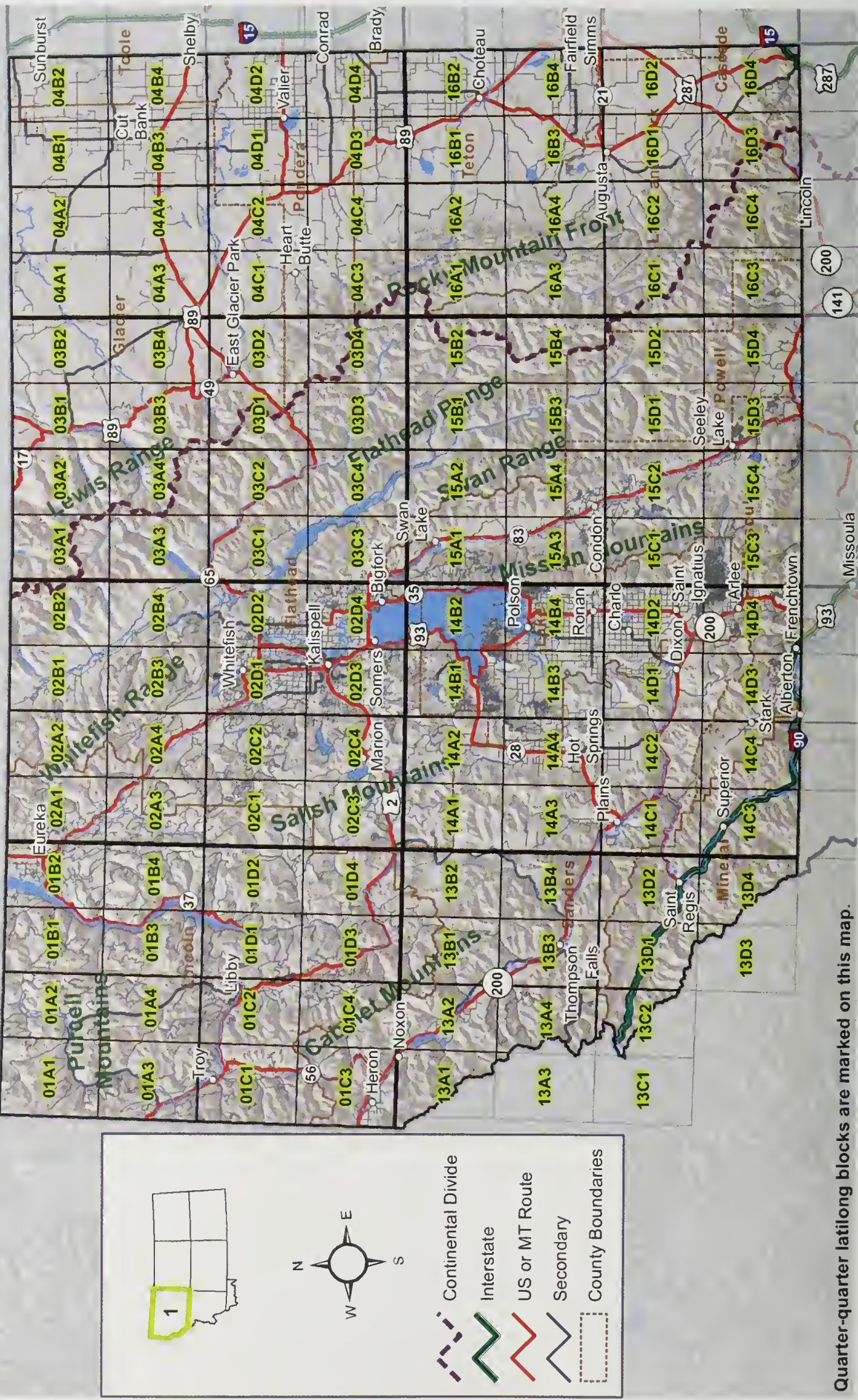




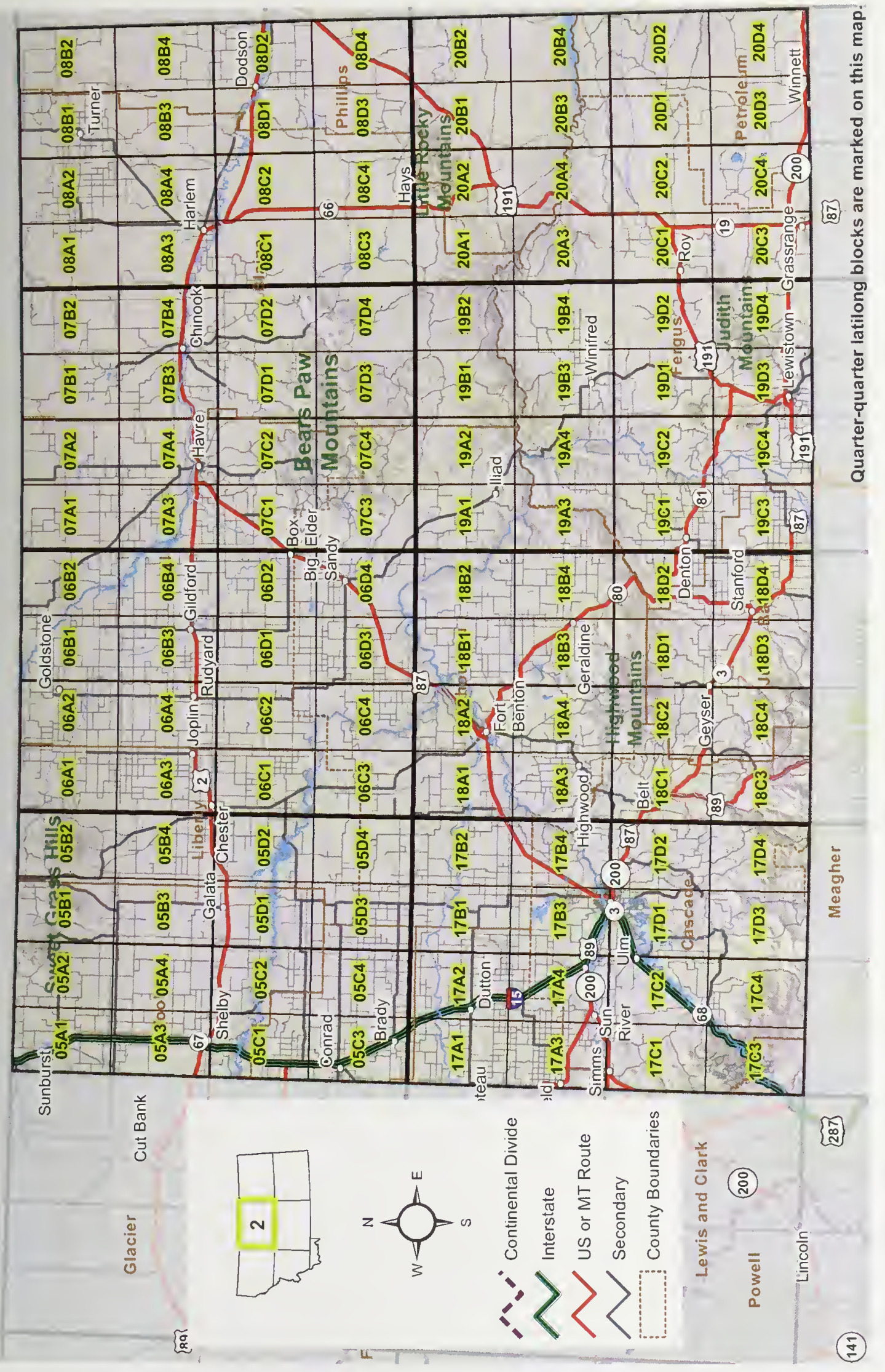




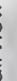

क

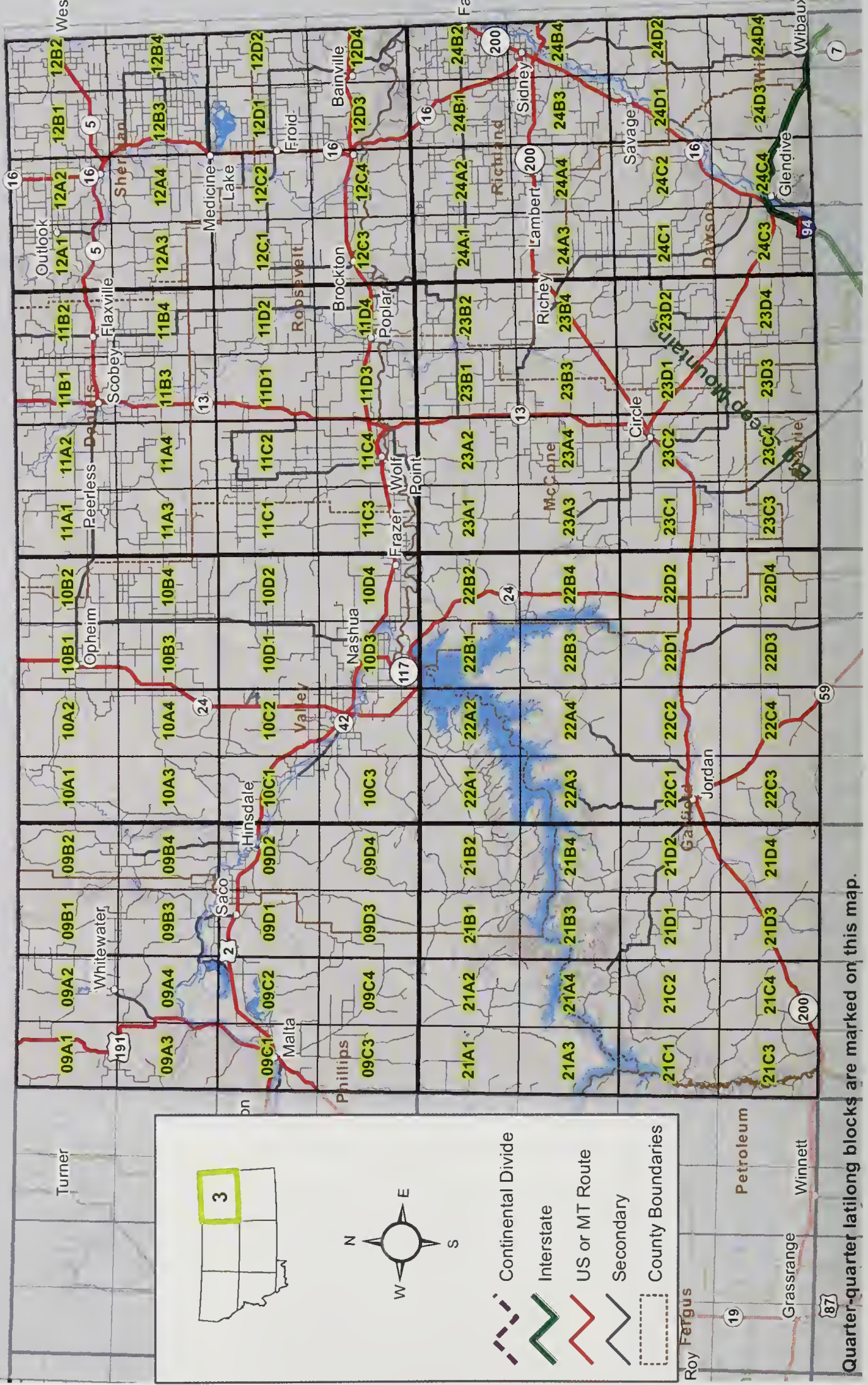




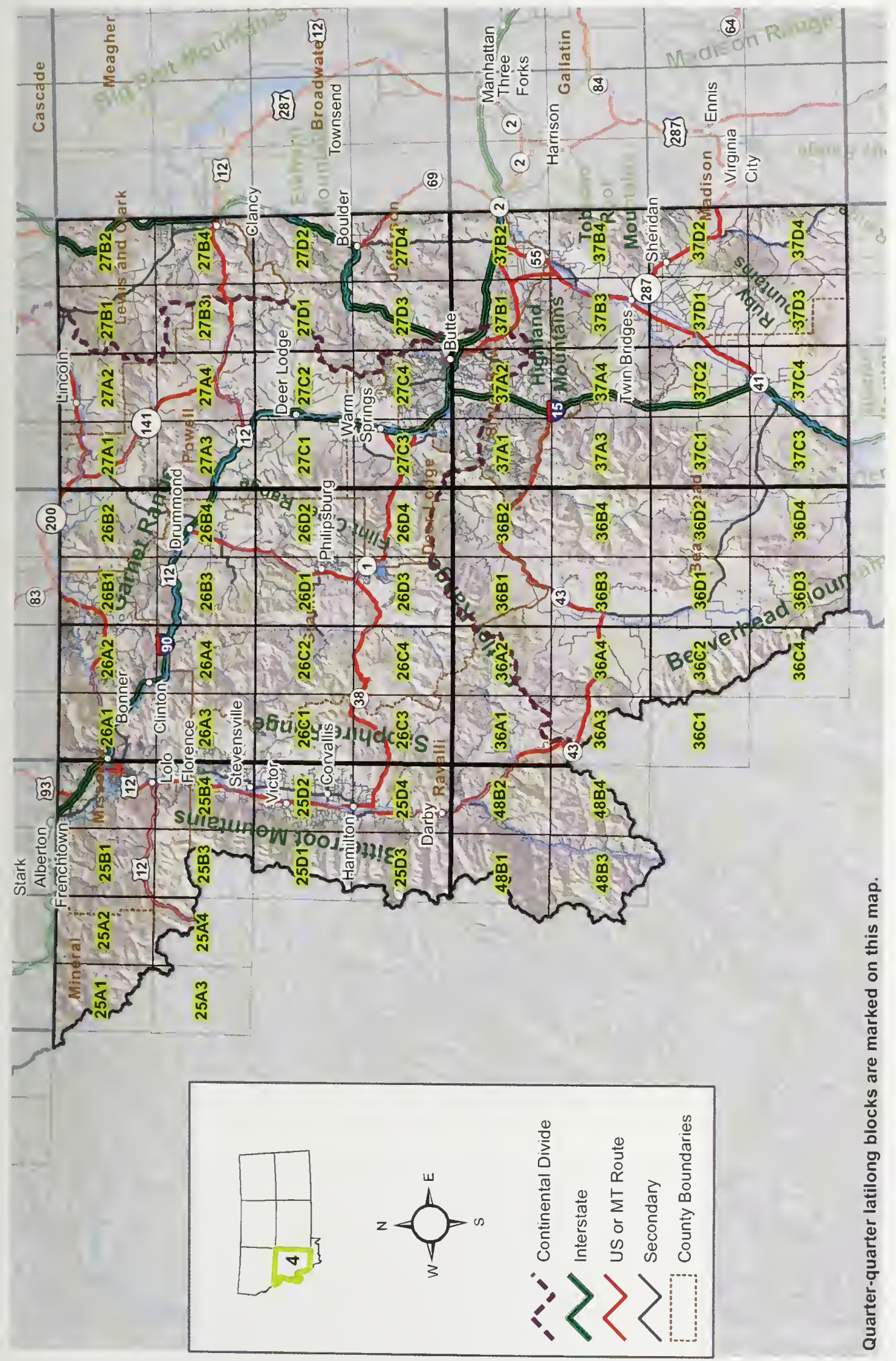




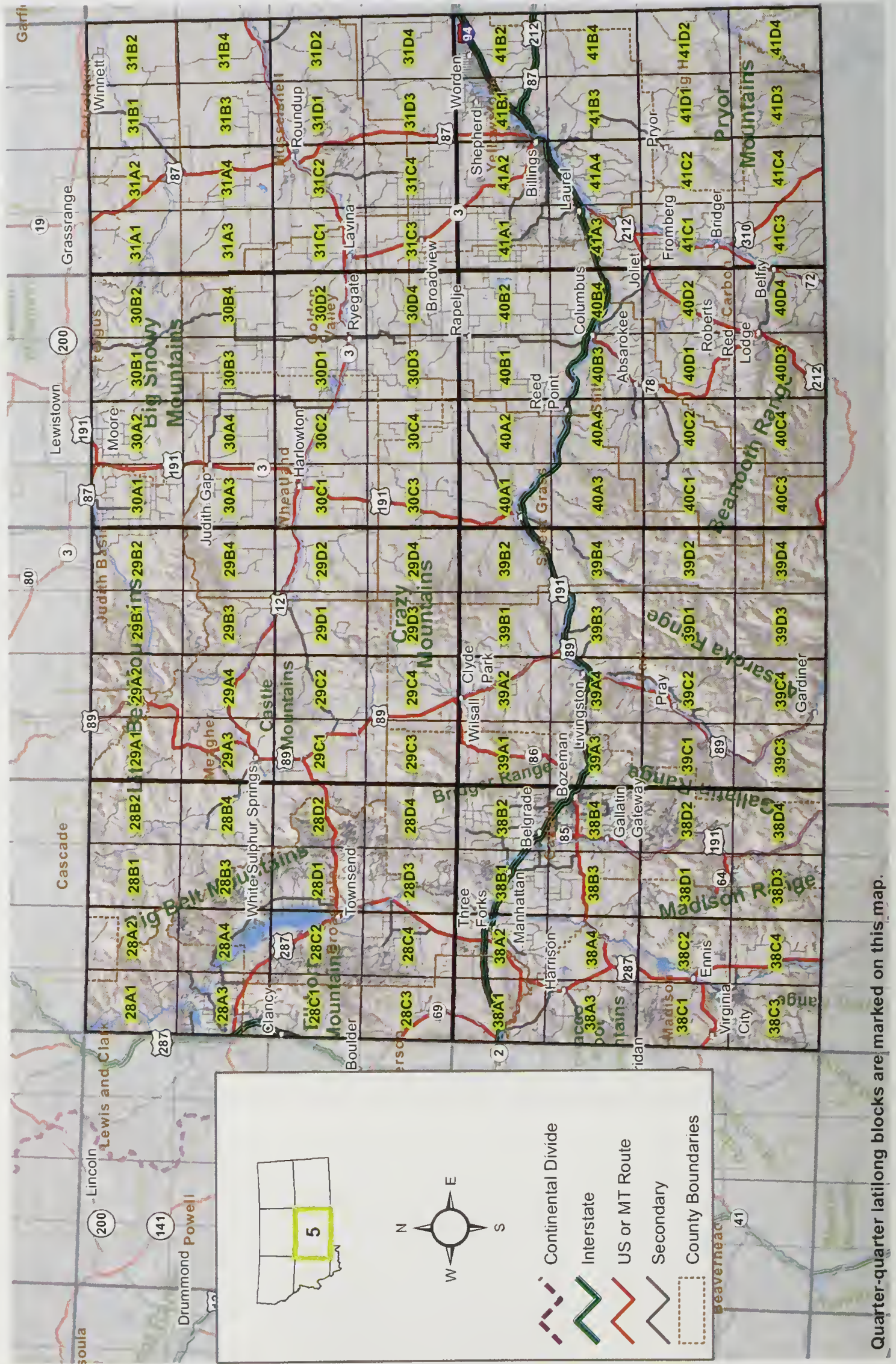




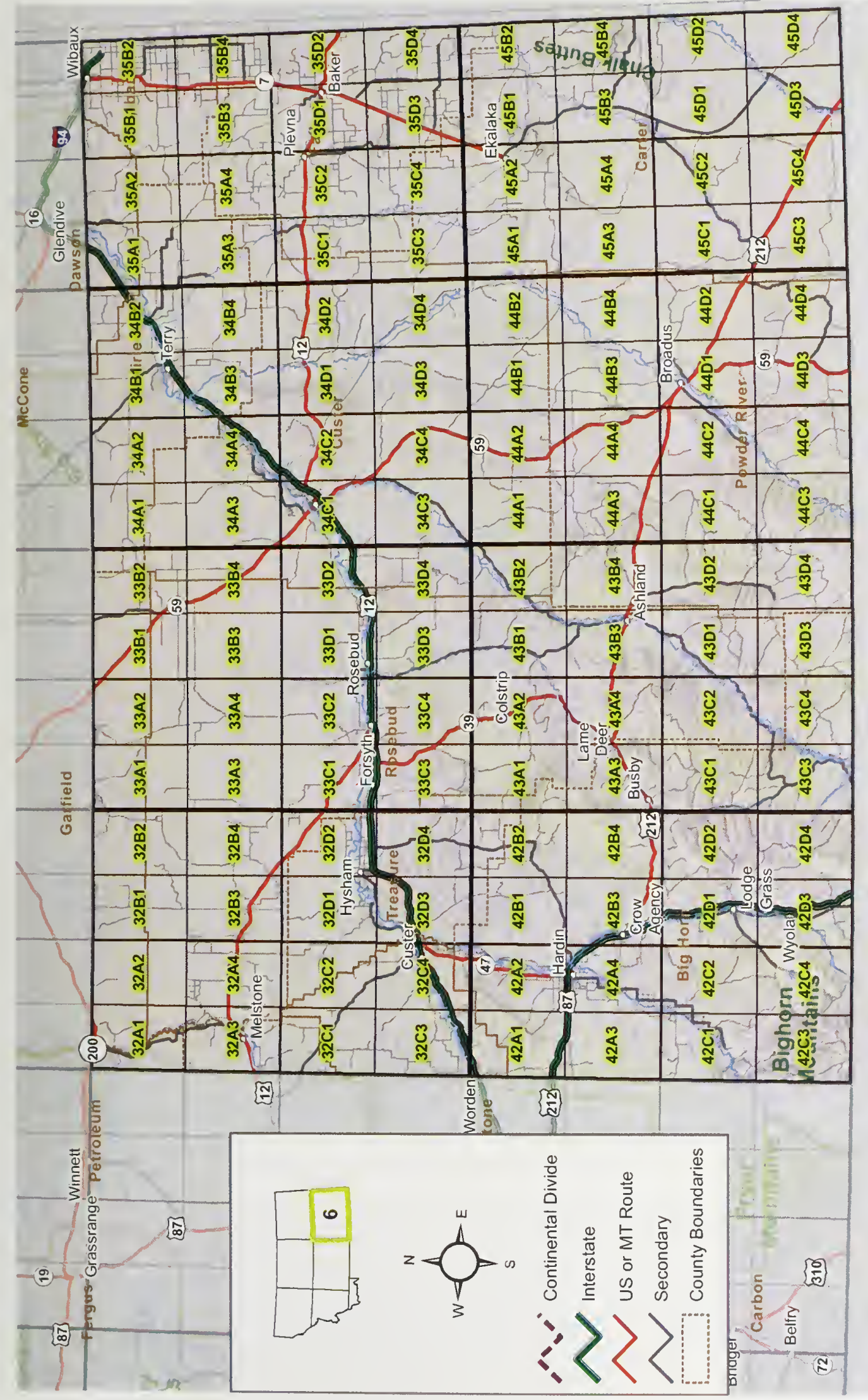

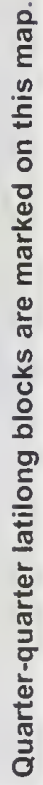




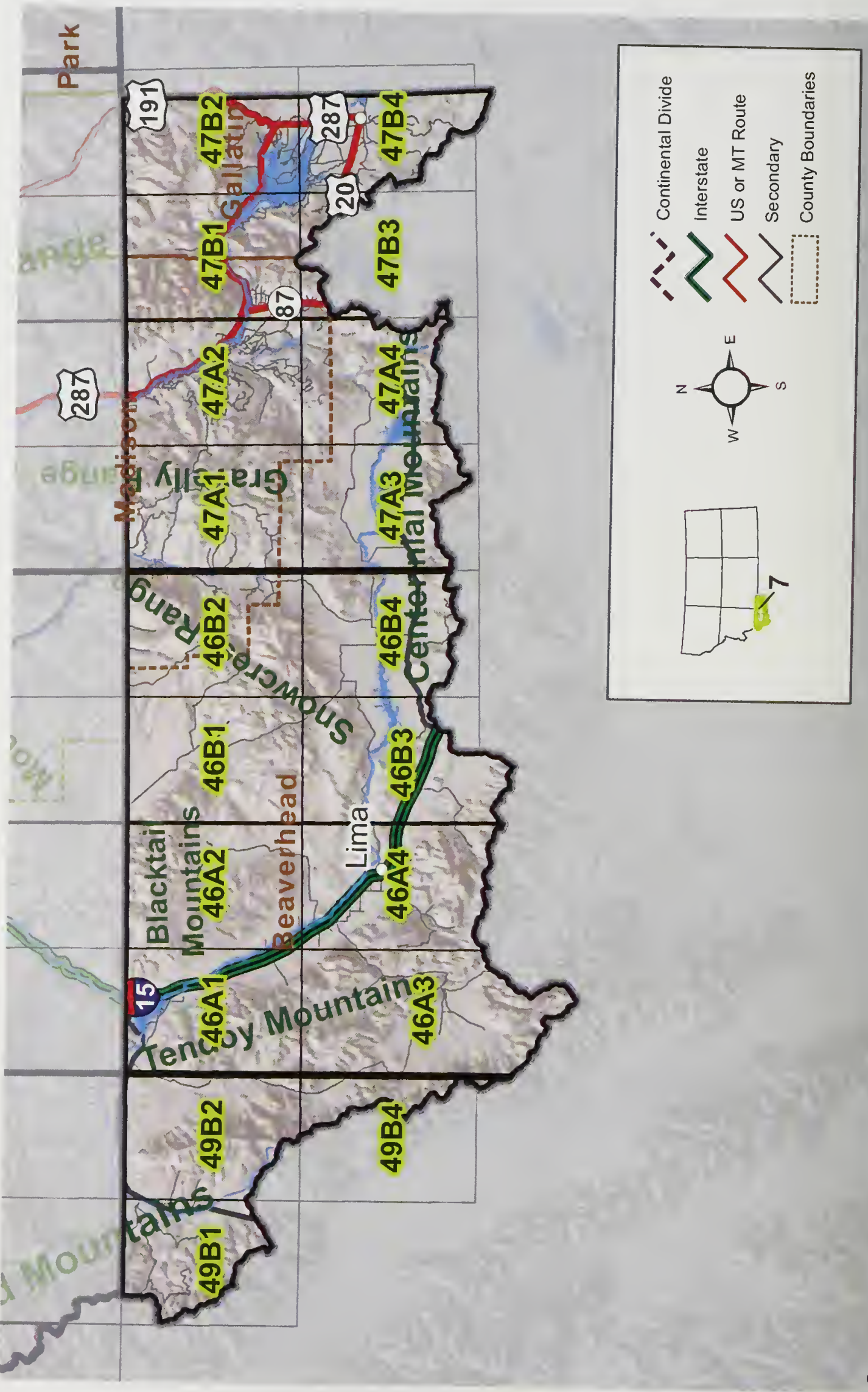

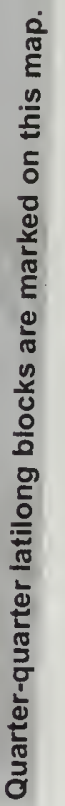




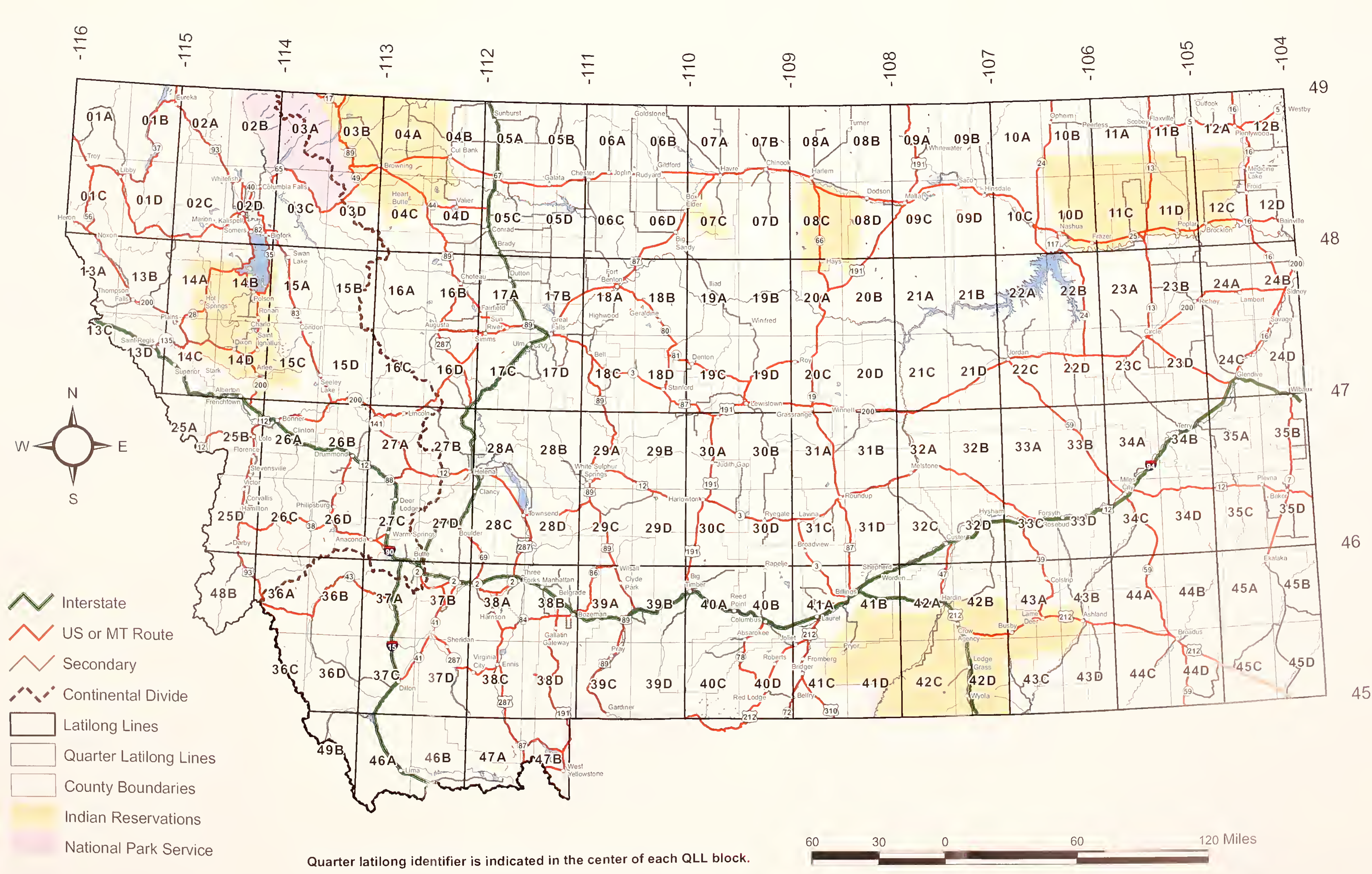




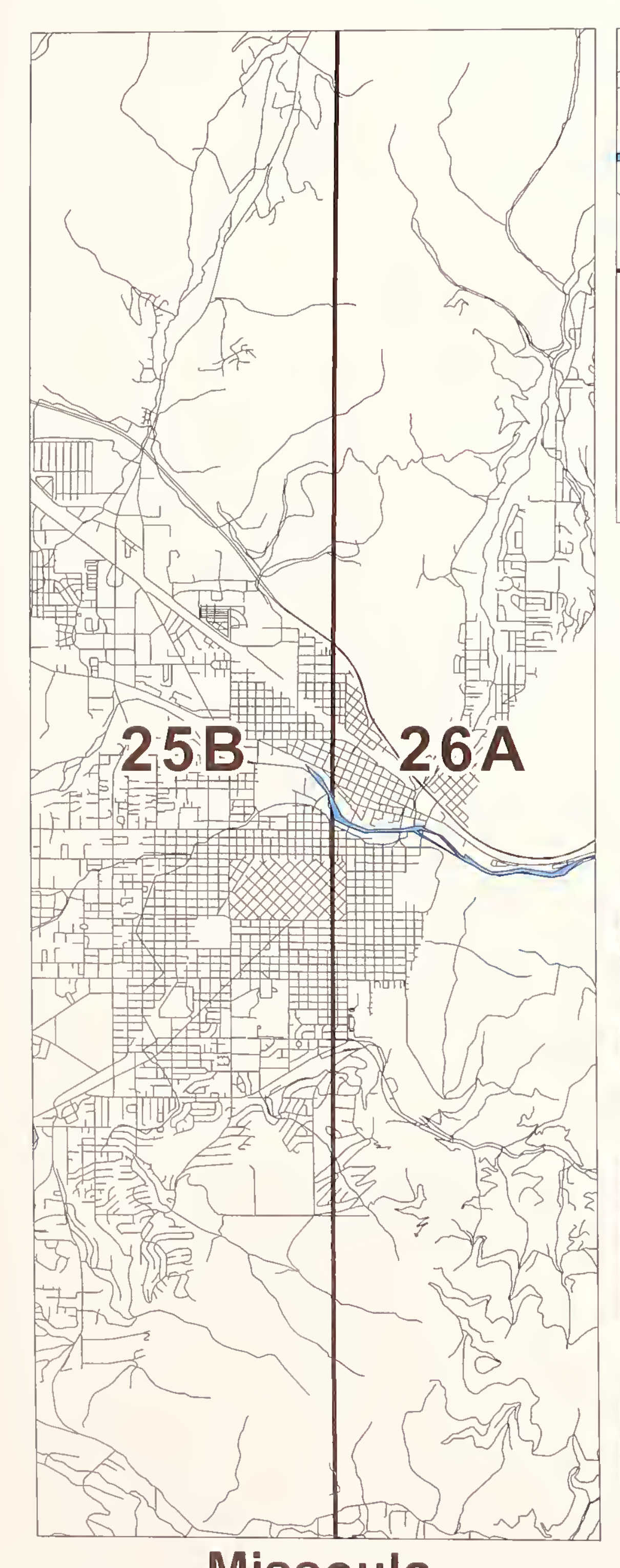

Great Falls

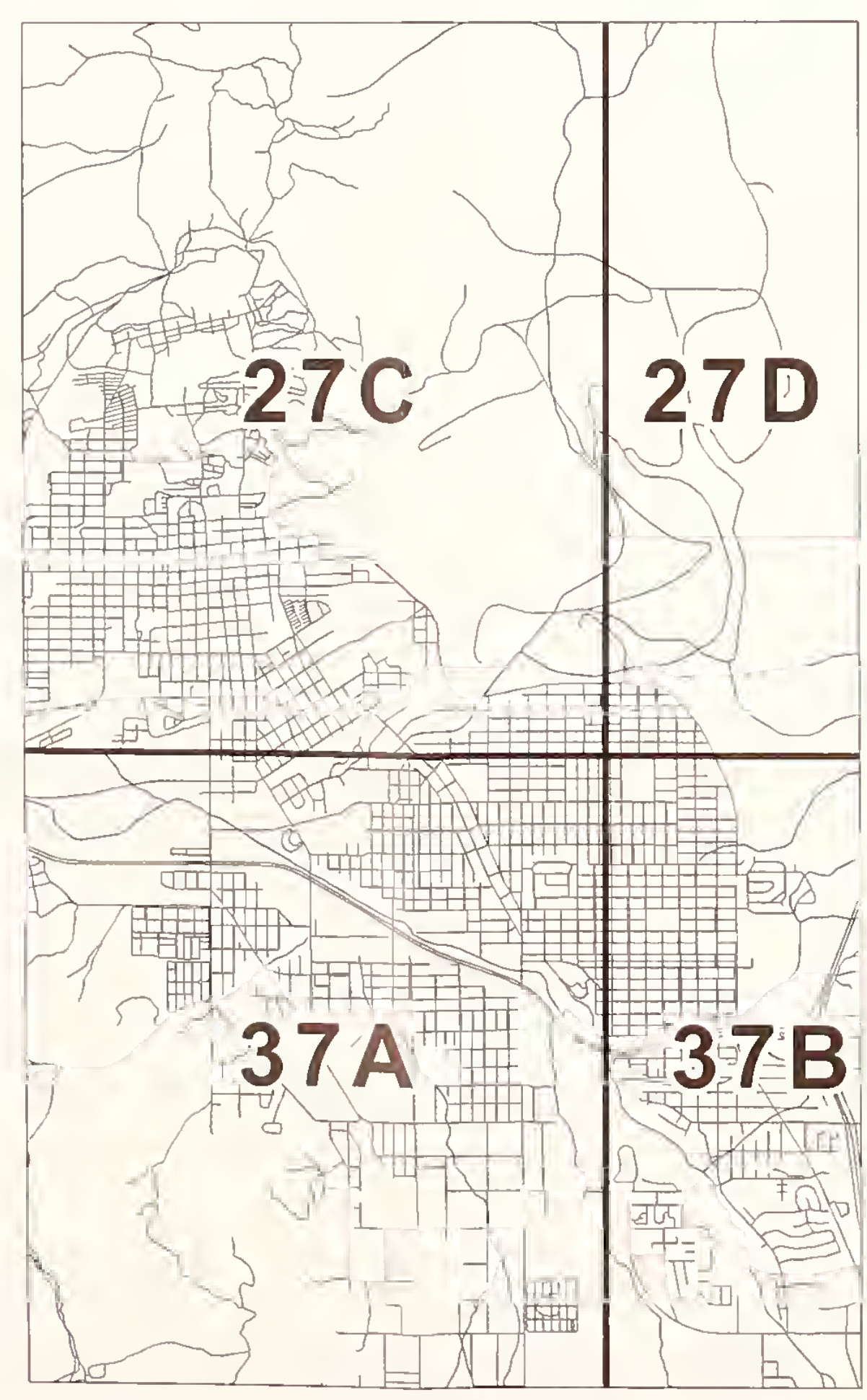

Butte
17B4

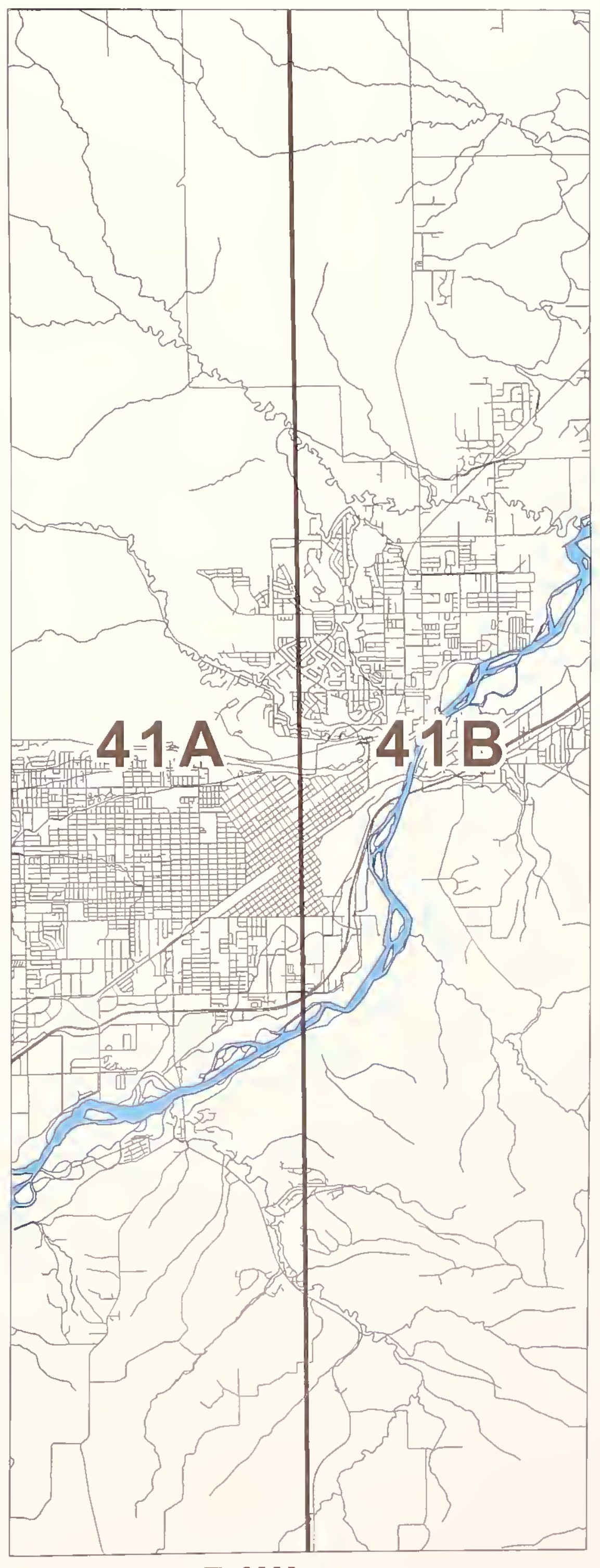

Billings 


\section{Legend}

For a complete legend and detailed descriptions of each legend item, see pages $10-12$.

B Direct evidence of breeding.

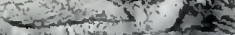

$-2 x^{2}+3 x-3=$

Fint $=0-25$

Mission Mountain

Audubon Society

TheNature Conservancy

Protecting nature. Preserving life."
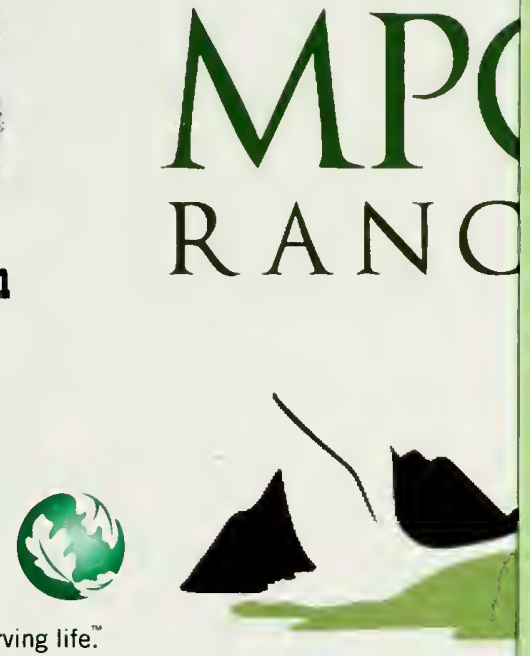

INTERMOUNTAIN V JOINT VENTURE

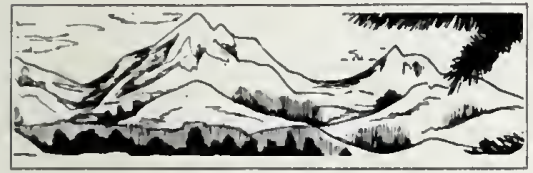

Pintler Audubon Society

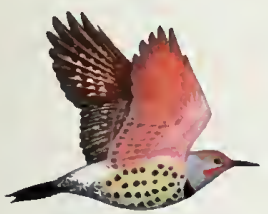

Sacajawea Audubon Society

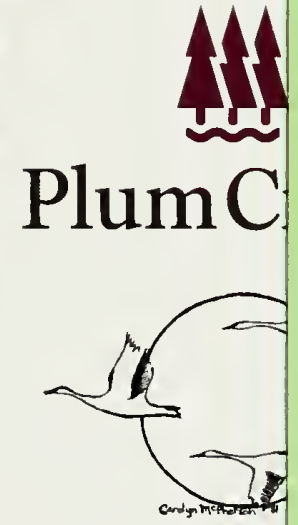

Upper Missouri Audubon Sc
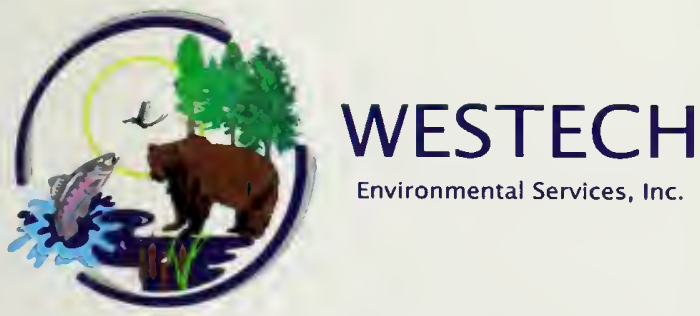

Environmental Services, Inc.

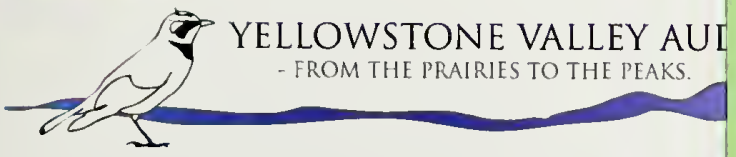

Observation records for years $1991-2002$ for $\mathbf{B}$.

b Indirect evidence of breeding.

Observation records for years $1991-2002$ for $\mathbf{b}$.

t No evidence of breeding, but observed between 16 February and 14 December.

Observation records for years $1991-2002$ for $t$.

Latilongs where the species was reported prior to 1991 and not since.

Q QLL where the species was reported prior to 1991 and not since.

W Overwintering; regular sightings between 15 December and 15 February.

Cumulative historical information for W.

w Observed during the wintering season (15 December to 15 February), but not confirmed as overwintering.

Cumulative historical information for $w$.

2003-2011 = total number of new sightings submitted for this edition.

T E C Threatened, endangered, or candidate species.

SOC Species of Concern

SI Species of Interest

$\mathrm{R} 1 / 2 / 3 \quad$ Rare bird status 


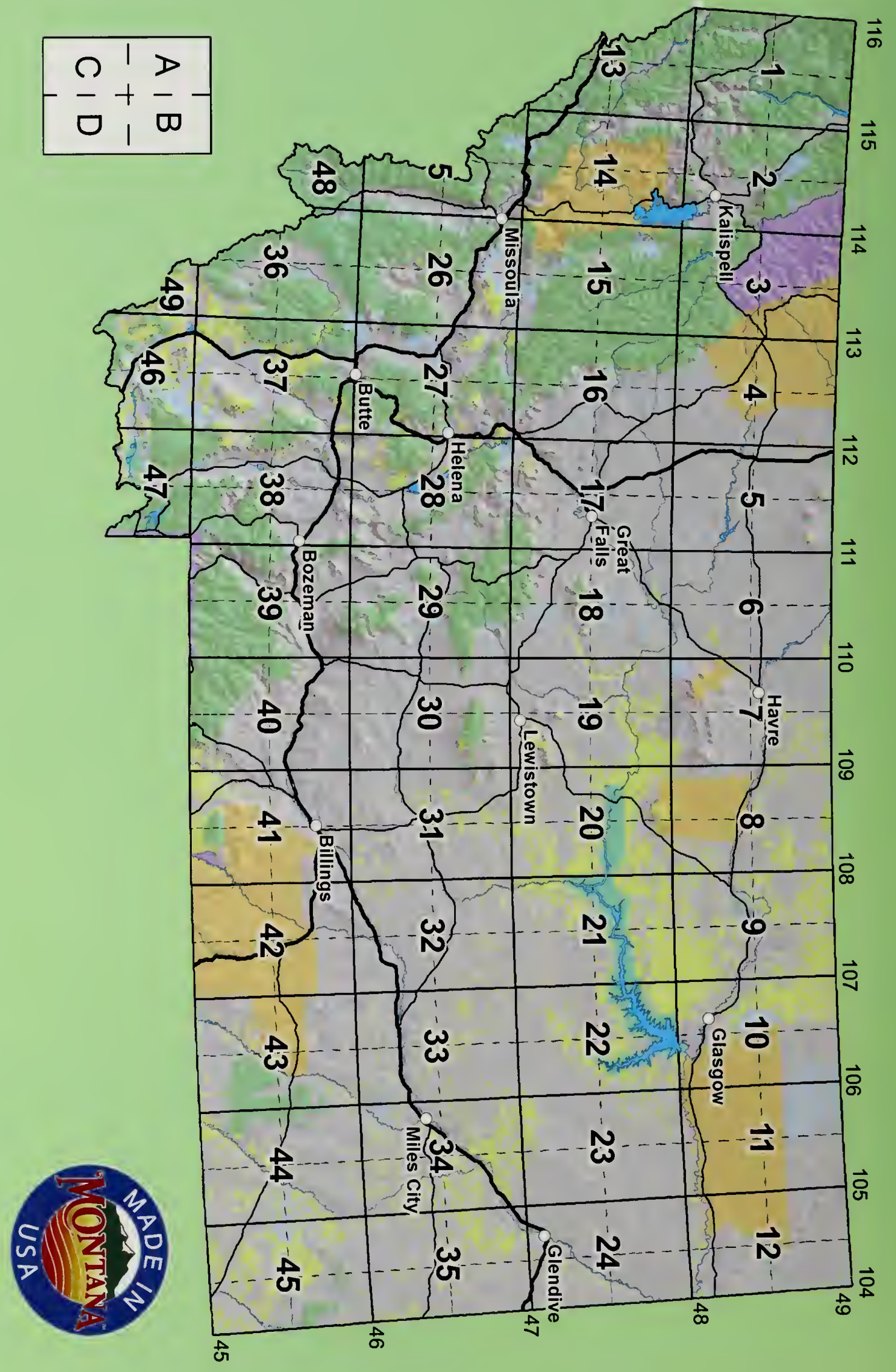

